EXPERIMENTAL STUDY OF RING-SHAPED STEEL PLATE SHEAR WALLS

Natalia Egorova

Thesis submitted to the faculty of the Virginia Polytechnic Institute and State University in partial fulfillment of the requirements for the degree of

Master of Science

in

Civil Engineering

\author{
Matthew R. Eatherton, Committee Chair \\ Finley A. Charney \\ Cristopher D. Moen
}

April 29, 2013

Blacksburg, Virginia

Keywords: steel plate shear wall, ring fuse, hysteretic behavior, plate buckling, seismic design, and experimentation

Copyright (C) 2013 by Natalia Egorova 


\title{
EXPERIMENTAL STUDY OF RING-SHAPED STEEL PLATE SHEAR WALLS
}

\author{
Natalia Egorova
}

\section{ABSTRACT}

A new type of steel plate shear wall has been devised which resists out-of-plane buckling without requiring stiffeners. The ring-shaped steel plate shear wall (RS-SPSW) includes a web plate that is cut with a pattern of holes leaving ring-shaped portions of steel connected by diagonal links. The ring shape resists out-of-plane buckling through the mechanics of how a circular ring deforms into an ellipse. It has been shown that the ring's compression diagonal will shorten a similar amount as the tension diagonal elongates, essentially eliminating the slack in the direction perpendicular to the tension field. Because of the unique features of the ring's mode of distortion, the load-deformation response of the resulting RS-SPSW system can exhibit full hysteretic behavior and possess greatly improved stiffness relative to thin unstiffened SPSW. The concept has been validated through testing on seven 34 in $\times 34$ in panels. General conclusions about influence of different geometric parameters on plate behavior have been made. 


\section{AKNOWLEDGEMENTS}

"If you want to build a ship, don't drum up the people to gather wood, divide up the work, and give orders. Instead, teach them to yearn for the vast and endless sea." Antoine de Saint-Exupery, French poet.

Spending two years in Virginia Tech and working on the research was a great, difficult and interesting time. First of all, I would like to thank a person who gave me the biggest support - my advisor Dr. Eatherton for his help and advise, patience and time during all that period. Thank you to Dr. Moen and Dr. Charney for being wonderful professors, for all the knowledge I earned from classes they teach, and for the feedback about my research. I am grateful to my classmates and labmates for their constant willingness to help and share their experience with me.

I would never be able to do my studies in Virginia Tech if not Fulbright scholarship, thank you for giving me such a unique opportunity to work with best professors and students and to know the world better.

I gratefully acknowledge support from the American Institute of Steel Construction though the Milek Faculty Fellowship Program. Thanks to Michael Wood, Abhilasha Maurya, Adam Phillips, Chris Galitz and Stuart Salmon for their contribution to this work.

At the last and special thank goes to my family and friends who always support and inspire me during all periods of my life. 


\section{TABLE OF CONTENTS}

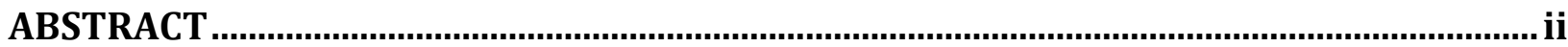

AKNOWLEDGEMENTS .....................................................................................................ii

LIST OF FIGURES

LIST OF TABLES............................................................................................................

1. INTRODUCTION

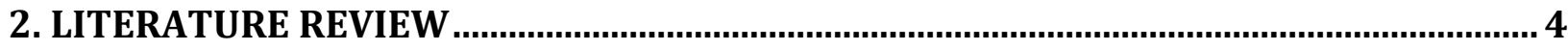

2.1. Experimental studies of solid SPSW ............................................................................

2.2. Experimental studies of perforated SPSW ……............................................................

2.3 Computational study of RS-SPSW …………….................................................................

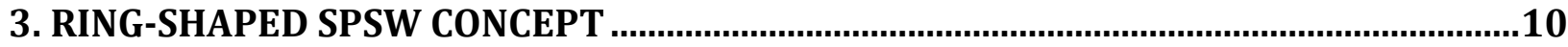

4. DEVELOPING A METHOD TO LIMIT LINK YIELDING ......................................................13

5. TESTING

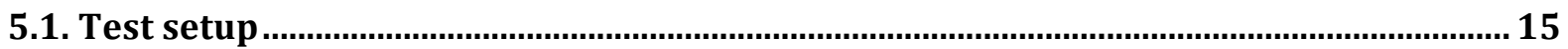

5.2. Loading protocol .......................................................................................................... 19

5.3. Specimen design ...................................................................................................... 23

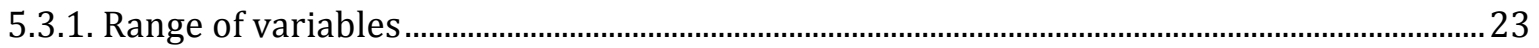

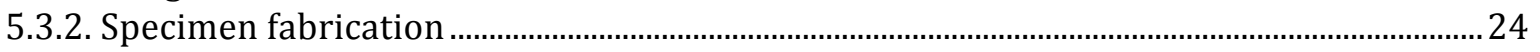

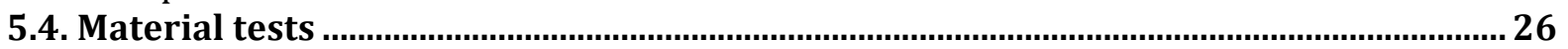

5.4.1. General .....................................................................................................................................26

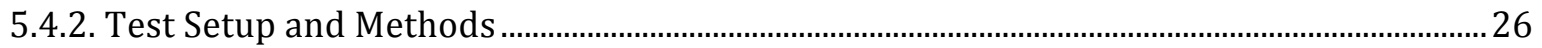

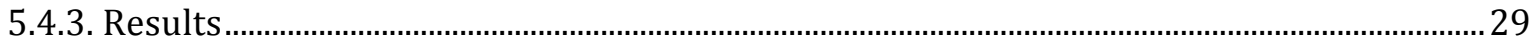

5.5. Data Acquisition, Instrumentation, and Associated Equipment .................................... 31

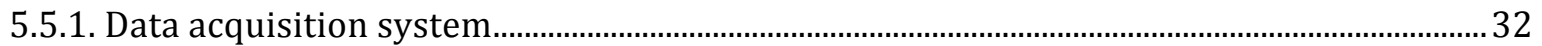

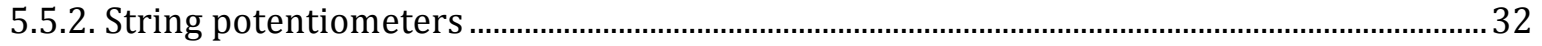

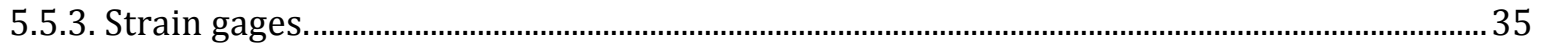

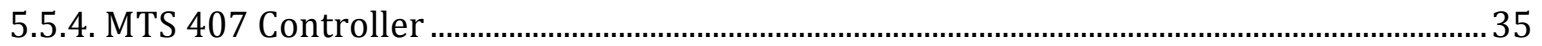

5.5.5. MTS Hydraulic Actuator ............................................................................................................ 35

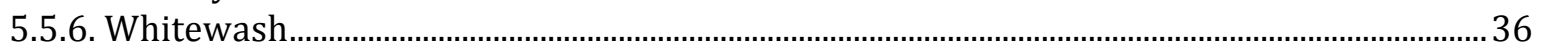

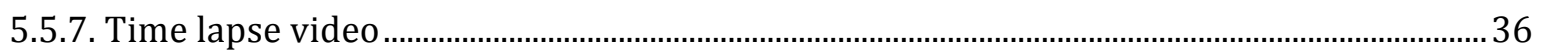

5.5.8. Photogrammetry ……………………………………………………………………………………...

6. TEST RESULTS

6.1. TEST 1 - Specimen 2-0.5-1 ………………………………………………….... 45

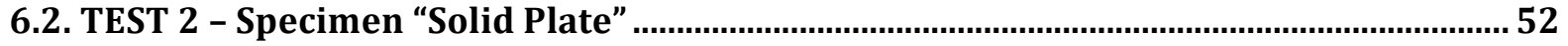

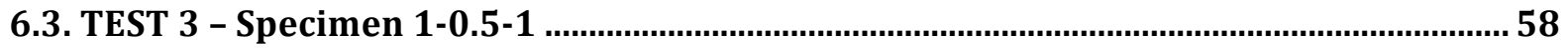

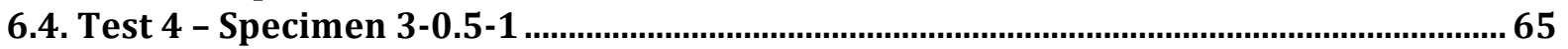

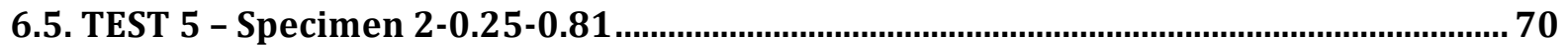

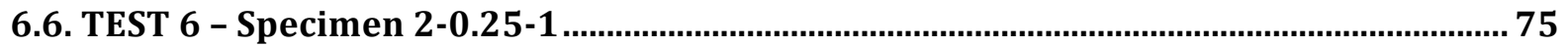

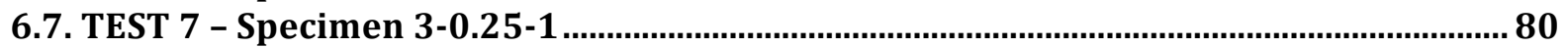

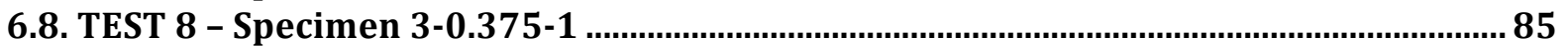

7. DISCUSSION OF RS-SPSW EXPERIMENTAL BEHAVIOR …............................................90

7.1. Comparison of Specimens behavior............................................................................90

7.2. Investigation of Ring Deformation Behavior ........................................................................ 93 


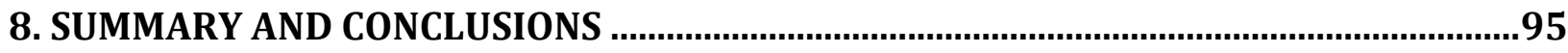

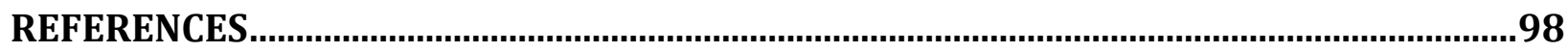

APPENDIX A: Instruments and channels .................................................................. 100

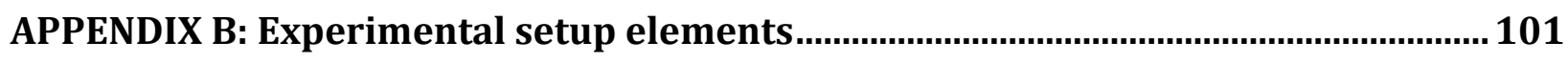

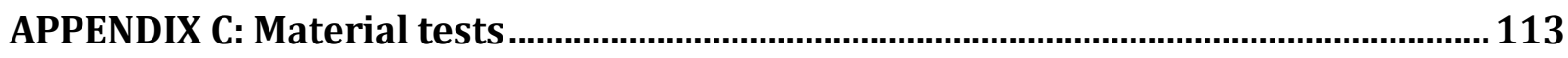

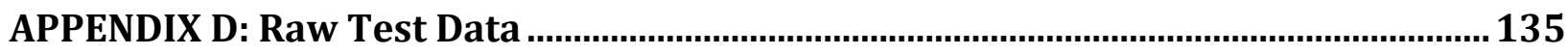

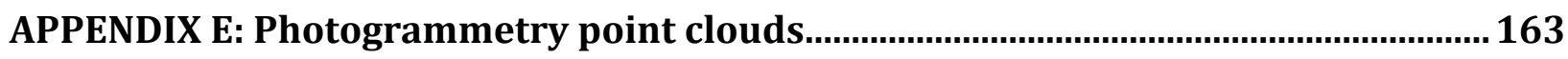

APPENDIX F: Citation of copyrighted works .................................................................. 177 


\section{LIST OF FIGURES}

Figure 1. Un-stiffened typical SPSW ........................................................................................... 1

Figure 2. Buckling resistant steel plate shear wall [from (Maurya 2012)] ................................ 2

Figure 3. Infill-to-boundary frame connection [from (Berman and Bruneau 2005)] .............. 5

Figure 4. Test setup and Specimen F2 infill-only hysteresis curve [from (Berman and

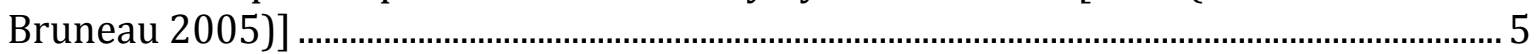

Figure 5. Test set up [from (Chen and Jhang 2011)] …………………………………………... 6

Figure 6. SPSW with slits concept [from (Hitaka and Matsui 2003)] ………………………......

Figure 7. Photograph of specimen prior to testing [from (Vian et al. 2009)] ............................. 8

Figure 8. SPSW with butterfly shapes fuses [from (Ma et al. 2010)] ……................................... 8

Figure 9. Circle and ellipse (result of ring deformation) geometry .......................................... 10

Figure 10. Concept graphical explanation [from (Maurya 2012)] ………………………….... 11

Figure 11. Computational model of full-scale BR-SPSW [from (Maurya 2012)] ..................... 12

Figure 12. Plastic mechanism analysis to determine shear yield capacity of the RS-SPSW 13

Figure 13. Side View of the Experimental Setup ........................................................................ 15

Figure 14. Horizontal and diagonal bracing .............................................................................. 16

Figure 15. Test frame with vertical displacement of 2.5 in (7.4\% drift ratio) ......................... 17

Figure 16. Top view: position on the reaction floor and bracing ............................................. 18

Figure 17. Bolted and pin connections..................................................................................... 19

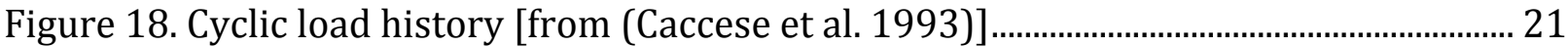

Figure 19. Cyclic load history [from (Li et al. 2011)] ………………………………………...... 22

Figure 20. Ring geometric parameters.......................................................................................... 23

Figure 21. Example of the specimen ……………………………………………………….... 25

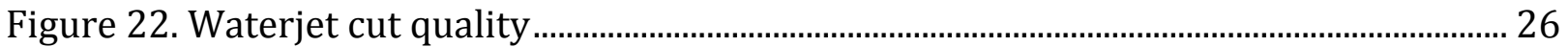

Figure 23. Design drawing for Plate tension specimens ............................................................. 27

Figure 24. United SHFM Servo-Controlled Hydraulic Floor Model Universal Testing

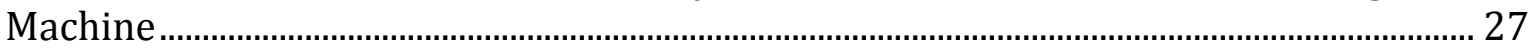

Figure 25. Quality of the cut (waterjet cut with highest quality) ............................................ 28

Figure 26. Extensometer EZ. 2-2 used for Plate Tension Coupons Tests.................................. 28

Figure 27. Tested Specimens from 1/2" Test 1 (bottom), Test 2 (middle), and Test 3 (top)

Figure 28. Tested Specimens from 0.075" Test 1 (bottom), Test 2 (middle), and Test 3

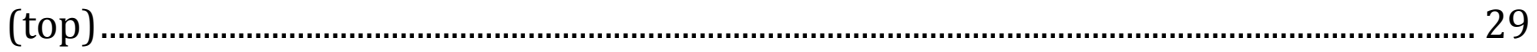

Figure 29. 1/2" Thick Plate - Test Number 1 Load - Displacement diagram ............................... 30

Figure 30. 1/2" Thick Plate - Test Number 1 Stress - Strain diagram using 2" Extensometer

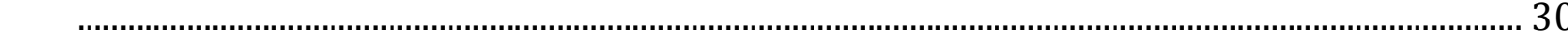

Figure 31. Test Number 1 Stress - Strain diagram using full gage length................................. 31

Figure 32. Photograph of test set up with string potentiometers shown ................................. 33

Figure 33. Calculation of plate vertical displacements using diagonal potentiometer......... 34

Figure 34. Horizontal and vertical separation of photos in photogrammetry.......................... 37

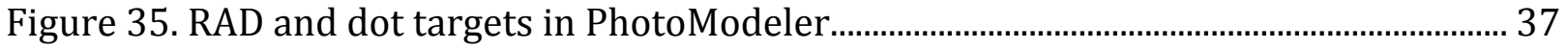


Figure 36. Photogrammetry targets placement - Specimens 2-0.5-1 and 2-0.25-1 ............ 38

Figure 37. Photogrammetry targets placement - Specimen "Solid Plate"............................... 39

Figure 38. Photogrammetry targets placements - Specimen 1-0.5-1 .................................... 40

Figure 39. Photogrammetry targets placement - Specimens 3-0.5-1, 3-0.25-1, 3-0.375-1 41

Figure 40. Photogrammetry targets placement - Specimen 2-0.25-0.81 ............................... 42

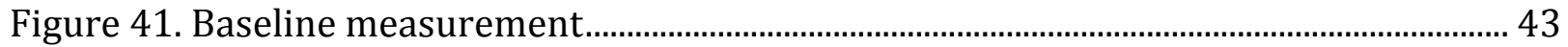

Figure 42. Photogrammetry side view - Test 1 - Specimen 2-05-1...................................... 45

Figure 43. Whitewash side view - Test 1 - Specimen 2-0.5-1 ................................................. 45

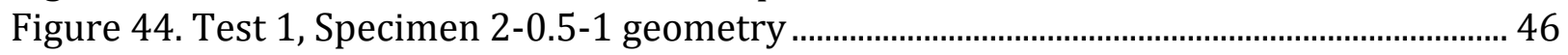

Figure 45. Point cloud and orthogonal regression plot - Specimen 2-0.5-1 initial shape.... 47

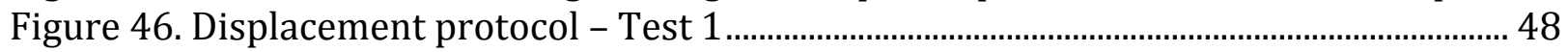

Figure 47. Shear Force versus Plate Drift Angle - Test 1 - Specimen 2-0.5-1 ...................... 49

Figure 48. Locations of initial yielding - Test 1 - Specimen 2-0.5-1........................................ 50

Figure 49. Point cloud - Specimen 2-05-0.1 - 6.7\% drift .......................................................... 50

Figure 50. Local yielding of W12 flanges - Test 1 ..................................................................... 51

Figure 51. Surrounding frame repair after Test 1................................................................... 51

Figure 52. Photogrammetry side view - Test 2 - Specimen "Solid Plate" ............................... 52

Figure 53. Whitewash side view - Test 2 - Specimen "Solid Plate" ............................................ 52

Figure 54. Test 2, Specimen "Solid Plate" geometry...................................................................... 53

Figure 55. Point cloud and orthogonal regression plot - Specimen "Solid Plate" initial shape

Figure 56. Displacement protocol - Test 2 …..................................................................... 54

Figure 57. Shear Force versus Plate Drift Angle - Test 2 - Specimen "Solid Plate"............... 56

Figure 58. Point cloud - Specimen "Solid Plate" - 0.5\% drift...................................................... 56

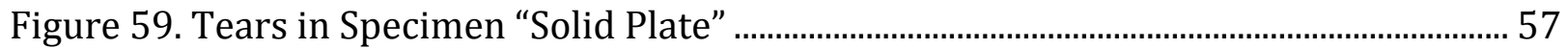

Figure 60. Photogrammetry side view - Test 3 - Specimen 1-0.5-1....................................... 58

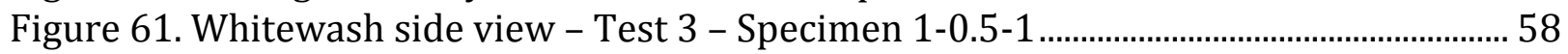

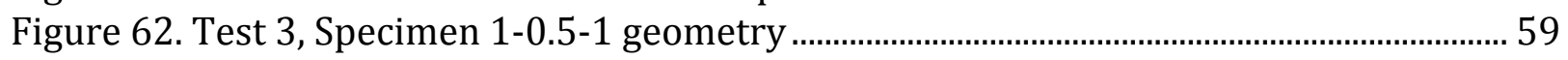

Figure 63. Point cloud and orthogonal regression plot - Specimen 1-0.5-1 initial shape.... 60

Figure 64. Yielding of the plate - result of wrong work of hydraulic machine - Test 3 ....... 61

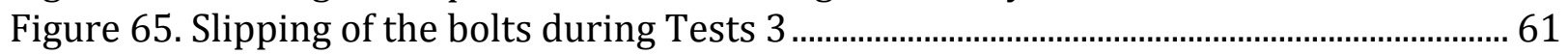

Figure 66. Shear Force versus Plate Drift Angle - Test 3 - Specimen 1-0.5-1 ........................ 62

Figure 67. Shear Force versus Actuator Drift Angle - Test 3 - Specimen 1-0.5-1................. 63

Figure 68. Location of initial yielding - Test 3-Specimen 1-0.5-1 ............................................. 63

Figure 69. Point cloud - Specimen 3-0.5-1 - 6.8\% drift ............................................................. 64

Figure 70. Photogrammetry side view - Test 4 - Specimen 3-0.5-1......................................... 65

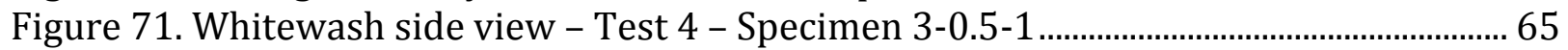

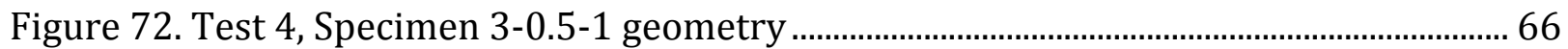

Figure 73. Point cloud and orthogonal regression plot - Specimen 3-0.5-1 initial shape.... 67

Figure 74. Shear Force versus Plate Drift Angle - Test 4 - Specimen 3-0.5-1 ........................ 68

Figure 75. Locations of initial yielding - Test 4 - Specimen 3-0.5-1......................................... 68

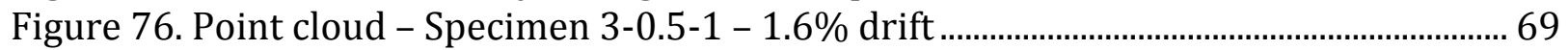

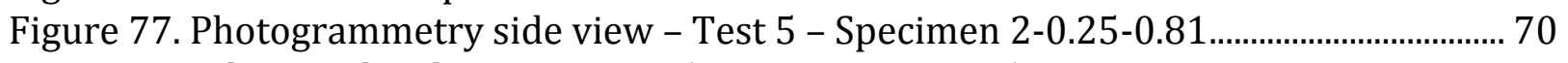

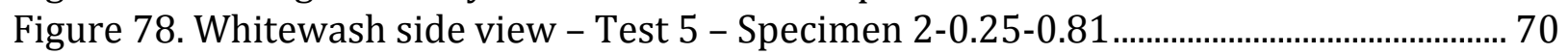

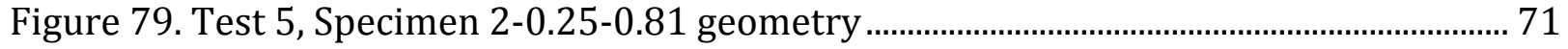


Figure 80. Point cloud and orthogonal regression plot - Specimen 2-0.25-0.81 initial shape

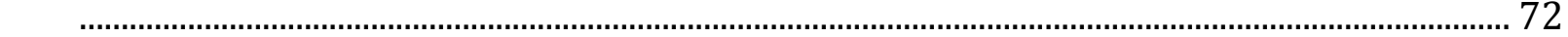

Figure 81. Shear Force versus Plate Drift Angle - Test 5 - Specimen 2-0.25-0.81 ............... 73

Figure 82. Locations of initial yielding - Specimen 2-0.25-0.81 ................................................ 73

Figure 83. Point cloud - Specimen 2-0.25-0.81 - 3\% drift............................................................ 74

Figure 84. Specimen 2-0.25-0.81 fracture at 10\% drift............................................................. 74

Figure 85. Photogrammetry side view - Test 6 - Specimen 2-0.25-1 ...................................... 75

Figure 86. Whitewash side view - Test 6 - Specimen 2-0.25-1 ............................................... 75

Figure 87. Test 6, Specimen 2-0.25-1 geometry …….................................................................. 76

Figure 88. Point cloud and orthogonal regression plot - Specimen 2-0.25-1 initial shape. 77

Figure 89. Shear Force versus Plate Drift Angle - Test 6 - Specimen 2-0.25-1 ..................... 78

Figure 90. Point cloud - Specimen 2-0.25-1 - 2.8\% drift ............................................................ 78

Figure 91. Specimen 2-0.25-1 fracture at 10\% drift .................................................................. 79

Figure 92. Photogrammetry side view - Test 7 - Specimen 3-0.25-1 ....................................... 80

Figure 93. Whitewash side view - Test 7 - Specimen 3-0.25-1 ................................................. 80

Figure 94. Test 7, Specimen 3-0.25-1 geometry …..................................................................... 81

Figure 95. Point cloud and orthogonal regression plot - Specimen 3-0.25-1 initial shape. 82

Figure 96. Shear Force versus Plate Drift Angle - Test 7 - Specimen 3-0.25-1 ..................... 83

Figure 97. Point cloud - Specimen 3-0.25-1 - 6.8\% drift .............................................................. 83

Figure 98. Specimen 3-0.25-1 fracture at 8\% drift...................................................................... 84

Figure 99. Photogrammetry side view - Test 8 - Specimen 3-0.375-1 ................................... 85

Figure 100. Whitewash side view - Test 8 - Specimen 3-0.375-1............................................ 85

Figure 101. Test 8, Specimen 3-0.375-1 geometry .................................................................. 86

Figure 102. Point cloud and orthogonal regression plot - Specimen 3-0.375-1 initial shape

Figure 103. Shear Force versus Plate Drift Angle - Test 8 .......................................................... 88

Figure 104. Point cloud - Specimen 3-0.375-1 - 6.1\% drift ........................................................ 88

Figure 105. Specimen 3-0.375-1 fracture locations at 8\% drift.................................................. 89

Figure 106. Energy dissipation during 4\% cycle ........................................................................ 91

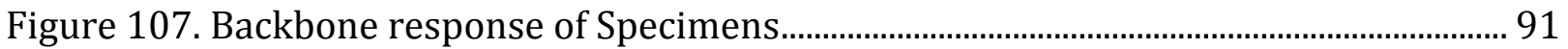

Figure 108. Energy dissipation energy versus ring slenderness .............................................. 92

Figure 109. Change of the ring inner and outer perimeter during the test - Specimen 2-

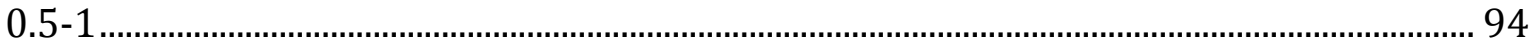

Figure 110. Change of the ring centerline perimeter during the test - Specimen 3-0.5-1.. 94

Figure 111. Side view of the experimental set up ...................................................................101

Figure 112. Horizontal and vertical bracing ............................................................................102

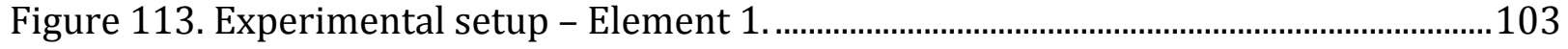

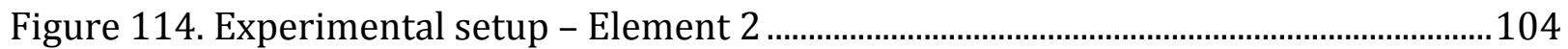

Figure 115. Experimental setup - Element 3 …............................................................................. 105

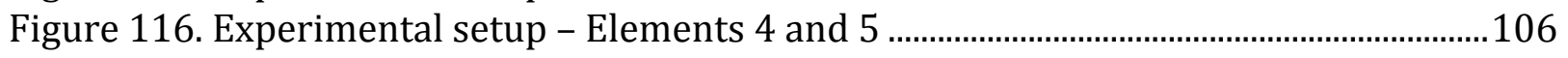

Figure 117. Experimental setup - Elements 6-9.......................................................................107

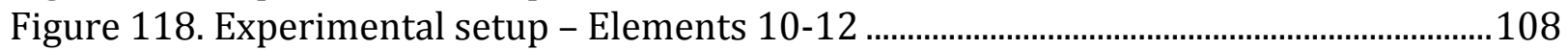

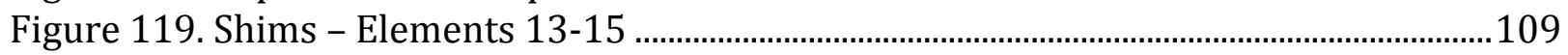

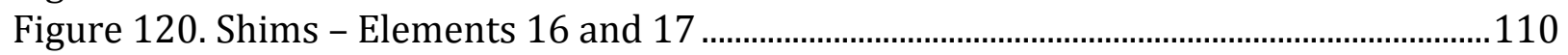

Figure 121. Upper part bracing - Elements 18-21 …...........................................................111

Figure 122. Lower part bracing - Elements 22-25 ..................................................................... 112 
Figure 123. 1/2" Thick Plate - Test Number 2 Load - Displacement diagram 113 Figure 124. 1/2" Thick Plate - Test Number 2 Stress - Strain diagram using 2" Extensometer 114

Figure 125. 1/2" Thick Plate - Test Number 2 Stress - Strain diagram using full gage length

Figure 126. 1/2" Thick Plate - Test Number 3 Load - Displacement diagram 114 Figure 127. 1/2" Thick Plate - Test Number 3 Stress - Strain diagram using 2" Extensometer 116

Figure 128. 1/2" Thick Plate - Test Number 3 Stress - Strain diagram using full gage length

Figure 129. 0.075" Thick Plate - Test Number 1 Load - Displacement diagram ...................117 Figure 130. 0.075 " Thick Plate - Test Number 1 Stress - Strain diagram using 2" Extensometer 118

Figure 131. 0.075" Thick Plate - Test Number 1 Stress - Strain diagram using full gage length 118

Figure 132. 0.075" Thick Plate - Test Number 2 Load - Displacement diagram 119

Figure 133. 0.075 " Thick Plate - Test Number 2 Stress - Strain diagram using 2" Extensometer. 120

Figure 134. 0.075" Thick Plate - Test Number 2 Stress - Strain diagram using full gage length 120

Figure 135. 0.075" Thick Plate - Test Number 3 Load - Displacement diagram ....................121 Figure 136. 0.075 " Thick Plate - Test Number 3 Stress - Strain diagram using 2" Extensometer . 122

Figure 137. 0.075" Thick Plate - Test Number 3 Stress - Strain diagram using full gage length

Figure 138. 0.25" Thick Plate - Test Number 1 Load - Displacement diagram 123

Figure 139. 0.25 " Thick Plate - Test Number 1 Stress - Strain diagram using 2" Extensometer 124

Figure 140. 0.25" Thick Plate - Test Number 1 Stress - Strain diagram using full gage length

Figure 141. 0.25" Thick Plate - Test Number 2 Load - Displacement diagram 125

Figure 142. 0.25 " Thick Plate - Test Number 2 Stress - Strain diagram using 2" Extensometer 126

Figure 143. 0.25" Thick Plate - Test Number 2 Stress - Strain diagram using full gage length 126

Figure 144. 0.25" Thick Plate - Test Number 3 Load - Displacement diagram.......................127

Figure 145. 0.25 " Thick Plate - Test Number 3 Stress - Strain diagram using 2" Extensometer

Figure 146. 0.25" Thick Plate - Test Number 3 Stress - Strain diagram using full gage length

Figure 147. 0.375" Thick Plate - Test Number 1 Load - Displacement diagram ....................129

Figure 148. 0.375 " Thick Plate - Test Number 1 Stress - Strain diagram using 2" Extensometer . 130

Figure 149. 0.375" Thick Plate - Test Number 1 Stress - Strain diagram using full gage length

Figure 150. 0.375" Thick Plate - Test Number 2 Load - Displacement diagram 131 
Figure 151. 0.375 " Thick Plate - Test Number 2 Stress - Strain diagram using 2"

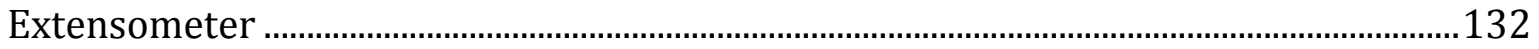

Figure 152. 0.375" Thick Plate - Test Number 2 Stress - Strain diagram using full gage

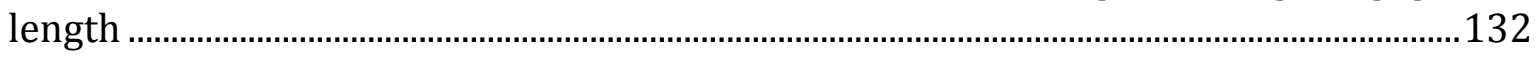

Figure 153. 0.375" Thick Plate - Test Number 2 Load - Displacement diagram ....................133

Figure 154. 0.375 " Thick Plate - Test Number 2 Stress - Strain diagram using 2"

Extensometer.

Figure 155. 0.375" Thick Plate - Test Number 2 Stress - Strain diagram using full gage length

Figure 156. Load versus Plate Vertical Displacement - Test 1................................................135

Figure 157. Load versus Time - Test 1 .....................................................................................135

Figure 158. Plate Drift Angle versus Time - Test 1 ..................................................................136

Figure 159. Diagonal and Horizontal Elongations of Plate versus Time - Test 1.................136

Figure 160. Plate Vertical Displacement versus Time- Test 1 ..................................................137

Figure 161. Actuator Vertical Displacement versus Time - Test 1..........................................137

Figure 162. Load versus Actuator Vertical Displacement - Test 1 ……………………….....138

Figure 163. 8 Strain Gauges versus Time - Test 1 ...................................................................138

Figure 164. Load versus Plate Vertical Displacement - Test 2 …………………………….....139

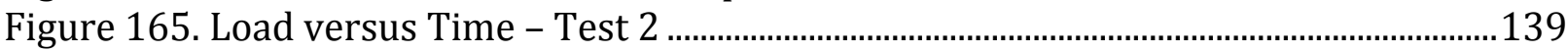

Figure 166. Diagonal and Horizontal Elongations of Plate versus Time - Test 2..................140

Figure 167. Plate Vertical Displacement versus Time - Test 2 ……………………………....140

Figure 168. Plate Drift Angle versus Time - Test 2 ………………………………………......141

Figure 169. Actuator Vertical Displacement versus Time - Test 2 ………………………….....141

Figure 170. Load versus Actuator Vertical Displacement - Test 2 .........................................142

Figure 171. Plate vertical displacement versus time - Test 3.................................................142

Figure 172. Actuator vertical displacement versus time - Test 3 ..........................................143

Figure 173. Load versus Actuator Vertical Displacement - Test 3 ............................................143

Figure 174. Load versus Plate Vertical Displacement - Test 3.................................................144

Figure 175. Load versus Time - Test 3 ..................................................................................144

Figure 176. Plate drift Angle versus Time - Test 3 ...............................................................145

Figure 177. Load versus Actuator Vertical Displacement - Test 4 ..........................................145

Figure 178. Load versus Plate Vertical Displacement - Test 4..................................................146

Figure 179. Load versus Time - Test 4 .......................................................................................146

Figure 180. Plate Drift Angle versus Time - Test 4 ……………………………………….......147

Figure 181. Plate Vertical Displacement versus Time - Test 4 ................................................147

Figure 182. Actuator Vertical Displacement versus Time - Test 4.........................................148

Figure 183. Diagonal and Horizontal Elongation of Plate versus Time - Test 4 ...................148

Figure 184. Diagonal and Horizontal Elongation of Plate versus Time - Test 5 ....................149

Figure 185. Plate vertical Displacement versus Time - Test 5................................................149

Figure 186. Actuator Vertical Displacement versus Time - Test 5.........................................150

Figure 187. Load versus Actuator Vertical Displacement - Test 5 ..........................................150

Figure 188. Load versus Plate Vertical Displacement - Test 5................................................151

Figure 189. Load versus Time - Test 5 .....................................................................................151

Figure 190. Plate Drift Angle versus Time - Test 5 ………………………………………......152

Figure 191. Plate Drift Angle versus Time - Test 6 ………………………………...................152

Figure 192. Plate Vertical Displacement versus Time - Test 6 .................................................153 
Figure 193. Actuator Vertical Displacement versus Time - Test 6..........................................153

Figure 194. Diagonal and Horizontal Elongation of Plate versus Time - Test 6....................154

Figure 195. Load versus Actuator Vertical Displacement - Test 6 ..........................................154

Figure 196. Load versus Plate Vertical Displacement - Test 6 ................................................155

Figure 197. Load versus Time - Test 6 ...................................................................................155

Figure 198. Plate Vertical Displacement versus Time - Test 7 ……………………………....156

Figure 199. Actuator Vertical Displacement versus Time - Test 7 ………………………......156

Figure 200. Load versus Actuator Vertical Displacement - Test 7 ..........................................157

Figure 201. Load versus Plate Vertical Displacement - Test 7 .................................................157

Figure 202. Load versus Time - Test 7 ...................................................................................158

Figure 203. Plate Drift Angle versus Time - Test 7 ………………………………………....158

Figure 204. Diagonal and Horizontal Elongation of Plate versus Time - Test 7 ...................159

Figure 205. Plate Vertical Displacement versus Time - Test 8 ...............................................159

Figure 206. Actuator Vertical Displacement versus Time - Test 8..........................................160

Figure 207. Diagonal and Horizontal Elongation of Plate versus Time - Test 8....................160

Figure 208. Load versus Actuator Vertical Displacement - Test 8 .........................................161

Figure 209. Load versus Plate Vertical Displacement - Test 8.................................................161

Figure 210. Load versus Time - Test 8 ......................................................................................162

Figure 211. Plate Drift Angle versus Time - Test 8 ………………………………………......162

Figure 212. Point cloud - Specimen 2-05-0.1 - 0.3\% drift.........................................................163

Figure 213. Point cloud - Specimen 2-05-0.1 - 0.8\% drift........................................................163

Figure 214. Point cloud - Specimen 2-05-0.1 - 2.8\% drift.........................................................164

Figure 215. Point cloud - Specimen 2-05-0.1 - 6.7\% drift.............................................................

Figure 216. Point cloud - Specimen "Solid Plate" - 0.5\% drift..................................................165

Figure 217. Point cloud - Specimen "Solid Plate" - 2.2\% drift...................................................165

Figure 218. Point cloud - Specimen "Solid Plate" - 6\% drift.....................................................166

Figure 219. Point cloud - Specimen 2-0.5-1 - 0.9\% drift ...........................................................166

Figure 220. Point cloud - Specimen 2-0.5-1 - 2.8\% drift ............................................................167

Figure 221. Point cloud - Specimen 2-0.5-1 - 6.8\% drift ..........................................................167

Figure 222. Point cloud - Specimen 2-0.5-1 - 11.9\% drift........................................................168

Figure 223. Point cloud - Specimen 3-0.5-1 - 0.4\% drift ...........................................................168

Figure 224. Point cloud - Specimen 3-0.5-1 - 1.6\% drift ............................................................169

Figure 225. Point cloud - Specimen 3-0.5-1 - 3.2\% drift ...........................................................169

Figure 226. Point cloud - Specimen 2-0.25-0.81 - 1.2\% drift ..................................................170

Figure 227. Point cloud - Specimen 2-0.25-0.81 - 3\% drift.....................................................170

Figure 228. Point cloud - Specimen 2-0.25-0.81 - 6.8\% drift ...................................................171

Figure 229. Point cloud - Specimen 2-0.25-0.81 - 11.9\% drift................................................171

Figure 230. Point cloud - Specimen 2-0.25-1 - 0.9\% drift.......................................................172

Figure 231. Point cloud - Specimen 2-0.25-1 - 2.8\% drift.........................................................172

Figure 232. Point cloud - Specimen 2-0.25-1 - 6.8\% drift.........................................................173

Figure 233. Point cloud - Specimen 2-0.25-1 - 11.9\% drift....................................................173

Figure 234. Point cloud - Specimen 3-0.25-1 - 1.1\% drift.......................................................174

Figure 235. Point cloud - Specimen 3-0.25-1 - 2.9\% drift........................................................174

Figure 236. Point cloud - Specimen 3-0.25-1 - 6.8\% drift........................................................175

Figure 237. Point cloud - Specimen 3-0.375-1 - 0.7\% drift......................................................175

Figure 238. Point cloud - Specimen 3-0.375-1 - 2.2\% drift.......................................................176 
Figure 239. Point cloud - Specimen 3-0.375-1 - 6.1\% drift 176

\section{LIST OF TABLES}

Table 1. Loading protocol [from (Chen and Jhang 2011)] ....................................................... 21

Table 2. Load cycles applied to the specimens …........................................................................ 22

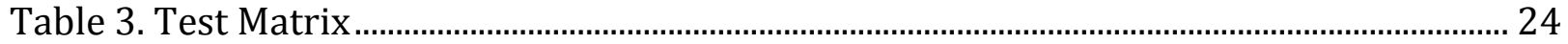

Table 4. 1/2" Thick Plate - Test Number 1 Measurements ............................................................... 30

Table 5. Material properties based on tension coupon tests...................................................... 31

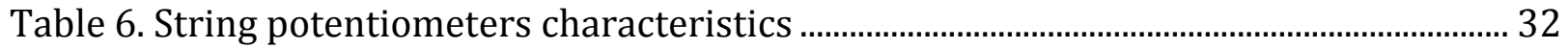

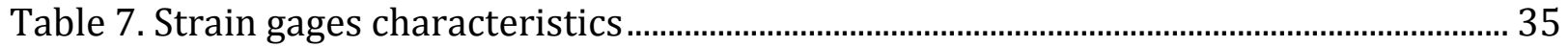

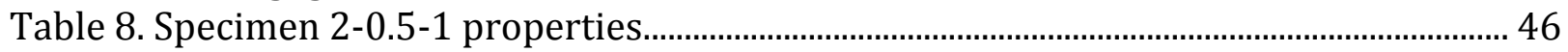

Table 9. Actuator vertical displacements and plate vertical displacement - Test 1 ............ 48

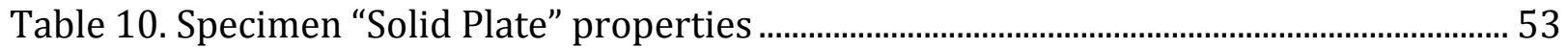

Table 11. Displacement protocol - Test 2 ….................................................................................. 55

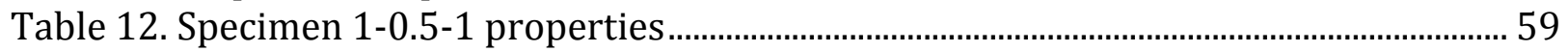

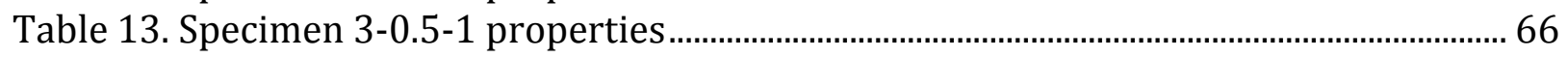

Table 14. Specimen 2-0.25-0.81 properties............................................................................... 71

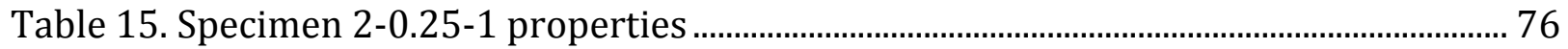

Table 16. Specimen 3-0.25-1 properties ...................................................................................... 81

Table 17. Specimen 3-0.375-1 properties …….............................................................................. 86

Table 18. Summary of specimen behavior ................................................................................ 90

Table 19. Comparison of shear yield force and shear capacity calculated using plastic

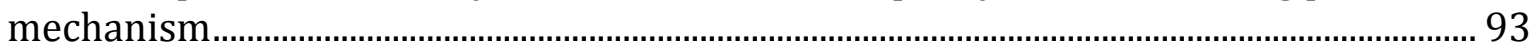

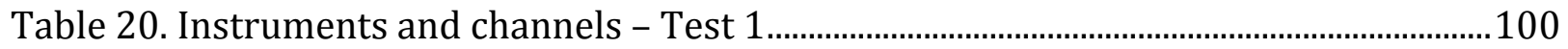

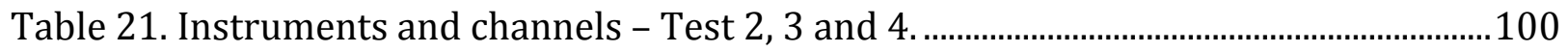

Table 22. 1/2" Thick Plate - Test Number 2 Measurements ...................................................113

Table 23. 1/2" Thick Plate - Test Number 3 Measurements ......................................................115

Table 24. 0.075" Thick Plate - Test Number 1 Measurements..................................................117

Table 25. 0.075" Thick Plate - Test Number 2 Measurements..................................................119

Table 26. 0.075" Thick Plate - Test Number 3 Measurements................................................121

Table 27. 0.25" Thick Plate - Test Number 1 Measurements ...................................................123

Table 28. 0.25" Thick Plate - Test Number 2 Measurements ....................................................125

Table 29. 0.25" Thick Plate - Test Number 2 Measurements ....................................................127

Table 30. 0.375" Thick Plate - Test Number 1 Measurements.................................................129

Table 31. 0.375" Thick Plate - Test Number 2 Measurements.................................................131

Table 32. 0.375" Thick Plate - Test Number 3 Measurements.................................................133 


\section{INTRODUCTION}

Steel plate shear walls (SPSW) are an attractive option for earthquake engineering design. In the past few decades a significant number of computational and experimental research programs have been conducted on SPSW. They have been widely used in practice for buildings located in the USA, Canada, Japan and some other countries. Even though SPSW has been used in practice, there are several challenges associated with the design, construction, and behavior of SPSW that limit its use.

The key component of a typical SPSW (Figure 1) is the thin web plate, which resists horizontal story shear through tension field action after shear buckling occurs at a relatively small shear load. The web plate then dissipates seismic energy as the web plate yields along the inclined tension filed direction. Although SPSW can develop significant post-buckling capacity, the web plate typically buckles early during the earthquake and there is even a possibility for it to buckle under large wind loads. Buckling of the SPSW leads to a significant loss of stiffness and a pinched hysteretic behavior. To mitigate the negative effects associated with SPSW web plate buckling, a surrounding moment frame is required through use of moment connections between the beams and columns. Beams also referred to horizontal boundary elements (HBE), and columns also referred to vertical boundary elements (VBE). The moment frame provides supplemental stiffness, energy dissipation, and resists lateral loads during load reversals. Using of surrounding moment frame only reduce the buckling, it doesn't fully eliminate it. These characteristics, such as pinched hysteretic behavior, low stiffness, and large boundary elements with moment connections are undesirable from an economic and behavioral standpoint.

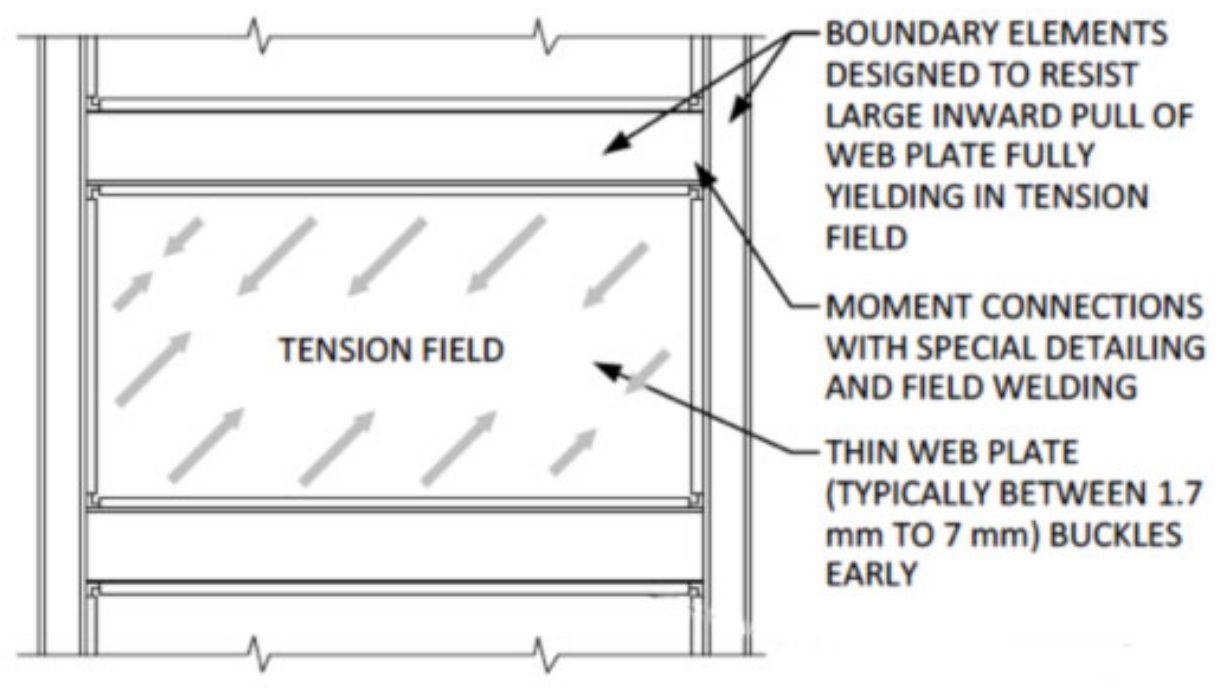

Figure 1. Un-stiffened typical SPSW 
A new buckling resistant steel plate shear wall (Figure 2) was proposed in Maurya (2012), and is the subject of this research.

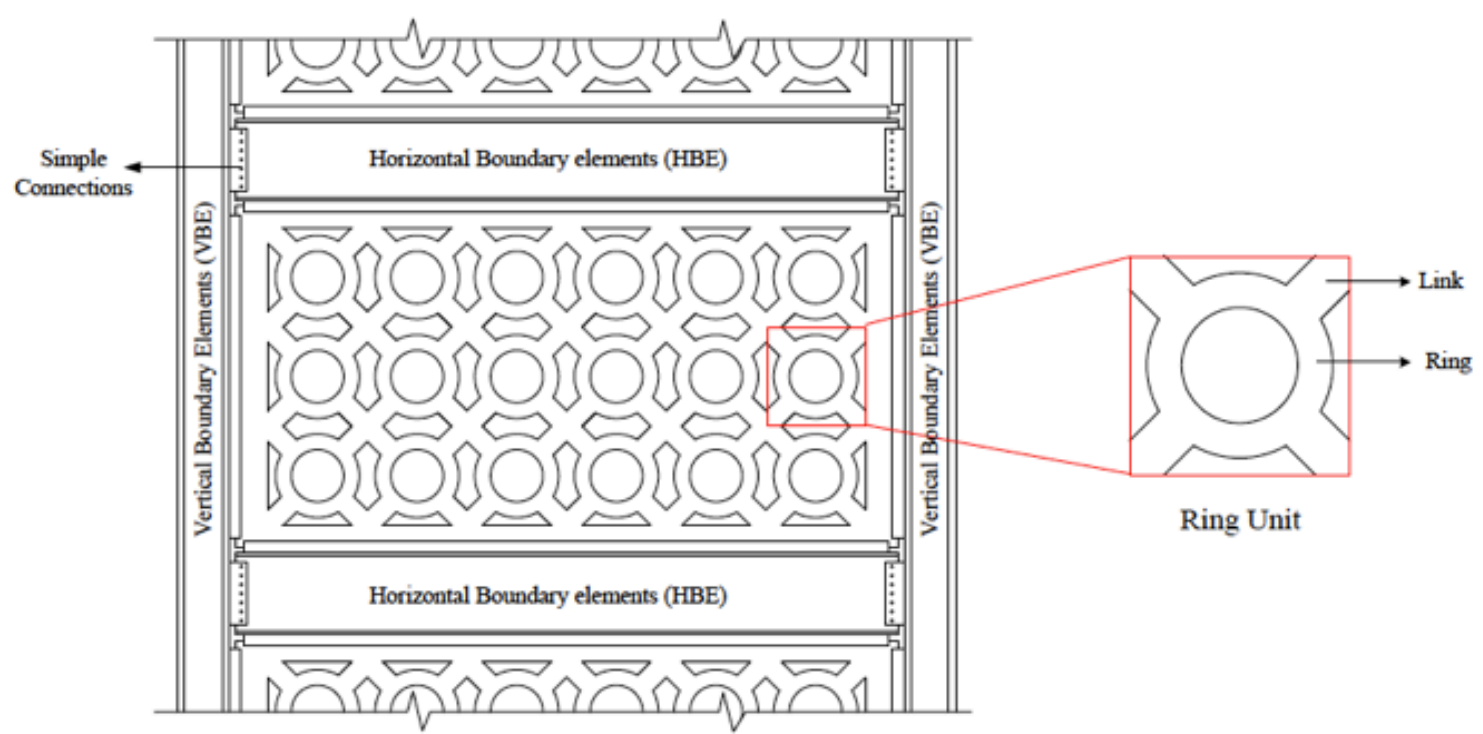

Figure 2. Buckling resistant steel plate shear wall [from (Maurya 2012)]

Maurya (2012) proposed that a unique pattern of cutouts would reduce buckling of the SPSW, would make energy-absorbing hysteresis loops fuller, and may result in smaller boundary elements.

The Ring-Shaped Steel Plate Shear Walls (RS-SPSW) concept was verified by Maurya (2012) using the finite element modeling software ABAQUS. The goal of the research described in this thesis is to verify the RS-SPSW concept experimentally. Different configurations of thicknesses, number of cutouts and geometry were chosen to understand the experimental behavior of the RS-SPSW, provide data for validation of computational models by others, and to explore which combinations of design variables produce the most desirable response. Seven one-quarter-scale specimens with different thicknesses, number of rings and geometry were designed. Also a solid plate of the same size and similar strength was designed and tested to compare its behavior with the RSSPSW.

The experimental set-up for cyclic testing of shear panels that are approximately $3 \mathrm{ft} \times 3 \mathrm{ft}$, was designed and built in the Structural Engineering Laboratory of Virginia Tech. An existing reaction frame was used in addition to a new shear testing frame that was designed and fabricated. An MTS actuator, MTS 407 Controller, and System 5000 data acquisition system were used to run the test and record measurements. Instrumentation included string potentiometers, strain gages and photogrammetry. Seven RS-SPSW specimens have been tested and results were obtained. A solid plate test has been run as well to compare its results with RS-SPSW results. 
RS-SPSW behavior was compared with solid plate behavior and also theoretical strength predictions. Furthermore, a deeper understanding of buckled shapes and buckling modes was obtained using the photogrammetry data. Conclusions about the buckling behavior were used to make suggestions about design parameters that prevent buckling.

The experimental study described herein coupled with the computational research by Maurya (2012) provided key information about the behavior of RS-SPSW, proved that they are a valid lateral load resisting system, and provided important information for selecting design variables that produce a desired behavior.

The report is divided into eight chapters and five appendices. The overview of the chapters is given below.

Chapter 2 summarizes some research work done in the field of steel plate shear walls in the last few decades. Experimental studies of solid and perforated SPSW are reviewed in this chapter. Additionally, the review of RS-SPSW computational study conducted is given.

Chapter 3 describes RS-SPSW concept. It explains how ring-shaped cutouts help to reduce the buckling of the plate, provides the mathematical proof of the concept.

Chapter 4 demonstrates the development of a method, which helps to limit yielding of the link. The optimal values of width of the ring and width of the ring are discussed.

Chapter 5 provides the description of experimental setup, specimens fabrication and specimens test matrix. Applied loading protocol is also included in the chapter. All instrumentation, data acquisition and associated equipment are described.

Chapter 6 focuses on test results. Hysteretic plots, description of the specimens behavior under the load are included. Some problems with the surrounding frame experienced during the test are also discussed.

Chapter 7 gives the comparison between specimens behavior. Additionally, deformation of the ring during the test is studied.

Chapter 8 summarizes the work presented in the report. It proposes some research that can be done in the future to prove the validity of the concept for full-scale RS-SPSW. 


\section{LITERATURE REVIEW}

A brief review of SPSW tests conducted by various researchers in the past is presented here. A main focus is made on the test setups used for experiments, specimens' connection to the surrounding frame, and failures that happened during the tests and their causes.

Even if there is no significant difference between tests set ups for solid and perforated plates the results of the tests are different because of the different concept for the SPSW, so they were separated into different sections in this literature review.

\subsection{Experimental studies of solid SPSW}

The main goal of the study by Berman and Bruneau (2005) was to prove experimentally that light-gauge SPSW, which is thinner than typical SPSW plate and thus does not require heavy reinforcement of the existing framing, is an effective engineering system for seismic retrofit of older building and is able to resist the necessary seismic forces.

Two specimens referred to as F1 and F2 with flat infill plates (thickness of $0.9 \mathrm{~mm}$ ) and a third specimen, C1, using a corrugated infill plate (thickness of $0.7 \mathrm{~mm}$ ), were designed as seismic retrofits for a hospital located in an area of high seismicity. Quasi-static loading was used during the experiment. Boundary elements for the frame were designed to remain elastic, resulting in W12x96 columns and W18x86 beams. Beams and columns were connected by L8x $4 \times 0.5$ angles. The angles were welded to the beam and bolted to the column flanges. Specimen F1 infill plates connected to the intermediate WT7x26.5 sections with epoxy (Figure 2). The F2 infill plate was fully welded to the WT7x26.5, and the corrugated infill plate for specimen $\mathrm{C} 1$ was connected to the beam and column sections using intermediate L6 $4 \times 3 / 4$ sections and epoxy as shown in Figure 2 . Due to the fact that infill elements should be removable for the test, WT7x26.5 sections were bolted to the boundary frame. Lateral load at the top of the wall was applied using a hydraulic actuator, which was attached to the reaction frame and specimen (Figure 3). ATC-24 (ATC 1992) loading protocol was followed [ATC-24 (1992)]. Specimen F1 suffered a premature failure while still exhibiting elastic behavior. The infill-only hysteretic plot for specimen F2 is shown in Figure 3. The component of resistance due to the moment frame was not included in Figure 4. Stable and ductile behavior was observed in specimen F2. It reached a ductility ratio of 12 , a drift of 3.7 percent, and provided $90 \%$ of the stiffness of the system. The failure happened due to the fracture in the infill plate propagating from the corners. 


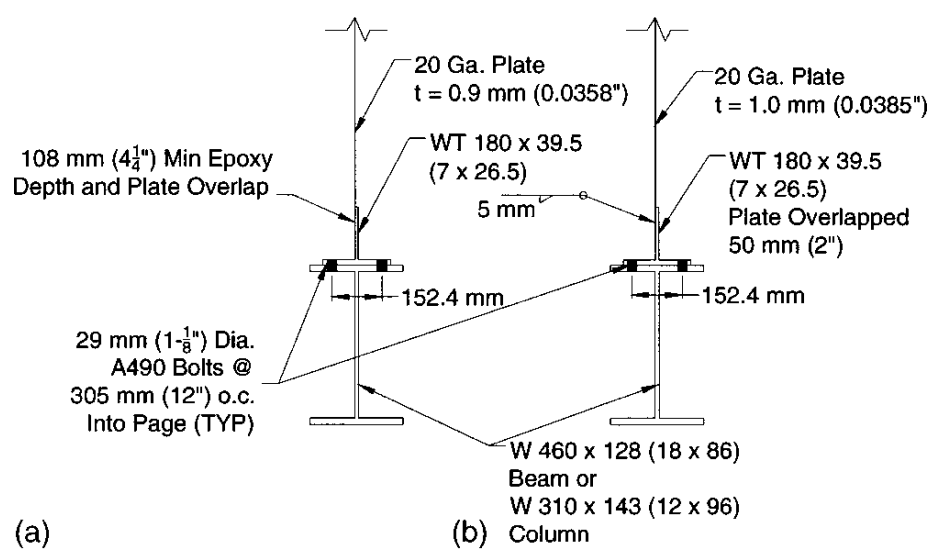

(a)

(b) Column
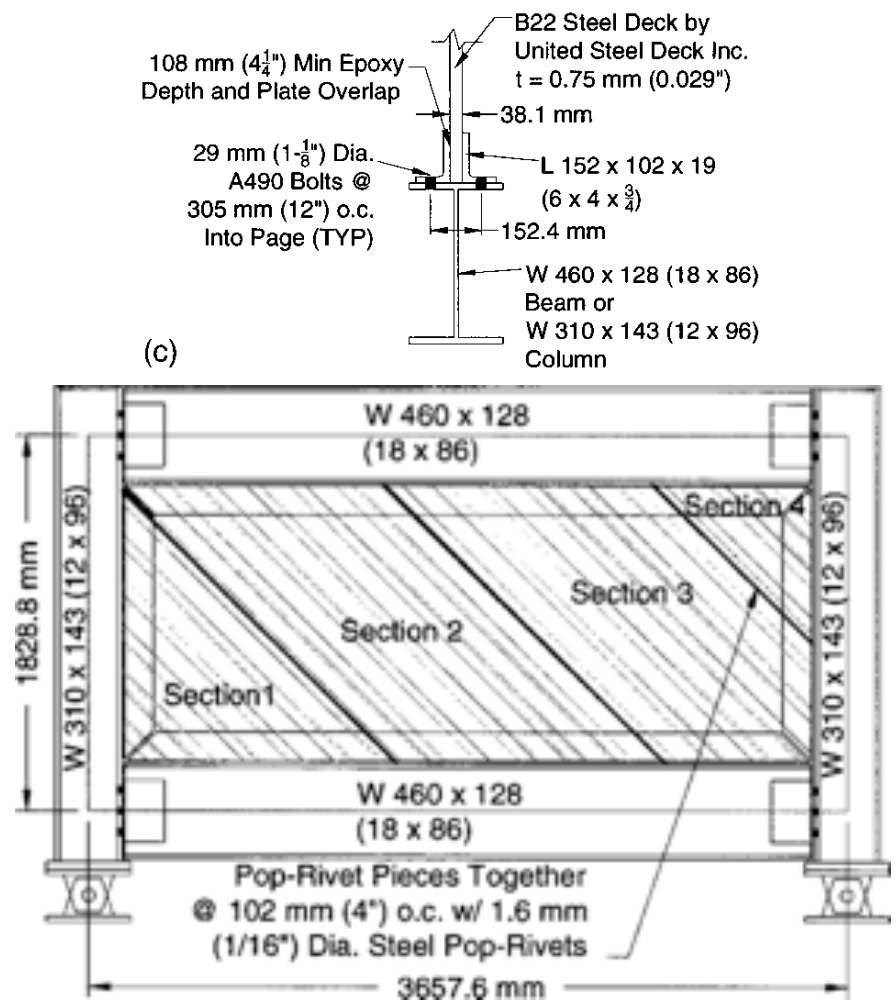

Figure 3. Infill-to-boundary frame connection [from (Berman and Bruneau 2005)]
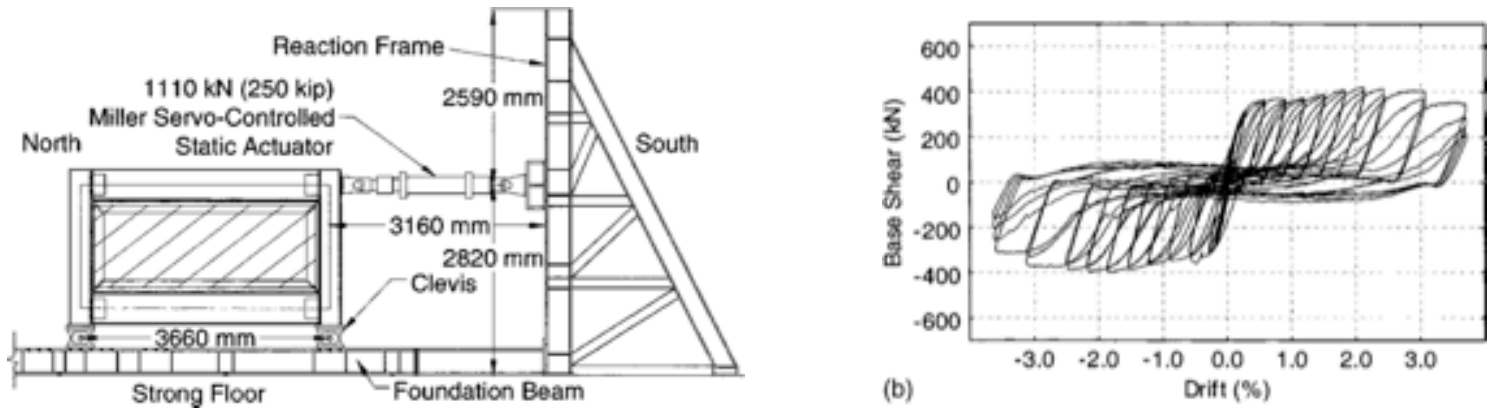

Figure 4. Test setup and Specimen F2 infill-only hysteresis curve [from (Berman and Bruneau 2005)] 
Caccese et al. (1993) investigated the seismic behavior of unstiffened thin plate shear walls. Cyclic testing of six, 1:4 scale specimens with various plate thicknesses was conducted. The specimens were a single-bay, three-story steel frames with thin unstiffened steel plates welded to the surrounding frame, which consisted of S3x5.7 horizontal members and W4x13 columns. Two different types of beam-column connections were tested: moment (entire beam section was welded to the column) and shear (only beam web was welded to the column).

Several important basic observations of SPSW behavior were made during the test. Experiments showed that the influence of beam-to-column connections is minor. A conclusion was made that welded infill plate acts as a moment-resisting connection even without welding the beam flanges to the column. The energy dissipation was observed to be approximately the same at the first cycles regardless of the thickness of the plate, and started to become bigger for thicker plates after 1 in displacement. Thickness of the plate has insignificant influence on the initial stiffness of the plate. More than that, in the slender specimen, the plate yielded long before inelastic behavior is observed in the column. In the thickest specimen, the failure was governed by elastic instability of the column. Once failure of the column occurred, the role of the plate in providing strength to the system is insignificant, so using of thicker plates is obviously a waste of material.

Chen and Jhang (2011) investigated SPSW made of low-yield-point steel, which was developed for seismic resistance. This kind of steel has low yield strength and high elongation capacity and can improve energy dissipation characteristics of the steel shear wall.

Four specimens were selected with width-to-thickness ratios from 50 to 150 . Width was defined as the distance between boundary elements. The test set-up is shown in Figure 5. Monotonic and cyclic loads were applied.

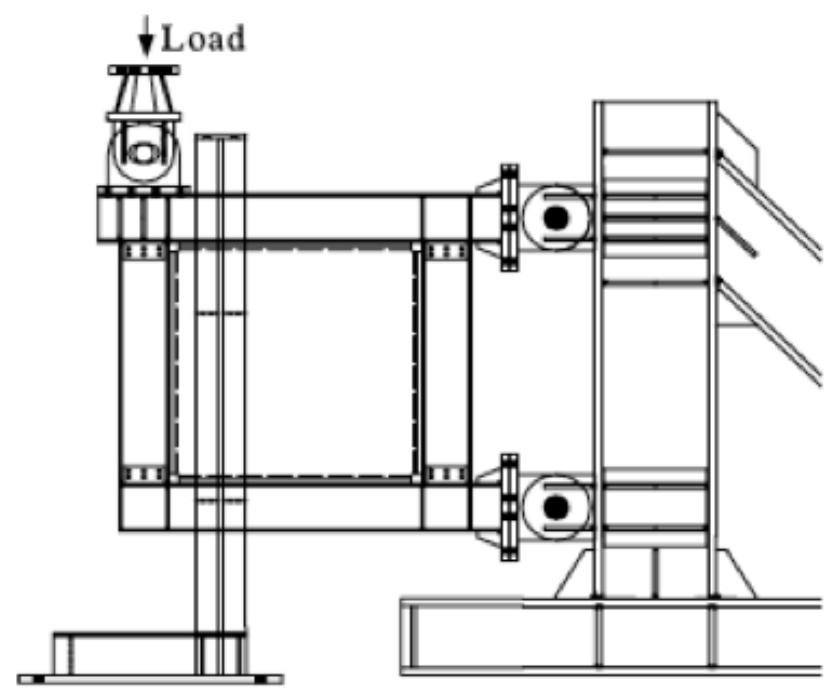

Figure 5. Test set up [from (Chen and Jhang 2011)] 
As a result of the test, width-to-thickness ratios less than 80 were suggested for better performance. Also it was proved that both moment and shear beam-to-column connections are able to provide good seismic performance of the system. However, moment connections give $10 \%$ more strength and energy dissipation than shear connections and therefore are preferable.

Many other researchers have conducted experimental studies on SPSW, but the ones described above were selected for this literature review because they represent some experiments conducted in the past two decades.

\subsection{Experimental studies of perforated SPSW}

SPSW with slits (Figure 5) was first introduced by Hitaka and Matsui (2003). Research showed good seismic behavior of this new type of shear wall because segments between slits behaved as flexural links and thus provided ductile behavior. As part of the research, 42 tests were conducted with specimens of different configurations. The effect of stiffening, specifically edge stiffeners and un-bonded mortar panel use, was also investigated during these tests. The width-to-thickness ratio was chosen as a basic test parameter, static monotonic and cyclic lateral loads were applied.

No cracks or strength degradation was observed for any specimens during the tests till $3 \%$ drift. All plates showed large ductility. Although the conclusion was made that width-tothickness ratio of the flexural links $b / t$ (Figure 6) should be greater than 0.25 for SPSW with slits to avoid lateral-torsional buckling of the flexural links (Figure 6). Welded edge stiffeners were found to be efficient for restraining shear buckling of the wall and had negligible effect on initial stiffness of the plate.

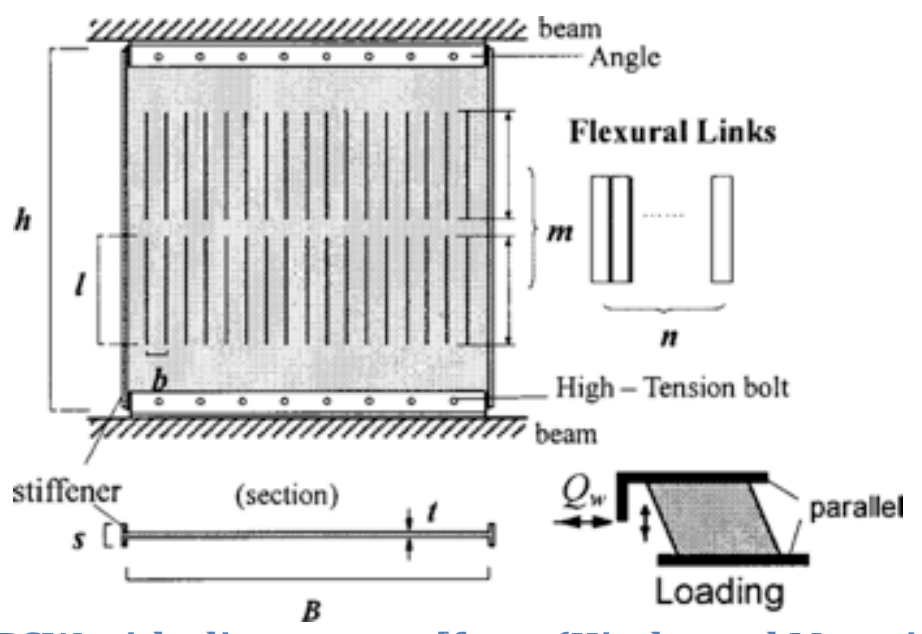

Figure 6. SPSW with slits concept [from (Hitaka and Matsui 2003)]

Vian et al. (2009) presented results of experiments on special perforated SPSW with reduced beam section anchor beams. Figure 7 shows a picture of perforated specimen prior to testing. Steel frame, $4 \mathrm{~m} \times 2 \mathrm{~m}$, was designed for the tests; the chosen beam and 
column sections were W18x65 and W18x71, respectively. The experiments showed that elastic stiffness and overall strength were reduced by $15 \%$ by using perforated SPSW instead of solid SPSW. The results of the tests also proved that no stiffeners around perforations were needed, as it was required before by seismic design specifications.

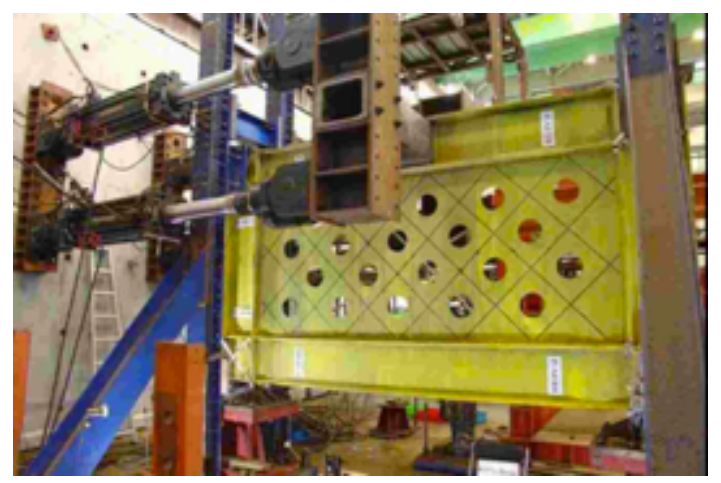

Figure 7. Photograph of specimen prior to testing [from (Vian et al. 2009)]

Ma et al. (2010) performed tests on specimens with butterfly shapes fuses (Figure 8). The test showed that SPSW with butterfly fuses can sustain more deformation and can sustain higher loads at larger drifts than the same plates without fuses. Yielding was initiated at the quarter height of the butterfly cut out.

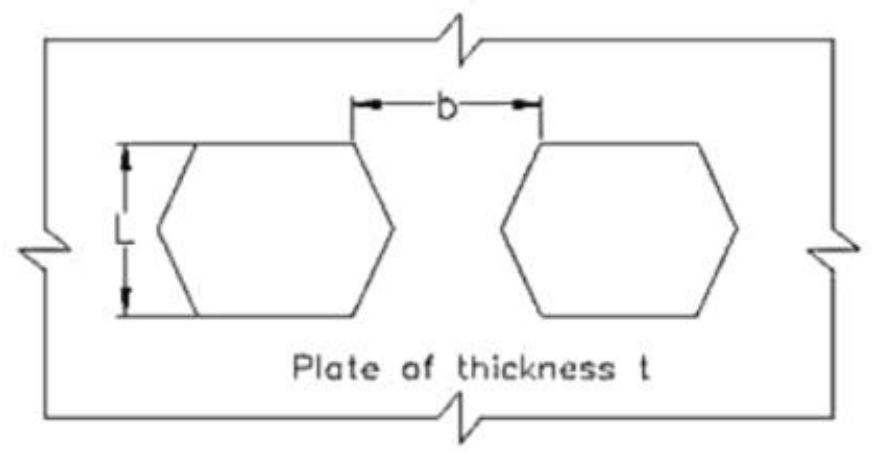

Figure 8. SPSW with butterfly shapes fuses [from (Ma et al. 2010)]

\subsection{Computational study of RS-SPSW}

Computational study of RS-SPSW was conducted by Maurya (2012). The finite element program ABAQUS was used to run full-scale models and prove that RS-SPSW experience less buckling and demonstrate fuller hysteretic behavior comparing with SPSW.

Constrained and unconstrained ring models were used to understand the yielding mechanism of the ring and for developing equations for the strength and stiffness of the full wall. It was found that up to elongation of $20 \%$ of the ring diameter the Poisson's ratio of the ring is equal to 1.0 . 
Four plastic mechanisms were proposed to explain the inelastic behavior of the ring in the full wall. Analytical solutions for the stiffness and strength of the full plate were derived. The effect of the initial imperfections on the RS-SPSW was studied. It was found that initial imperfections larger than $\mathrm{L} / 100$ result in significant reduction in the strength and stiffness.

The parametric study was done to understand the influence of geometric parameters of the plate on the plate behavior. The specimens with larger rings experienced a combination of global tension field buckling and lateral torsional buckling of the rings, while the specimens with the smaller rings experienced mostly global shear buckling. It was found that thicker plates exhibit fuller hysteretic behavior and larger energy dissipation.

In summary, the computational simulations showed that the RS-SPSW concept was highly promising. Future experimental study was recommended to prove the computational results. 


\section{RING-SHAPED SPSW CONCEPT}

The main goal of this research was to reduce buckling of SPSW. One of the ways to do it is to use ring shaped cutouts. When any object is stretched in one direction, elongation in the longitudinal direction is typically accompanied by contraction in the transverse direction. The ratio between these two values for a material is defined by the Poisson's ratio. For structural steel this value is typically equal to 0.3. It means that the elongation of the SPSW in the tension field direction is significantly bigger than the transverse contraction, which causes buckling in the plate. Observing the transformation of a ring undergoing stretching, it can be noticed that its extension in the direction of the applied load is almost equal to the contraction of the ring in the transverse direction. It can also be proven mathematically.

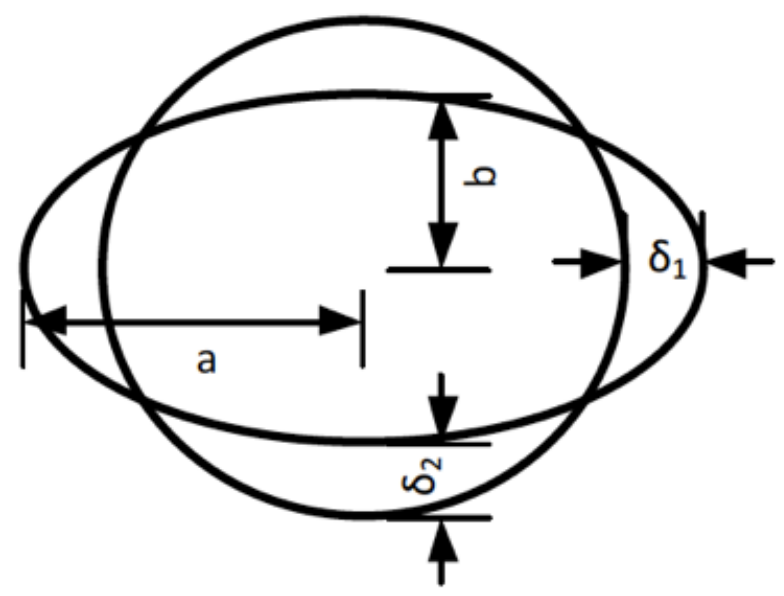

Figure 9. Circle and ellipse (result of ring deformation) geometry

Consider a circle with radius $r$, and an ellipse (resulting from ring elongation) with dimensions $a$ and $b$ as shown in Figure 9. The elongation of the circle in the longitudinal direction and its shortening in the transverse direction can be given by the following equations:

$$
\begin{aligned}
& \delta_{1}=a-r \\
& \delta_{2}=b-r
\end{aligned}
$$

Now the task is to find a relationship between $\delta_{1}$ and $\delta_{2}$ (elongation of the ring in one direction and shortening in the transverse direction).

The perimeter of the circle and ellipse are given by the following equations:

$$
P_{\text {circle }}=2 \pi r
$$




$$
P_{\text {ellipse }} \approx 2 \pi \sqrt{\left(a^{2}+b^{2}\right) / 2}
$$

As far as perimeter should not change just because the change of the shape, these two perimeters should be equal resulting in the following set of equations:

$$
\begin{aligned}
& 2 \pi r=2 \pi \sqrt{\left(a^{2}+b^{2}\right) / 2} \\
& r^{2}=\left(a^{2}+b^{2}\right) / 2 \\
& r^{2}=\frac{\left(\delta_{1}+r\right)^{2}+\left(\delta_{2}+r\right)^{2}}{2} \\
& \delta_{2}=r-\frac{1}{2} \sqrt{4 r^{2}-4 \delta_{1}^{2}-8 r \delta_{1}}
\end{aligned}
$$

The graph representing the comparison of the Poisson ratios for the solid steel plate and ring is given below (Figure 10). The green line "Ring" shows Eq.3.8, and red line "Solid Plate" represents 0.3 Poisson ratio for standard SPSW. The green line "Ring" shows that during small deformation, elongation of the ring in one direction is almost equal to the shortening of the ring in the transverse direction. This is a improved behavior compared with a solid plate, as the Poisson's ratio is almost equal to 1.0, which means buckling should be reduced.

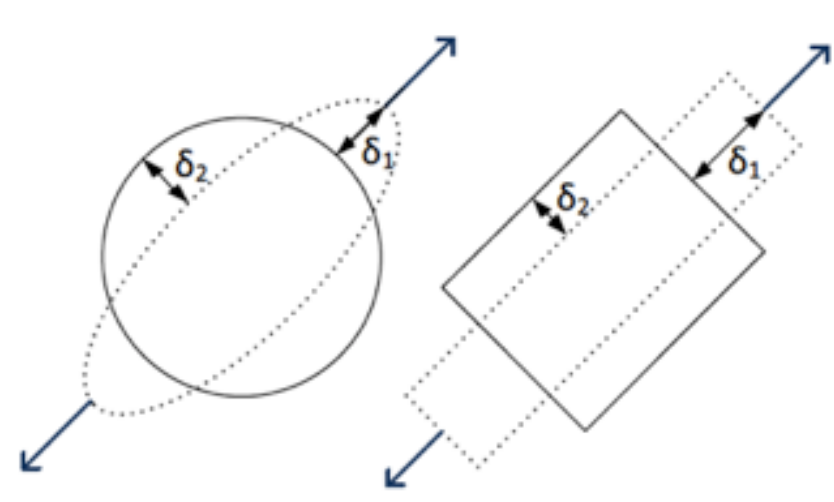

a. Ring

b. Solid Plate

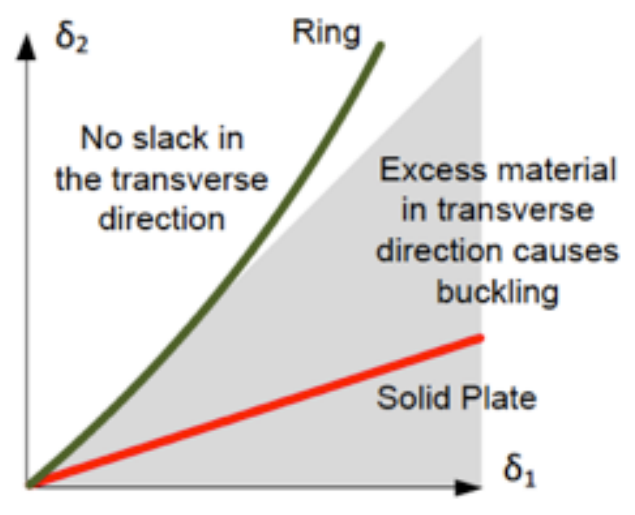

c. Relationship Between $\bar{\delta}_{1}$ and $\bar{\delta}_{2}$

Figure 10. Concept graphical explanation [from (Maurya 2012)]

Maurya (2012) conducted a computational study of the ring-shaped SPSW concept (example shown in Figure 11). Full-scale models with different configurations and ring sizes have been analyzed using finite element analysis program ABAQUS. Furthermore two ring units with different boundary conditions have been studied for better understanding of deformation ring behavior. Analytical and empirical equations for the stiffness of the full wall were derived. Analytical solution for the strength of the unit ring and full wall was developed. Random imperfections and imperfections in the shape of 
buckling modes of the web plate were studied and considered while running the models. The computational study showed that BR-SPSW exhibits fuller hysteresis than solid SPSW. According to Maurya (2012), two buckling modes were predicted to occur. The specimens with larger rings showed a combination of global tension field buckling and lateral torsional buckling of the rings, while the specimens with smaller rings mostly showed global tension field buckling. It was also found out that up to $20 \%$ of elongation of the ring diameter, the effective Poisson's ratio of the ring was approximately 1.0.

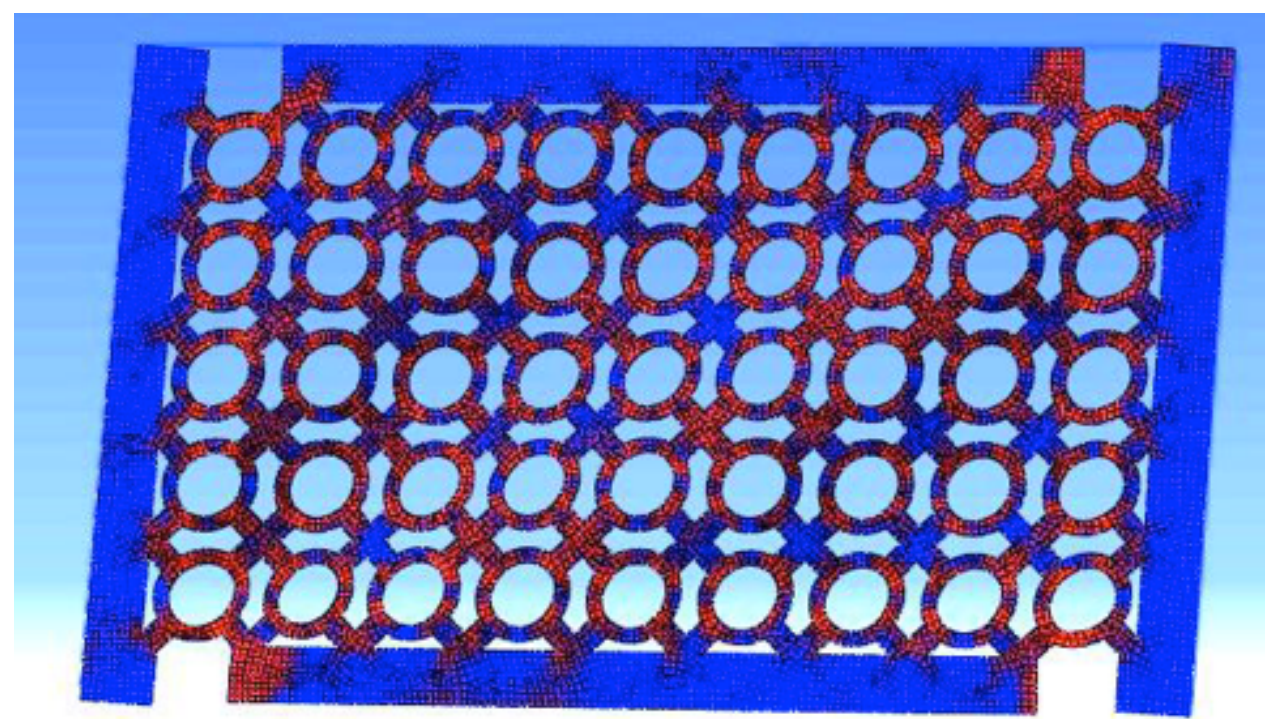

Figure 11. Computational model of full-scale BR-SPSW [from (Maurya 2012)]

A parametric study was done to understand the sensitivity of the system behavior on the geometric features of BR-SPSW followed by detailed finite element analysis. In summary, the computational simulations showed that the RS-SPSW concept was highly promising but experimental testing was necessary to prove the theoretical concept. 


\section{DEVELOPING A METHOD TO LIMIT LINK YIELDING}

Understanding the ring behavior as subjected to shear load was found to be important for determining the strength of the RS-SPSW system and its yield capacity. Preliminary analytical expressions for strength have been derived using plastic mechanism analysis as provided in Maurya (2012). Interaction between axial forces and flexure were neglected because the effects were generally found to be small [Maurya (2012)]. The mechanism considered (shown in Figure 12b), assumes that four plastic hinges will form. The shear loads (horizontal component of the diagonal force) associated with this mechanism were derived in Maurya (2012) and are given by Eq.(4.2) The equation is based on the geometry of the ring shown in Figure 12a including the centerline radius, $R$, the ring width, $w_{c}$, the link width, $w_{L}$, and the plate thickness, $t$. The plastic moment capacity, $M_{p}$, is given by Eq. (4.1) as a function of the plate yield strength, $F_{y}$.
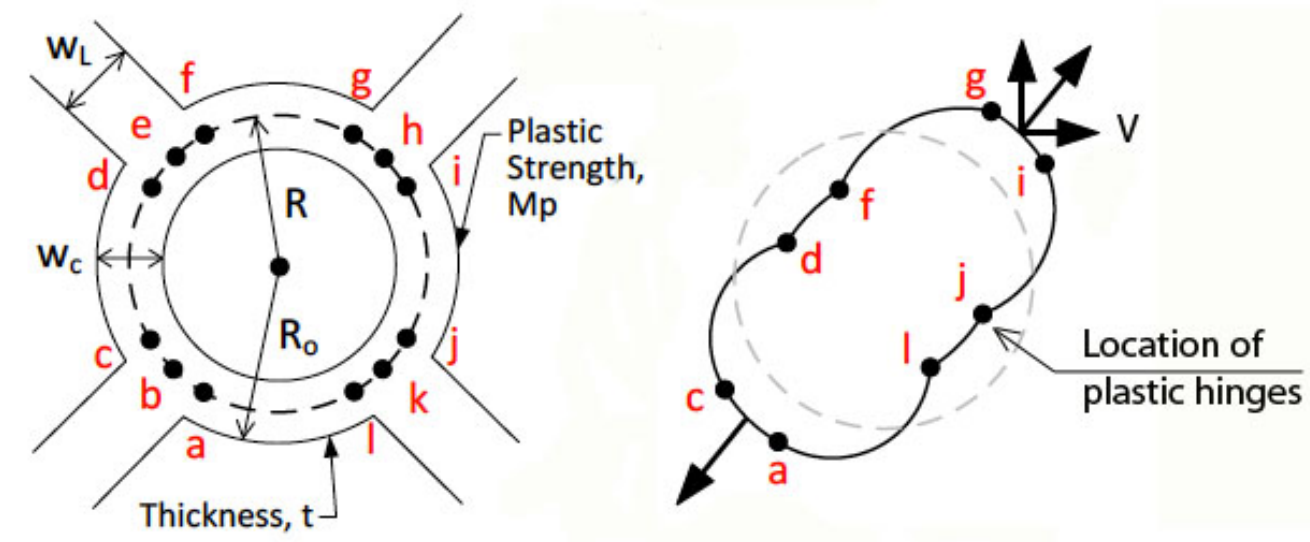

a. Ring geometry

b. Plastic Mechanism

Figure 12. Plastic mechanism analysis to determine shear yield capacity of the RSSPSW

$$
\begin{gathered}
M_{p}=\frac{w_{c}^{2} t}{4} F_{y} \\
V=\frac{4 * M_{p}}{\sqrt{2} *\left(R-{ }^{W_{L}} / 2\right)}
\end{gathered}
$$

Choosing the width of link $w_{L}$ and the width of the ring $w_{c}$ relative to the ring outer diameter $R_{0}$ is an important part of design in that an improper choice could lead to yielding of the links rather than plastic deformation of the rings. If the diagonal force in the ring becomes bigger than the link tension yield capacity, it means that the link will yield. If the link starts to yield before the ring itself, it means that the whole ring mechanism described before cannot be developed and the plate doesn't deform in a desirable way. That is why it is important that the link proportional ratio, $Y$, satisfy the following condition: 


$$
Y=\frac{T_{c}}{T_{d}} \geq 1
$$

where $T_{c}$ - tension capacity of the link, $T_{d}$ - tension demand of the link equal to the diagonal force in the ring due to ring yielding.

$$
\begin{aligned}
& T_{c}=t_{w} * w_{L} * F_{y} \\
& T_{d}=V / \cos 45^{\circ} \\
& Y=\frac{t_{w^{*} w_{L} * F_{y}}}{\frac{V}{\cos 45}^{\circ}}=\frac{t_{w^{*} w_{L} * F_{y} *\left(R \sqrt{2}-\frac{w_{L}}{\sqrt{2}}\right) * \cos 45^{\circ}}}{4 * M_{p}} \geq 1
\end{aligned}
$$

After simplification Eq. (4.6) becomes:

$$
Y=\frac{w_{L} * R_{O}-w_{L}^{2}}{w_{c}^{2}} \geq 1
$$

Eq. (4.7) is useful for a design, because for a given $R_{0}$ it can always be checked if $w_{L}$ and $w_{C}$ chosen for a design satisfy this equation and thus the ring mechanism is working. Eq. (4.7) demonstrates the ratio of tension capacity of the link to the tension demand of the link. Thus it is desirable to make the yield ratio, $Y$ as large as possible. It is possible to calculate the value of $w_{L}$ that maximizes the numerator, and thus maximize the yield ratio. By taking the first derivative of the numerator and setting it equal to zero, the value for $w_{L}$ that maximizes the yield ration is

$$
w_{L}=R_{0} / 2
$$

After maximizing the numerator, it is desirable to minimize the denominator. This means using the least ring width, $w_{c}$. If the optimal value of $w_{L}=R_{0} / 2$ is used, the maximum $w_{c}$ that will just satisfy the target value is

$$
w_{c}=R_{0} / 2
$$

In this case, the value of $w_{c}$ should be less than or equal to $R_{0} / 2$. 


\section{TESTING}

\subsection{Test setup}

The test setup shown in Figure 13 was designed to apply shear deformations to a steel plate. The fabrication of the frame was performed in the Structural Laboratory of Virginia Tech. The free end shown on the left of Figure 12 was moved up and down using an MTS Model 243.60 Hydraulic Actuator. The fixed end, shown on the right of Figure 13, was anchored to the strong floor. The web plate was sandwiched between angles on all four edges. The horizontal angles were attached to the vertical elements using 2 in diameter steel pins. The horizontal angles therefore acted as pinned struts that hold a constant distance between the pins creating an arc motion of the free side. The shear panel specimen was bolted to the test frame (instead of welded) to allow many specimens to be tested. The pins were aligned with the centerline of the bolts. Out-of-plane lateral bracing was provided for the actuator and at the middle of the W12x26 free end vertical element (Figure 14). The fixed side of the SPSW panel was restrained from out-of-plane motion by minor axis flexural rigidity of the reaction frame and its connection to the strong floor.

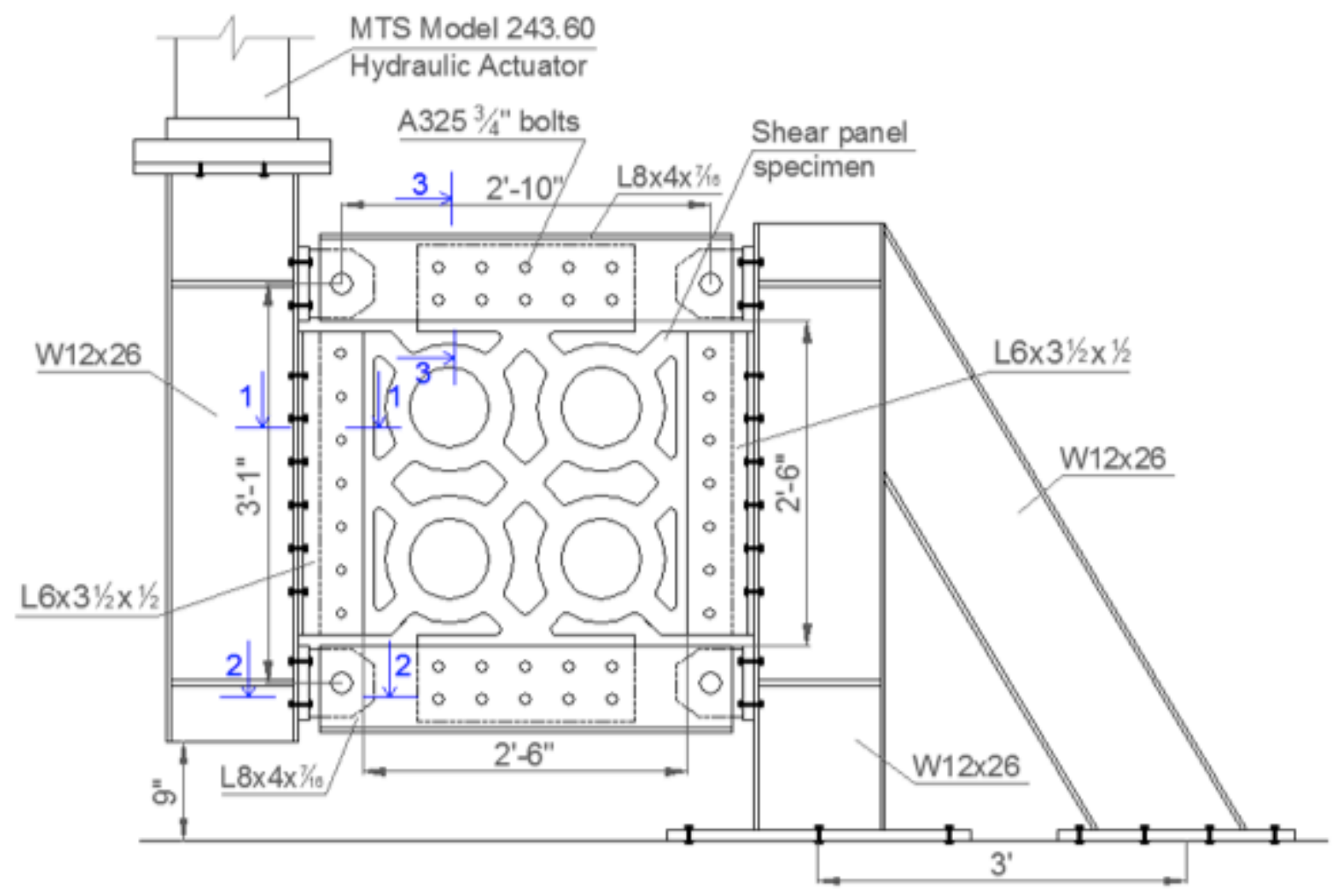

Figure 13. Side View of the Experimental Setup 


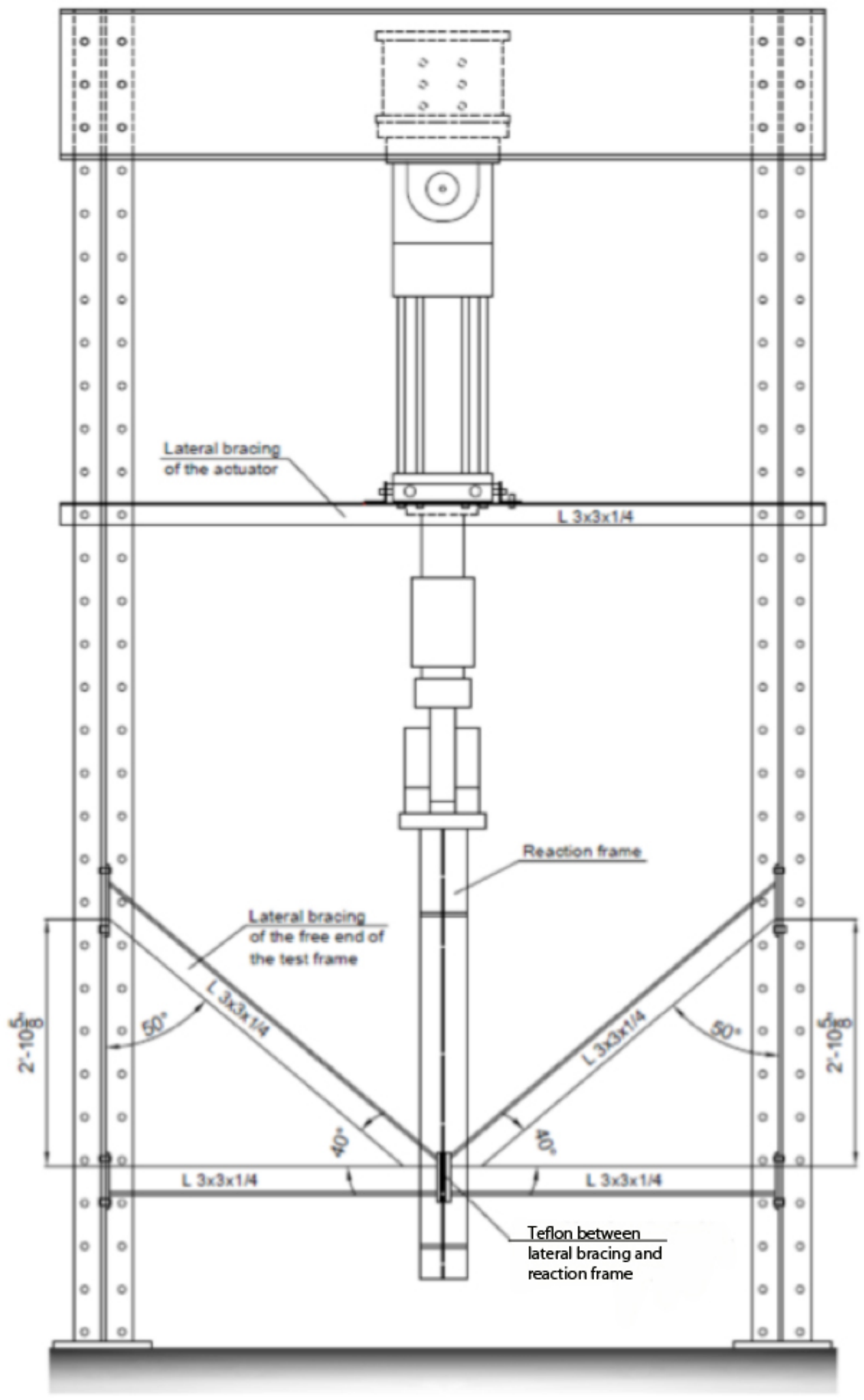

Figure 14. Horizontal and diagonal bracing 


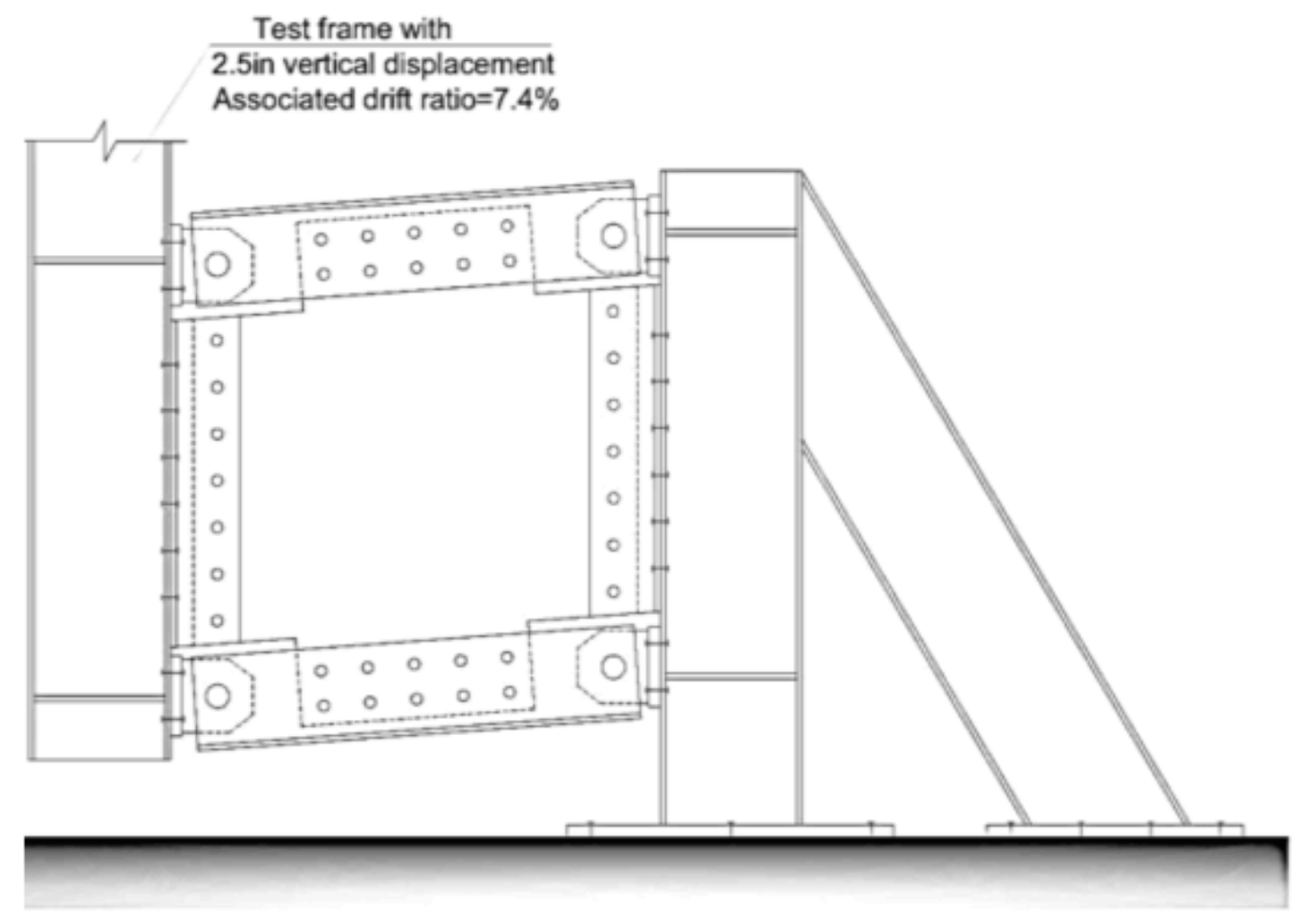

Figure 15. Test frame with vertical displacement of 2.5 in (7.4\% drift ratio)

Figure 15 shows the test frame with a vertical displacement of $2.5 \mathrm{in}$, which corresponds to 7.4\% panel drift ratio. The distance between free end of the frame and the floor at "no shear" position was 9 in. Thus the maximum displacement that was possible with this set up was 9 in up and down. But the test frame configuration was not the only parameter that dictated the limits for maximum displacements during the test. The other limit state was the stroke of the actuator. The detailed description of actuator characteristics is provided in Chapter 5.5.5. That model of the actuator allowed the stroke to move 10 in up and down. At beginning of the test stroke was already 3 in down (the way the actuator was connected to the surrounding frames dictated that). Thus it could move only 7 more inches down during the test. The last limit state that ended up to be the most strict and governed the maximum possible displacements during the test was the length of string potentiometer, connected to the bottom of the free end of the frame (see Chapter 5.5.2 for string potentiometers location). The stroke of the string potentiometer was $10 \mathrm{in}$, which meant that only 5 in up and down displacements were possible during the test. 
Figure 16 shows the position of the test frame on the reaction floor in the Structural Lab of Virginia Tech. The reaction floor consists of continuous beams built-in into the floor with $8 \mathrm{ft}$ spacing and $5 \mathrm{ft}$ long beams built-in between them.

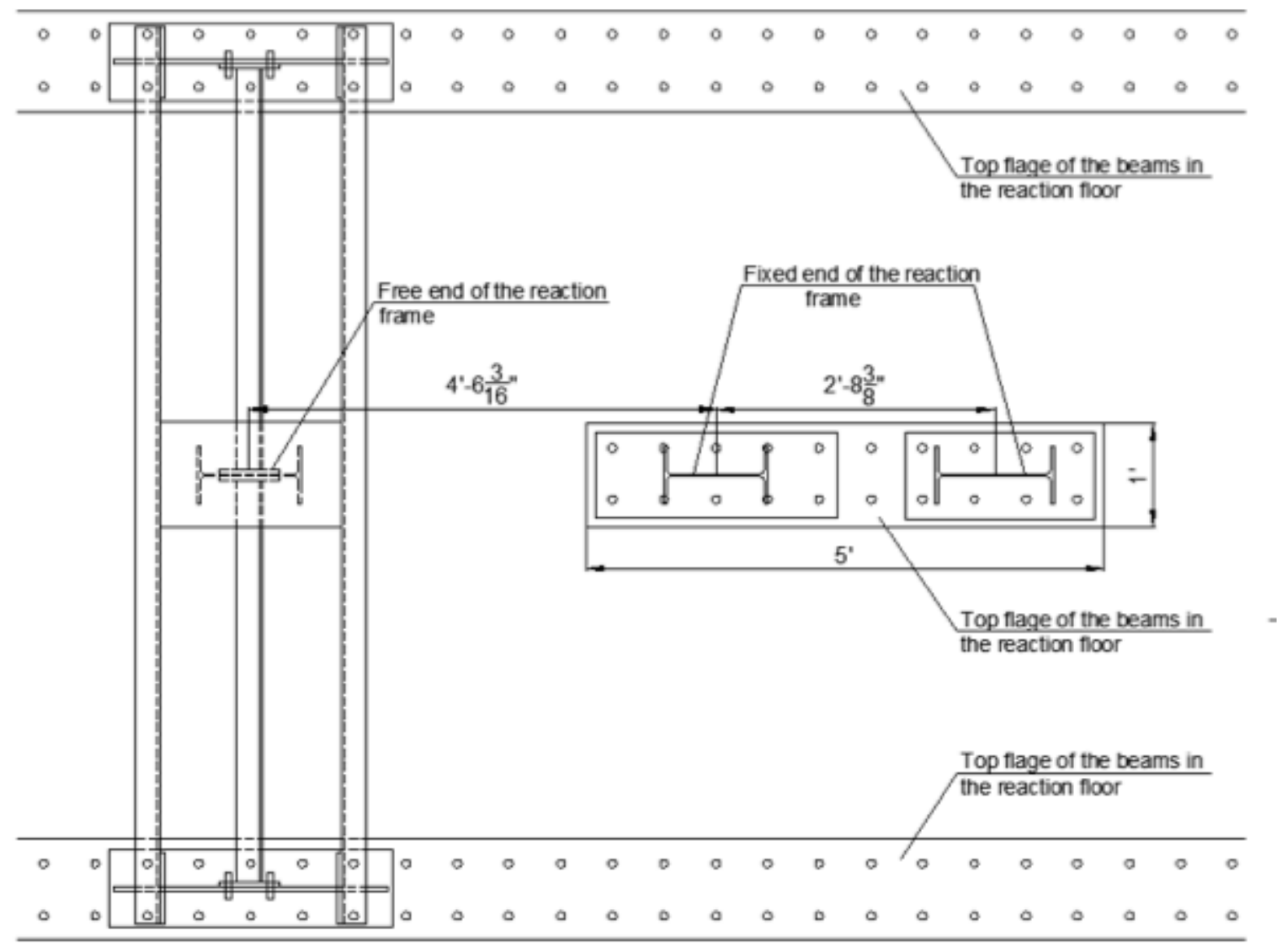

Figure 16. Top view: position on the reaction floor and bracing

Detailed bolted and pin connections are shown in Figure 17. All bolts were fully pretensioned before every test. 


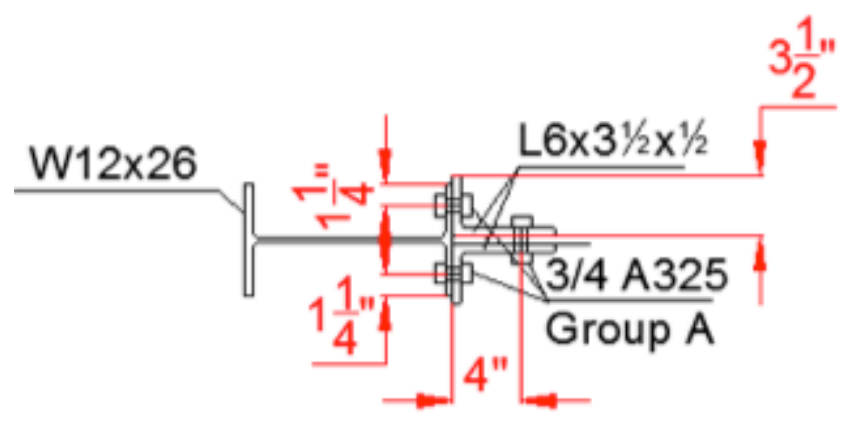

a. Section 1-1

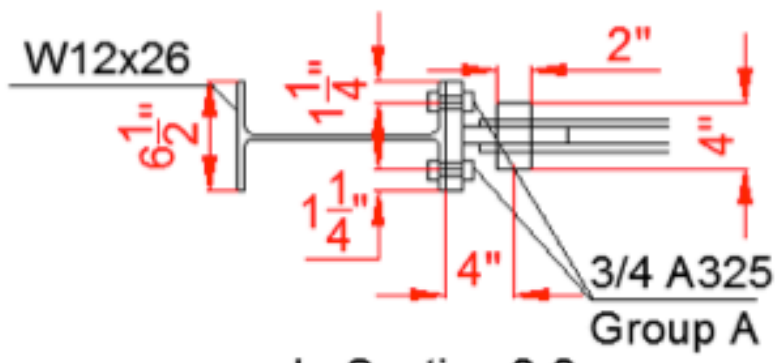

b. Section $2-2$

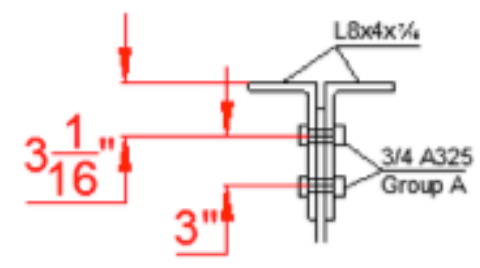

c. Section 3-3

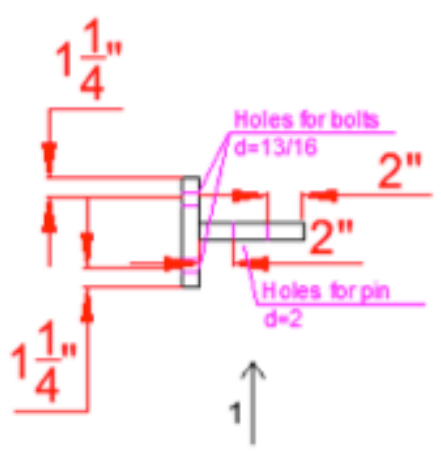

d. Pin connection elements top view

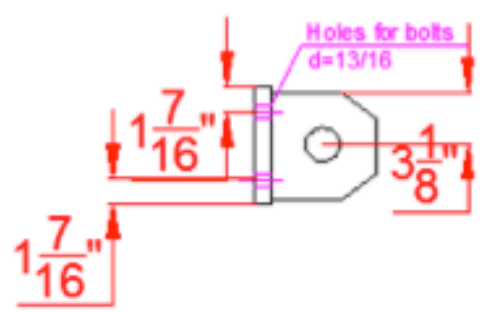

e. Pin connection elements view 1

Note: See the previous figures for location of these sections

Figure 17. Bolted and pin connections

\subsection{Loading protocol}

Today's seismic design codes, standards and guidelines reference the need for performance assessment through testing, but with few exceptions they remain mostly silent on testing and acceptable criteria to be used for this purpose. Therefore there is no standard loading protocol. This issue was discussed in detail by Krawinkler (2009).

In earthquake engineering, strength and deformation capacities depend on cumulative damage, which implies that every component has a permanent memory of past damaging events and at any instance in time it will remember all the past cycles that have contributed to the deterioration in its state of health. Thus performance depends on the 
history of previously applied damaging cycles, and the only reasonable way to assess the consequences of history is to replicate, as best as possible, the load and deformation histories a structure will undergo in an earthquake. The object of a loading protocol is to achieve this in a conservative, but also statically representative manner.

There is no "best" loading history, because no two earthquakes are alike and because the specimen may be representative of a subassembly in many different structural configurations. Any load protocol will always be a compromise that will provide deformations whose realism will depend on earthquake magnitude, distance from epicenter and soil type. The number and amplitude of cycles the component will experience depend on the configuration, strength, stiffness, and modal properties of the structure, and also on the deterioration characteristics of the structural systems and its components. Moreover, on most practical cases the actual sequence of cycles as experienced in an earthquake has to be rearranged into cycles of increasing magnitude in order to avoid commitment to a single maximum amplitude that may have meaning only for a specific combination of ground motion and structural configuration.

For reasons just cited, there are several decisions required to come up with a compromise loading history. Several loading protocols for steel structures have been proposed in the literature and used in testing programs. These protocols recommend somewhat different histories, but in most cases they differ more in detail than in concept.

The description of two most often used protocols for steel structures are the ATC-24 and the SAC [Clark et al. (1997)] protocols. The ATC-24 loading protocol is anchored to the yield deformation of the subassembly $\Delta_{\text {yield }}$. The ATC-24 loading history contains 6 elastic cycles, which amplitude is smaller than $\Delta_{\text {yield }}$, followed by $\Delta_{\text {yield }}, 2 \Delta_{\text {yield }}, 3 \Delta_{\text {yield }}$ amplitude cycles repeated for three times, followed by $4 \Delta_{\text {yield }}, 5 \Delta_{\text {yield }}, 6 \Delta_{\text {yield }}$, etc. amplitude cycles repeated for 2 times, until the severe damage of the component will occur.

Conversely, the controlling parameter for the SAC loading protocol is story drift angle. The SAC protocol contains more elastic cycles than the ATC-24 Protocol, two cycles of intermediate amplitude and much fewer cycles of larger amplitudes, but otherwise is similar to the ATC-24 Protocol.

As there is no strict requirement for which protocol should be used, the literature on SPSW testing is examined to determine which protocol has been used for previous experimental programs.

The cyclic load history used by Caccese et al. (1993) (see Figure 18) consisted of displacement peaks that were increased in 8 increments of 0.25 in each up to a maximum of 2 in ( $2 \%$ drift). Each displacement cycle was repeated 3 times, for a total of 24 cycles. The entire displacement history was repeated for another 24 cycles. Finally the specimen was pulled monotonically to the displacement limit of the MTS actuator. 


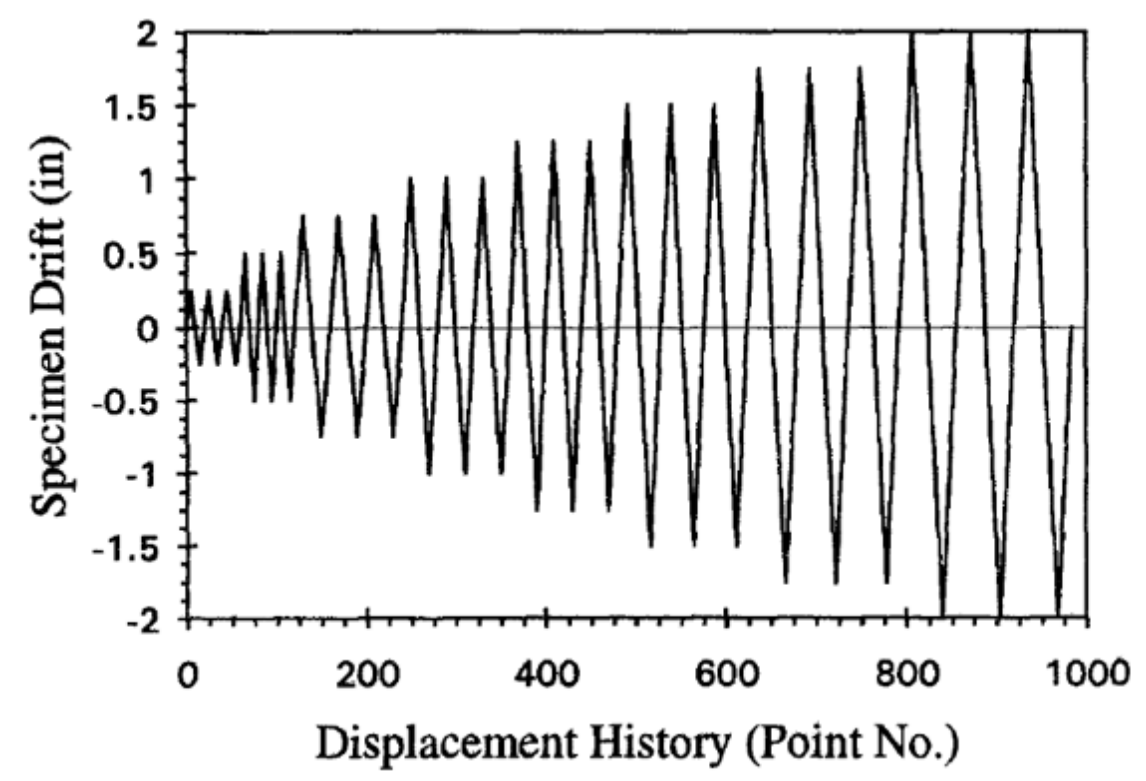

Figure 18. Cyclic load history [from (Caccese et al. 1993)]

The loading protocol applied by Chen and Jhang (2011) in the experimental study is shown in the Table 1 . The loading rate was kept under $0.7 \mathrm{~mm} / \mathrm{s}$. The structural tests were stopped when either the floor story drift angle exceeded $6 \%$, or if the strength of the specimen degraded to a value less than $80 \%$ of it's ultimate strength.

Table 1. Loading protocol [from (Chen and Jhang 2011)]

\begin{tabular}{|l|l|l|l|l|l|l|l|l|l|l|}
\hline Drift angle (\% rad) & 0.375 & 0.5 & 0.75 & 1 & 1.5 & 2.0 & 3.0 & 4.0 & 5.0 & 6.0 \\
\hline Number of cycles & 3 & 3 & 3 & 3 & 2 & 2 & 2 & 2 & 2 & 2 \\
\hline
\end{tabular}

Cyclic displacements used by Li et al. (2010) were imposed according to the loading history (Figure 19), starting from small drift and increasing to 5\%. The first three peak displacements were repeated for three times, next 10 peak displacements were repeated for 2 times. Altogether 29 cycles were applied to the structure. 


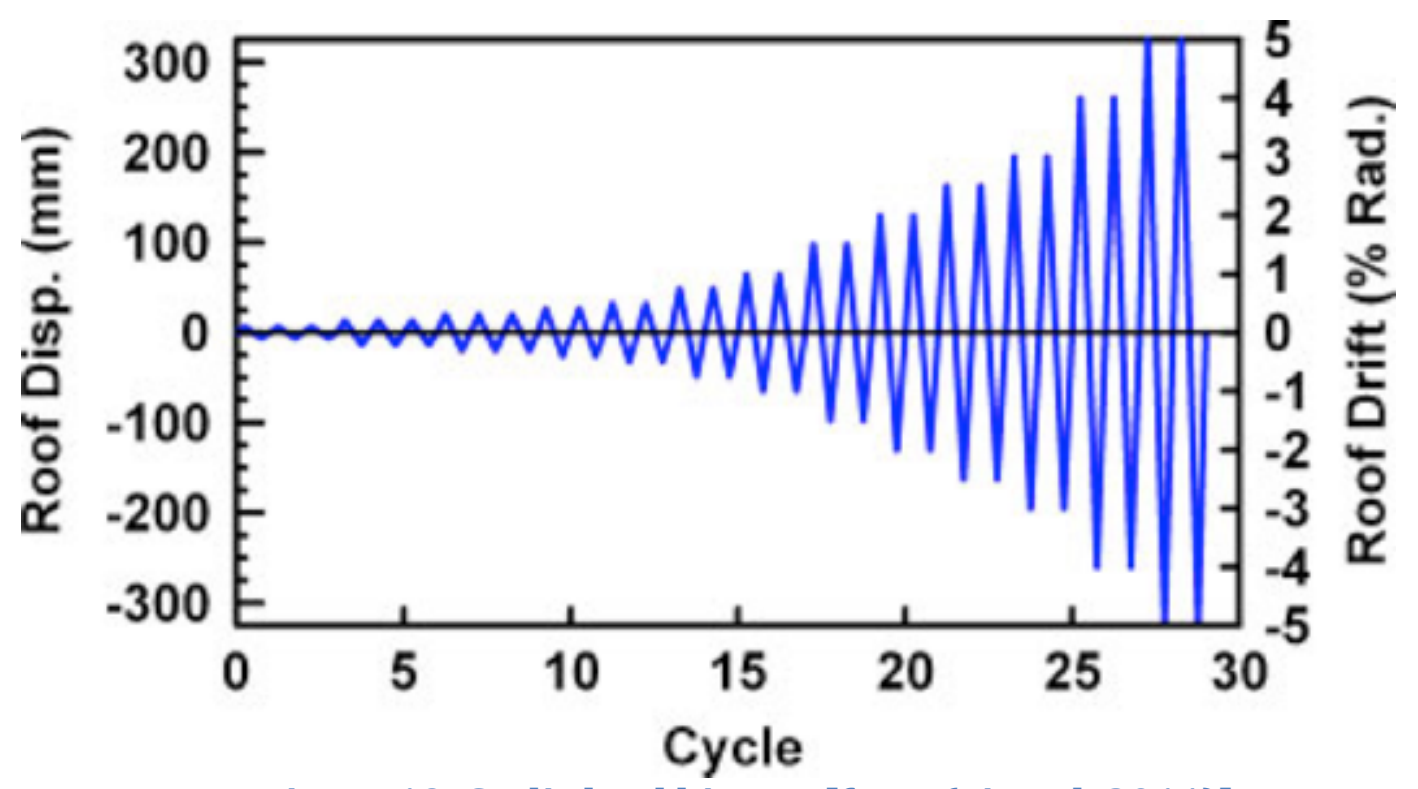

Figure 19. Cyclic load history [from (Li et al. 2011)]

Vian et al. (2009) developed loading protocol as a hybrid/combination of the ATC-24 (ATC-1992) protocol and the AISC Seismic provisions [AISC (2005)] requirements for loading sequence in cyclic testing.

Berman and Bruneau (2005) used FEMA 302 [FEMA (1997)] protocol in the experiments.

For this research the loading protocol was based on ATC-24 (ATC-1992), however it was decided to assume that $\Delta_{\text {yield }}=0.5 \%$ for all specimens in order not to make a new loading protocol for every single test. The cycles applied to the structure are shown in Table 2 . The actual applied displacements will be given in Chapter 6 .

\section{Table 2. Load cycles applied to the specimens}

\begin{tabular}{|l|l|}
\hline $0.5 \Delta_{\text {yield }}$ & 3 cycles \\
\hline $0.75 \Delta_{\text {yield }}$ & 3 cycles \\
\hline$\Delta_{\text {yield }}$ & 3 cycles \\
\hline $2 \Delta_{\text {yield }}$ & 3 cycles \\
\hline $3 \Delta_{\text {yield }}$ & 3 cycles \\
\hline $4 \Delta_{\text {yield }}$ & 2 cycles \\
\hline $5 \Delta_{\text {yield }}$ & 2 cycles \\
\hline $6 \Delta_{\text {yield }}$ & 2 cycles \\
\hline $8 \Delta_{\text {yield }}$ & 2 cycles \\
\hline $10 \Delta_{\text {yield }}$ & 2 cycles \\
\hline $12 \Delta_{\text {yield }}$ & 2 cycles \\
\hline
\end{tabular}




\subsection{Specimen design}

\subsubsection{Range of variables}

Eight different plates with various configurations were chosen in order to study the influence of different parameters on the behavior of the plate, such as number of rings in a row, thickness of the plate, ring diameter, ring width, and associated nondimensional slenderness parameters. Ring geometric parameters are shown on Figure 20. The range of variables (Table 3) was chosen in such a way that it would be possible to investigate the following questions:

- Is the RS-SPSW concept valid, and are there configurations of the RS-SPSW that can minimize buckling and develop full hysteretic behavior?

- Do plates with similar ring slenderness $\left(R_{0} / t_{w}\right)$ but different $R_{0}$ have similar buckling behavior or different?

- How sensitive is the buckling magnitude and buckling mode to three different nondimensional slenderness parameters (global slenderness ratio: $a / t_{w}$, and local slenderness ratios: $\left.R_{0} / t_{w}, R_{0} / w_{c}\right)$ ?

- How well does the plastic mechanism equation predict the shear strength of the panels over the range of tested configurations?

- How does the stiffness vary from one ring to a $3 \times 3$ grid of rings?

- Are the RS-SPSW plates prone to fracture?

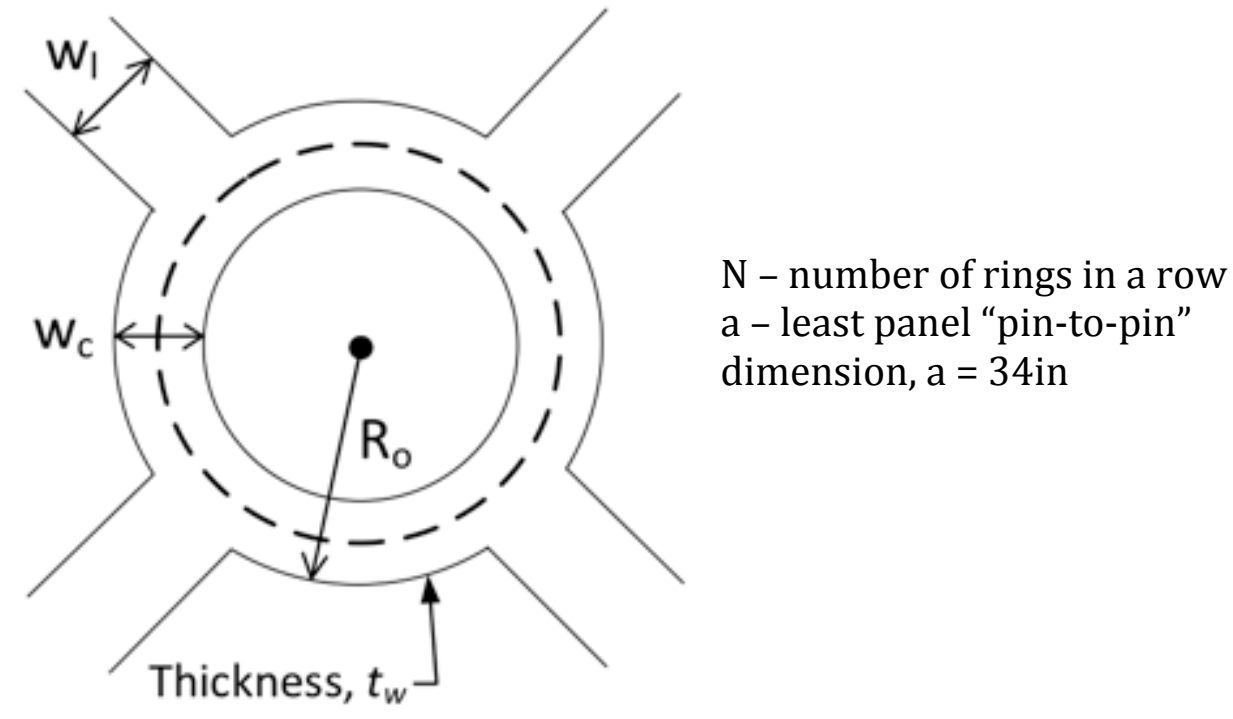

Figure 20. Ring geometric parameters 
Table 3. Test Matrix

\begin{tabular}{|c|c|c|c|c|c|c|c|c|c|c|c|}
\hline & & \multicolumn{4}{|c|}{ Basic dimensions } & \multicolumn{4}{c|}{ Nondimensional ratios } \\
\hline Test \# & Specimen \# & $t_{w}$ (in) & $R_{0}$ (in) & $w_{c}$ (in) & $w_{L}$ (in) & $N$ & $\begin{array}{l}R_{0} \\
/ t_{w}\end{array}$ & $\begin{array}{l}R_{0} \\
/ w_{c}\end{array}$ & $\begin{array}{l}R_{0} \\
/ w_{L}\end{array}$ & $\begin{array}{l}a \\
/ t_{w}\end{array}$ & $\begin{array}{l}w_{c} \\
/ t_{w}\end{array}$ \\
\hline 1 & $2-0.5-1$ & 0.5 & 5.89 & 2.21 & 2.21 & 2 & 11.8 & 2.67 & 2.67 & 68 & 4.42 \\
\hline 2 & Solid Plate & 0.075 & - & - & - & - & - & - & - & 453 & - \\
\hline 3 & $1-0.5-1$ & 0.5 & 11.79 & 4.42 & 4.42 & 1 & 23.6 & 2.67 & 2.67 & 68 & 8.84 \\
\hline 4 & $3-0.5-1$ & 0.5 & 3.93 & 1.47 & 1.47 & 3 & 7.9 & 2.67 & 2.67 & 68 & 2.94 \\
\hline 5 & $2-0.25-0.81$ & 0.25 & 5.89 & 1.8 & 2.21 & 2 & 23.6 & 3.27 & 2.67 & 136 & 7.20 \\
\hline 6 & $2-0.25-1$ & 0.25 & 5.89 & 2.21 & 2.21 & 2 & 23.6 & 2.67 & 2.67 & 136 & 8.84 \\
\hline 7 & $3-0.25-1$ & 0.25 & 3.93 & 1.47 & 1.47 & 3 & 15.7 & 2.67 & 2.67 & 136 & 5.88 \\
\hline 8 & $3-0.375-1$ & 0.375 & 3.93 & 1.47 & 1.47 & 3 & 10.5 & 2.67 & 2.67 & 91 & 3.92 \\
\hline
\end{tabular}

Specimen number (Table 3) is based of the specimen geometry, where the first number is the number of rings in a row, the second number is the thickness of the plate in inches, and the third number is the width of the ring to width of the link ratio. For example, Specimen 2-0.5-1 has 2 rings in a row, thickness of $0.5 \mathrm{in}$, and ratio of ring width to width of the link is 1.0 .

A solid plate with the same width and length dimensions was also tested in order to compare solid plate behavior with the behavior of the RS-SPSW. The thickness of the solid plate $(0.075 \mathrm{in})$ was chosen such that the strength of the solid plate would be approximately equal to the strength of the 0.5 " thick RS-SPSW panels. Furthermore, 0.075 in is representative of thinner SPSW web plates using in practice. Solid plate strength calculation was based on following equation [Berman and Bruneau (2005)]:

$$
V=\frac{1}{2} F_{y} * t_{w} * a * \sin 2 \alpha
$$

In the calculation of strength for the solid plate specimen it was assumed that $\alpha=45^{\circ}$, and that $F_{y}=43 \mathrm{ksi}$, based on tension coupon tests (See Chapter 5.4.3.)

\subsubsection{Specimen fabrication}

RS-SPSW specimens (example shown in Figure 21) were cut using a waterjet cutting machine at Waterjet Cutting Services, Inc. in Lynchburg, Virginia. The highest cutting quality available (level 5) was used for all cutting and was achieved by slowing down the speed at which the cutting was done. Figure 22 shows the waterjet cut quality. The price of cutting was based of the thickness of the plate and number of minutes it took to cut the plate (it takes more time to make a curved cut than a straight cut of the same length). The cost of all 7 RS-SPSW specimens (including both the material cost and the cost of cutting) 
was $\$ 3100$. The solid plate was cut using laser technology. The cost of solid plate was $\$ 200$ (including both the material cost and the cost of cutting).

In order to compare the cost of using SPSW and BR-SPSW in a building as a primary lateral load resisting system, let's assume 3-story building. Assume one story to be a square 120 $\mathrm{ft} x 120 \mathrm{ft}$ and steel weight to be $7 \mathrm{lb} / \mathrm{ft}^{2}$. The average cost of steel on the market today is $\$ 0.5 / \mathrm{lb}$ for material and $\$ 0.5 / \mathrm{lb}$ for fabrication. The cost of gravity framing can be calculated as $3 \times 120 \times 120 \times 7 \times(0.5+0.5)=\$ 302,400$. Assume that the boundary elements for SPSW and RS-SPSW are the same. Reduction of cost due to no moment connection and no special inspection for RS-SPSW can be roughly assumed to be $\$ 1,000$ per connection, which means $\$ 1000 \times 6=\$ 6,000$ for a given building per frame (one frame has 6 conncetions). Assume SPSW to be $12 \mathrm{ft} \times 12 \mathrm{ft}$ floors. Cost of SPSW per $\mathrm{ft}^{2}$ can be calculated as: $\$ 200 /\left(34\right.$ in $\left.\times 34 / 144 \mathrm{ft}^{2}\right)=\$ 24.91 / \mathrm{ft}^{2}$. Total cost of SPSW per frame is $\$ 24.91 / \mathrm{ft}^{2} \times 12 \mathrm{ft} \times 12 \mathrm{ft} \times 3=\$ 10,762$. Cost of RS-SPSW per $\mathrm{ft}^{2}$ is: $\$ 3100 / 7 /(34 \times 34 / 144$ $\left.\mathrm{ft}^{2}\right)=\$ 55.16 / \mathrm{ft}^{2}$. Total cost of RS-SPSW per frame is $\$ 55.16 / \mathrm{ft}^{2} \times 12 \mathrm{ft}$ x $12 \mathrm{ft} \times 3=\$ 23,831$. The total structure cost with SPSW is $\$ 302,400+\$ 10,762 * 4$ frames $=\$ 345,448$. The total structure cost with RS-SPSW is $\$ 302,400+(\$ 23,831-\$ 6000) * 4$ frames $=\$ 373,724$. Use of RS-SPSW instead of SPSW increases the structure cost by $8 \%$.

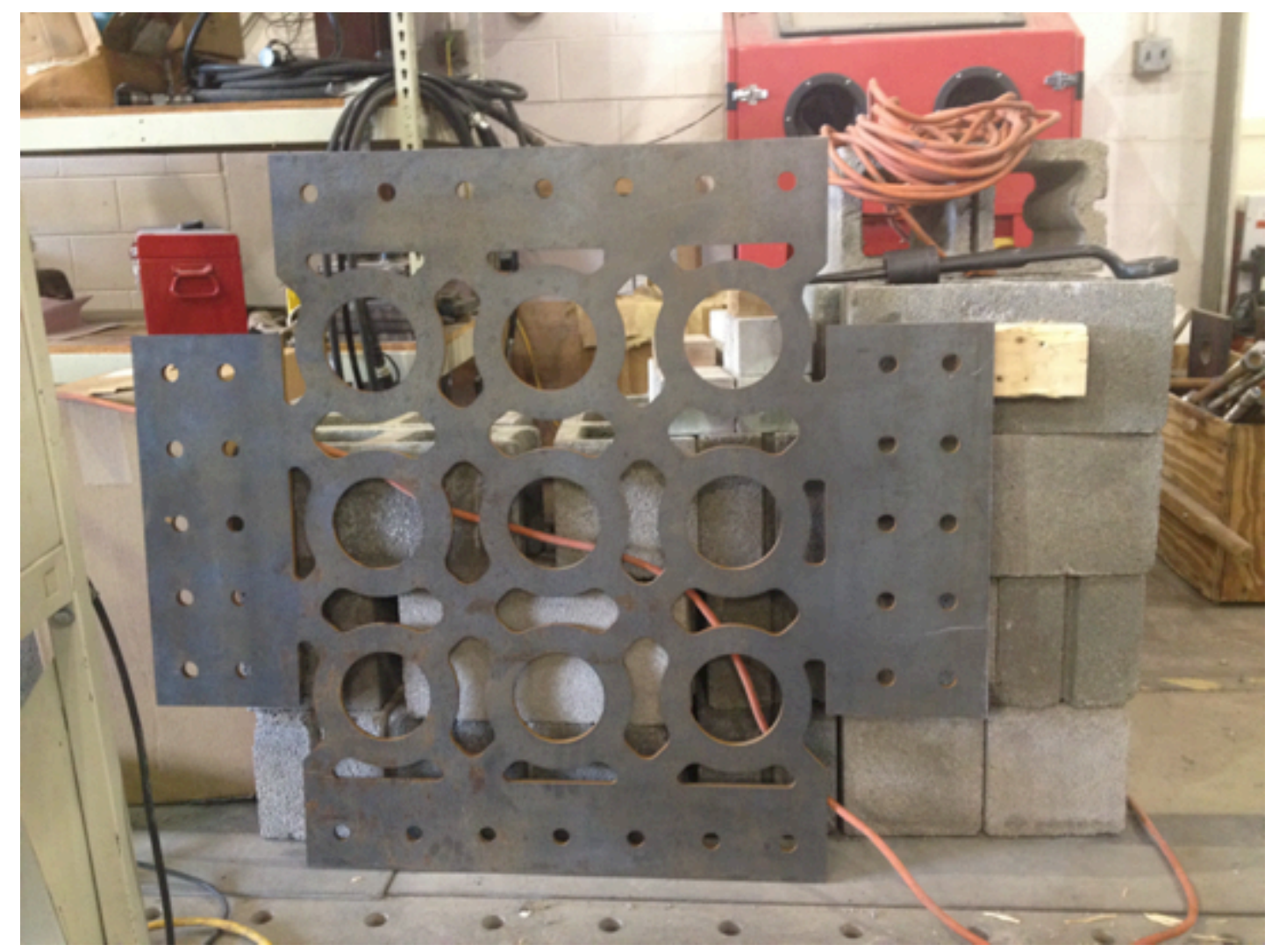

Figure 21. Example of the specimen 


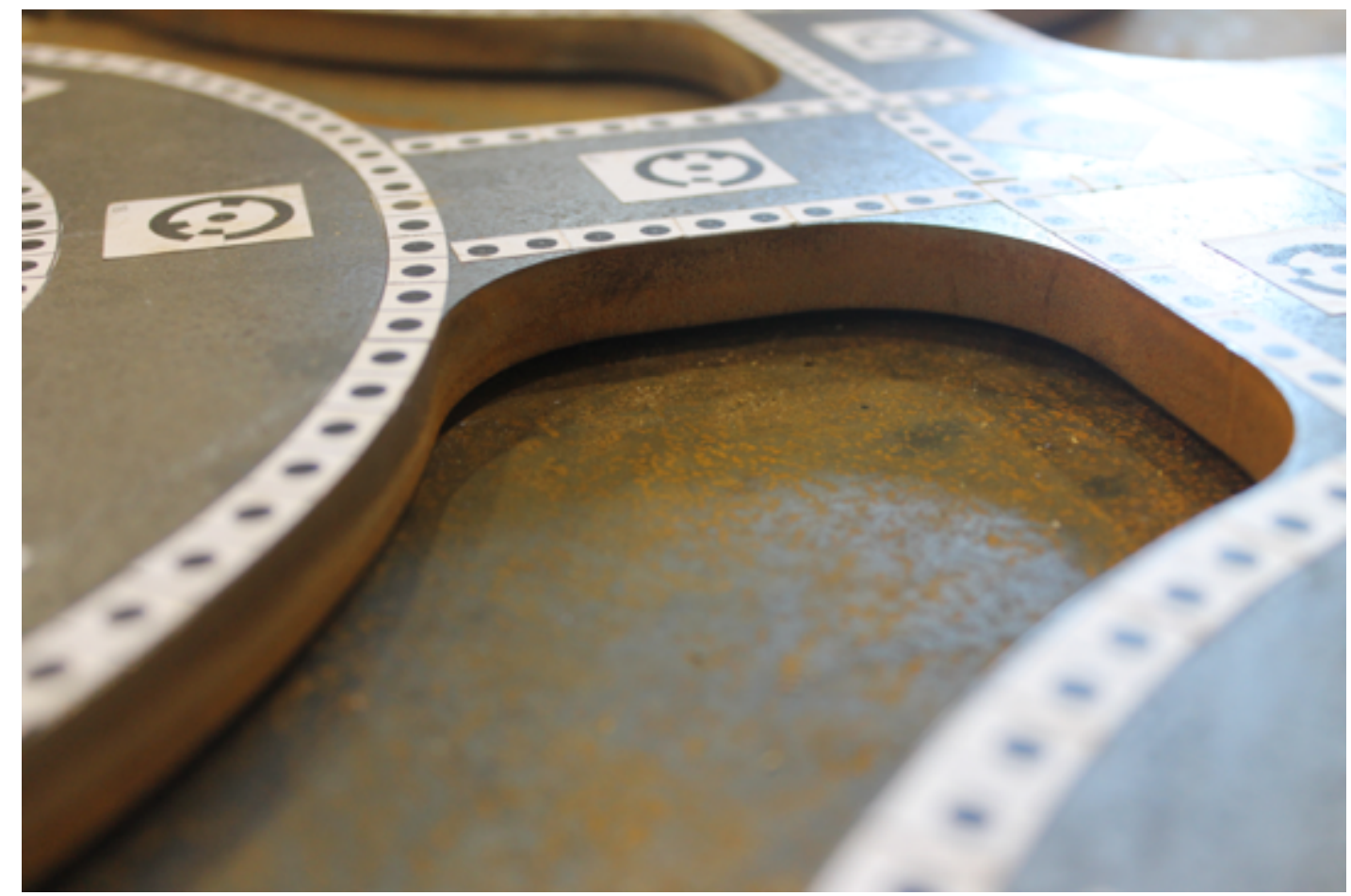

Figure 22. Waterjet cut quality

\subsection{Material tests}

Material properties were determined from ancillary tests on coupons cut from the same heat of steel as the test specimens. The coupons were cut from the same piece of steel plate as the test specimens. This chapter describes the test methods and results of the ancillary material tests.

\subsubsection{General}

Three tension tests on dogbone-shaped tension coupons for each material thickness $(1 / 2$ ", $\left.0.075 ", 3 / 8^{\prime \prime}, 1 / 4^{\prime \prime}\right)$ were conducted to measure material properties. The coupons were tested using a $600 \mathrm{kN}$ capacity MTS uniaxial testing machine. ASTM standard A370-07a was followed, which outlines the method for tension testing of metals, and also ASTM standard E8-01, which summarizes the test methods for tension testing of metallic materials.

\subsubsection{Test Setup and Methods}

Figure 23 shows the tension specimens. The specimens were cut using a waterjet cutting machine at Waterjet Cutting Services, Inc. in Lynchburg, Virginia. The highest level of cutting was used (Figure 25). 


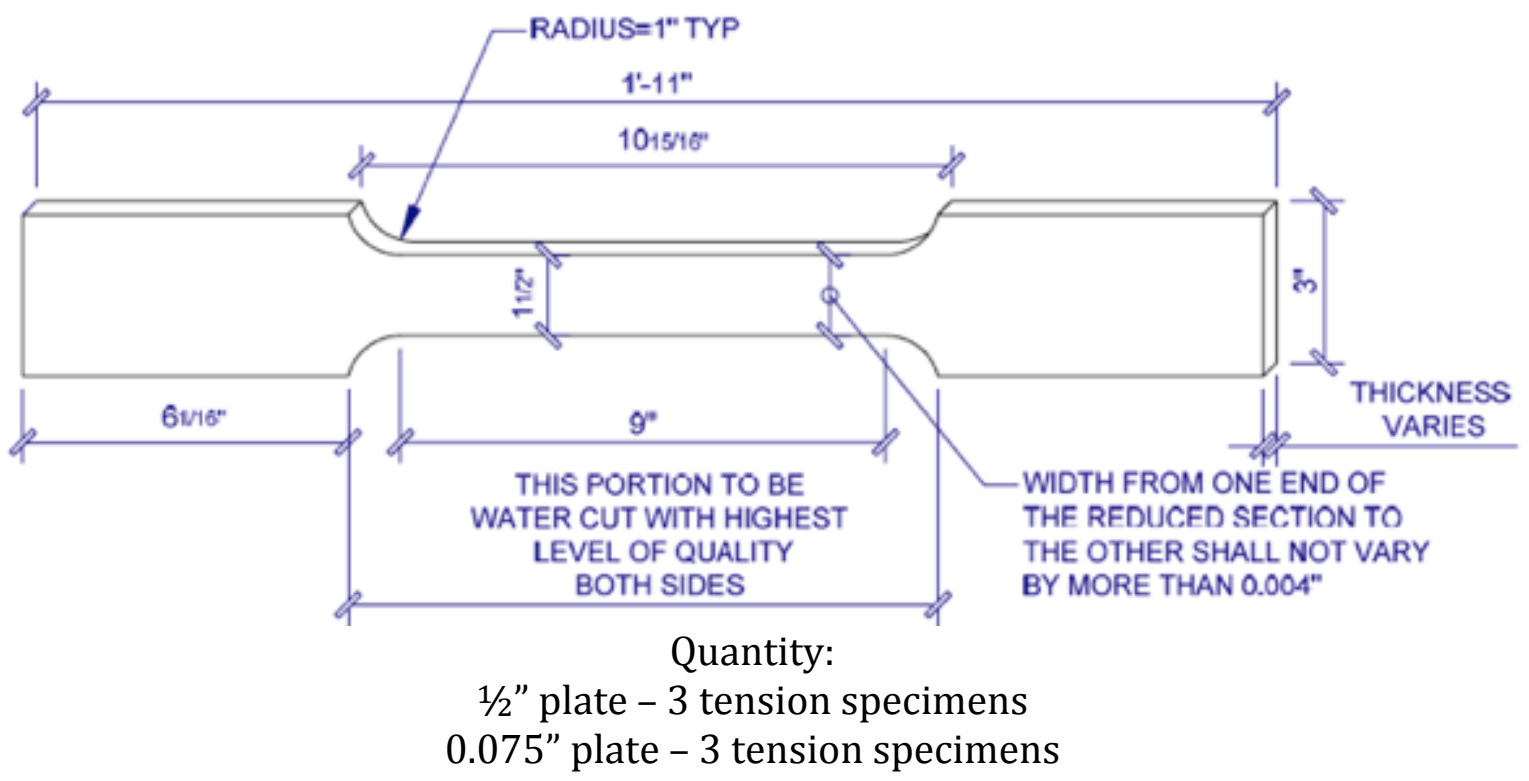

Figure 23. Design drawing for Plate tension specimens

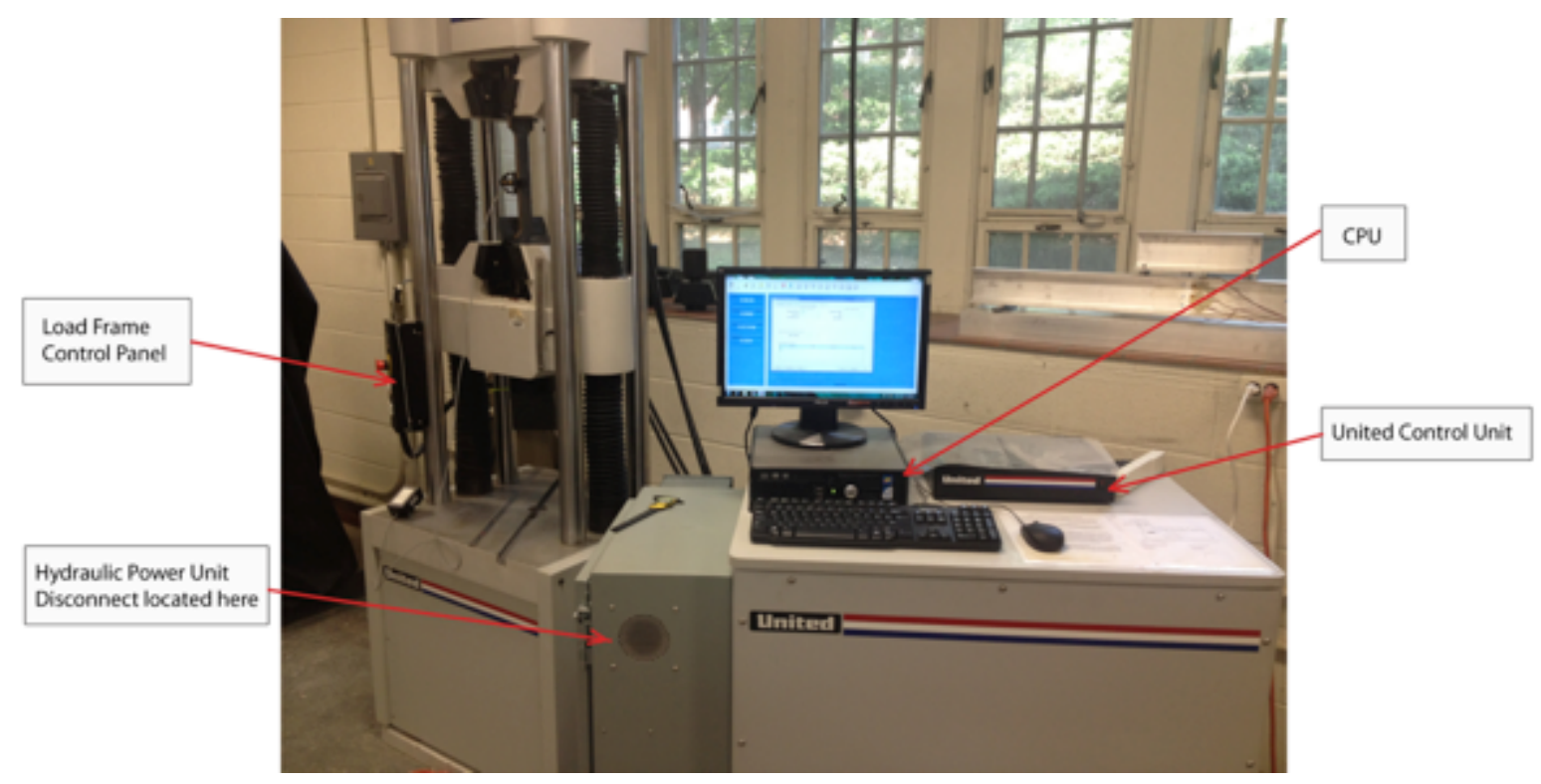

Figure 24. United SHFM Servo-Controlled Hydraulic Floor Model Universal Testing Machine 


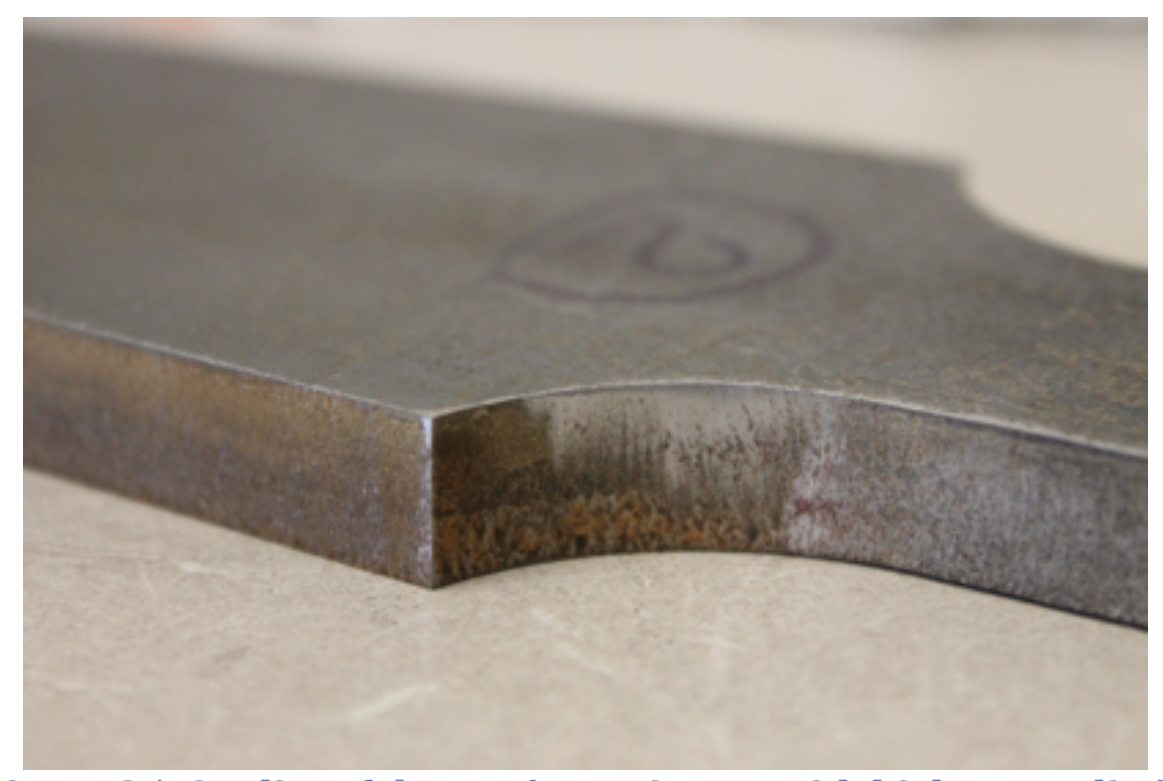

Figure 25. Quality of the cut (waterjet cut with highest quality)

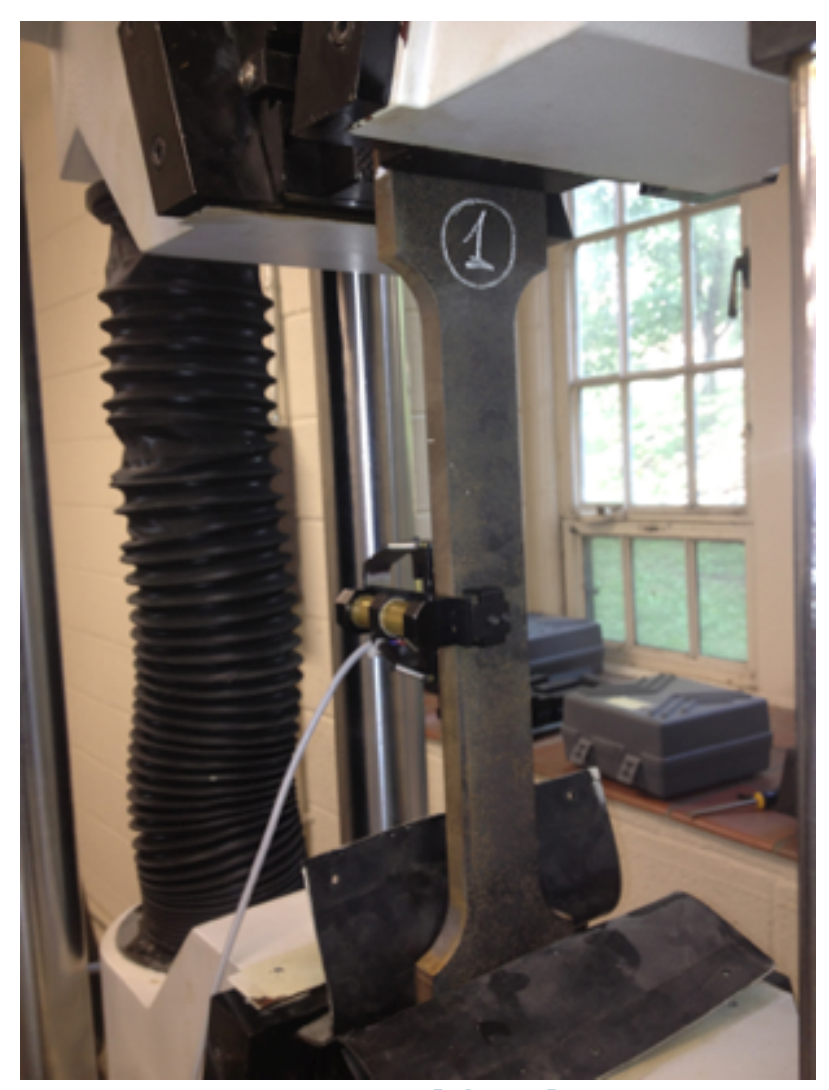

Figure 26. Extensometer EZ. 2-2 used for Plate Tension Coupons Tests

The testing machine is produced by the United Calibration Corporation, Model SHFM-600 $\mathrm{kN}$ (Figure 24). The extensometer was from United Calibration Corporation, Model EZ. 2-2 
(Figure 26). The load rate was 0.25 inches per minute up until 3\% strain, then 0.5 inches per minute to failure.

\subsubsection{Results}

The photographs of the tested specimens are shown in Figure 27 and Figure 28. Dimensions of the specimens were obtained by measuring specimens before and after the test (Table 4). Based on the data from tension coupon tests some important graphs were plotted. Figure 29 shows load-displacement diagram for 0.5 in thick tension coupons. The stress-strain diagram in Figure 30 helps to obtain the yield strength by identifying the yield plateau on the graph, and modulus of elasticity. The stress-stain diagram in Figure 31 allows getting ultimate stress of the steel. Figure 29 and Figure 31 have nonlinearity at the beginning of the plot due to slight slack in a system that fully engages the grip at the beginning of the test. All material properties obtained are summarized in Table 5 (the numbers were rounded till the nearest whole number).

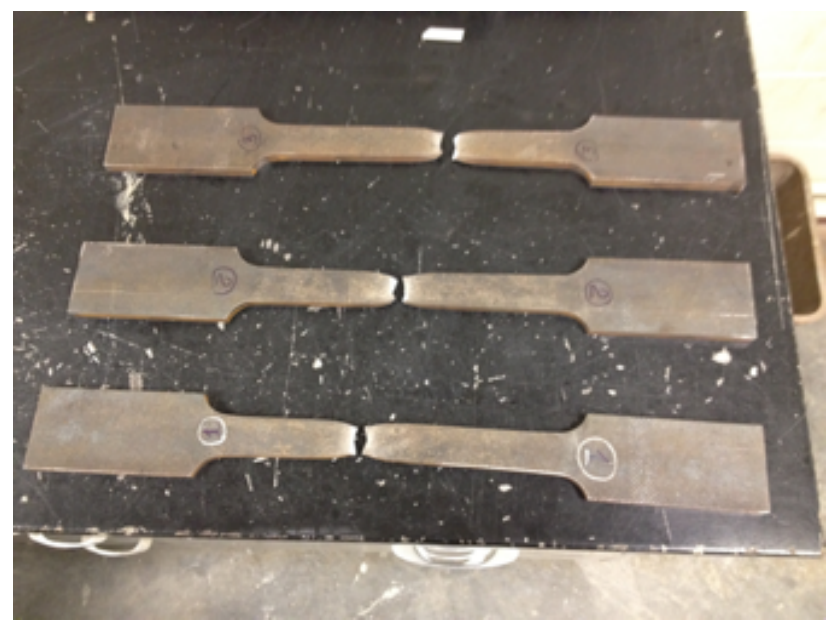

Figure 27. Tested Specimens from 1/2" Test 1 (bottom), Test 2 (middle), and Test 3 (top)

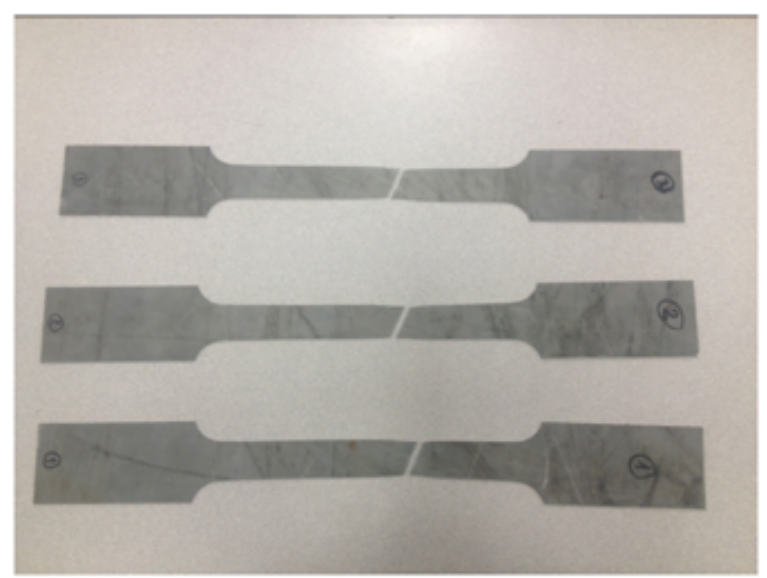

Figure 28. Tested Specimens from 0.075" Test 1 (bottom), Test 2 (middle), and Test 3 (top) 
Table 4. 1/2" Thick Plate - Test Number 1 Measurements

\begin{tabular}{|l|l|l|}
\hline $1 / 2$ " Test & $\begin{array}{l}\text { Initial at two } \\
\text { locations }\end{array}$ & $\begin{array}{l}\text { Final at } \\
\text { necking }\end{array}$ \\
\hline \multirow{2}{*}{ Width } & 1.500 & \multirow{2}{*}{1.026} \\
\cline { 2 - 2 } & 1.500 & \multirow{2}{*}{0.228} \\
\hline \multirow{2}{*}{ Thickness } & 0.500 & \\
\cline { 2 - 3 } & 0.500 & \\
\hline
\end{tabular}

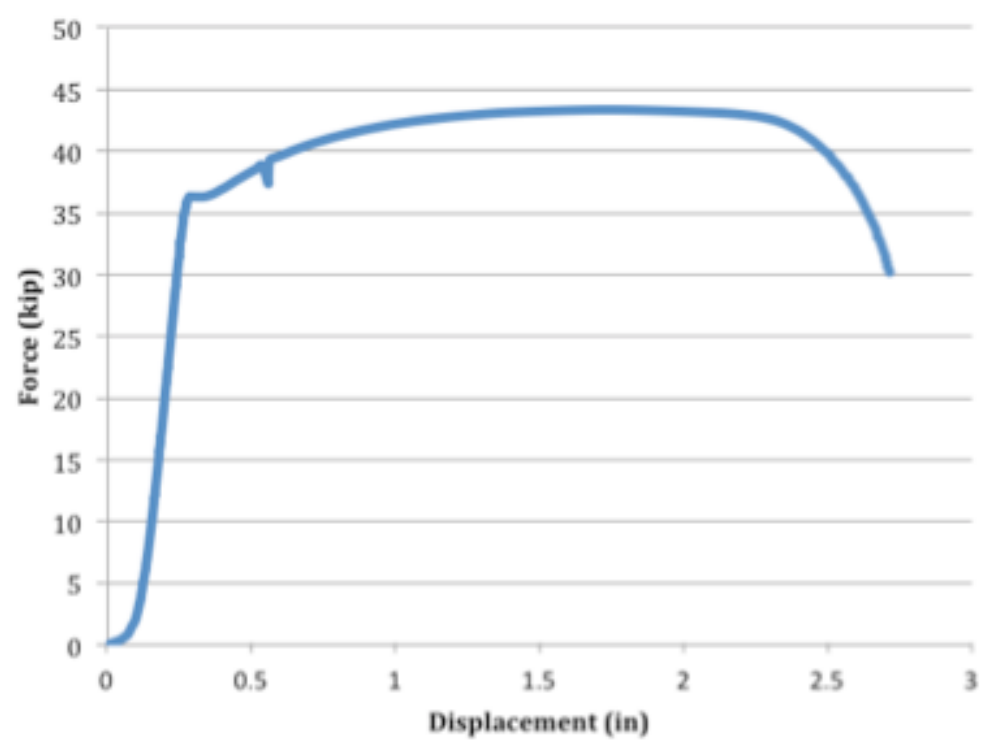

Figure 29. 1/2" Thick Plate - Test Number 1 Load - Displacement diagram

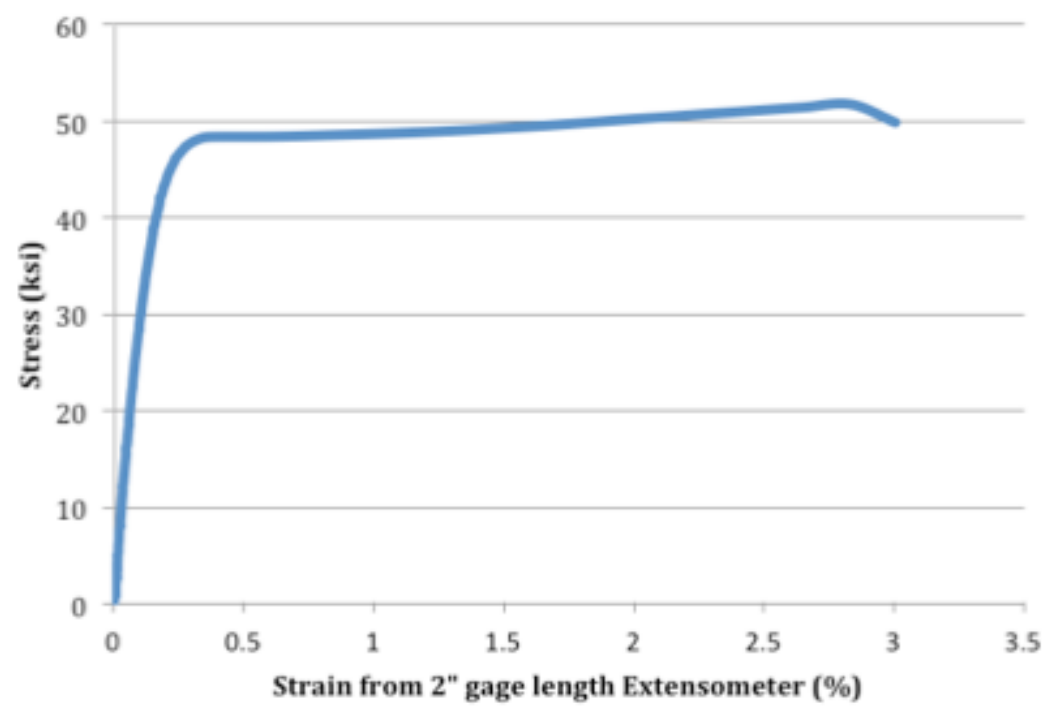

Figure 30. 1/2" Thick Plate - Test Number 1 Stress - Strain diagram using 2" Extensometer 


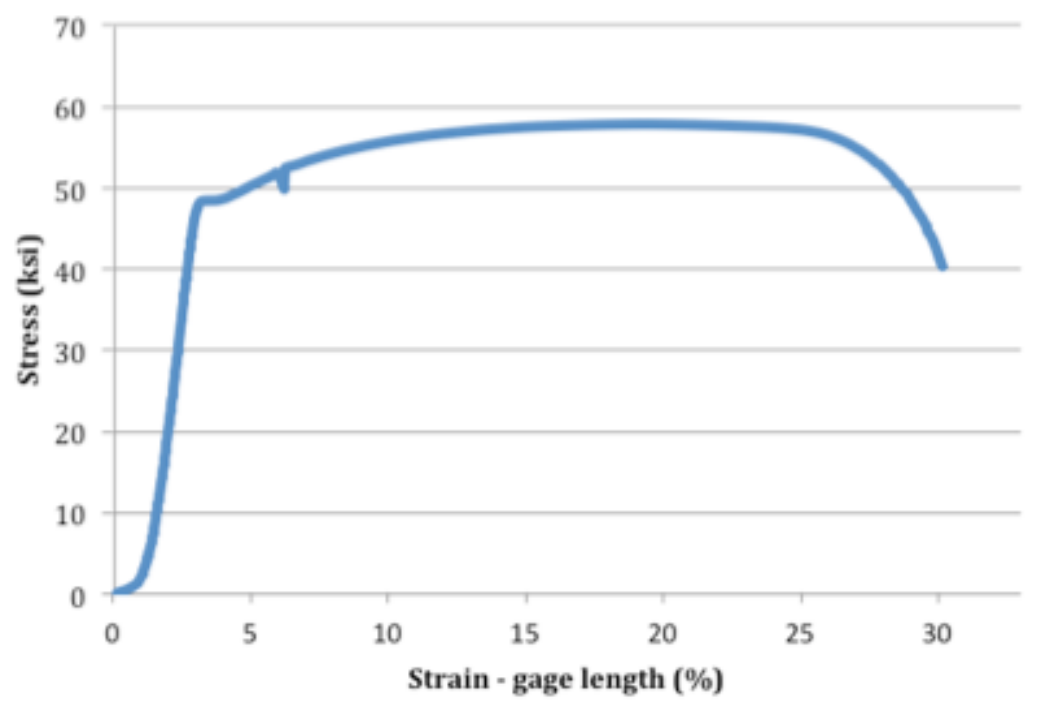

Figure 31. Test Number 1 Stress - Strain diagram using full gage length

Table 5. Material properties based on tension coupon tests

\begin{tabular}{|c|c|c|c|}
\hline Specimen & $\begin{array}{l}\text { Modulus of elasticity } \\
\text { (ksi) }\end{array}$ & $\begin{array}{l}\text { Yield Strength } \\
\text { (ksi) }\end{array}$ & $\begin{array}{c}\text { Ultimate } \\
\text { strength (ksi) }\end{array}$ \\
\hline $1 / 2 "$ Test 1 & 30500 & 48 & 58 \\
\hline $1 / 2 "$ Test 2 & 30500 & 48 & 58 \\
\hline $1 / 2 "$ Test 3 & 30500 & 48 & 57 \\
\hline $1 / 2 "$ Average & 30500 & 48 & 58 \\
\hline $0.075^{\prime \prime}$ Test 1 & 29000 & 44 & 53 \\
\hline $0.075^{\prime \prime}$ Test 2 & 29000 & 42 & 52 \\
\hline $0.075^{\prime \prime}$ Test 3 & 29000 & 43 & 54 \\
\hline $\begin{array}{c}0.075 " \\
\text { Average }\end{array}$ & 29000 & 43 & 53 \\
\hline $1 / 4 "$ Test 1 & 30500 & 46 & 70 \\
\hline $1 / 4 "$ Test 2 & 30500 & 46 & 70 \\
\hline $1 / 4 "$ Test 3 & 30500 & 46 & 70 \\
\hline $1 / 4$ "Average & 30500 & 46 & 70 \\
\hline $3 / 8$ " Test 1 & 29500 & 42 & 62 \\
\hline $3 / 8 "$ Test 1 & 29500 & 43 & 63 \\
\hline $3 / 8^{\prime \prime}$ Test 1 & 29500 & 43 & 63 \\
\hline 3/8" Average & 29500 & 43 & 63 \\
\hline
\end{tabular}

\subsection{Data Acquisition, Instrumentation, and Associated Equipment}

This section includes a detailed description of data acquisition, instrumentation and associated equipment used during the tests. A MTS Hydraulic actuator was used for 
loading, and Data Acquisition system recorded loading and associated deformations. Four string potentiometers and photogrammetry were used to measure the displacements. Eight strain gages were applied to obtain the strain for Test 1 . Whitewash helped to indicate the locations of yielding. Photos were taken at regular intervals and assembled into time-lapse videos. The following subsections will give more information about each type of equipment.

\subsubsection{Data acquisition system}

The data acquisition System 5000-A, Vishay Micro-Measurements (Software - Strain Smart data Systems Version 3.10 Build 3.1.14.490) was used to record load, actuator vertical displacement and deformation of the plate. The data was read and recorded every $0.1 \mathrm{sec}$ for Test 1 and every $0.2 \mathrm{sec}$ for all other tests (which was decided to be more than enough to get accurate results from the test). All channels were zeroed before the test except of MTS load cell.

\subsubsection{String potentiometers}

Three string potentiometers, pictured in Figure 32 (String Pot. 1, String Pot. 2 and String Pot. 3) were used to measure horizontal and diagonal elongation/shortening of the steel plate. Another sting potentiometer (String Pot. 4 - Figure 32) measured vertical displacement of the actuator. Characteristics of string potentiometers used in the tests are given in Table 6.

\section{Table 6. String potentiometers characteristics}

\begin{tabular}{|c|c|}
\hline $\begin{array}{l}\text { String potentiometer } 1 \\
\text { CELESCO, Chatsworth, California } \\
\text { PT101-0010-111-5110 } \\
\text { Pos. Sens.: } 0.20061 \mathrm{mV} / \mathrm{V} / \mathrm{Inch} \\
\text { Serial No: D2203933B }\end{array}$ & $\begin{array}{l}\text { String potentiometer } 2 \\
\text { CELESCO, Chatsworth, California } \\
\text { PT101-0010-111-5110 } \\
\text { Pos. Sens.: } 0.20260 \mathrm{mV} / \mathrm{V} / \mathrm{Inch} \\
\text { Serial No: D2203939B }\end{array}$ \\
\hline $\begin{array}{l}\text { String potentiometer } 3 \\
\text { CELESCO, Chatsworth, California } \\
\text { PT101-0010-111-5110 } \\
\text { Pos. Sens.: } 0.20335 \mathrm{mV} / \mathrm{V} / \mathrm{Inch} \\
\text { Serial No: D2203937B }\end{array}$ & $\begin{array}{l}\text { String potentiometer } 4 \\
\text { CELESCO, Chatsworth, California } \\
\text { PT101-0010-111-5110 } \\
\text { Pos. Sens.: } 0.20382 \mathrm{mV} / \mathrm{V} / \mathrm{Inch} \\
\text { Serial No: D2203935B }\end{array}$ \\
\hline
\end{tabular}




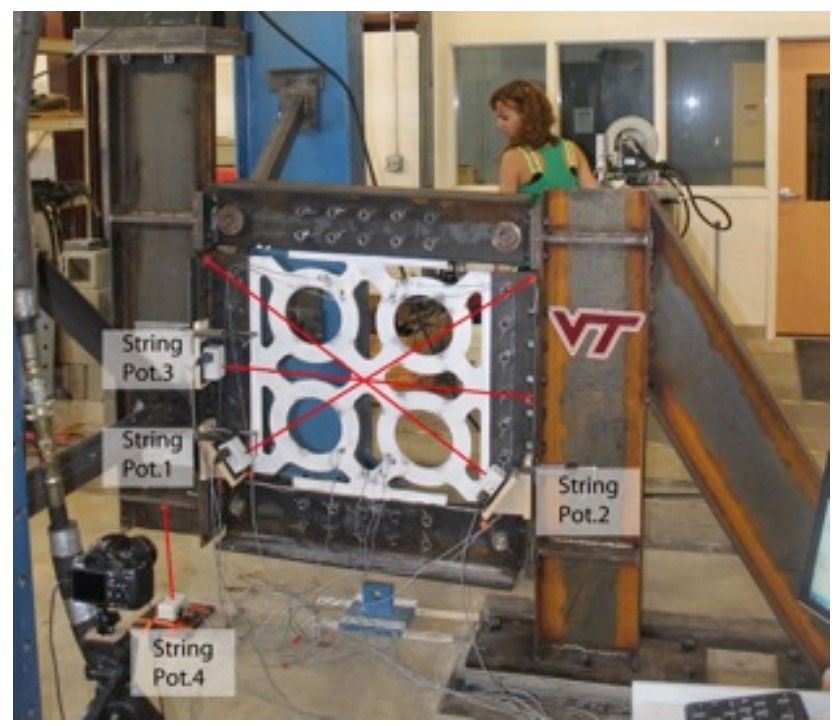

Figure 32. Photograph of test set up with string potentiometers shown

The method described below was used to determine the actual vertical displacement of the plate and shear.

Assumptions:

1. Rotation of one side of the plate relative to the other is small.

2. Consider that diagonal and horizontal strings of potentiometers are a good approximation of the real diagonals and centerline of the plate.

Basic concept:

Diagonal string potentiometer can be considered as a diagonal of the cube with dimensions $\mathrm{a}, \mathrm{b}$ and $\mathrm{d}$ respectively (Figure 33), where

$$
\begin{aligned}
& a=x_{2}-x_{1} \\
& b=y_{2}-y_{1} \\
& d=z_{2}-z_{1}
\end{aligned}
$$

$x_{1}, y_{1}, z_{1}$ and $x_{2}, y_{2}, z_{2}$ - measured coordinates of the beginning and the end of sting respectively to the plate. Coordinates were measured before every test. 


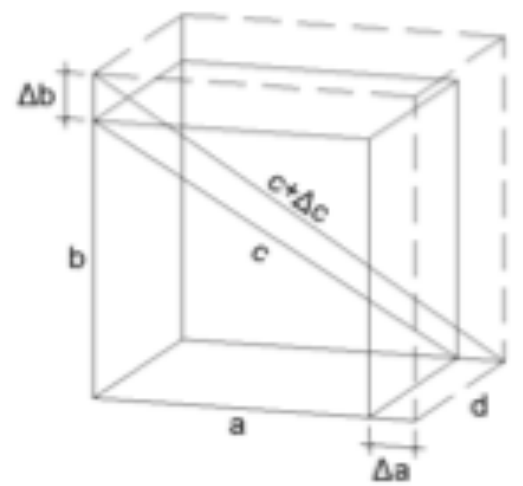

Figure 33. Calculation of plate vertical displacements using diagonal potentiometer

Let $\mathrm{c}$ be the length of the diagonal:

$$
c^{2}=a^{2}+b^{2}+d^{2}
$$

Let's assume that during the elongation/shortening only dimensions $a$ and $b$ change, and coordinate $d$ stays the same (the change is very small and will not affect the result). The length of the diagonal after elongation is:

$$
(c+\Delta c)^{2}=(a+\Delta a)^{2}+(b+\Delta b)^{2}+d^{2}
$$

where $\Delta c$ is elongation/shortening of the diagonal string, $\Delta a$ - elongation/shortening of the horizontal string, $\Delta b$ - vertical plate displacement, the value we need to find. From equation (5.6):

$$
\Delta b=\sqrt{(c+\Delta c)^{2}-(a+\Delta a)^{2}-d^{2}}-b
$$

The vertical plate displacement basing on diagonal string potentiometer 1 data:

$$
\Delta b_{1}=\sqrt{\left(c_{1}+\Delta c_{1}\right)^{2}-\left(a_{1}+\Delta a_{1}\right)^{2}-d_{1}^{2}}-b_{1}
$$

The vertical plate displacement basing on diagonal string potentiometer 2 data:

$$
\Delta b_{2}=\sqrt{\left(c_{2}+\Delta c_{2}\right)^{2}-\left(a_{2}+\Delta a_{2}\right)^{2}-d_{2}{ }^{2}}-b_{2}
$$

It is noted that $\Delta b_{1}$ and $\Delta b_{2}$ always have the opposite signs, because one of them is corresponding to diagonal shortening of the plate, and the one to the diagonal elongation of the plate. Final vertical plate displacement can be found as an average between $\Delta b_{1}$ and $\Delta b_{2}$ :

$$
\Delta b=\left(\Delta b_{1}-\Delta b_{2}\right) / 2
$$




\subsubsection{Strain gages.}

Eight strain gages (Table 7) were applied on Specimen 2-0.5-1 to measure strain on positions of possible steel fractures predicted by theoretical analysis. The preparation and application of the gages was completed as directed in the instruction bulletins supplied by Measurements Group, Inc. Strain gages were discounted on later tests because their measurement limits were less than the stain experienced by the specimen, and because of high cost related to buying different strain gages.

Table 7. Strain gages characteristics

\begin{tabular}{|l|l|}
\hline Manufacturing company & Tokyo Sokki Kenkyujo Co., Ltd. \\
\hline Type & YEFLA-5-1LT \\
\hline Lot no & Y510611 \\
\hline Gauge length & $5 \mathrm{~mm}$ \\
\hline Gauge factor & $2.14+/-\%$ \\
\hline Gauge resistance & $119.5+/-0.5$ \\
\hline Temp. compensation for & - \\
\hline Test conditions & $23^{0} \mathrm{C} 50 \% \mathrm{RH}$ \\
\hline Transverse sensitivity & $-0.1 \%$ \\
\hline Batch NO. & LD27M \\
\hline Lead Wires & $10 / 0.123 \mathrm{~W} 1 \mathrm{~m}$ \\
\hline
\end{tabular}

\subsubsection{MTS 407 Controller}

Displacement control was applied during the test. Every peak displacement point was typed manually in MTS 407 Controller Firmware Version 5.3 (MTS Systems Corporation); ramp loading was used. The loading rate was determined based on the fact that it was desirable to take at least three pictures every quarter cycle, and the first peak displacement was 0.085 in. Taking into consideration the fact that maximum possible speed of the cameras is 1 picture per $2 \mathrm{sec}$, the first quarter cycle should last at least $2 \mathrm{sec} x$ $2=4 \mathrm{sec} .5 \mathrm{sec}$ was selected to be conservative resulting in the maximum possible load rate $\mathrm{R}=0.085 \mathrm{in} * 60 / 5 \mathrm{sec}=1.02 \mathrm{in} / \mathrm{min}$. A rate of $\mathrm{R}=1 \mathrm{in} / \mathrm{min}$ was selected. If the load rate is $1 \mathrm{in} / \mathrm{min}$, the duration of the whole test is predicted to be more that 1 hour, not considering time for photogrammetry pauses and time for entering peak displacement point in MTS 407 Controller. This was deemed to be fast enough to complete a test in a reasonable amount of time.

\subsubsection{MTS Hydraulic Actuator}

The MTS Model 243.60 Hydraulic Actuator used for loading had maximum tension force of $146,000 \mathrm{lbs}(648 \mathrm{kN})$, maximum compression force of 228,000 lbs (1013 kN). The stroke length was 20 inches (500 mm) 


\subsubsection{Whitewash}

Whitewash is a mixture of lime and water, essentially paint with no binder, that is applied to a steel specimen that will be tested to strains beyond yield. During testing, mill scale (tightly adherent surface rust) flakes off and with it, the whitewash. Visually distinct patterns of yielding result. The sequential development of the whitewash cracks may be recorded to indicate the yielding pattern during loading.

The following proportion was used for a whitewash mixture: $1.25 \mathrm{lb}$. of hydrated lime for 8 cups of water. Before applying the whitewash, a wire brush was used to remove loose rust and clean the specimen, followed by water and alcohol surface cleaning. Three coats of whitewash were applied; every individual coat was allowed to dry for about two hours.

\subsubsection{Time lapse video}

GBTimelapseEOS is a Microsoft Windows application for the capture of time-lapse image sequences. A Canon EOS digital camera was tethered to the control computer by a USB cable. Using the camera settings images were captured using the computer rather than the camera controls and collected automatically while computer and camera were left unattended. One camera for Test 1 and two cameras for all other tests were taking pictures every few seconds. The rate of taking pictures was every 2 seconds for first test (maximum possible speed for the cameras), but then was changed to every 3 seconds for other tests due to huge amount of pictures, which is difficult to process. Adobe Premiere Elements 10 software was used to assemble the pictures into a time lapse videos.

\subsubsection{Photogrammetry}

Close-range photogrammetry is a method for measuring three-dimensional coordinates and displacements. Photogrammetry measurements can be used to characterize the relative magnitude of lateral torsional buckling and global tension field buckling. This kind of measurement is more efficient than single point measurements (e.g. string potentiometers) as it allows a much larger number of displacement measurements in a small amount of time. However, the measurements were not recorded continuously. To take photogrammetry measurements, the test was paused in order to take the required pictures, which usually takes around 10 minutes.

The camera, NIKON D7000 with NIKKON 20mm, f/2.8 fixed focal length lens, were used for taking pictures. This DSLR camera features a high resolution 16.2 megapixel DX-format sensor, with available autofocus. The process of photogrammetry used a series of pictures taken from different locations and angles to create a three-dimensional point cloud obtained using automated triangulation calculations.

In order to obtain a good point cloud, the following rules were followed while taking the pictures:

- Camera must be consistent during the shoot (no changes in zoom or focus)

- The camera position must cover a wide range of angles 
- Horizontal separation of photos depicted in Figure 34

- Vertical separation of the photos depicted in Figure 34

- Must assure good redundancy of photos (points appearing on numerous photos from different angles)

- Photos should be taken from both landscape and portrait camera orientation (rolled camera position)

- Minimize obstructions in line of sight of photo

- Always assure the green focus box on camera is aimed where you are trying to take the picture as well as focusing correctly

- Take photos from no more than 2' away from farthest target

- Make sure to take 3 photos at each station to ensure at least 1 photo of high clarity
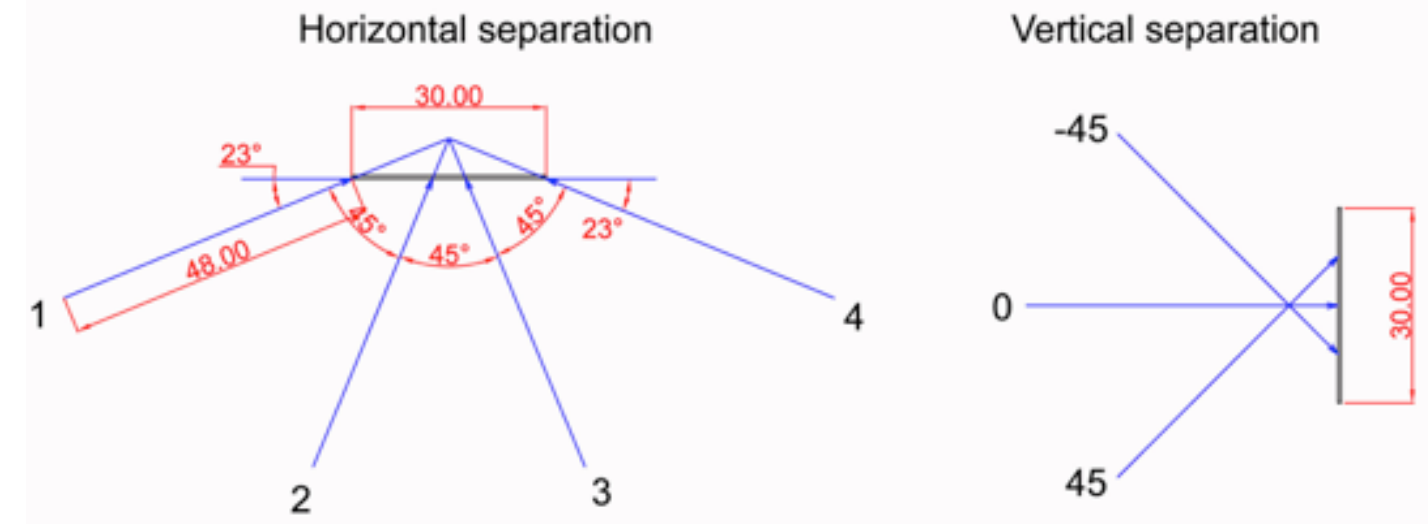

Figure 34. Horizontal and vertical separation of photos in photogrammetry.

Software called PhotoModeler (Eos Systems, Inc., 2011) was used for processing the photographs. Ringed Automatically Detected (RAD) targets and dot targets were attached to the specimen before making pictures (Figure 35). Each RAD target has a unique shape and helps to reference points to each other while detected in the multiple pictures [Orsa et al. (2011)]. All dots are the same and are just used to obtain additional measurements locations. They are not used for referencing. Dot targets diameter is the same as inner RAD targets diameters.

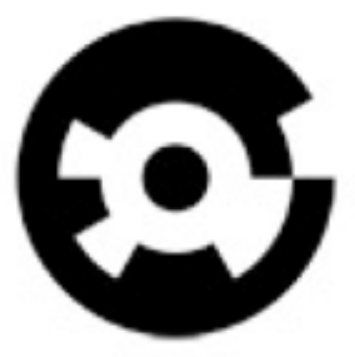

Figure 35. RAD and dot targets in PhotoModeler 
It is important that targets be attached to the specimen securely. Special sticky paper was used to print out targets from PhotoModeler software. Targets were applied in such a manner that each picture will contain at least 10 RAD targets. Photogrammetry targets placement for specimens 1-4 is shown on Figure 36 - Figure 40.

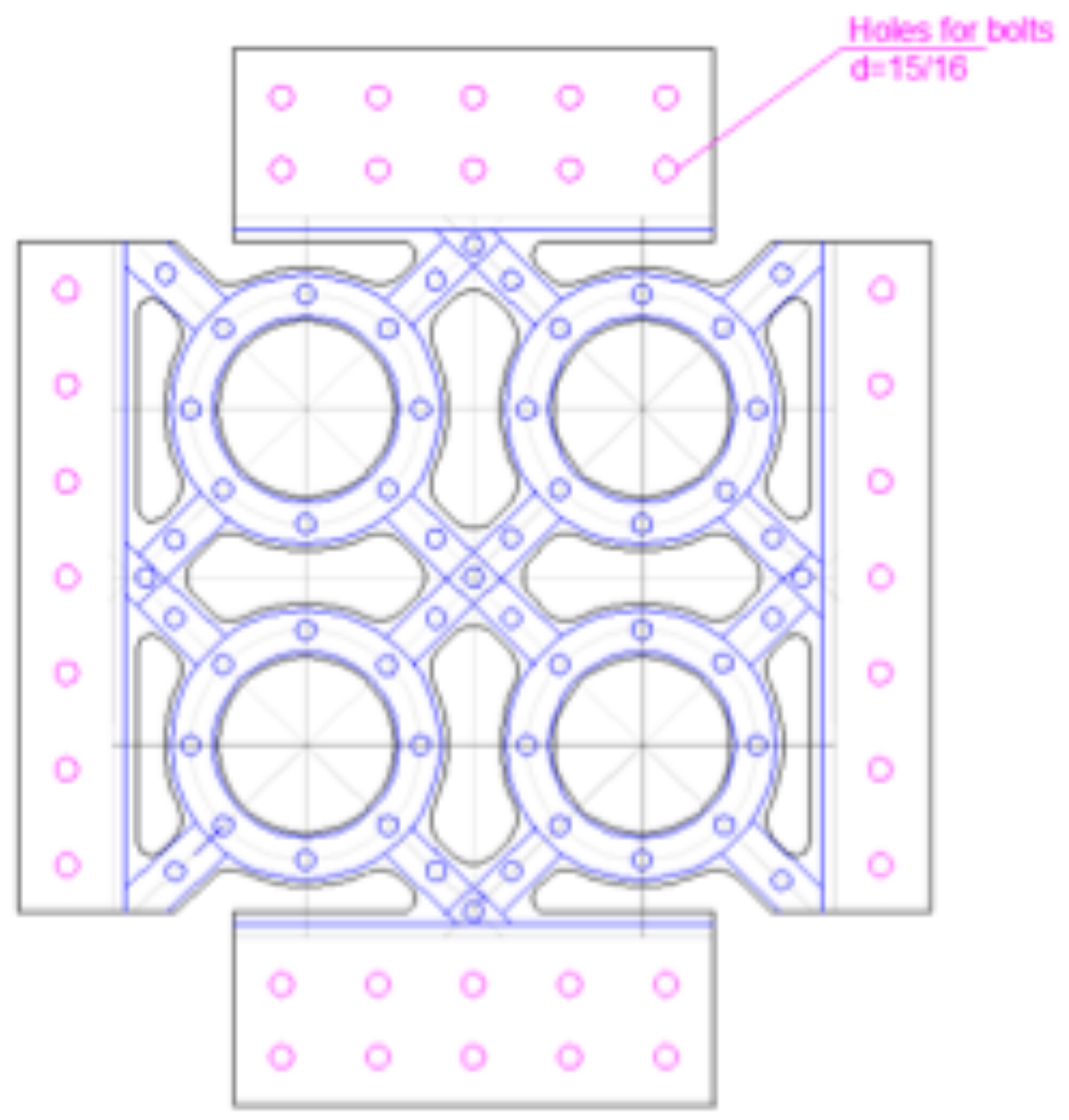

- RAD targets

Line of dot targets at approximately dot diameter

spacing

Figure 36. Photogrammetry targets placement - Specimens 2-0.5-1 and 2-0.25-1 


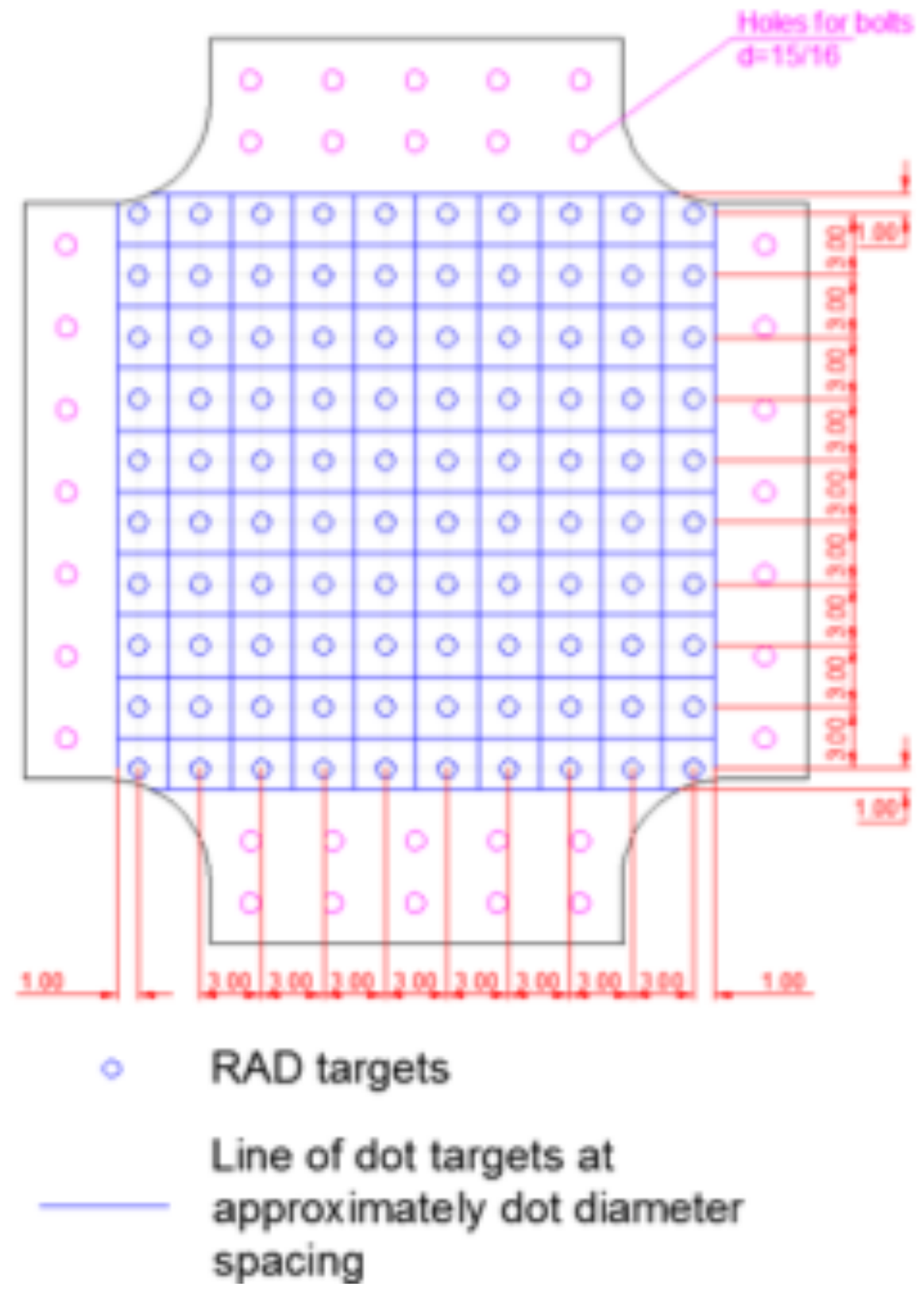

Figure 37. Photogrammetry targets placement - Specimen "Solid Plate" 


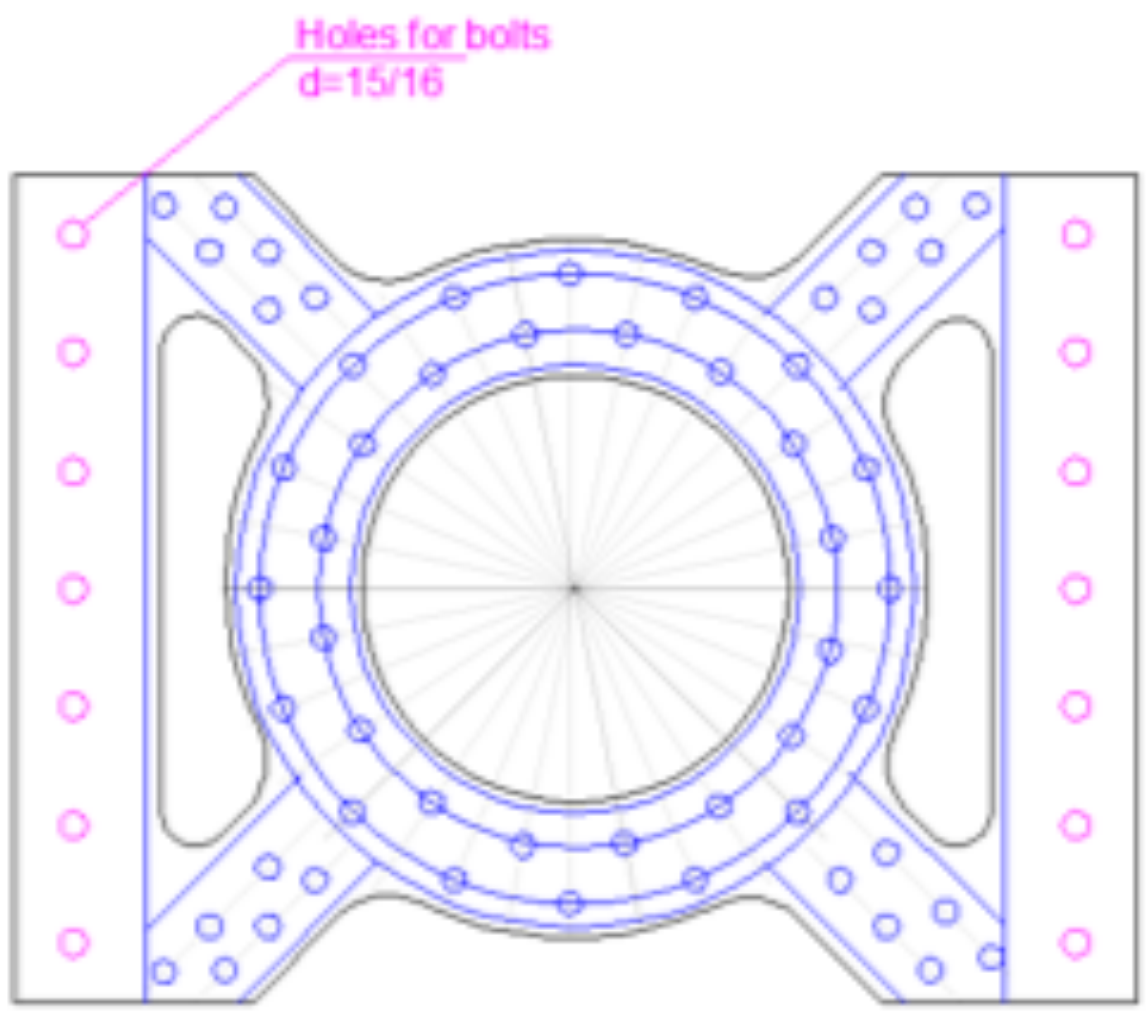

- RAD targets

\section{Line of dot targets at approximately dot diameter spacing}

Figure 38. Photogrammetry targets placements - Specimen 1-0.5-1 


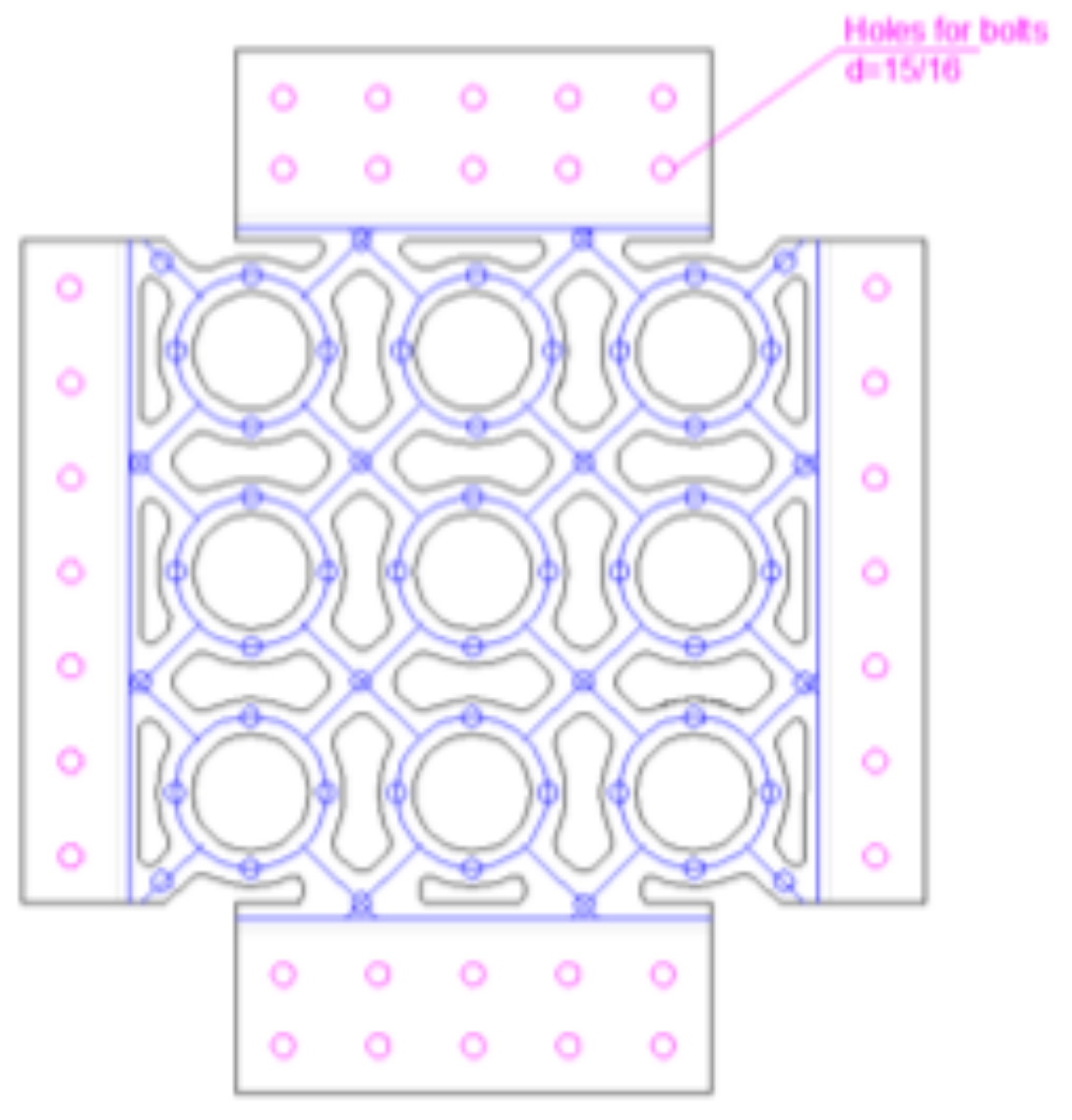

RAD targets

Line of dot targets at - approximately dot diameter spacing

Figure 39. Photogrammetry targets placement - Specimens 3-0.5-1, 3-0.25-1, 3-

0.375-1 


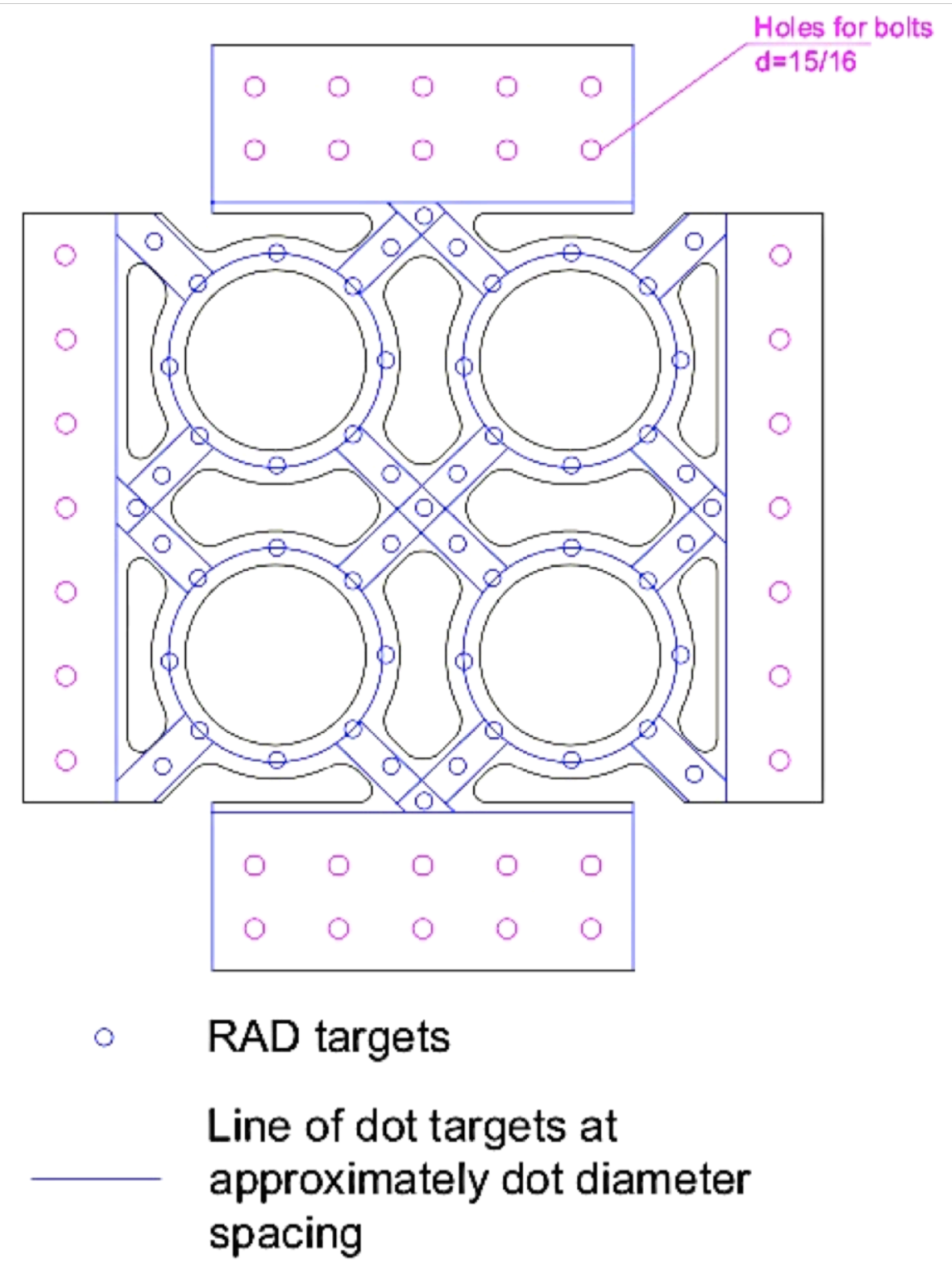

Figure 40. Photogrammetry targets placement - Specimen 2-0.25-0.81

In order to give a point cloud proper scale, a known dimension was provided to the PhotoModeler software. This dimension was recorded before the test between two Rad targets as far apart as possible (typically 23.15in) in order to minimize relative error. The potential error due to inaccurate baseline measurement was $1 / 112$ in as far as ruler with the value of the smallest graduation of $1 / 64$ in was used (Figure 41). This represents a possible error of $(1 / 112) / 23.15=0.0004$ or $0.04 \%$, which means that the possible error due to inaccuracy in the baseline measurement was not expected to exceed $0.04 \%$ of the distance or displacement being measured. 


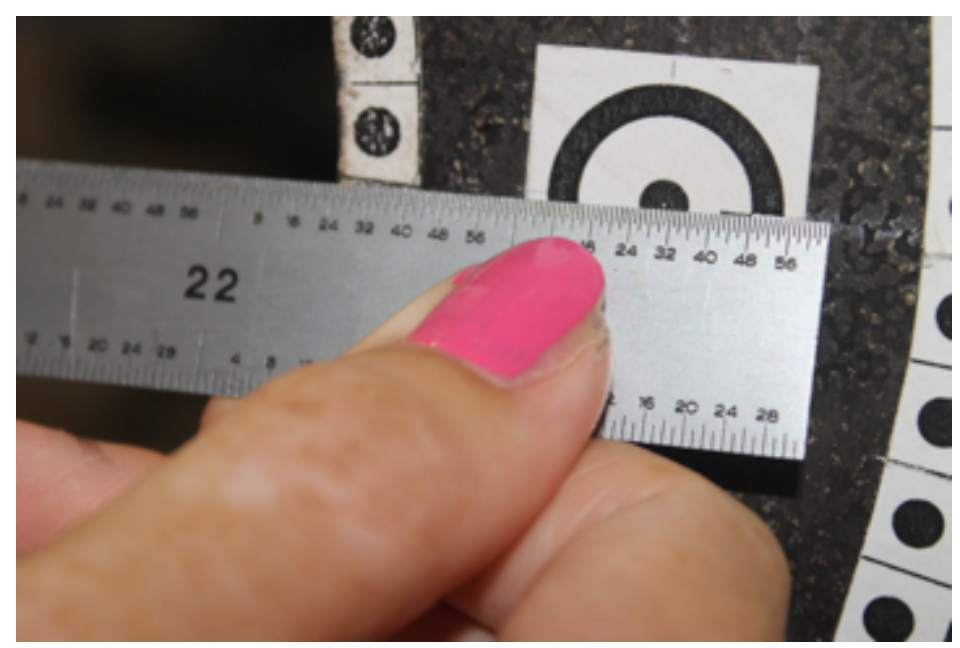

Figure 41. Baseline measurement

To increase accuracy, high residual removal option was used in the PhotoModeler software. High residual removal is an option available when processing a photogrammetry project. Once bundle adjustment has minimized error as much as possible, the high residual removal will remove points from the project that exceed a maximum specified error tolerance. This can be useful to eliminate points that have been incorrectly referenced between pictures. The high residual removal option simply unreferences the point in the photograph where the high residual is present. Residual error measured in PhotoModeler is in pixels.

Two values obtained from photogrammetry are a good indicator of photogrammetry accuracy including residual error and total error. Residual error is the difference between where the user marked it and where the program expects it to be measured in pixels. When a point has a 3D position, PhotoModeler can project it back onto the photograph (along its light ray) to determine where, in the ideal case, that point should be on the photograph. Due to various errors and averages etc. the $\mathrm{x}, \mathrm{y}$ point that the user marked and this projected $\mathrm{x}, \mathrm{y}$ position will not be the same. The distance beteen these two is called residual error.

Residual errors are one of the best indicators of the project quality and were checked after processing. All projects should have the largest point marking residual of under 10 pixels. For projects with known and calibrated cameras, the largest residual should be less than 3 pixels, and for projects done with all sub-pixels targets, the largest residual should be less than 1 pixel and smaller if possible. A large marking residual means that the 3D location of the point and mark position of the point do not "agree". The lack of the agreement points to problems in the project that should not be ignored. If there are only a few points with high residuals then they are probably not marked well (or misreferenced). If there are many points with high residuals then perhaps there is a camera calibration (or parameterization problem) or 1 or more of the camera stations are not oriented correctly.

The total error appears beside the 3D Process Dialog during Global Potimiation. The dialog 
shows a set of vertical bars where each bar shows the accumulated errors within the 3D model in one iteration. A new vertical bar is added to the Total Error Dialog for every iteration of the global optimization solution. The bars should get shorter as processing proceeds (which shows reducing erros in the model). The errors shown in this dialog are technically referred as the "standard error of unit weight". These values are unitless and combine information about image residuals, camera parameters residuals, edge residuals, constraint residuals, and control point residuals. If there are no control points, no constraints and self-calibration or full-calibration is not being done, these values reflect closely the total weight residual error in the photo markings.

If the bars get bigger instead of smaller or there are more that 10 vertical bars of the same height, then PhotoModeler is having trouble solving. A few bigger bars are generally ok as long as the bars start to get smaller. If the bars do not get smaller then the processing is failing. The total error dialog usually shows two sets of bars separated by a gray area. The first set corresponds to the first optimization phase amd the second set of bars to the second optimization and self-calibration phase. Sometimes the first vertical bar in the second set will be larger than the last bar in the first set. This is normal. If the automatic high residual point removal feature is turned on, the second set of bars at right may include a single green bar. This indicates that PhotoModeler has made changes to the project with the unreferencing of the high residual point. The project Status Report will explain the change by indicating the point ID and the photo on which the unreference occurred.

Residual and total error don't exceed 3 pixels for all obtained point clouds used in this research. 


\section{TEST RESULTS}

The following sections present results of all 8 tests, observations, plots of hysteretic behavior of the plates, and also describes details of issues experienced during the test and solutions that were made to solve them. Comparison between the test results and further analysis of the test results are provided in Chapter 7.

\subsection{TEST 1 - Specimen 2-0.5-1}

Figure 42 and Figure 43 show the side of the plate with photogrammetry targets and the side with whitewash, respectively, before the test was started. Table 8 and Figure 44 represent the Specimen 2-0.5-1 geometric properties. All dimensions are given in inches.

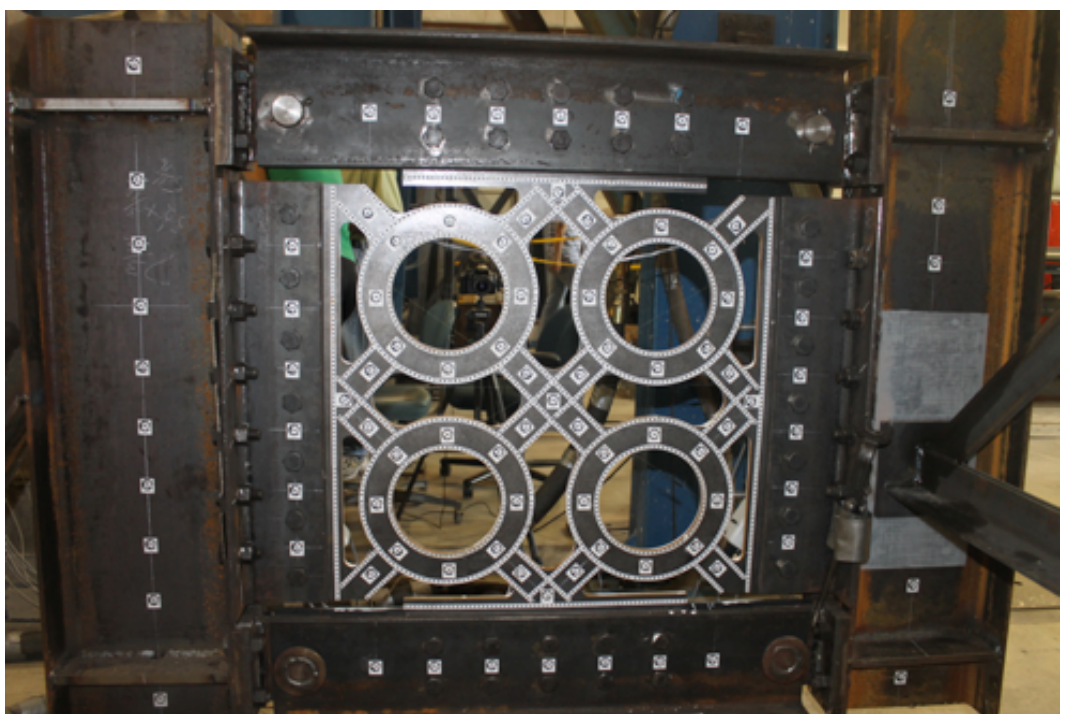

Figure 42. Photogrammetry side view - Test 1 - Specimen 2-05-1

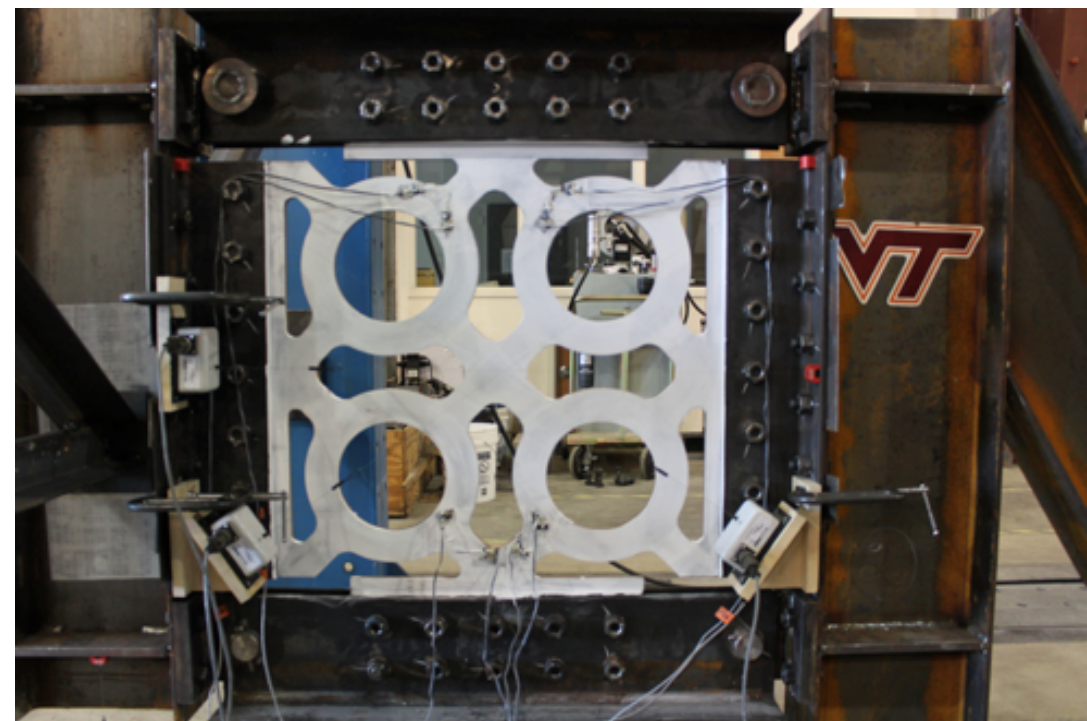

Figure 43. Whitewash side view - Test 1 - Specimen 2-0.5-1 
Table 8. Specimen 2-0.5-1 properties

\begin{tabular}{|l|l|}
\hline Thickness of the plate $\left(t_{w}\right)$ & 0.5 in \\
\hline Outer ring radius $\left(R_{0}\right)$ & 5.89 in \\
\hline Ring width $\left(w_{c}\right)$ & 2.21 in \\
\hline Link width $\left(w_{L}\right)$ & 2.21 in \\
\hline Number of rings in a row $(N)$ & 2 \\
\hline Pin to pin dimensions (a) & 34 in x 34in \\
\hline
\end{tabular}

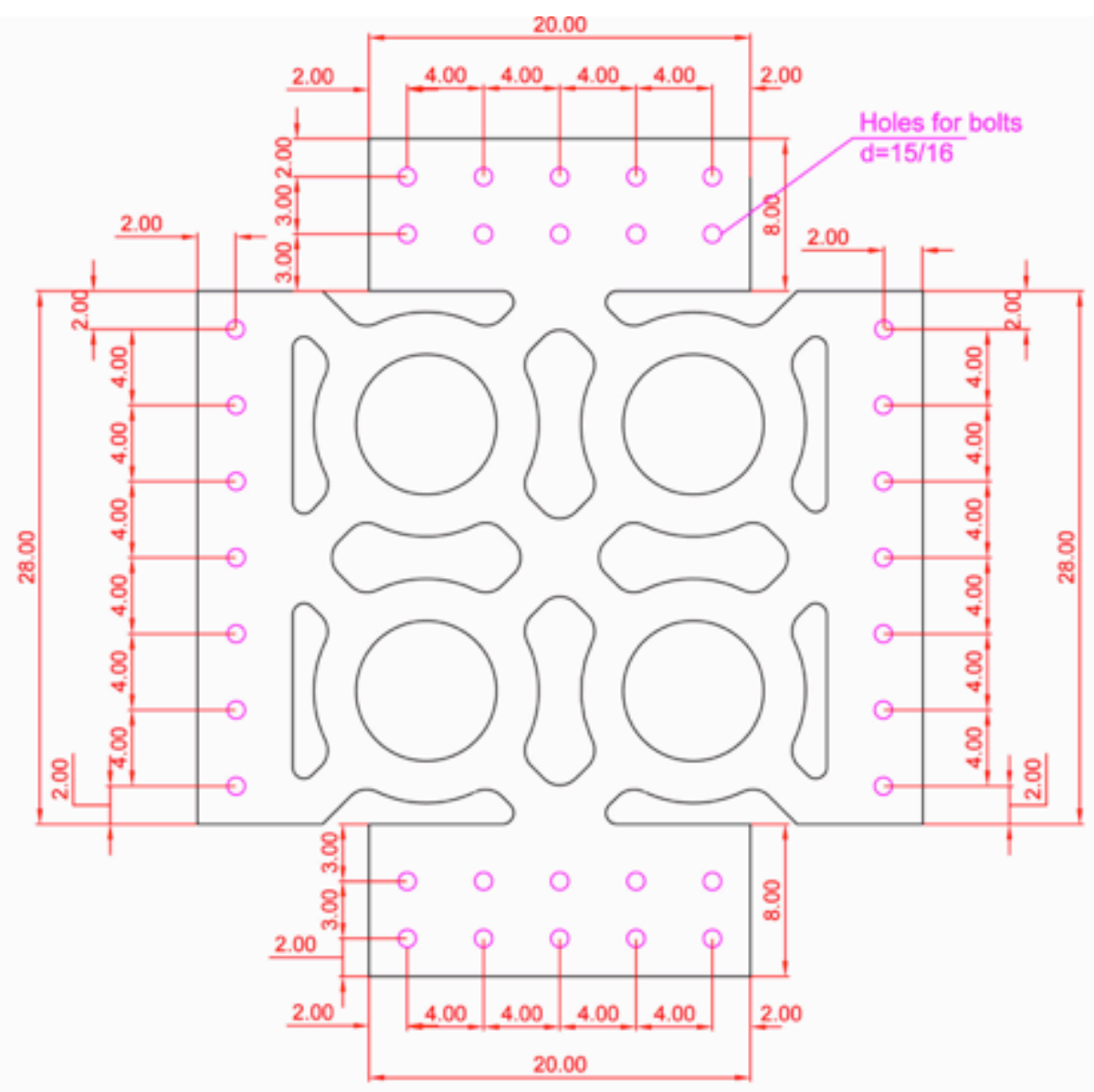

\section{Plate thickness $=0.5$}

Figure 44. Test 1, Specimen 2-0.5-1 geometry

The initial shape of Specimen 2-0.5-1 was obtained by exporting initial photogrammetry data to MATLAB and is shown in Figure 45. In order to compare the 3D point cloud to an assumed ideal flat surface of the plate (to find initial imperfections of the plate), an orthogonal regression was created for the 3D point cloud. This orthogonal regression results in a plane that is used to represent the assumed perfectly planar surface of the plate. The orthogonal regression is implemented using principal component analysis. This 
procedure uses the variance of a set of 3D points to assign representative vectors called principal components. The first principal component is the vector that represents the 3 dimensional direction, in which the largest variance of the 3D point cloud lies. The second principal component represents the direction in which the largest variance of the 3D point cloud lies, with the constraint that it must be orthogonal to the first principal component. The third principal component is the vector that is orthogonal to both the first and second principal components. The orthogonal regression can be defined by its normal vector, which in this case is the third principal component. Since this vector is normal to the orthogonal regression, it will also be taken as normal to the planar surface of the slab. The magnitude of maximum initial imperfection of Specimen 2-0.5-1 was measured to be 0.11 in. It was calculated like a difference between the biggest and the smallest $Z$ coordinate of all targets.

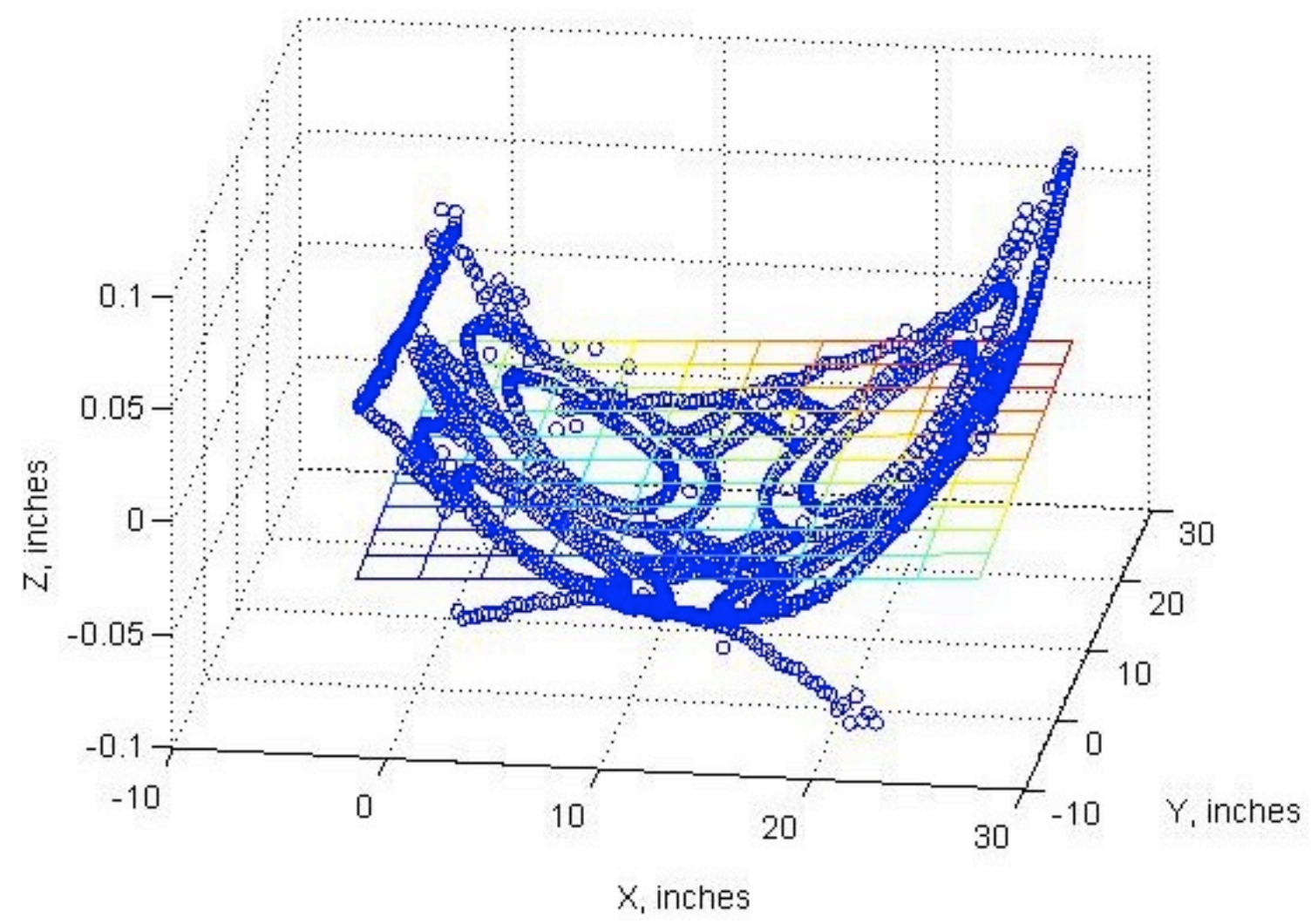

Figure 45. Point cloud and orthogonal regression plot - Specimen 2-0.5-1 initial shape 


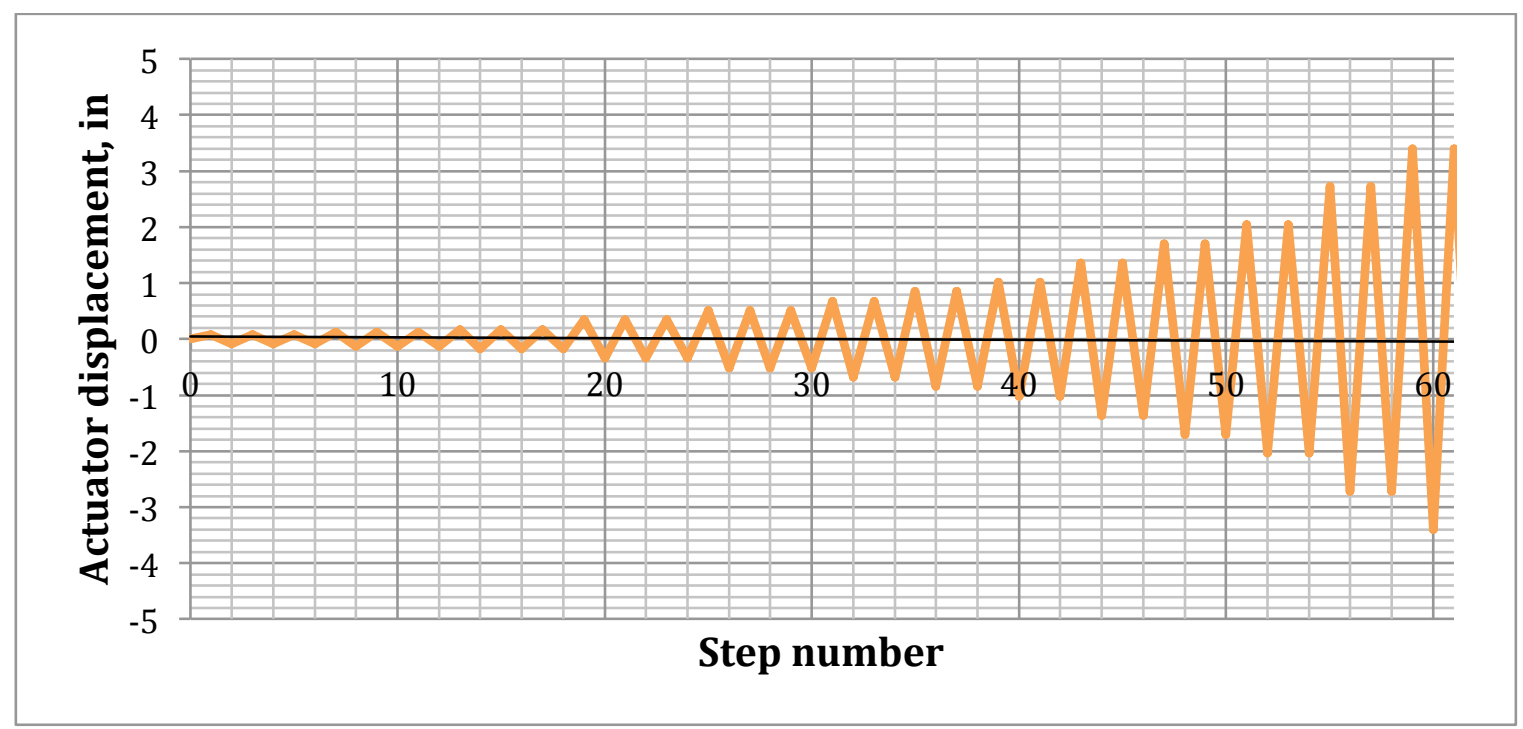

Figure 46. Displacement protocol - Test 1

Displacement protocol (Figure 46 and Table 9) was applied to the plate. During the experiment it was realized that proposed vertical displacements are displacements of the actuator, and the actual displacements and drift angle of the specimen are smaller partially due to elastic deformations in the reaction frames. The actual vertical displacement and drift angle of the plate (Table 9) was found based on string potentiometers readings.

Table 9. Actuator vertical displacements and plate vertical displacement - Test 1

\begin{tabular}{|c|c|c|c|c|c|}
\hline $\begin{array}{l}\text { Interstory } \\
\text { drift angle }\end{array}$ & $\begin{array}{l}\mathrm{N} \text { of } \\
\text { cycles }\end{array}$ & $\begin{array}{l}\text { Actuator } \\
\text { displacement, } \\
\text { in }\end{array}$ & $\begin{array}{l}\text { Actuator } \\
\text { drift angle, } \\
\%\end{array}$ & $\begin{array}{l}\text { Specimen shear } \\
\text { displacement } \Delta, \\
\text { in }\end{array}$ & $\begin{array}{l}\text { Specimen } \\
\text { drift angle, \% }\end{array}$ \\
\hline $0.5 \delta y$ & 3 & 0.0850 & 0.25 & 0.0136 & 0.04 \\
\hline $0.75 \delta y$ & 3 & 0.1275 & 0.38 & 0.0204 & 0.06 \\
\hline$\delta y$ & 3 & 0.1700 & 0.50 & 0.0238 & 0.07 \\
\hline $2 \delta y$ & 3 & 0.3400 & 1.00 & 0.0051 & 0.15 \\
\hline $3 \delta y$ & 3 & 0.5100 & 1.50 & 0.0986 & 0.29 \\
\hline $4 \delta y$ & 2 & 0.6800 & 2.00 & 0.1326 & 0.39 \\
\hline $5 \delta y$ & 2 & 0.8500 & 2.50 & 0.1836 & 0.54 \\
\hline $6 \delta y$ & 2 & 1.0200 & 3.00 & 0.2618 & 0.77 \\
\hline $8 \delta y$ & 2 & 1.3600 & 4.00 & 0.4556 & 1.34 \\
\hline $10 \delta y$ & 2 & 1.7000 & 5.00 & 0.6698 & 1.97 \\
\hline $12 \delta y$ & 2 & 2.0400 & 6.00 & 0.9316 & 2.74 \\
\hline $16 \delta y$ & 2 & 2.7200 & 8.00 & 1.564 & 4.60 \\
\hline $20 \delta y$ & 2 & 3.400 & 10.00 & 2.210 & 6.65 \\
\hline
\end{tabular}


Figure 47 shows the behavior of Specimen 2-0.5-1 is the Shear displacement of the plate $(\Delta)$ was calculated using the diagonal string potentiometers (see Chapter 5.5.2). Pin to pin dimension of the plate $(a)$ was equal to 34 in. First the specimen deformed elastically. Then there was some initiation of yielding at inner radius of the ring at the locations where ring is connected to the link (Figure 48). Then the yielding spread to outer radius at well. Plastic hinges appeared to form at the locations of initial yielding. Then buckling started to occur at second cycle at $4 \%$ drift. The buckling increased in amplitude as the shear angle was increased. The mode of buckling was global shear with some lateral torsional buckling of the rings. The buckling shape is well observed at $6.7 \%$ drift on the photogrammetry point cloud obtained during the test (Figure 49). The amount of buckling that specimen 2-0.5-1 experienced might be acceptable in a practical design since the web plate might only buckle under extreme earthquake motions and even then, the energy dissipation and strength are only mildly affected. The specimen demonstrated excellent ductility, no fracture was observed.

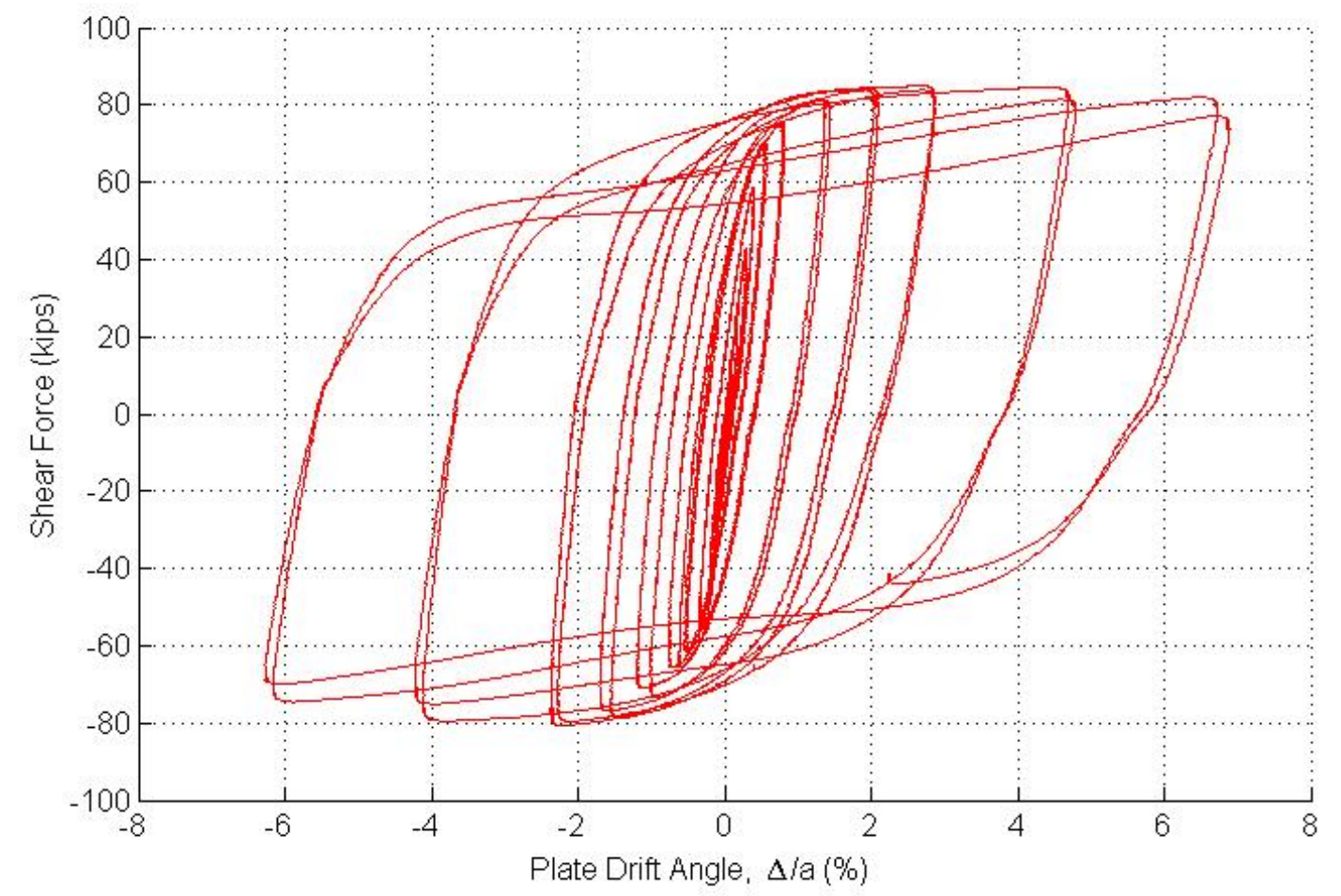

Figure 47. Shear Force versus Plate Drift Angle - Test 1 - Specimen 2-0.5-1 


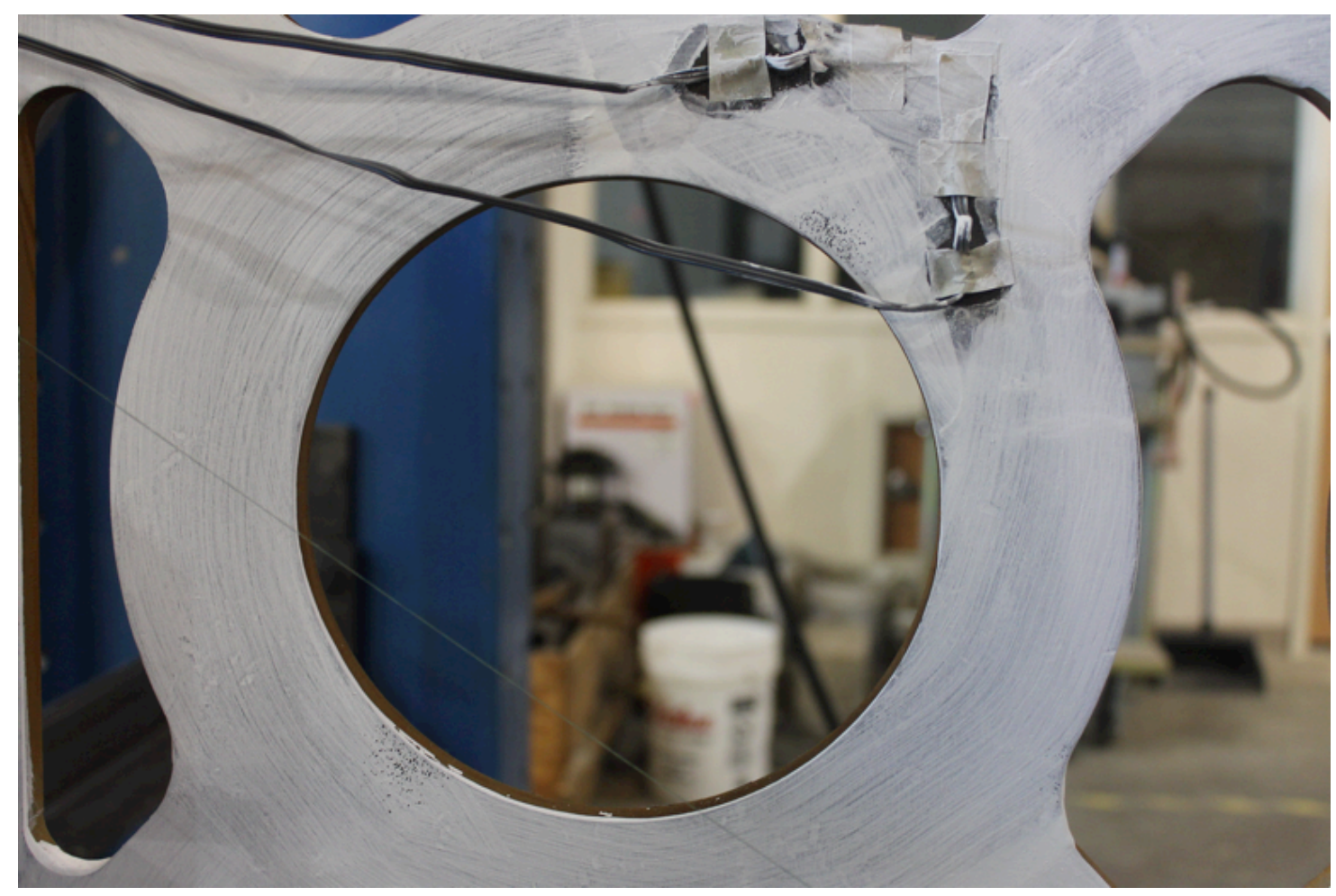

Figure 48. Locations of initial yielding - Test 1 - Specimen 2-0.5-1

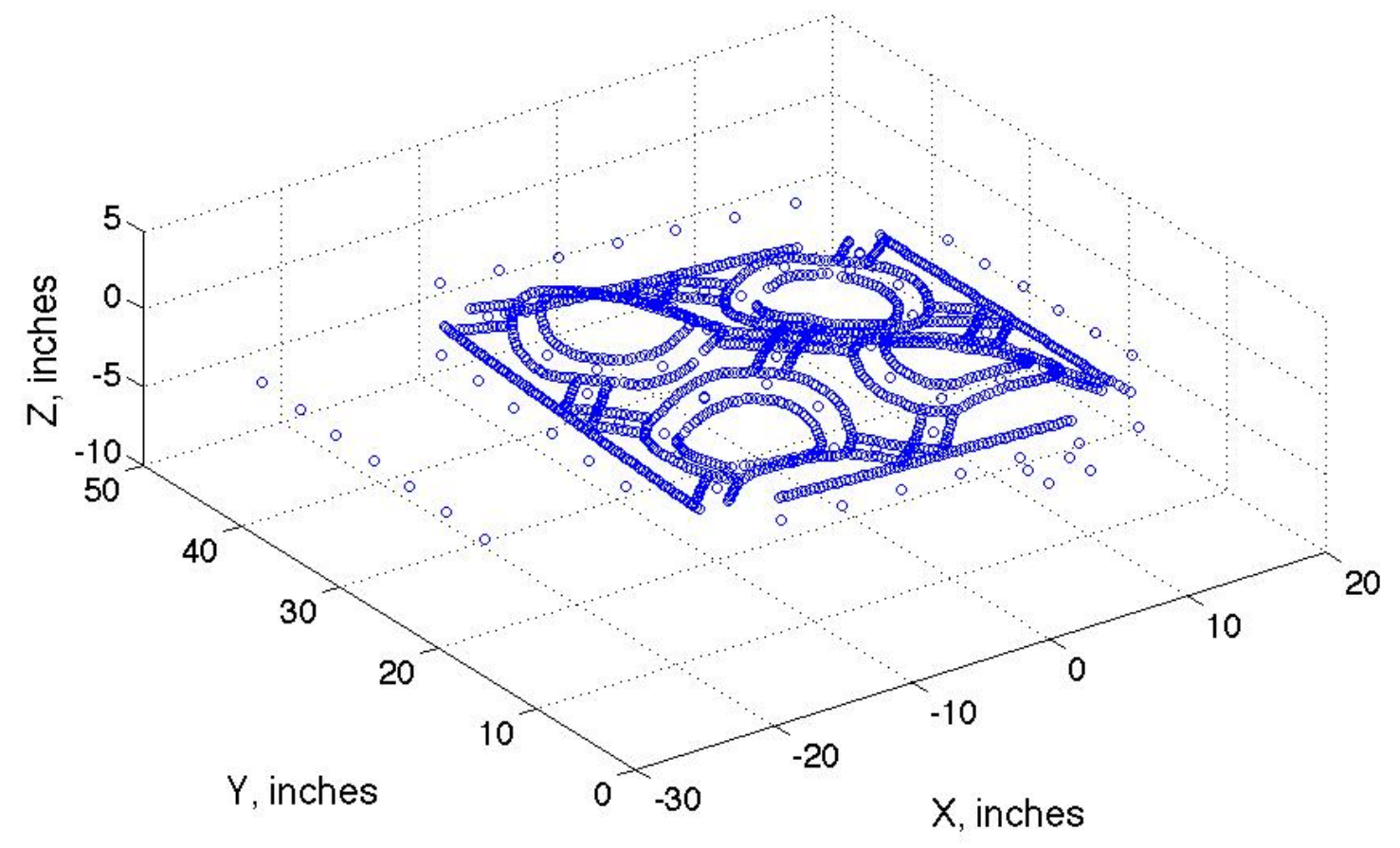

Figure 49. Point cloud - Specimen 2-05-0.1 - 6.7\% drift 
Some problems with surrounding frame were experienced during the test. During the $54^{\text {th }}$ cycle a load noise occurred. Local yielding of flanges of W12 reaction frame was noticed (Figure 50). There was even a fracture of the right bottom flange of the reaction frame. The test was stopped. Yielding of the actuator bracing angles also occurred. For the next test, these problems were solved. Stiffeners were welded to the actuator brace angles to prevent their rotation. 1 in thick steel plates were welded or bolted to the W12 frame flanges and additional rods were provided (Figure 51).

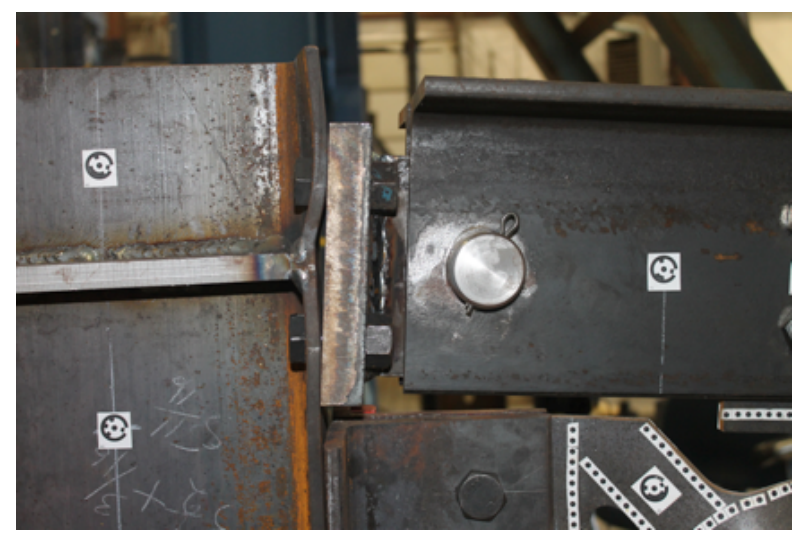

Figure 50. Local yielding of W12 flanges - Test 1
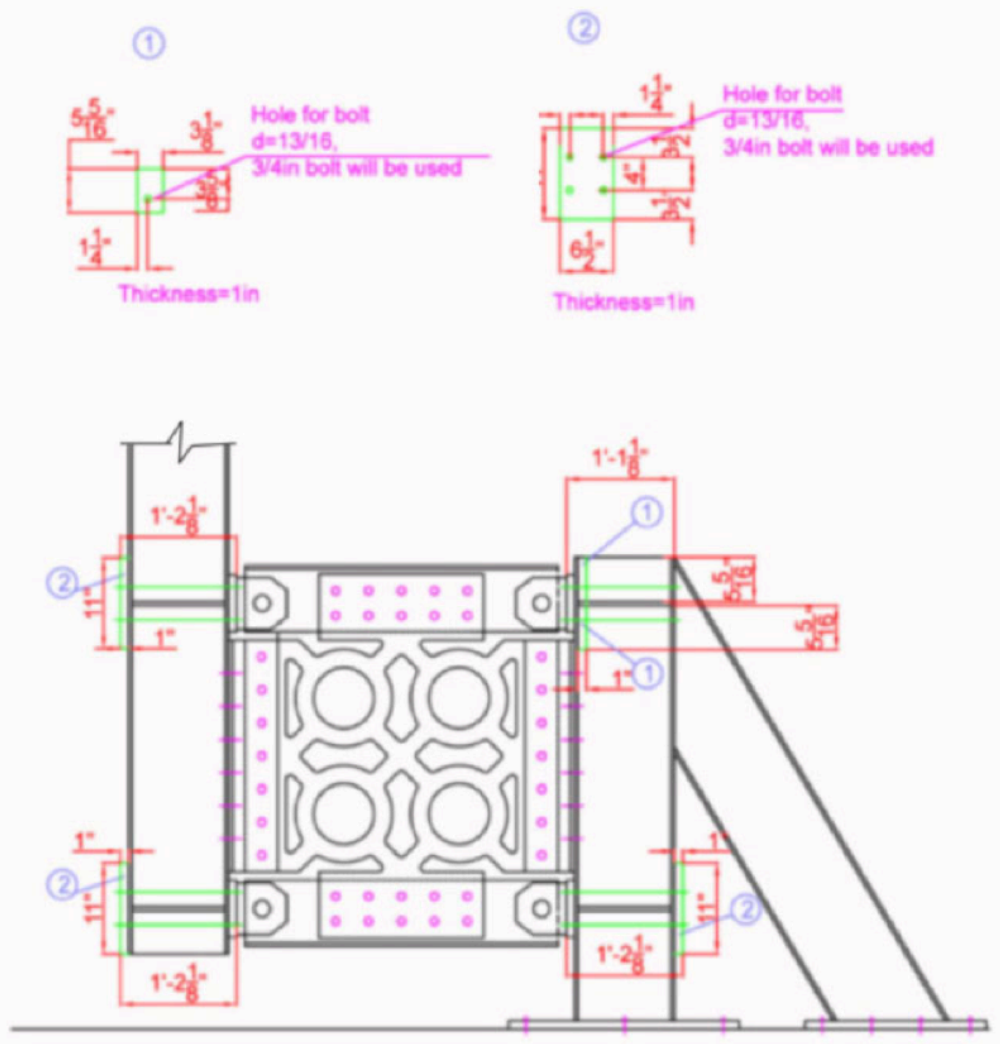

Figure 51. Surrounding frame repair after Test 1 


\subsection{TEST 2 - Specimen "Solid Plate"}

Figure 52 and Figure 53 show the side of the plate with photogrammetry targets and the side with whitewash, respectively, before the test was started. Table 10 and Figure 54 represent the Specimen "Solid Plate" geometric properties. All dimensions are given in inches.

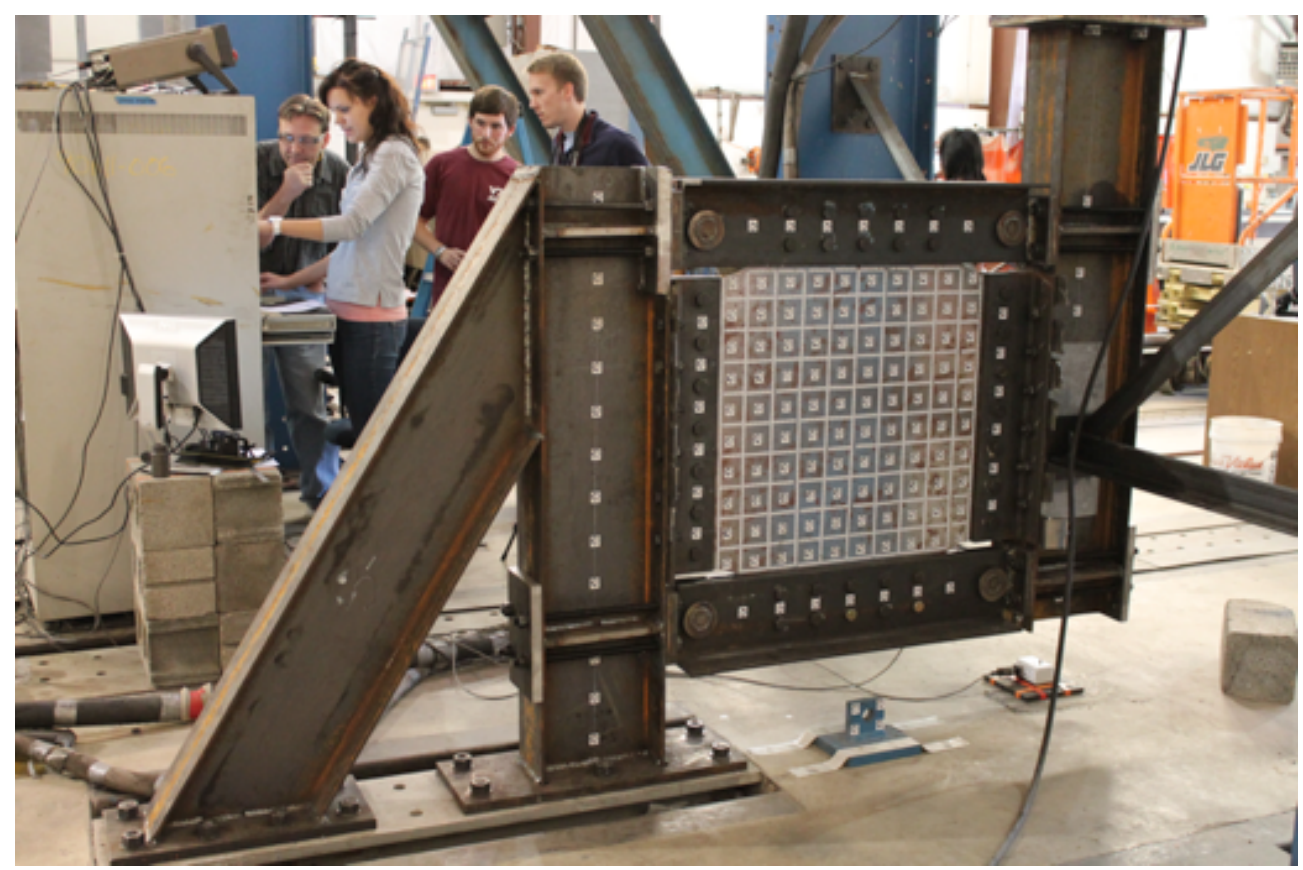

Figure 52. Photogrammetry side view - Test 2 - Specimen "Solid Plate"

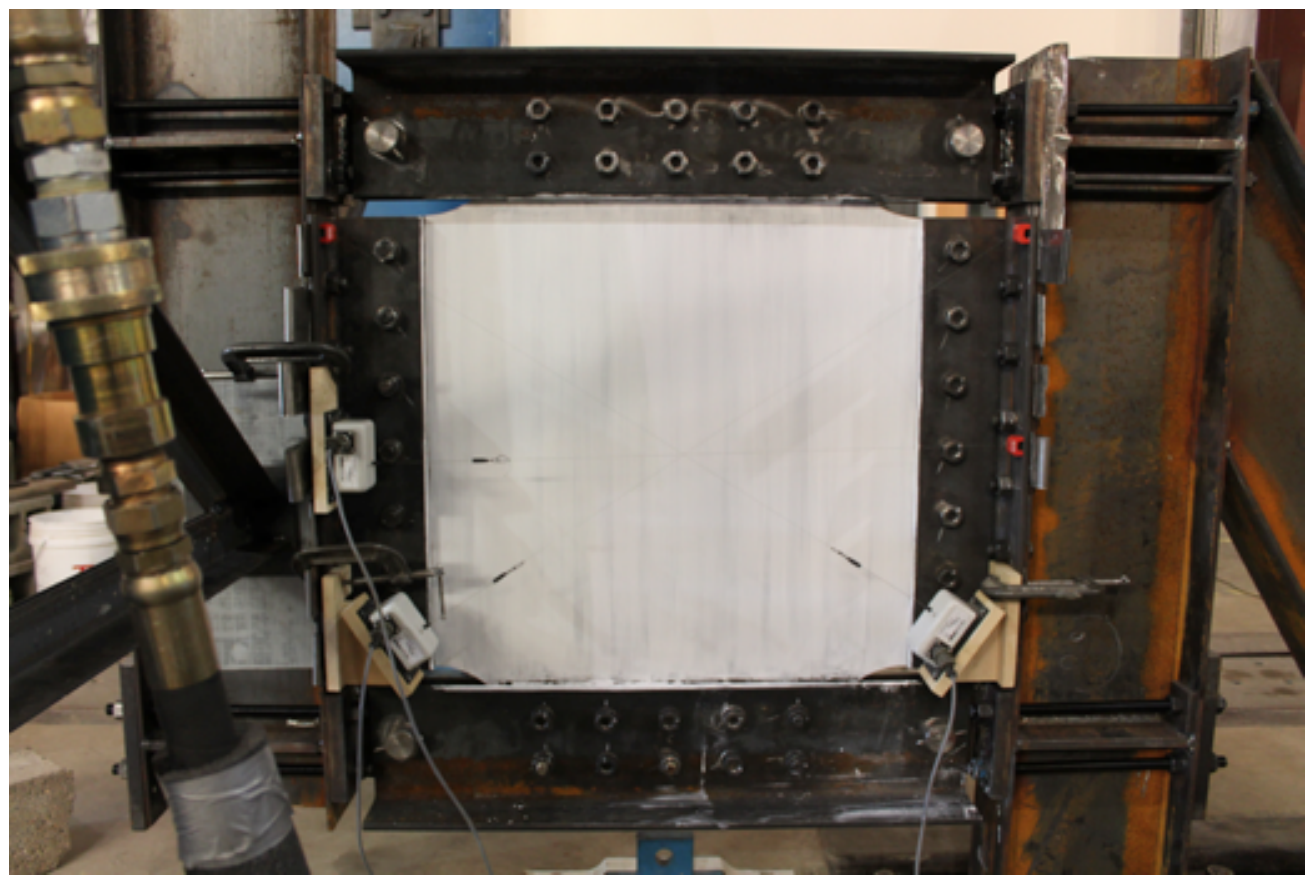

Figure 53. Whitewash side view - Test 2 - Specimen "Solid Plate" 
Table 10. Specimen "Solid Plate" properties

\begin{tabular}{|l|l|}
\hline Thickness of the plate $\left(t_{w}\right)$ & 0.075 in \\
\hline Outer ring radius $\left(R_{0}\right)$ & - \\
\hline Ring width $\left(w_{c}\right)$ & - \\
\hline Link width $\left(w_{L}\right)$ & - \\
\hline Number of rings in a row $(N)$ & - \\
\hline Pin to pin dimensions (a) & 34in x 34in \\
\hline
\end{tabular}

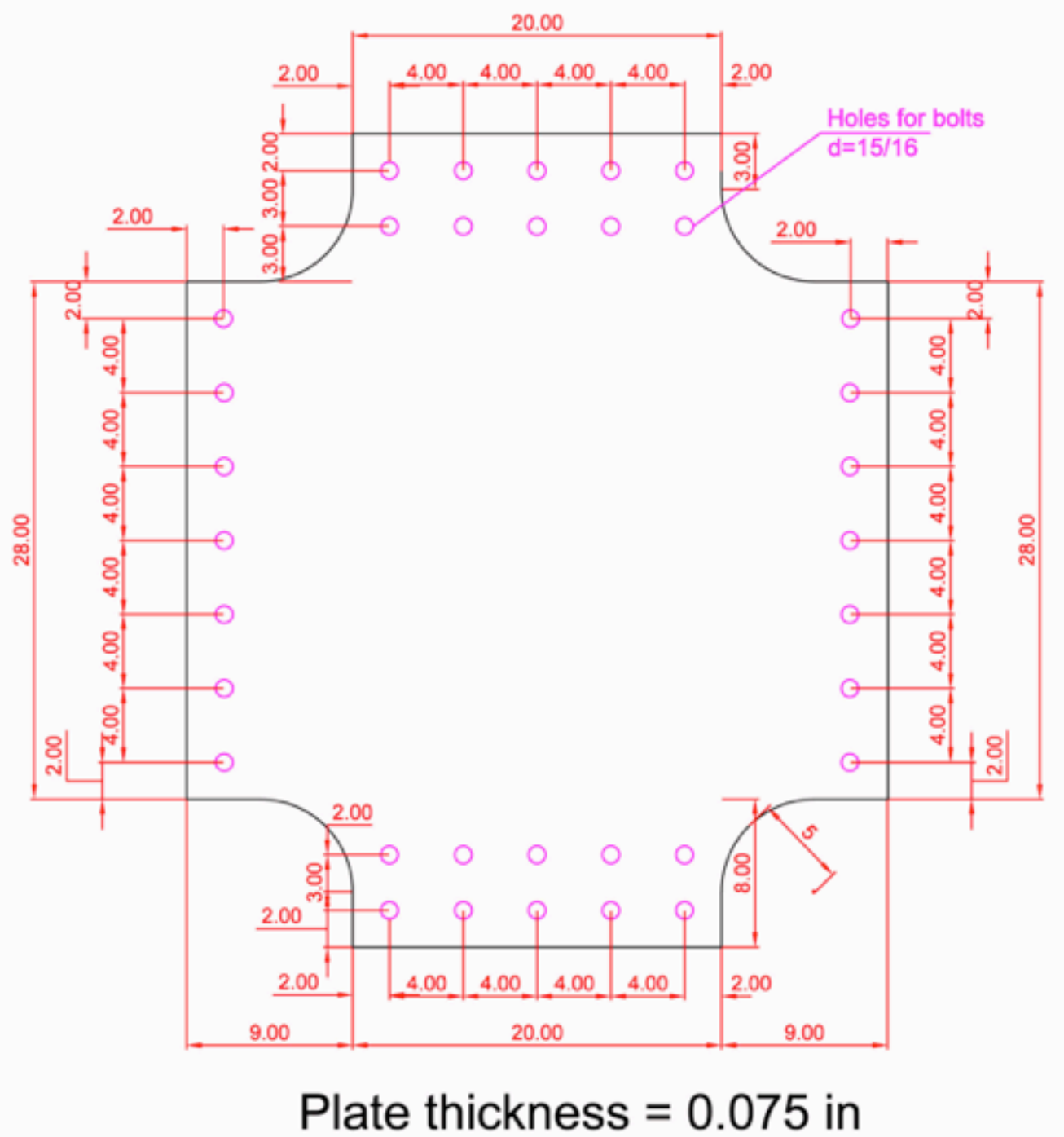

Figure 54. Test 2, Specimen "Solid Plate" geometry

The initial shape of the Specimen "Solid Plate" was obtained by exporting initial photogrammetry data to MATLAB and is shown in Figure 55. The magnitude of maximum initial imperfection of Specimen "Solid Plate" was measured to be $0.08 \mathrm{in}$. It was calculated like a difference between the biggest and the smallest $Z$ coordinate of all targets. 


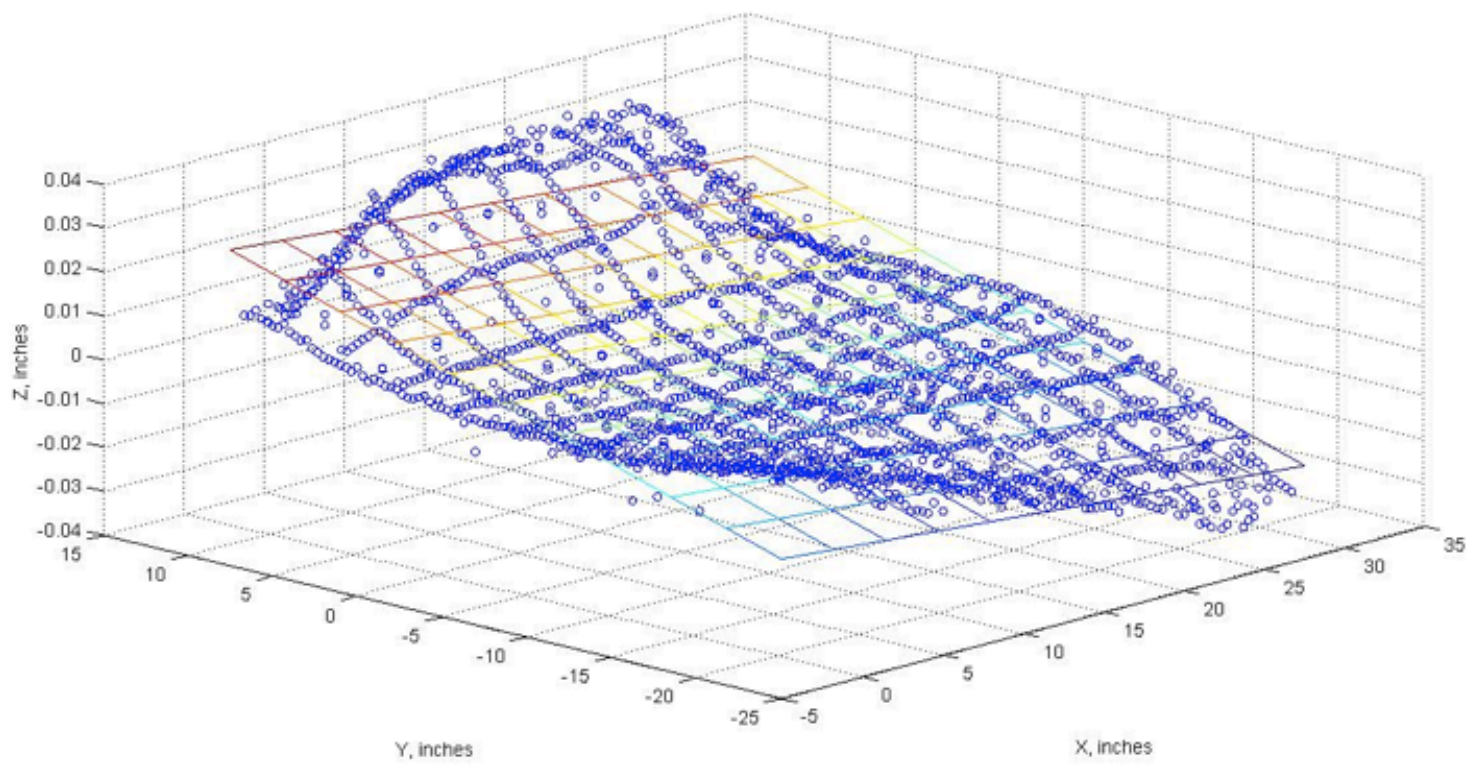

Figure 55. Point cloud and orthogonal regression plot - Specimen "Solid Plate" initial shape

During Test 1 it was observed that actuators vertical displacement is always bigger than plate vertical displacement. So in order to get peak displacements for the remaining seven tests, it was decided to multiply all peak displacements applied for Test 1 by a certain factor. A factor of 1.3 was found to be reasonable based on the results of Test 1 . Displacements applied during Test 2 are shown in Figure 56 and Table 11.

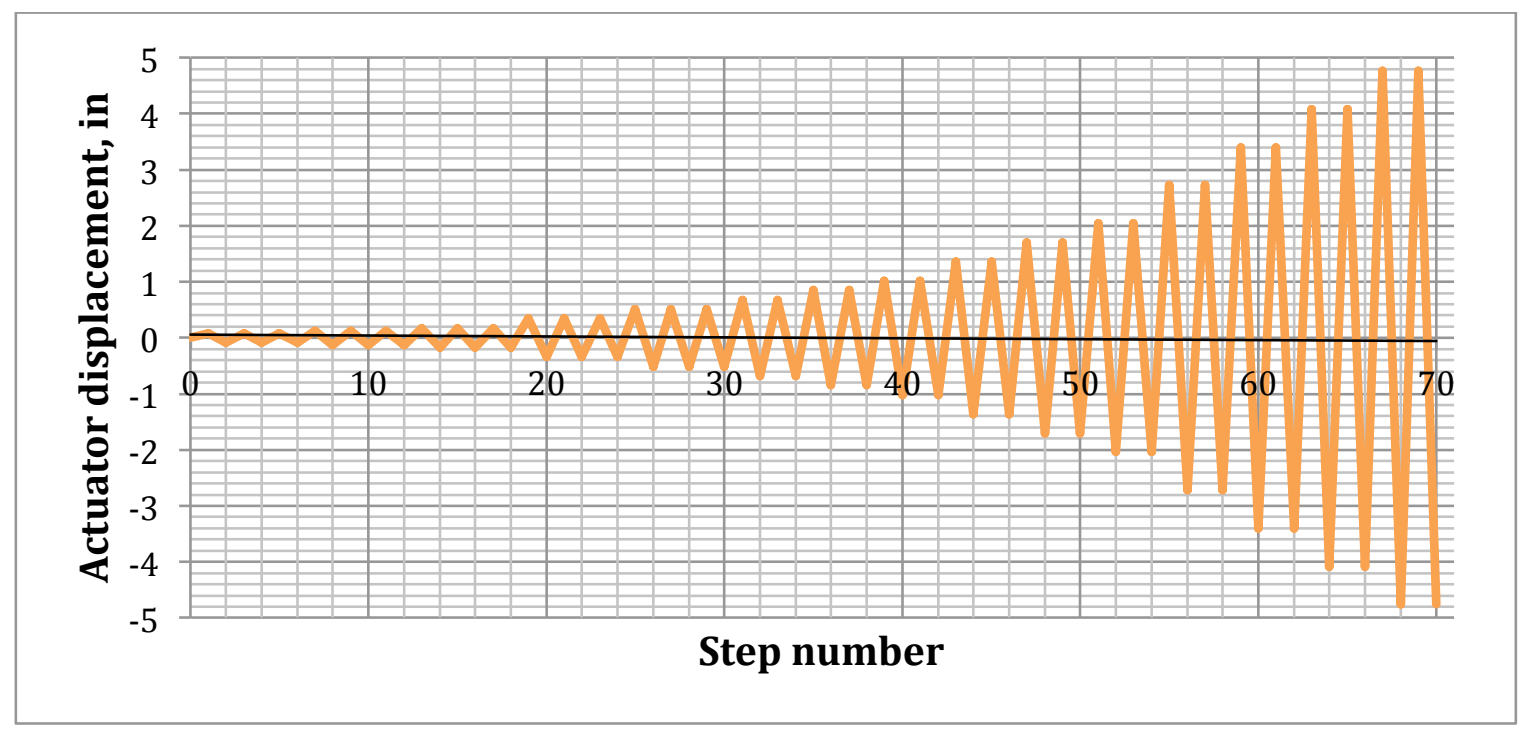

Figure 56. Displacement protocol - Test 2 
Table 11. Displacement protocol - Test 2

\begin{tabular}{|c|c|}
\hline $\begin{array}{c}\text { Applied actuator } \\
\text { displacement, in }\end{array}$ & N of cycles \\
\hline 0.11 & 3 \\
\hline 0.17 & 3 \\
\hline 0.22 & 3 \\
\hline 0.44 & 3 \\
\hline 0.66 & 3 \\
\hline 0.88 & 2 \\
\hline 1.11 & 2 \\
\hline 1.33 & 2 \\
\hline 1.77 & 2 \\
\hline 2.21 & 2 \\
\hline 2.65 & 2 \\
\hline 3.54 & 2 \\
\hline 4.42 & 2 \\
\hline & \\
\hline
\end{tabular}

Figure 57 shows the behavior of Specimen "Solid Plate". The shear displacement of the plate $(\Delta)$ was calculated using the diagonal string potentiometers (see Chapter 5.5.2). Pin to pin dimension of the plate $(a)$ was equal to $34 \mathrm{in}$. As expected, yielding and buckling of the specimen occurred early during the test. Photogrammetry point cloud shows that significant buckling of the plate occurred already at $0.5 \%$ of the drift (Figure 58). The corresponding hysteretic behavior was highly pinched as the plate acted in a manner similar to tension-only bracing producing little resistance during load reversal. The buckling increased in amplitude as the shear angle was increased. Tearing of the plate started at 6\% drift at the point close to the plate corner (Figure 59a) and propagated fast. The cause of the tearing could be sharp folds in the plate at this location. Small tearing of the plate at points close to other corners was observed later during the test. Furthermore, bearing failure of the plate at the bolts holes happened. The bearing deformations were noted as the plate began to pull out of the bolted perimeter connections at the corners. Another tearing was noticed at 8\% drift. Tearing occurred at the bottom of the plate and started to propagate fast all along the bottom connection (Figure 59b). There was a significant drop in capacity associated with the tearing. The test was stopped because of significant tearing developed in the web plate. The mode of buckling was global shear buckling. 


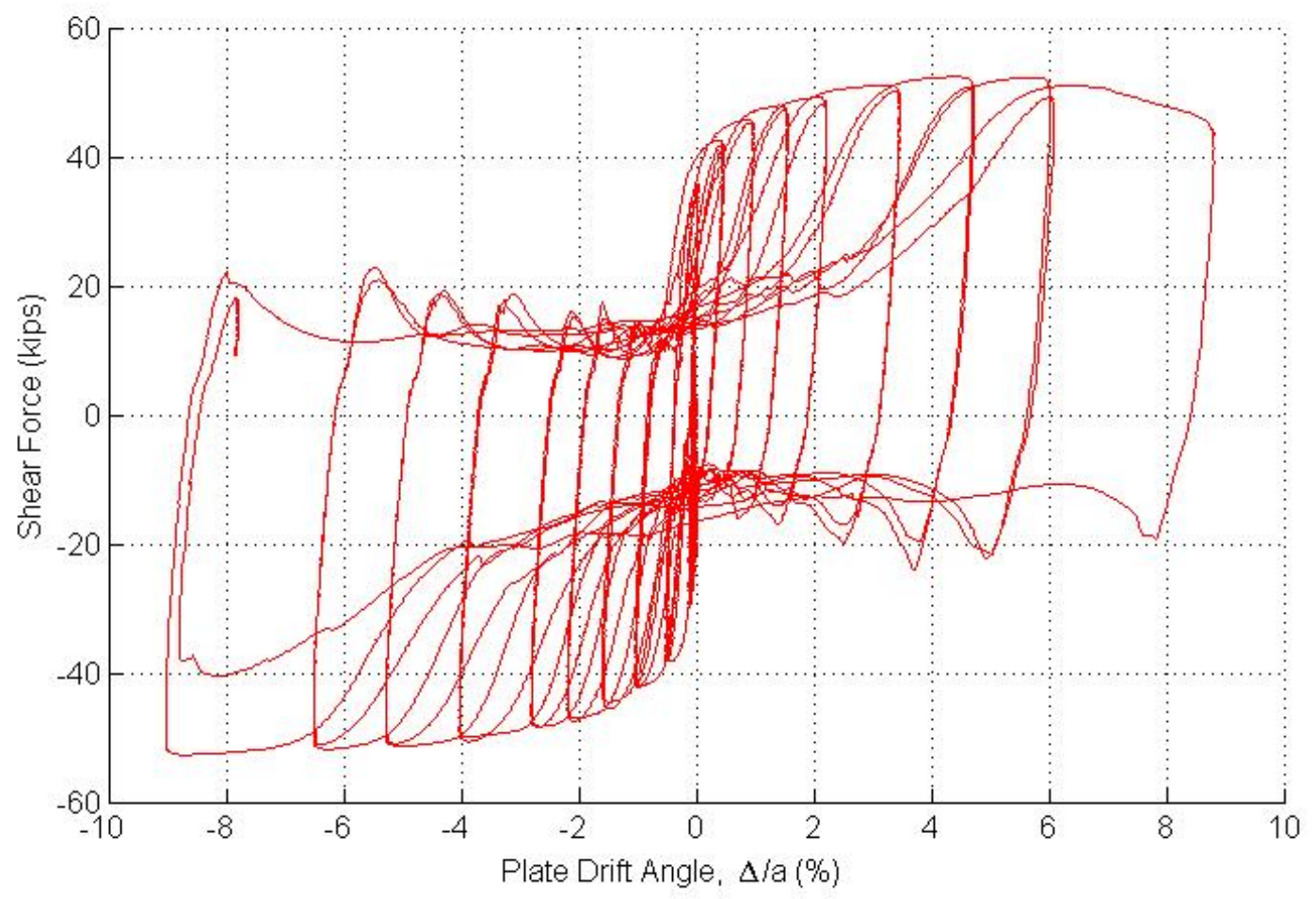

Figure 57. Shear Force versus Plate Drift Angle - Test 2 - Specimen "Solid Plate"

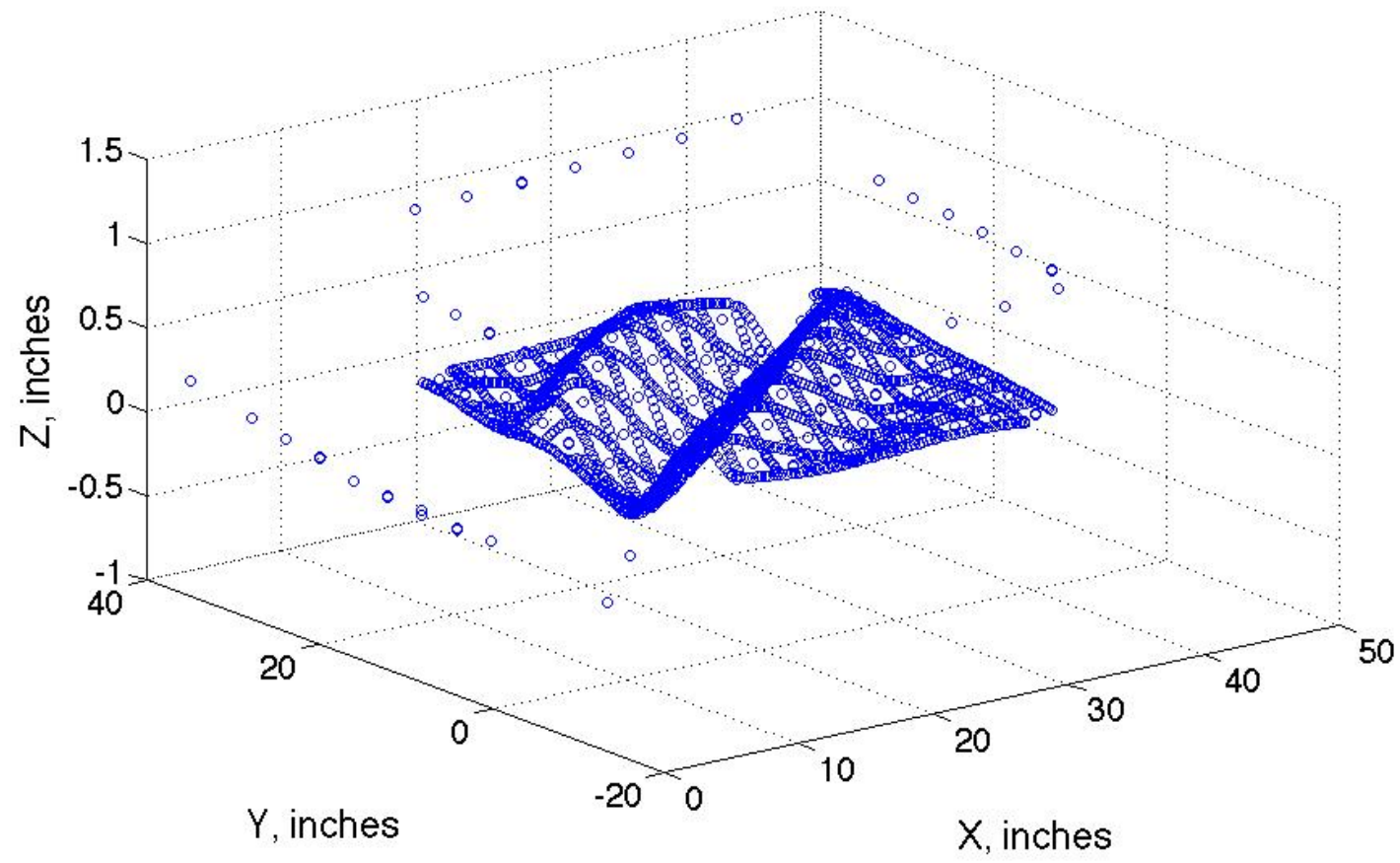

Figure 58. Point cloud - Specimen "Solid Plate" - 0.5\% drift 


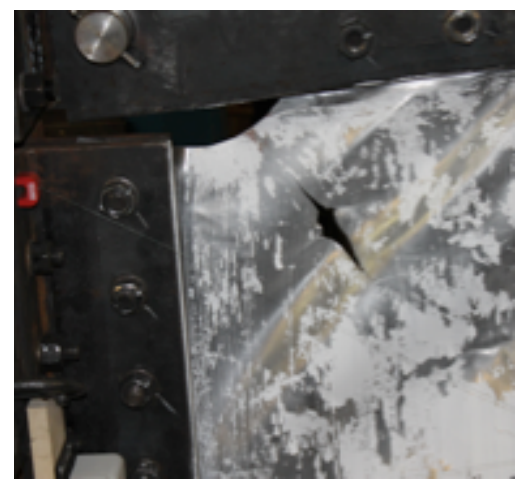

(a)

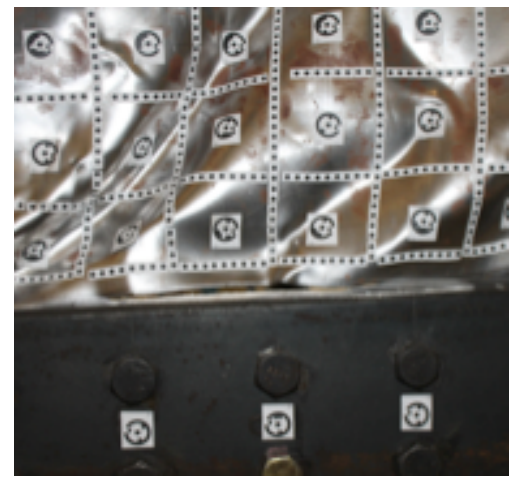

(b)

Figure 59. Tears in Specimen "Solid Plate"

During the test it was noticed that load versus actuator vertical displacement plot is significantly unsymmetrical about $\mathrm{x}$ axis. It was assumed that the initial load (before the test was started) was not 0 . But as far as the load channel was zeroed before the test, the data acquisition system showed 0. After the test was finished and specimen "Solid Plate" was removed from the frame, this hypothesis was checked and confirmed. That time the MTS load channel was not zeroed before test was started, and data acquisition system showed 8.0 kips. Later this value was subtracted from all data for load obtained from the test, which gave symmetric load versus actuator vertical displacement plots (Figure 57). For all future tests MTS load channel was not zeroed before the test to be able to get a real value of the initial load. 


\subsection{TEST 3 - Specimen 1-0.5-1}

Figure 60 and Figure 61 show the side of the plate with photogrammetry targets and the side with whitewash, respectively, before the test was started. Table 12 and Figure 62 represent the Specimen "Solid Plate" geometric properties. All dimensions are given in inches.

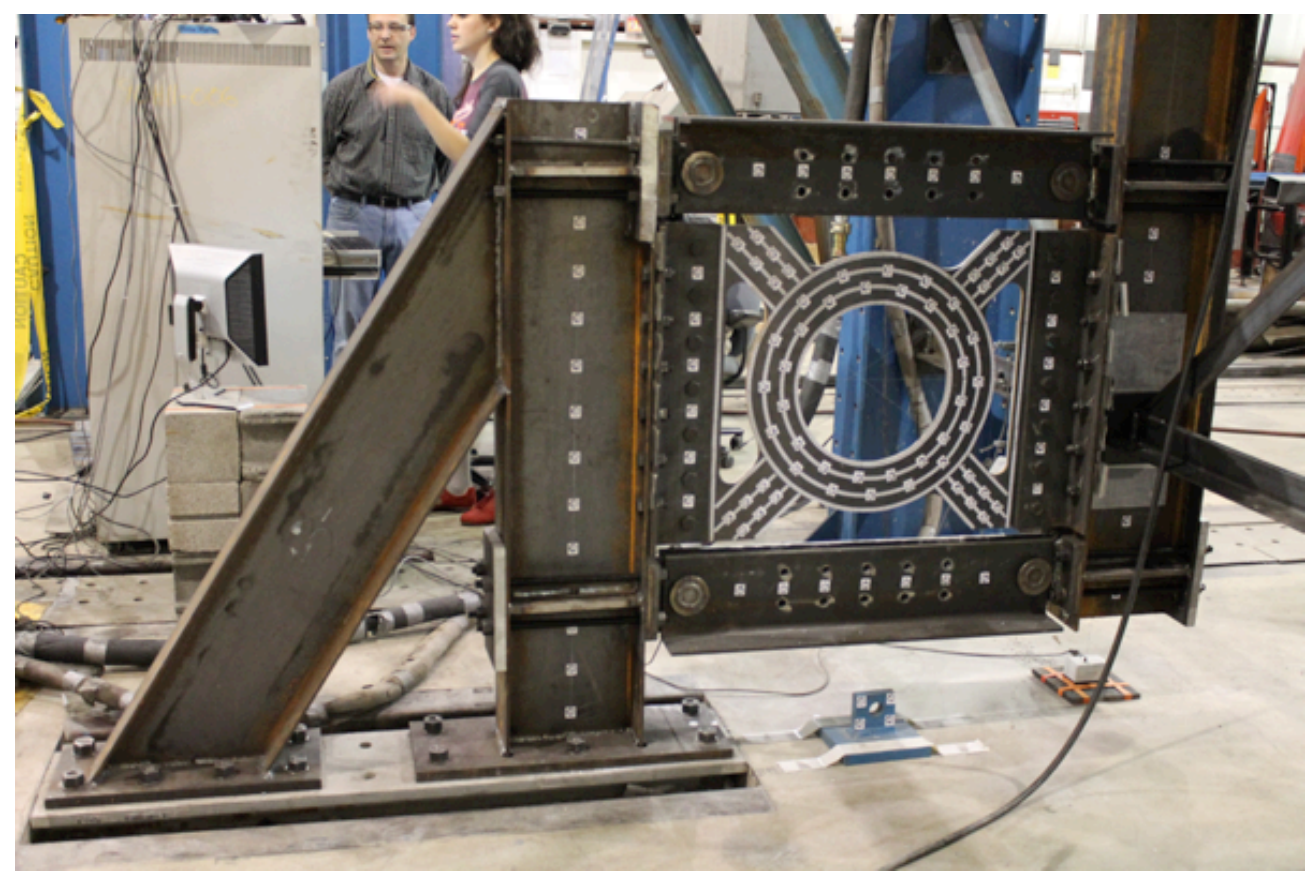

Figure 60. Photogrammetry side view - Test 3 - Specimen 1-0.5-1

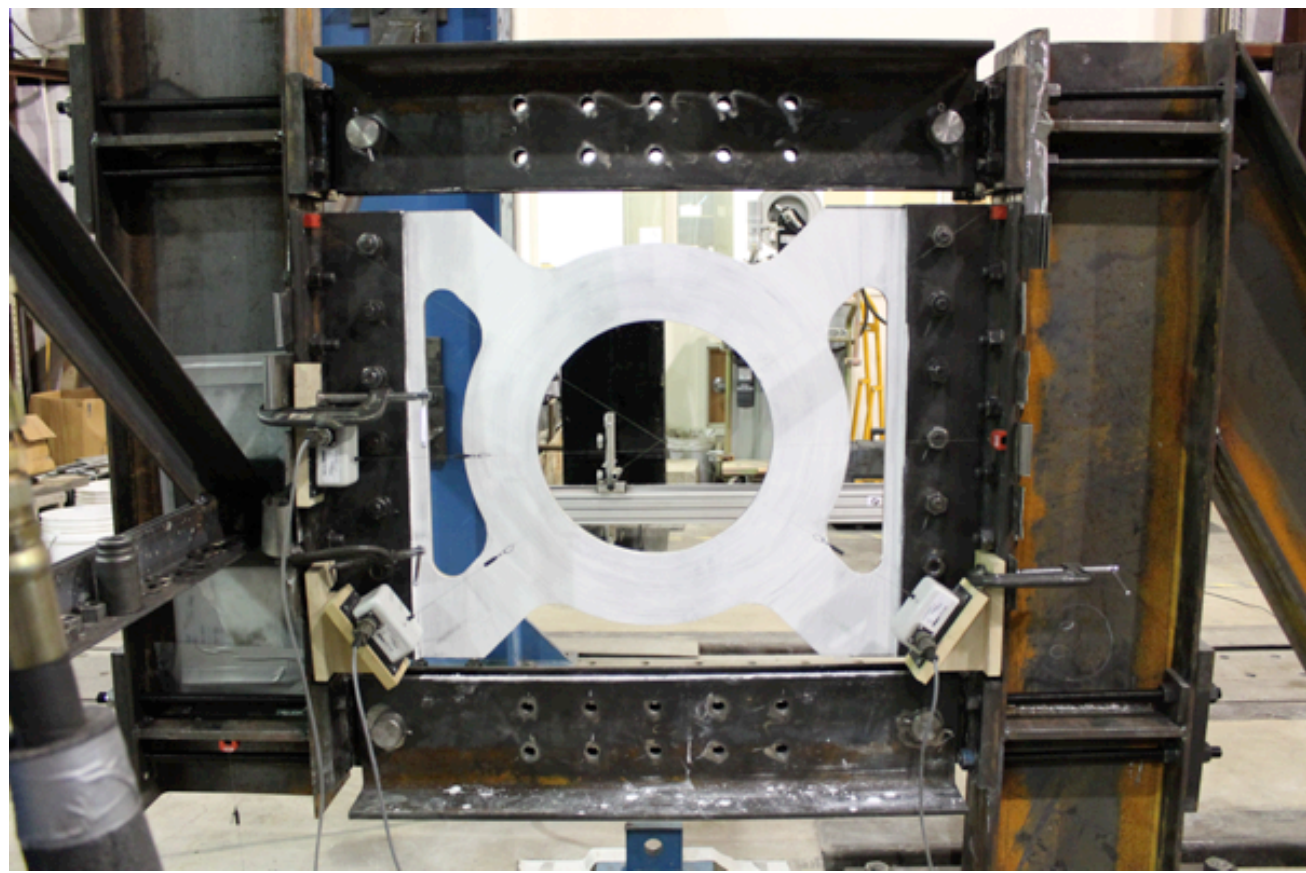

Figure 61. Whitewash side view - Test 3 - Specimen 1-0.5-1 


\section{Table 12. Specimen 1-0.5-1 properties}

\begin{tabular}{|l|l|}
\hline Thickness of the plate $\left(t_{w}\right)$ & 0.5 in \\
\hline Outer ring radius $\left(R_{0}\right)$ & 11.79 in \\
\hline Ring width $\left(w_{c}\right)$ & 4.42 in \\
\hline Link width $\left(w_{L}\right)$ & 4.42 in \\
\hline Number of rings in a row $(N)$ & 1 \\
\hline Pin to pin dimensions (a) & 34 in x 34in \\
\hline
\end{tabular}

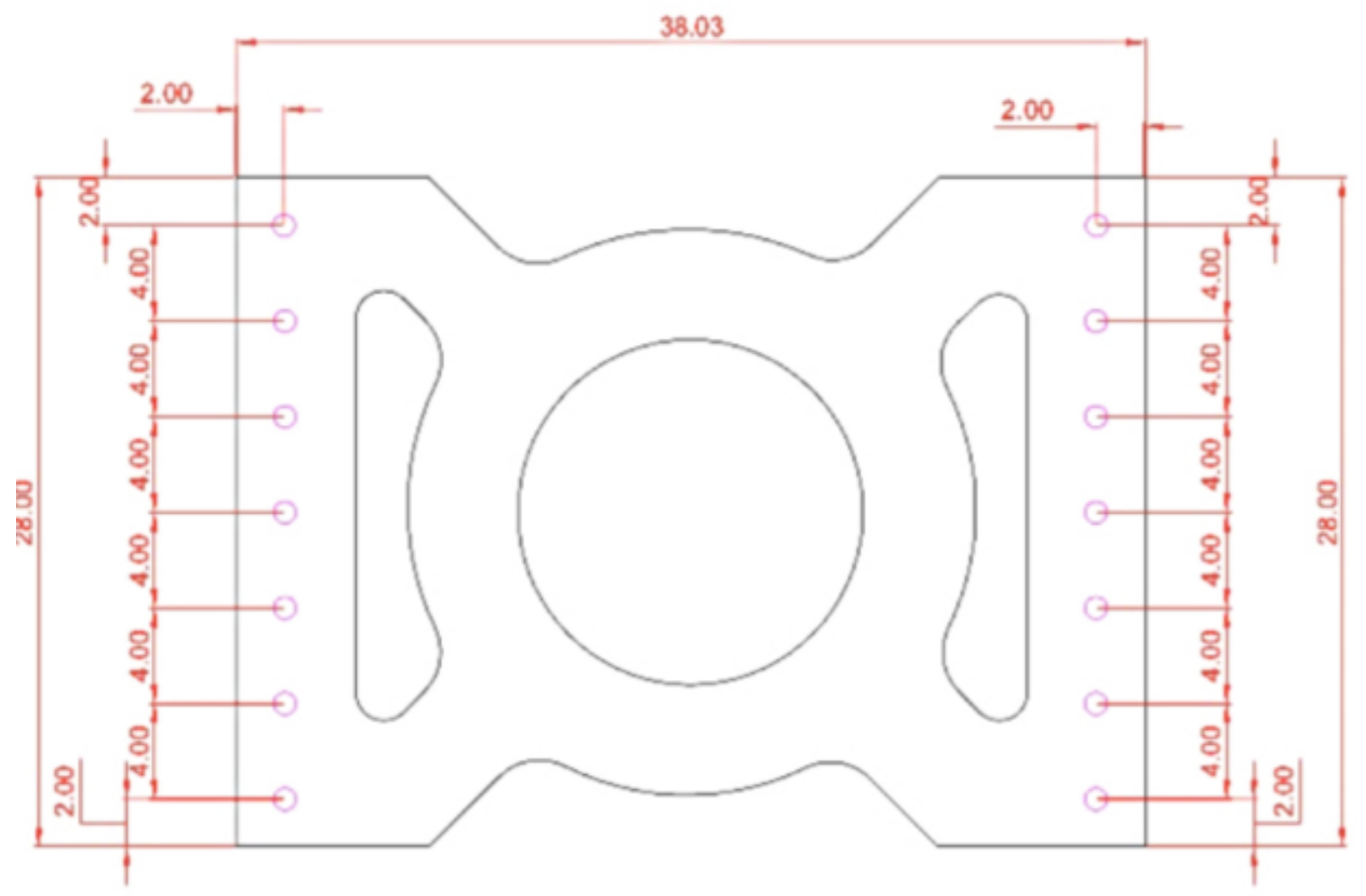

Plate thickness $=0.5$

Figure 62. Test 3, Specimen 1-0.5-1 geometry

The initial shape of the Specimen 1-0.5-1 was obtained by exporting initial photogrammetry data to MATLAB and is shown in Figure 63. Magnitude of maximum initial imperfection of Specimen 1-0.5-1 was measured to be $0.0691 \mathrm{in}$. It was calculated like a difference between the biggest and the smallest $Z$ coordinate of all targets. 


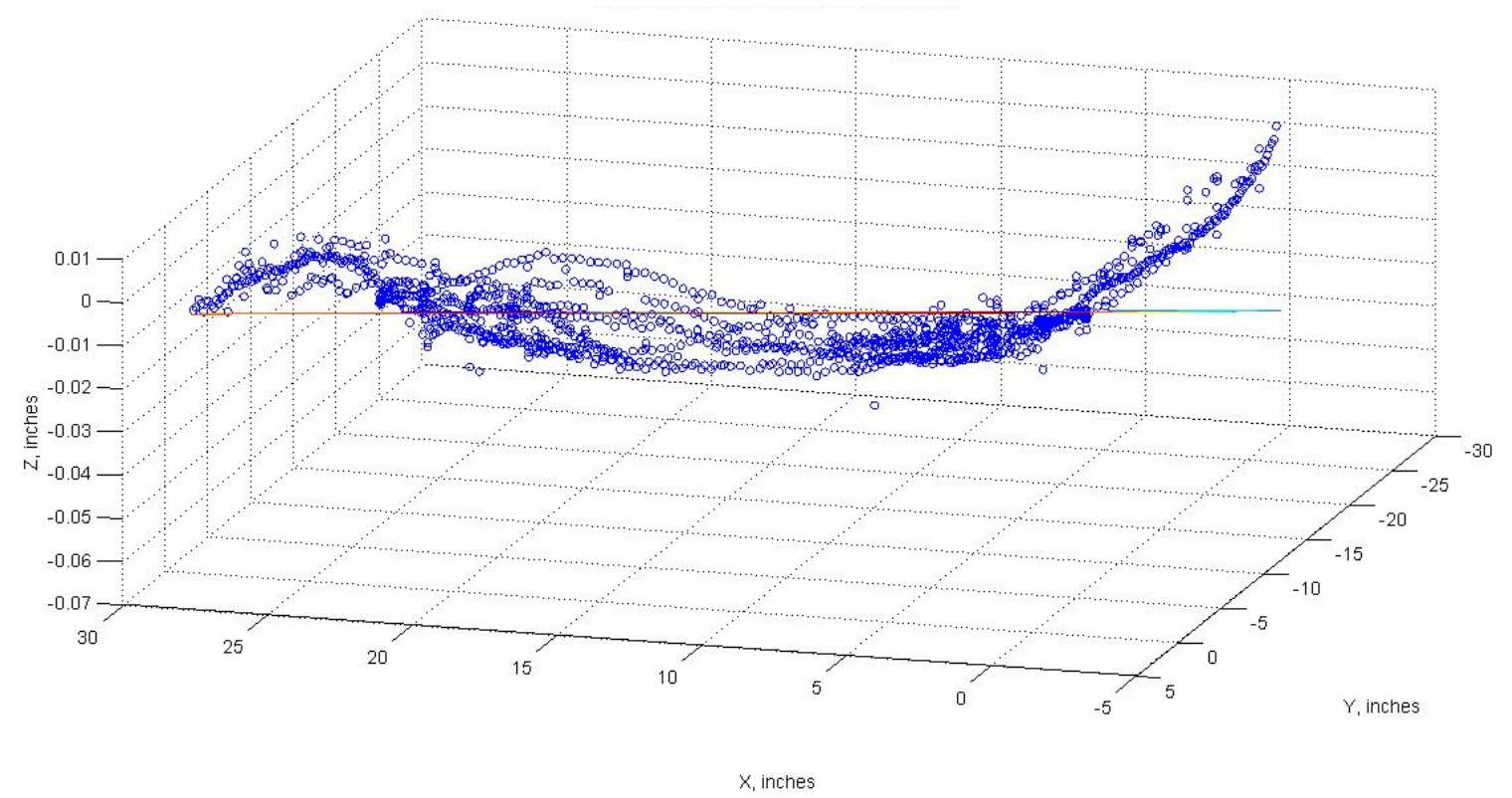

Figure 63. Point cloud and orthogonal regression plot - Specimen 1-0.5-1 initial shape

When Test 3 was started, it was noticed that actuator was not moving correctly. Instead of holding on the same position when "hold" bottom was pressed, it kept moving down. As a result actuator already moved 1 in down before the hydraulics could be shut down. The test was stopped and the actuator was moved to its initial position. As a result of this 1 in down movement, the plate yielded. The yield can be seen on picture (Figure 64) made from the whitewash side. As it was found out later, one of the hoses connecting hydraulic and pump machine was disconnected and caused wrong behavior of the actuator.

The test was restarted from the initial position. During the first cycles, loud bangs started, which were concluded to be result of slipping of the bolts. It was also noticed that line of whitewash is no more parallel to the frame angle and it rotates to the right or to the left depending on the position of the actuator (Figure 65). At $3 \%$ of the drift position the test was paused and more powerful impact ranch was used to pretension all the bolts. After that the test was continued and much less bangs were heard after that. No more slipping of the bolts was noticed.

During the test because of slipping of the bolts, the plate experienced significant shakes, which resulted in the slight movement of the horizontal and diagonal string potentiometers. 


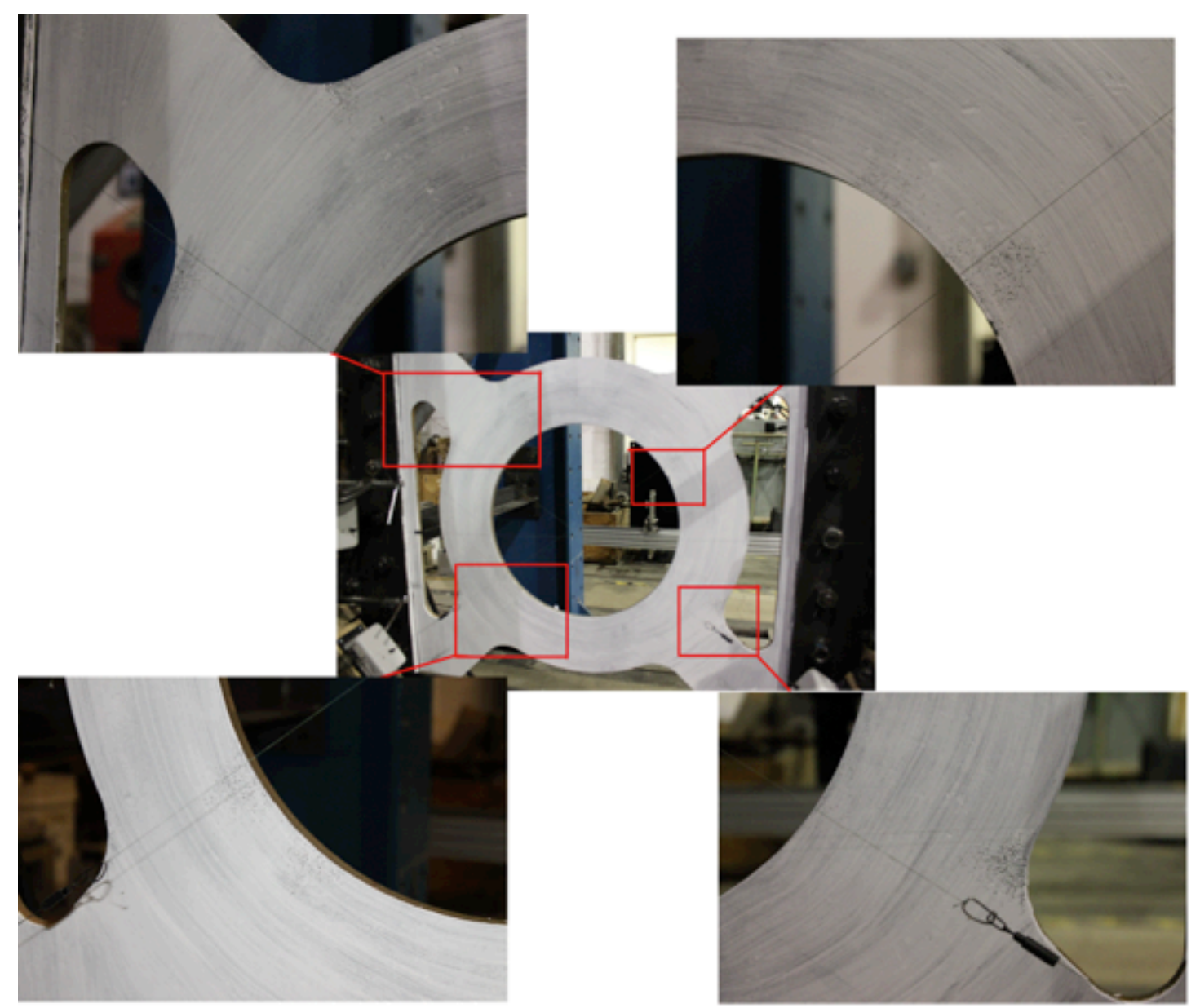

Figure 64. Yielding of the plate - result of wrong work of hydraulic machine - Test 3

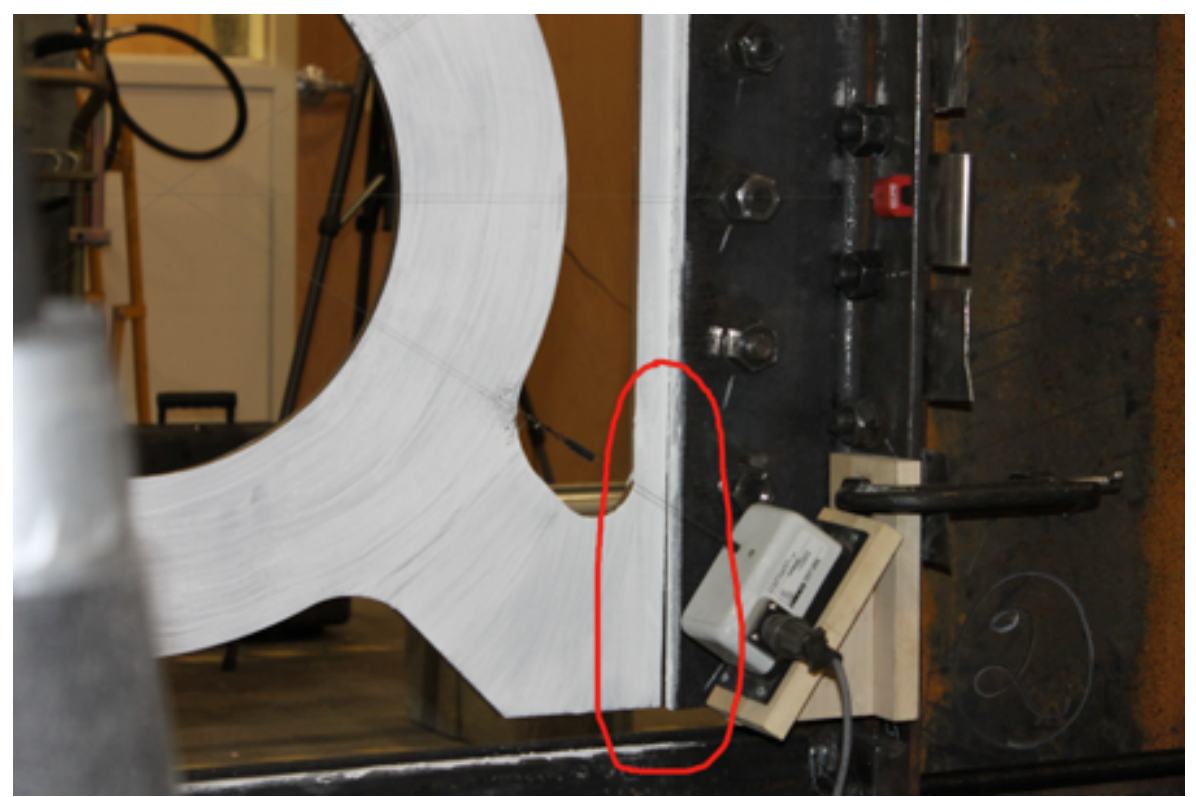

Figure 65. Slipping of the bolts during Tests 3 
Displacements applied during Test 3 are shown in Figure 56 and Table 11. Figure 66 and Figure 67 show the behavior of Specimen 1-0.5-1. Two plots, one that uses diagonal string potentiometers and the other that uses vertical sting potentiometer, are provided here because of the problems with string potentiometers mentioned above. The shear displacement of the plate $(\Delta)$ was calculated using the diagonal string potentiometers (see Chapter 5.5.2). Pin to pin dimension of the plate (a) was equal to 34 in. First, the specimen deformed elastically. Then there was some initiation of yielding at outer radius of the ring at the locations close to where the ring is connected to the link (Figure 68). The buckling started to occur at $2.5 \%$ drift. The buckling increased in amplitude as the shear angle was increased. The ring experienced lateral torsional buckling of the top and bottom legs of the ring. The shape of the buckling can be seen on photogrammetry point cloud at $6.8 \% \mathrm{drift}$ (Figure 69). The lateral torsional buckling caused considerable deterioration in the strength and energy dissipation capability. No fracture occurred in the plate.

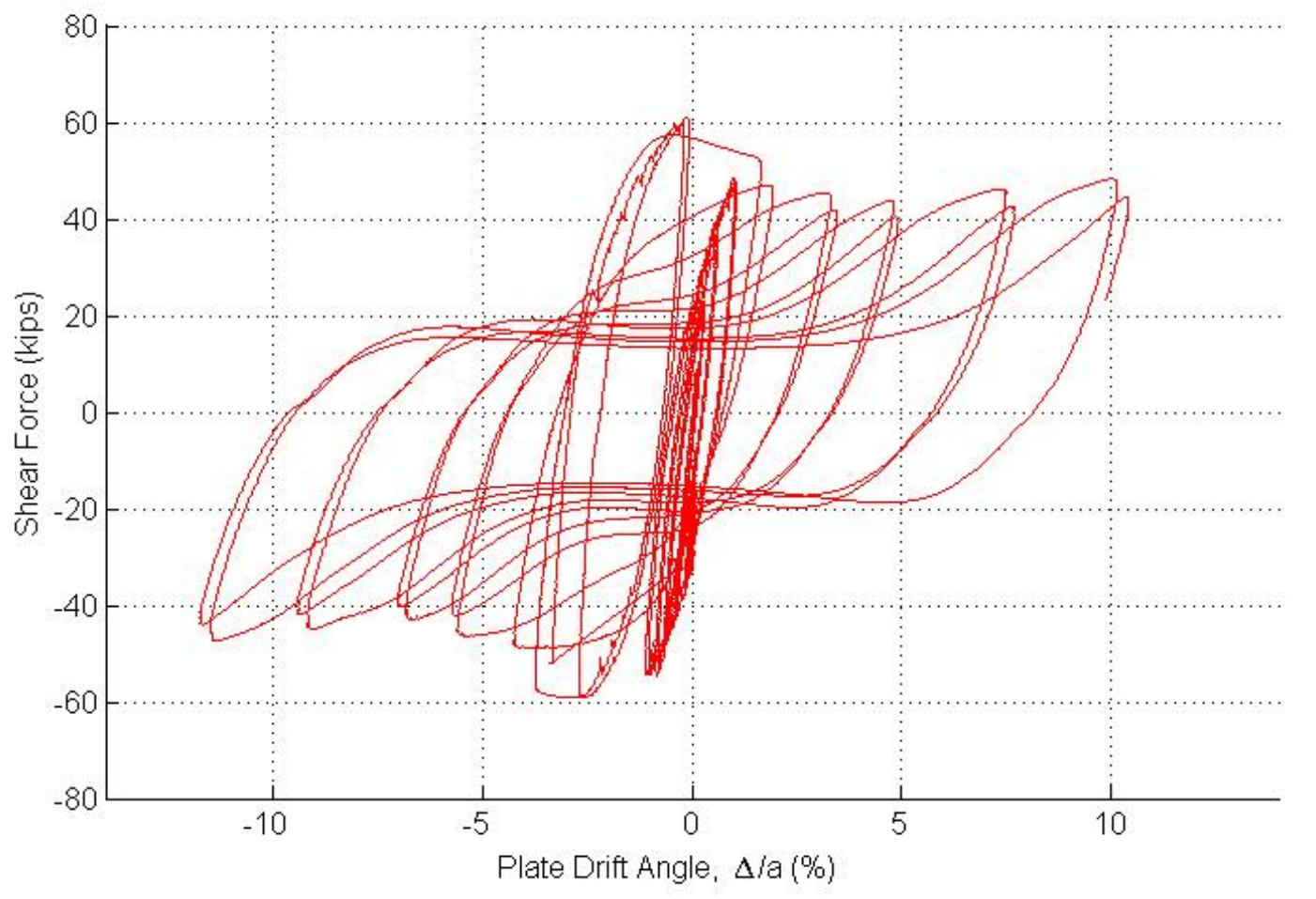

Figure 66. Shear Force versus Plate Drift Angle - Test 3 - Specimen 1-0.5-1 


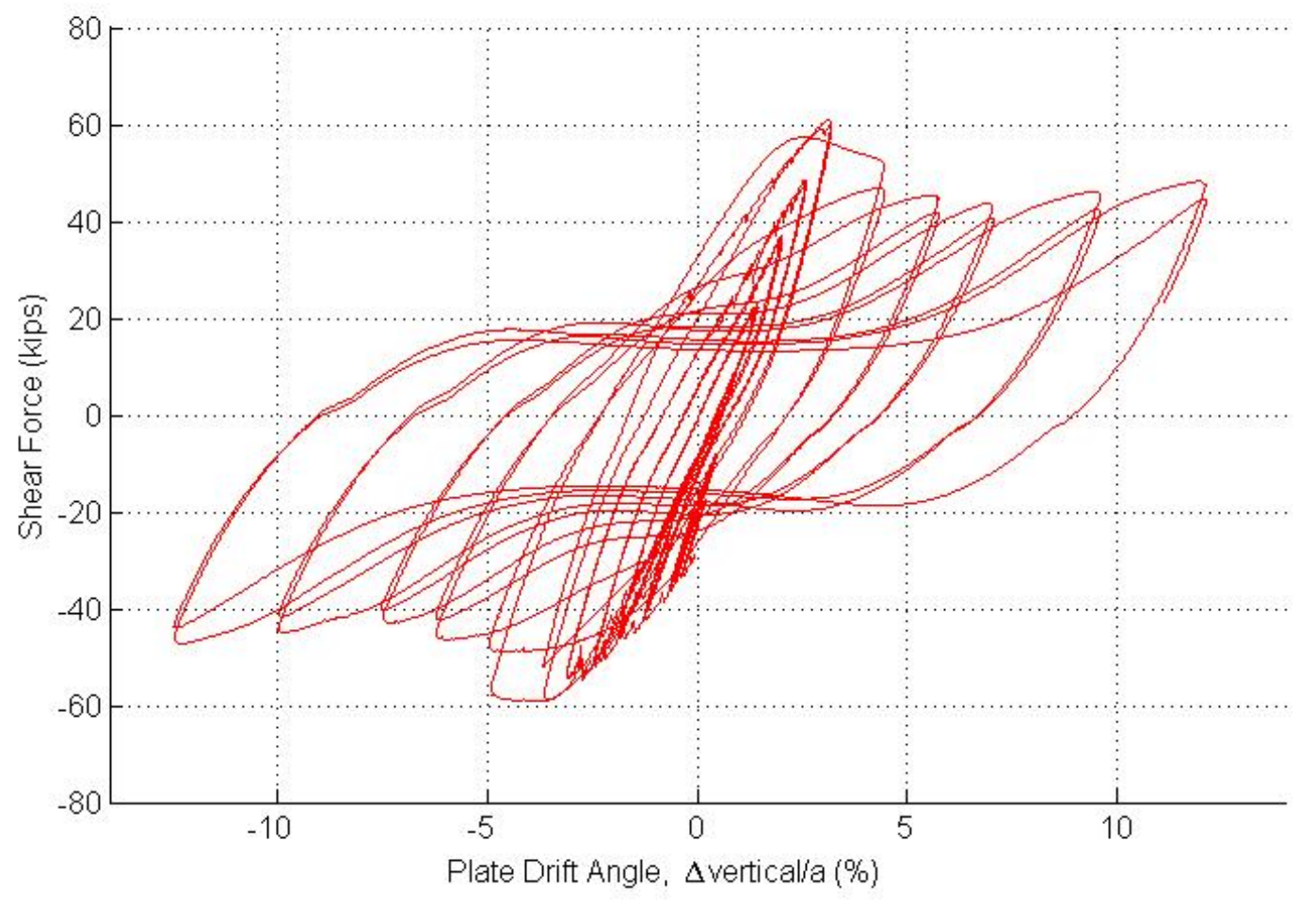

Figure 67. Shear Force versus Actuator Drift Angle - Test 3 - Specimen 1-0.5-1

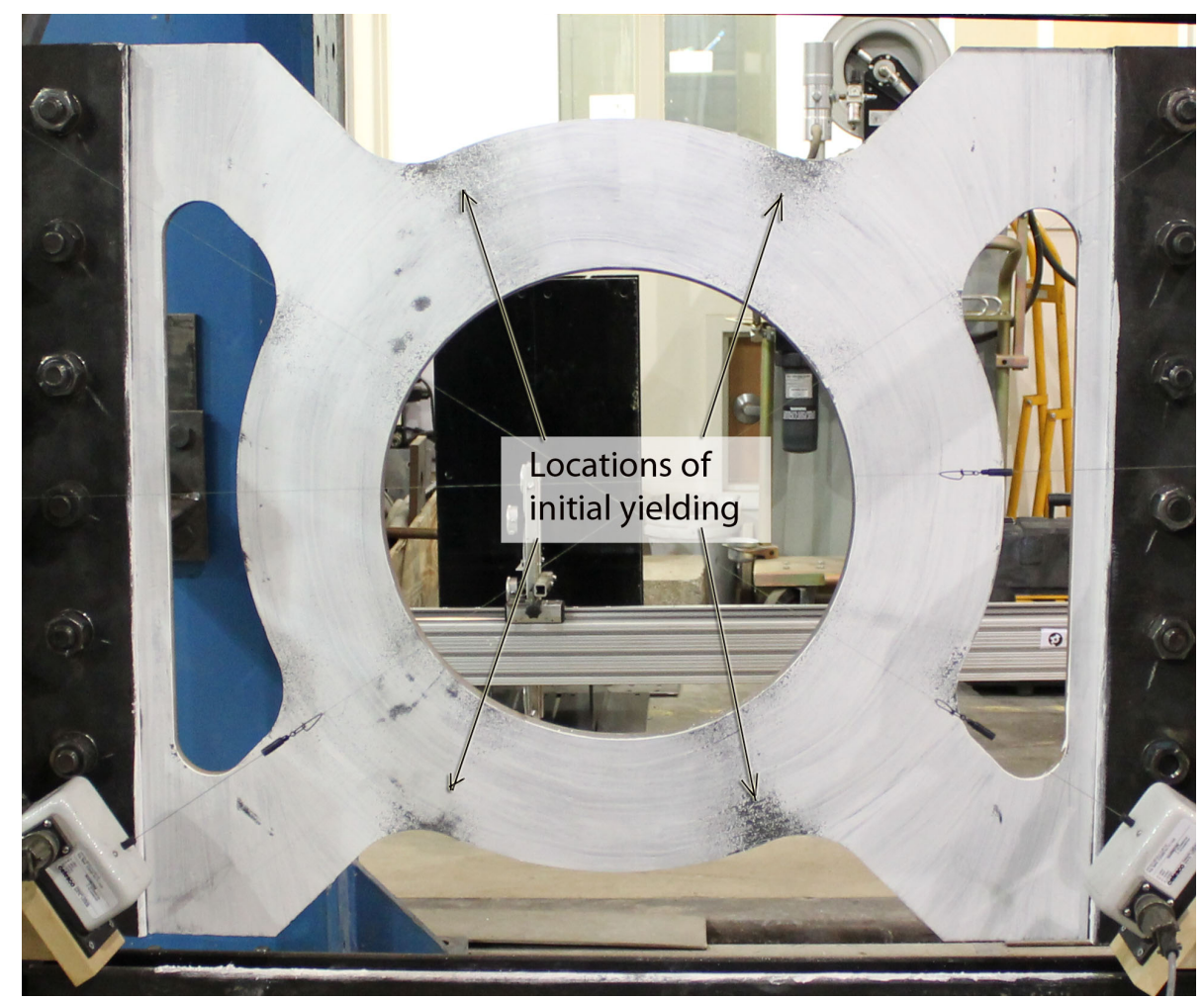

Figure 68. Location of initial yielding - Test 3-Specimen 1-0.5-1 


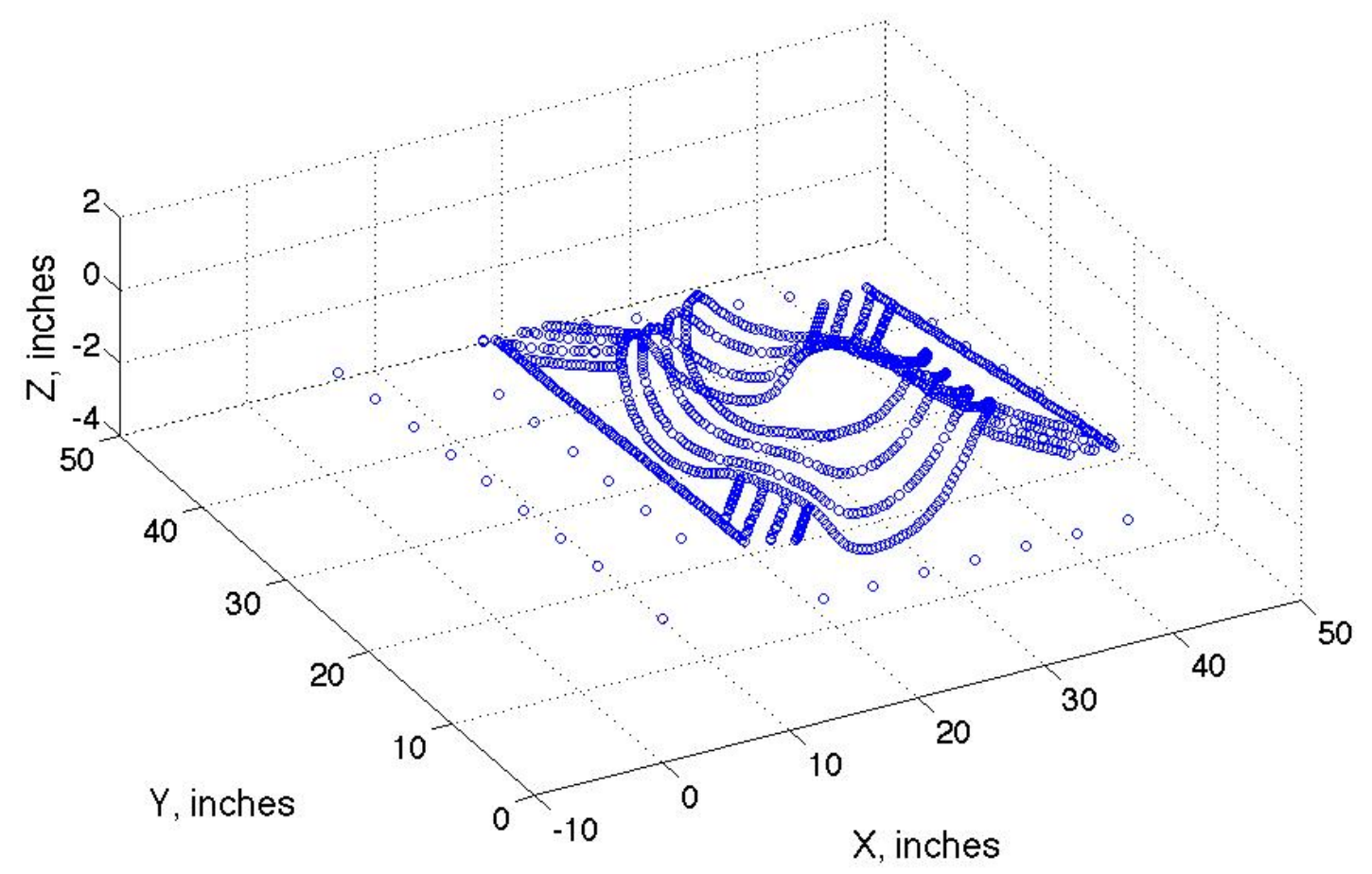

Figure 69. Point cloud - Specimen 1-0.5-1 - 6.8\% drift 


\subsection{Test 4 - Specimen 3-0.5-1}

Figure 70 and Figure 71 show the side of the plate with photogrammetry targets and the side with whitewash, respectively, before the test was started. Table 13 and Figure 72 represent the Specimen 3-0.5-1 geometric properties. All dimensions are given in inches.

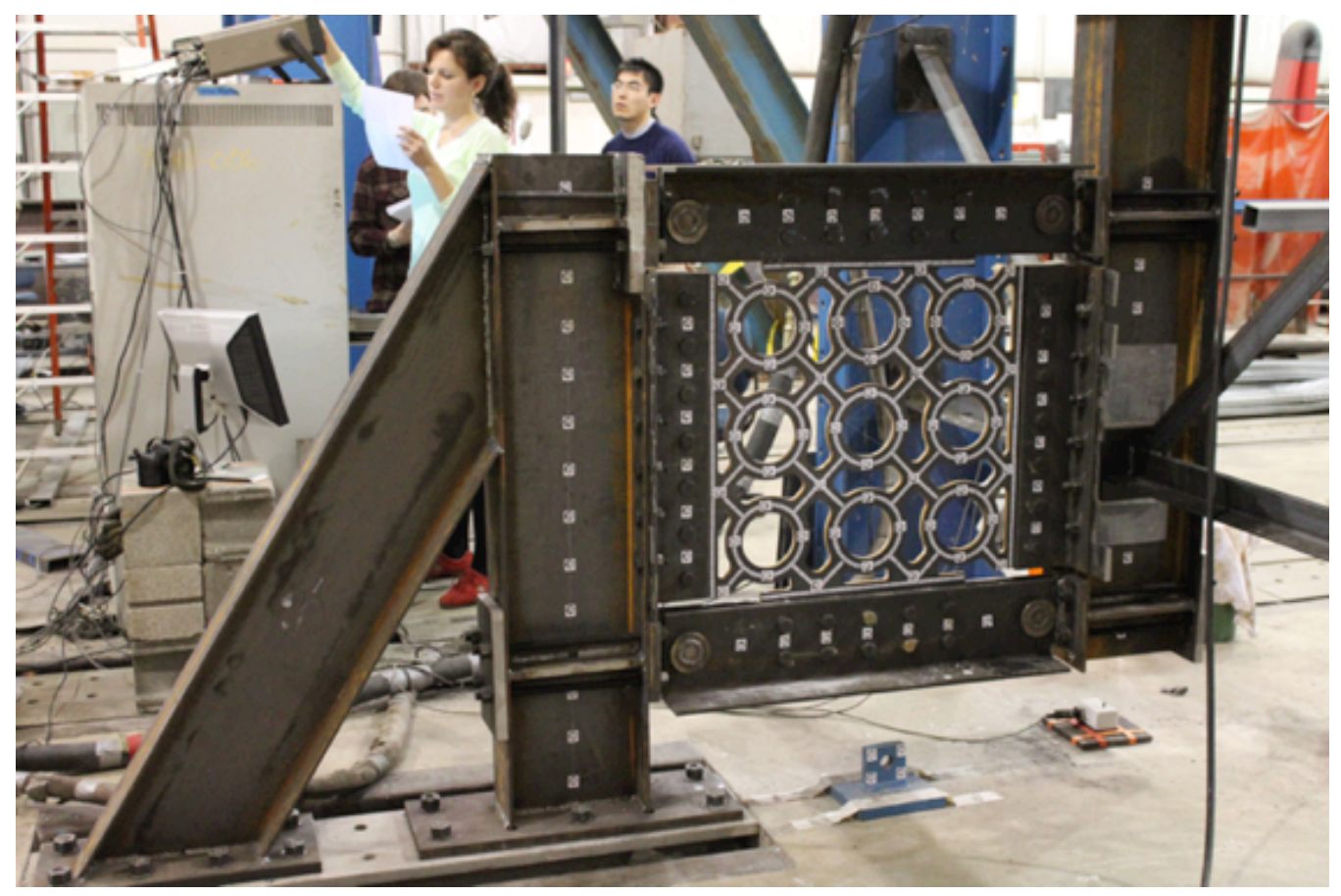

Figure 70. Photogrammetry side view - Test 4 - Specimen 3-0.5-1

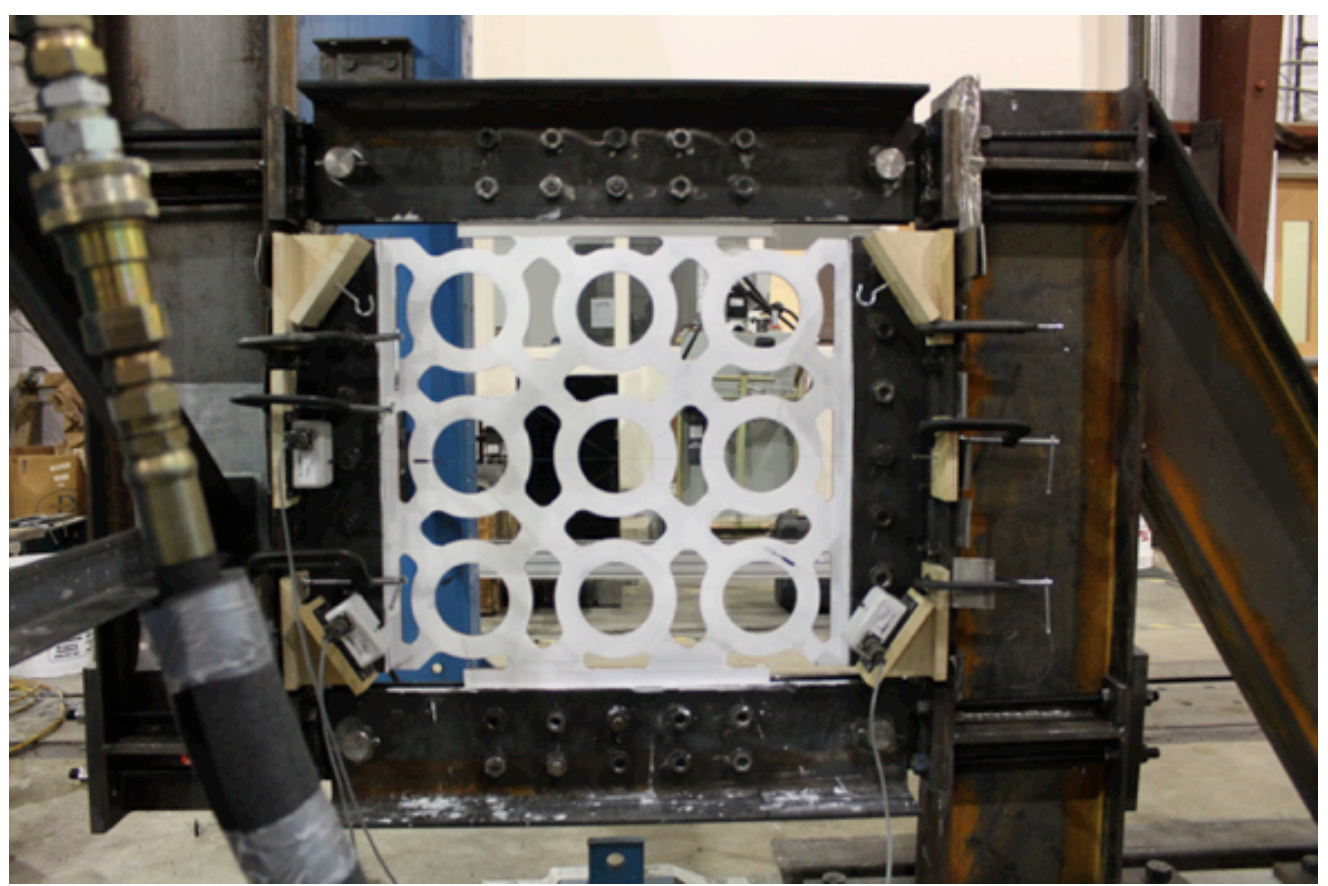

Figure 71. Whitewash side view - Test 4 - Specimen 3-0.5-1 
Table 13. Specimen 3-0.5-1 properties

\begin{tabular}{|l|l|}
\hline Thickness of the plate $\left(t_{w}\right)$ & 0.5 in \\
\hline Outer ring radius $\left(R_{0}\right)$ & 3.93 in \\
\hline Ring width $\left(w_{c}\right)$ & 1.47 in \\
\hline Link width $\left(w_{L}\right)$ & 1.47 in \\
\hline Number of rings in a row $(N)$ & 3 \\
\hline Pin to pin dimensions $(\mathrm{a})$ & $34 \mathrm{in} \times$ 34in \\
\hline
\end{tabular}

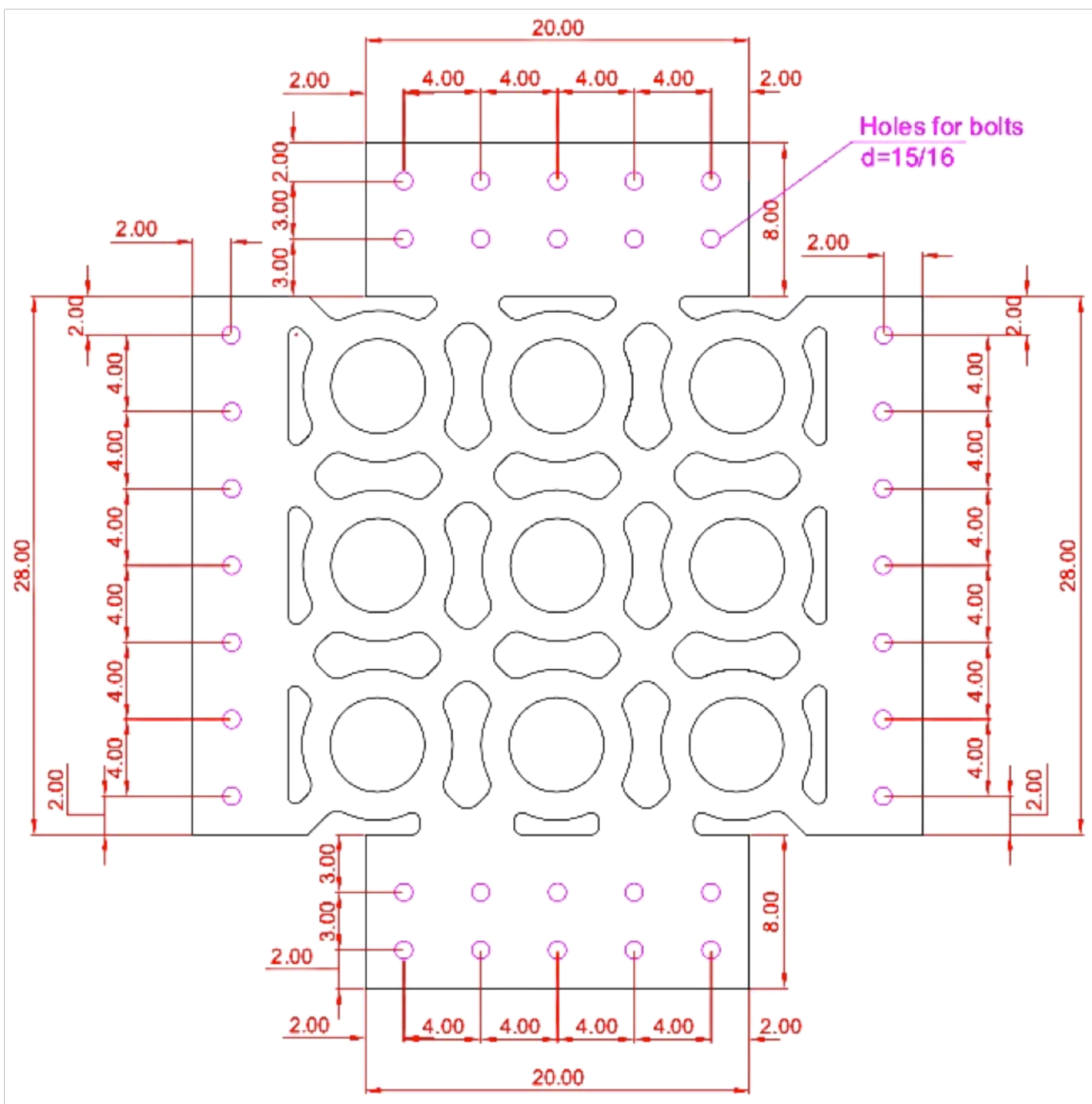

Plate thickness $=0.5$

Figure 72. Test 4, Specimen 3-0.5-1 geometry 
The initial shape of the Specimen 3-0.5-1 was obtained by exporting initial photogrammetry data to MATLAB and is shown in Figure 73. The magnitude of maximum initial imperfection of Specimen 3-0.5-1 was measured to be 0.0689 in. It was calculated like a difference between the biggest and the smallest $Z$ coordinate of all targets.

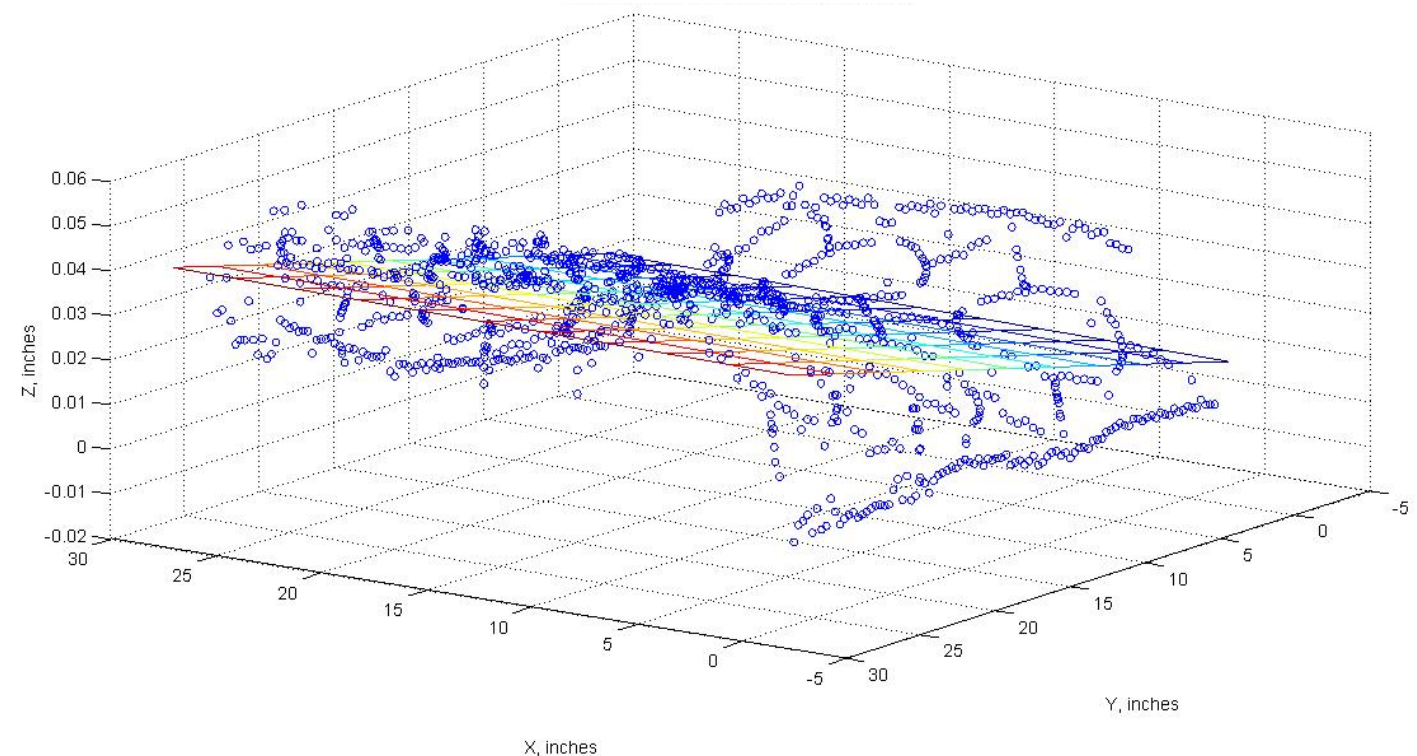

Figure 73. Point cloud and orthogonal regression plot - Specimen 3-0.5-1 initial shape

Displacements applied during Test 4 are shown in Figure 56 and Table 11. Figure 74 shows the behavior of Specimen 3-0.5-1. The shear displacement of the plate $(\Delta)$ was calculated using the diagonal string potentiometers (see Chapter 5.5.2). Pin to pin dimension of the plate $(a)$ was equal to 34 in. First the specimen deformed elastically. Then there was some initiation of yielding at inner and outer radius of the ring at the locations close to where the ring is connected to the link (Figure 75). Figure 76 shows no buckling of the plate at $1.6 \%$. The buckling started to occur at $3 \%$ drift. The plate experienced very small global shear buckling. No lateral torsional buckling of the rings was noticed. Specimen 3-0.5-1 showed excellent hysteretic behavior. No fracture occurred in the plate. 


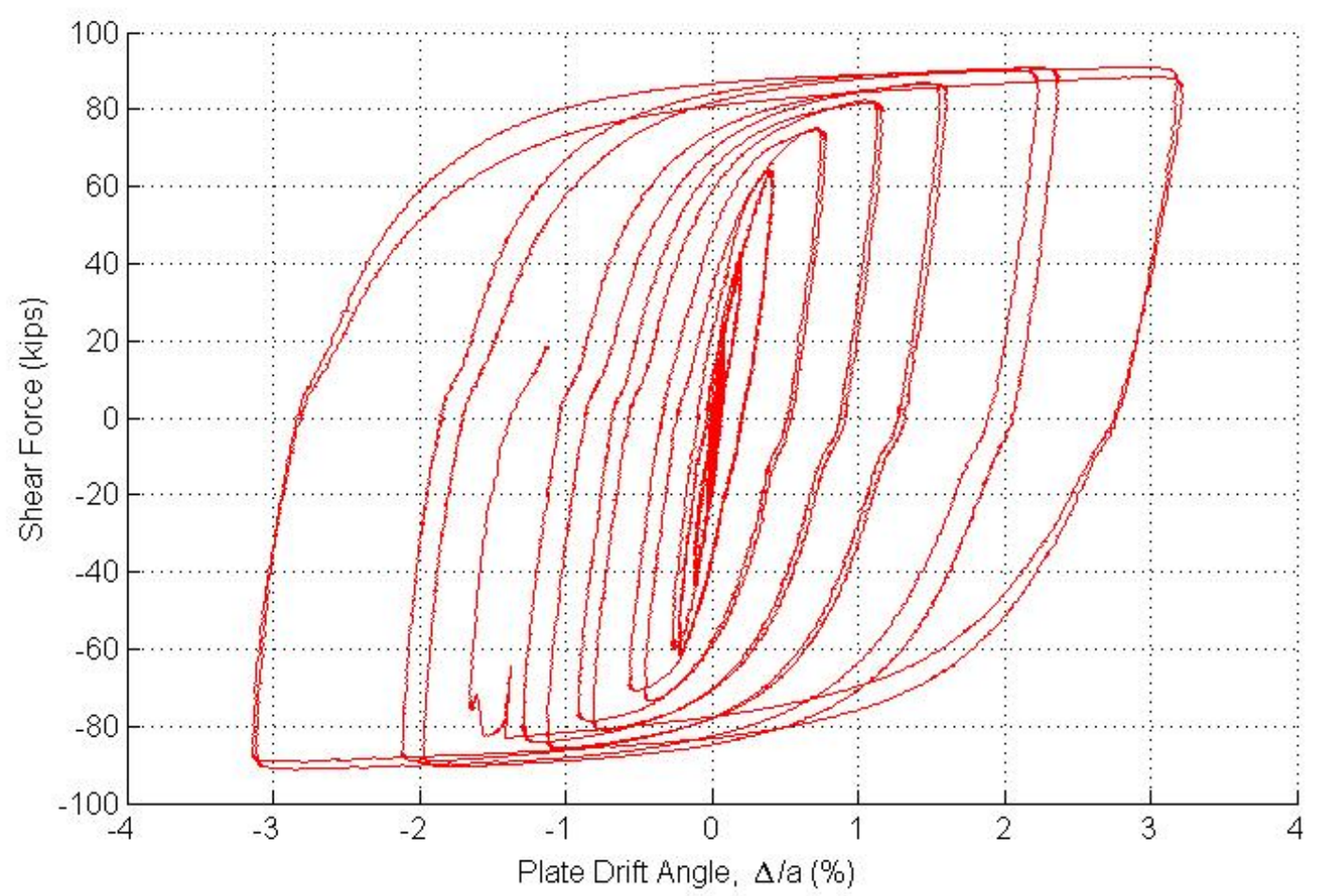

Figure 74. Shear Force versus Plate Drift Angle - Test 4 - Specimen 3-0.5-1

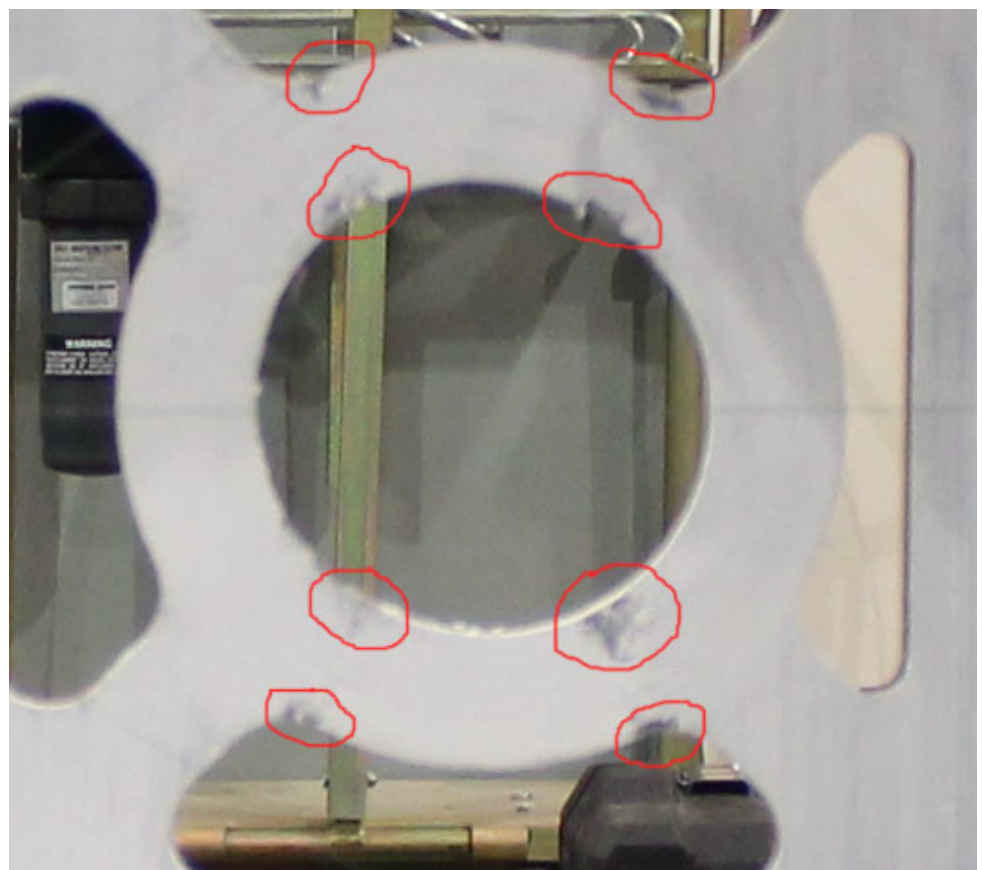

Figure 75. Locations of initial yielding - Test 4 - Specimen 3-0.5-1 


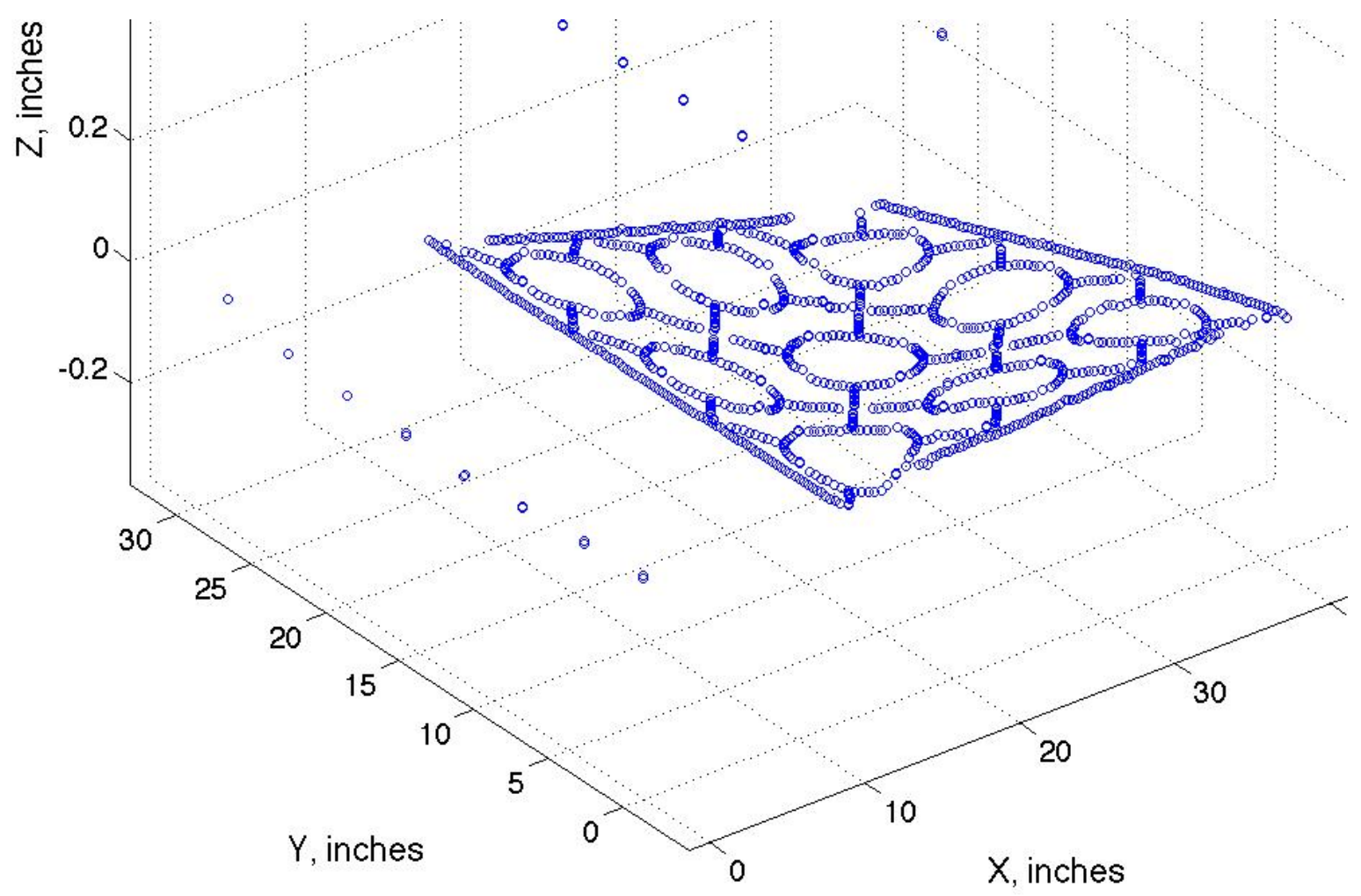

Figure 76. Point cloud - Specimen 3-0.5-1 - 1.6\% drift 


\subsection{TEST 5 - Specimen 2-0.25-0.81}

Figure 77 and Figure 78 show the side of the plate with photogrammetry targets and the side with whitewash, respectively, before the test was started. Table 14 and Figure 79 represent the Specimen 2-0.25-0.81 geometric properties. All dimensions are given in inches.

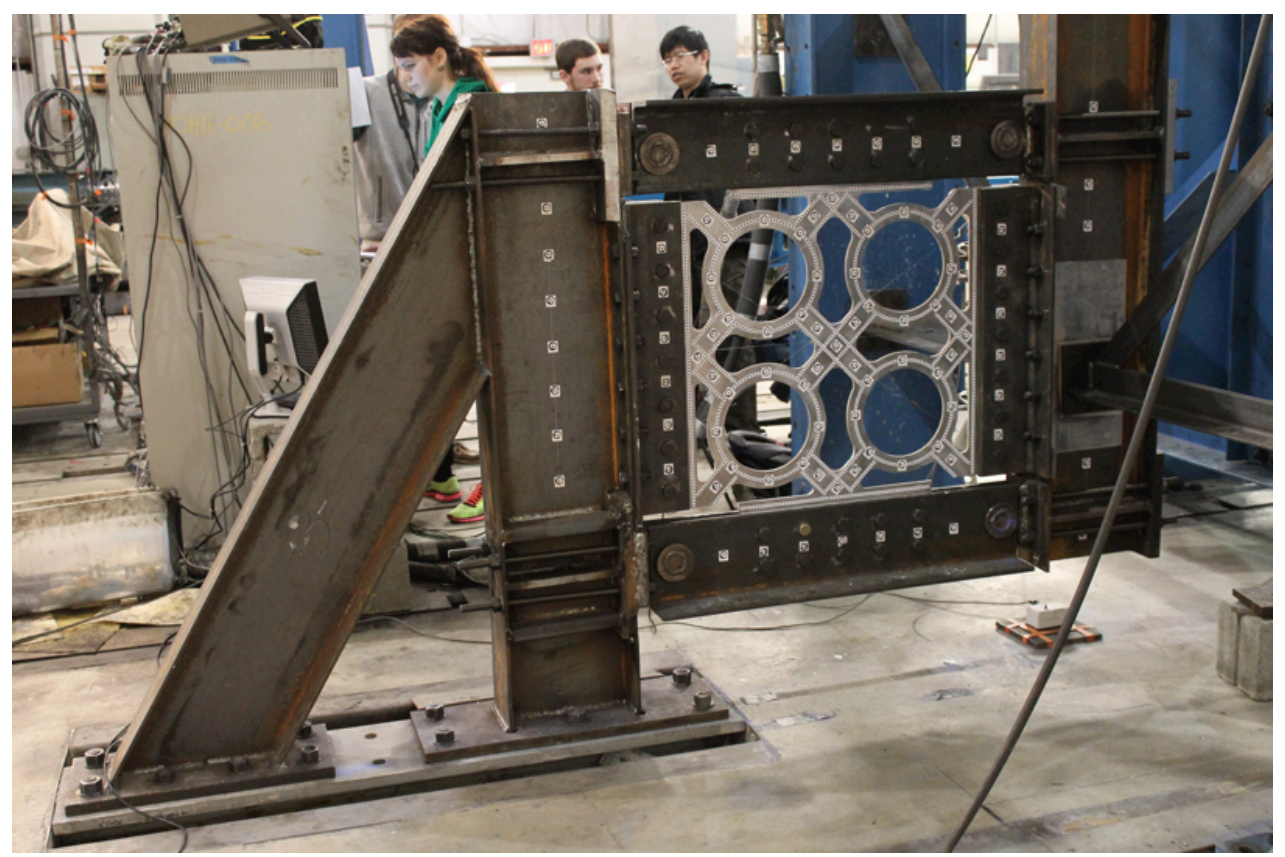

Figure 77. Photogrammetry side view - Test 5 - Specimen 2-0.25-0.81

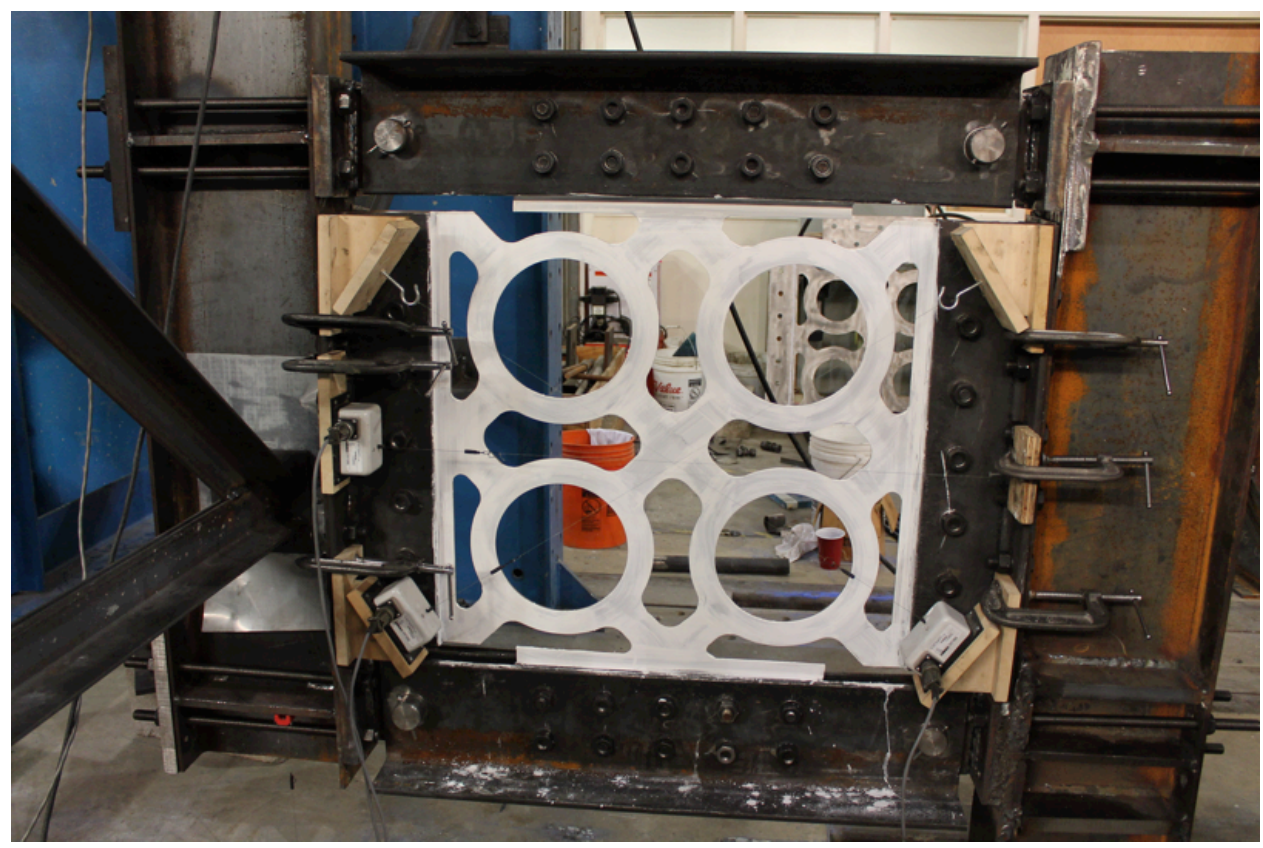

Figure 78. Whitewash side view - Test 5 - Specimen 2-0.25-0.81 
Table 14. Specimen 2-0.25-0.81 properties

\begin{tabular}{|l|l|}
\hline Thickness of the plate $\left(t_{w}\right)$ & $0.25 \mathrm{in}$ \\
\hline Outer ring radius $\left(R_{0}\right)$ & $5.89 \mathrm{in}$ \\
\hline Ring width $\left(w_{c}\right)$ & $1.8 \mathrm{in}$ \\
\hline Link width $\left(w_{L}\right)$ & $2.21 \mathrm{in}$ \\
\hline Number of rings in a row $(N)$ & 2 \\
\hline Pin to pin dimensions (a) & $34 \mathrm{in} \times 34 \mathrm{in}$ \\
\hline
\end{tabular}

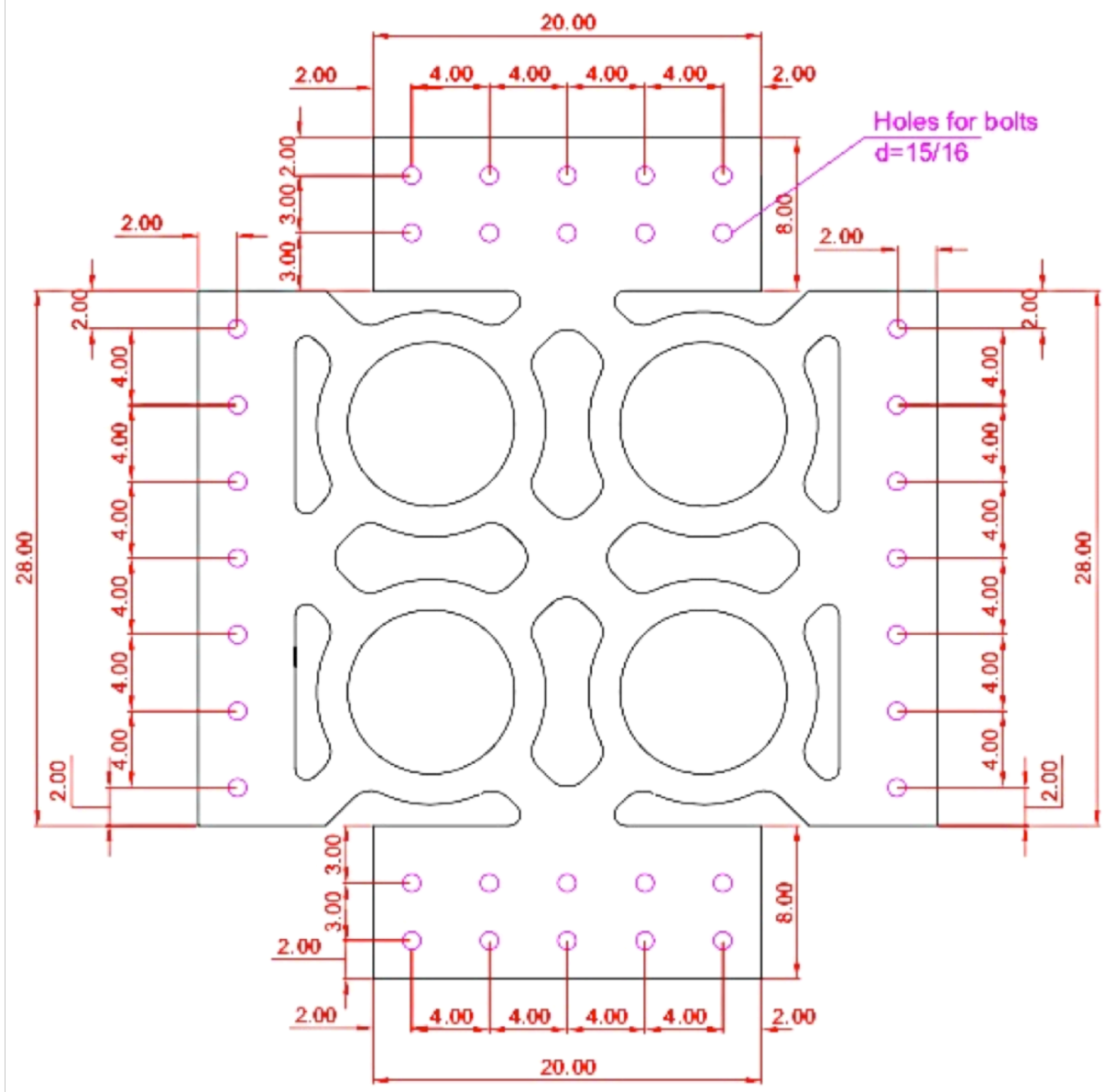

\section{Plate thickness $=0.25$}

Figure 79. Test 5, Specimen 2-0.25-0.81 geometry

The initial shape of the Specimen 2-0.25-0.81 was obtained by exporting initial photogrammetry data to MATLAB and is shown in Figure 80. The magnitude of maximum initial imperfection of Specimen 2-0.25-0.81 was measured to be 0.0836 in. It was 
calculated like a difference between the biggest and the smallest $Z$ coordinate of all targets.

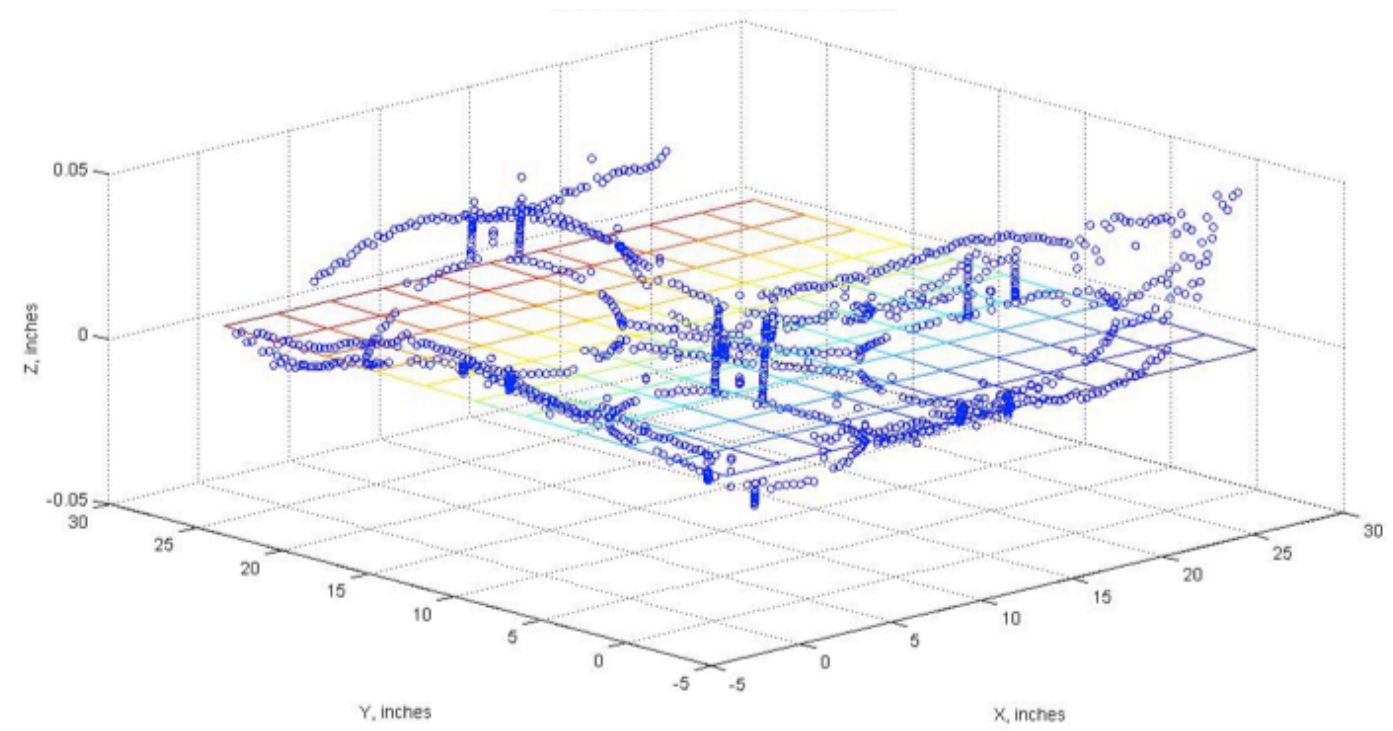

Figure 80. Point cloud and orthogonal regression plot - Specimen 2-0.25-0.81 initial shape

Displacements applied during Test 5 are shown in Figure 56 and Table 11. Figure 81 shows the behavior of Specimen 2-0.25-0.81. The shear displacement of the plate $(\Delta)$ was calculated using the diagonal string potentiometers (see Chapter 5.5.2). Pin to pin dimension of the plate $(a)$ was equal to 34 in. First the specimen deformed elastically. Then there was some initiation of yielding at inner and outer radius of the ring at the locations close to where the ring is connected to the link (Figure 82). The buckling started to occur at $2 \%$ drift. The plate experienced global shear buckling with some lateral torsional buckling of the rings (Figure 83). The fracture of the specimen occurred at $10 \%$ drift at the ring point close to the link, at the right top ring looking from whitewash side of the plate (Figure 84). It propagated fast, and the test was stopped. 


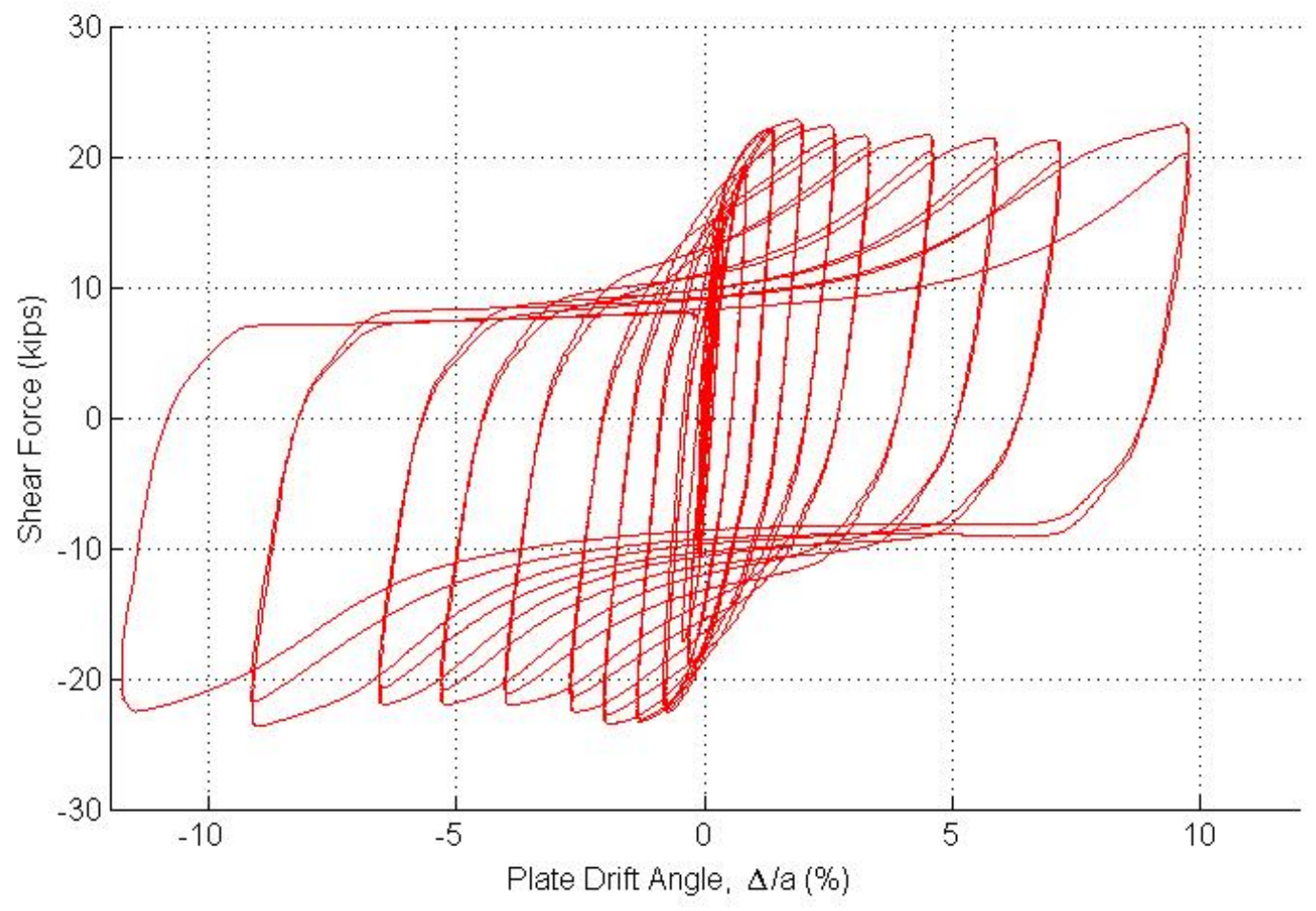

Figure 81. Shear Force versus Plate Drift Angle - Test 5 - Specimen 2-0.25-0.81

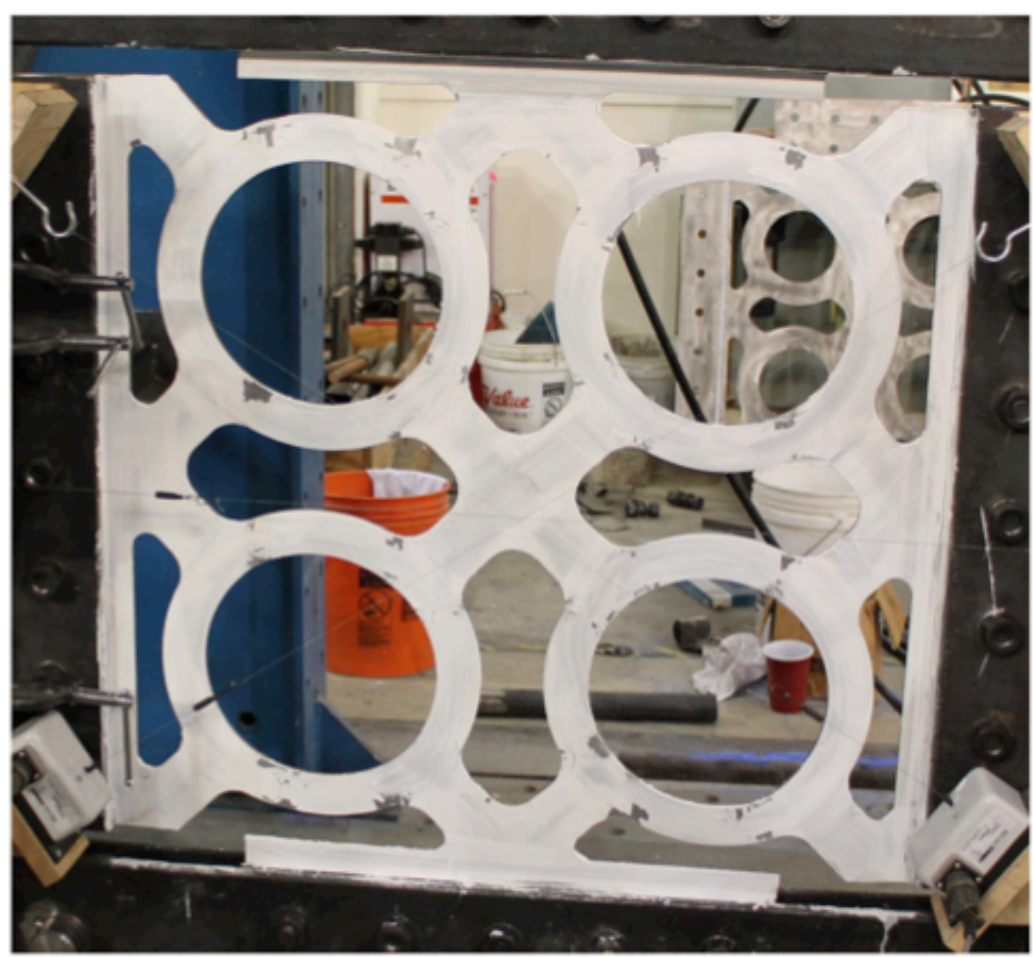

Figure 82. Locations of initial yielding - Specimen 2-0.25-0.81 


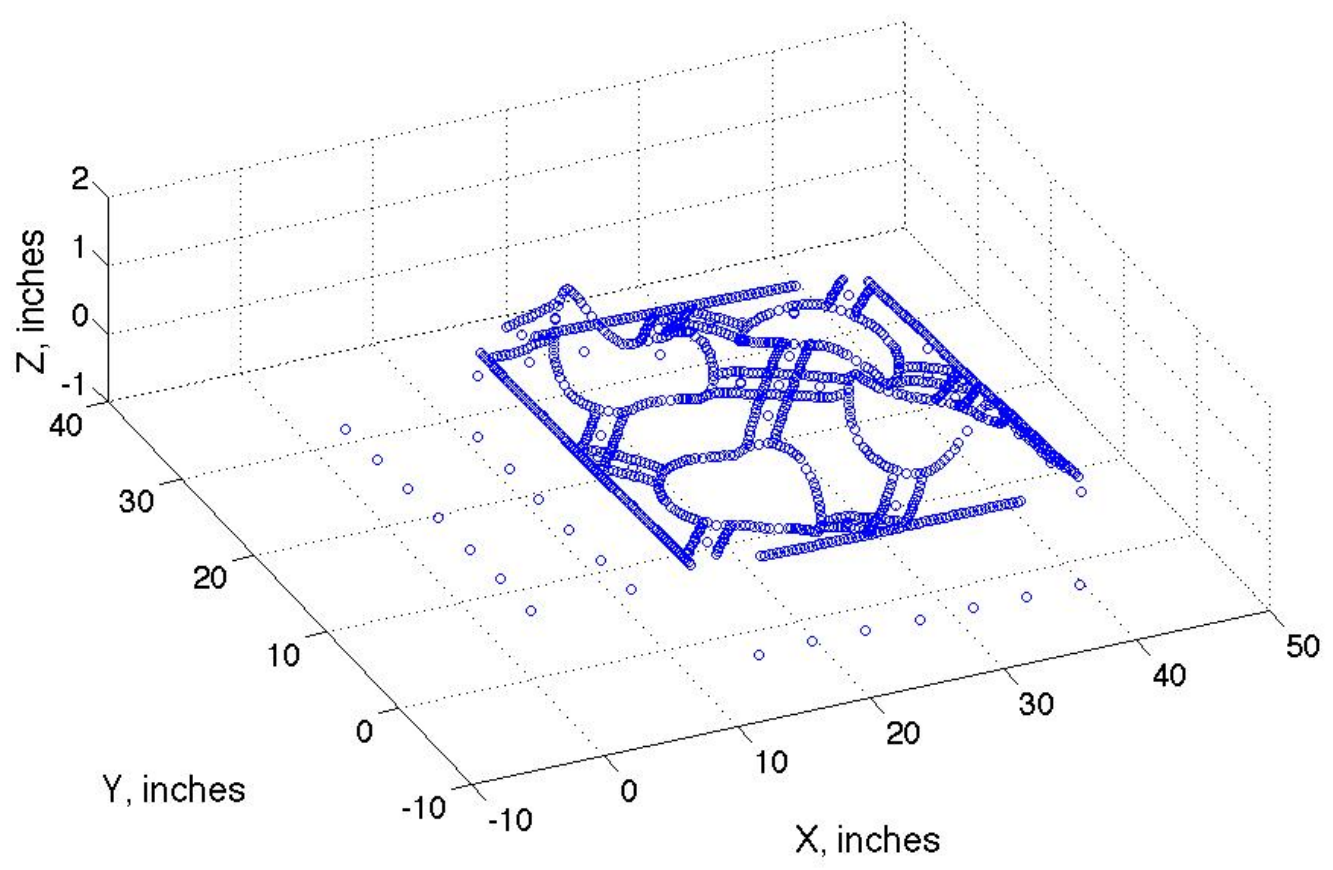

Figure 83. Point cloud - Specimen 2-0.25-0.81 - 3\% drift

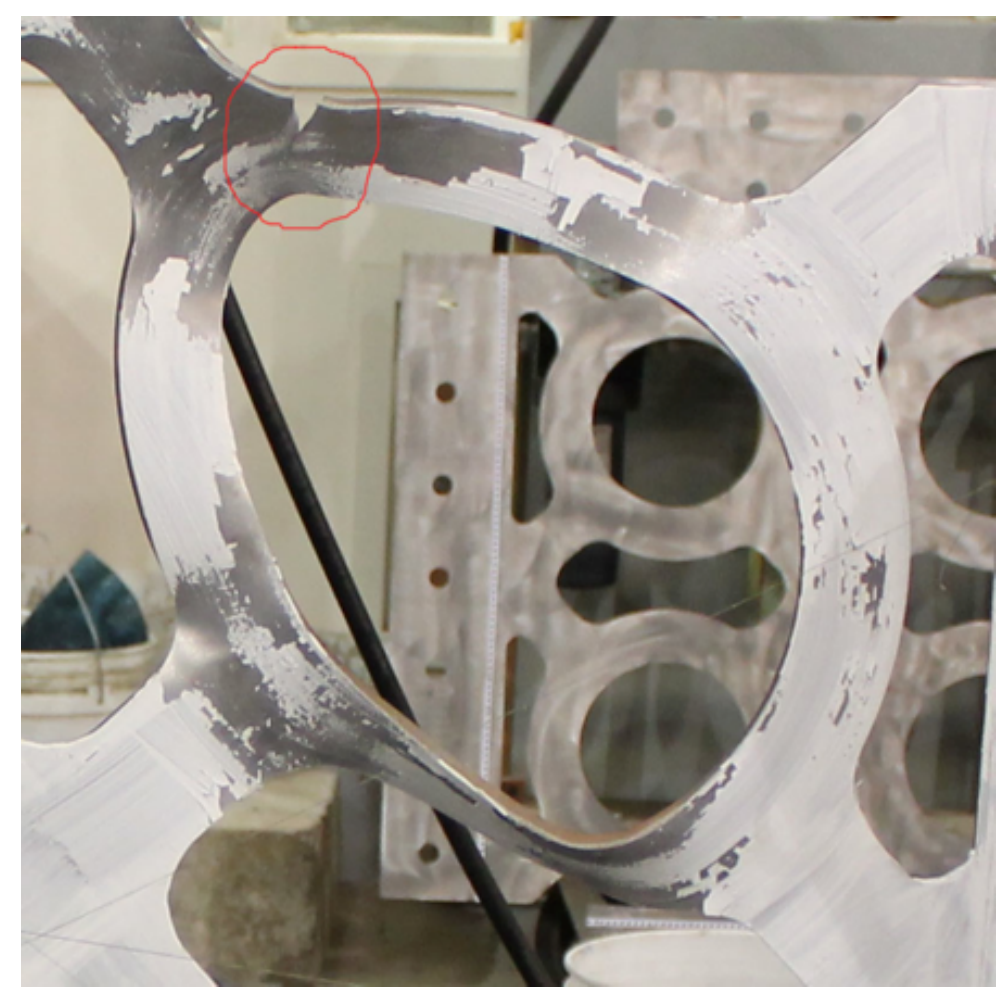

Figure 84. Specimen 2-0.25-0.81 fracture at 10\% drift 


\subsection{TEST 6 - Specimen 2-0.25-1}

Figure 85 and Figure 86 show the side of the plate with photogrammetry targets and the side with whitewash, respectively, before the test was started. Table 15 and Figure 87 represent the Specimen 2-0.25-1 geometric properties. All dimensions are given in inches.

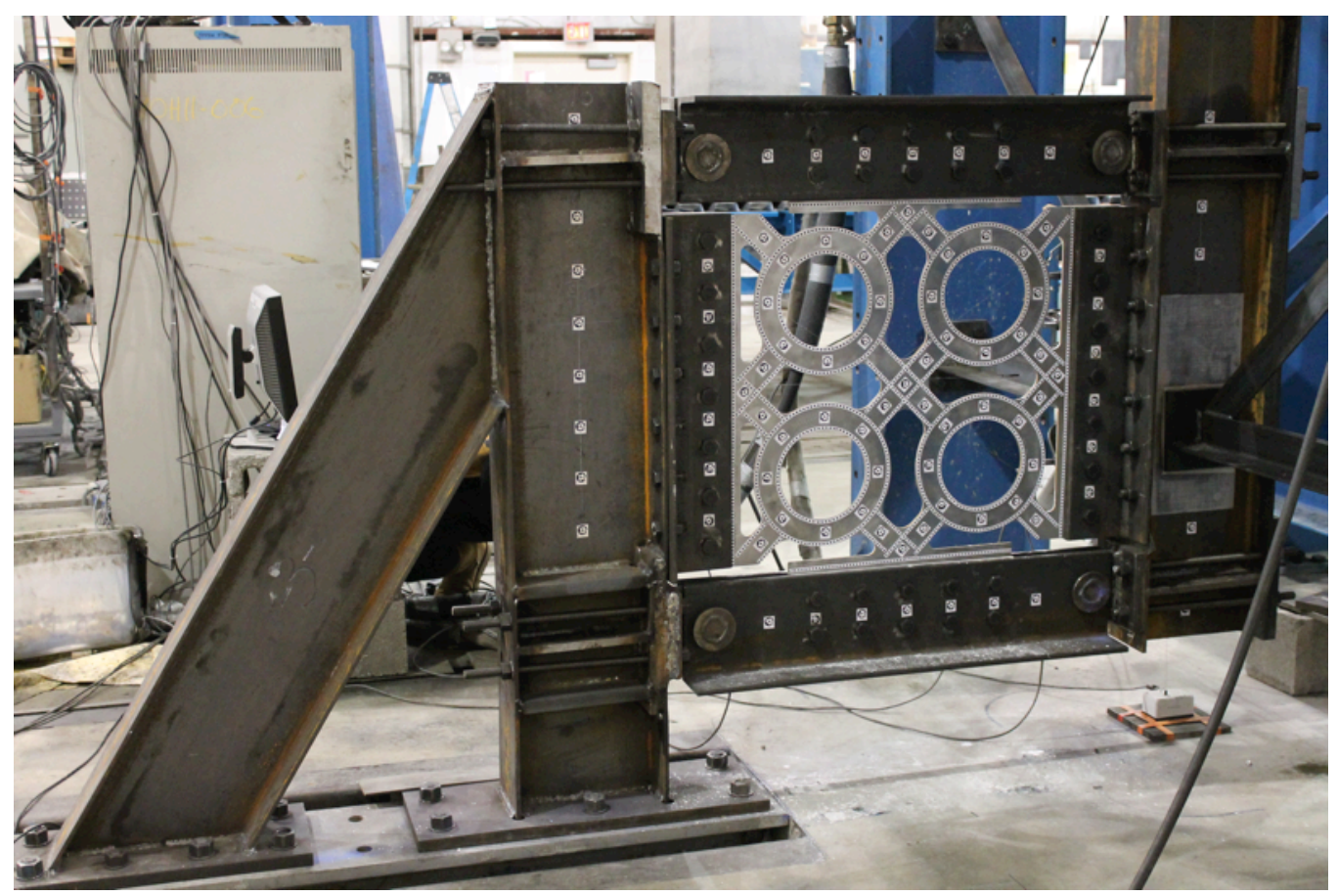

Figure 85. Photogrammetry side view - Test 6 - Specimen 2-0.25-1

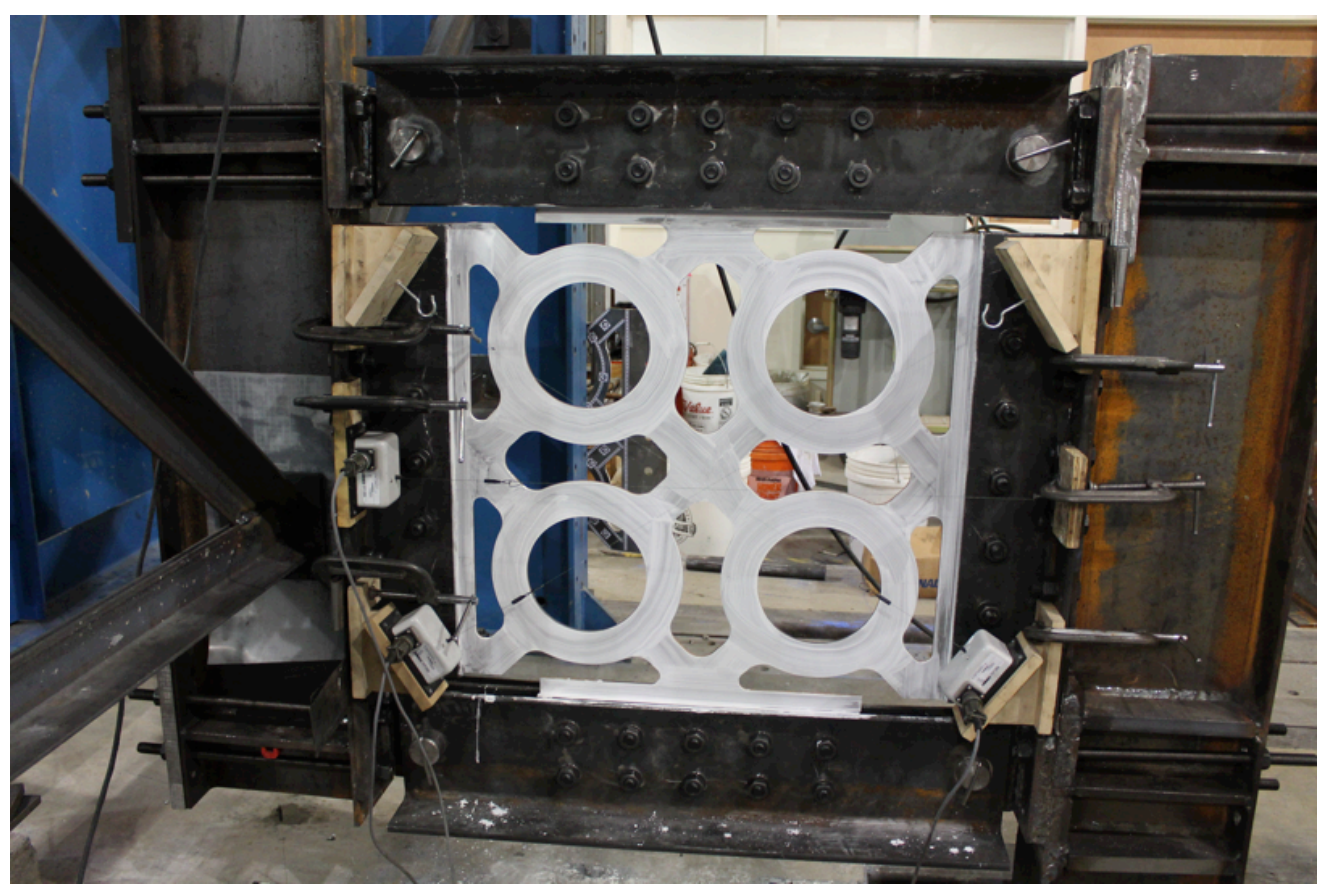

Figure 86. Whitewash side view - Test 6 - Specimen 2-0.25-1 
Table 15. Specimen 2-0.25-1 properties

\begin{tabular}{|l|l|}
\hline Thickness of the plate $\left(t_{w}\right)$ & 0.25 in \\
\hline Outer ring radius $\left(R_{0}\right)$ & 5.89 in \\
\hline Ring width $\left(w_{c}\right)$ & 2.21 in \\
\hline Link width $\left(w_{L}\right)$ & 2.21 in \\
\hline Number of rings in a row $(N)$ & 2 \\
\hline Pin to pin dimensions (a) & 34 in x 34in \\
\hline
\end{tabular}

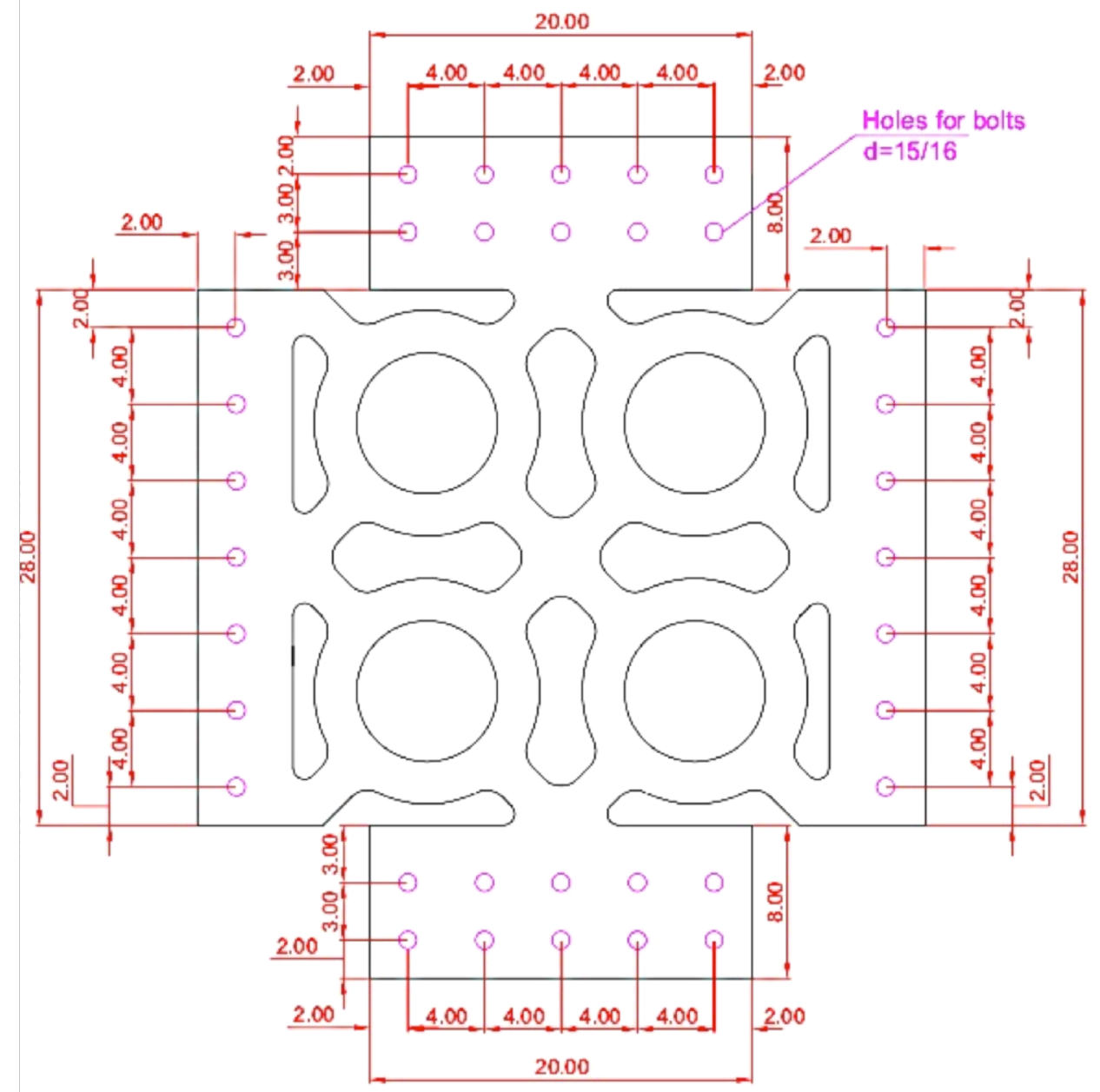

\section{Plate thickness $=0.25$}

Figure 87. Test 6, Specimen 2-0.25-1 geometry

The initial shape of the Specimen 2-0.25-0.81 was obtained by exporting initial photogrammetry data to MATLAB and is shown in Figure 88. The magnitude of maximum initial imperfection of Specimen 2-0.25-1 was measured to be $0.1098 \mathrm{in}$. It was calculated like a difference between the biggest and the smallest $Z$ coordinate of all targets. 


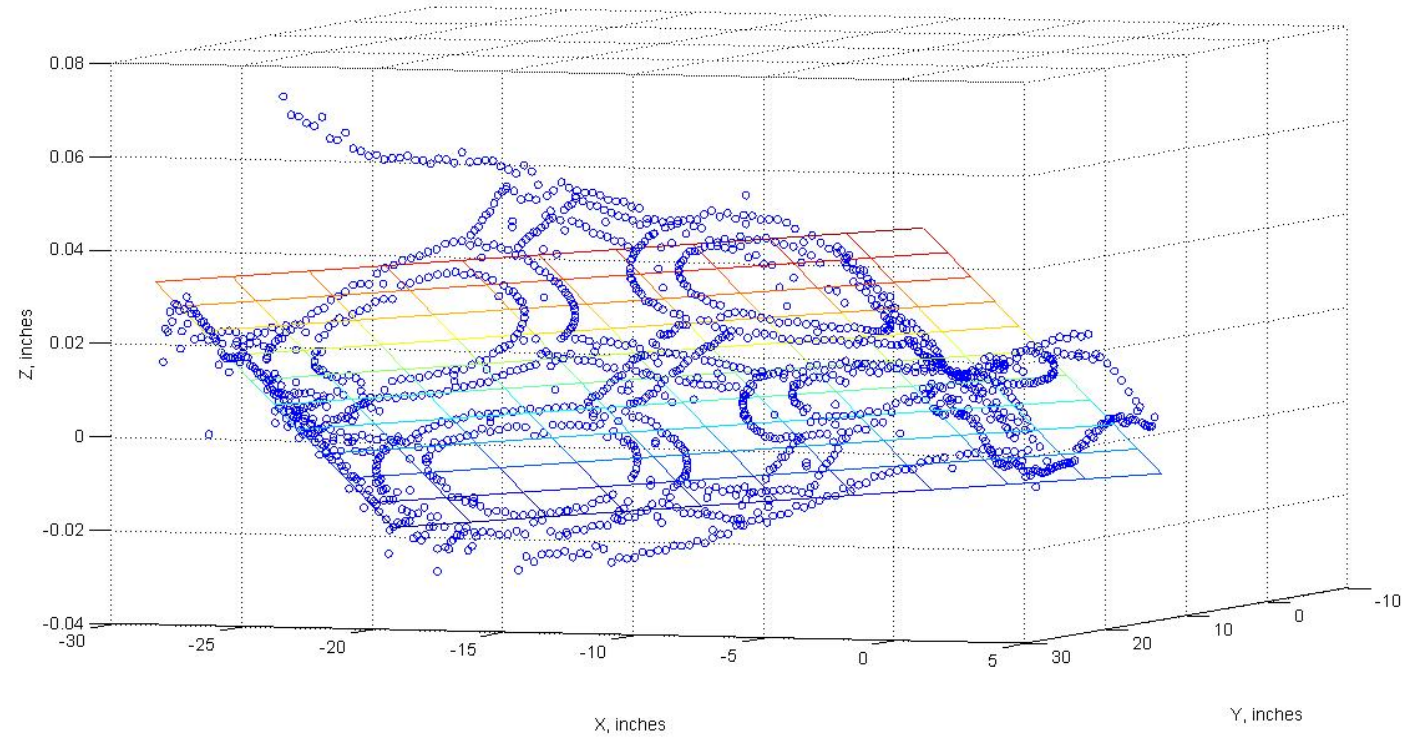

Figure 88. Point cloud and orthogonal regression plot - Specimen 2-0.25-1 initial shape

Displacements applied during Test 6 are shown in Figure 56 and Table 11. Figure 89 shows the behavior of Specimen 2-0.25-1. The shear displacement of the plate $(\Delta)$ was calculated using the diagonal string potentiometers (see Chapter 5.5.2). Pin to pin dimension of the plate $(a)$ was equal to $34 \mathrm{in}$. First the specimen deformed elastically. Then there was some initiation of yielding at inner and outer radius of the ring at the locations close to where the ring is connected to the link. The buckling started to occur at $2 \%$ drift. The plate experienced global shear buckling with some lateral torsional buckling of the rings (Figure 90). The fracture of the specimen occurred at $10 \%$ drift at the ring point close to the link, at the right bottom ring looking from whitewash side of the plate (Figure 91). It propagated fast, and the test was stopped. 


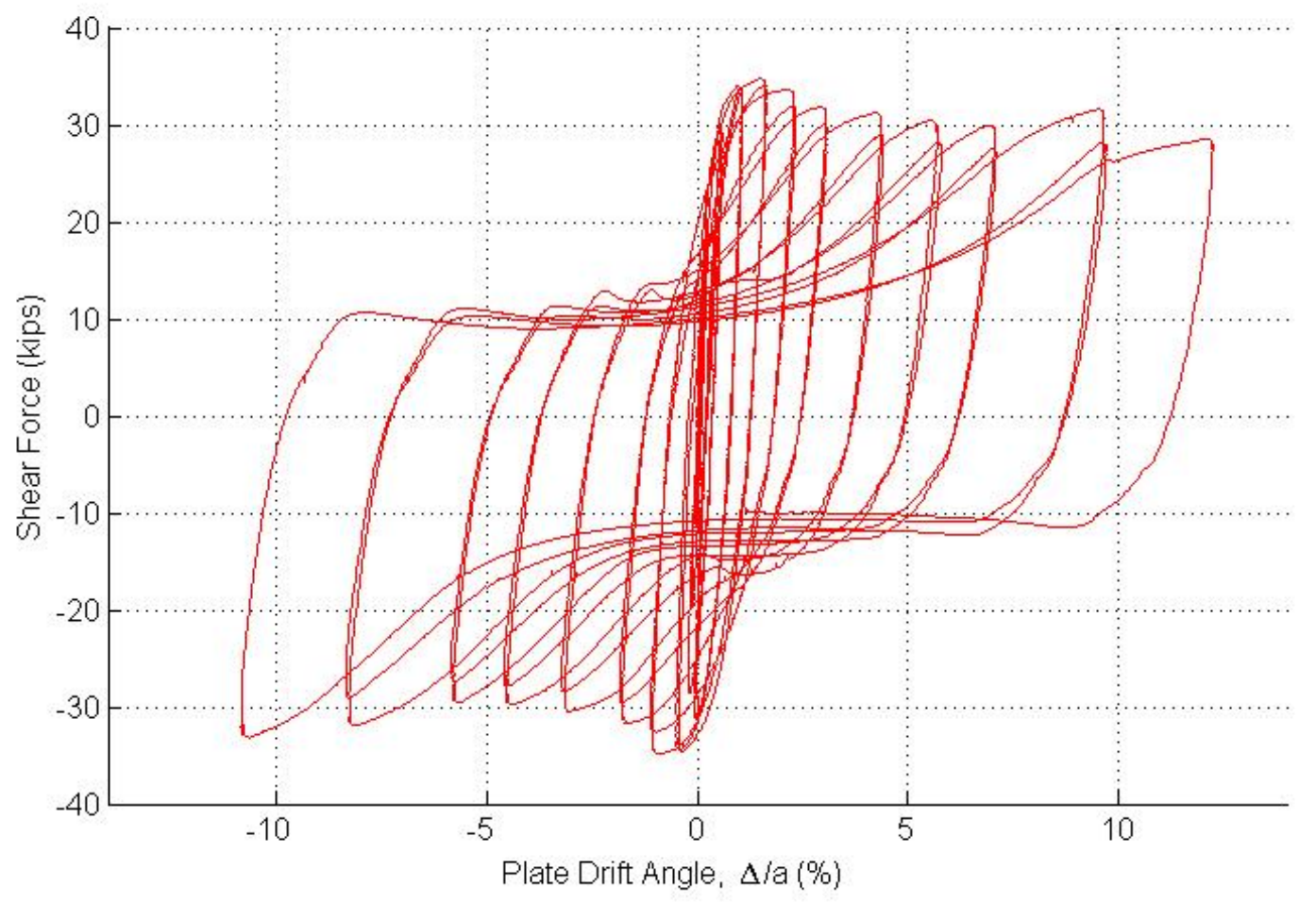

Figure 89. Shear Force versus Plate Drift Angle - Test 6 - Specimen 2-0.25-1

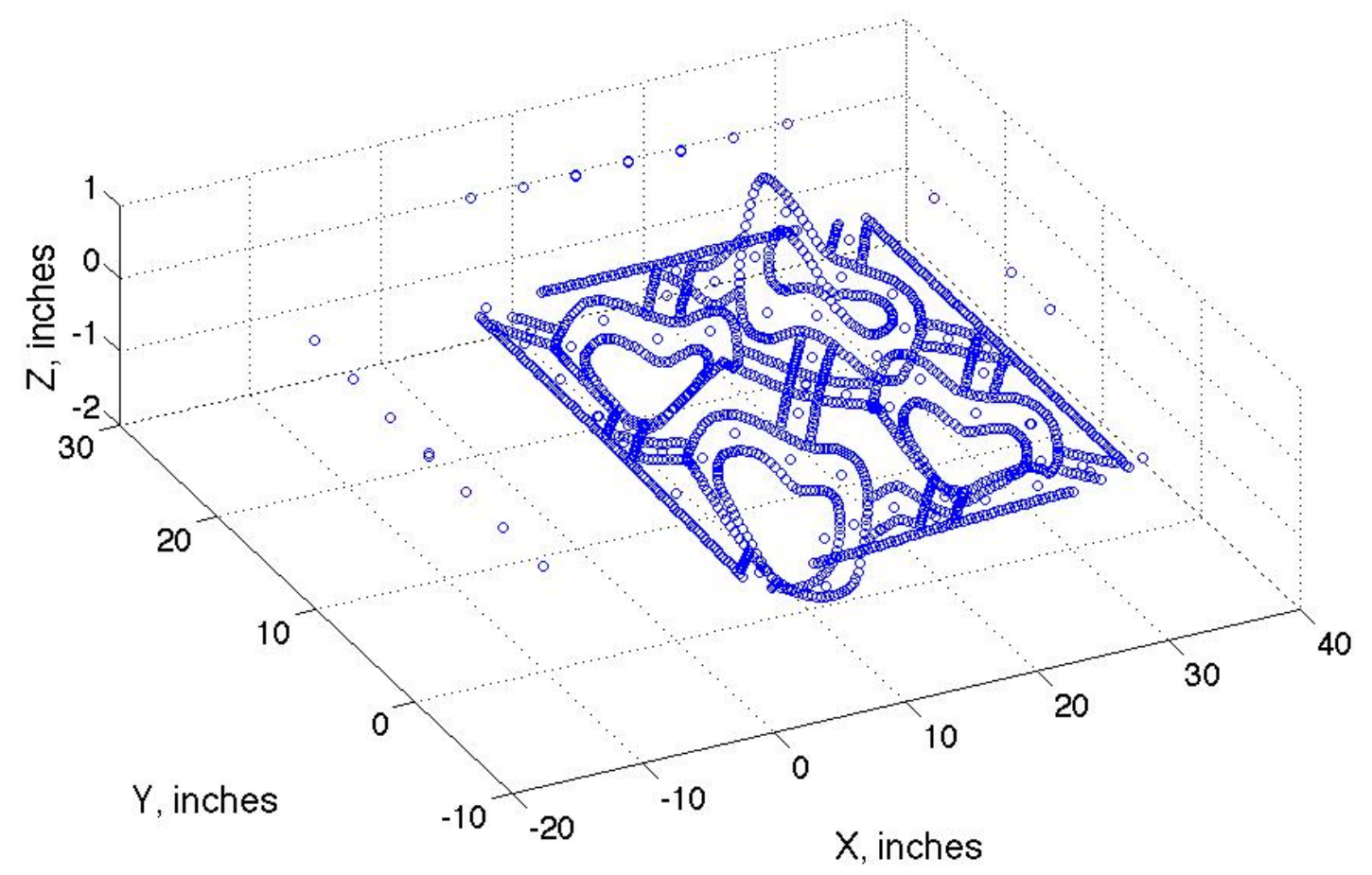

Figure 90. Point cloud - Specimen 2-0.25-1 - 2.8\% drift 


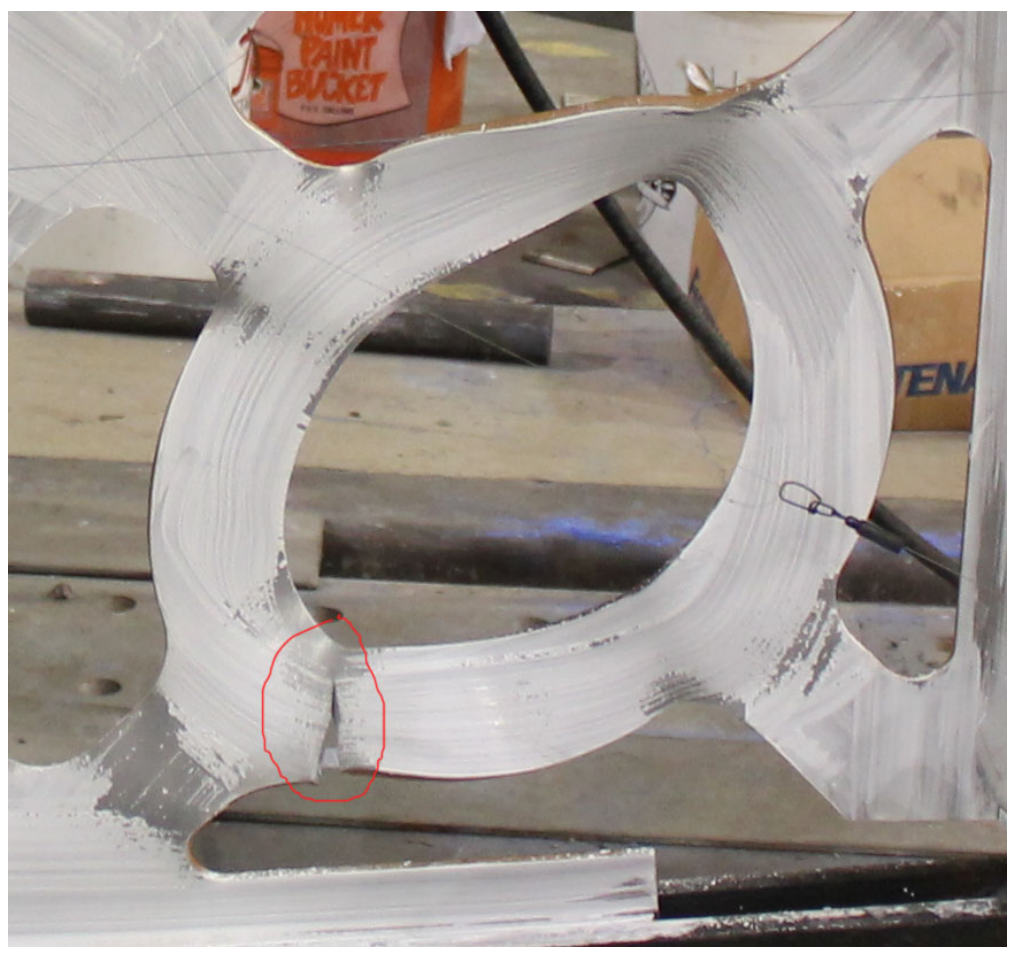

Figure 91. Specimen 2-0.25-1 fracture at 10\% drift 


\subsection{TEST 7 - Specimen 3-0.25-1}

Figure 92 and Figure 93 show the side of the plate with photogrammetry targets and the side with whitewash, respectively, before the test started. Table 16 and Figure 94 represent the Specimen 3-0.25-1 geometric properties. All dimensions are given in inches.

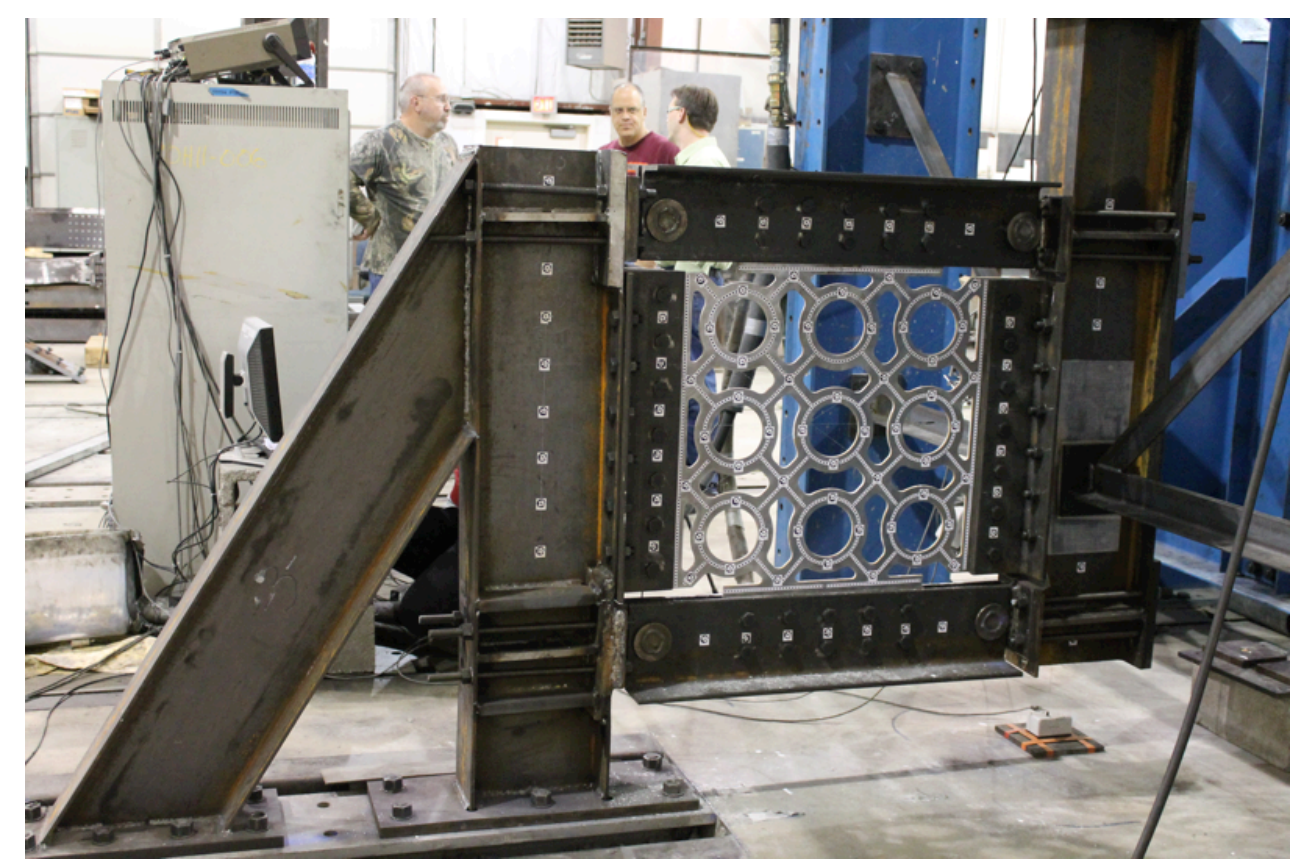

Figure 92. Photogrammetry side view - Test 7 - Specimen 3-0.25-1

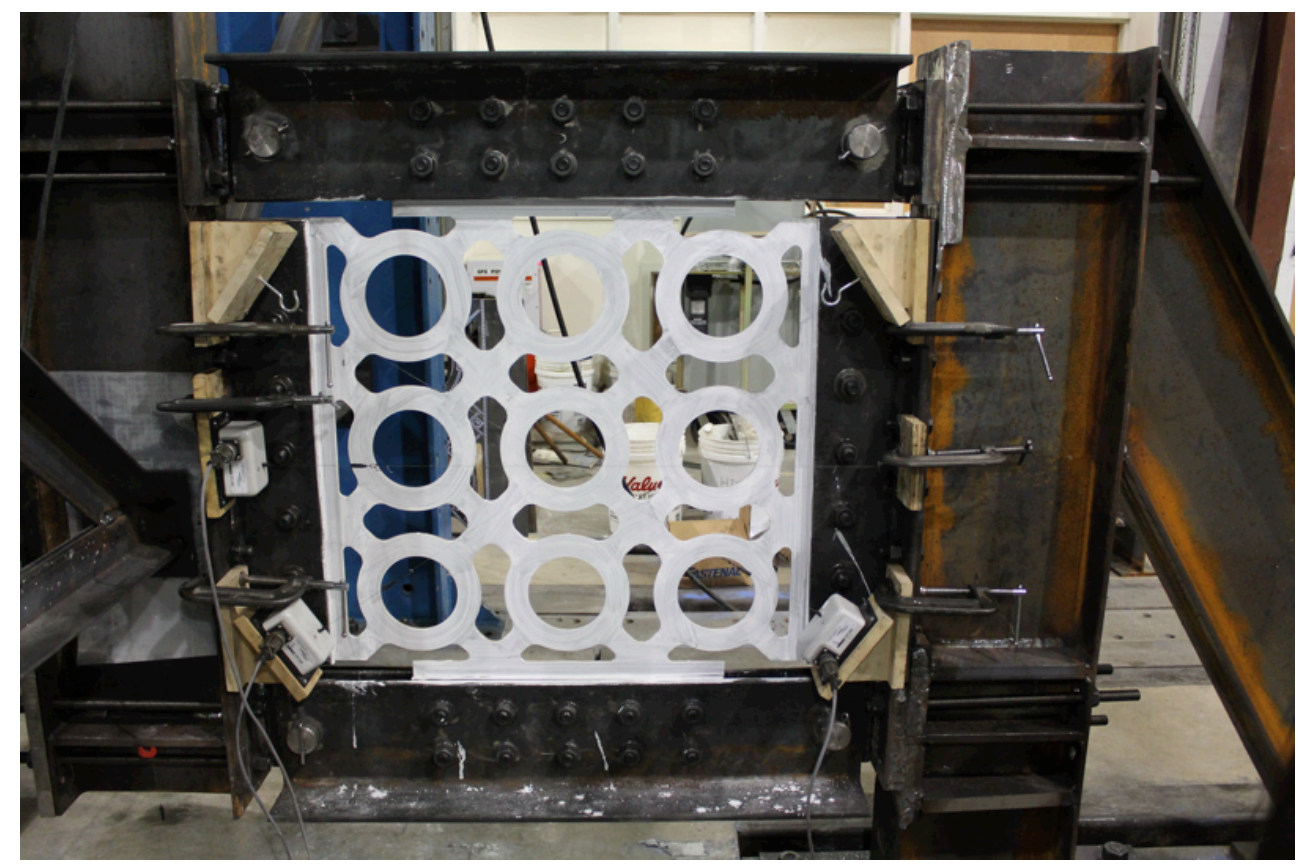

Figure 93. Whitewash side view - Test 7 - Specimen 3-0.25-1 
Table 16. Specimen 3-0.25-1 properties

\begin{tabular}{|l|l|}
\hline Thickness of the plate $\left(t_{w}\right)$ & $0.25 \mathrm{in}$ \\
\hline Outer ring radius $\left(R_{0}\right)$ & $3.93 \mathrm{in}$ \\
\hline Ring width $\left(w_{c}\right)$ & $1.47 \mathrm{in}$ \\
\hline Link width $\left(w_{L}\right)$ & $1.47 \mathrm{in}$ \\
\hline Number of rings in a row $(N)$ & 3 \\
\hline Pin to pin dimensions $(\mathrm{a})$ & $34 \mathrm{in} \times$ 34in \\
\hline
\end{tabular}

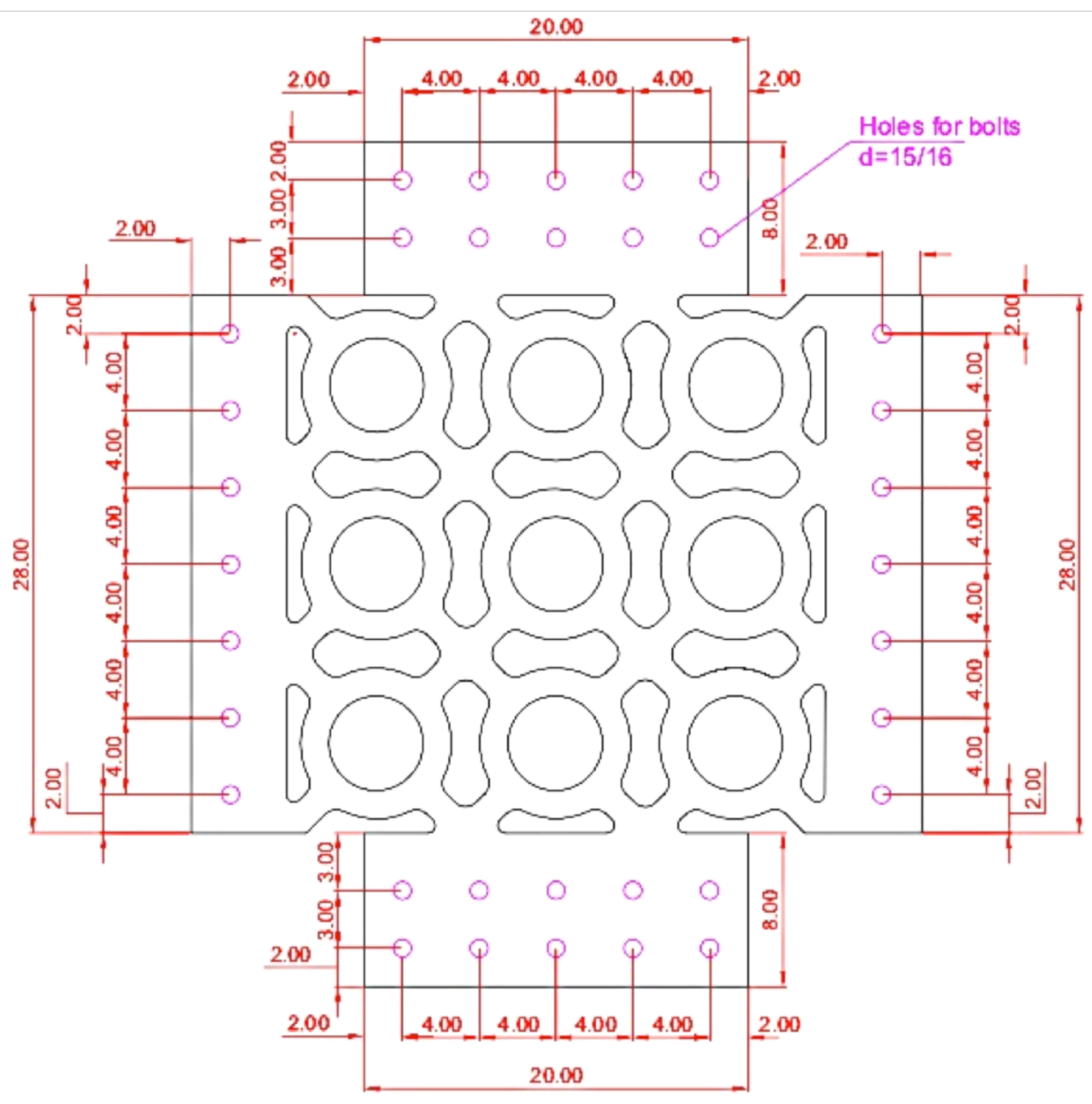

\section{Plate thickness $=0.25$}

Figure 94. Test 7, Specimen 3-0.25-1 geometry

The initial shape of the Specimen 3-0.25-1 was obtained by exporting initial photogrammetry data to MATLAB and is shown in Figure 95. The magnitude of maximum initial imperfection of Specimen 3-0.25-1 was measured to be $0.1071 \mathrm{in}$. It was calculated like a difference between the biggest and the smallest $Z$ coordinate of all targets. 


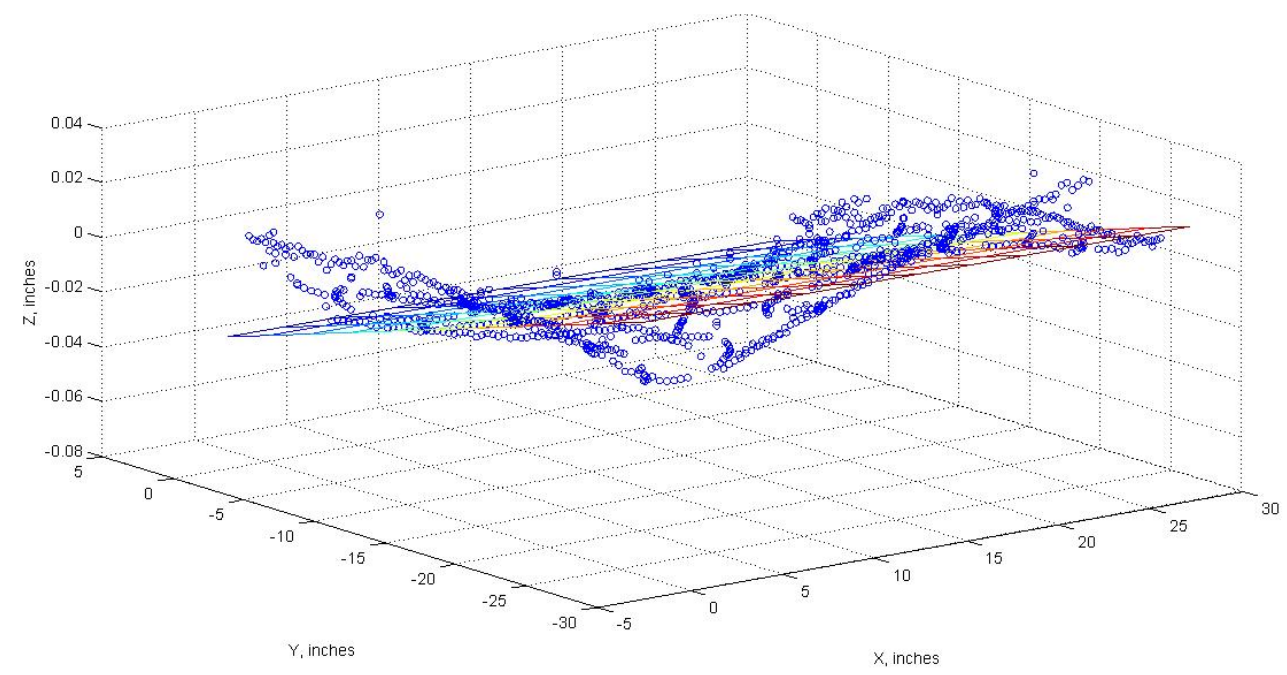

Figure 95. Point cloud and orthogonal regression plot - Specimen 3-0.25-1 initial shape

Displacements applied during Test 7 are shown in Figure 56 and Table 11. Figure 96 shows the behavior of Specimen 3-0.25-1. The shear displacement of the plate $(\Delta)$ was calculated using the diagonal string potentiometers (see Chapter 5.5.2). Pin to pin dimension of the plate $(a)$ was equal to 34 in. First the specimen deformed elastically. Then there was some initiation of yielding at inner and outer radius of the ring at the locations close to where the ring is connected to the link. The buckling started to occur at $2 \%$ drift. The plate experienced global shear buckling (Figure 97). Lateral torsional buckling of the rings was insignificant. Two rings of the specimen experienced fracture at $8 \%$ drift. The fracture of the specimen occurred at the ring point close to the link, at the right bottom and right top rings looking from whitewash side of the plate (Figure 98). It propagated fast, and the test was stopped. 


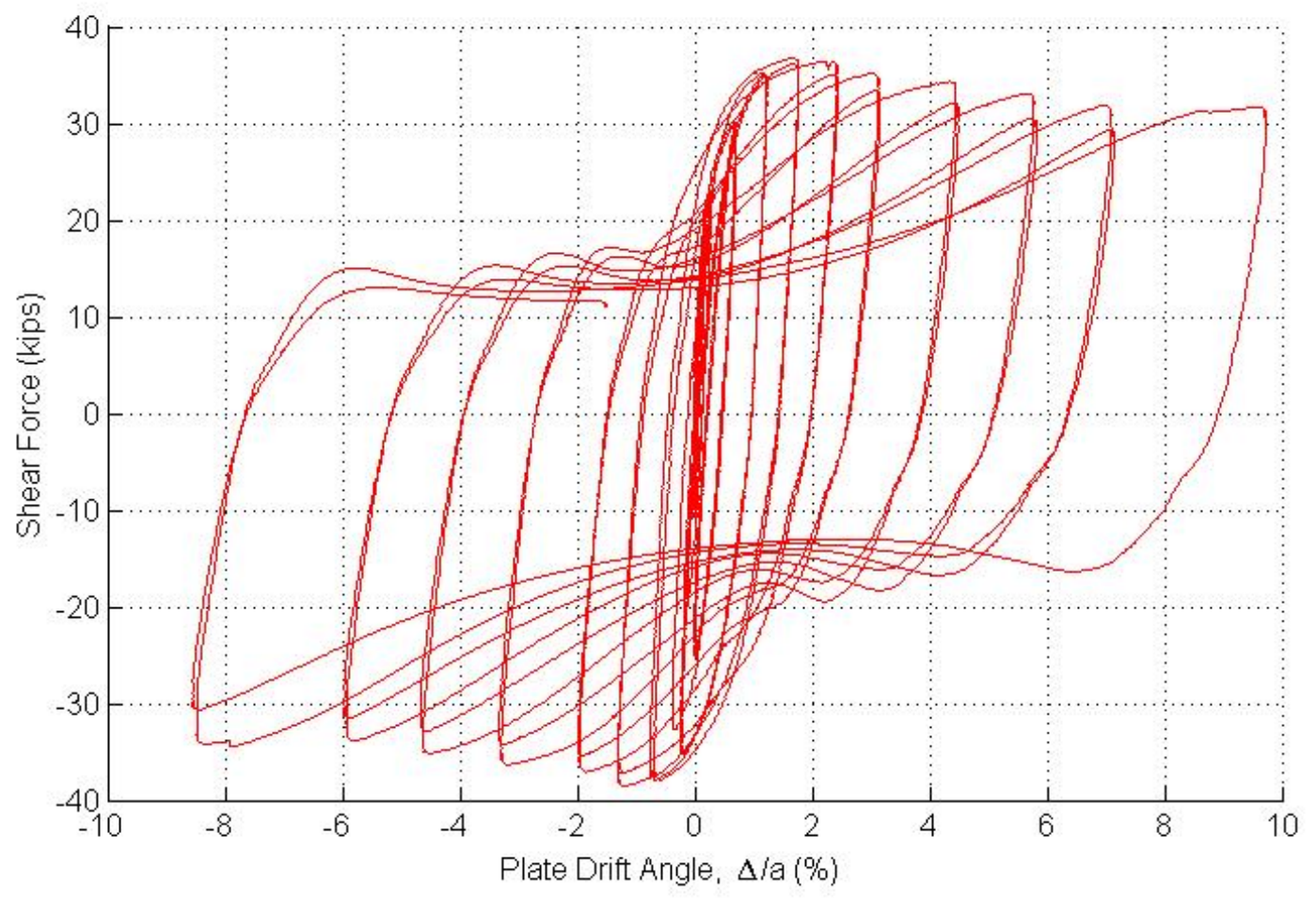

Figure 96. Shear Force versus Plate Drift Angle - Test 7 - Specimen 3-0.25-1

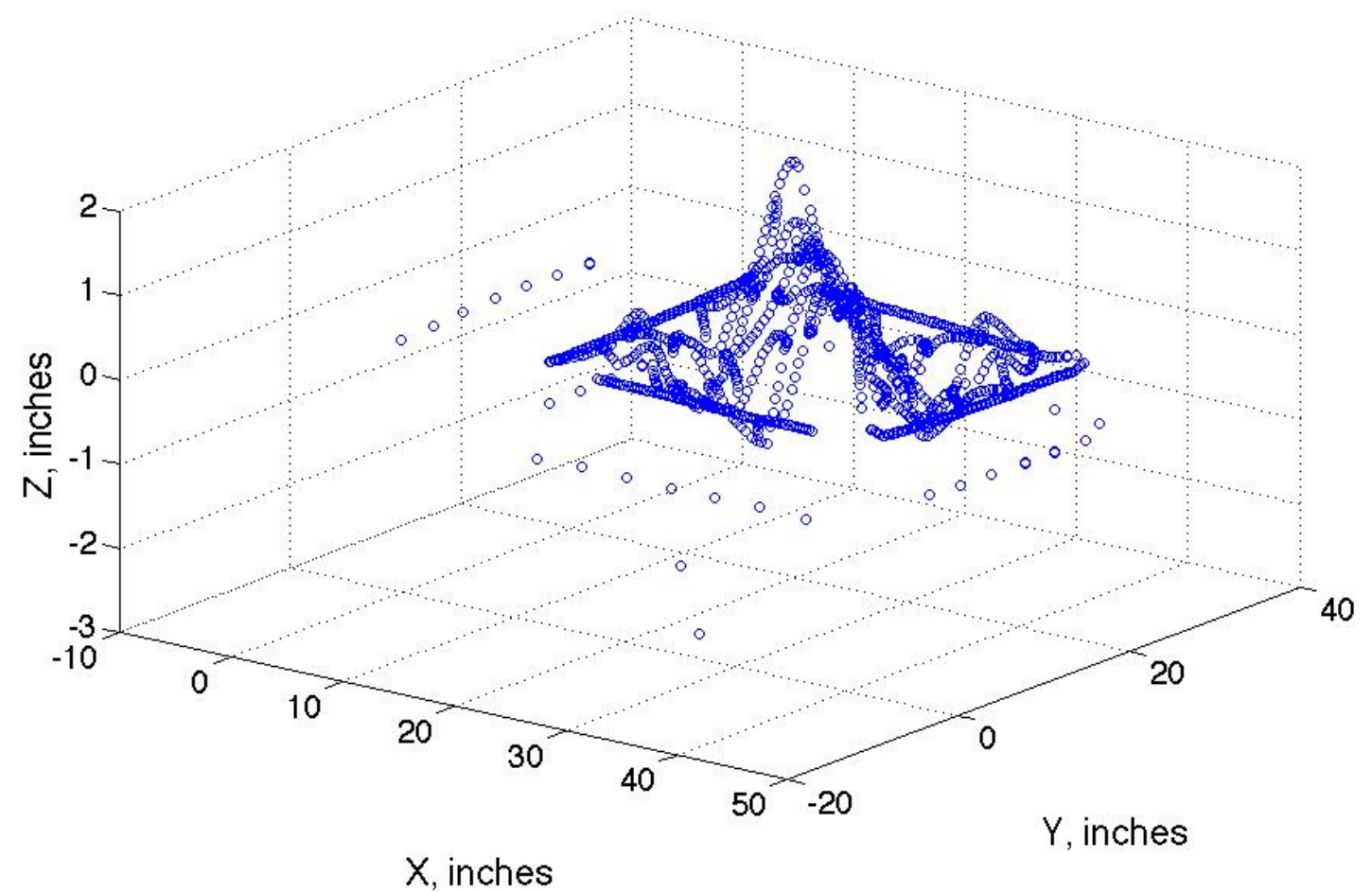

Figure 97. Point cloud - Specimen 3-0.25-1 - 6.8\% drift 


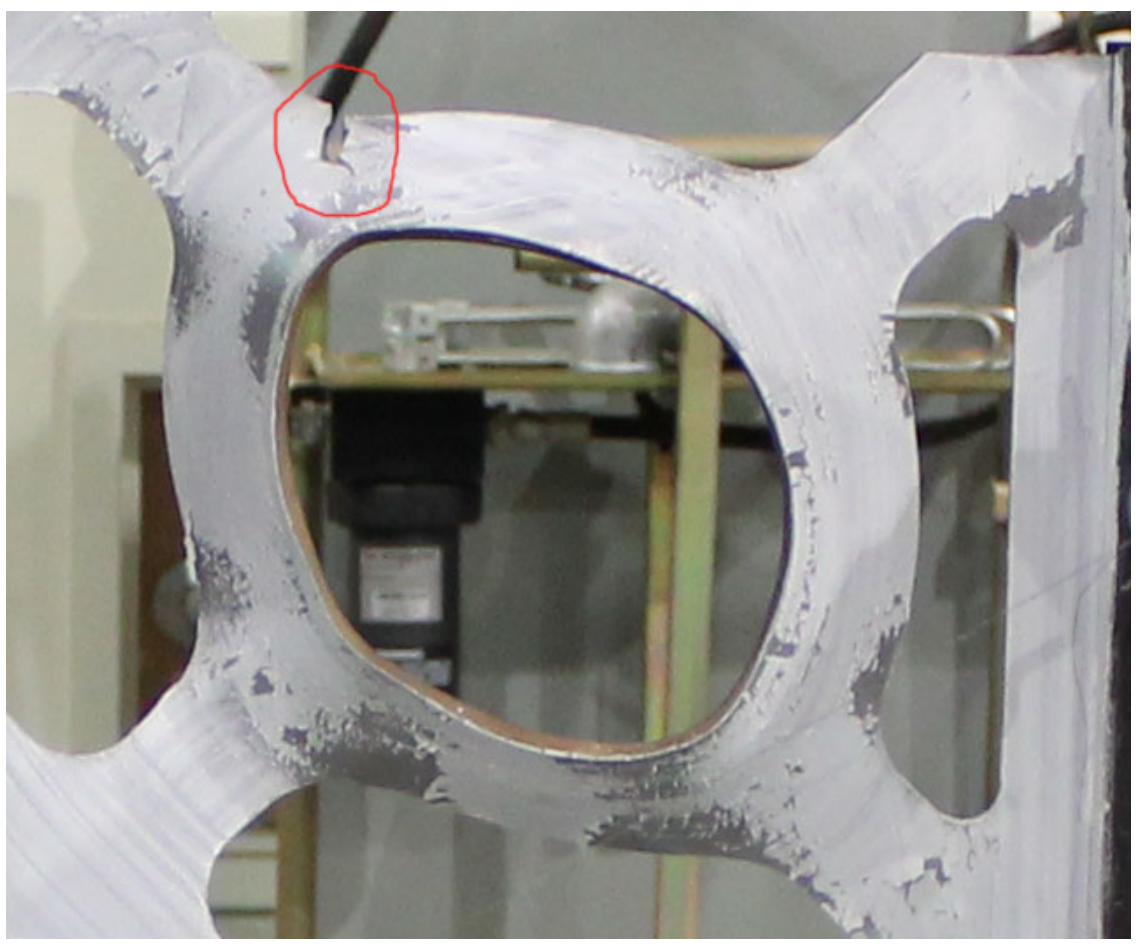

Figure 98. Specimen 3-0.25-1 fracture at 8\% drift 


\subsection{TEST 8 - Specimen 3-0.375-1}

Figure 99 and Figure 100 show the side of the plate with photogrammetry targets and the side with whitewash, respectively, before the test was started. Table 17 and Figure 101 represent the Specimen 3-0.375-1 geometric properties. All dimensions are given in inches.

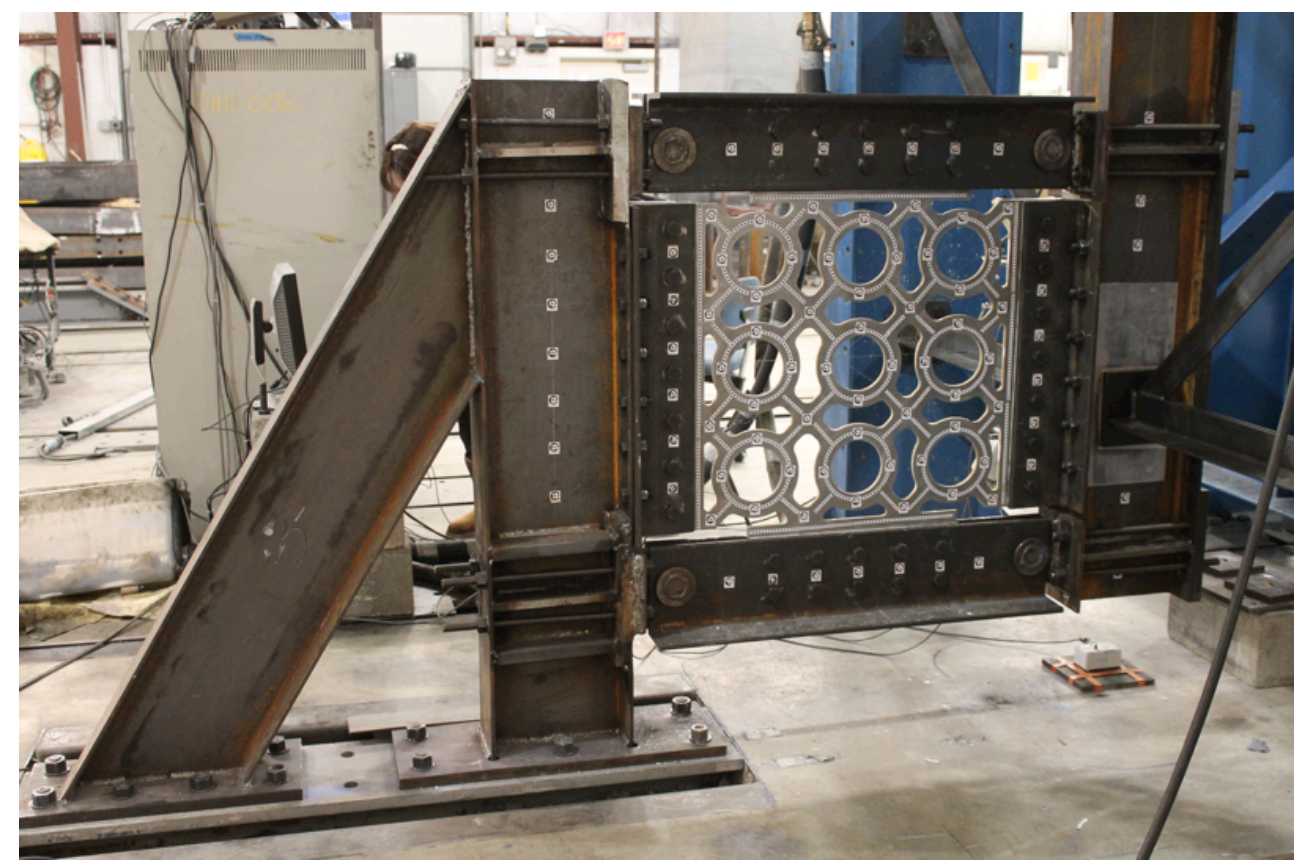

Figure 99. Photogrammetry side view - Test 8 - Specimen 3-0.375-1

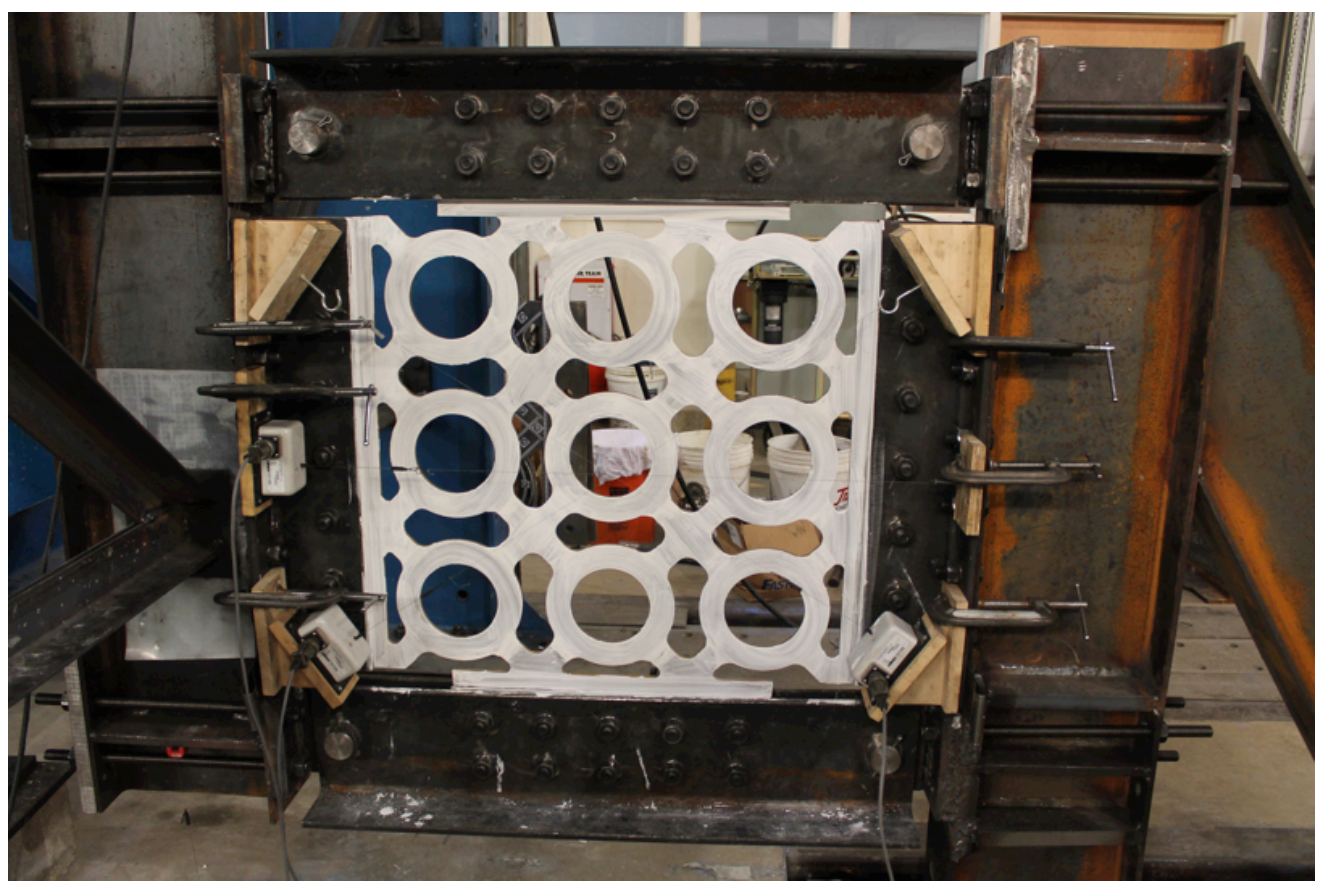

Figure 100. Whitewash side view - Test 8 - Specimen 3-0.375-1 
Table 17. Specimen 3-0.375-1 properties

\begin{tabular}{|l|l|}
\hline Thickness of the plate $\left(t_{w}\right)$ & 0.375 in \\
\hline Outer ring radius $\left(R_{0}\right)$ & 3.93 in \\
\hline Ring width $\left(w_{c}\right)$ & 1.47 in \\
\hline Link width $\left(w_{L}\right)$ & 1.47 in \\
\hline Number of rings in a row $(N)$ & 3 \\
\hline Pin to pin dimensions $(\mathrm{a})$ & $34 \mathrm{in} \times$ 34in \\
\hline
\end{tabular}

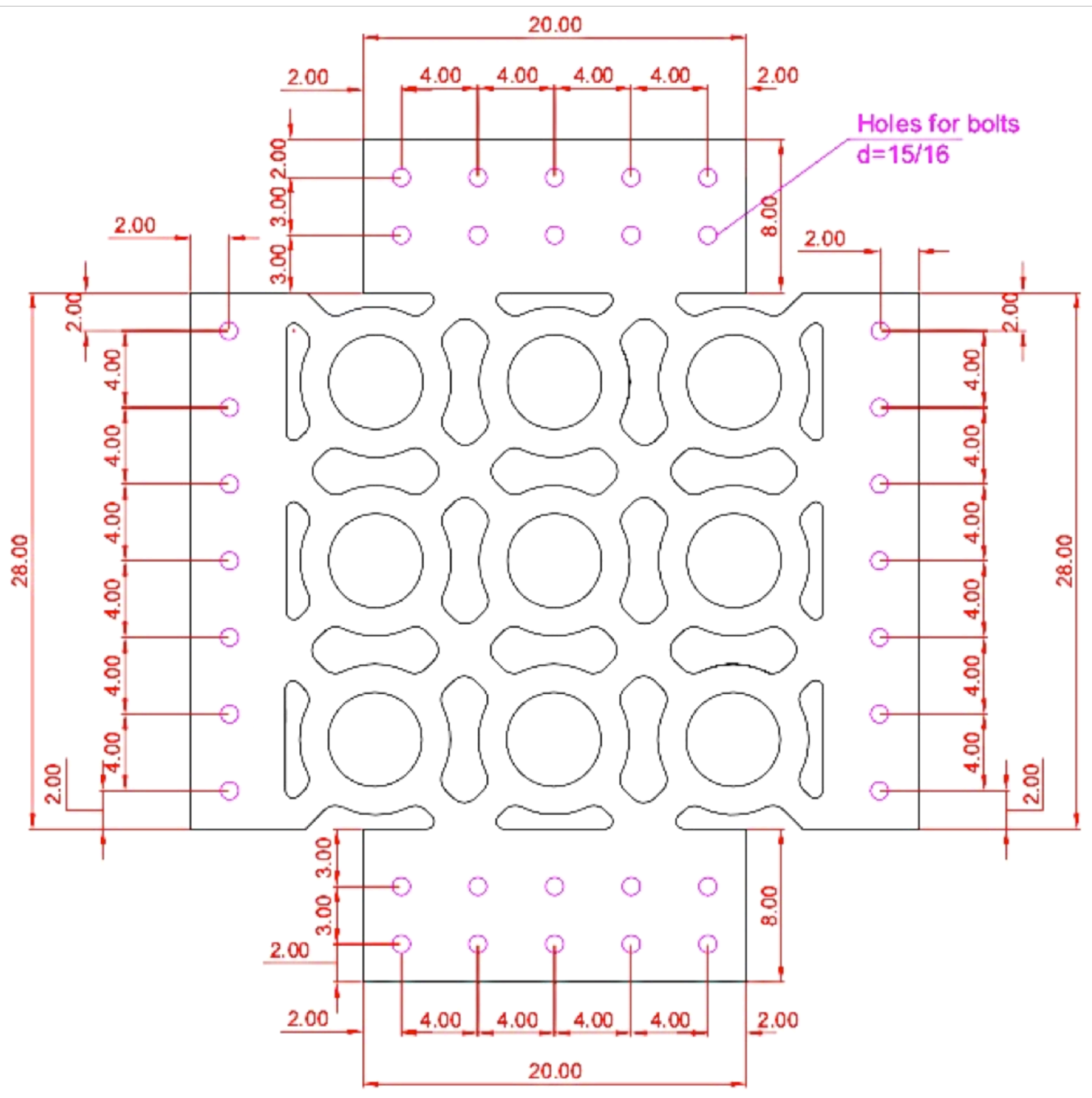

Plate thickness $=0.375$

Figure 101. Test 8, Specimen 3-0.375-1 geometry 
The initial shape of the Specimen 3-0.375-1 was obtained by exporting initial photogrammetry data to MATLAB and is shown in Figure 102. The magnitude of maximum initial imperfection of Specimen 3-0.375-1 was measured to be $0.1026 \mathrm{in}$. It was calculated like a difference between the biggest and the smallest $Z$ coordinate of all targets.

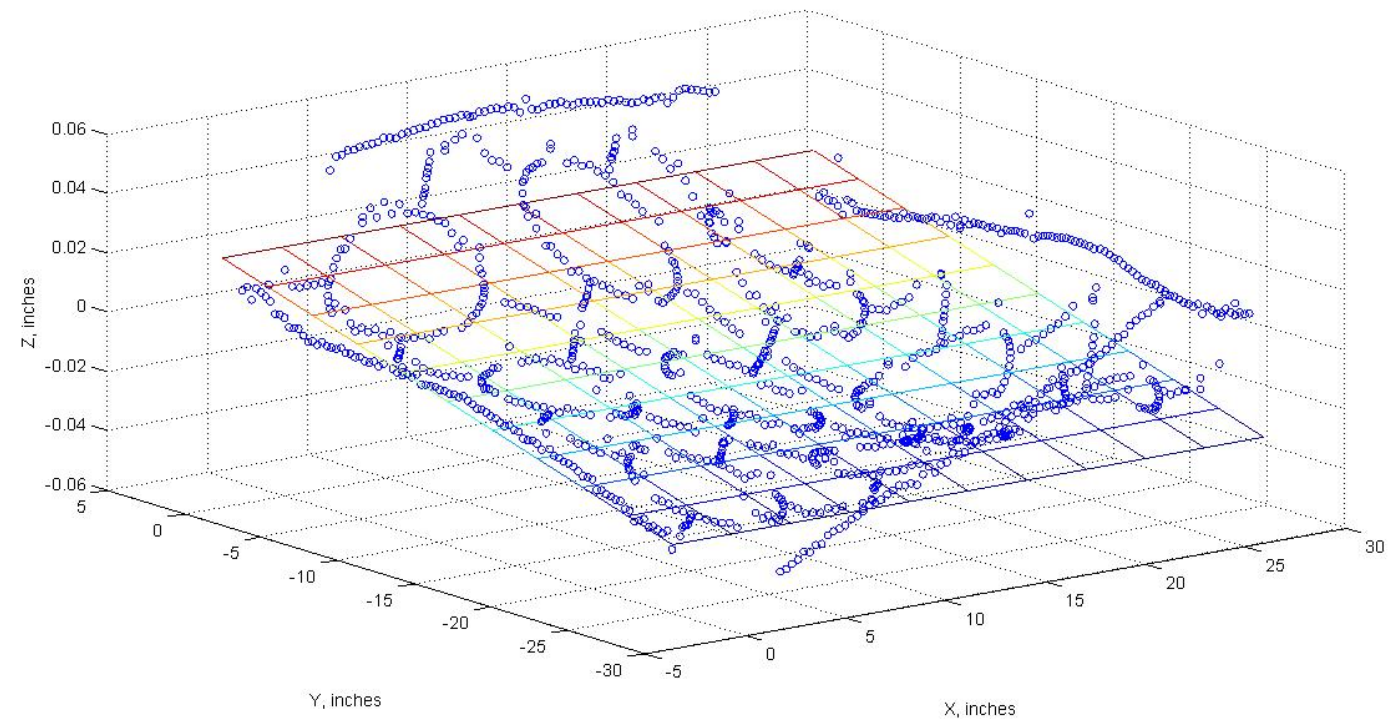

Figure 102. Point cloud and orthogonal regression plot - Specimen 3-0.375-1 initial shape

Displacements applied during Test 8 are shown in Figure 56 and Table 11. Figure 103 shows the behavior of Specimen 3-0.375-1. The shear displacement of the plate $(\Delta)$ was calculated using the diagonal string potentiometers (see Chapter 5.5.2). Pin to pin dimension of the plate $(a)$ was equal to 34 in. First the specimen deformed elastically. Then there was some initiation of yielding at inner and outer radius of the ring at the locations close to where the ring is connected to the link. The buckling started to occur at almost $4 \%$ drift. The plate experienced global shear buckling (Figure 104). Lateral torsional buckling of the rings was insignificant. Two rings of the specimen experienced fracture at $8 \%$ drift. The fracture of the specimen occurred at the ring point close to the link, at the right bottom and right top rings looking from whitewash side of the plate (Figure 105). It propagated fast, and the test was stopped. 


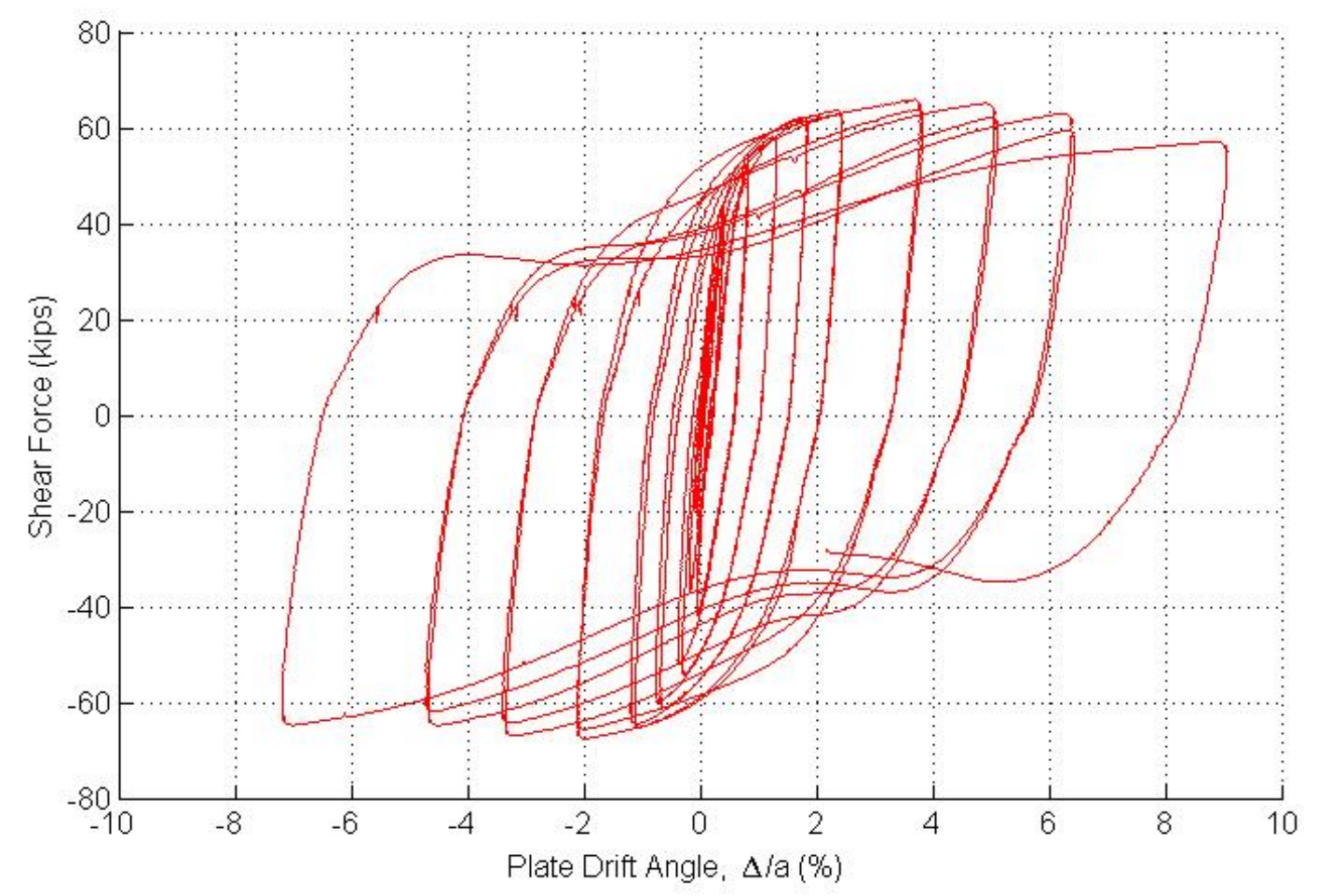

Figure 103. Shear Force versus Plate Drift Angle - Test 8

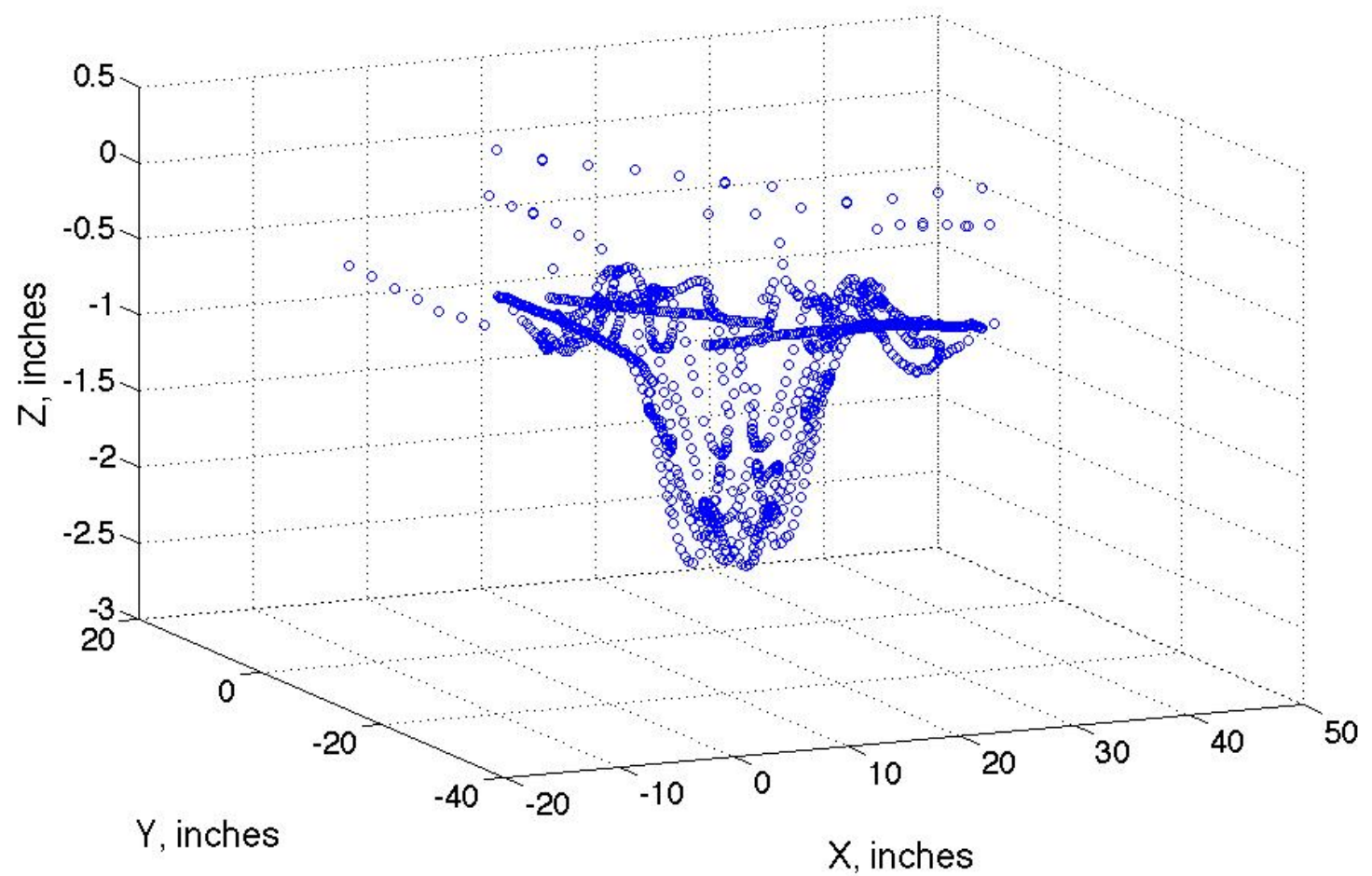

Figure 104. Point cloud - Specimen 3-0.375-1 - 6.1\% drift 


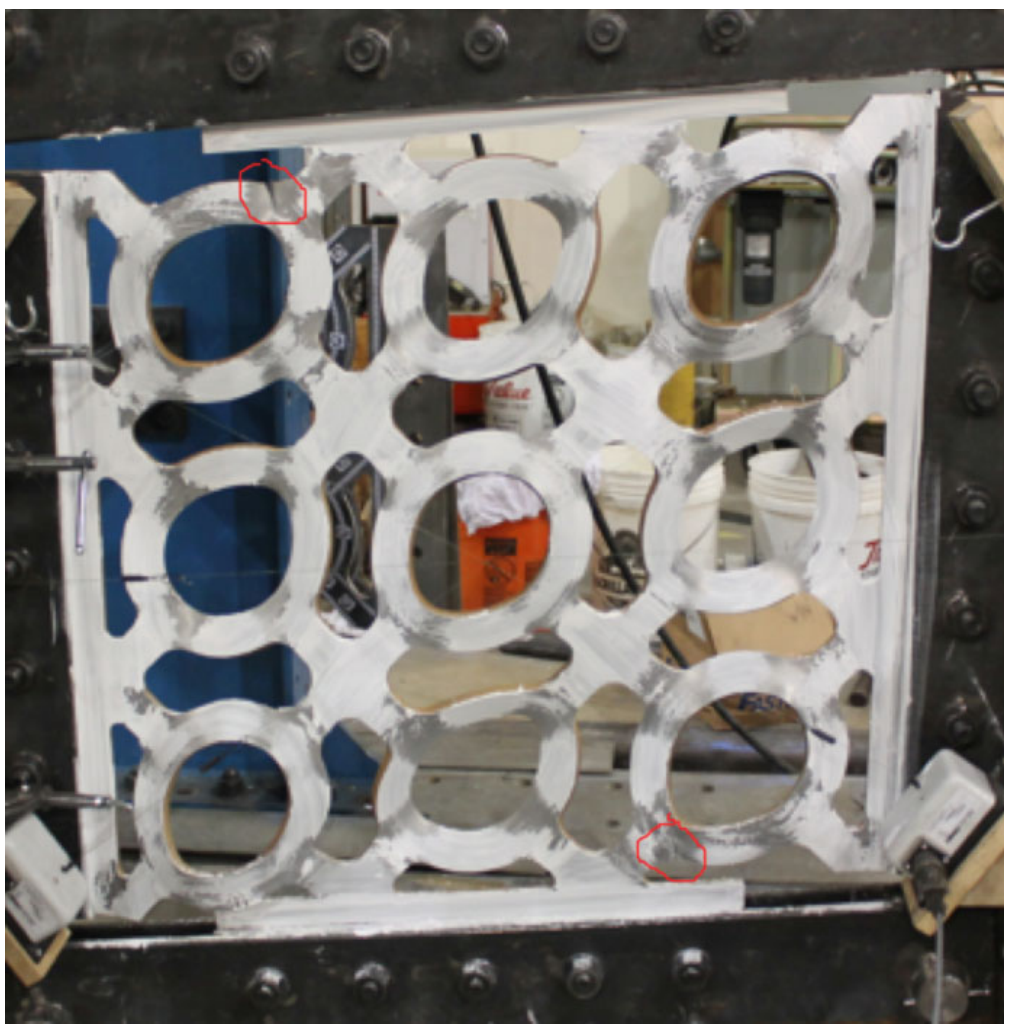

Figure 105. Specimen 3-0.375-1 fracture locations at 8\% drift 


\section{DISCUSSION OF RS-SPSW EXPERIMENTAL BEHAVIOR}

\subsection{Comparison of Specimens behavior}

Summary of all specimens' behavior is represented in Table 18. The energy dissipated during the $4 \%$ drift cycle was computed, $E_{d}$, and was compared with the energy that would be dissipated by an equivalent elastic perfectly plastic system, EEPP. The comparison is shown graphically in Figure 106 and tabulated in Table 18. Backbone response of the specimens is shown in Figure 107. Backbone graphs were also used to calculate shear yield strength for every specimen. Shear yield strength was computed as an approximate load at which significant nonlinearity began occurring.

Table 18. Summary of specimen behavior

\begin{tabular}{|c|c|c|c|c|c|c|c|}
\hline $\begin{array}{c}\text { Test } \\
\#\end{array}$ & $\begin{array}{c}\text { Specimen } \\
\#\end{array}$ & $\begin{array}{c}\text { Shear } \\
\text { yield } \\
\text { strength, } \\
V y \text { (kips) }\end{array}$ & $\begin{array}{c}\text { Ultimate } \\
\text { force, } V u \\
\text { (kips) }\end{array}$ & $\begin{array}{c}\text { Shear } \\
\text { distortion } \\
\text { at Ultimate } \\
\text { force (\%) }\end{array}$ & $\begin{array}{c}\text { Energy } \\
\text { dissipation, } \\
\text { Ed/EEPP } \\
(\%)\end{array}$ & Buckling mode & Fracture \\
\hline 1 & $2-0.5-1$ & 81 & 85 & 3.1 & 79 & $\begin{array}{c}\text { Global shear + } \\
\text { Local torsional }\end{array}$ & No \\
\hline 2 & $\begin{array}{c}\text { Solid } \\
\text { Plate }\end{array}$ & 45 & 52.5 & 4.5 & 44 & Global shear & Yes \\
\hline 3 & $1-0.5-1$ & 53 & 61 & & 46 & Local torsional & No \\
\hline 4 & $3-0.5-1$ & 87 & 91 & 3.4 & 86 & Global shear & No \\
\hline 5 & $\begin{array}{c}2-0.25- \\
0.81\end{array}$ & 20 & 23.6 & 9 & 64 & $\begin{array}{c}\text { Global shear+ } \\
\text { Local torsional }\end{array}$ & Yes \\
\hline 6 & $2-0.25-1$ & 30 & 34.9 & 1 & 54 & $\begin{array}{c}\text { Global shear+ } \\
\text { Local torsional }\end{array}$ & Yes \\
\hline 7 & $3-0.25-1$ & 30 & 38.5 & 1.3 & 64 & Global shear & Yes \\
\hline 8 & $3-0.375-1$ & 61 & 67.8 & 2.1 & 71 & Global shear & Yes \\
\hline
\end{tabular}




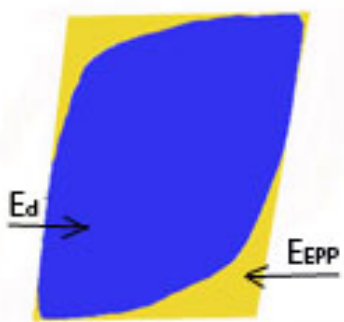

Specimen 2-0.5-1

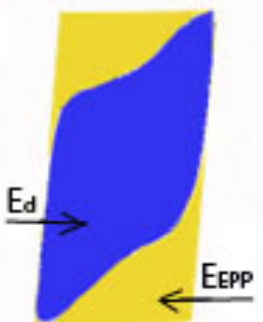

Specimen 2-0.25-0.81

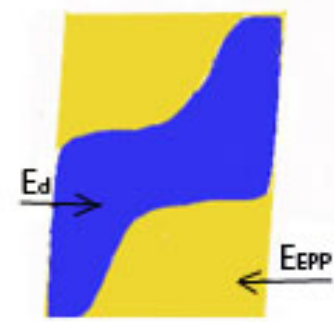

Specimen "Solid Plate"

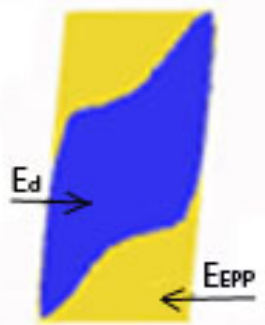

Specimen 2-0.25-1

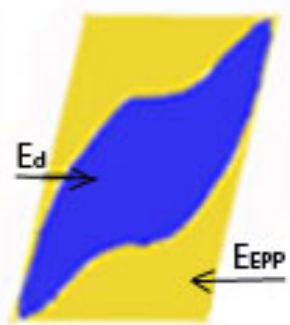

Specimen 1-0.5-1

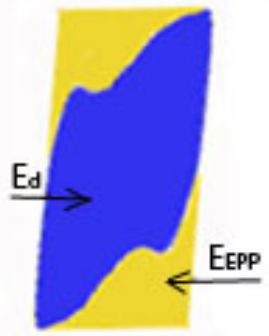

Specimen 3-0.25-1

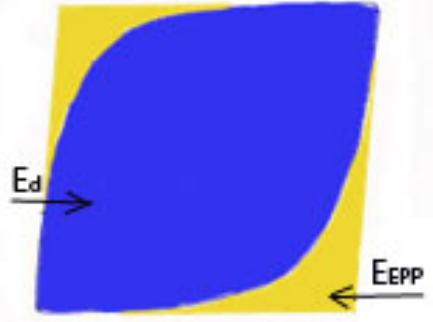

Specimen 3-0.5-1

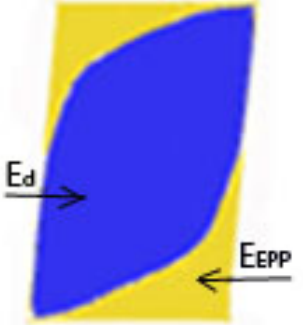

Specimen 3-0.375-1

Figure 106. Energy dissipation during 4\% cycle

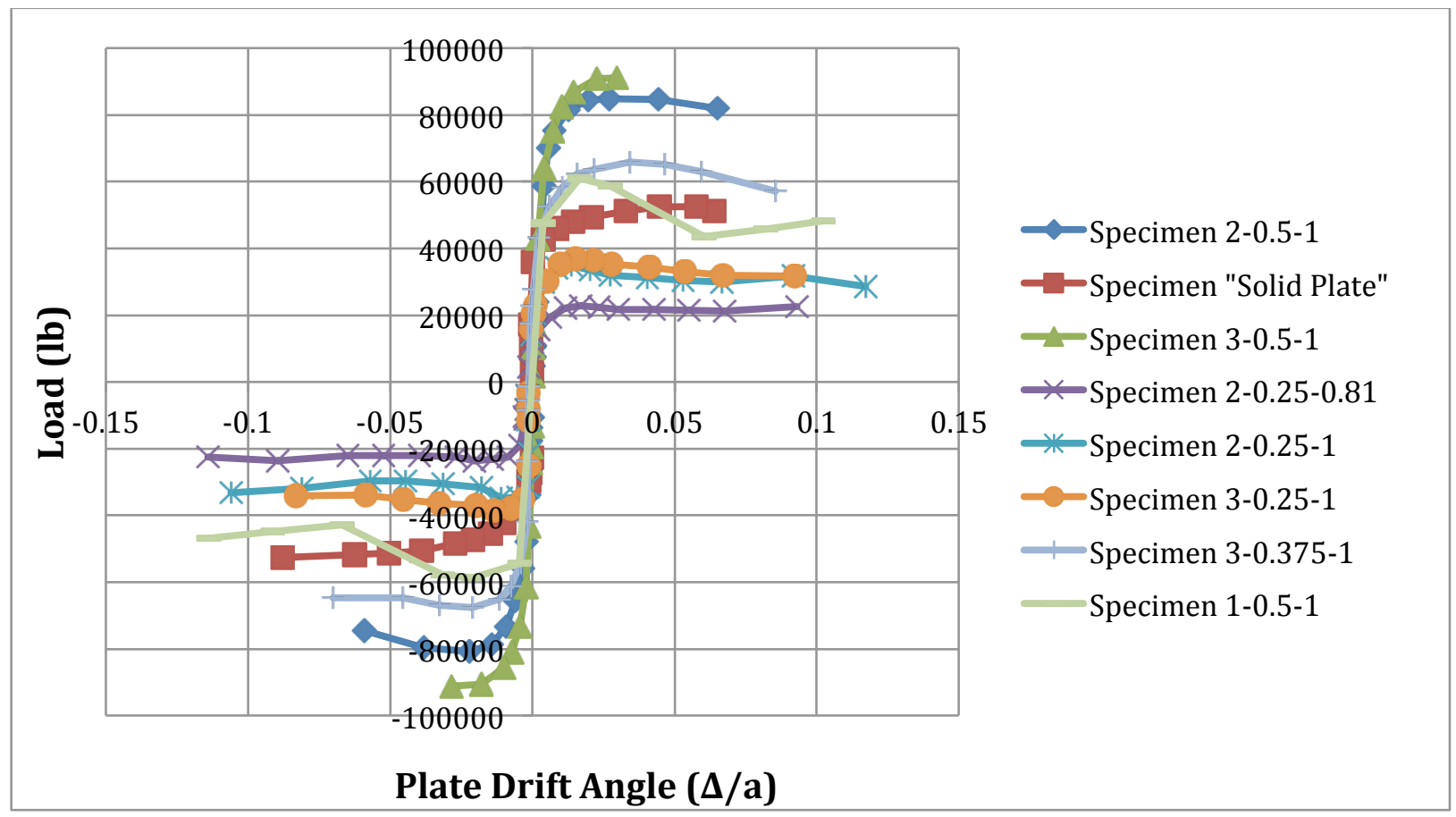

Figure 107. Backbone response of Specimens

Specimen 2-0.5-1, 3-0.5-1 and 3-0.375-1 experienced limited buckling. They validated the RS-SPSW concept. The nondimensional parameters that led to this desirable response were $a / t_{w} \leq 91, R_{o} / t_{w} \leq 12$, and $w_{c} / t_{W} \leq 4.4$. 
The effect of lateral torsional buckling can be understood by comparing the response of specimens 1-0.5-1, 2-0.5-1 and 3-0.5-1. These specimens have the same global slenderness $a / t_{w}=68$. But the ring slenderness of the specimens is different. Figure 108 demonstrates how the energy dissipation ratio $E_{d} /$ EEPP changes versus the ring slenderness $R_{o} / t_{w}$. Decreasing of the ring slenderness leads to increasing of amount of lateral torsional buckling in the specimen, which decreases the energy dissipation ratio.

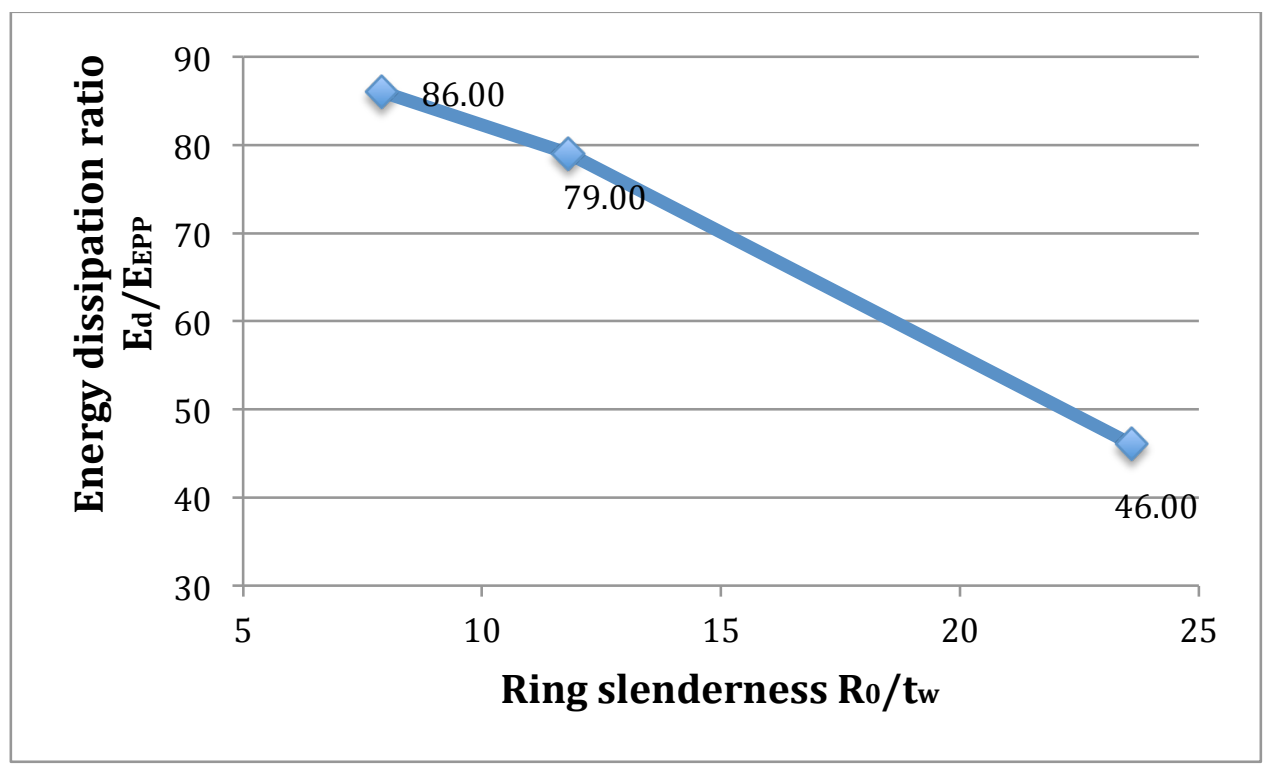

Figure 108. Energy dissipation energy versus ring slenderness

Furthermore, lateral torsional buckling can be studied while comparing the response of specimens 2-0.25-0.81 and 2-0.25-1. These specimens have identical global slenderness $a / t_{w}=136$ and ring slenderness $R_{o} / t_{w}=23.6$, but different $w_{c} / t_{W}$. From the hysteretic behavior of these specimens and their energy dissipation, it appears than Specimen 20.25-1 degraded more than Specimen 2-0.25-0.81. It means that $w_{c} / t_{W}$ parameter has an influence on the amount of lateral torsional buckling. While designing a plate it is an important parameter for predicting severity of lateral torsional buckling.

Global shear buckling is a buckling mode that every specimen experienced. For Specimens 3-0.5-1, 3-0.25-1 and 3-0.375-1 it was the only buckling mode observed during the test. Other specimens, including even those with small global slenderness $a / t_{w}=68$, experienced global shear buckling after large shear distortions. However, the global shear buckling did not cause much energy dissipation ability. It means that some amount of global shear buckling may probably be acceptable for RS-SPSW.

Specimens 1-0.5-1 and 2-0.25-1 had exactly the same ring slenderness values $R_{o} / t_{w}=23.6$ and $w_{c} / t_{W}=8.84$, but different global slenderness ratio $a / t_{w}$. From the hysteretic behavior of these specimens and their energy dissipation, it appears than Specimen 1-0.51 degraded more than Specimen 2-0.25-1. 
Specimens 3-0.25-1, 3-0.375-1 and 3-0.5-1 all have the same $R_{0}$ and $w_{c}$, but varying plate thickness. From the hysteretic behavior of these specimens and their energy dissipation ratio, it appears that decreasing of plate thickness leaded to decreasing of energy dissipation of the specimen.

The influence of geometric parameters on plate behavior can be summarized in a following way:

$w_{c} / t_{W}$ : bigger $w_{c} / t_{W}$ leads to higher degradation of the specimen.

$a / t_{w}$ : higher $a / t_{w}$ leads to higher energy dissipation

$t_{W}$ : higher $t_{W}$ lead to higher energy dissipation and less probability of lateral torsional buckling of the ring

$R_{o} / t_{w}$ : higher $R_{o} / t_{w}$ means higher probability of lateral torsional buckling of the rings

RS-SPSWs are not prone to fracture at the drift angles normally experiences by structures. Specimens 1-0.5-1, 2-0.5-1 and 3-0.5-1 did not fracture at all. Other specimens only fracture at large drift angles ( $8 \%$ and 10\%).

Comparison of the measures shear yield force $V_{y}$ and the computed shear capacity calculated using plastic mechanism is given at Table 19. $Q_{1}$ is computed shear capacity using centerline radius, $R_{0} . Q_{2}$ is computed shear capacity using inner radius, $R_{i}$. Table 19 shows that the analytical solution for the RS-SRSW shear capacity can be quite conservative underestimating the capacity by approximately $60 \%$. Capacity computed by using centerline radius $R_{0}$ gave more accurate result than the one obtained using inner radius $R i$. On the other hand, the shear capacity of the solid plate specimen was fairly well predicted by calculating capacity assuming a tension field angle of $45^{\circ}$.

Table 19. Comparison of shear yield force and shear capacity calculated using plastic mechanism

\begin{tabular}{|c|c|c|c|c|}
\hline Test \# & Specimen \# & $\mathrm{V}_{\mathrm{y}}$ (kips) & $\mathrm{Q}_{1}$ (kips) & $\mathrm{Q}_{2}$ (kips) \\
\hline 1 & $2-0.5-1$ & 81 & 34.7 & 28.1 \\
\hline 2 & Solid Plate & 45 & 49.5 & - \\
\hline 3 & $1-0.5-1$ & 53 & 34.6 & 28.1 \\
\hline 4 & $3-0.5-1$ & 87 & 34.4 & 28.0 \\
\hline 5 & $2-0.25-0.81$ & 20 & 11.0 & 9.3 \\
\hline 6 & $2-0.25-1$ & 30 & 16.6 & 13.5 \\
\hline 7 & $3-0.25-1$ & 30 & 16.5 & 13.4 \\
\hline 8 & $3-0.375-1$ & 61 & 23.1 & 18.8 \\
\hline
\end{tabular}

\subsection{Investigation of Ring Deformation Behavior}

Behavior of the ring like a unit during the test was investigated for specimens with 2 and 3 rings in the row respectively, but having the same thickness 0.5in and the same width of 
the ring to width of the link ratio 1 . The perimeter of the ring was calculated based on photogrammetry targets coordinates measured during certain moments of the test.

First of all, investigation showed that the perimeter of the ring increases during the test, but not significant. Dependence between ring perimeter and plate drift angle is mostly linear before 6\% drift (Figure 109 and Figure 110). Then, it showed that ring inner and outer perimeters are changing with approximately same rate (Figure 109).

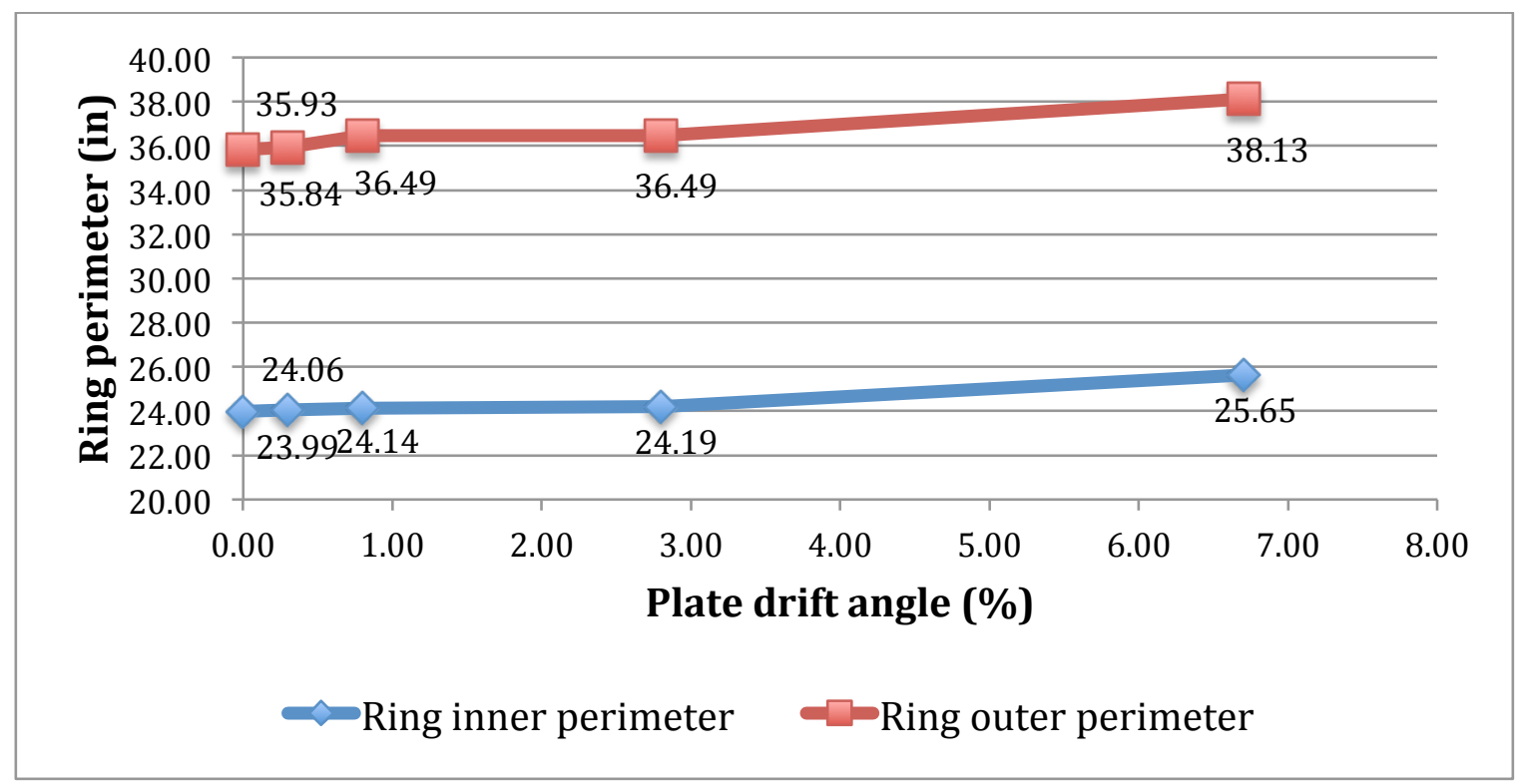

Figure 109. Change of the ring inner and outer perimeter during the test Specimen 2-0.5-1

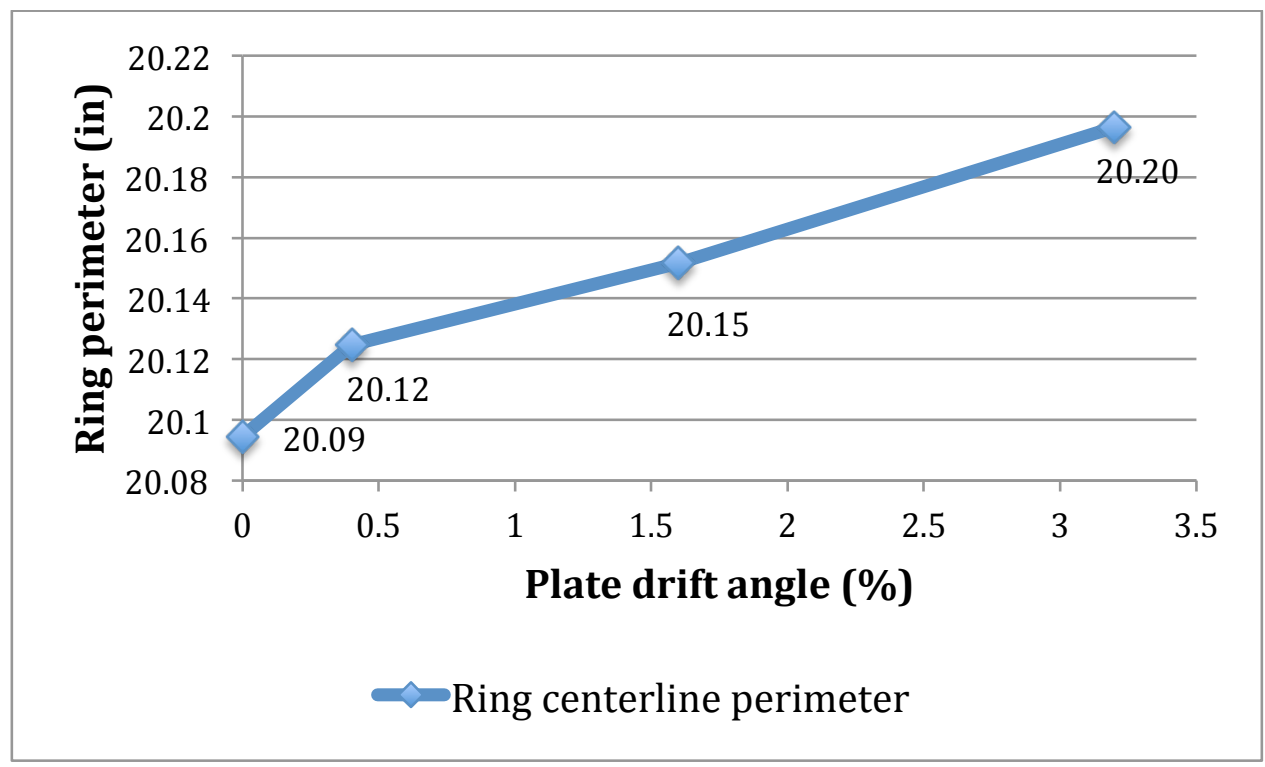

Figure 110. Change of the ring centerline perimeter during the test - Specimen 30.5-1 


\section{SUMMARY AND CONCLUSIONS}

Steel plate shear walls (SPSW) are widely used structures for earthquake engineering design, especially in USA, Canada and Japan. However, they have some significant behavioral and economic disadvantages such as pinched hysteretic behavior, low stiffness, and large boundary elements with moment connections. These disadvantages can be improved by using new type of SPSW - ring-shaped steel plate shear wall (RS-SPSW)

RS-SPSW includes a web plate that is cut with a pattern of holes leaving ring-shaped portions of steel connected by diagonal links. The ring shape resists out-of-plane buckling through the mechanics of how a circular ring deforms into an ellipse. It has been shown that the ring's compression diagonal will shorten a similar amount as the tension diagonal elongates, essentially eliminating the slack in the direction perpendicular to the tension field.

Yielding of the link was found to be an important parameter of the RS-SPSW behavior. The yield of the link equation was derived. The most desirable geometric characteristics of the plate in terms of link yielding were found. The value of width of the link that maximizes the link ratio was found to be equal to $R_{0} / 2$. If the optimal value of width of the link is being used, the width of the ring that will maximize the link ration, should be less than or equal to $R_{0} / 2$.

The testing program was conducted at the Thomas M. Murray Structural Engineering Laboratory at Virginia Tech to validate the RS-SPSW concept, investigate the cyclic hysteretic behavior of these panels, verify strength predictions, and study the buckling modes associated with these plate configurations. The tests were performed on 34 in x 34 in plates. This size plate was found to be adequate to investigate the behavior of full scale ring units. The cyclic loading was applied by hydraulic actuator, casing the shear deformation of the specimens. The load cell was attached in line with the actuator. Four string potentiometers were used to measure the in-plane displacements of the test setup. Photogrammetry was used to track the out-of-plane displacement of the web plates. Data from photogrammetry was used to understand the buckling modes of the RS-SPSW and quantify the magnitude of out-of-plane displacements.

Seven specimens with different geometric parameters were tested to investigate the influence of geometry on the behavior of the plate. The specimens varied in thickness, number of rings in the row and width of the ring. 34 in $\times 34$ in solid plate was tested to compare it behavior with RS-SPSW specimens behavior.

Some general observations and conclusions can be made based on the work presented herein. First of all, all RS-SPSW specimens demonstrated excellent ductility. Some of the specimens (Specimen 1-05-1, Specimen 2-0.5-1, Specimen 1-0.5-1) didn't fracture at all, some experienced fracture only during $8-10 \%$ shear drift (Specimen 2-0.25-0.81, Specimen 2-0.25-1, Specimen 3-0.25-1, Specimen 3-0.375-1). The same loading for solid plate resulted in significant tearing early during the test. Second, it is evident that an 
energy dissipation of the RS-SPSW is significantly higher than solid plates. Different configurations of RS-SPSW have been tested. Specimen 3-0.5-1 showed that it is possible to proportionate the plate such that it won't buckle and will produce full hysteretic behavior. Two distinct buckling modes have been identified associated with RS-SPSW. The global shear buckling experienced by the solid plate specimen can occur in RS-SPSW for large values of the global panel slenderness. Lateral torsional buckling of ring legs was investigated in the experiments presented herein and was found to be related to the ring slenderness. Furthermore, test proved the whole concept of RS-SPSW and confirmed that theoretical predictions of the behavior made by Maurya (2012) are accurate, computational results match very well with experimental results.

Further development of the buckling resistant steel plate shear wall is necessary to allow implementation in real buildings. Experimental study of full scale RS-SPSW will be done at Virginia Tech in the near future. This work will include large-scale experimentation, development of calibrated computational models, and their application to a wide range of prototype buildings. Within the next year, the large-scale tests are expected to begin. The purpose of the large scale tests will be to adapt the concept to full-scale structures and to gather data on the spread of inelasticity and buckling deformations in larger plates. The test matrix will include six different configurations of plates by varying ring spacing, ring width, ring diameter, and plate thickness.

The data collected from the tests will then be used to construct a computationally efficient model. The plan is to utilize the OpenSEES open source structural analysis software platform in conjunction with Matlab for data manipulation, calculations, and plotting. Once the behavior of a single plate and its boundary elements are modeled and appropriately calibrated, the model will be expanded to subject a variety of low, mid, and high-rise systems to different ranges of simulated earthquakes. The models will be ran on isolated multi-story walls as well as inter-connected walls using simulated floor diaphragm action. Using the data obtained from the experimental and computation tests, in conjunction with sound mechanics theory, sets of design guidelines and recommendations will be produced.

It is expected that the large-scale tests will validate the ring concept. Based on Abaqus models and initial small-scale tests, the plates with rings will have improved seismic behavior as compared to thin-gauge SPSW. It is anticipated that due to the ability to modify parameters such as ring diameter, ring width, and plate thickness, individual aspects of the system response, such as stiffness, strength, ductility, and energy dissipation can be individually tuned. This would be a substantial improvement for SPSW systems because it would give designers the ability to tune the performance of the seismic load resisting system.

The computational model will be used to simulate the performance of multi-story structures including the BR-SPSW. It is expected that these structures will exhibit better energy dissipation, smaller peak drifts, and thus lower residual drifts at the conclusion of the seismic events. It is also expected that the use of bolted shear connections, in place of 
moment connections, will make the BR-SPSW cost competitive. The computation model will assist with quantifying the exact demand applied to the boundary elements and connections. The design guidelines created by the end of this project will produce solutions that safely resist these demands.

The future work can also be done on examining the effect of different loading histories on behavior of the specimens. As it was mentioned above there is no standard loading protocol for cyclic loading tests. 


\section{REFERENCES}

- AISC, 2005. "Seismic Provisions for Structural Steel Buildings," ANSI/AISC 341-05, American Institute for Steel Construction, Chicago, Illinois

- ATC-24, 1992. "Guidelines for Cyclic Seismic Testing of Components of Steel Structures for Buildings," Report No. ATC-24, Applied Technology Council, Redwood City, CA.

- Berman, J. W., and Bruneau, M. (2005) "Experimental Investigation of Light-Gauge Steel Plate Shear Walls", ASCE Journal of Structural Engineering, Vol. 131, No. 2.

- Caccese, V., Elgaaly, M., and Chen, R. (1993) "Experimental Study of Thin Steel-Plate Shear Walls Under Cyclic Load", Journal of Structural Engineering, ASCE, Vol. 119, No. 2.

- Chen, S.-J., and Jhang, C. (2011) "Experimental Study of Los-Yield-Point Steel Plate Shear Wall Under In-Plane Load", Journal of Constructional Steel Research, Vol. 67, pp. 977-985.

- Clark, P., Frank, K., Krawinkler, H., and Shaw, R., 1997. "Protocol for Fabrication, Inspection, Testing, and Documentation of Beam-Column Connection Tests and Other Experimental Specimens," SAC Steel Project Background Document. October, Report No. SAC/BD-97/02.

- Federal Emergency Management Agency (FEMA). (1997). "NEHRP Recommended Provisions for Seismic Regulations for New Buildings and Other Structures, Part-1Provisions." FEMA 302, Building Seismic Safety Council for the FEMA, Washington, D.C.

- Hitaka, T. and Matsui, C. (2003) "Experimental Study on Steel Shear Wall With Slits", Journal of Structural Engineering, ASCE, Vol. 129, No. 5.

- Krawinkler, H. (2009). "Loading histories for cyclic tests in support of performance assessment of structural components" The 3rd International Conference on Advances in Experimental Structural Engineering, October 15-16, 2009, San Francisco, CA.

- Li, C.-H., Tsai, K.-C, Lin, C.-H., and Chen, P.-C. (2010) "Cyclic Tests of Four Two-Story Narrow Steel Plate Shear Walls - Part 2: Experimental Results and Design Implications", Earthquake Engineering and Structural Dynamics, Vol. 39, pp. 801826.

- Ma, X., Borchers, E., Peña, A., Krawinkler, H., and Deierlein, G. (2010). Design and Behavior of Steel Shear Plates With Openings as Energy-Dissipating Fuses, Blume Earthquake Engineering Center, TR 173, Stanford University, Stanford, CA.

- Maurya A. (2012) Computational Simulation and Analytical Development of Buckling Resistant Steel Plate Shear Wall (BR-SPSW), Report, The Charles E. Via, Jr. Department of Civil and Environmental Engineering, Blacksburg, VA, USA.

- Orsa, R.J. , Eatherton,, M.R., Moen, C.D. (2011) Using Close-range Photogrammetry to Characterize Initial Imperfections of Cold Formed Steel Members, Report No. CE/VPIST-11/03, The Charles E. Via, Jr. Department of Civil and Environmental Engineering, Blacksburg, VA, USA. 
- Vian, D., Bruneau, M., Tsai K.C., Lin, Y.C. (2009) "Special Perforated Steel Plate Shear Walls with Reduced Beam Section Anchor Beam. I: Experimental Investigation", Journal of Structural Engineering, ASCE, Vol. 135, No. 3, pp. 211-220. 


\section{APPENDIX A: Instruments and channels}

Table 20. Instruments and channels - Test 1

\begin{tabular}{|c|c|}
\hline Channel & Instrument \\
\hline 1 & Strain gage 1 \\
\hline 2 & Strain gage 2 \\
\hline 3 & Strain gage 3 \\
\hline 4 & Strain gage 4 \\
\hline 5 & Strain gage 5 \\
\hline 6 & Strain gage 6 \\
\hline 7 & Strain gage 7 \\
\hline & \\
\hline 9 & Strain gage 8 \\
\hline & \\
\hline 11 & String pot 1 \\
\hline 12 & String pot 2 \\
\hline 13 & String pot 3 \\
\hline 14 & String pot 4 \\
\hline & \\
\hline & \\
\hline 26 & MTS Load cell \\
\hline
\end{tabular}

Table 21. Instruments and channels - Test 2, 3 and 4.

\begin{tabular}{|c|c|}
\hline Channel & Instrument \\
\hline & \\
\hline 11 & String pot 1 \\
\hline 12 & String pot 2 \\
\hline 13 & String pot 3 \\
\hline 14 & String pot 4 \\
\hline & \\
\hline & \\
\hline & $\begin{array}{c}\text { MTS Load } \\
\text { cell }\end{array}$ \\
\hline 26 & \\
\hline
\end{tabular}


APPENDIX B: Experimental setup elements

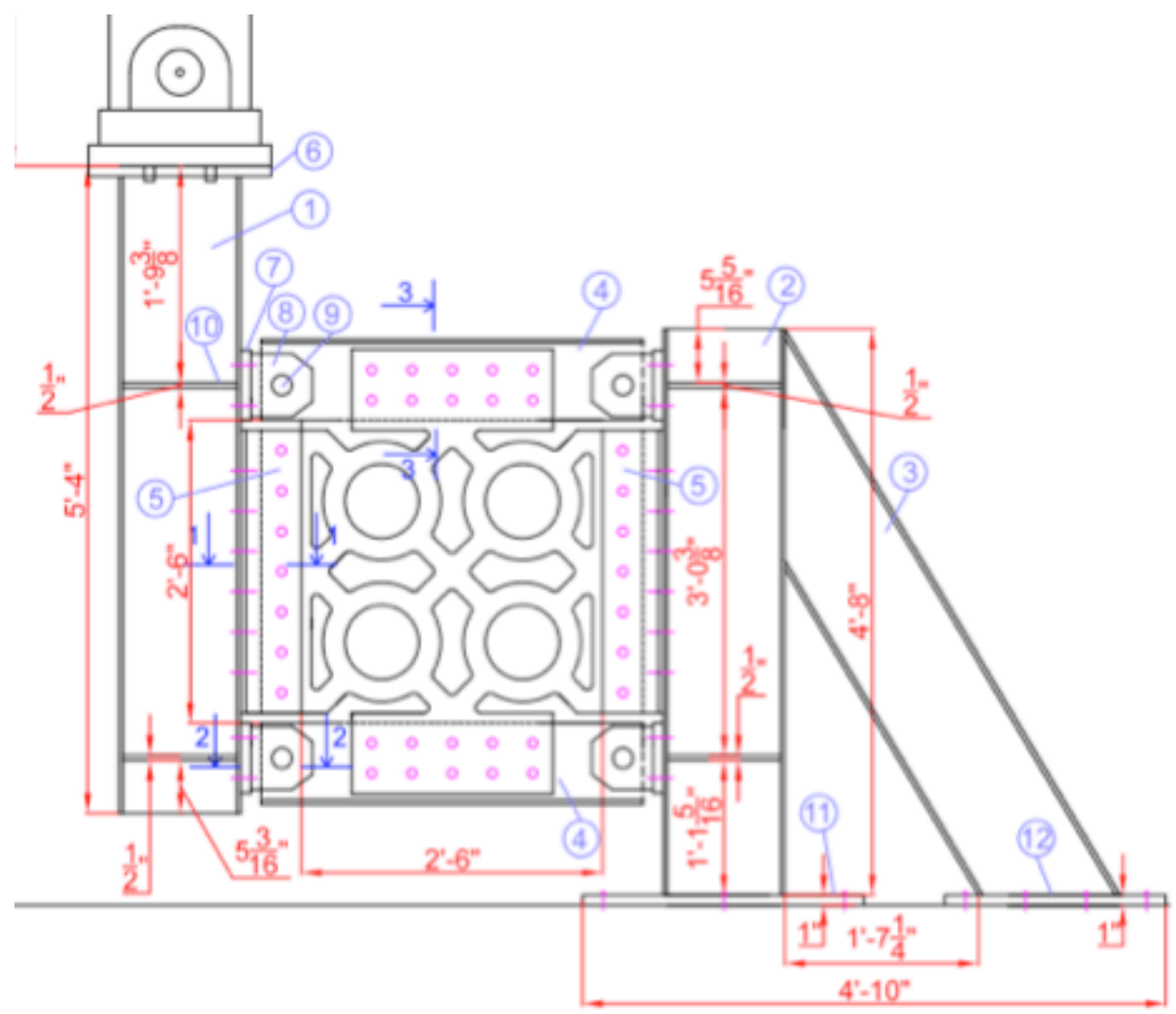

Figure 111. Side view of the experimental set up 


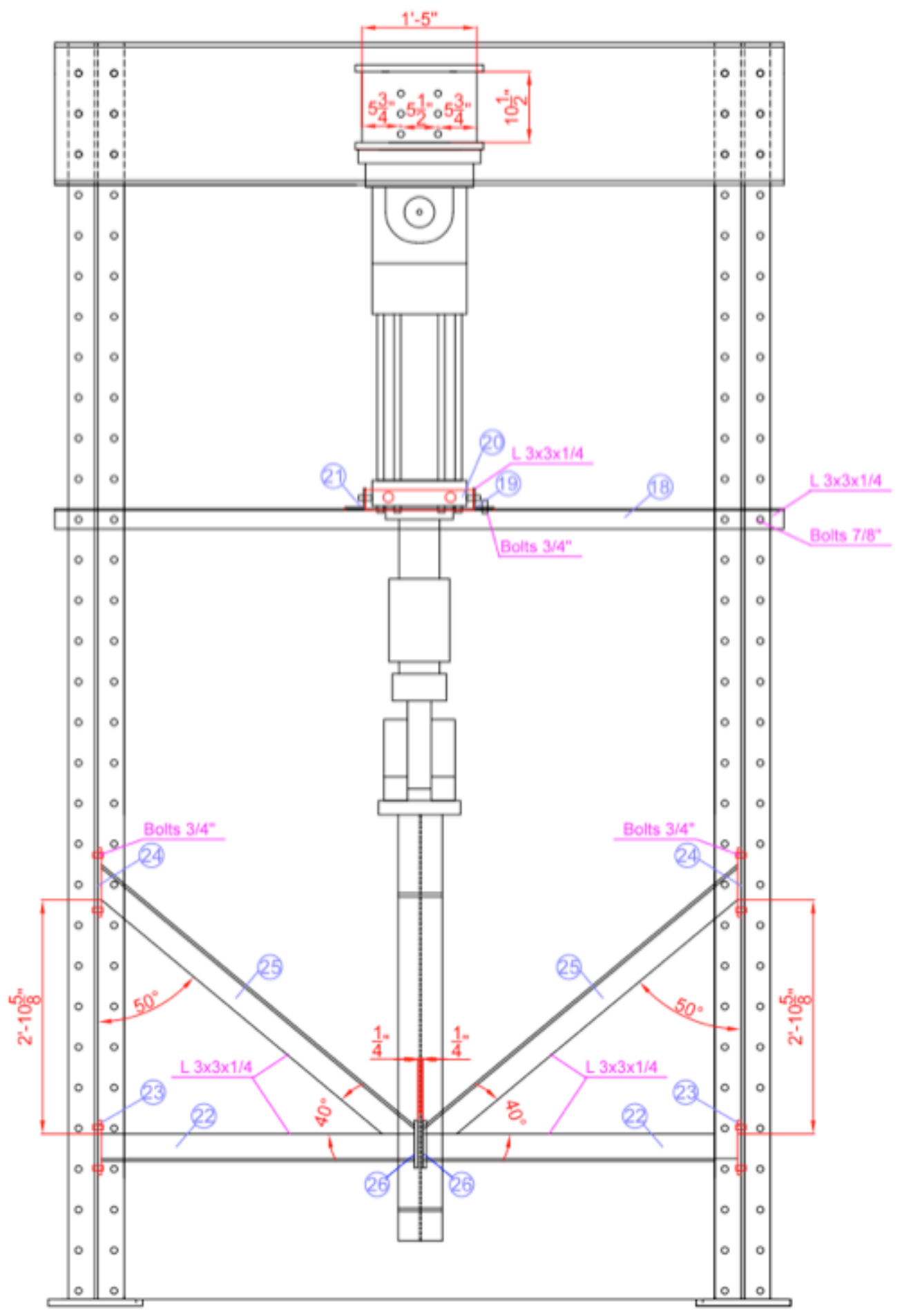

Figure 112. Horizontal and vertical bracing 


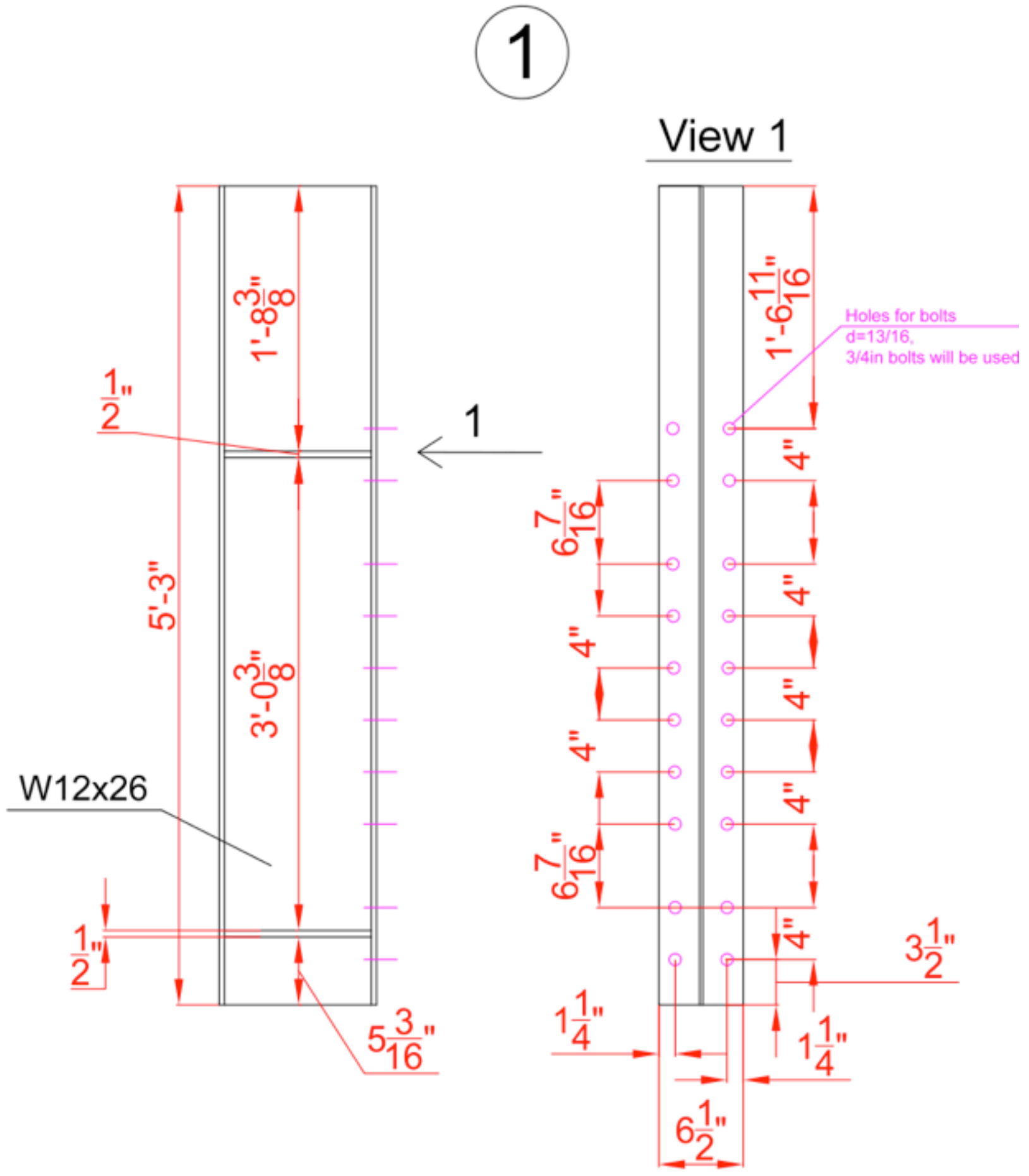

Figure 113. Experimental setup - Element 1. 


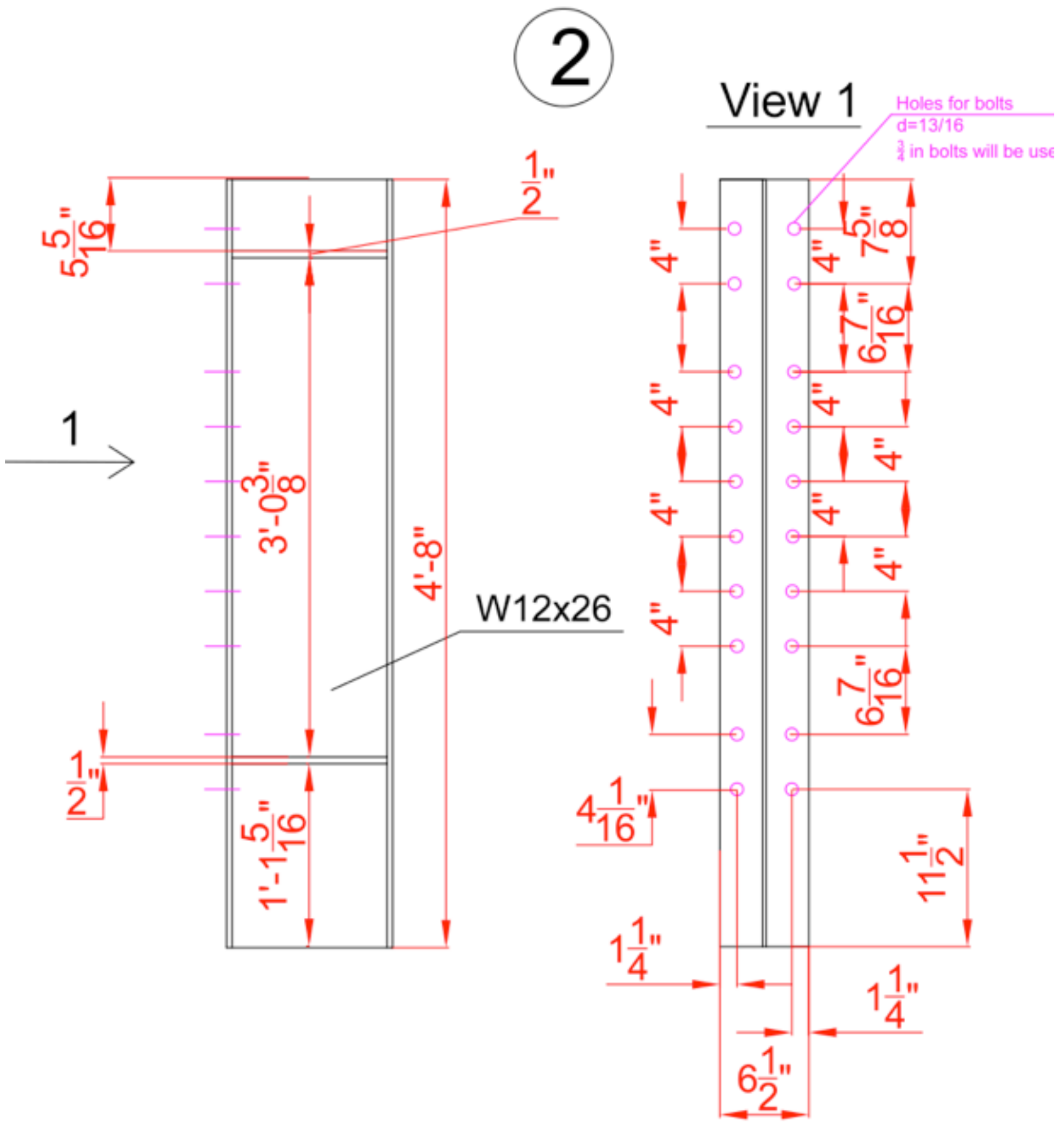

Figure 114. Experimental setup - Element 2 

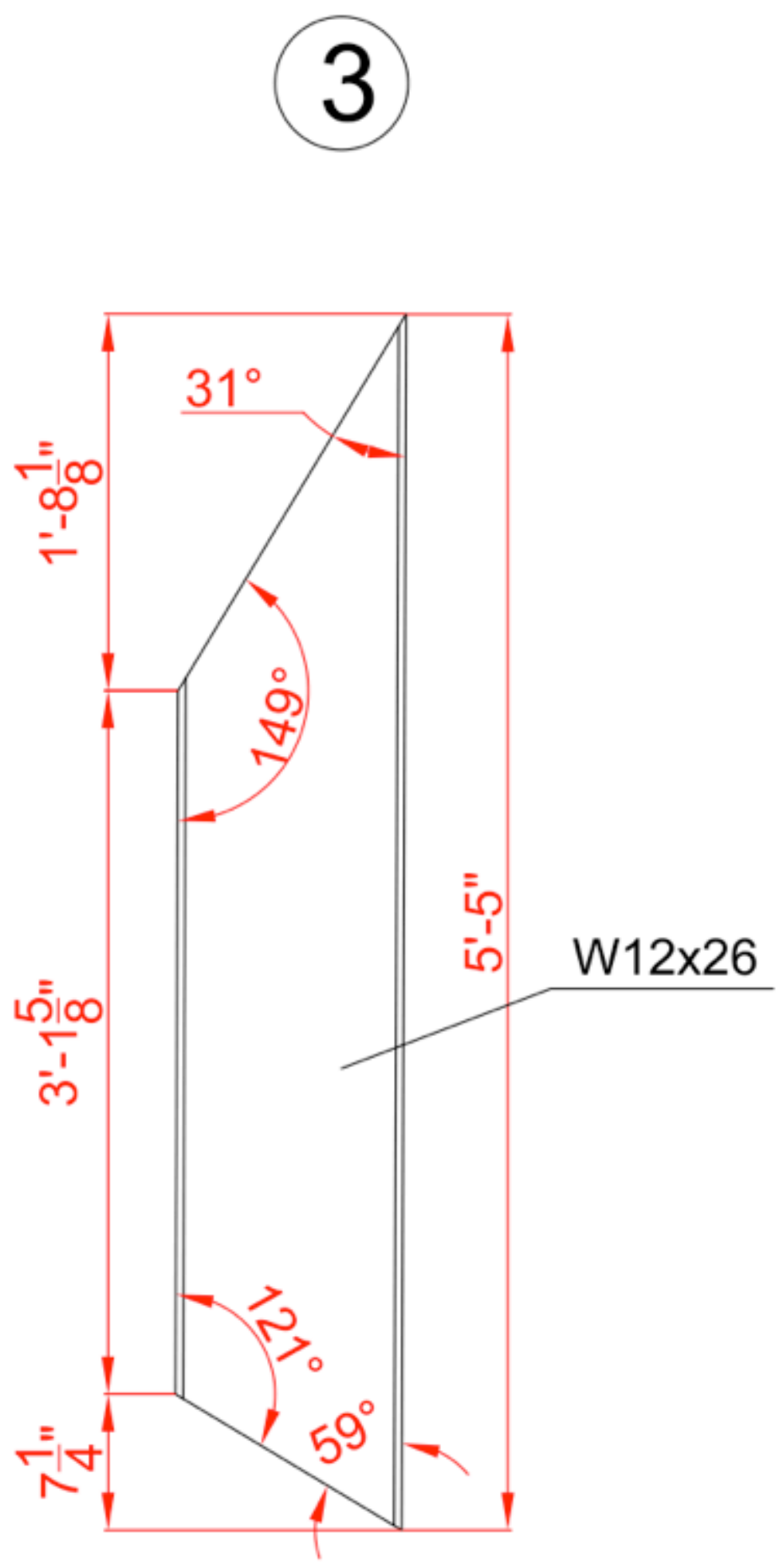

Figure 115. Experimental setup - Element 3 


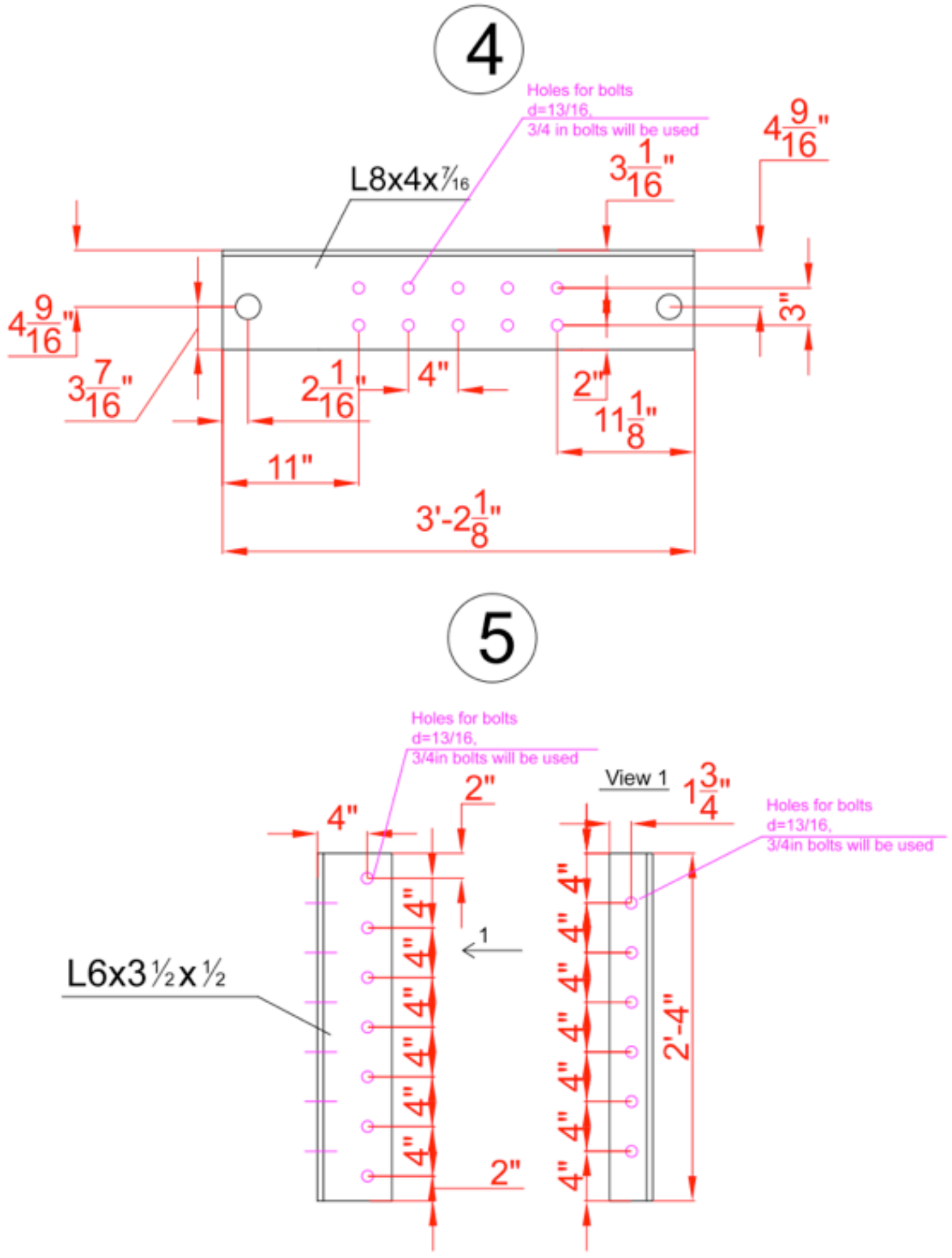

Figure 116. Experimental setup - Elements 4 and 5 

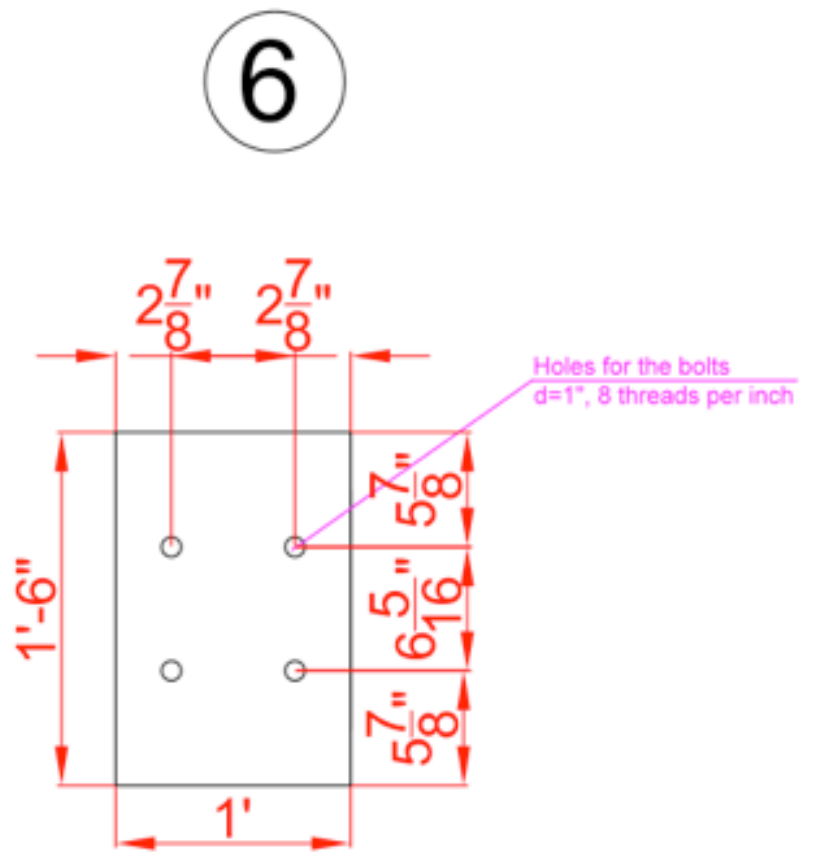

Thickness $=1 \mathrm{in}$

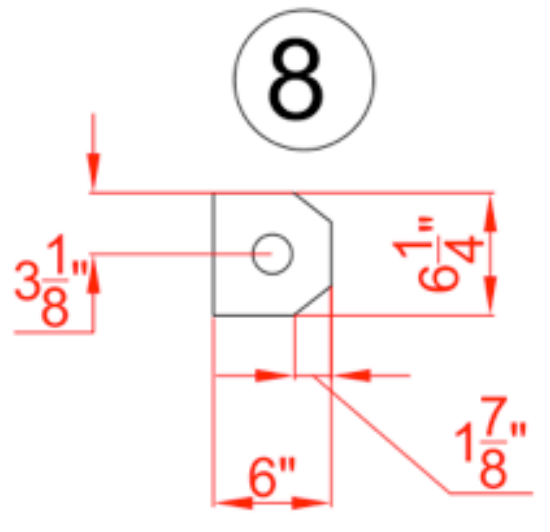

Thickness $=1 \mathrm{in}$
(7)

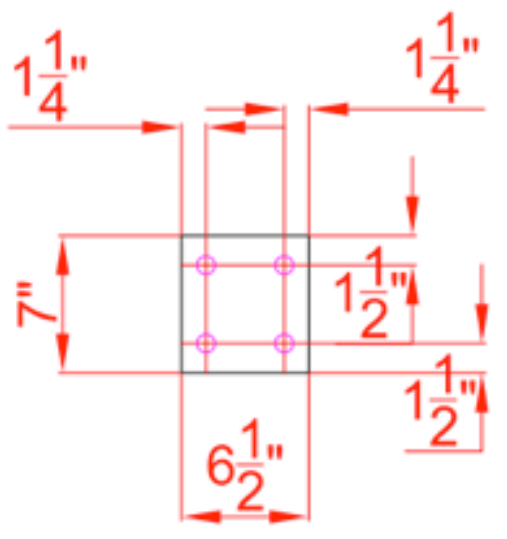

Thickness $=1$ in

9

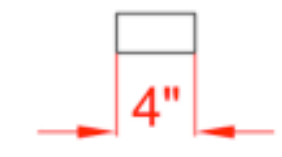

Cylinder $\mathrm{D}=2$ in

Figure 117. Experimental setup - Elements 6-9 


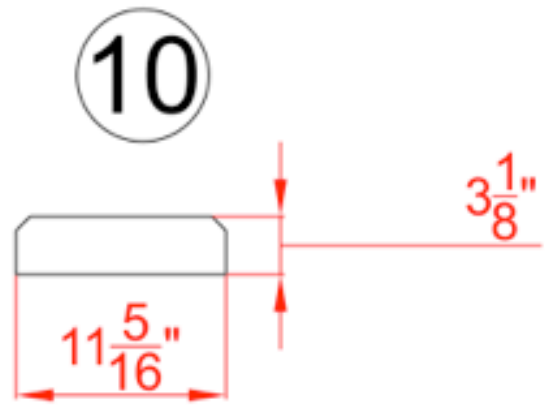

Thickness $=0.5 \mathrm{in}$

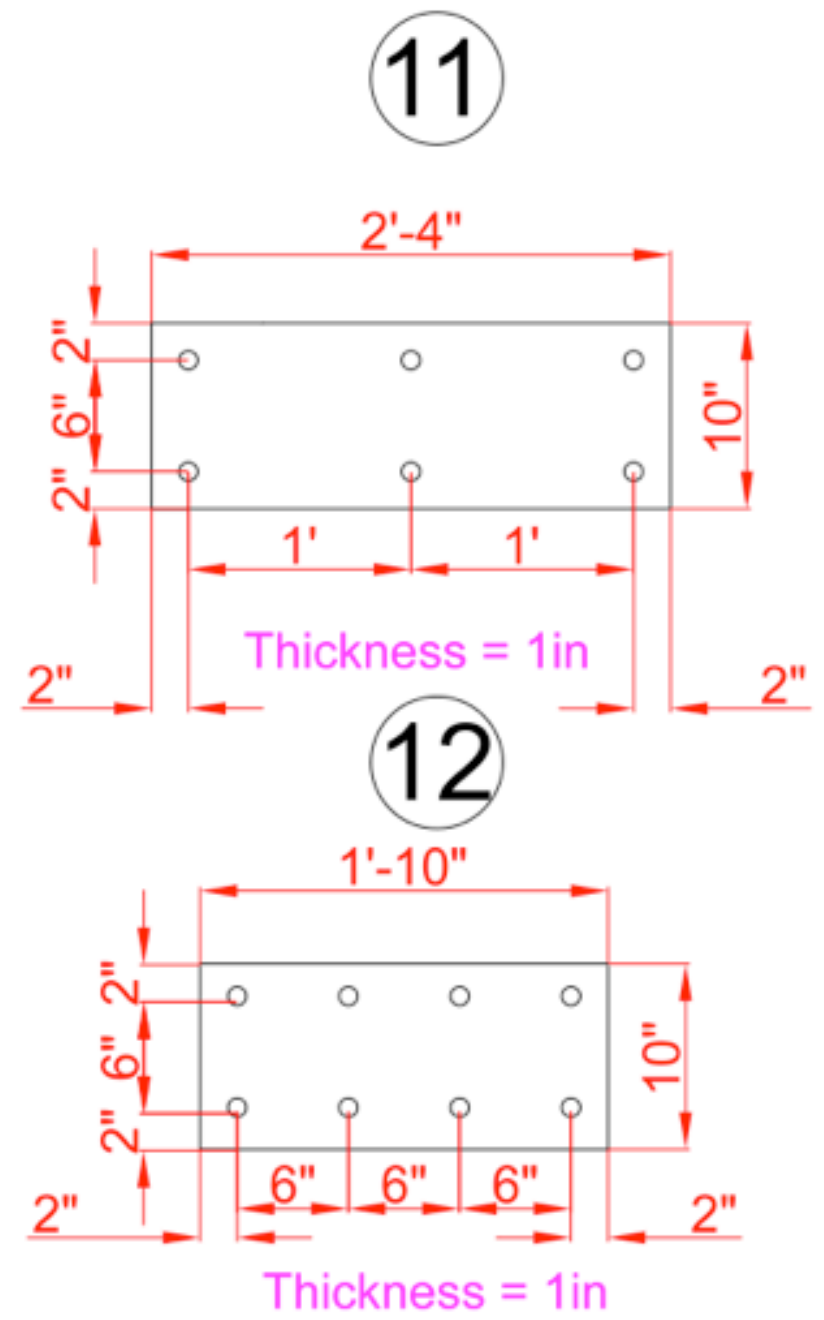

Figure 118. Experimental setup - Elements 10-12 


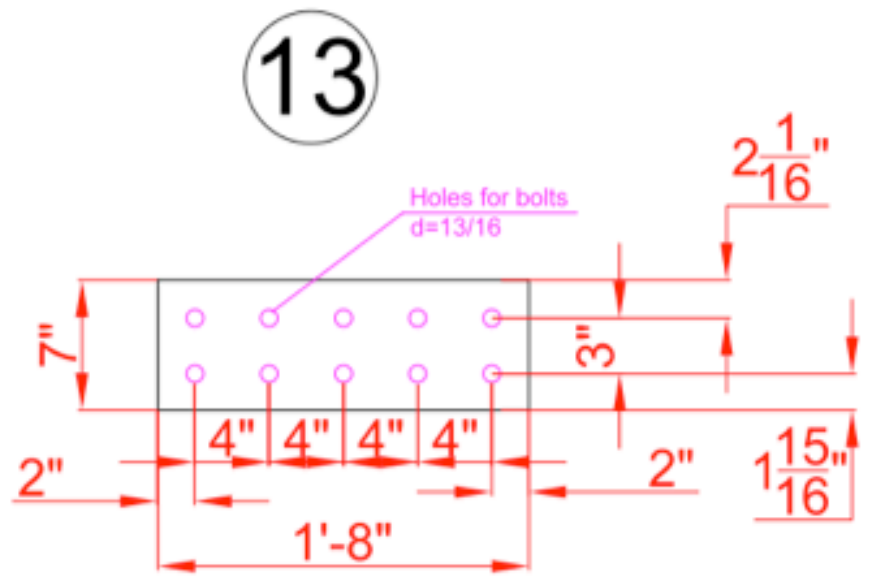

Thickness $=1 / 4 \mathrm{in}$

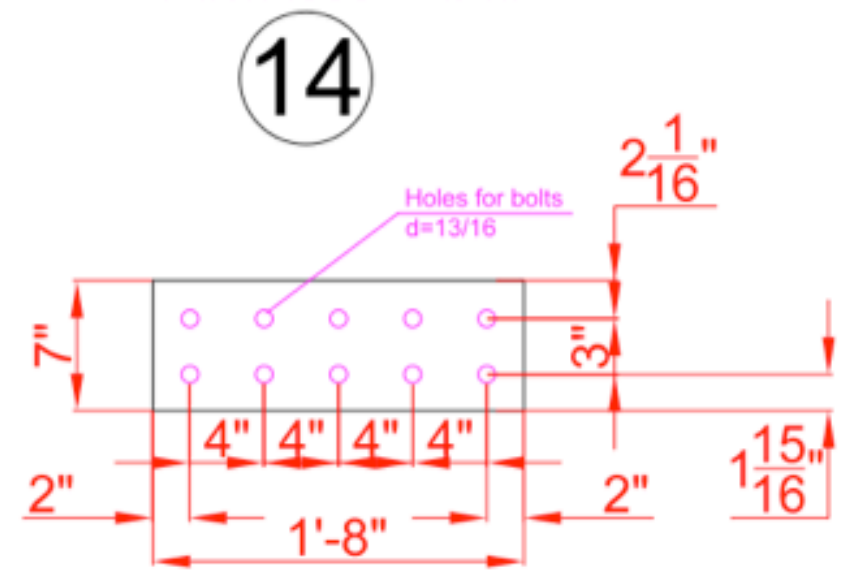

Thickness $=1 / 8 \mathrm{in}$

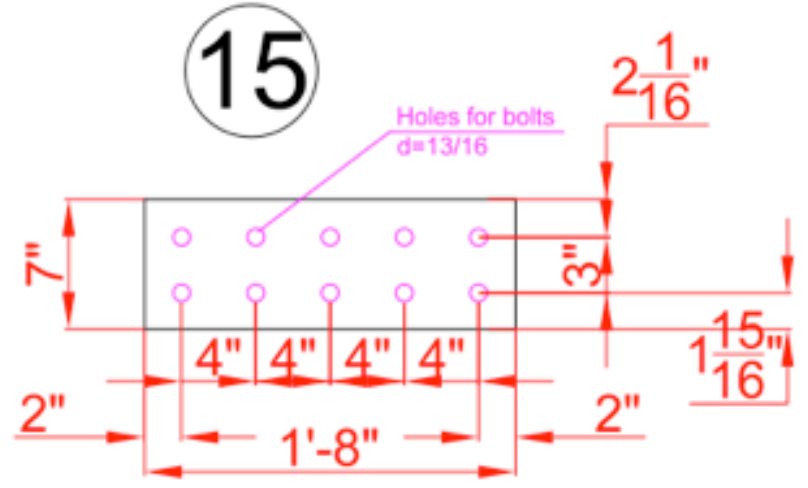

Thickness $=3 / 16 \mathrm{in}$

Figure 119. Shims - Elements 13-15 
16

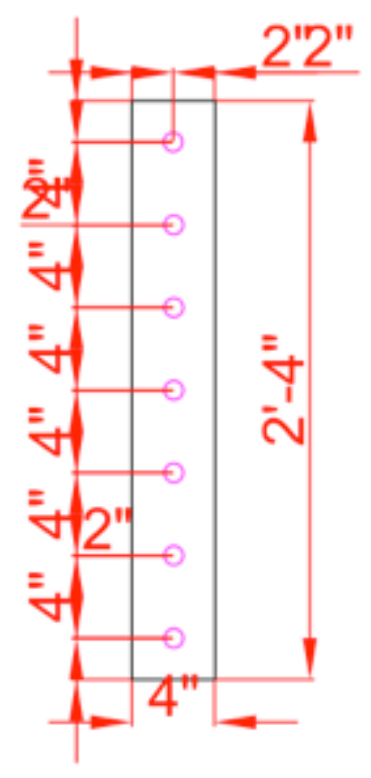

Thickness $=1 / 8$ in
17

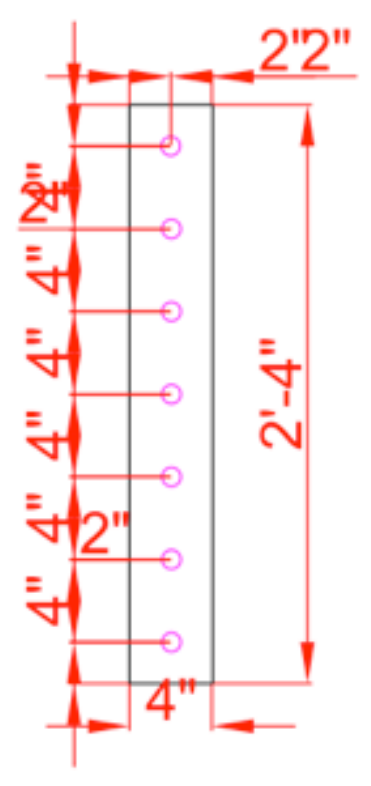

Thickness $=3 / 16 \mathrm{in}$

Figure 120. Shims - Elements 16 and 17 


\section{8}

19

20

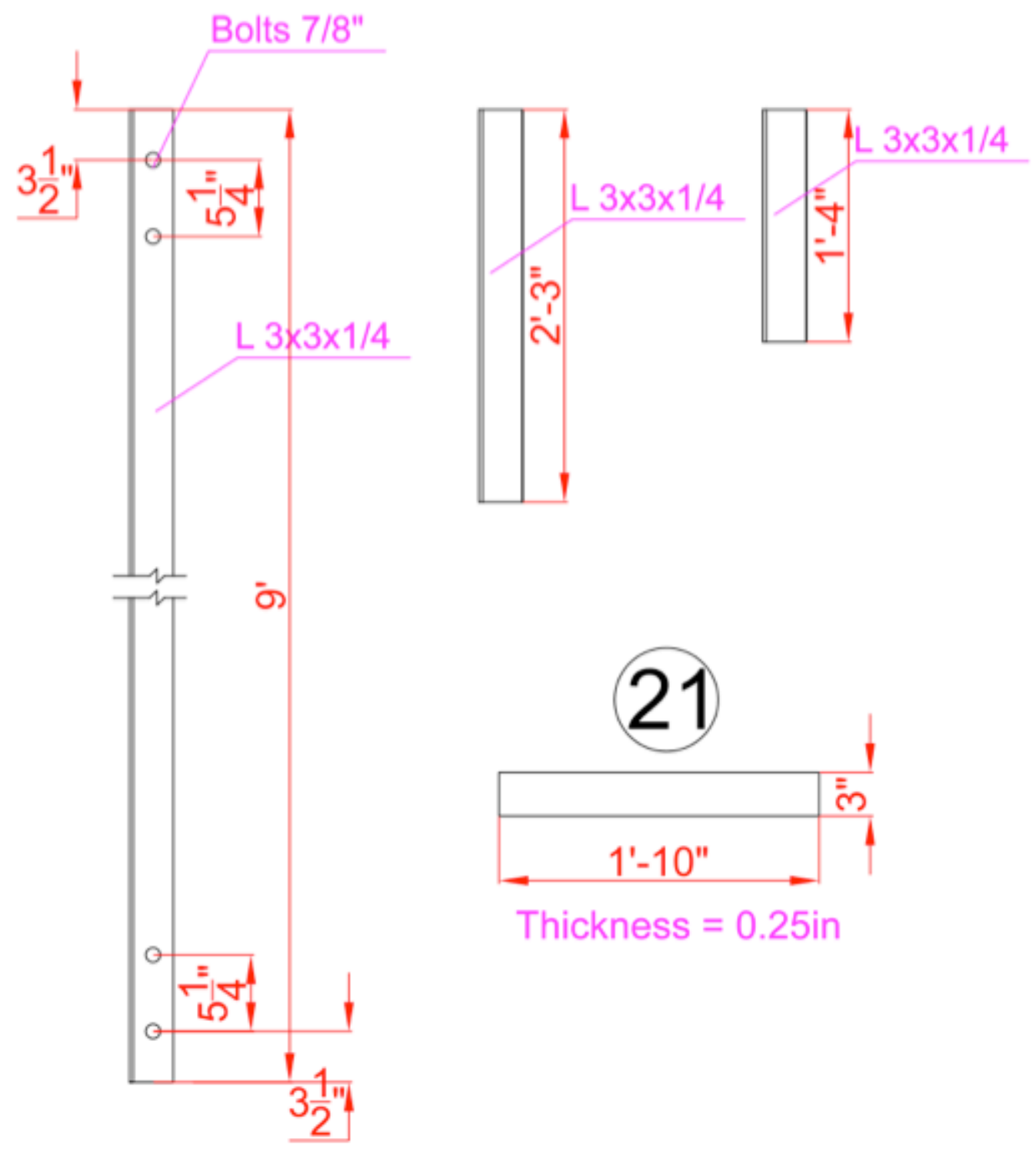

Figure 121. Upper part bracing - Elements 18-21 


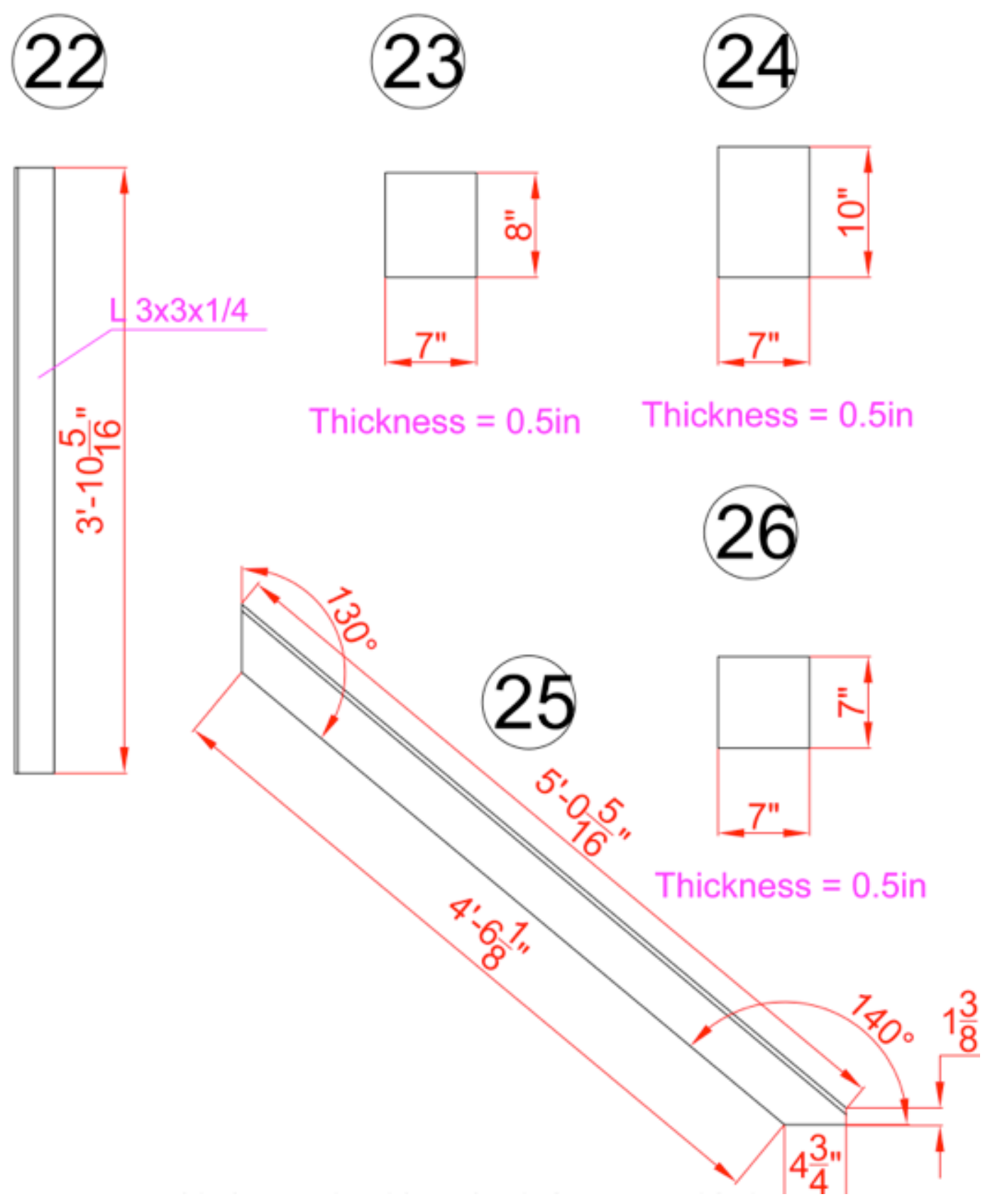

Figure 122. Lower part bracing - Elements 22-25 


\section{APPENDIX C: Material tests}

Table 22. 1/2" Thick Plate - Test Number 2 Measurements

\begin{tabular}{|c|c|c|}
\hline \multirow{2}{*}{$1 / 2$ " Test 2} & $\begin{array}{c}\text { Initial at two } \\
\text { locations }\end{array}$ & $\begin{array}{c}\text { Final at } \\
\text { necking }\end{array}$ \\
\hline \multirow{2}{*}{ Width } & 1.500 & \multirow{2}{*}{1.03} \\
\cline { 2 - 2 } & 1.500 & \\
\hline \multirow{2}{*}{ Thickness } & 0.500 & \multirow{2}{*}{0.224} \\
\cline { 2 - 2 } & 0.500 & \\
\hline
\end{tabular}

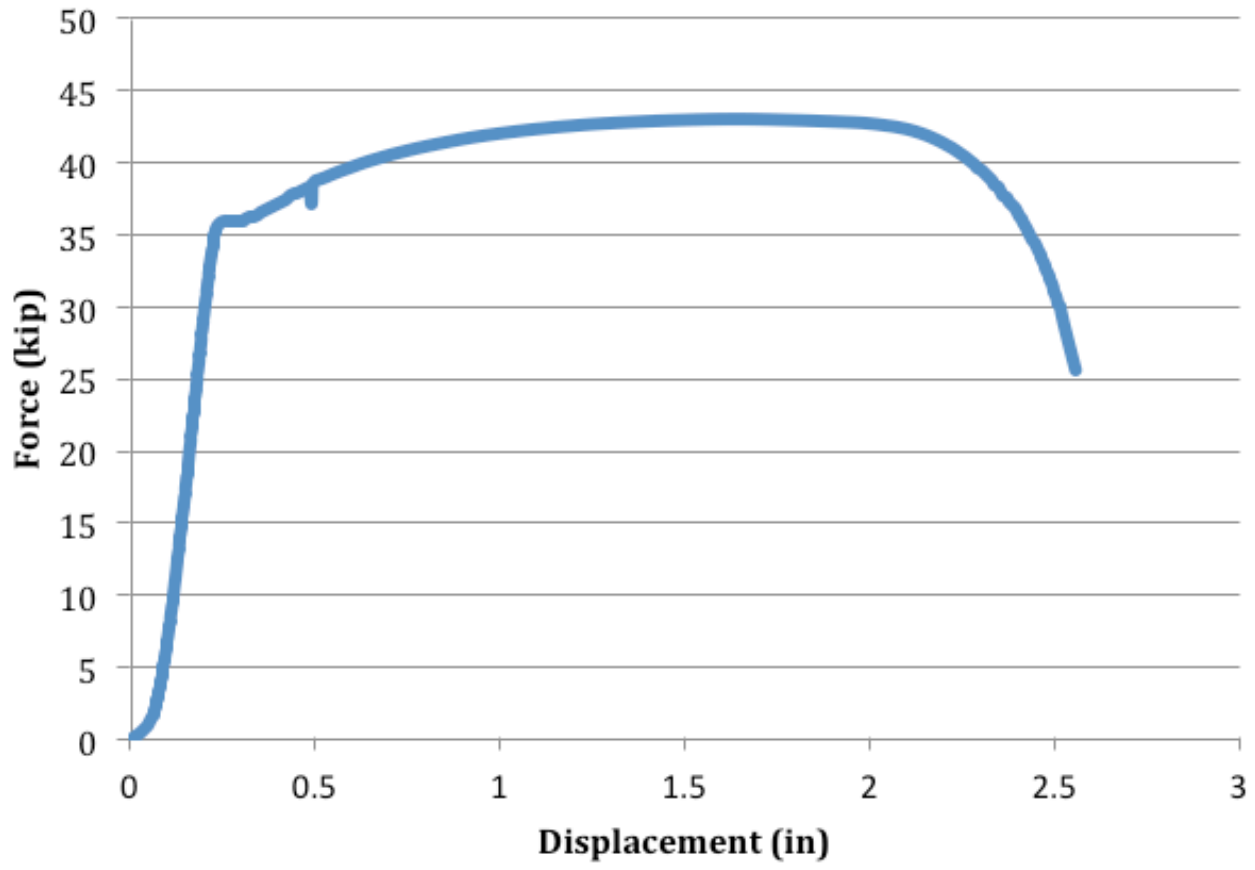

Figure 123. 1/2" Thick Plate - Test Number 2 Load - Displacement diagram 


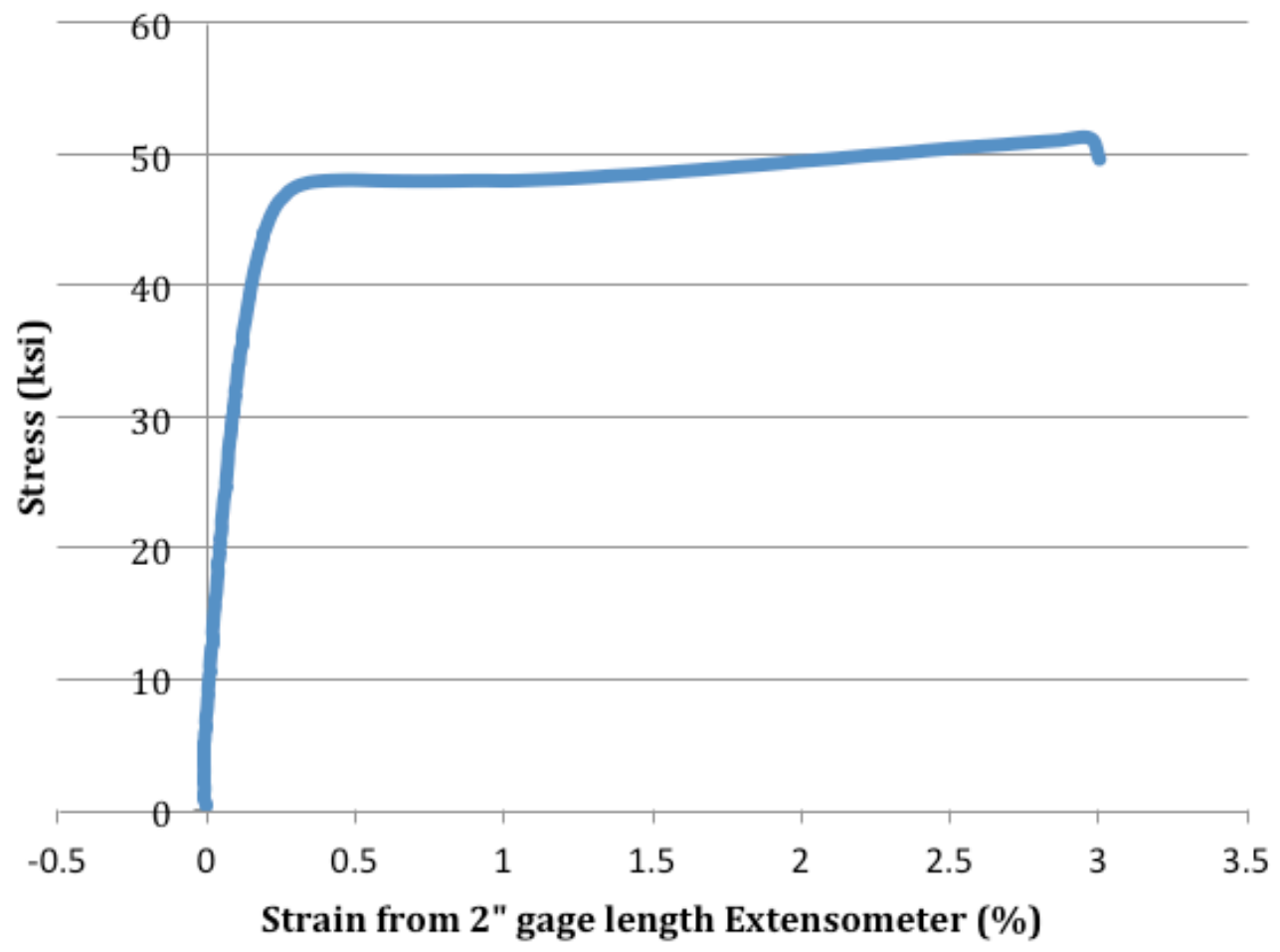

Figure 124. 1/2" Thick Plate - Test Number 2 Stress - Strain diagram using 2" Extensometer

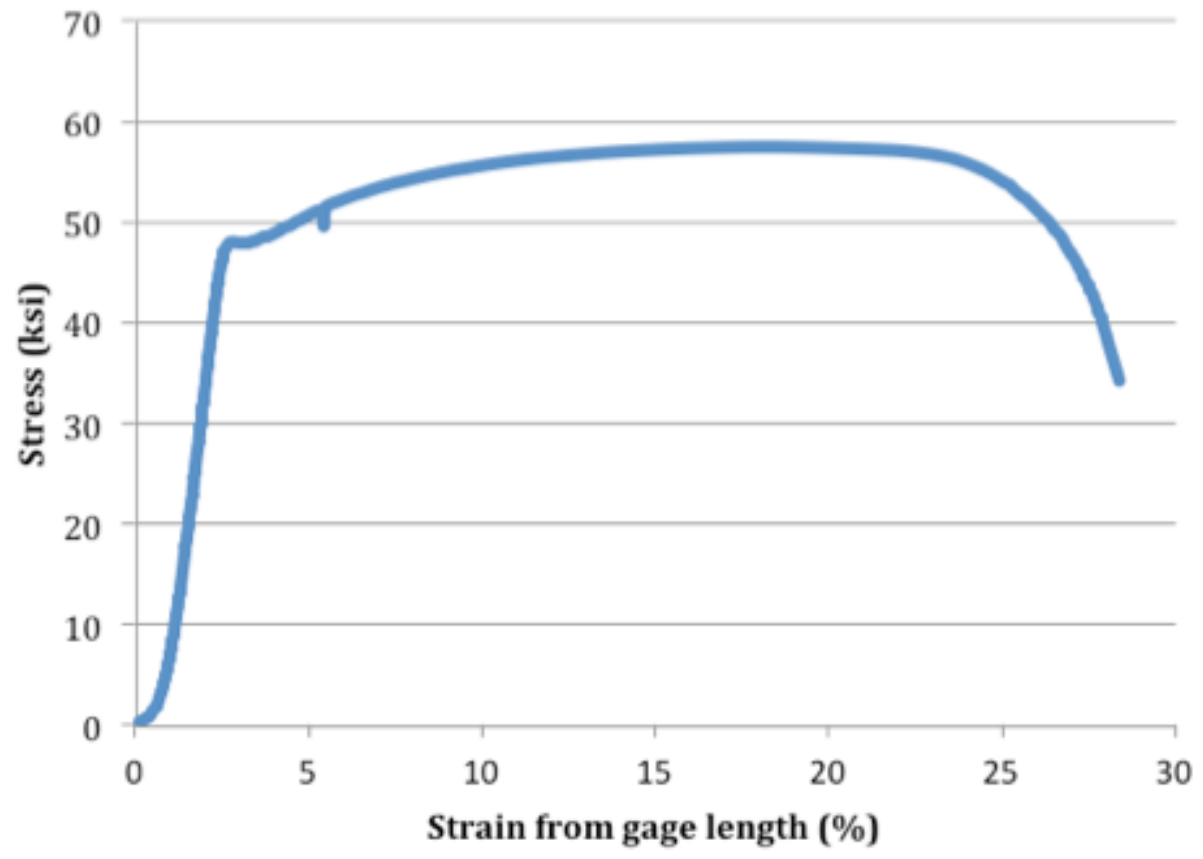

Figure 125. 1/2" Thick Plate - Test Number 2 Stress - Strain diagram using full gage length 
Table 23. 1/2" Thick Plate - Test Number 3 Measurements

\begin{tabular}{|c|c|c|}
\hline \multirow{2}{*}{$1 / 2$ " Test 3 } & $\begin{array}{c}\text { Initial at two } \\
\text { locations }\end{array}$ & $\begin{array}{c}\text { Final at } \\
\text { necking }\end{array}$ \\
\hline \multirow{2}{*}{ Width } & 1.500 & \multirow{2}{*}{1.026} \\
\cline { 2 - 2 } Thickness & 1.500 & \\
\cline { 2 - 2 } & 0.500 & \multirow{2}{*}{0.249} \\
\hline
\end{tabular}

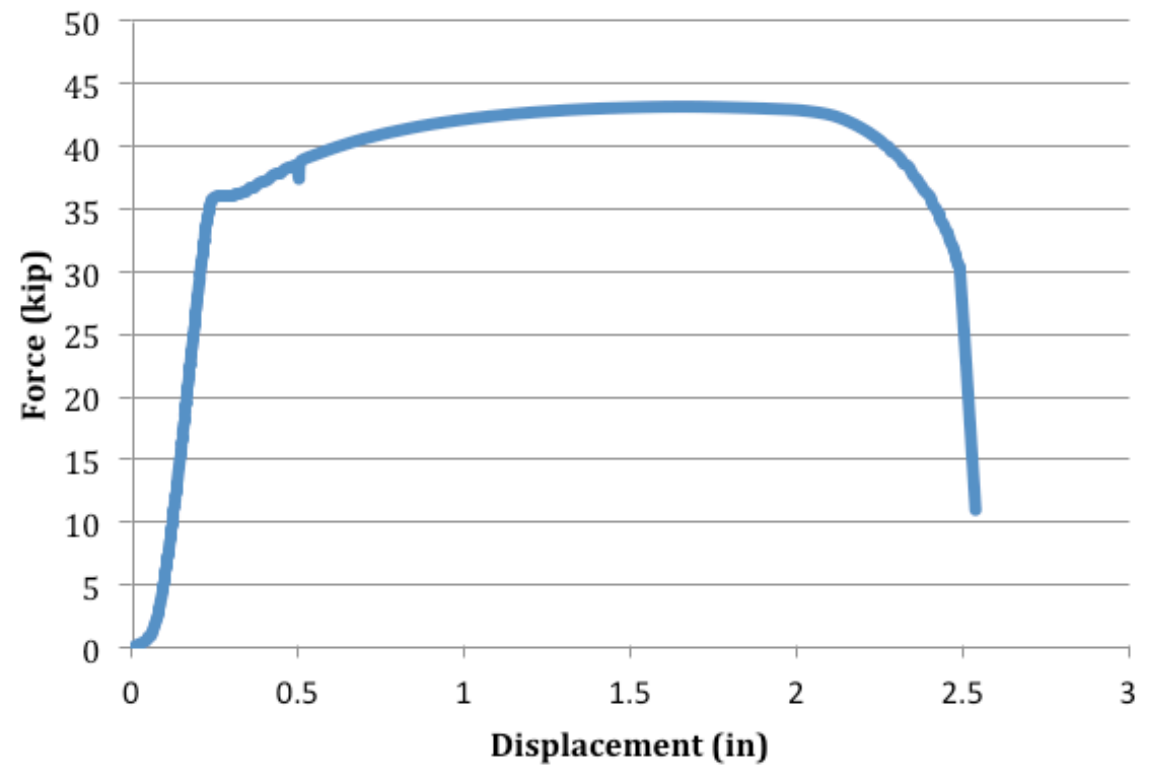

Figure 126. 1/2" Thick Plate - Test Number 3 Load - Displacement diagram 


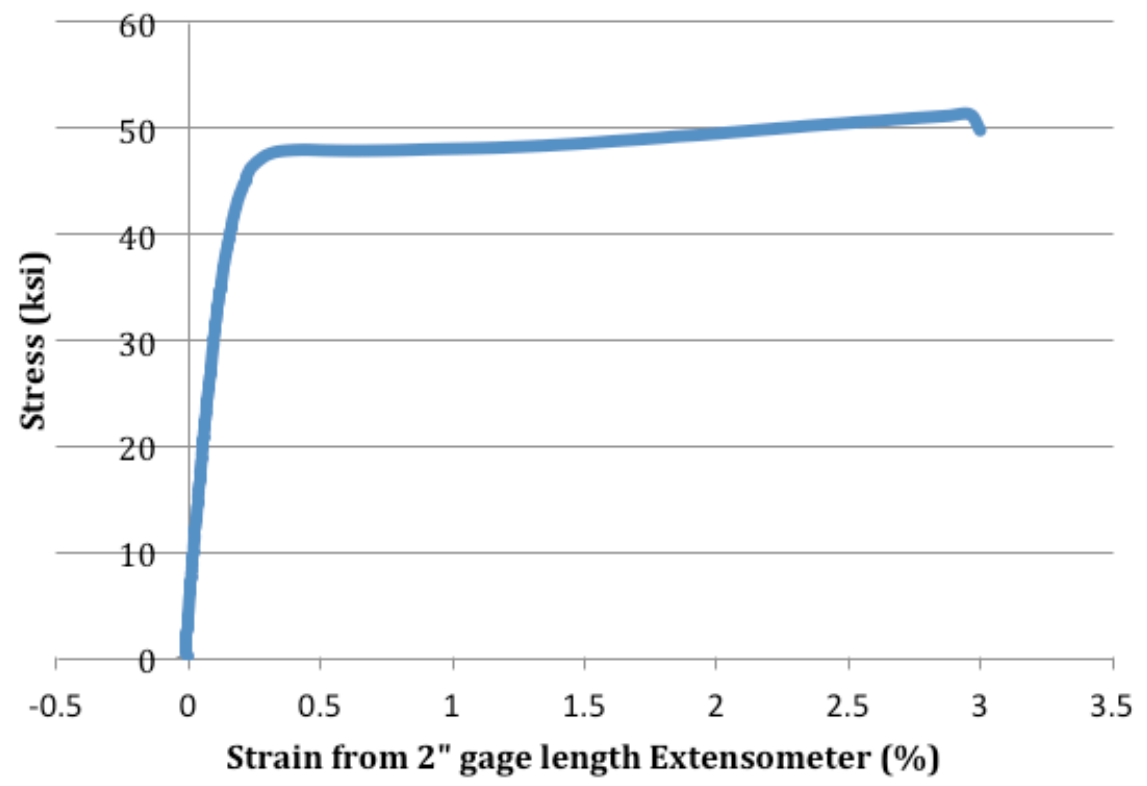

Figure 127. 1/2" Thick Plate - Test Number 3 Stress - Strain diagram using 2" Extensometer

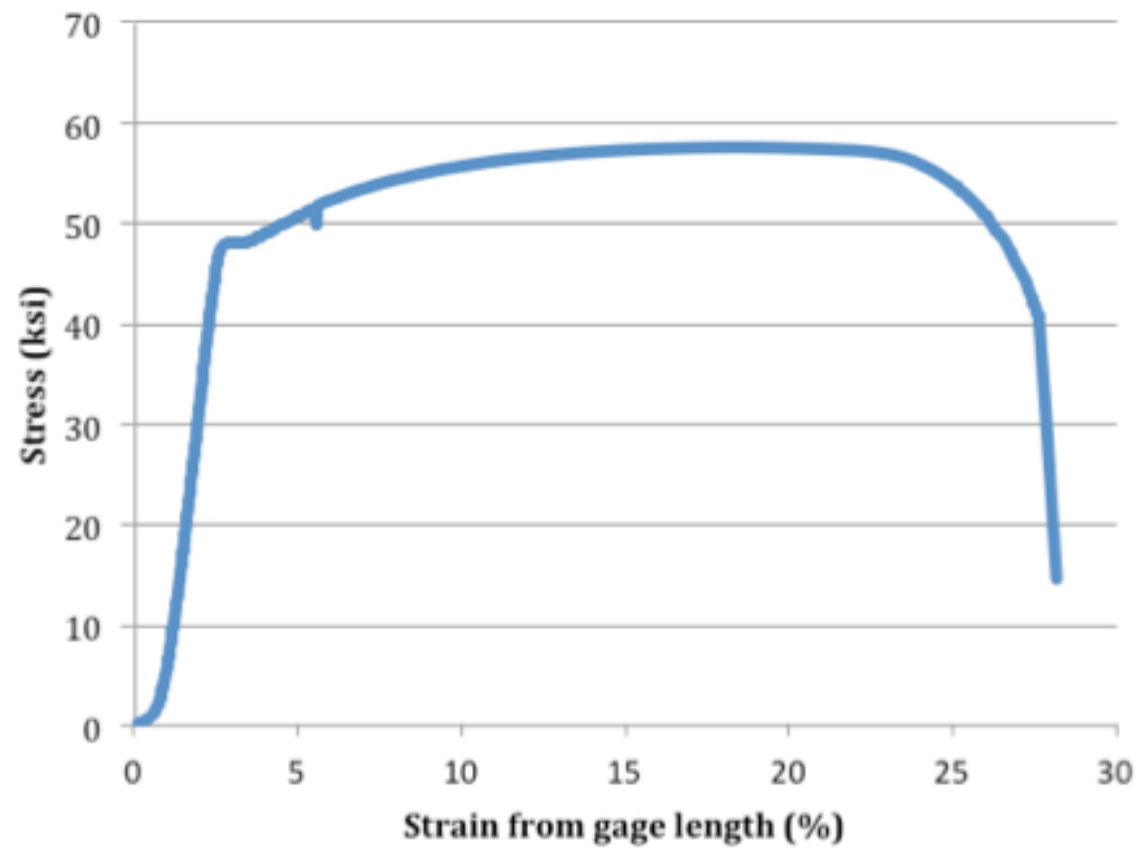

Figure 128. 1/2" Thick Plate - Test Number 3 Stress - Strain diagram using full gage length 
Table 24. 0.075" Thick Plate - Test Number 1 Measurements

\begin{tabular}{|c|c|c|}
\hline 0.08 " Test 1 & Initial, in & Final, in \\
\hline \multirow{2}{*}{ Width } & 1.503 & \multirow{2}{*}{$\begin{array}{c}1.242 \text { (at } \\
\text { necking) }\end{array}$} \\
\cline { 2 - 2 } & 1.501 & \multirow{2}{*}{0.051 (at } \\
nhickness & 0.074 & \\
\cline { 2 - 2 } & 0.075 & \multirow{2}{*}{14.0625} \\
\cline { 2 - 2 } & 0.08 & \\
\hline $\begin{array}{c}\text { Length } \\
\text { between } \\
\text { punch } \\
\text { marks, in }\end{array}$ & 11.82 & \\
\hline $\begin{array}{c}\text { Specimen } \\
\text { elongation, } \\
\text { in }\end{array}$ & 2.2425 & \\
\hline
\end{tabular}

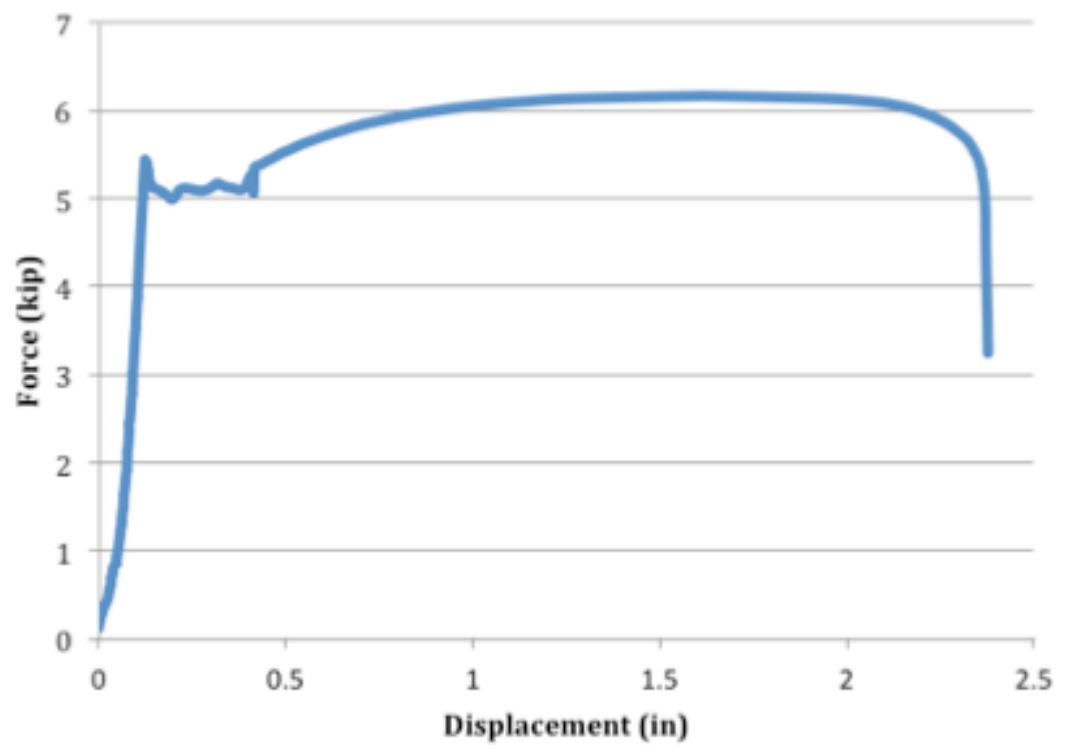

Figure 129. 0.075" Thick Plate - Test Number 1 Load - Displacement diagram 


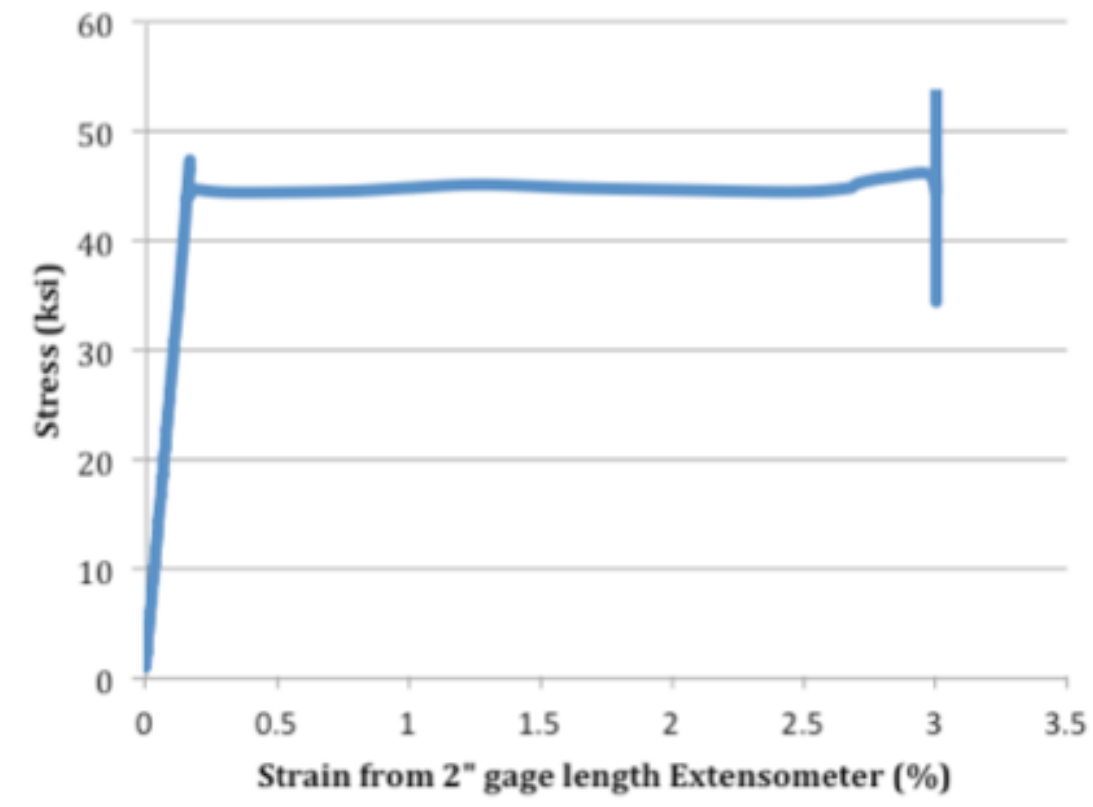

Figure 130. 0.075 " Thick Plate - Test Number 1 Stress - Strain diagram using 2" Extensometer

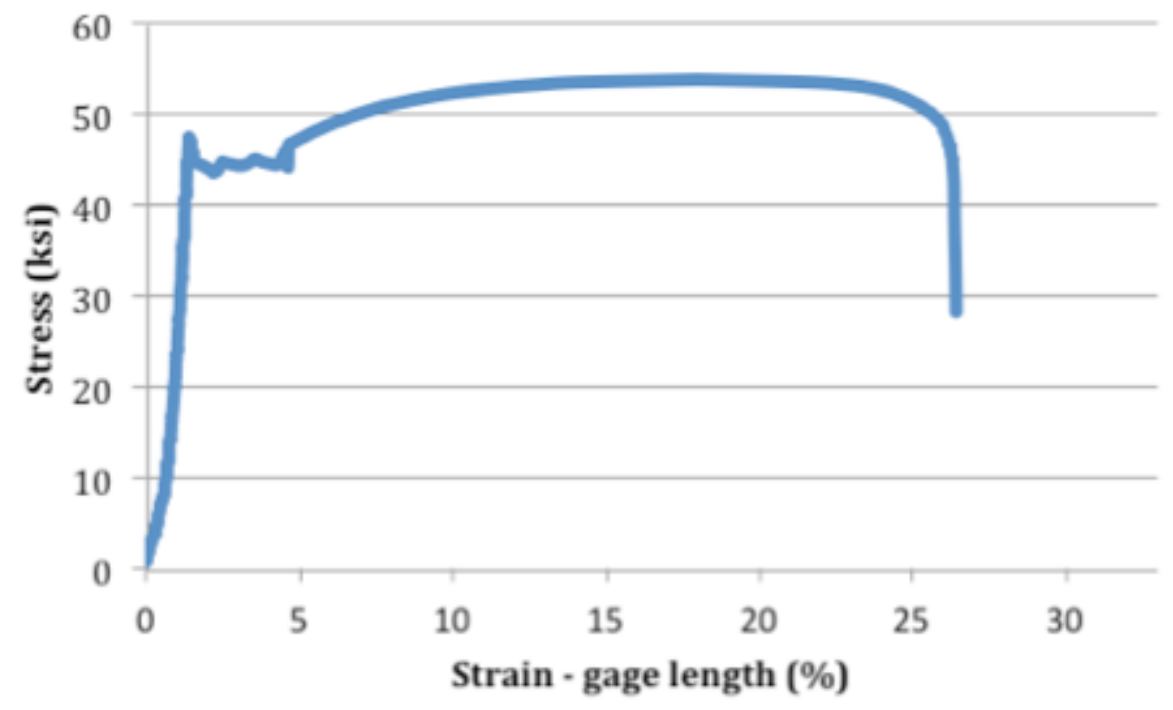

Figure 131. 0.075" Thick Plate - Test Number 1 Stress - Strain diagram using full gage length 
Table 25. 0.075" Thick Plate - Test Number 2 Measurements

\begin{tabular}{|c|c|c|}
\hline 0.08 " Test 2 & Initial, in & Final, in \\
\hline \multirow{2}{*}{ Width } & 1.502 & \multirow{2}{*}{$\begin{array}{c}1.235 \text { (at } \\
\text { necking) }\end{array}$} \\
\cline { 2 - 2 } & 1.516 & \multirow{2}{*}{$\begin{array}{c}0.055 \text { (at } \\
\text { necking) }\end{array}$} \\
\hline \multirow{2}{*}{ Thickness } & 1.515 & \\
\cline { 2 - 2 } & 0.075 & 14.3906 \\
\hline $\begin{array}{c}\text { Length } \\
\text { between } \\
\text { punch } \\
\text { marks, in }\end{array}$ & 0.082 & \\
\hline $\begin{array}{c}\text { Specimen } \\
\text { elongation, } \\
\text { in }\end{array}$ & 11.899 & \\
\hline
\end{tabular}

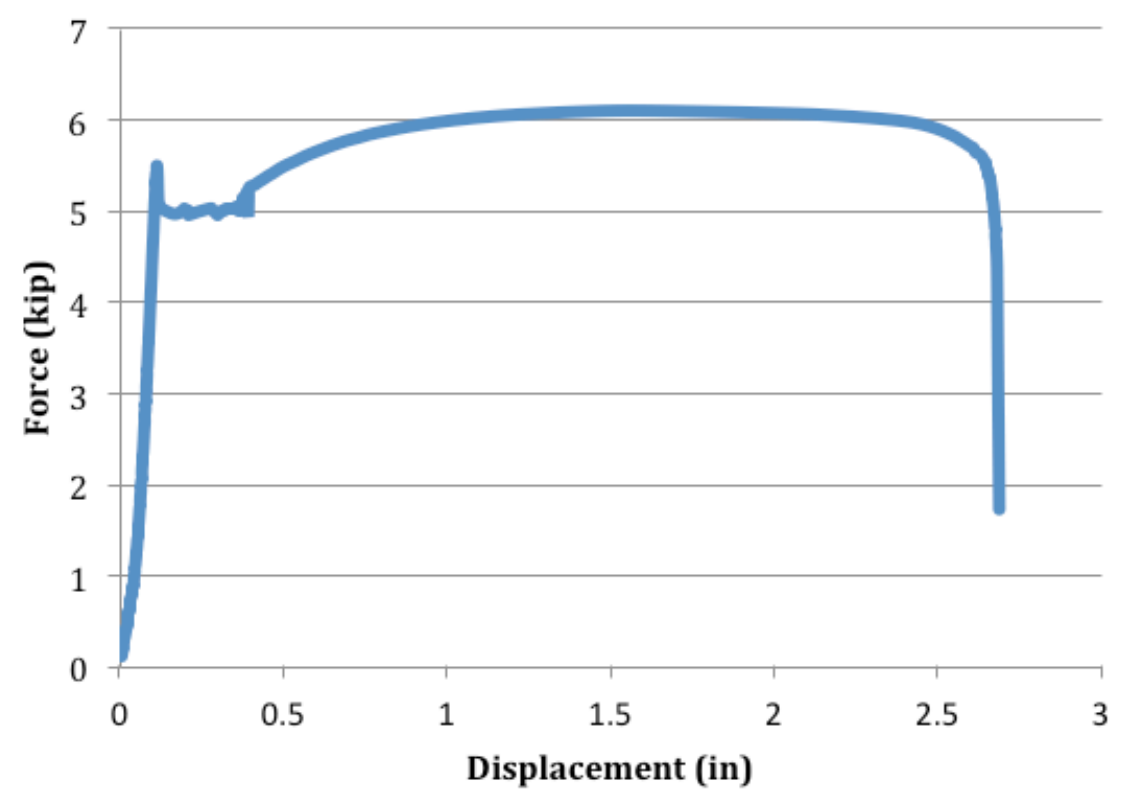

Figure 132. 0.075" Thick Plate - Test Number 2 Load - Displacement diagram 


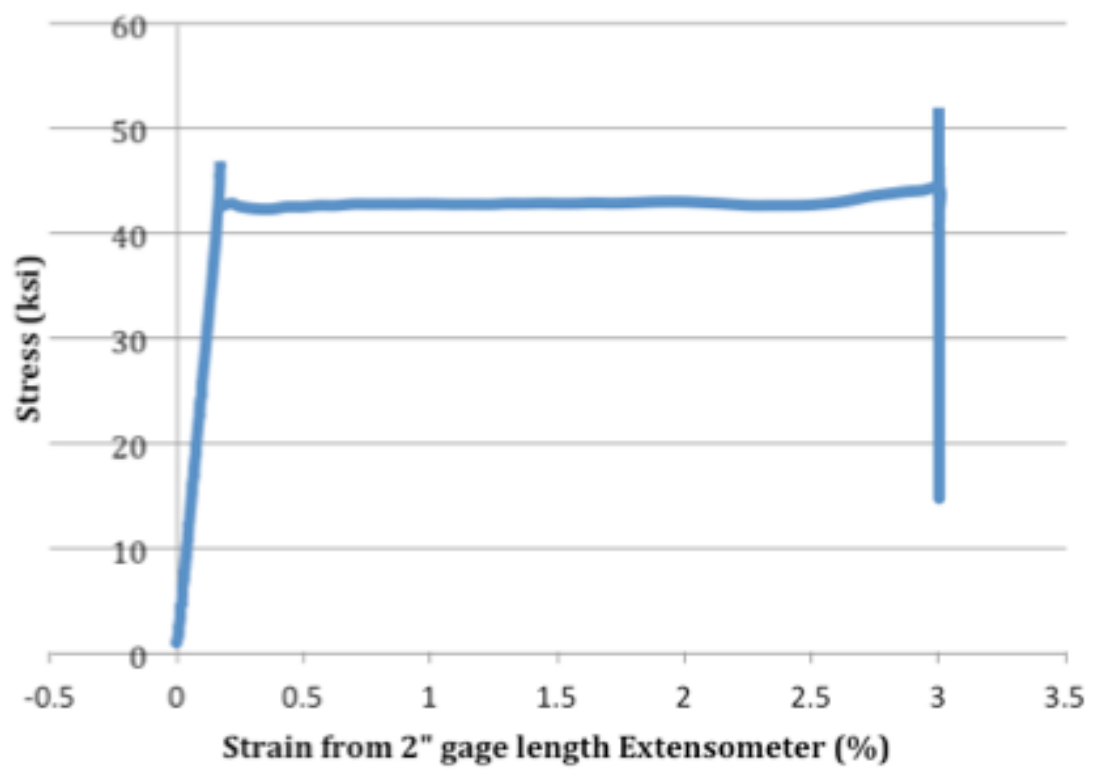

Figure 133. 0.075 " Thick Plate - Test Number 2 Stress - Strain diagram using 2" Extensometer

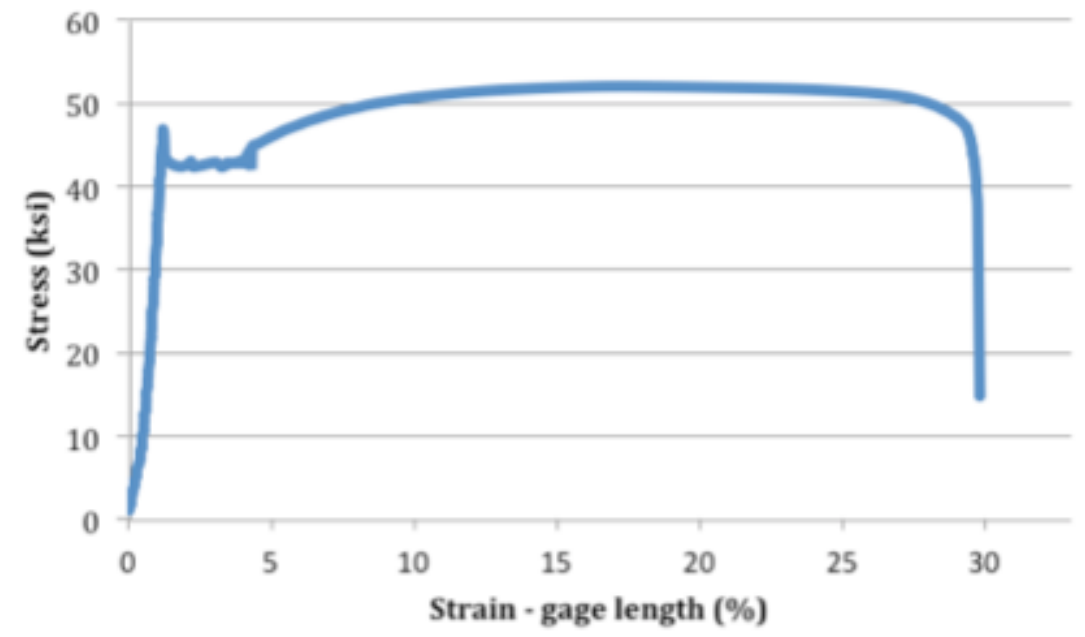

Figure 134. 0.075" Thick Plate - Test Number 2 Stress - Strain diagram using full gage length 
Table 26. 0.075" Thick Plate - Test Number 3 Measurements

\begin{tabular}{|c|c|c|}
\hline 0.08 " Test 3 & Initial, in & Final, in \\
\hline \multirow{2}{*}{ Width } & 1.502 & \multirow{2}{*}{$\begin{array}{c}1.111 \text { (at } \\
\text { necking) }\end{array}$} \\
\cline { 2 - 2 } Thickness & 1.501 & \multirow{2}{*}{$\begin{array}{c}0.053 \text { (at } \\
\text { necking) }\end{array}$} \\
\cline { 2 - 2 } & 0.075 & \\
\cline { 2 - 2 } & 0.073 & \multirow{2}{*}{14.9375} \\
\hline $\begin{array}{c}\text { Length } \\
\text { between } \\
\text { punch } \\
\text { marks, in }\end{array}$ & 11.777 & \\
\hline $\begin{array}{c}\text { Specimen } \\
\text { elongation, } \\
\text { in }\end{array}$ & 3.1605 & \\
\hline
\end{tabular}

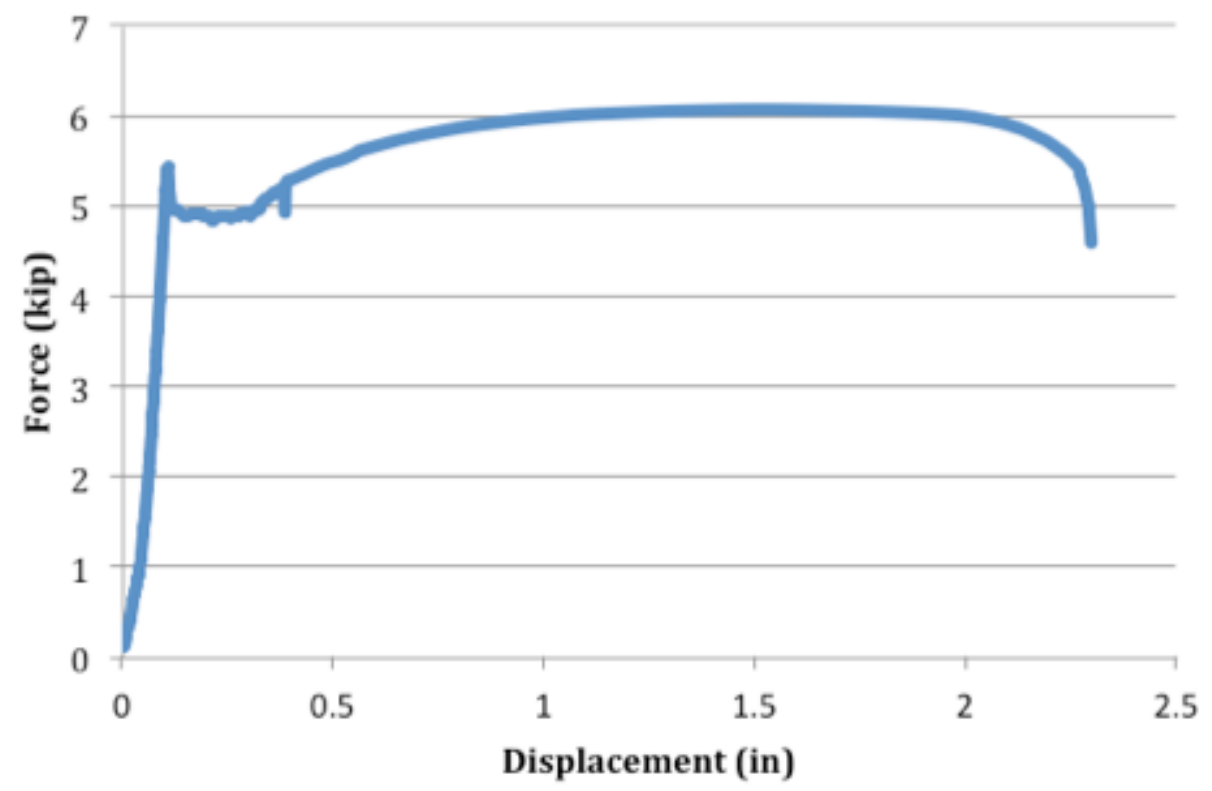

Figure 135. 0.075" Thick Plate - Test Number 3 Load - Displacement diagram 


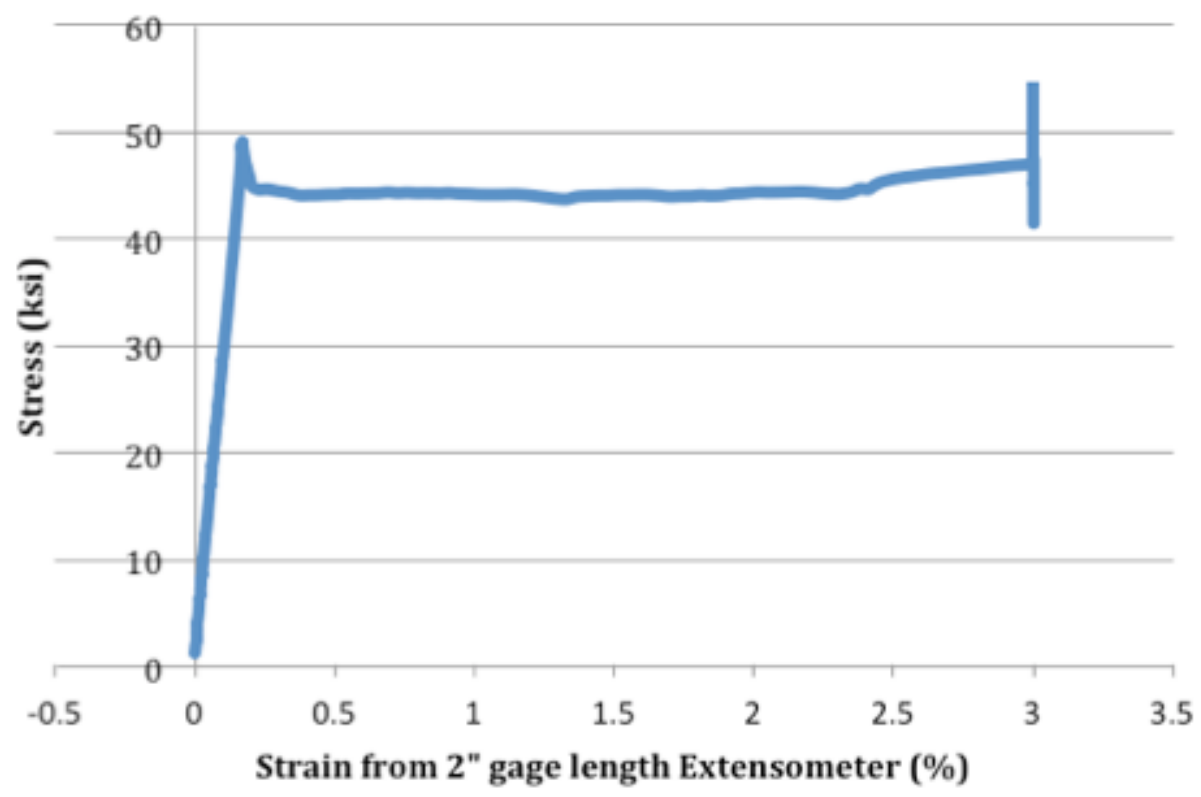

Figure 136. 0.075 " Thick Plate - Test Number 3 Stress - Strain diagram using 2" Extensometer

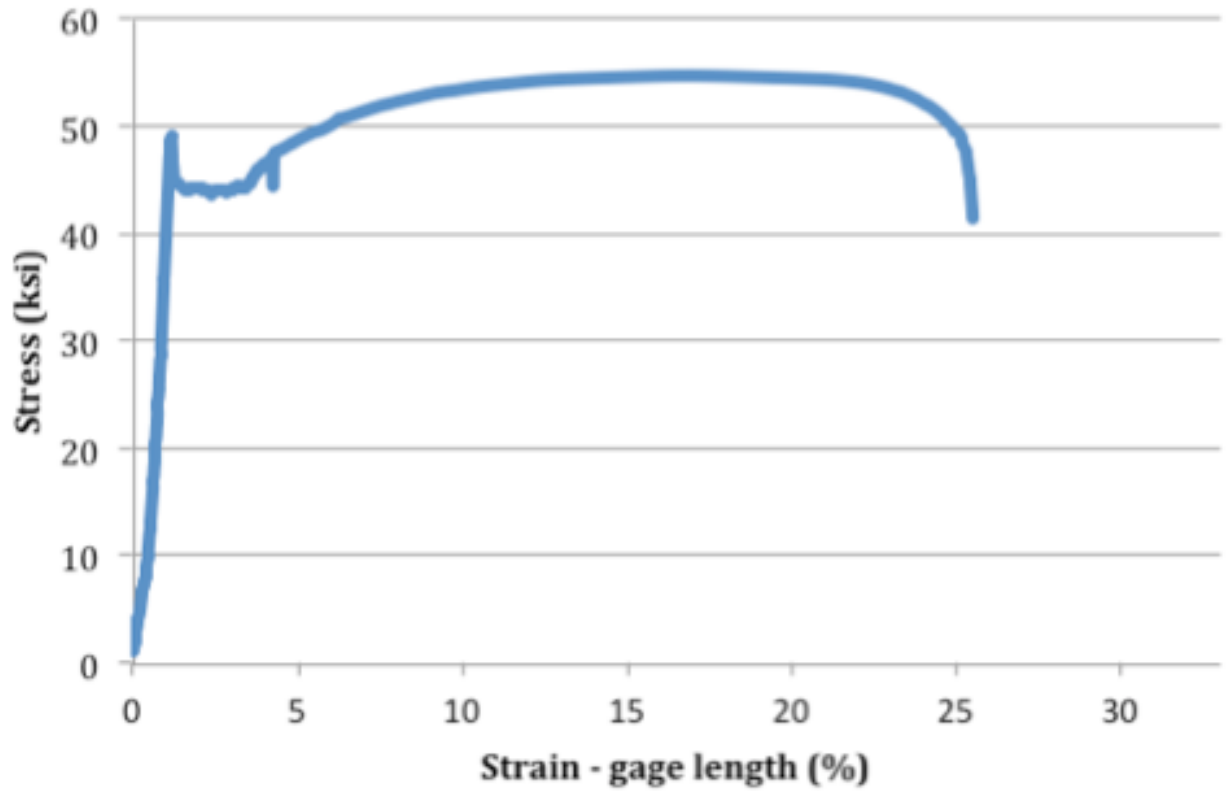

Figure 137. 0.075" Thick Plate - Test Number 3 Stress - Strain diagram using full gage length 
Table 27. 0.25" Thick Plate - Test Number 1 Measurements

\begin{tabular}{|c|c|c|}
\hline $\begin{array}{c}0.25 \text { " Test } \\
1\end{array}$ & Initial, in & Final, in \\
\hline \multirow{3}{*}{ Width } & 1.495 & \multirow{3}{*}{1.11 (at necking) } \\
\hline & 1.5 & \\
\hline & 1.495 & \\
\hline \multirow{3}{*}{ Thickness } & 0.255 & \multirow{3}{*}{0.175 (at necking) } \\
\hline & 0.251 & \\
\hline & 0.253 & \\
\hline $\begin{array}{l}\text { Length } \\
\text { between } \\
\text { punch } \\
\text { marks, in }\end{array}$ & 6.78 & 8.57 \\
\hline $\begin{array}{l}\text { Specimen } \\
\text { elongation, } \\
\text { in }\end{array}$ & 1.79 & \\
\hline
\end{tabular}

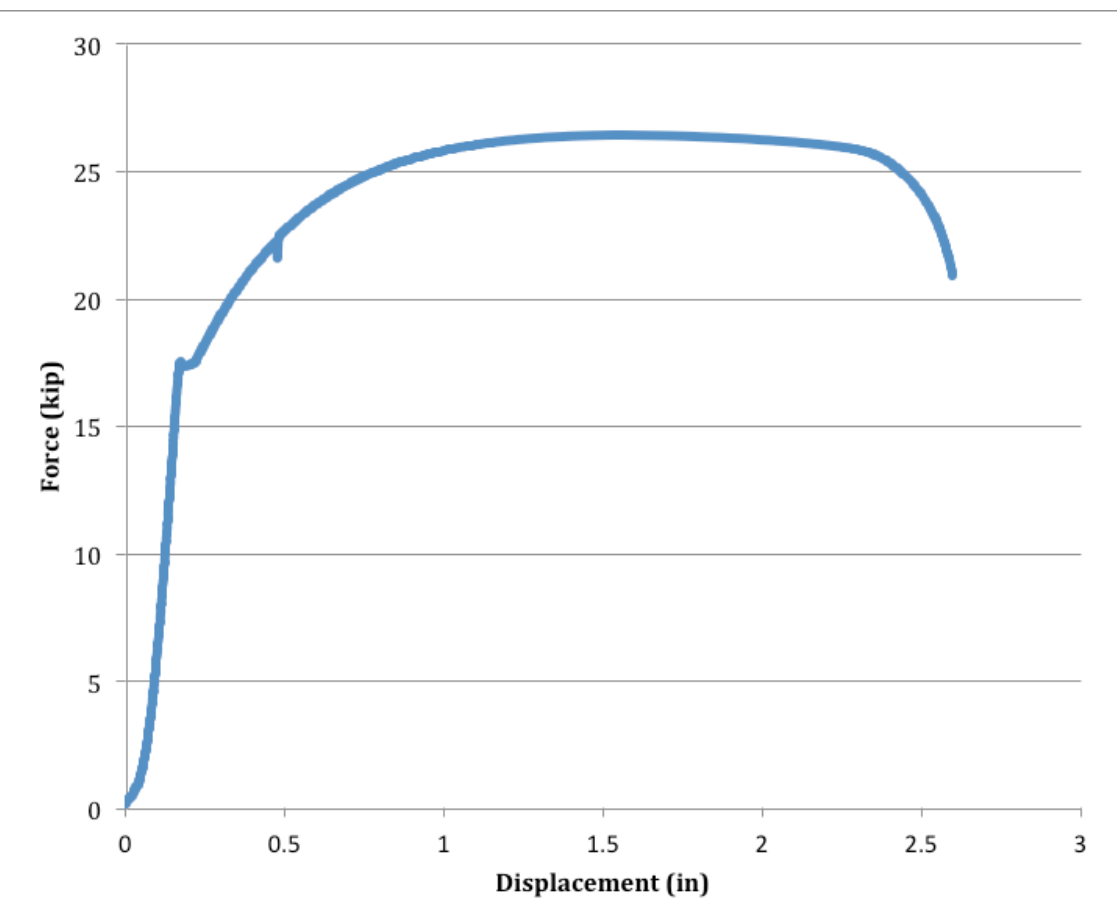

Figure 138. 0.25" Thick Plate - Test Number 1 Load - Displacement diagram 


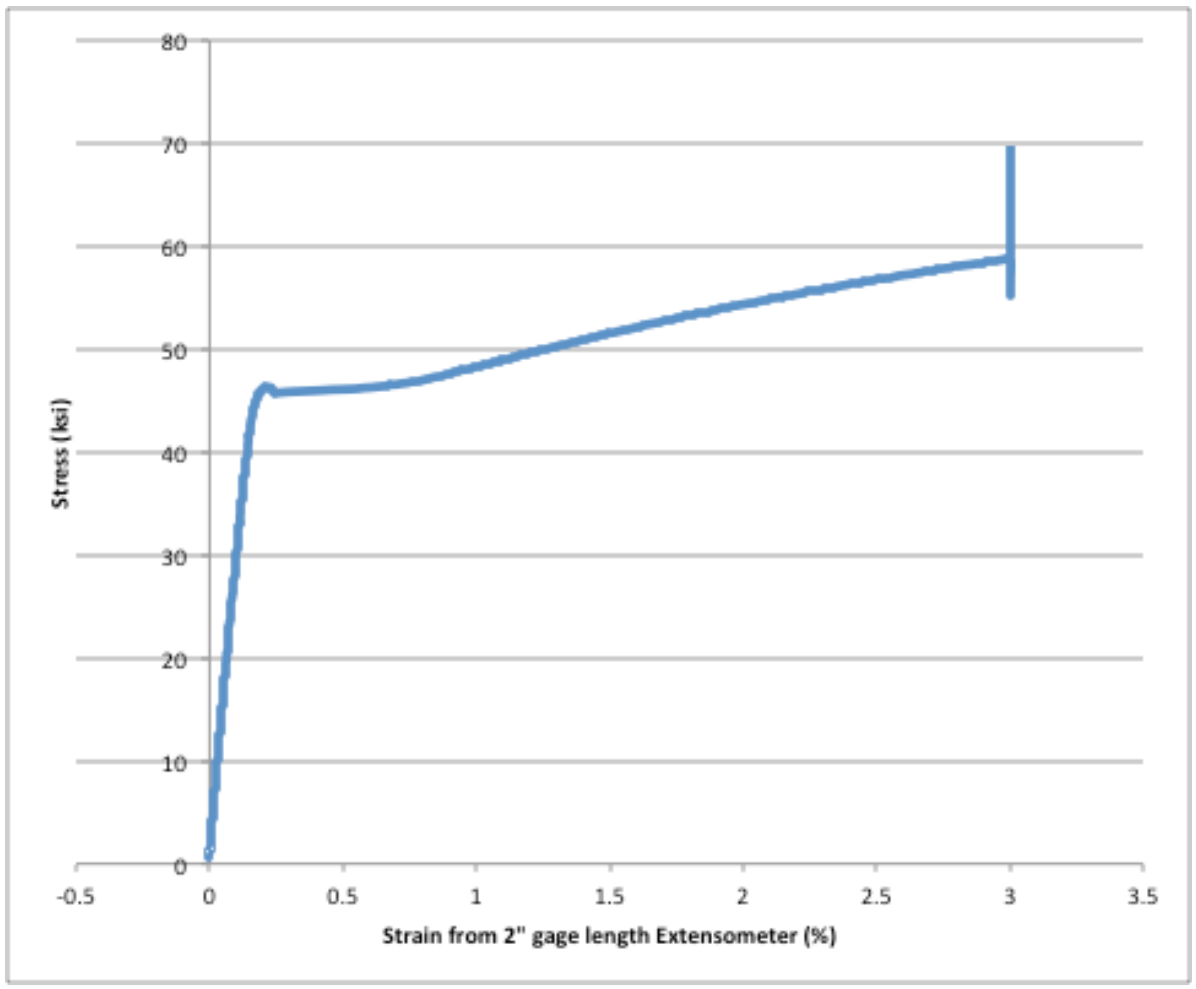

Figure 139. 0.25" Thick Plate - Test Number 1 Stress - Strain diagram using 2" Extensometer

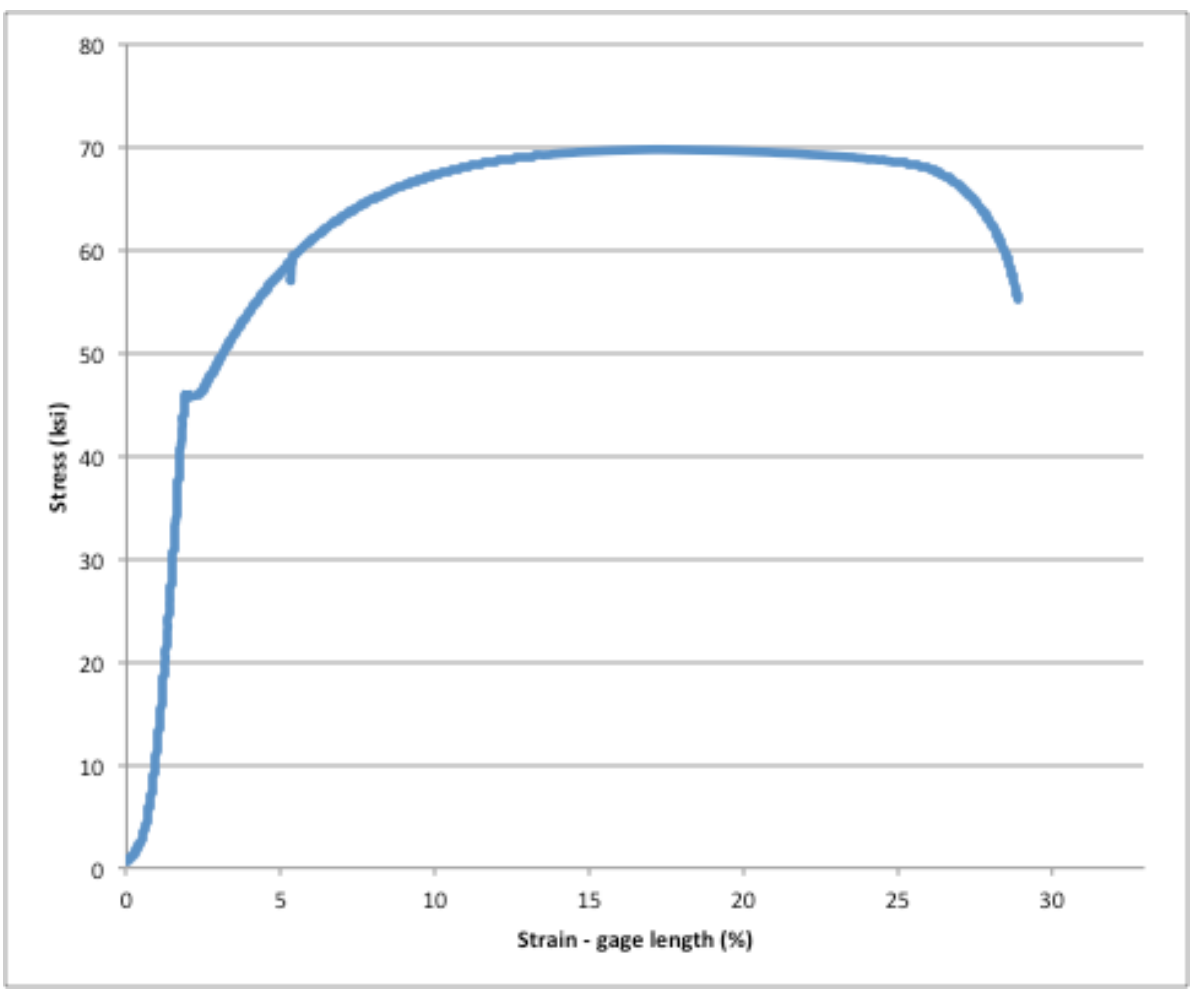

Figure 140. 0.25" Thick Plate - Test Number 1 Stress - Strain diagram using full gage length 
Table 28. 0.25" Thick Plate - Test Number 2 Measurements

\begin{tabular}{|c|c|c|}
\hline $\begin{array}{c}0.25 \text { " Test } \\
2\end{array}$ & Initial, in & Final, in \\
\hline \multirow{2}{*}{ Width } & 1.498 & \multirow{2}{*}{1.1 (at necking) } \\
\cline { 2 - 2 } & 1.495 & \multirow{2}{*}{0.185 (at necking) } \\
\cline { 2 - 2 } Thickness & 1.505 & \\
\cline { 2 - 2 } & 0.255 & \\
\cline { 2 - 2 } $\begin{array}{c}\text { Length } \\
\text { between } \\
\text { punch } \\
\text { marks, in }\end{array}$ & 0.25 & \\
\hline $\begin{array}{c}\text { Specimen } \\
\text { elongation, } \\
\text { in }\end{array}$ & 1.805 & \\
\hline
\end{tabular}

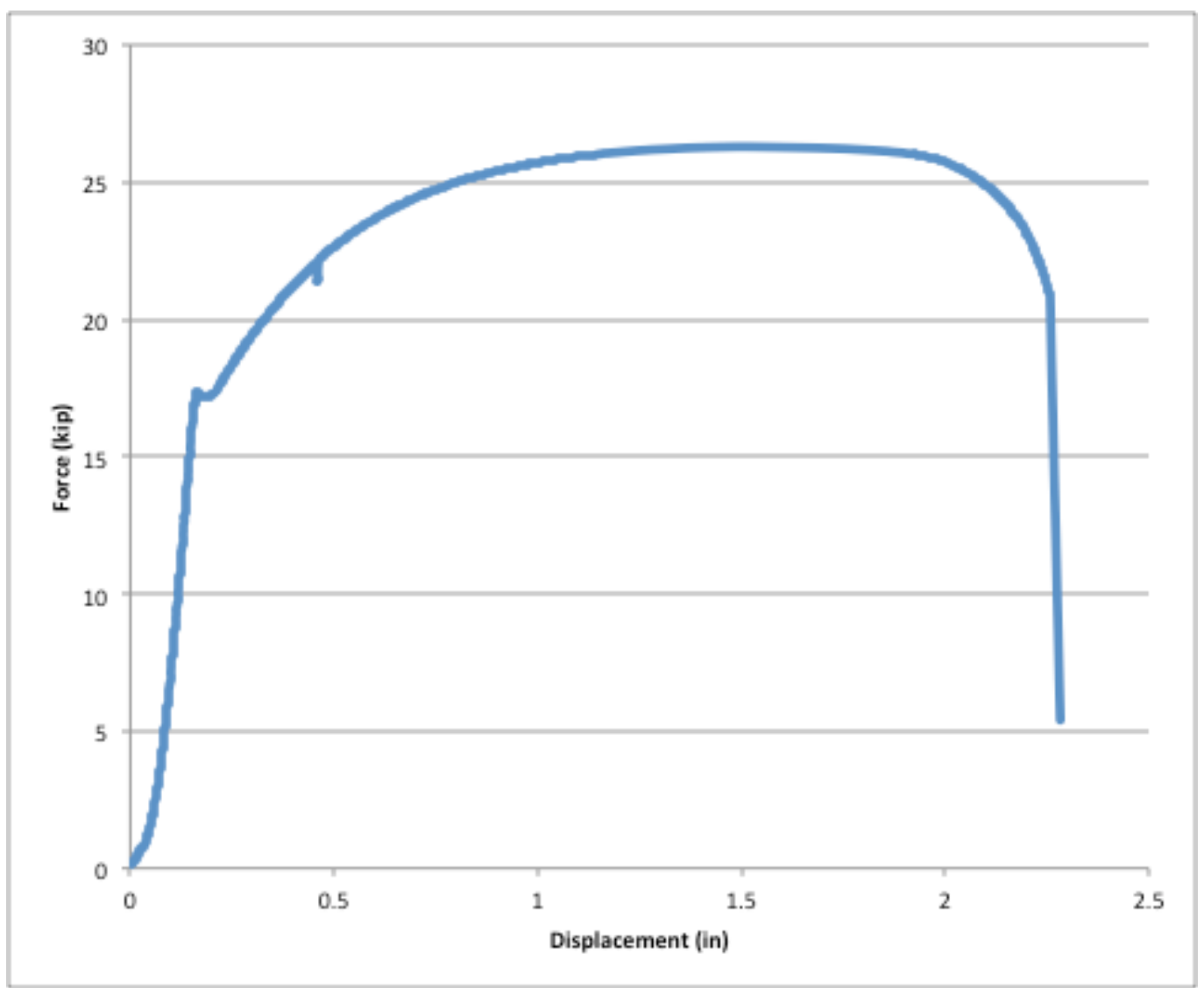

Figure 141. 0.25" Thick Plate - Test Number 2 Load - Displacement diagram 


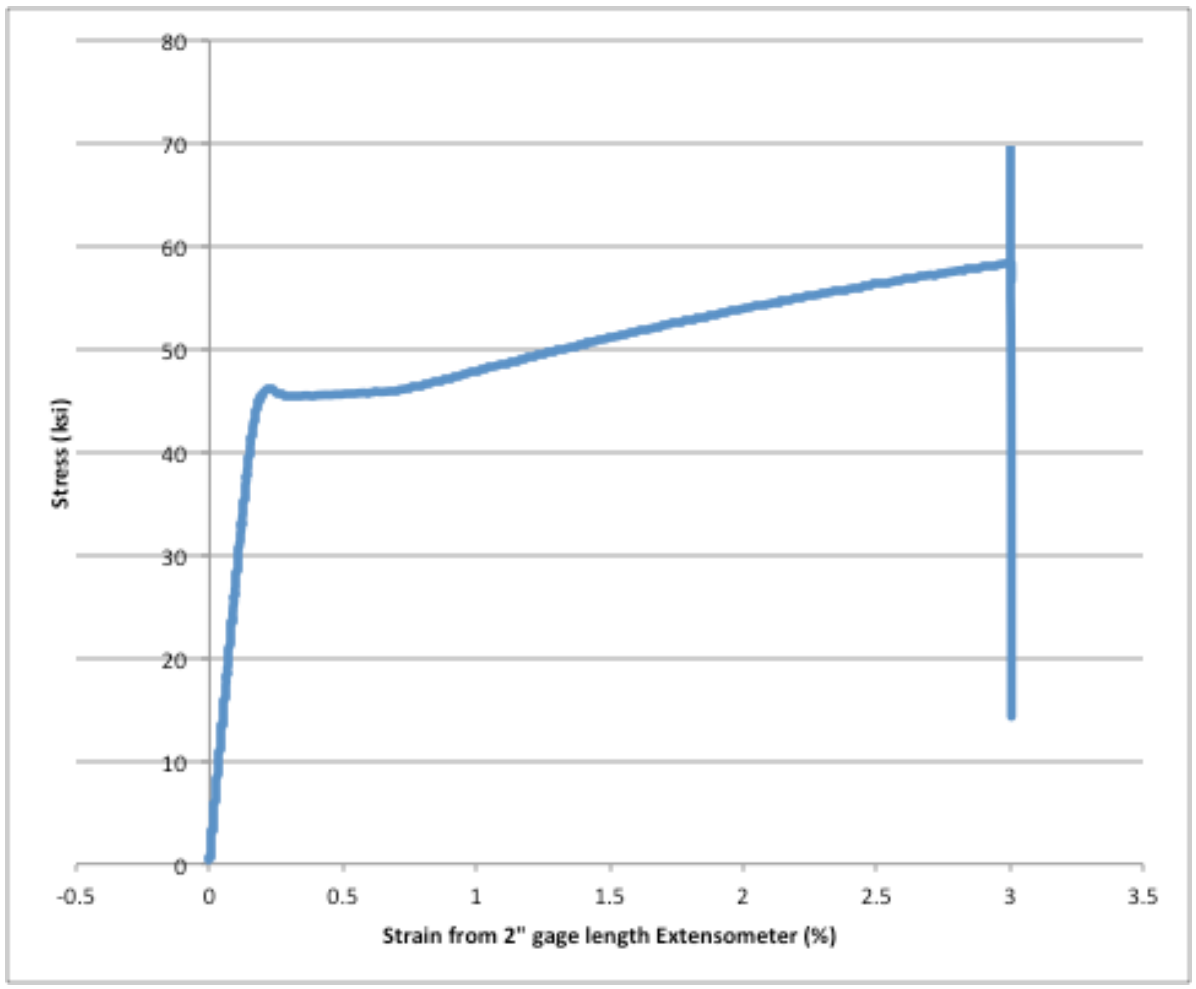

Figure 142. 0.25 " Thick Plate - Test Number 2 Stress - Strain diagram using 2" Extensometer

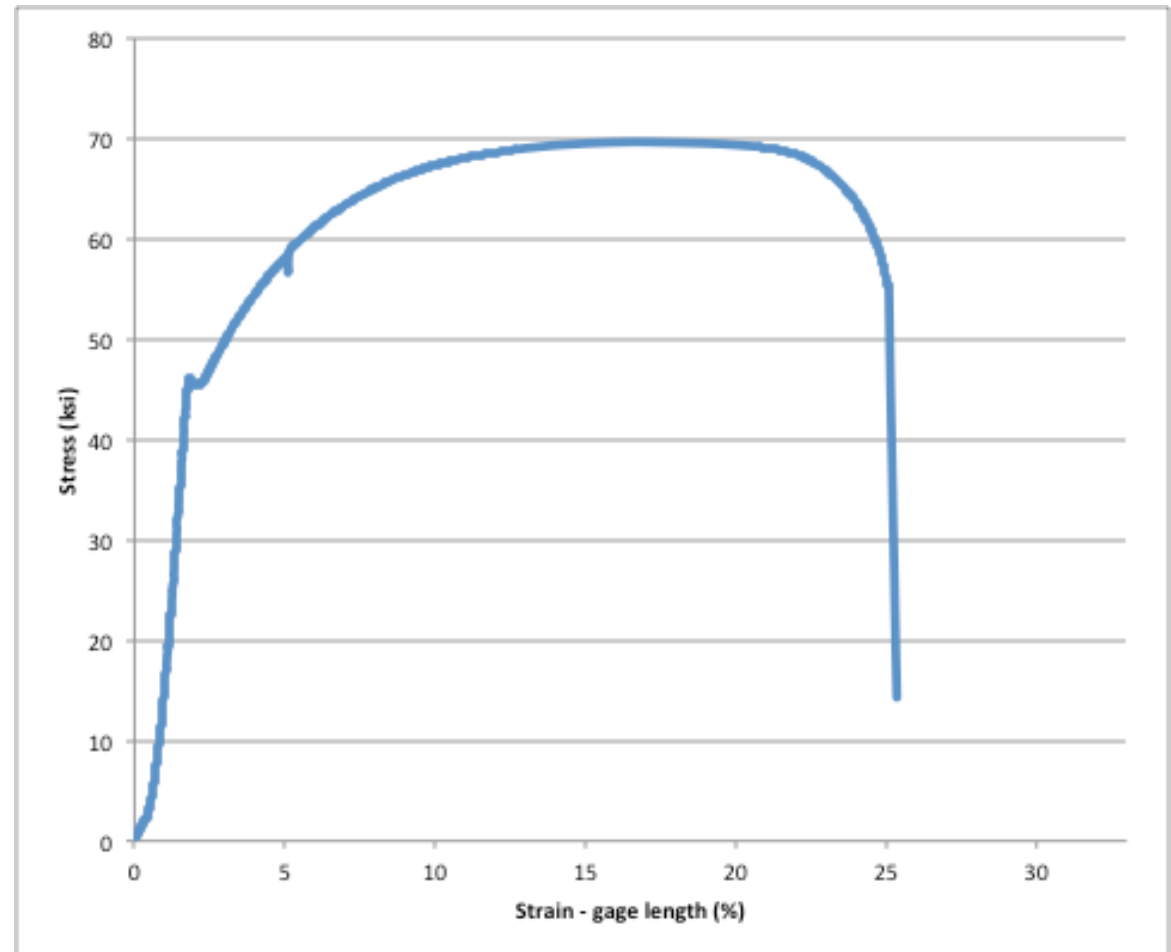

Figure 143. 0.25" Thick Plate - Test Number 2 Stress - Strain diagram using full gage length 
Table 29. 0.25" Thick Plate - Test Number 2 Measurements

\begin{tabular}{|c|c|c|}
\hline $\begin{array}{c}0.25 \text { " Test } \\
3\end{array}$ & Initial, in & Final, in \\
\hline \multirow{3}{*}{ Width } & 1.51 & \multirow{3}{*}{1.09 (at necking) } \\
\hline & 1.502 & \\
\hline & 1.55 & \\
\hline \multirow{3}{*}{ Thickness } & 0.255 & \multirow{3}{*}{0.17 (at necking) } \\
\hline & 0.245 & \\
\hline & 0.245 & \\
\hline $\begin{array}{l}\text { Length } \\
\text { between } \\
\text { punch } \\
\text { marks, in }\end{array}$ & 6.94 & 8.57 \\
\hline $\begin{array}{l}\text { Specimen } \\
\text { elongation, } \\
\text { in }\end{array}$ & 1.63 & \\
\hline
\end{tabular}

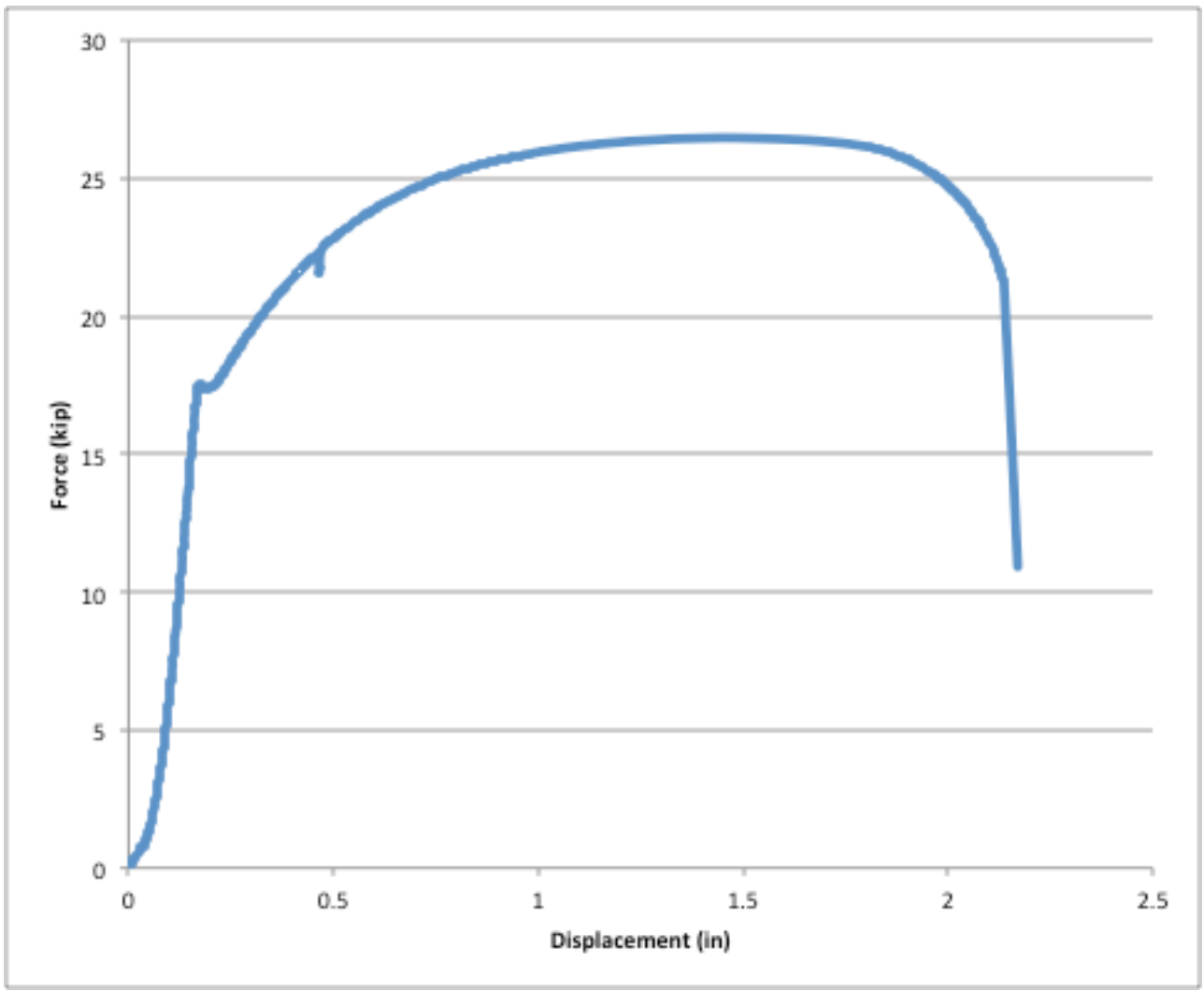

Figure 144. 0.25" Thick Plate - Test Number 3 Load - Displacement diagram 


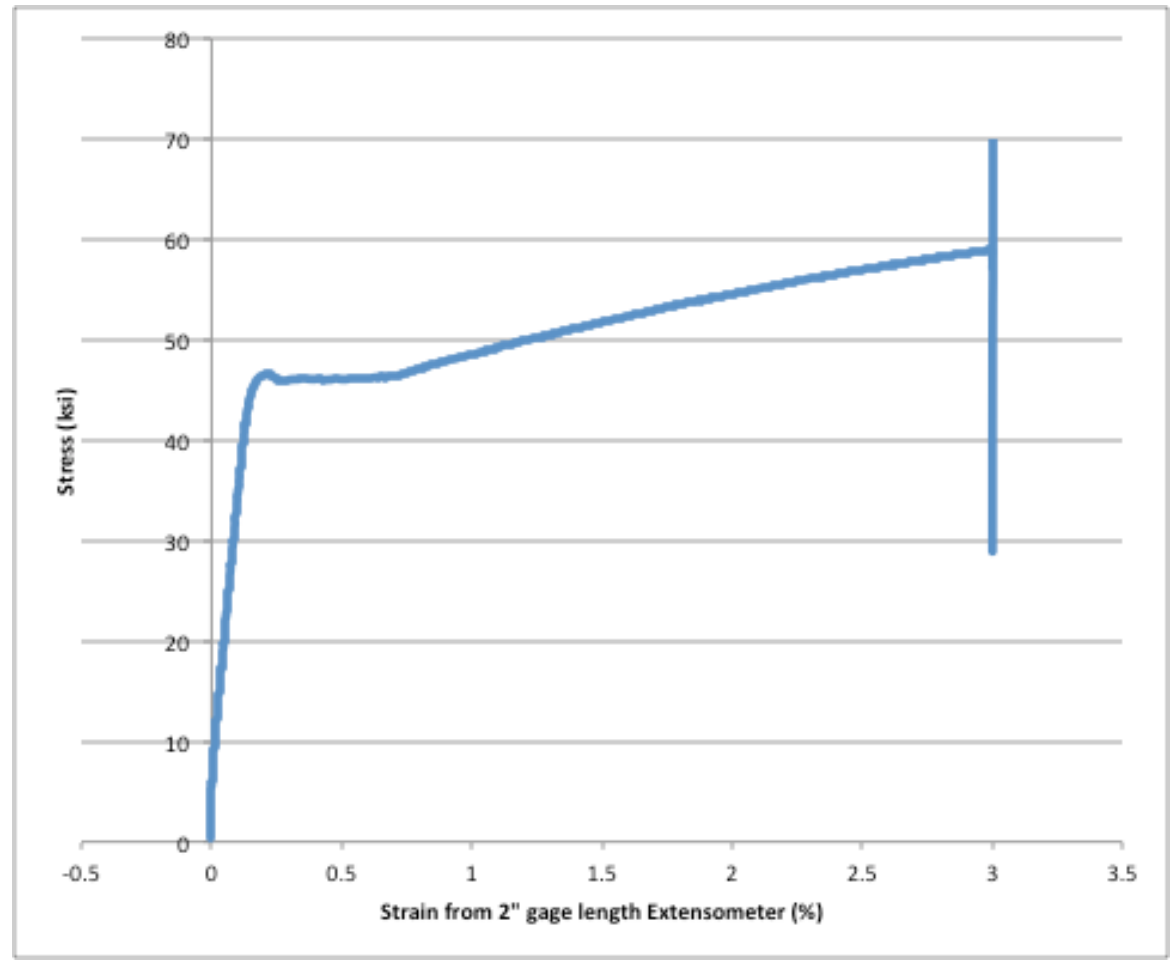

Figure 145. 0.25" Thick Plate - Test Number 3 Stress - Strain diagram using 2" Extensometer

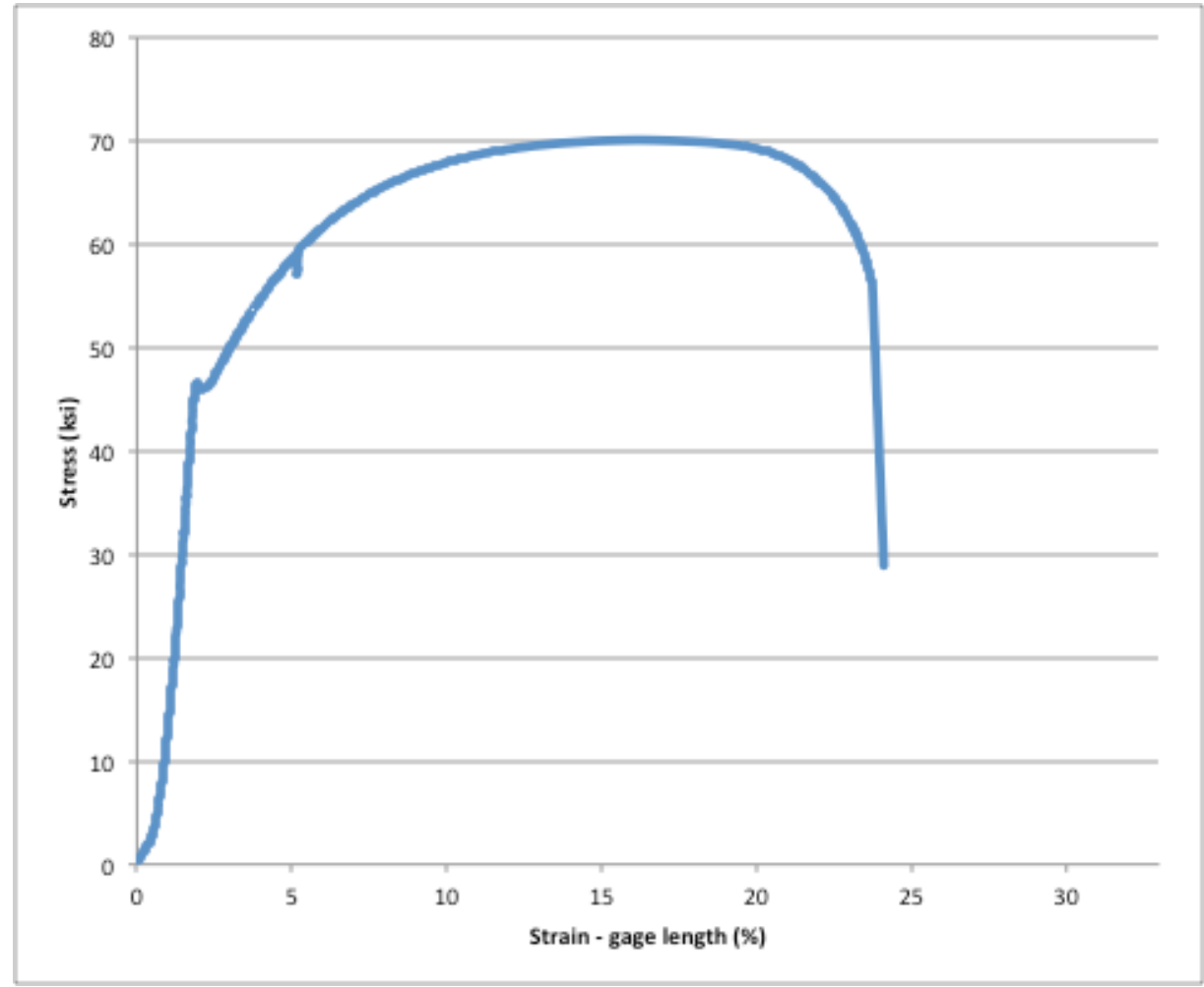

Figure 146. 0.25" Thick Plate - Test Number 3 Stress - Strain diagram using full gage length 
Table 30. 0.375" Thick Plate - Test Number 1 Measurements

\begin{tabular}{|c|c|c|}
\hline 0.375 " Test 1 & Initial, in & Final, in \\
\hline \multirow{3}{*}{ Width } & 1.505 & \multirow{3}{*}{1.13 (at necking) } \\
\hline & 1.506 & \\
\hline & 1.505 & \\
\hline \multirow{3}{*}{ Thickness } & 0.3745 & \multirow{3}{*}{0.23 (at necking) } \\
\hline & 0.375 & \\
\hline & 0.375 & \\
\hline $\begin{array}{c}\text { Length } \\
\text { between } \\
\text { punch marks, } \\
\text { in }\end{array}$ & 7.52 & 9.85 \\
\hline $\begin{array}{l}\text { Specimen } \\
\text { elongation, in }\end{array}$ & 2.33 & \\
\hline
\end{tabular}

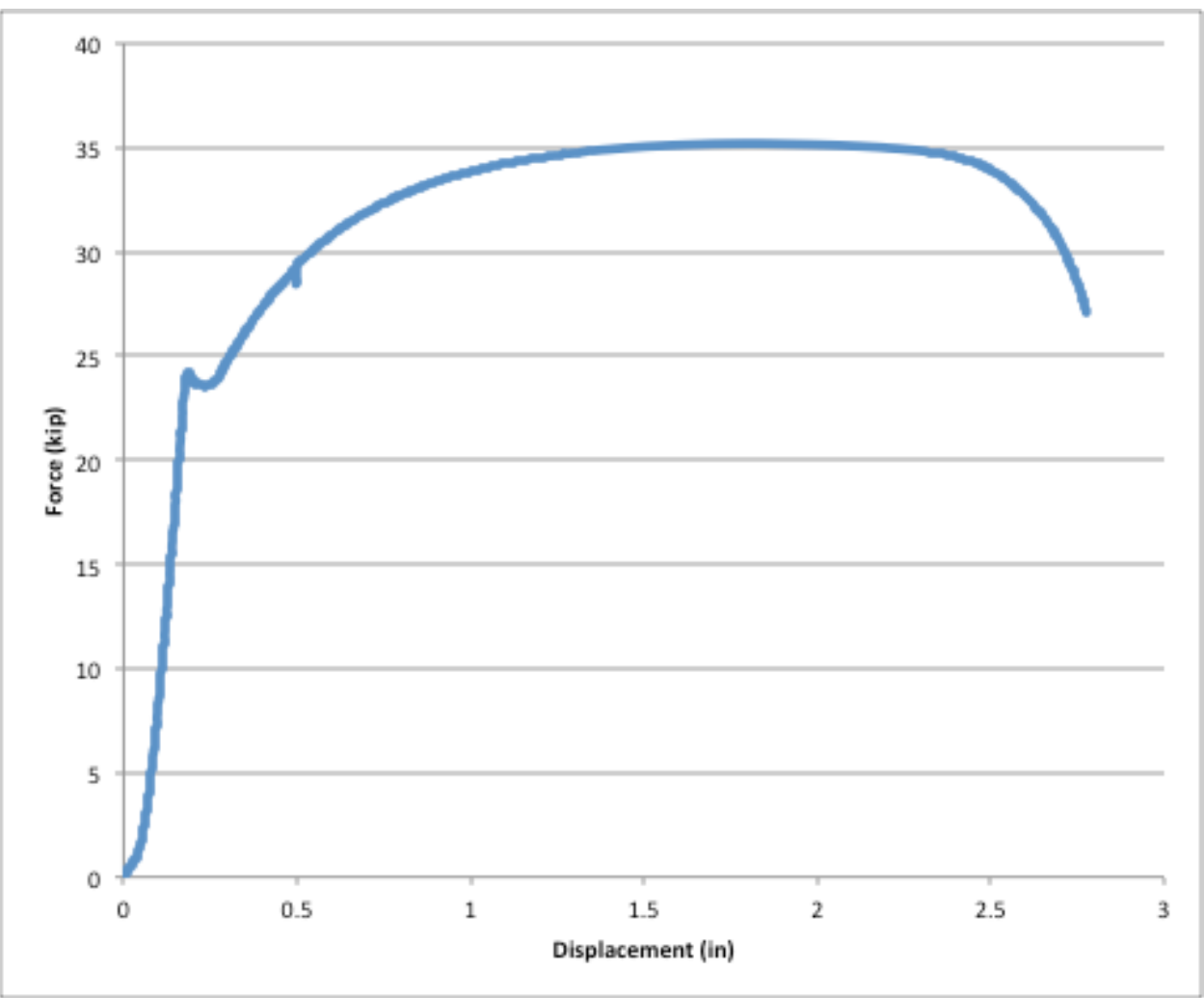

Figure 147. 0.375" Thick Plate - Test Number 1 Load - Displacement diagram 


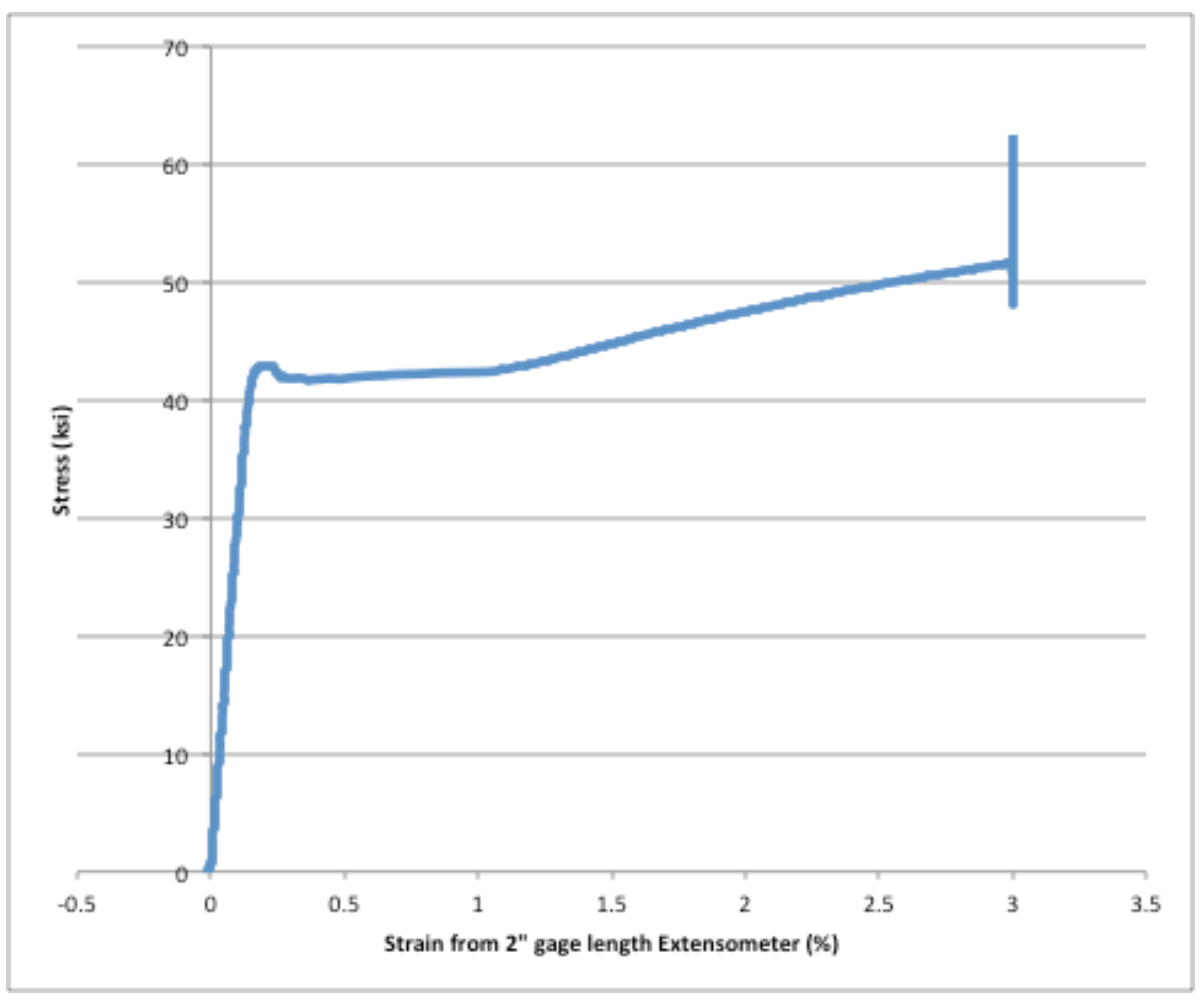

Figure 148. 0.375 " Thick Plate - Test Number 1 Stress - Strain diagram using 2" Extensometer

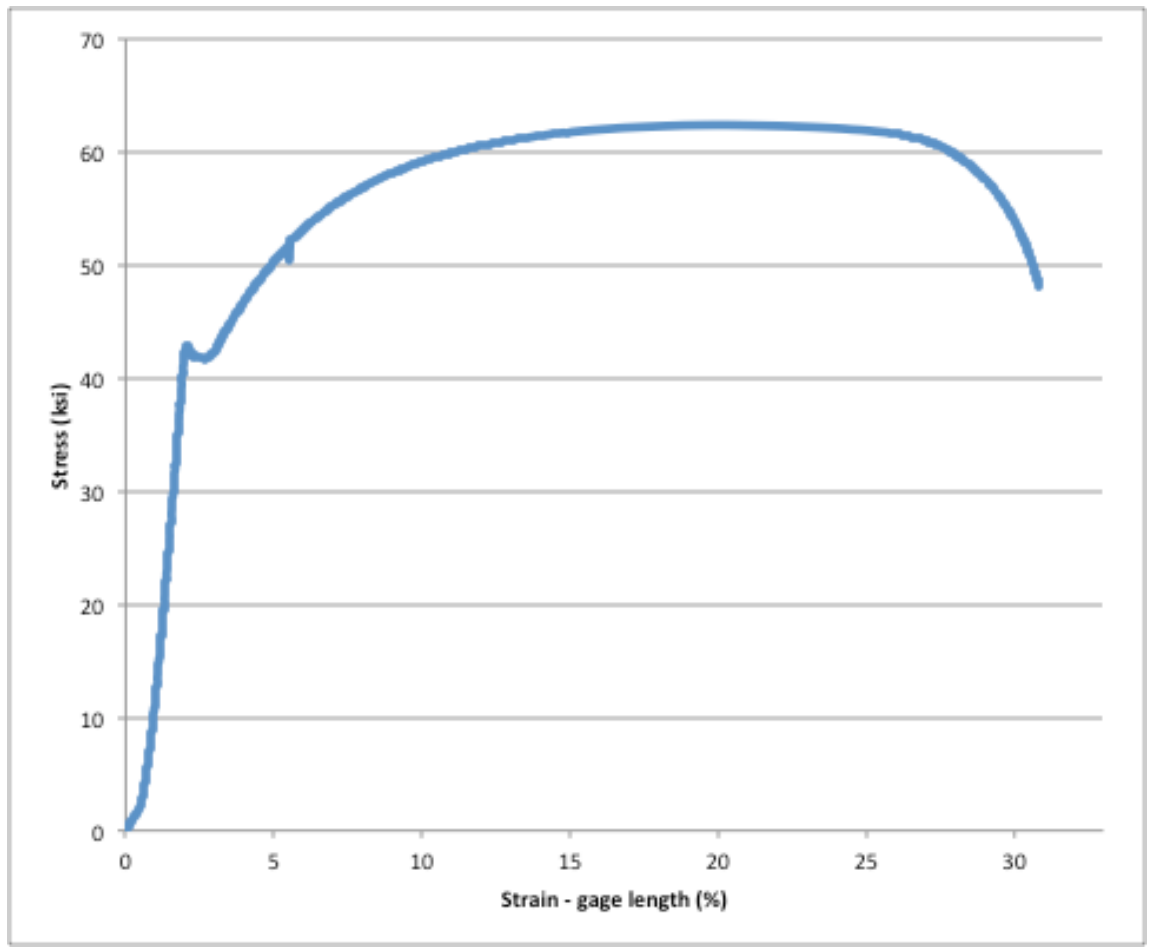

Figure 149. 0.375" Thick Plate - Test Number 1 Stress - Strain diagram using full gage length 
Table 31. 0.375" Thick Plate - Test Number 2 Measurements

\begin{tabular}{|c|c|c|}
\hline 0.375 " Test 2 & Initial, in & Final, in \\
\hline \multirow{3}{*}{ Width } & 1.51 & \multirow{3}{*}{1.12 (at necking) } \\
\hline & 1.51 & \\
\hline & 1.51 & \\
\hline \multirow{3}{*}{ Thickness } & 0.375 & \multirow{3}{*}{0.25 (at necking) } \\
\hline & 0.37 & \\
\hline & 0.37 & \\
\hline $\begin{array}{c}\text { Length } \\
\text { between } \\
\text { punch marks, } \\
\text { in }\end{array}$ & 7.81 & 10.07 \\
\hline $\begin{array}{l}\text { Specimen } \\
\text { elongation, in }\end{array}$ & 2.26 & \\
\hline
\end{tabular}

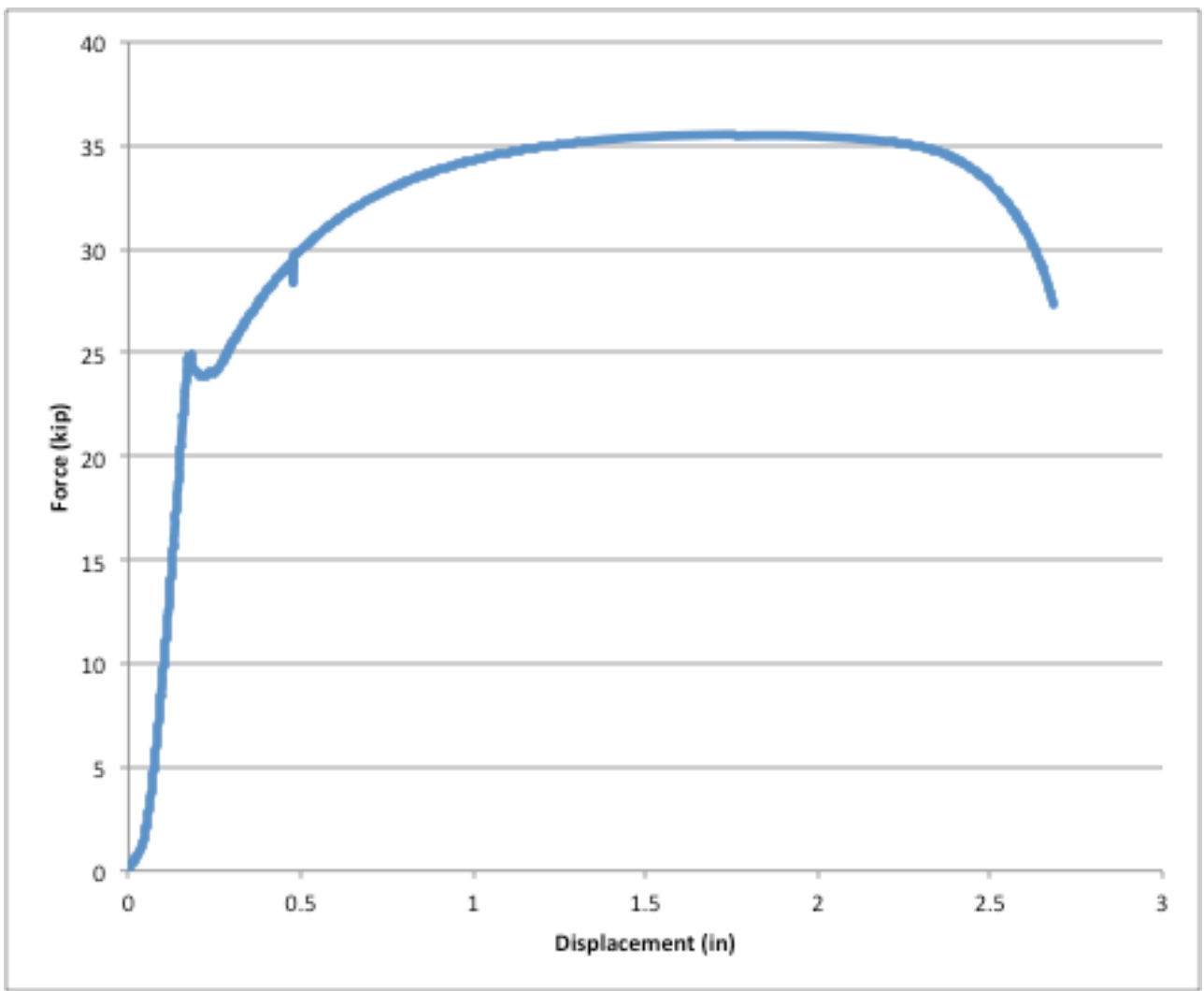

Figure 150. 0.375" Thick Plate - Test Number 2 Load - Displacement diagram 


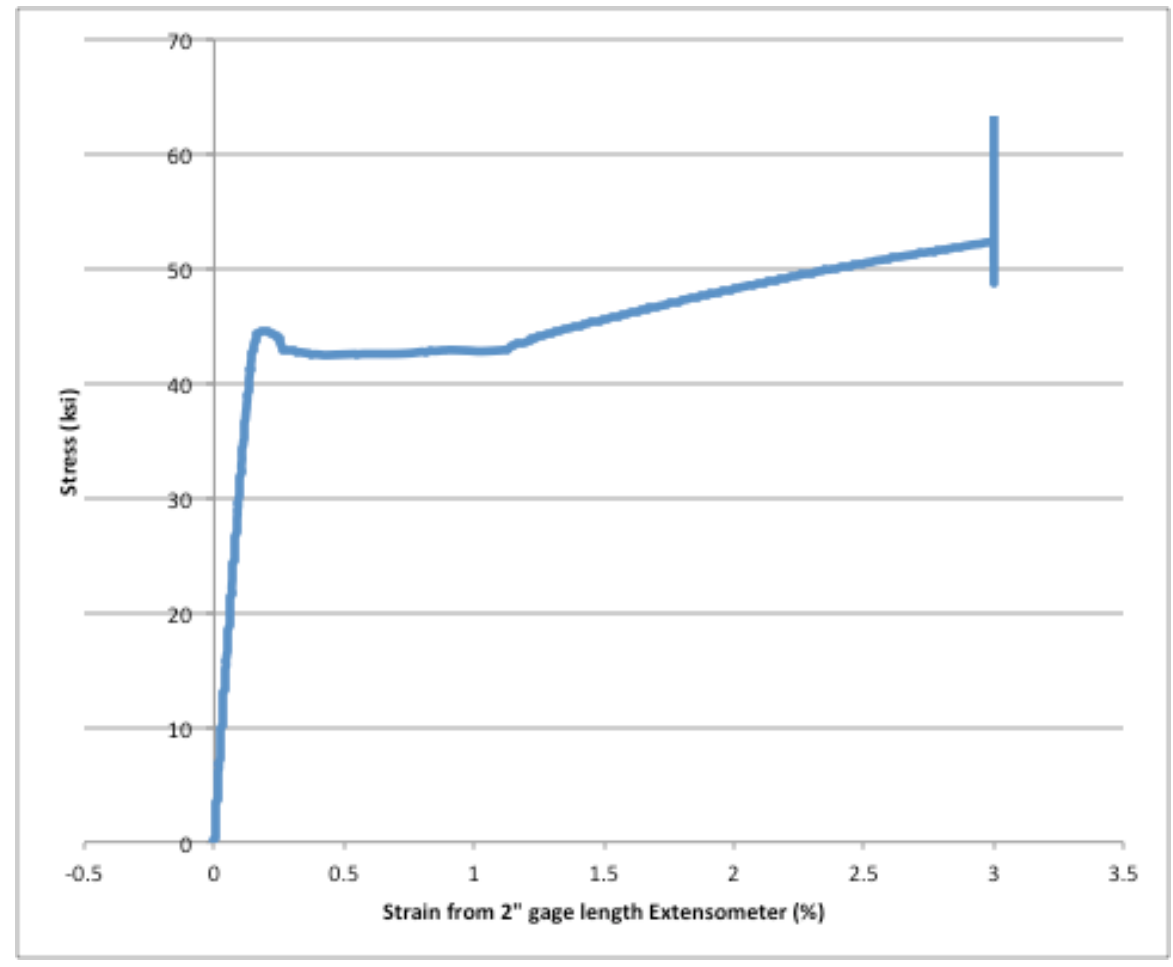

Figure 151. 0.375 " Thick Plate - Test Number 2 Stress - Strain diagram using 2" Extensometer

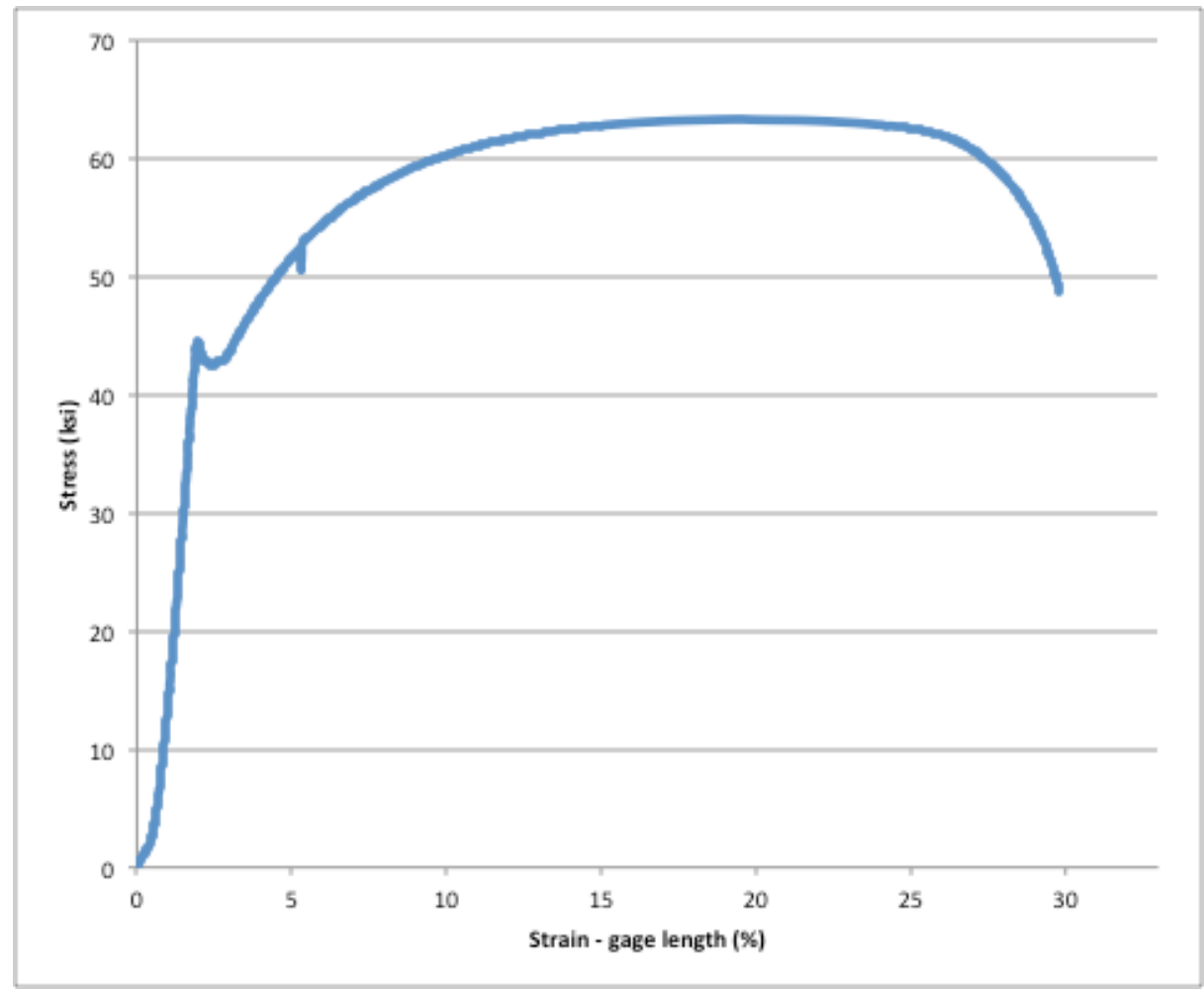

Figure 152. 0.375" Thick Plate - Test Number 2 Stress - Strain diagram using full gage length 
Table 32. 0.375" Thick Plate - Test Number 3 Measurements

\begin{tabular}{|c|c|c|}
\hline 0.375 " Test 3 & Initial, in & Final, in \\
\hline \multirow{3}{*}{ Width } & 1.51 & \multirow{3}{*}{1.07 (at necking) } \\
\hline & 1.51 & \\
\hline & 1.51 & \\
\hline \multirow{3}{*}{ Thickness } & 0.37 & \multirow{3}{*}{0.25 (at necking) } \\
\hline & 0.37 & \\
\hline & 0.375 & \\
\hline $\begin{array}{l}\text { Length } \\
\text { between } \\
\text { punch marks, } \\
\text { in }\end{array}$ & 8.165 & 10.33 \\
\hline $\begin{array}{l}\text { Specimen } \\
\text { elongation, in }\end{array}$ & 2.165 & \\
\hline
\end{tabular}

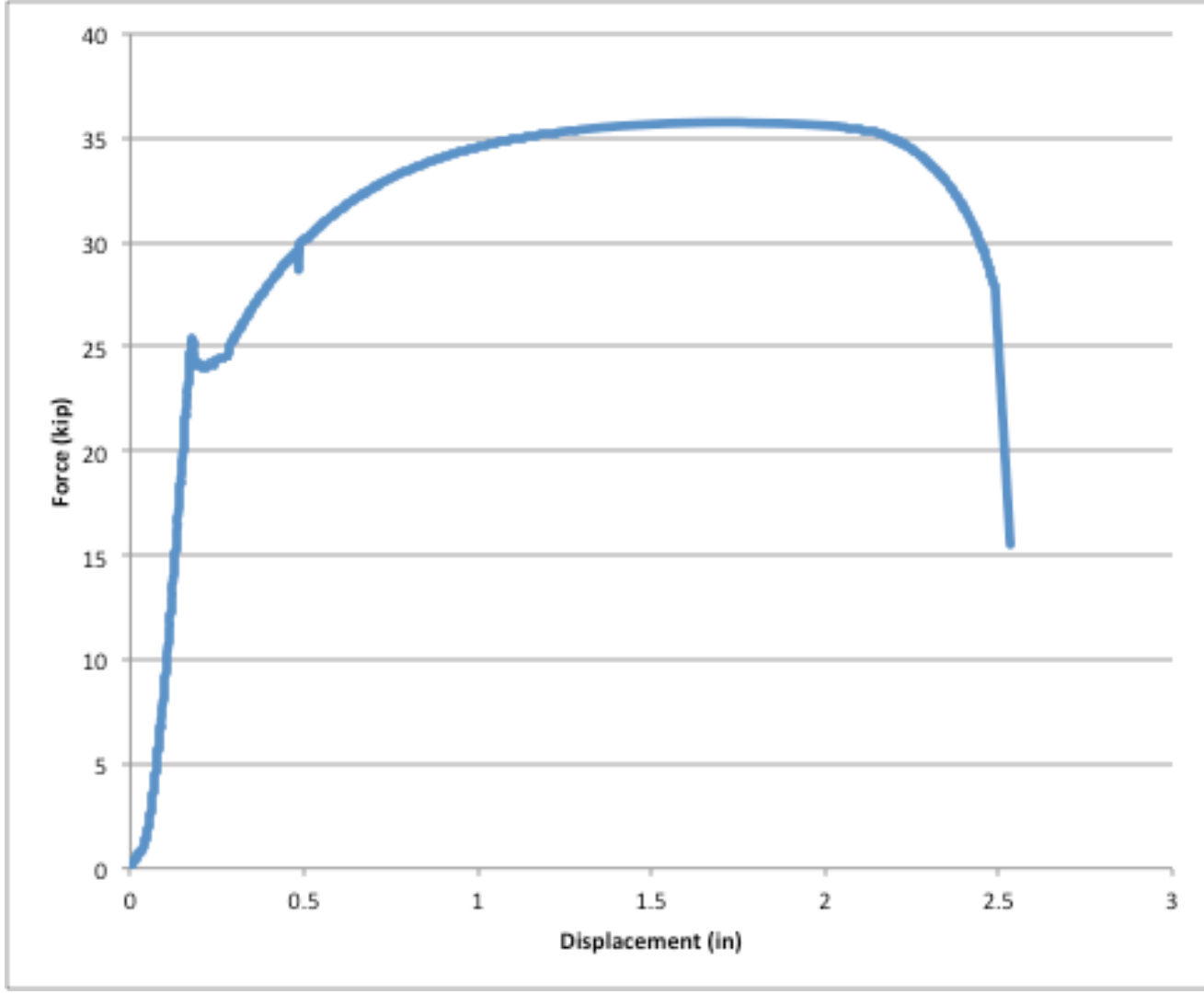

Figure 153. 0.375" Thick Plate - Test Number 2 Load - Displacement diagram 


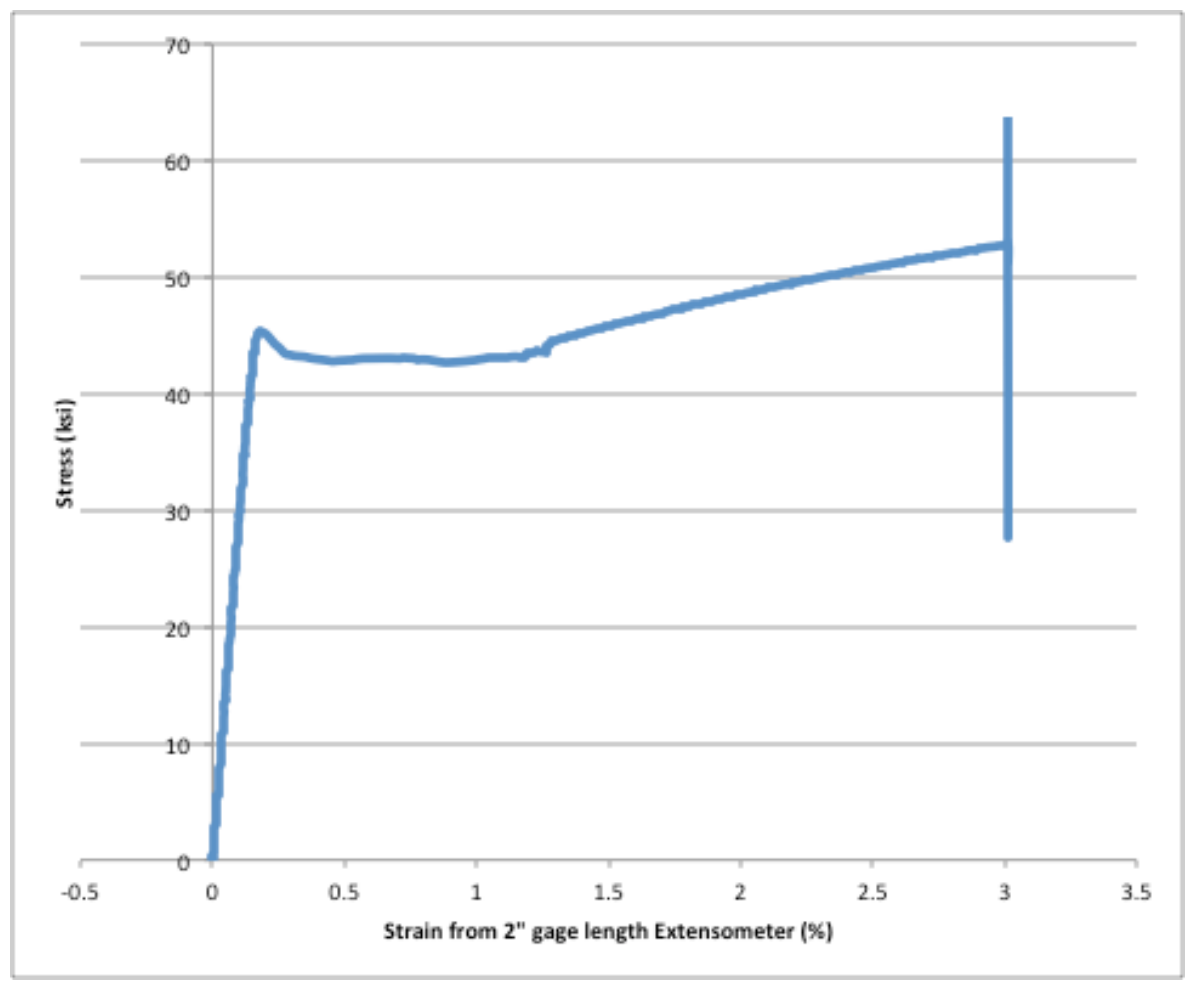

Figure 154. 0.375 " Thick Plate - Test Number 2 Stress - Strain diagram using 2" Extensometer

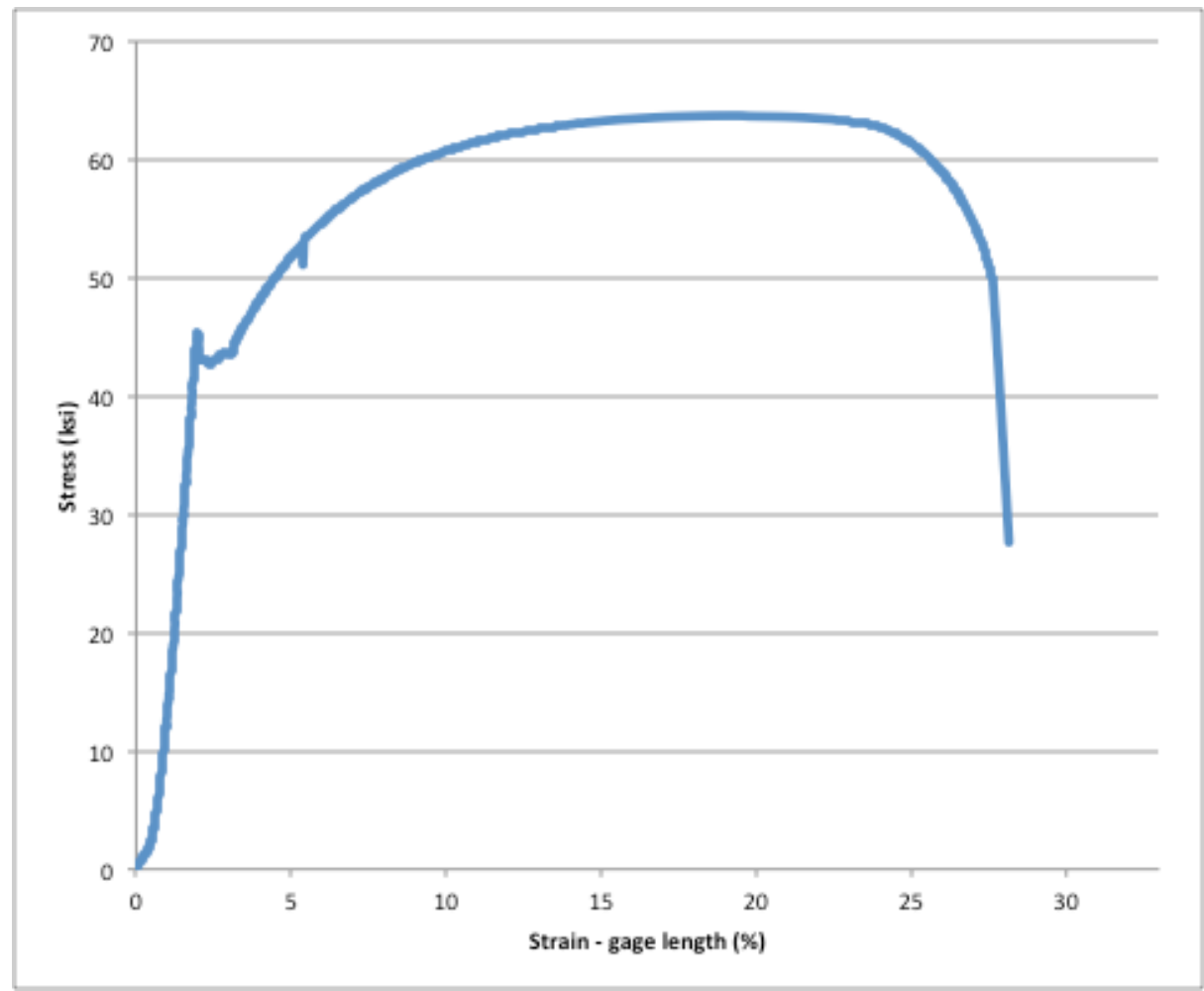

Figure 155. 0.375" Thick Plate - Test Number 2 Stress - Strain diagram using full gage length 


\section{APPENDIX D: Raw Test Data}

\section{TEST 1}

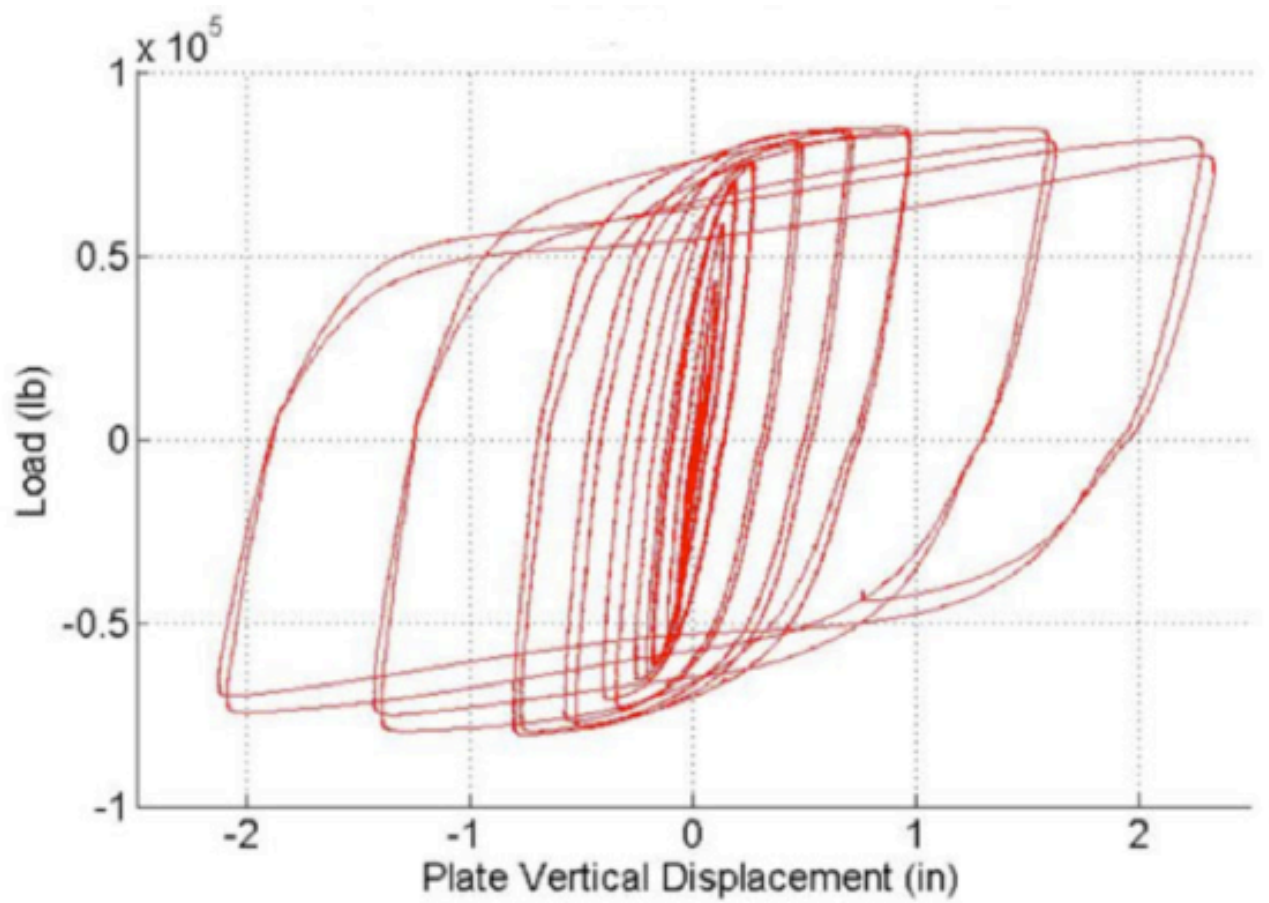

Figure 156. Load versus Plate Vertical Displacement - Test 1

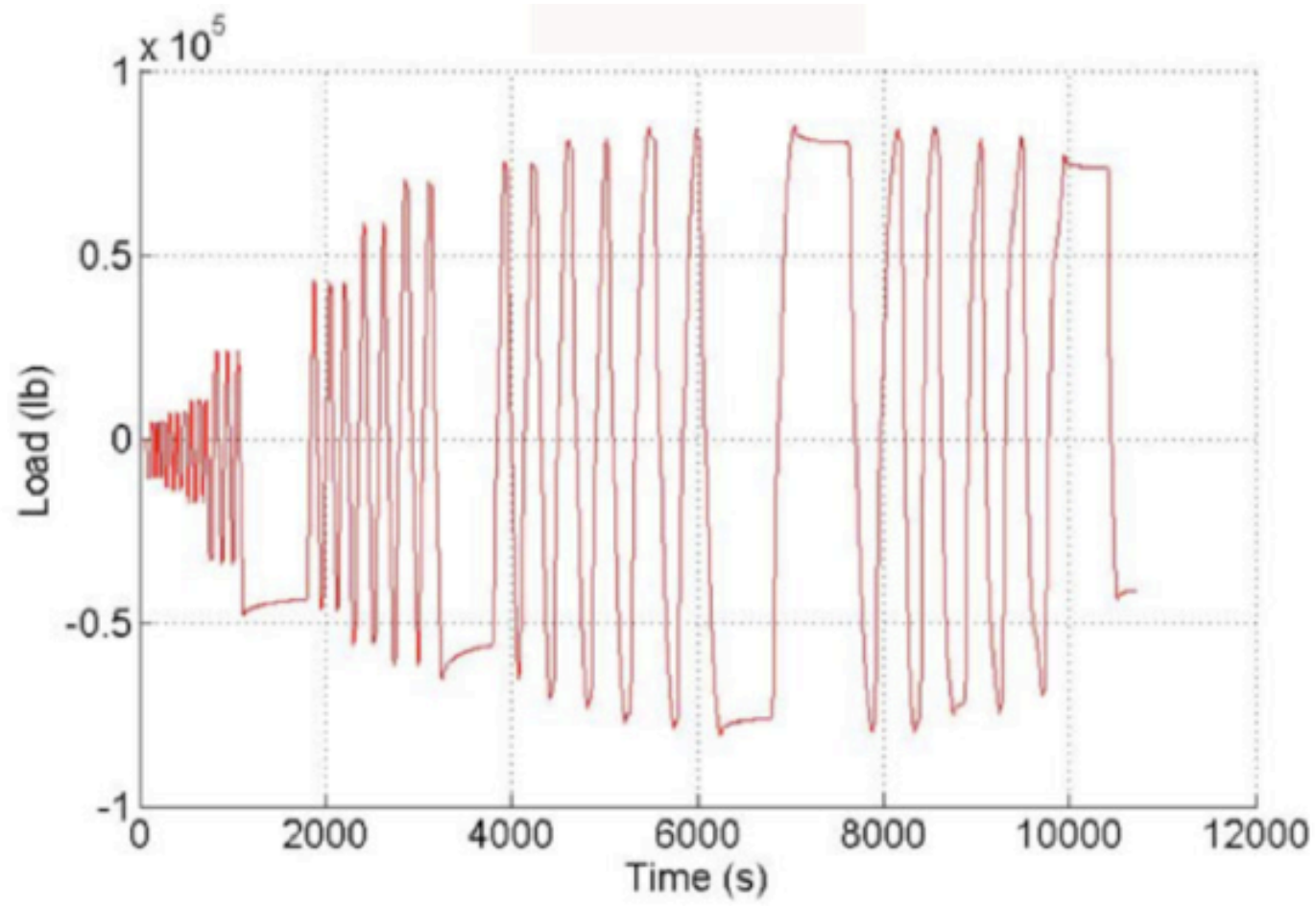

Figure 157. Load versus Time - Test 1 


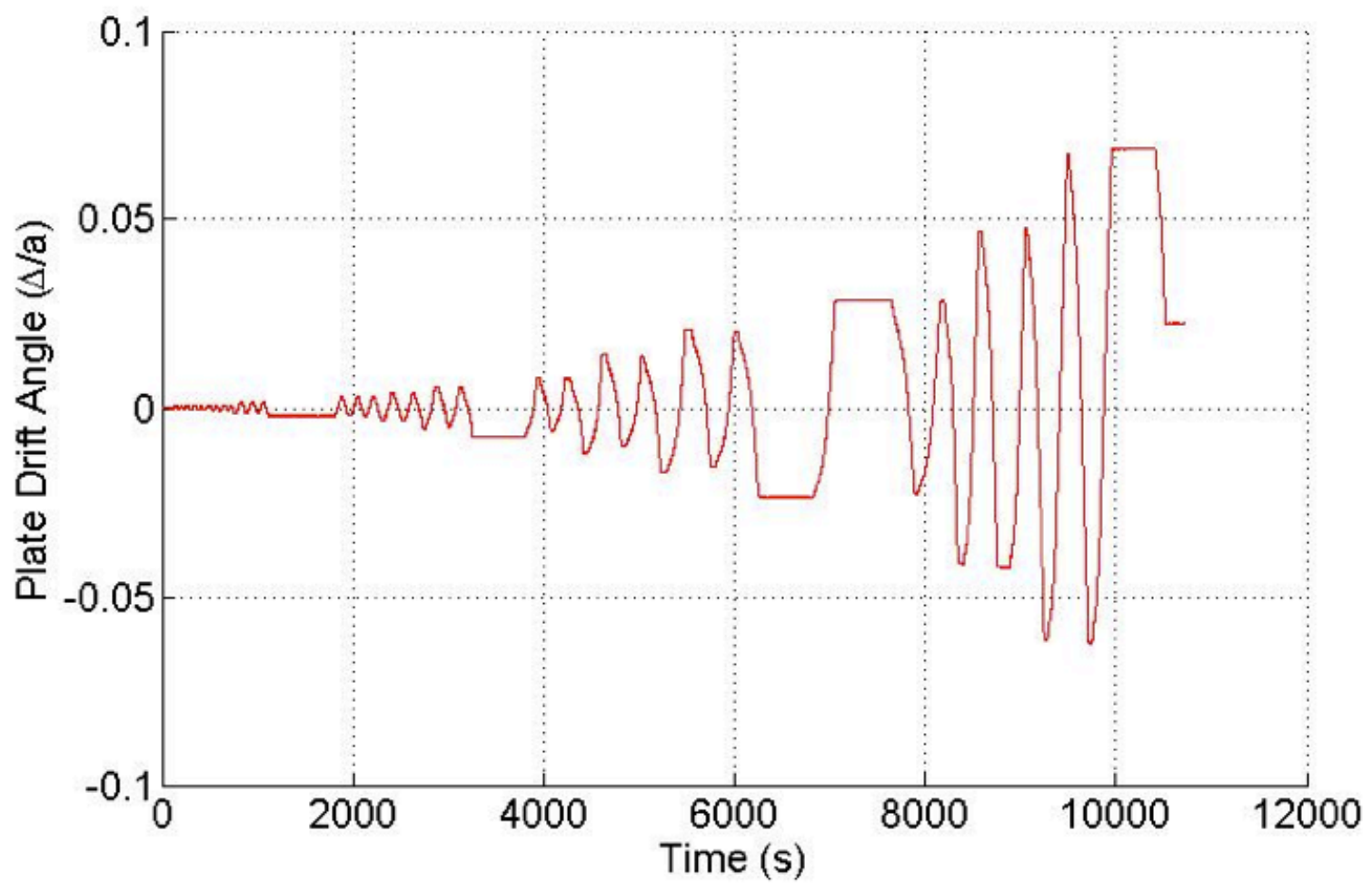

Figure 158. Plate Drift Angle versus Time - Test 1

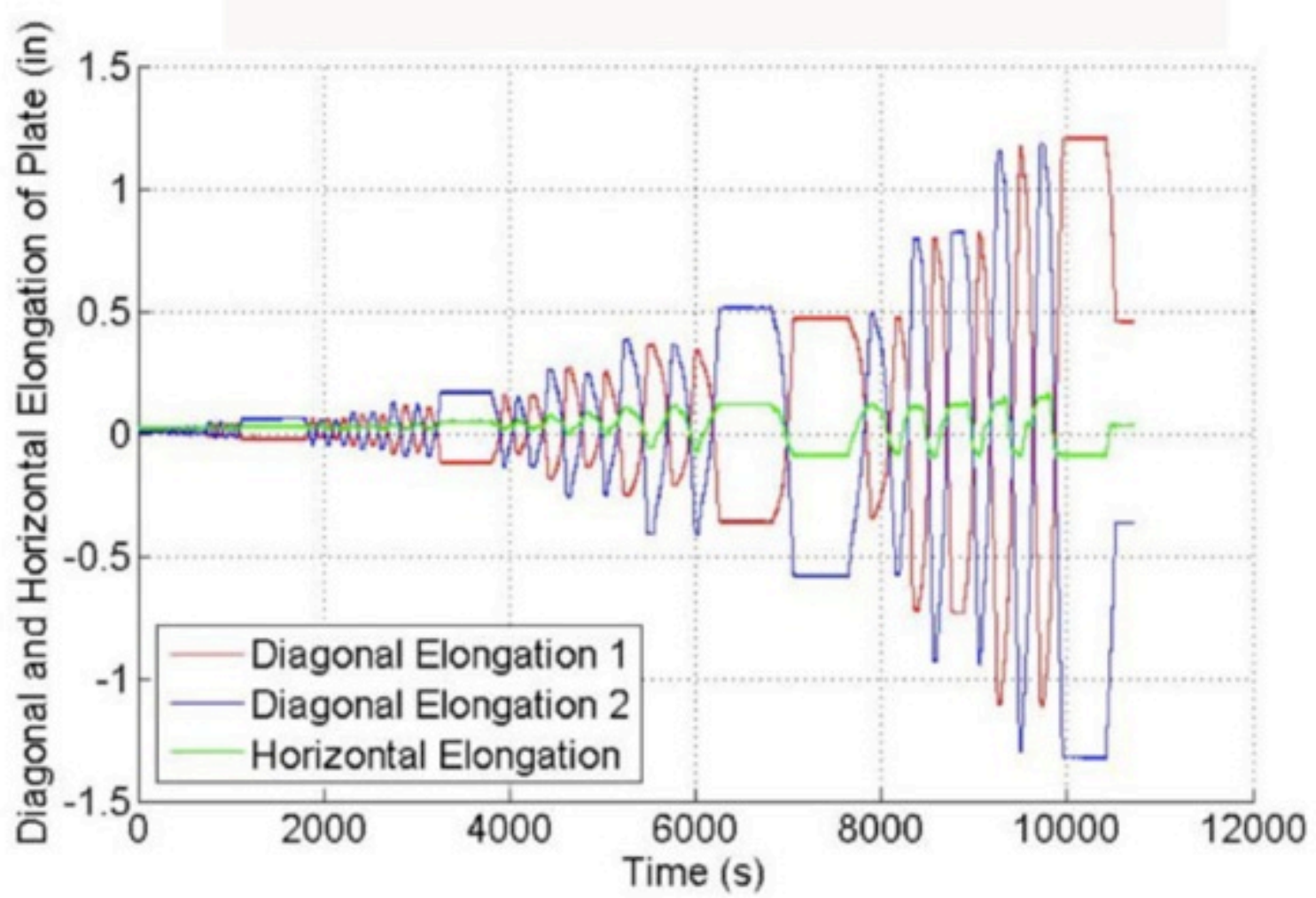

Figure 159. Diagonal and Horizontal Elongations of Plate versus Time - Test 1 


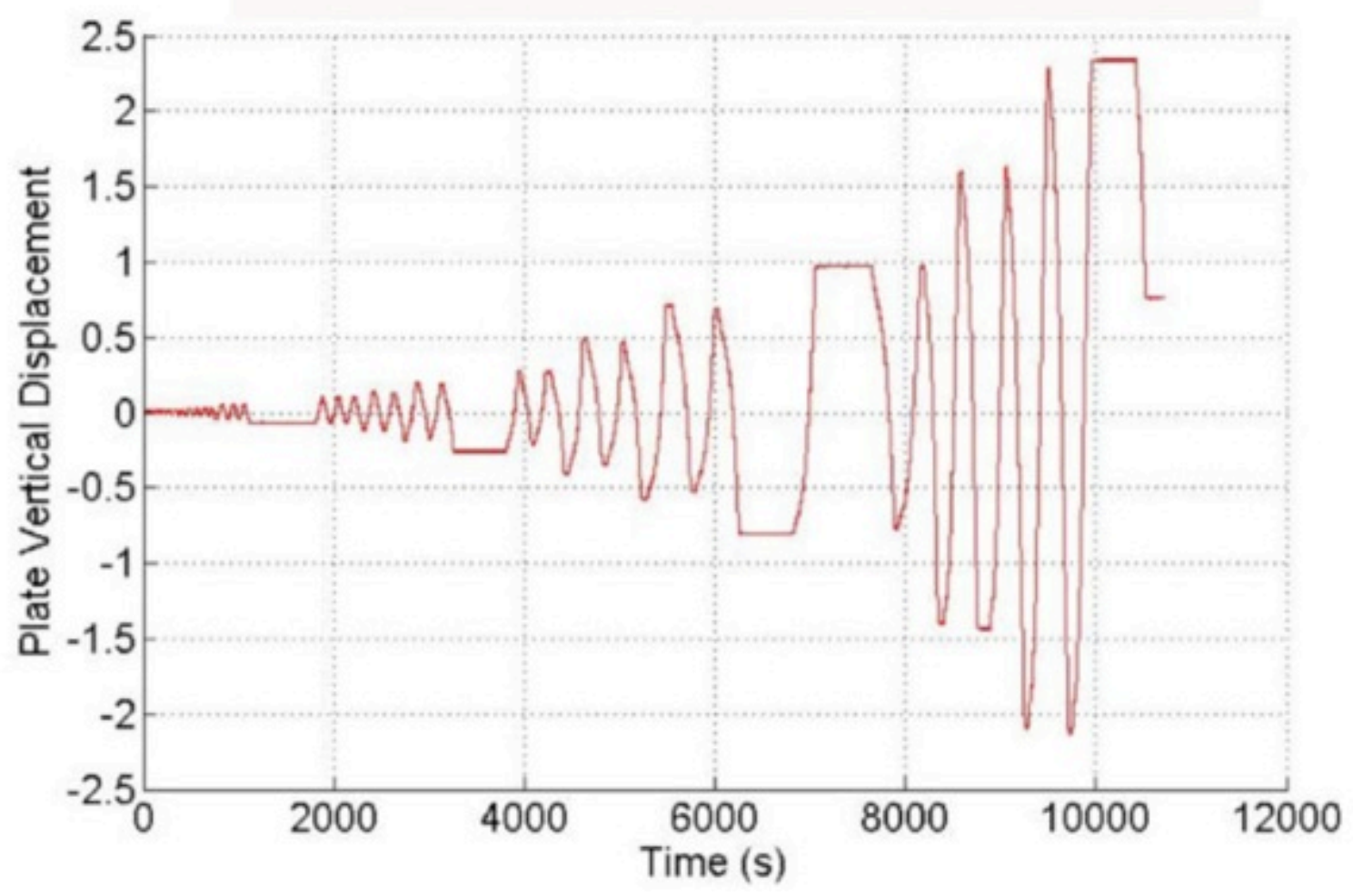

Figure 160. Plate Vertical Displacement versus Time- Test 1

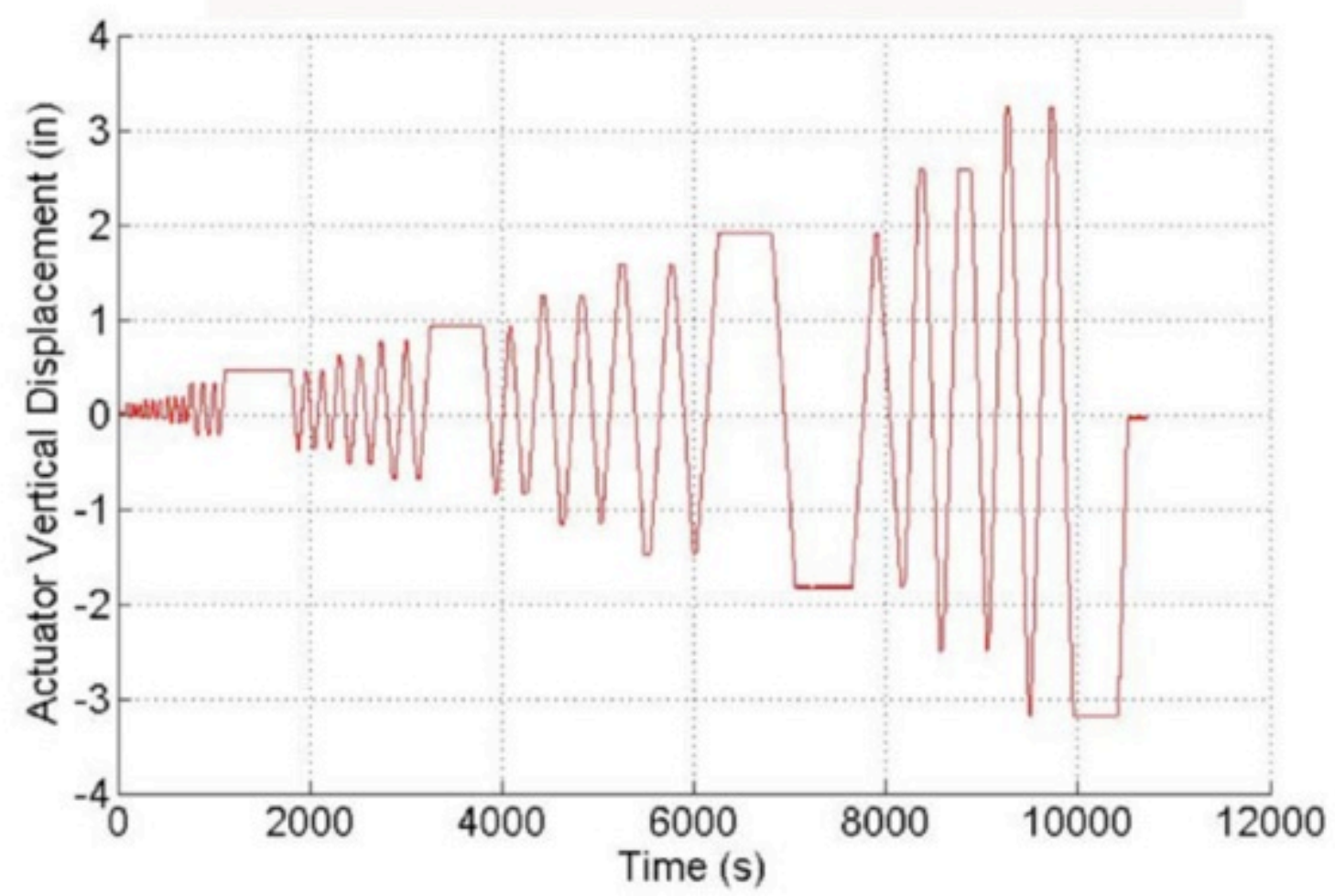

Figure 161. Actuator Vertical Displacement versus Time - Test 1 


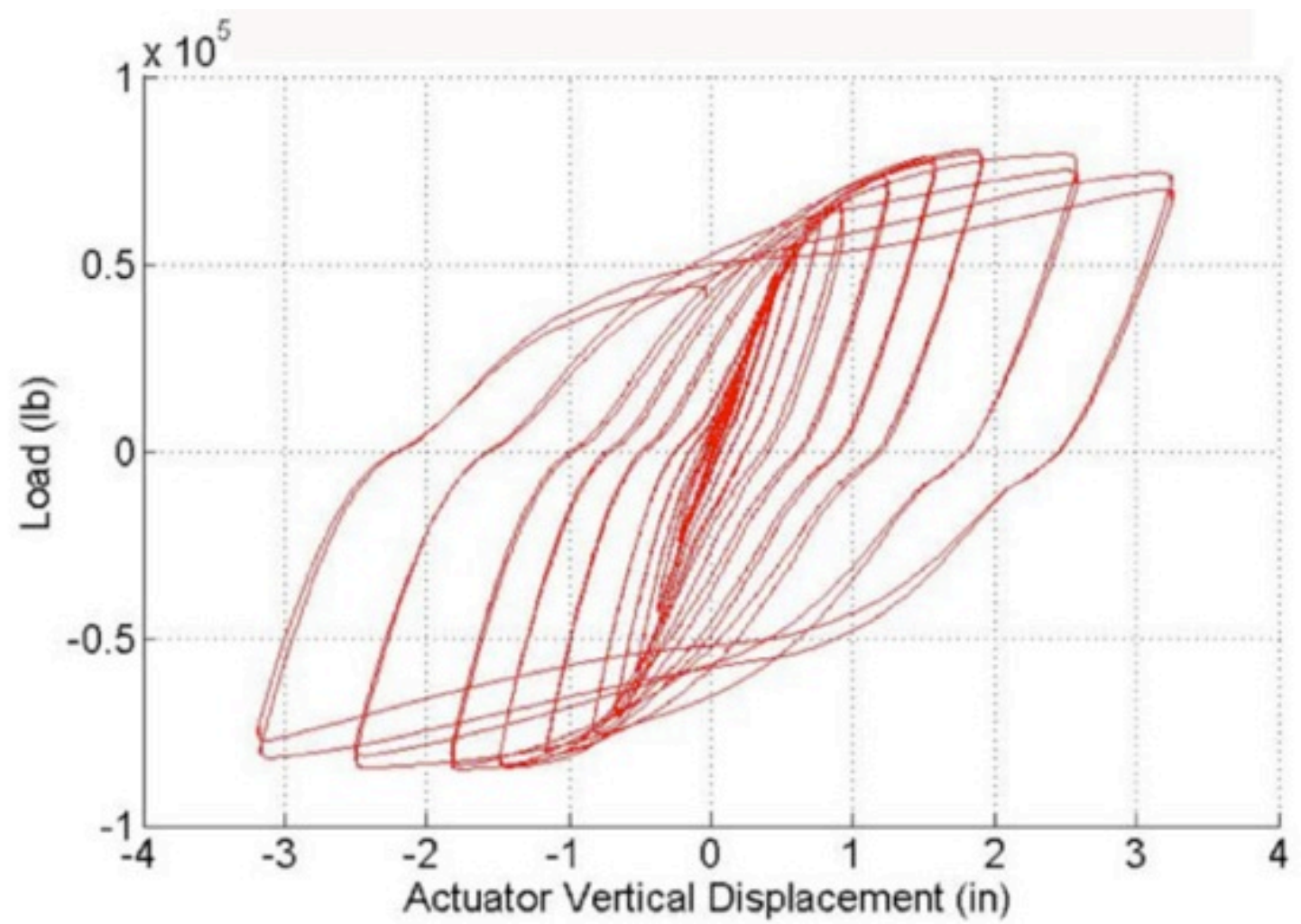

Figure 162. Load versus Actuator Vertical Displacement - Test 1

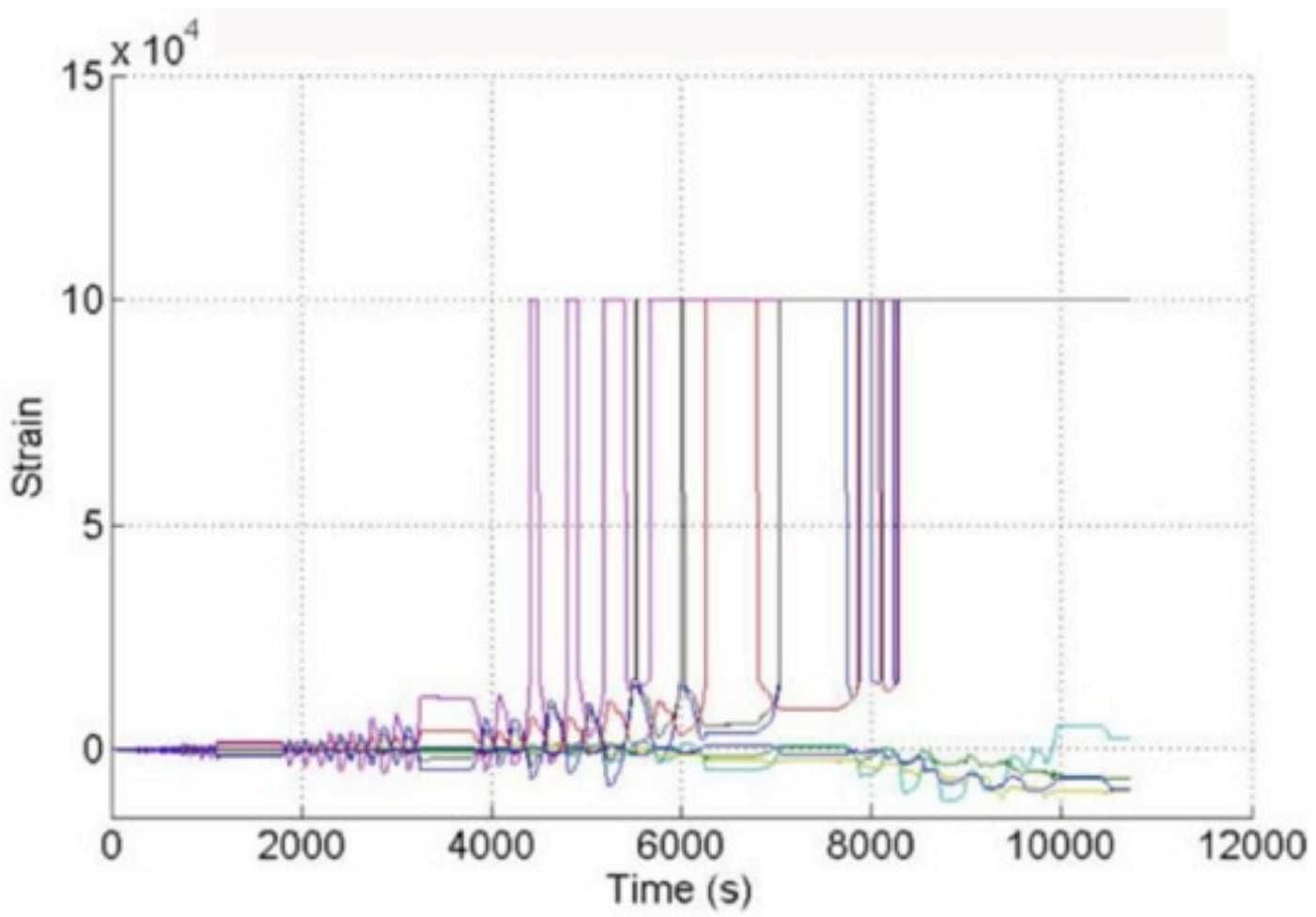

Figure 163. 8 Strain Gauges versus Time - Test 1 


\section{TEST 2}

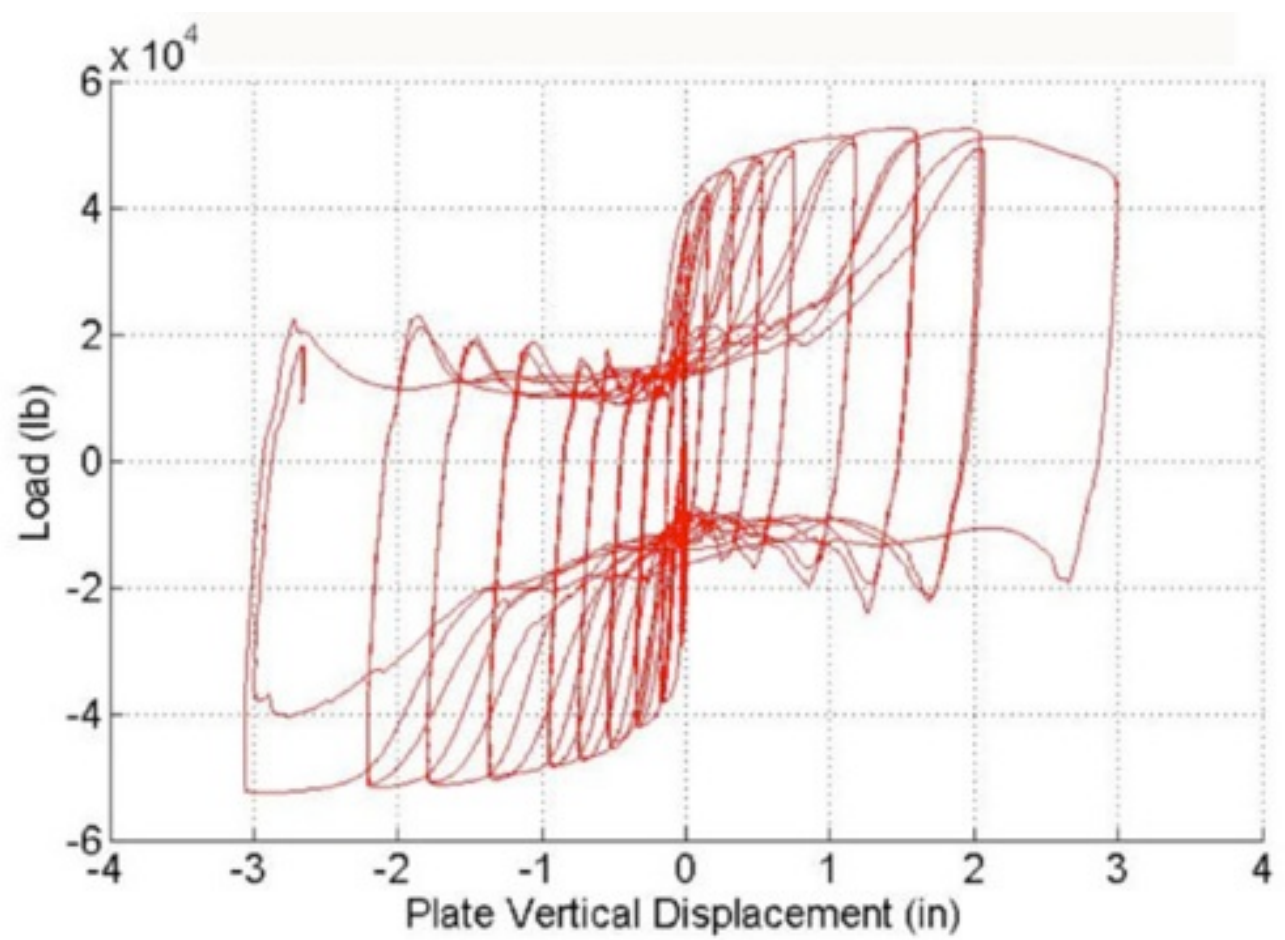

Figure 164. Load versus Plate Vertical Displacement - Test 2

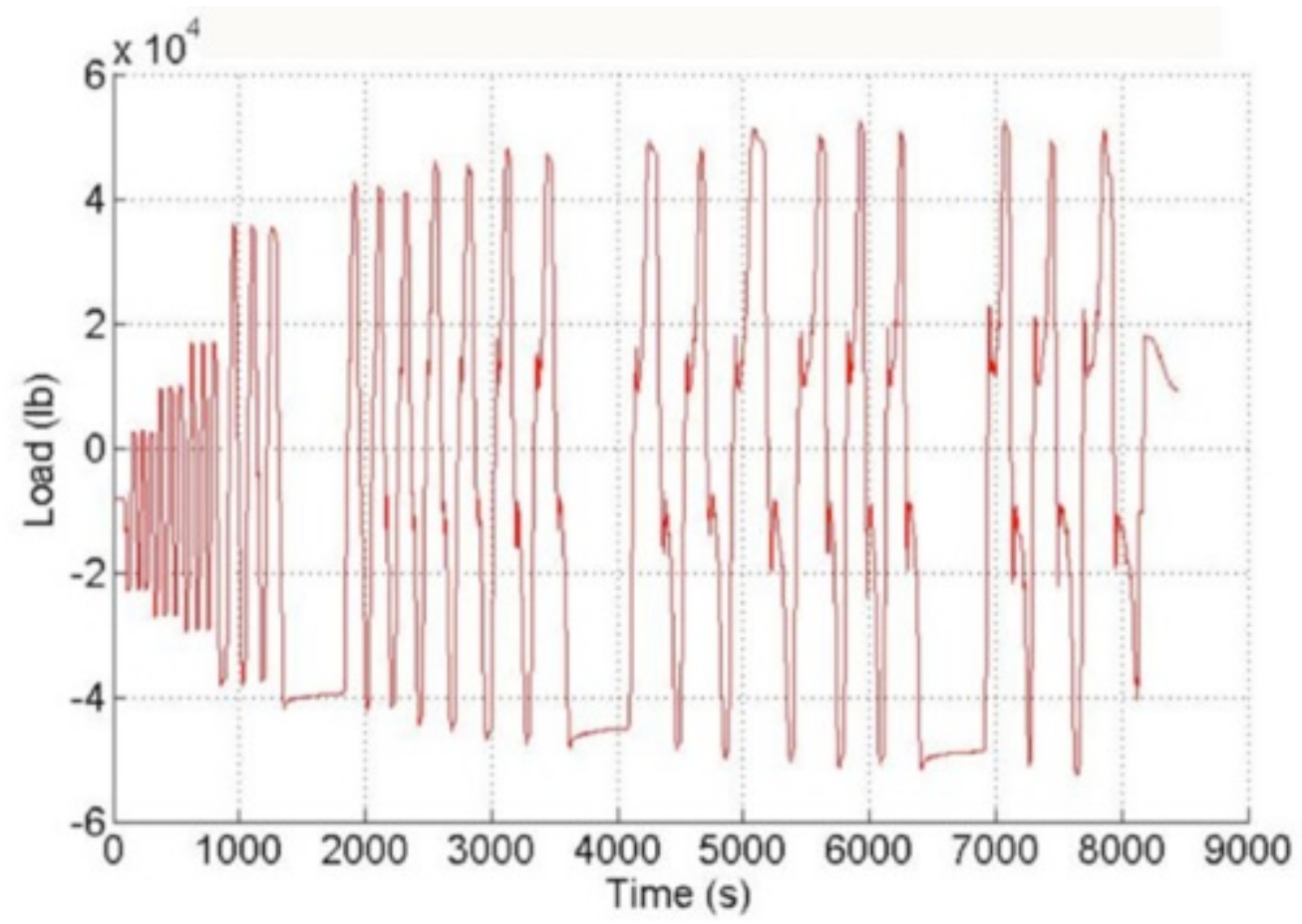

Figure 165. Load versus Time - Test 2 


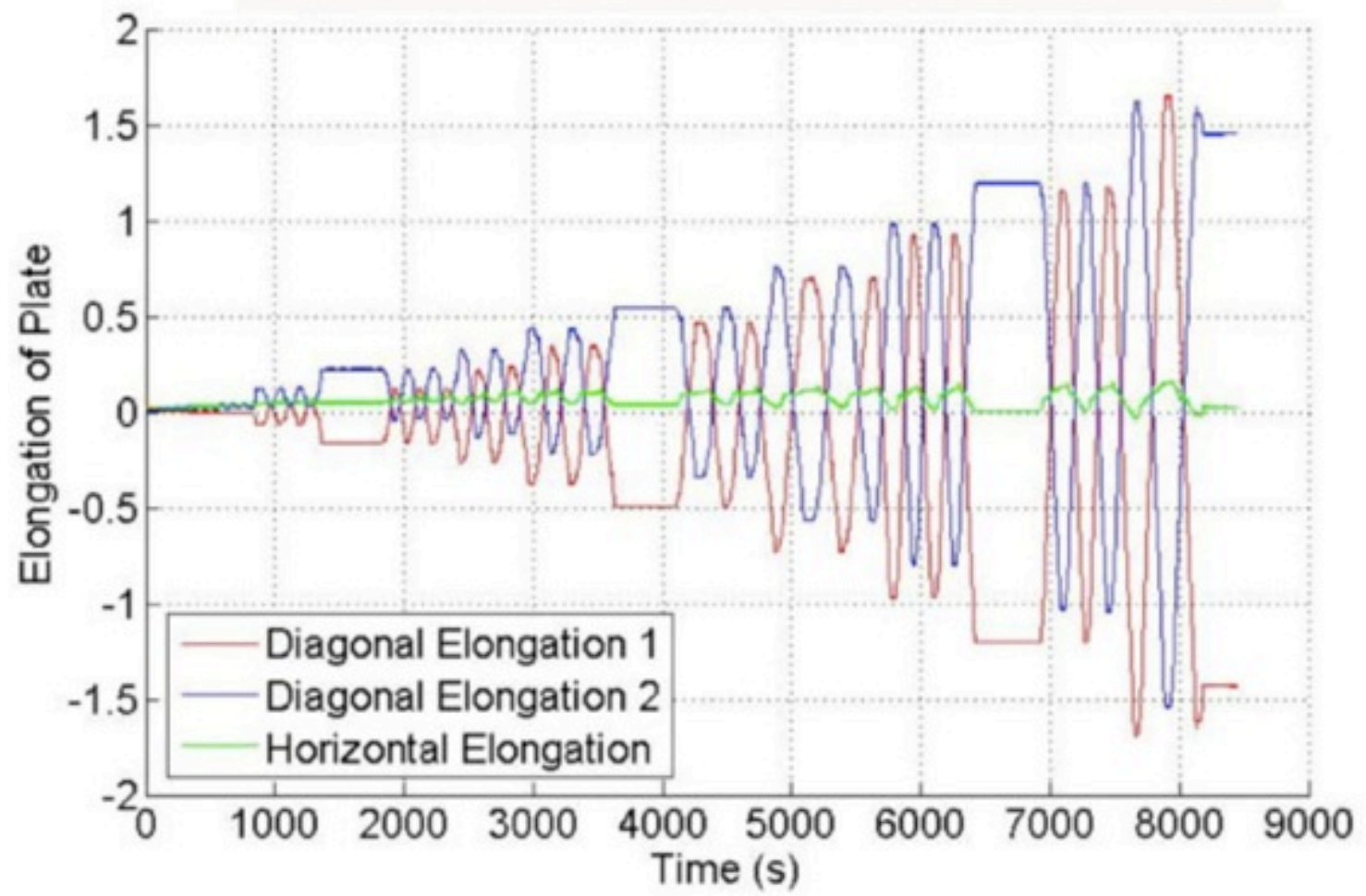

Figure 166. Diagonal and Horizontal Elongations of Plate versus Time - Test 2

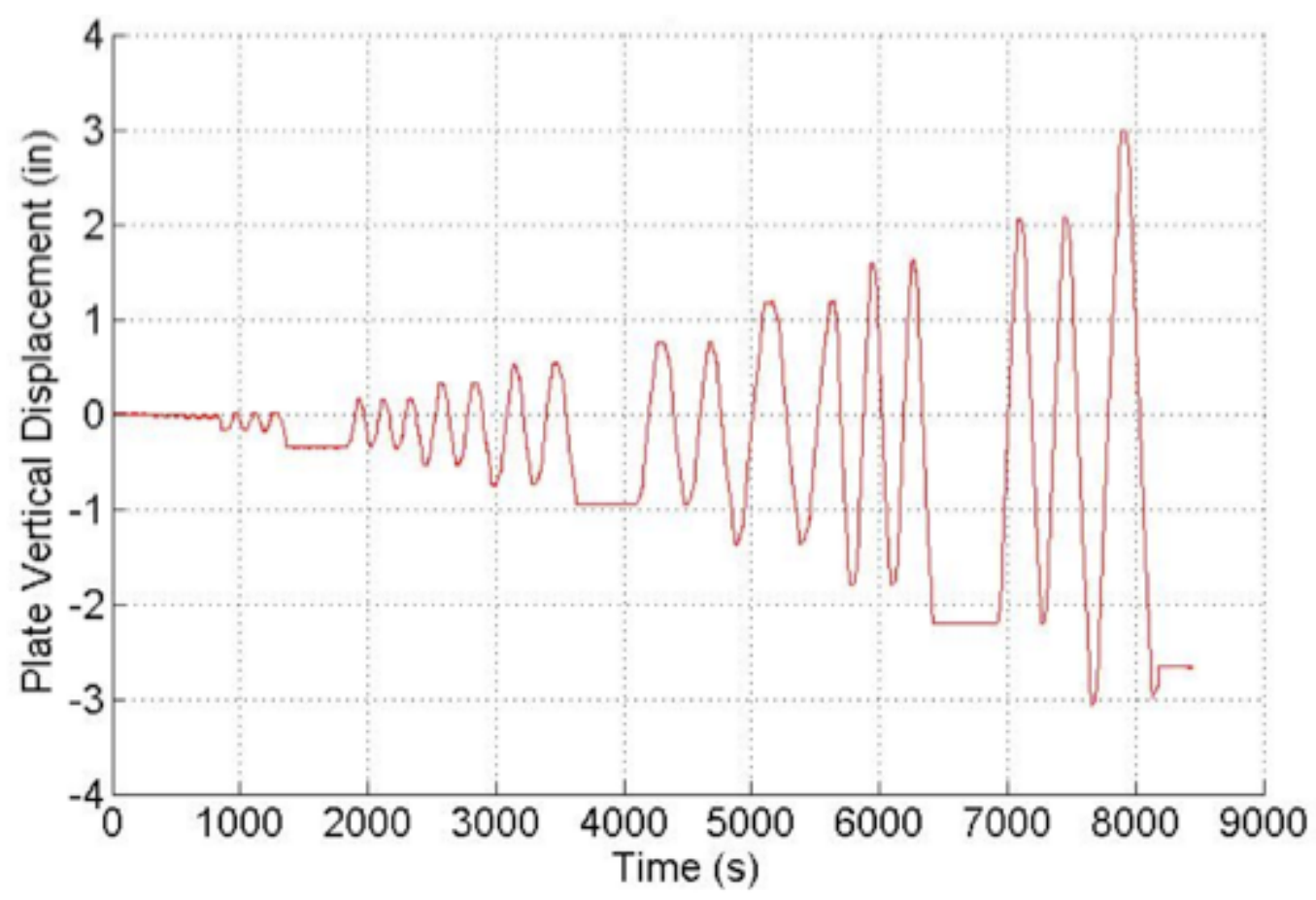

Figure 167. Plate Vertical Displacement versus Time - Test 2 


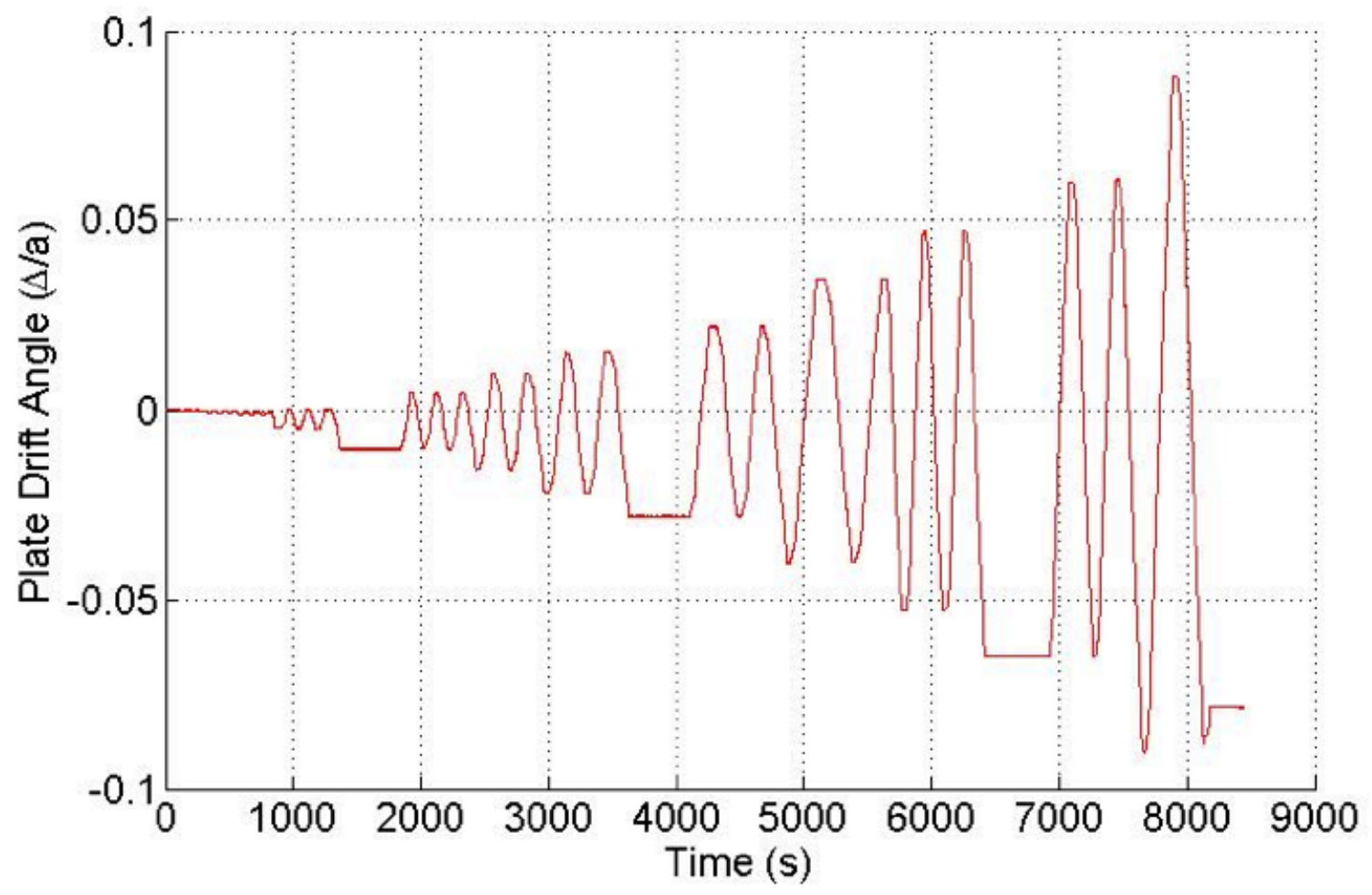

Figure 168. Plate Drift Angle versus Time - Test 2

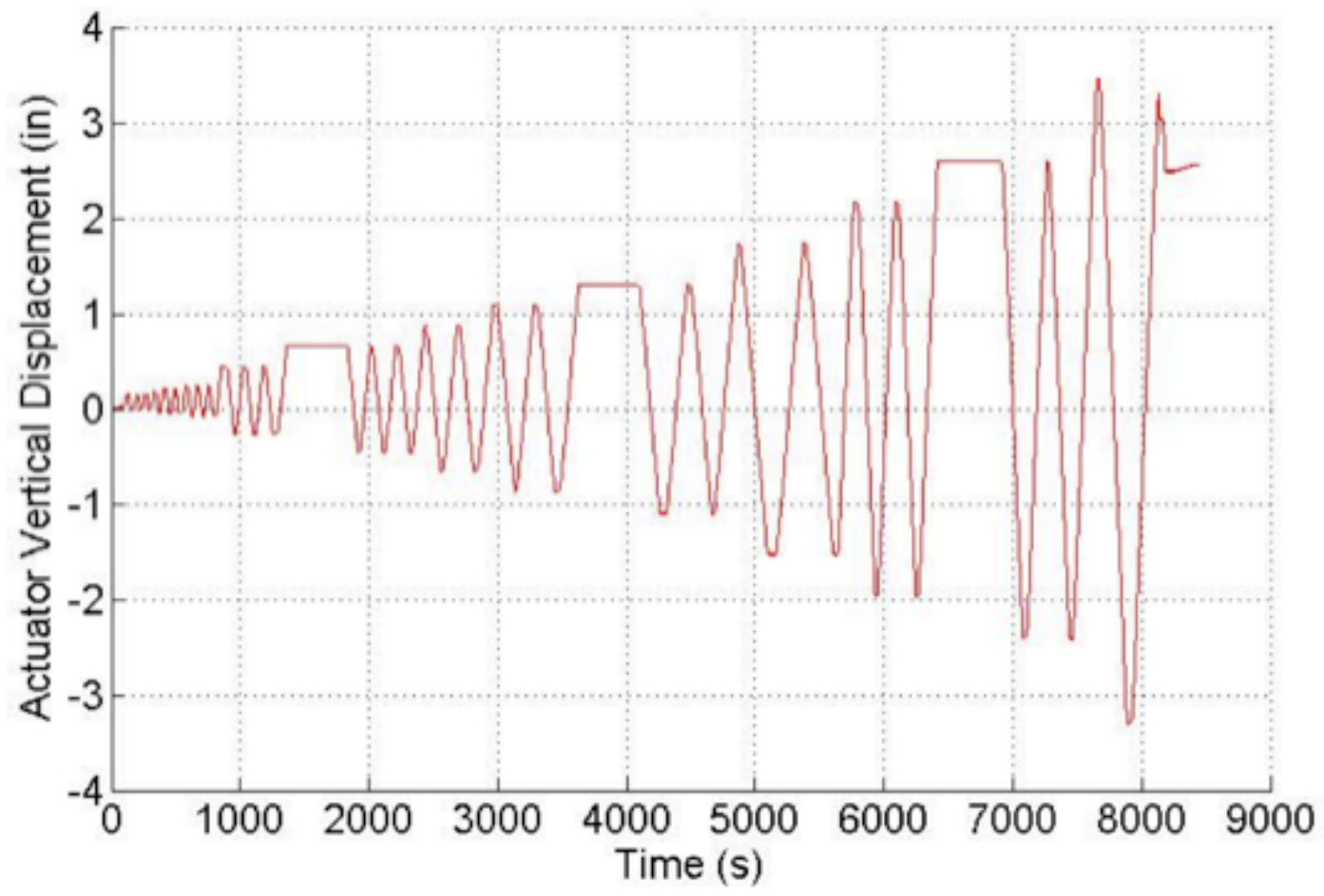

Figure 169. Actuator Vertical Displacement versus Time - Test 2 


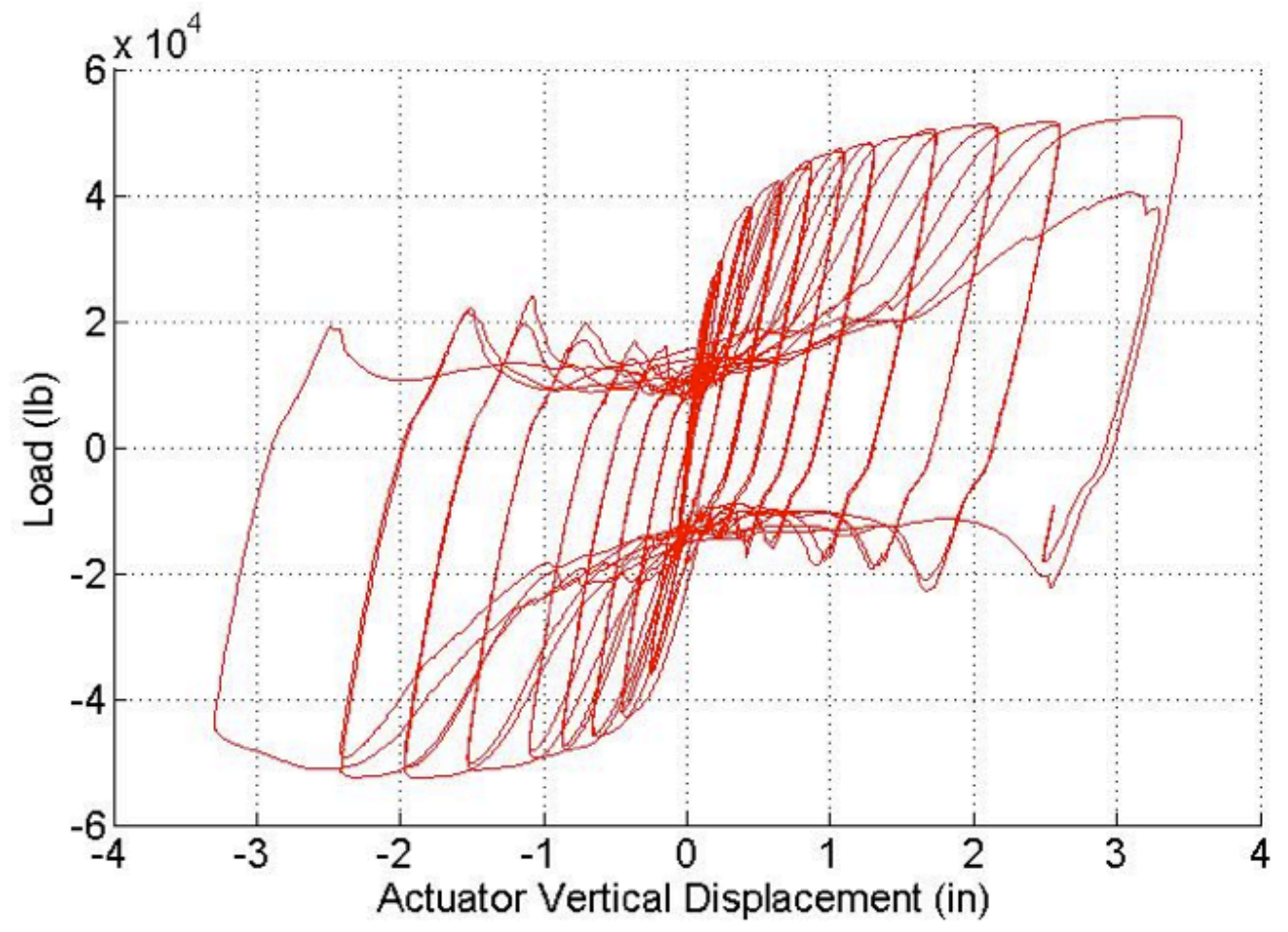

Figure 170. Load versus Actuator Vertical Displacement - Test 2

\section{TEST 3}

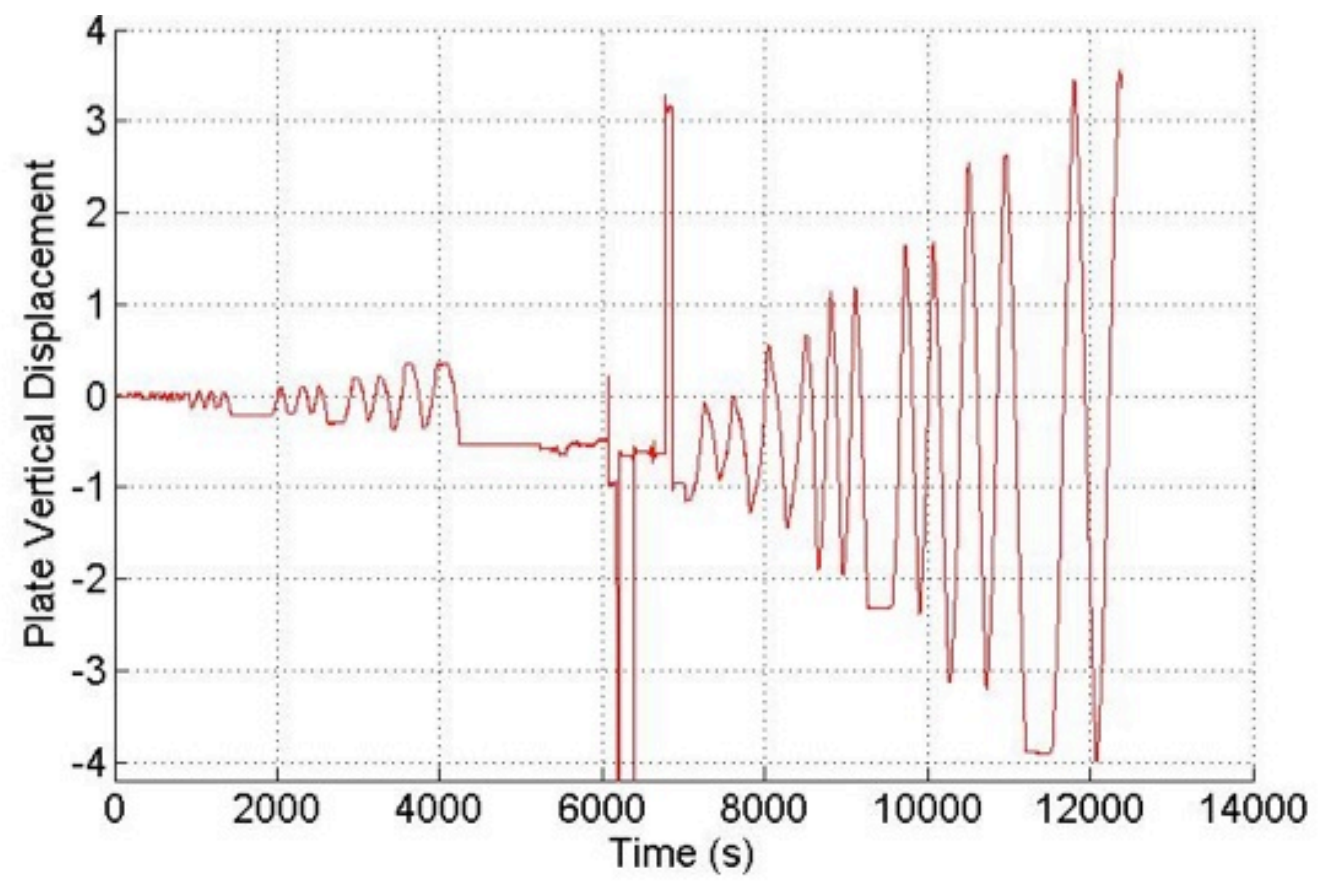

Figure 171. Plate vertical displacement versus time - Test 3 


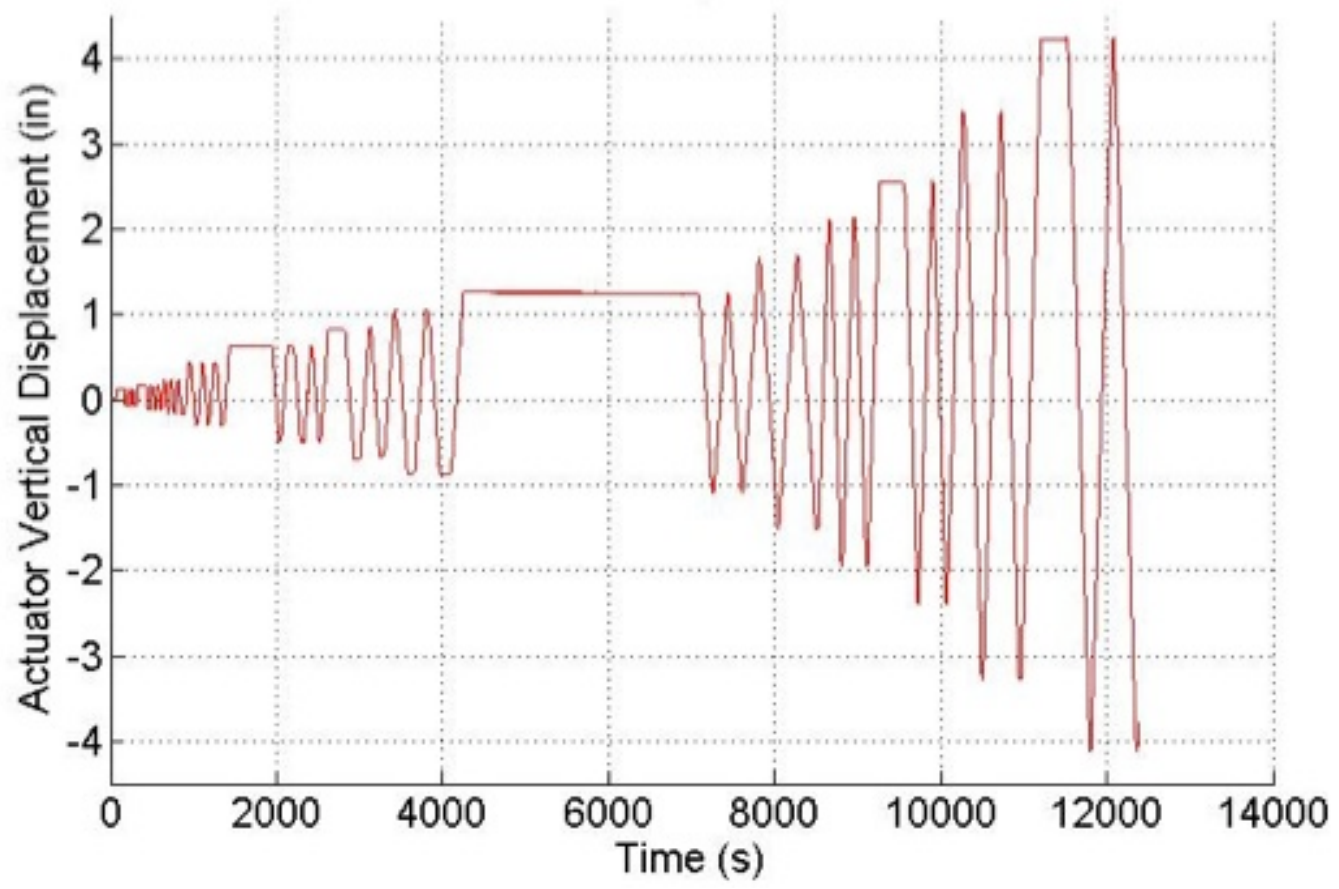

Figure 172. Actuator vertical displacement versus time - Test 3

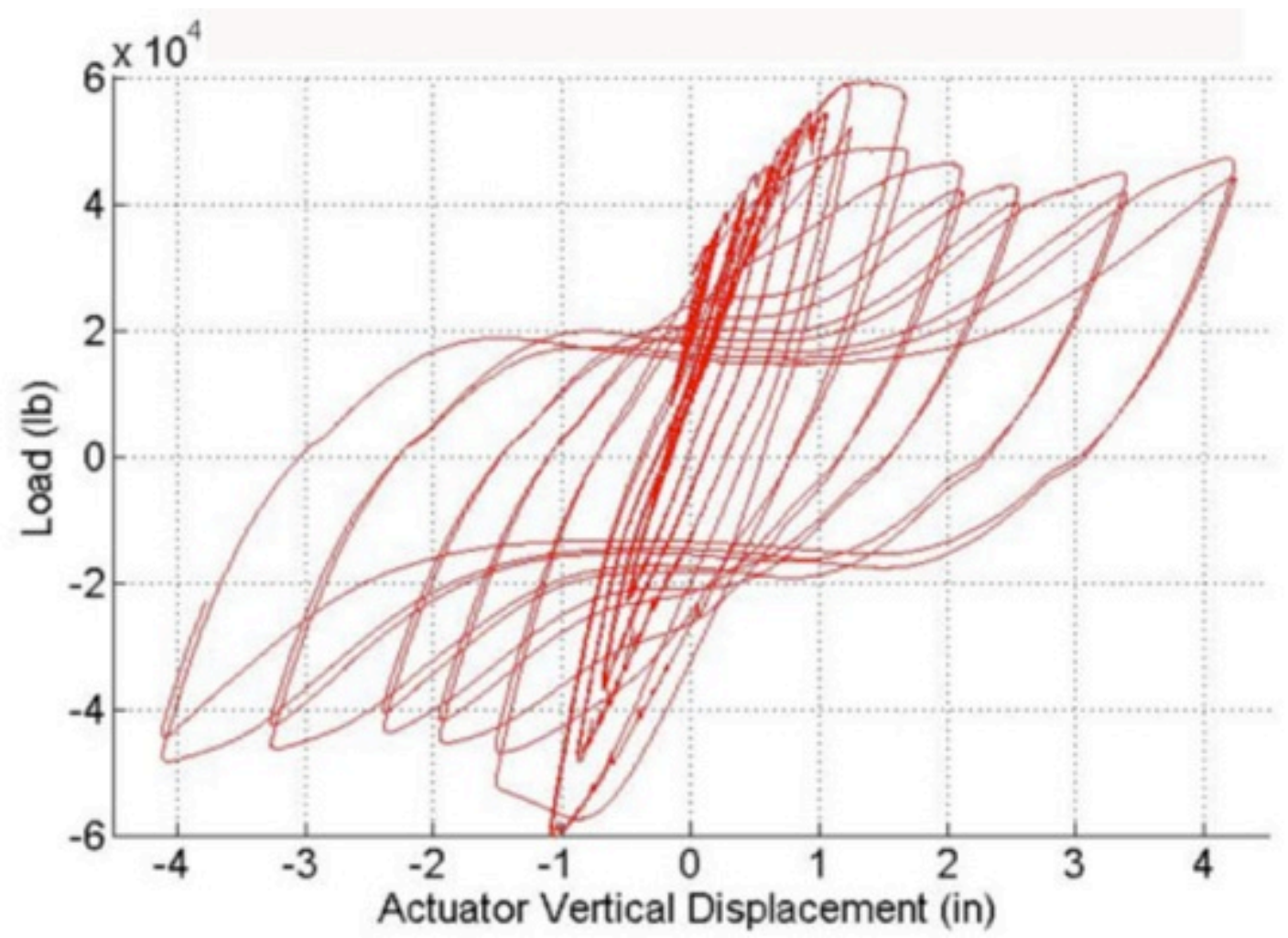

Figure 173. Load versus Actuator Vertical Displacement - Test 3 


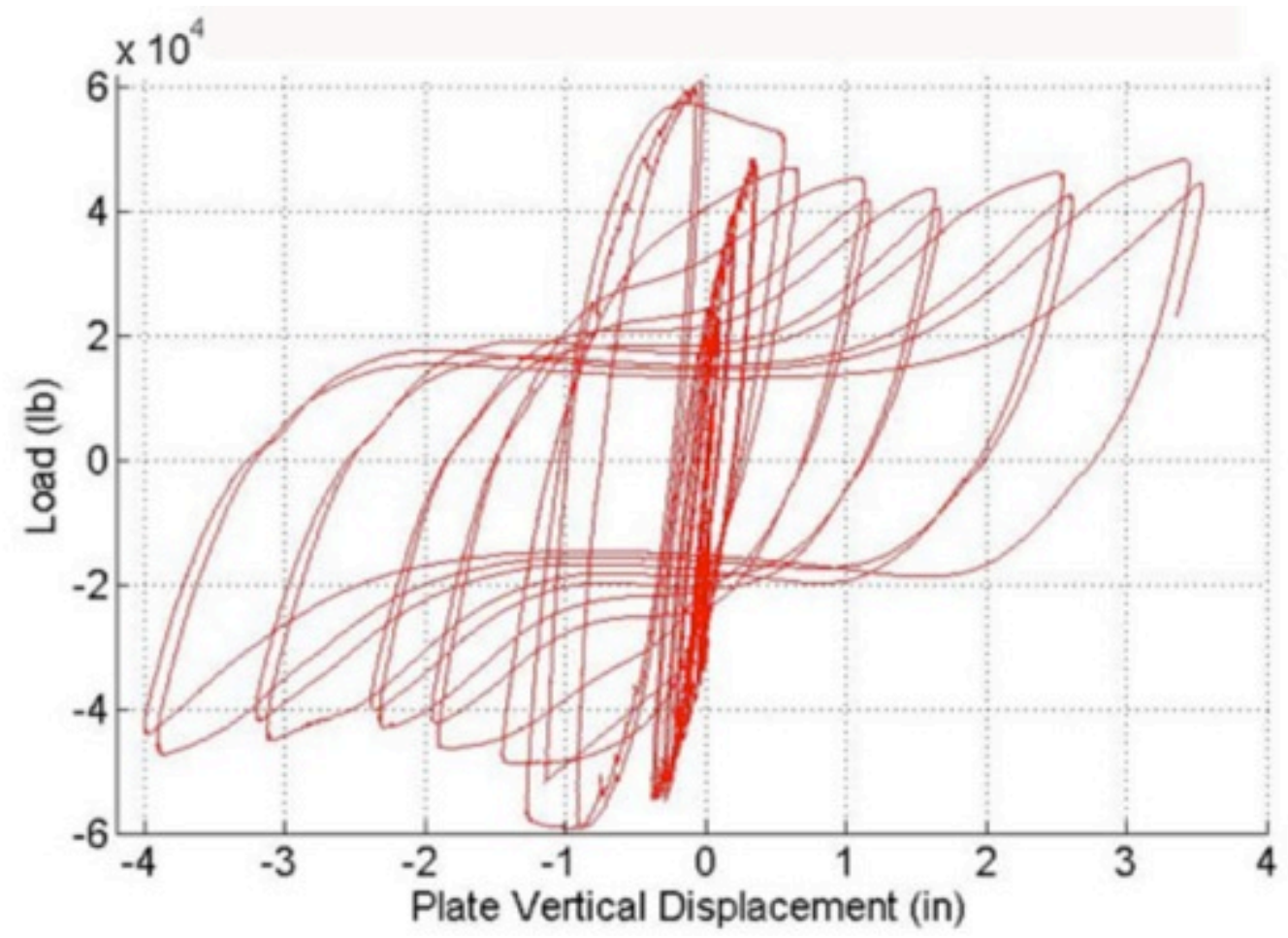

Figure 174. Load versus Plate Vertical Displacement - Test 3

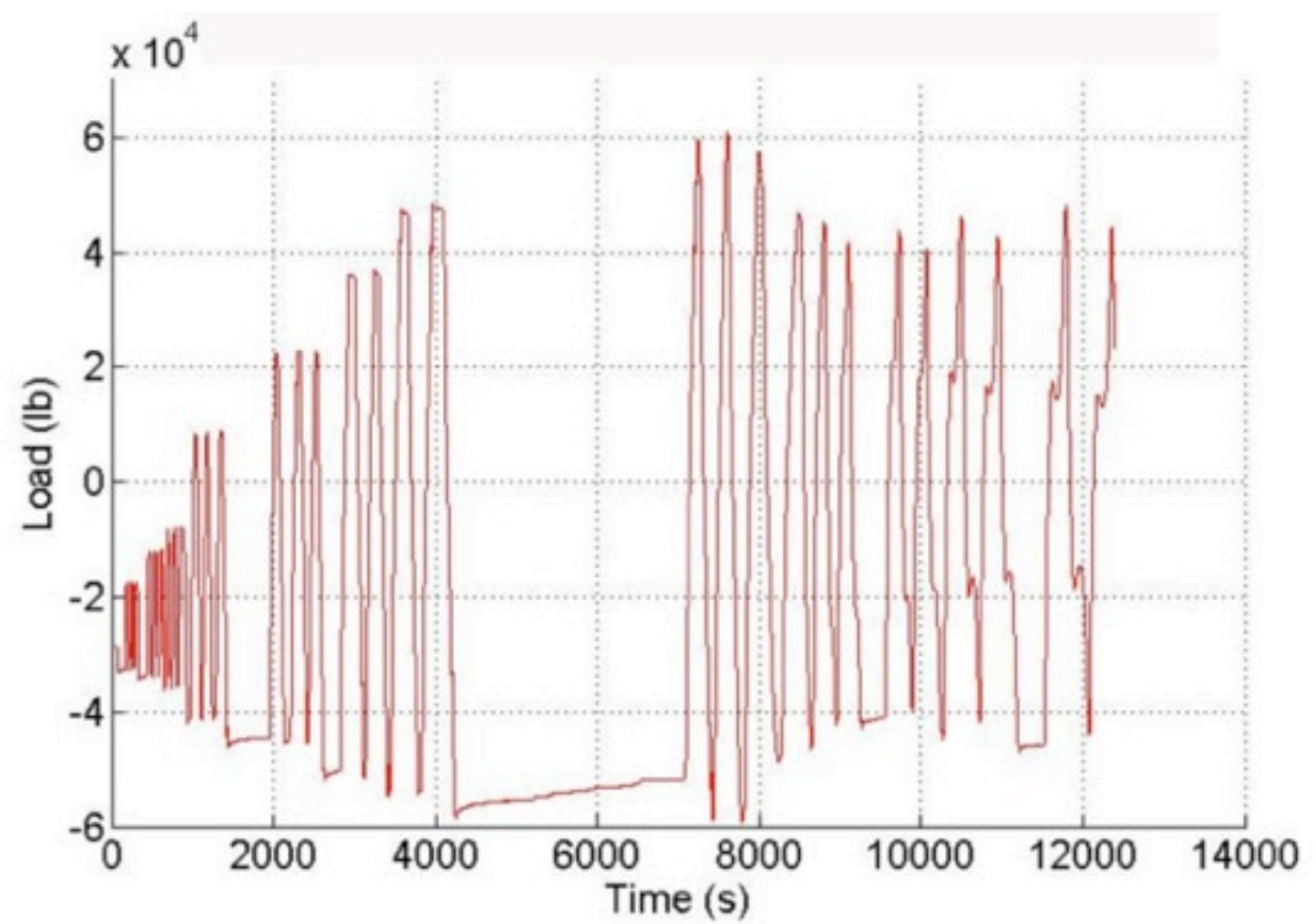

Figure 175. Load versus Time - Test 3 


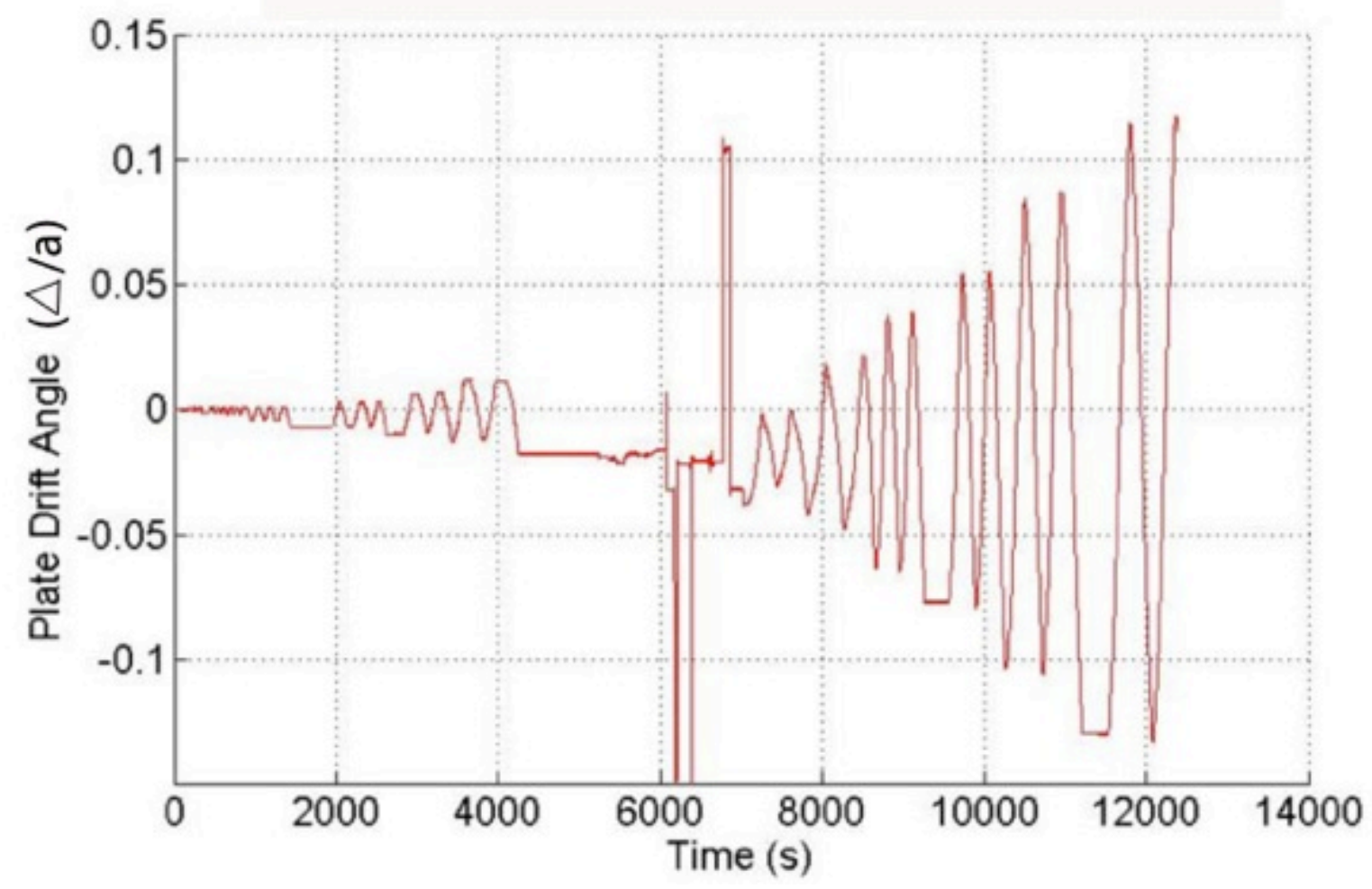

Figure 176. Plate drift Angle versus Time - Test 3

TEST 4.

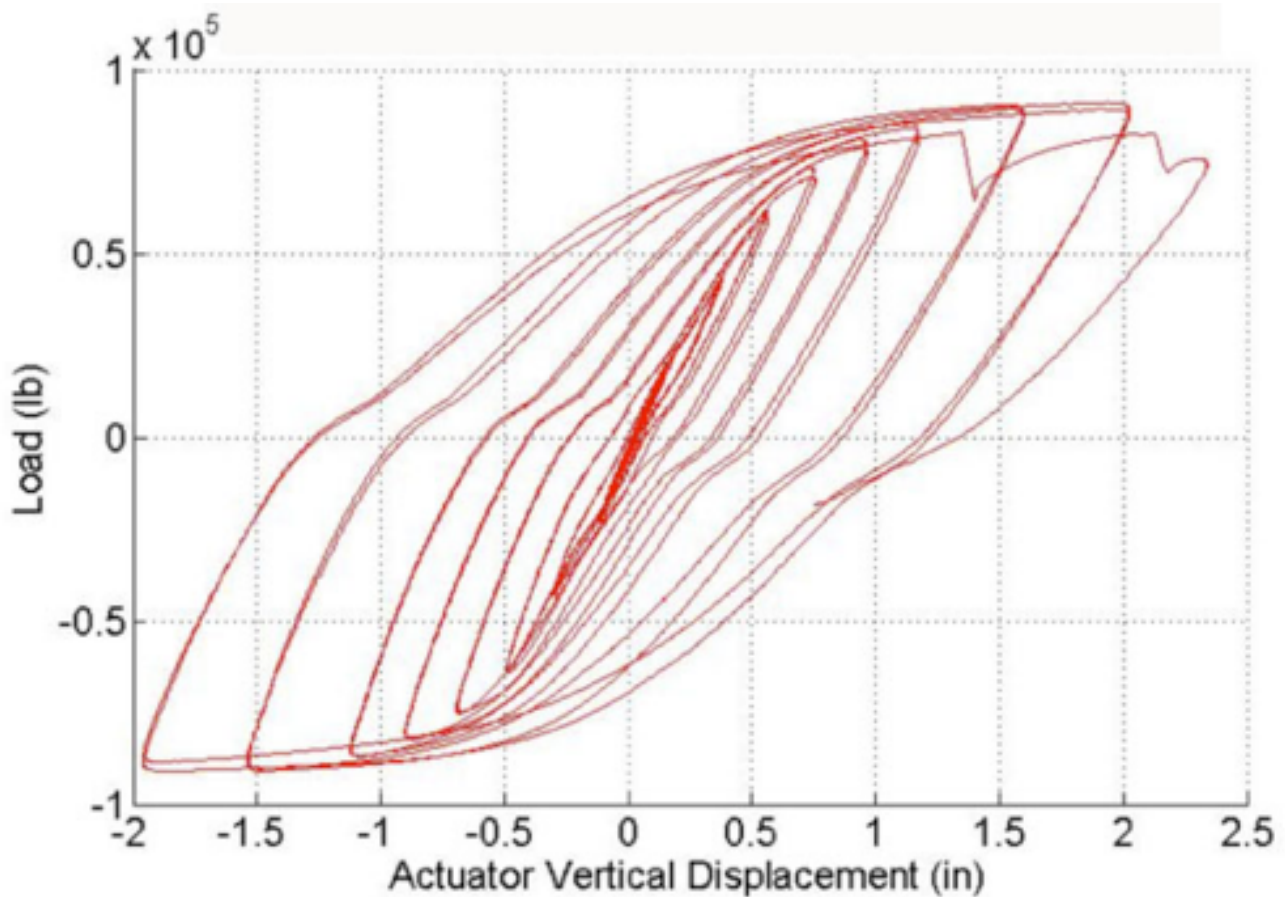

Figure 177. Load versus Actuator Vertical Displacement - Test 4 


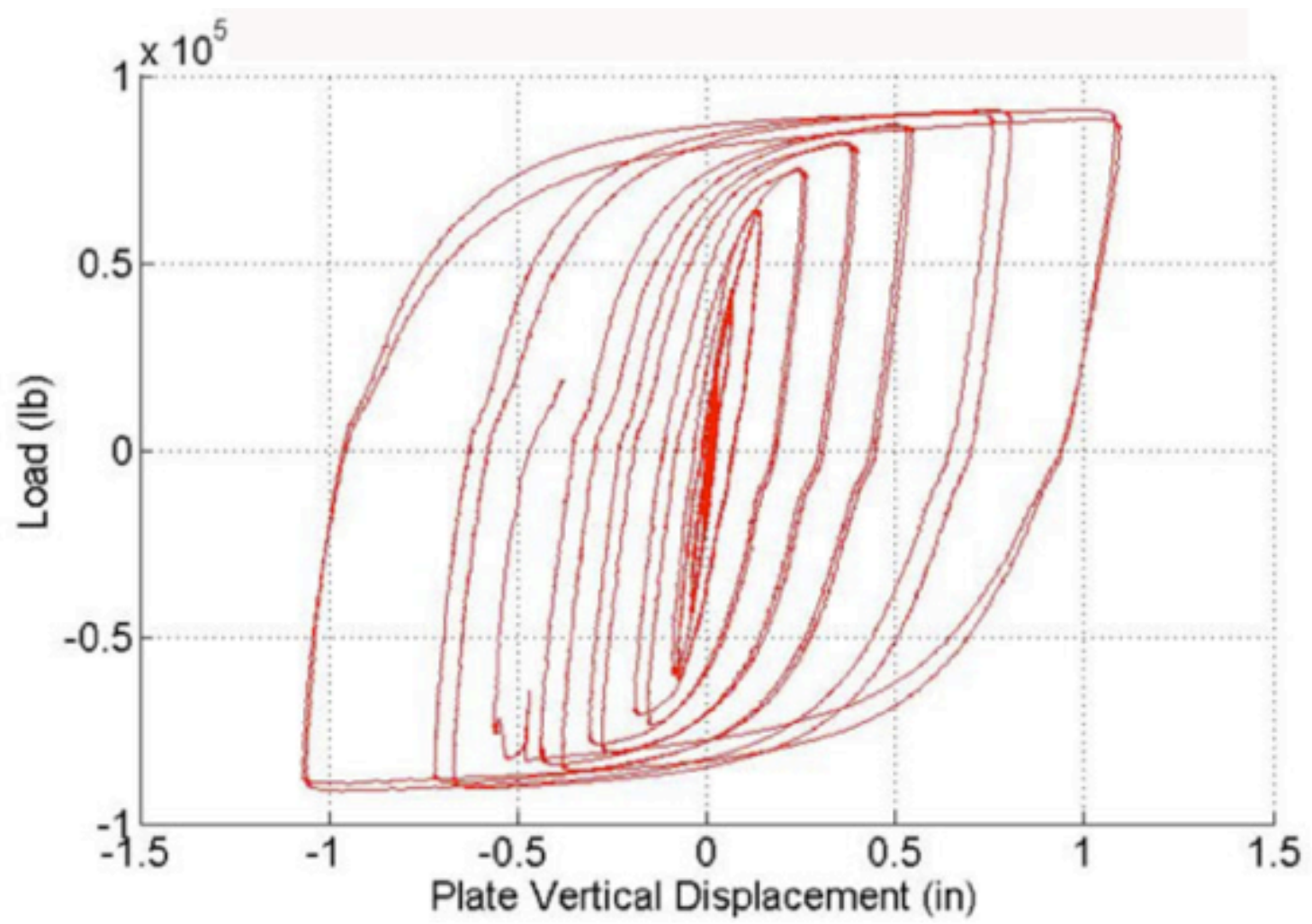

Figure 178. Load versus Plate Vertical Displacement - Test 4

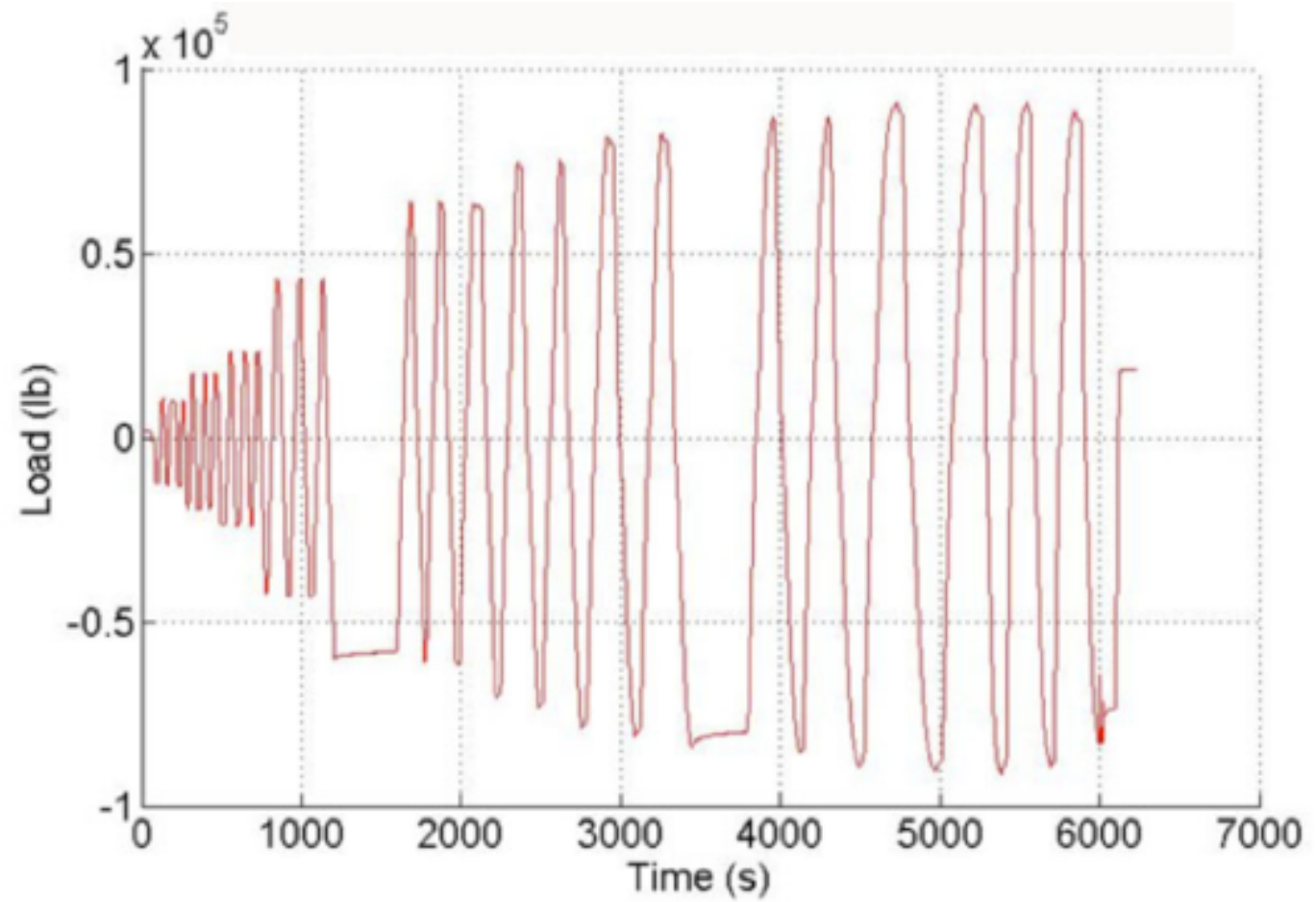

Figure 179. Load versus Time - Test 4 


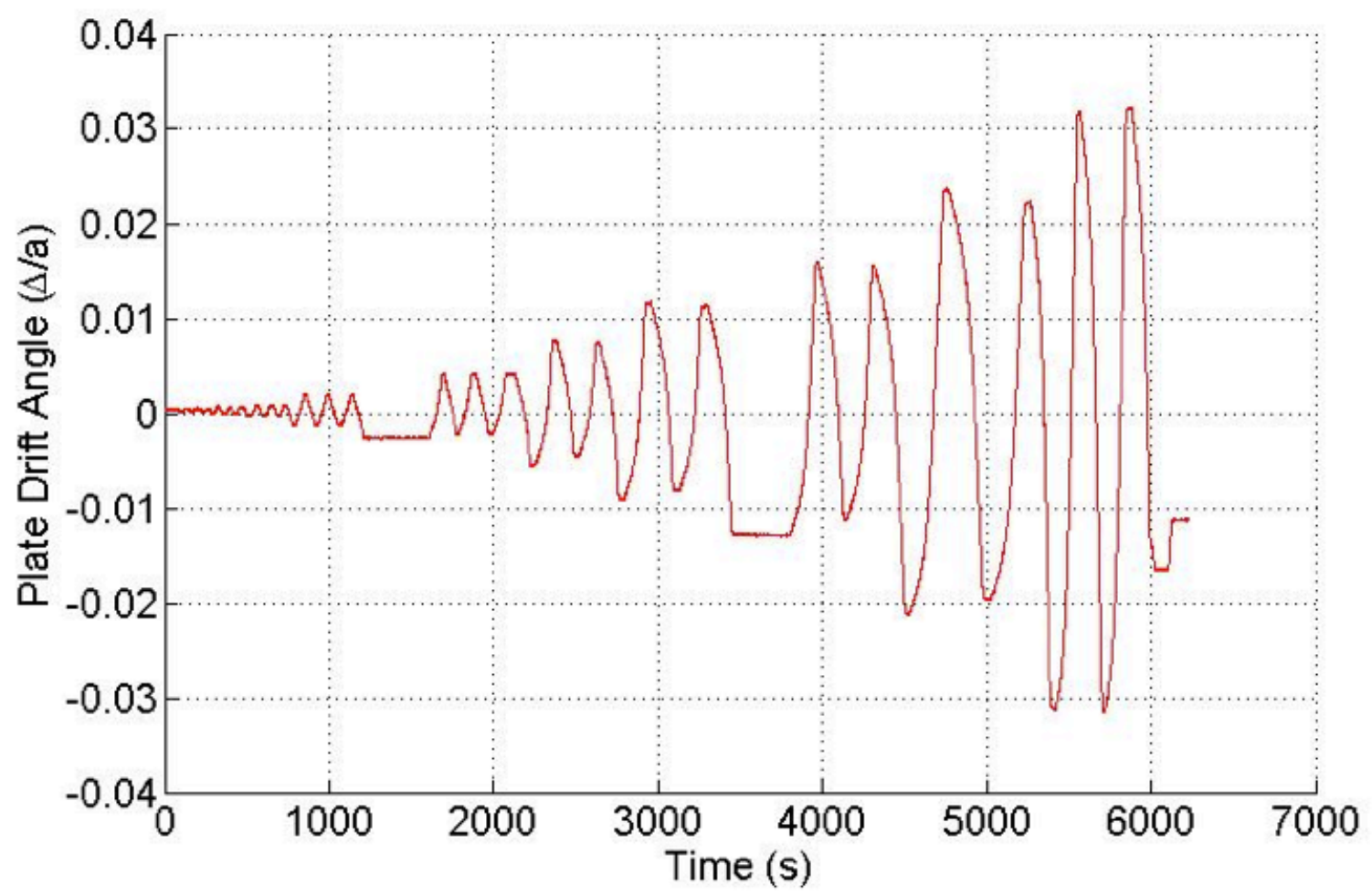

Figure 180. Plate Drift Angle versus Time - Test 4

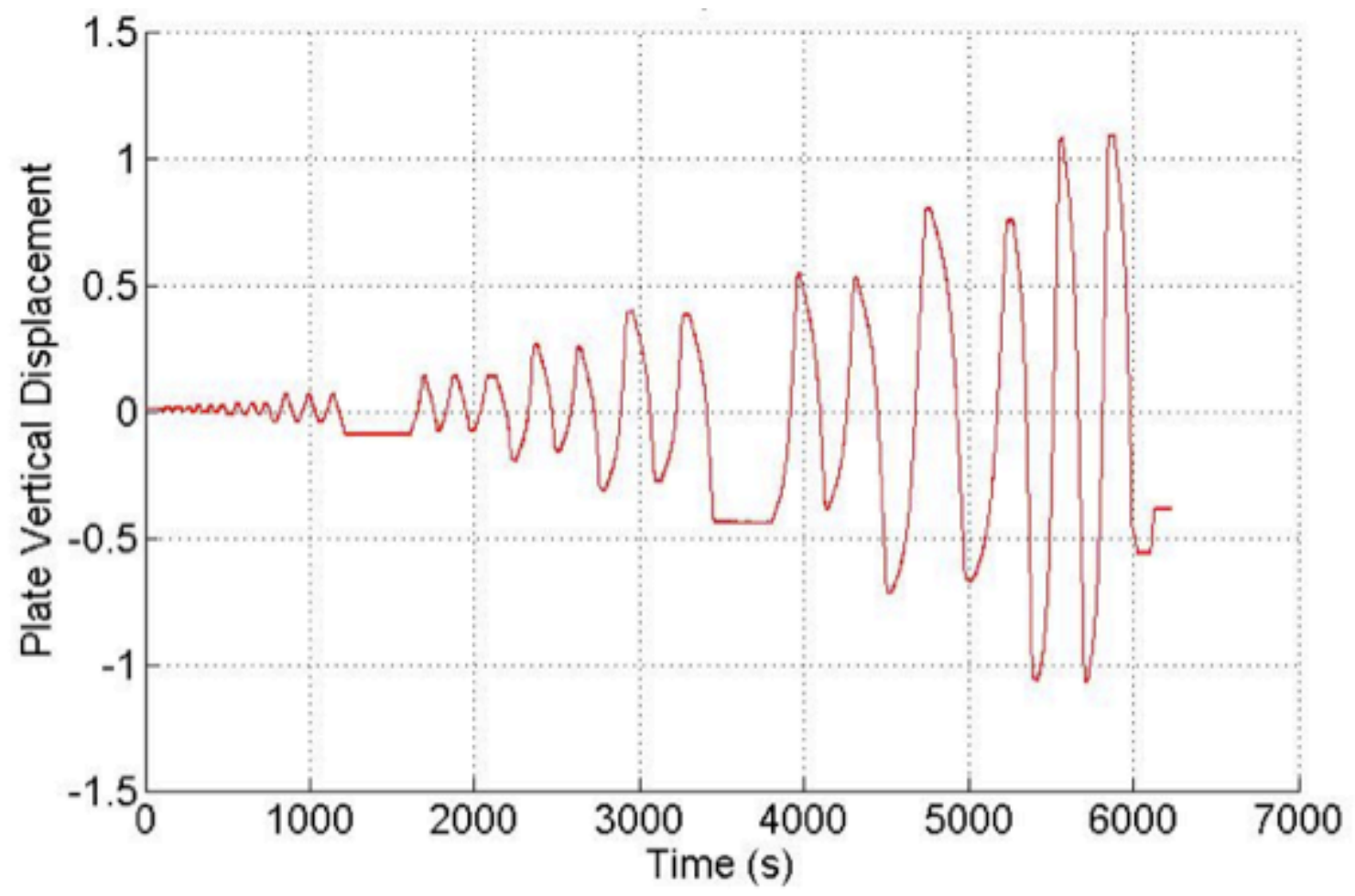

Figure 181. Plate Vertical Displacement versus Time - Test 4 


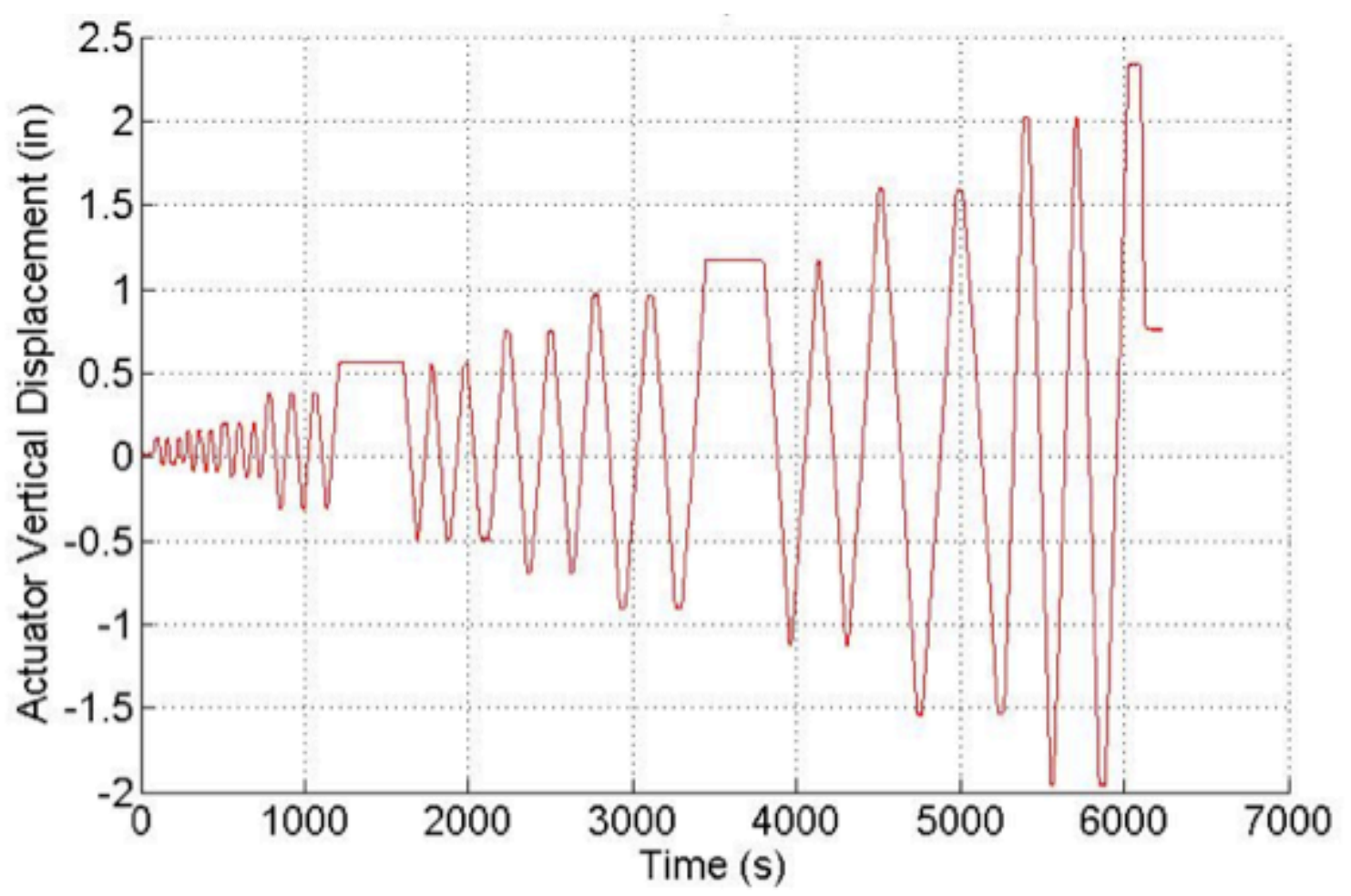

Figure 182. Actuator Vertical Displacement versus Time - Test 4

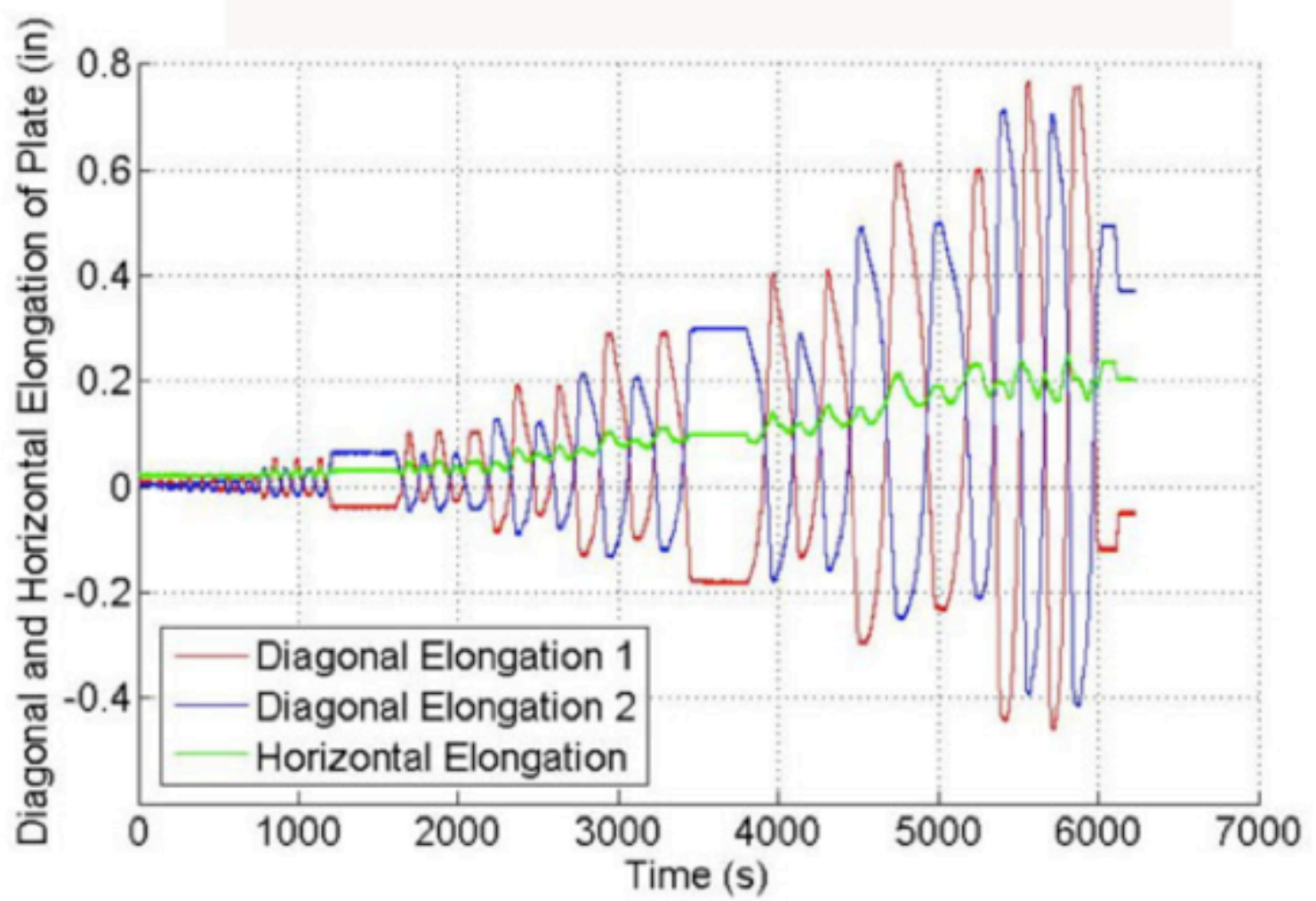

Figure 183. Diagonal and Horizontal Elongation of Plate versus Time - Test 4 
TEST 5.

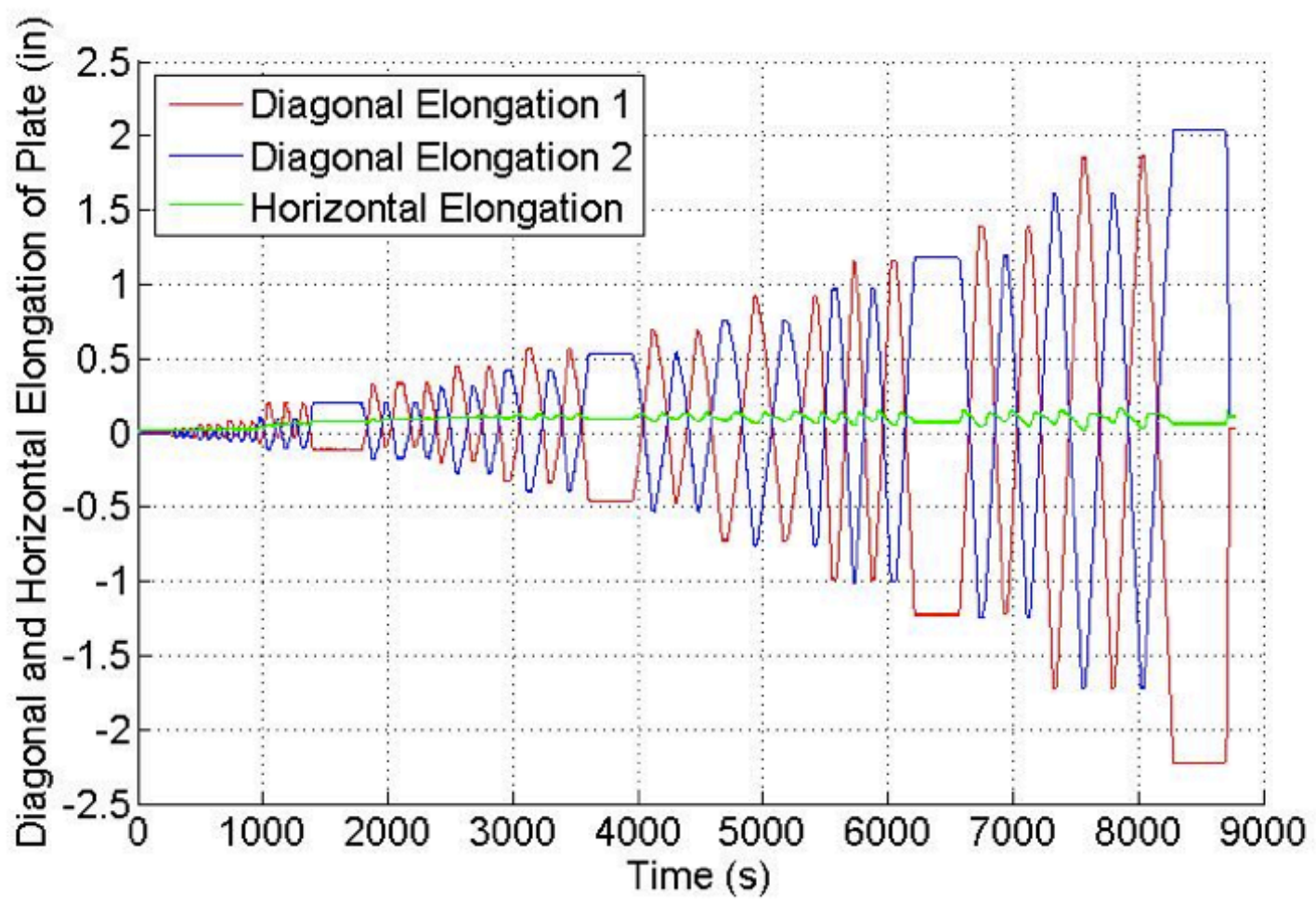

Figure 184. Diagonal and Horizontal Elongation of Plate versus Time - Test 5

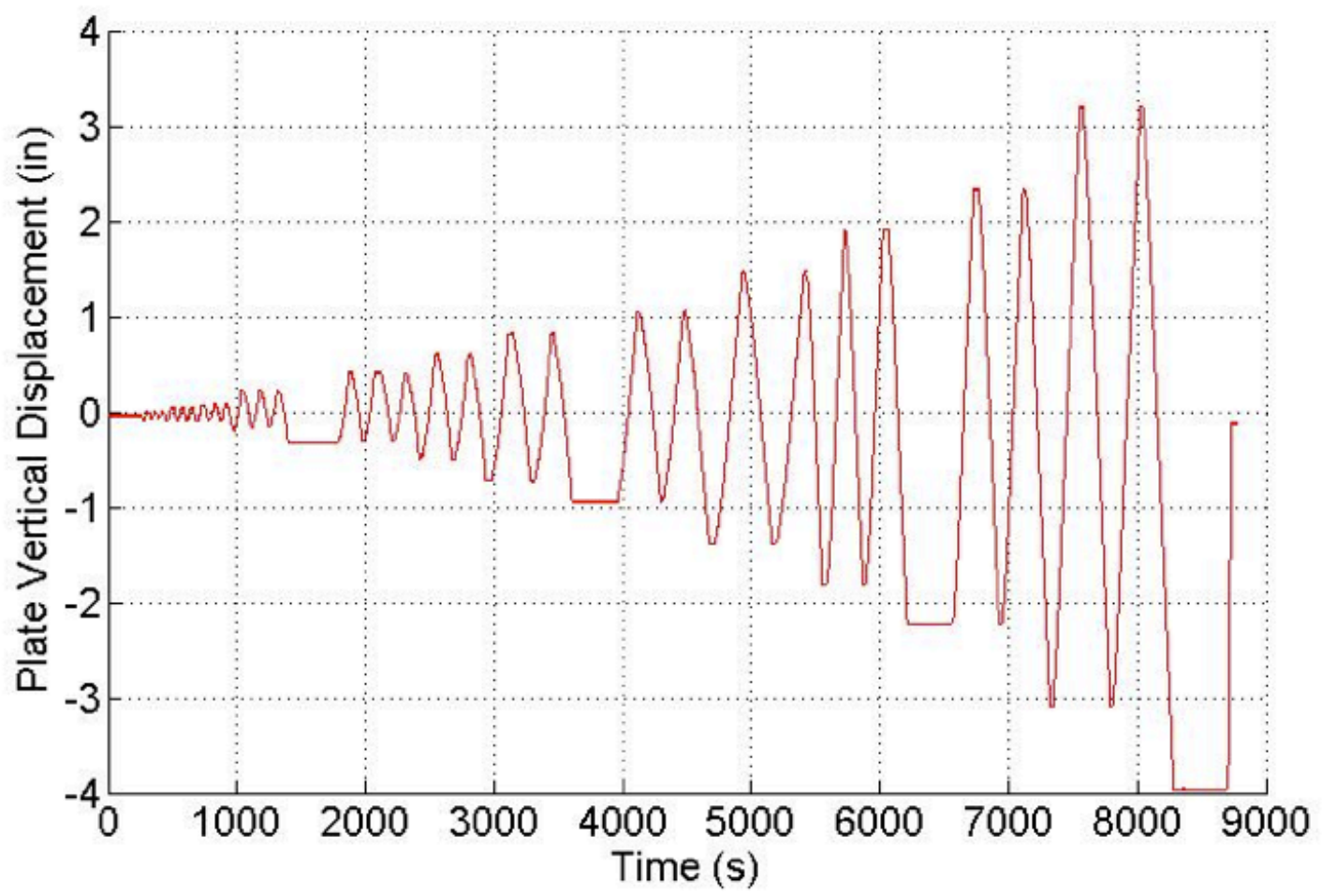

Figure 185. Plate vertical Displacement versus Time - Test 5 


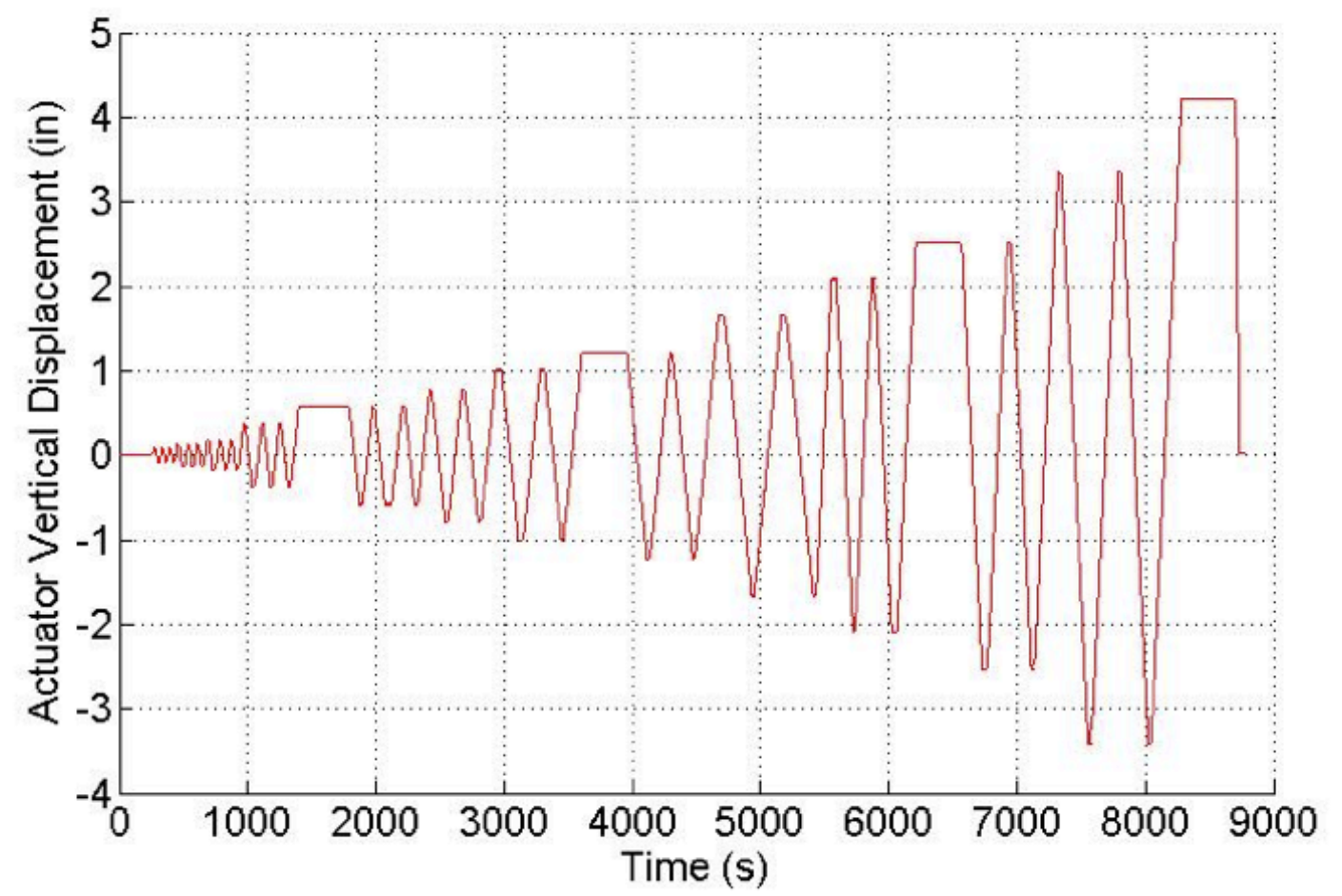

Figure 186. Actuator Vertical Displacement versus Time - Test 5

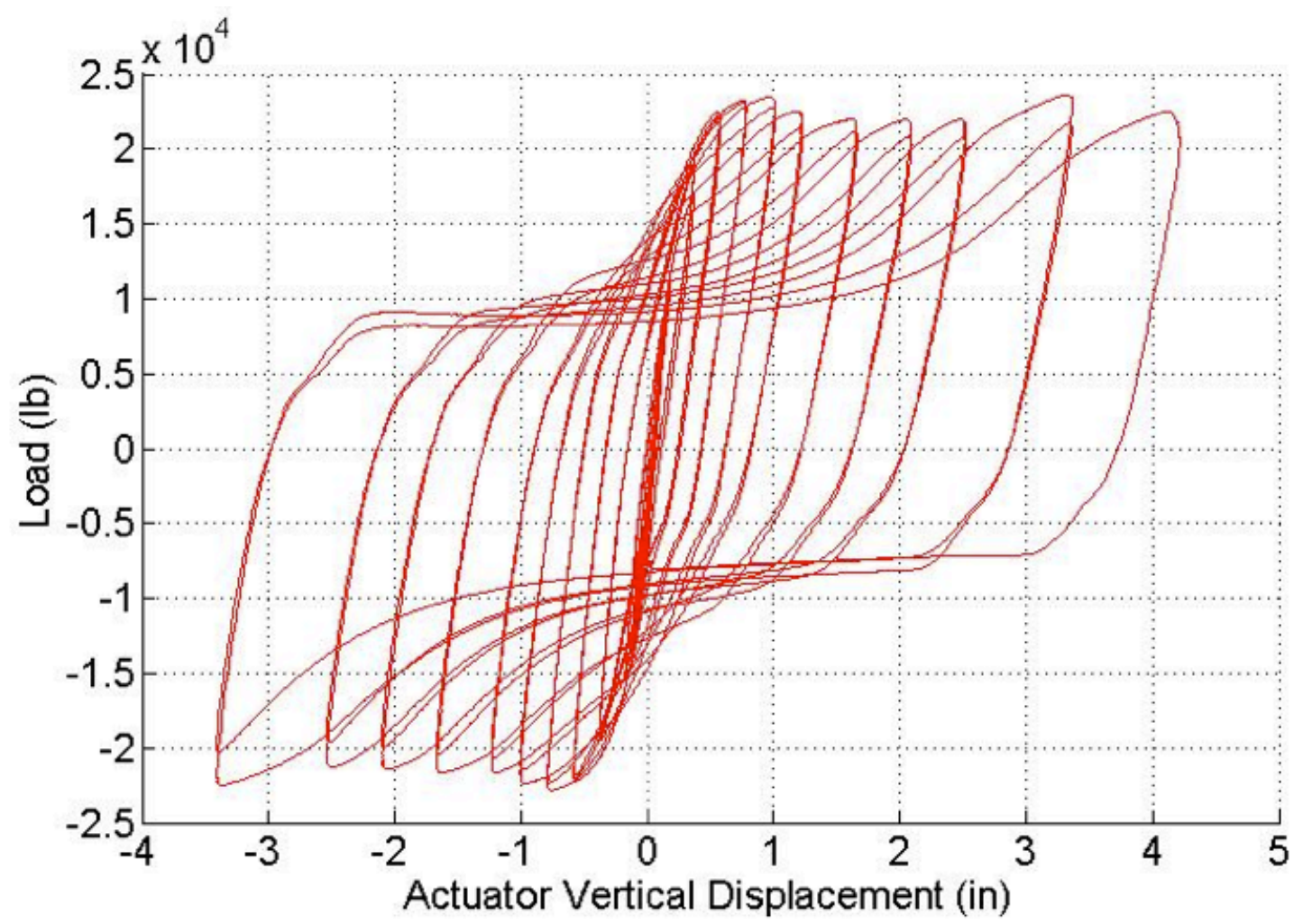

Figure 187. Load versus Actuator Vertical Displacement - Test 5 


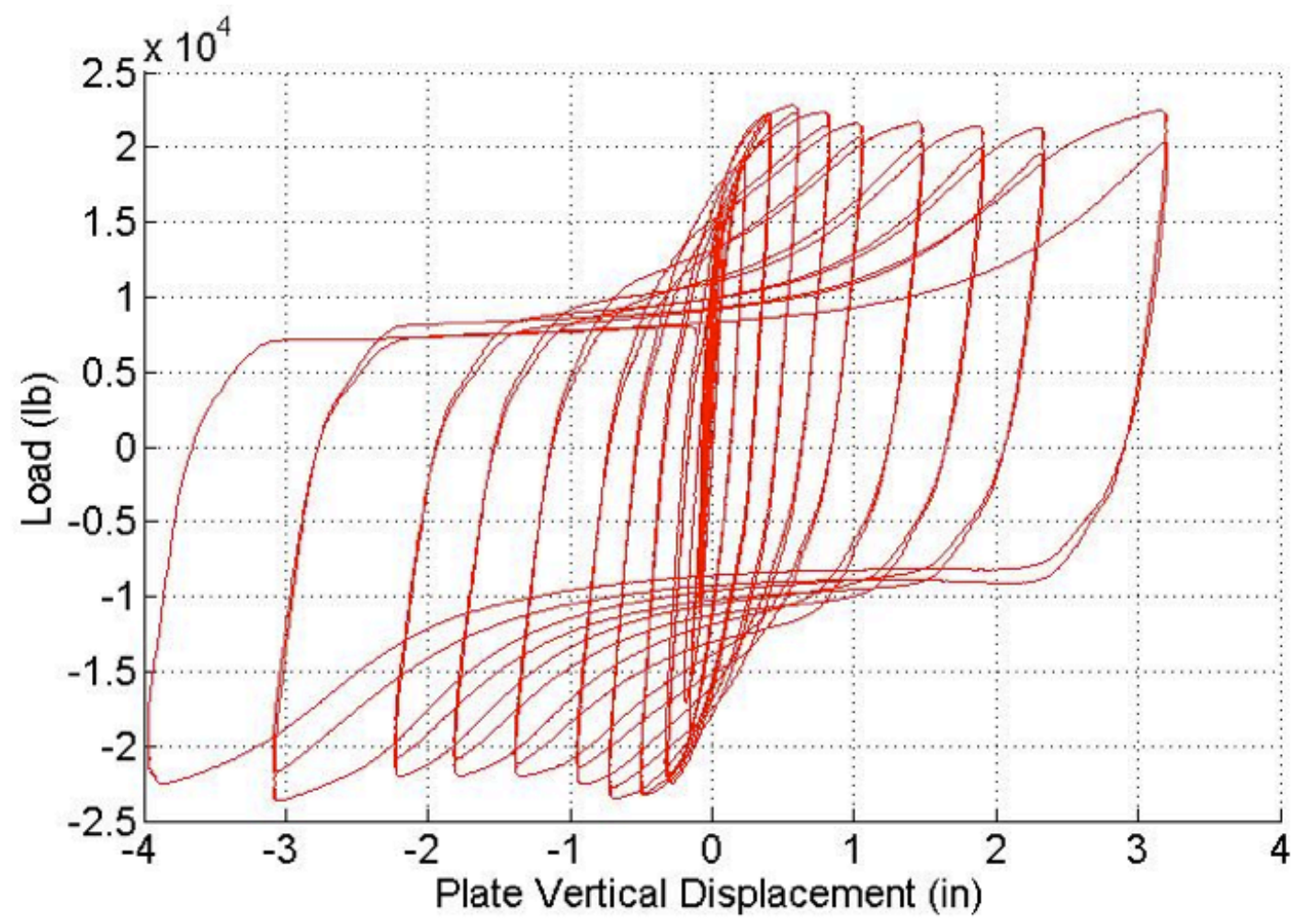

Figure 188, Load versus Plate Vertical Displacement - Test 5

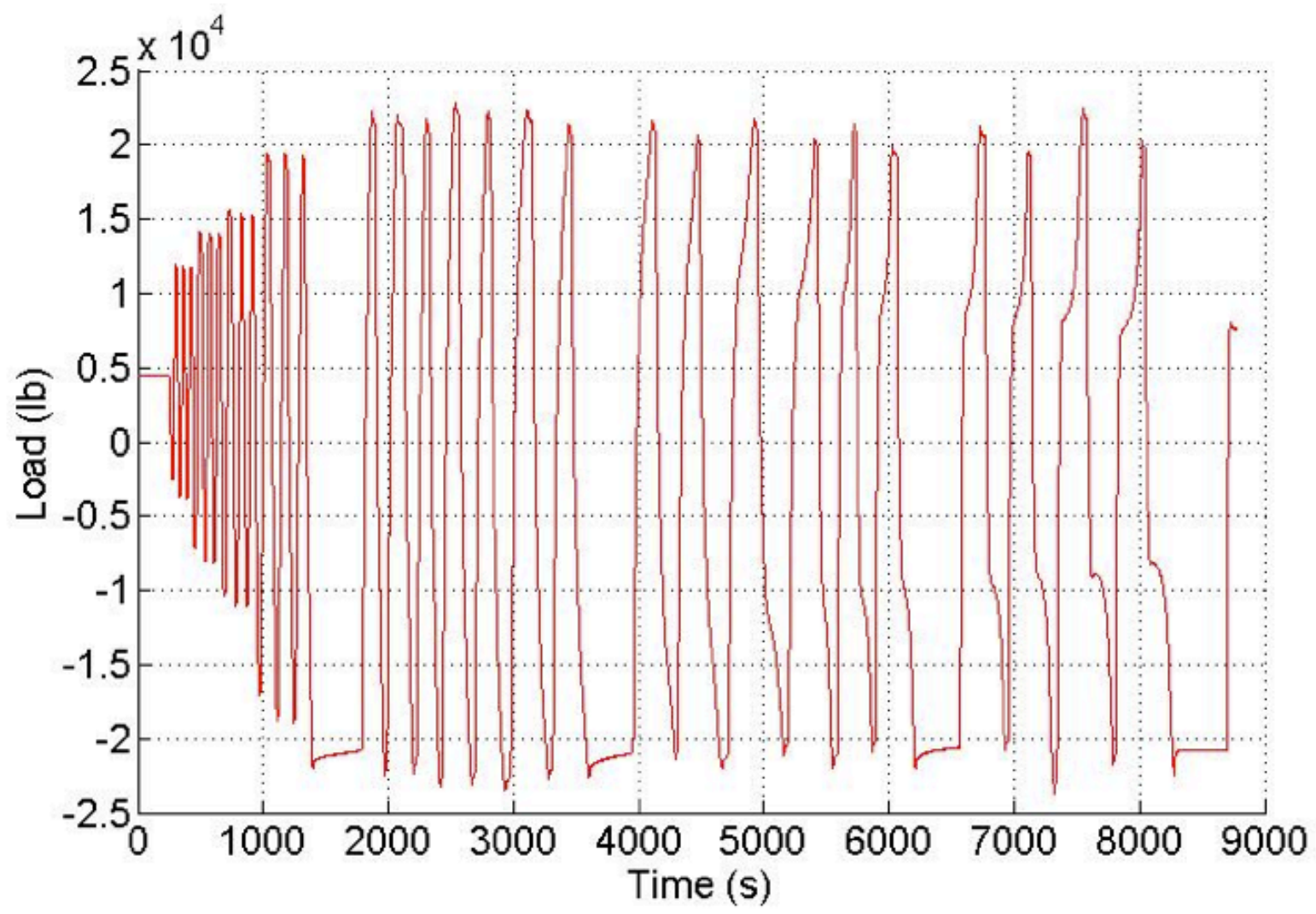

Figure 189. Load versus Time - Test 5 


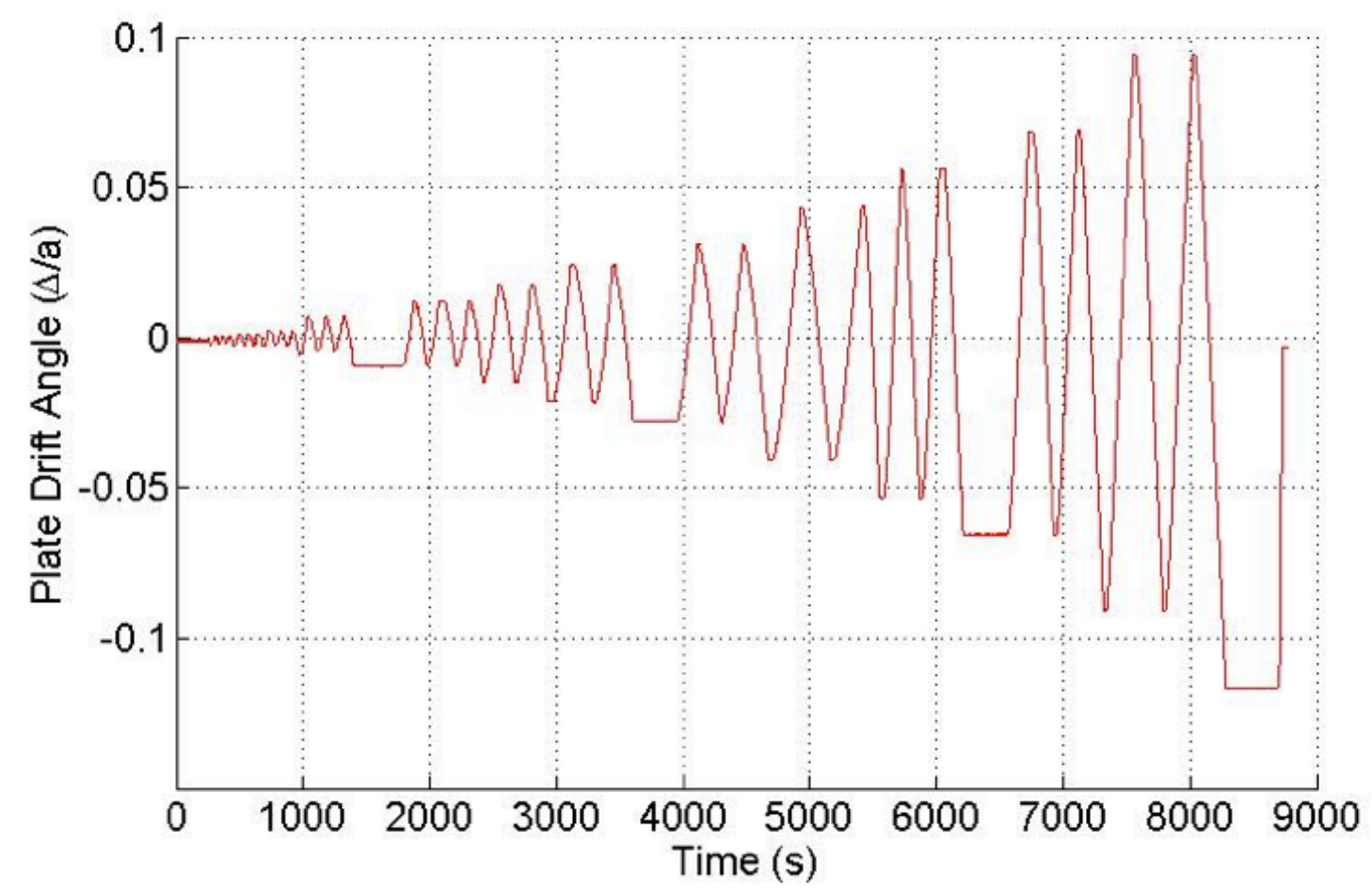

Figure 190. Plate Drift Angle versus Time - Test 5

TEST 6.

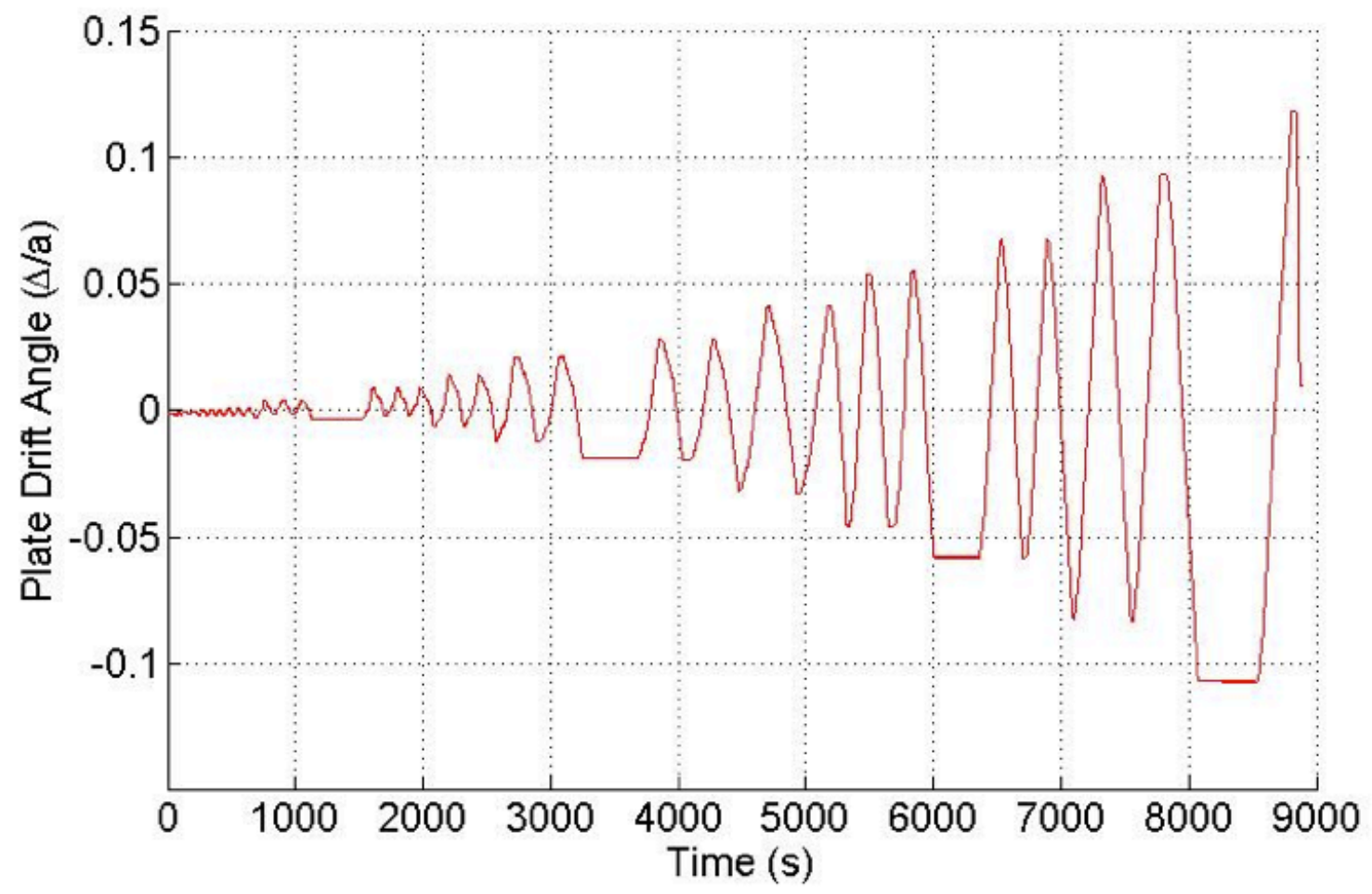

Figure 191. Plate Drift Angle versus Time - Test 6 


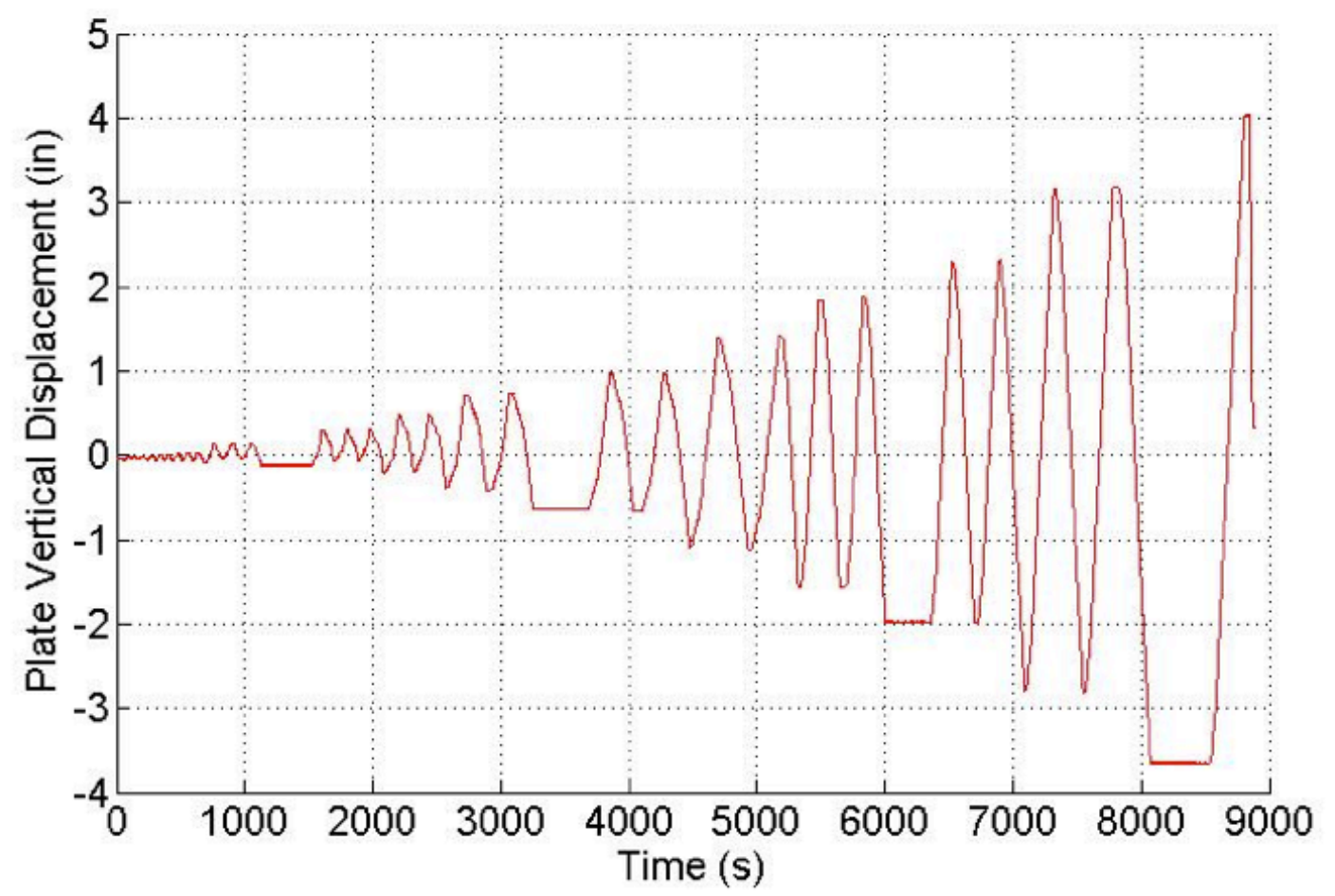

Figure 192. Plate Vertical Displacement versus Time - Test 6

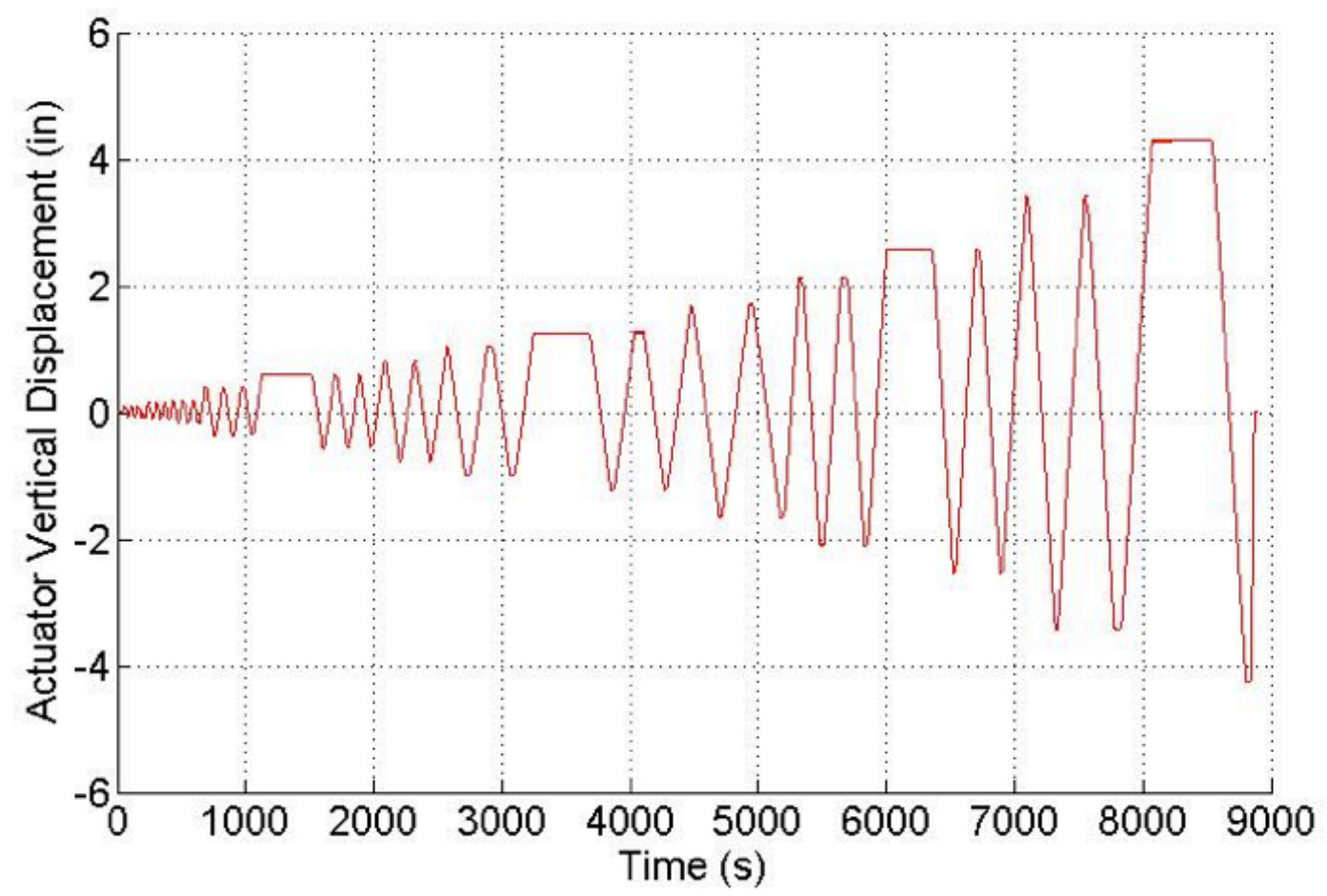

Figure 193. Actuator Vertical Displacement versus Time - Test 6 


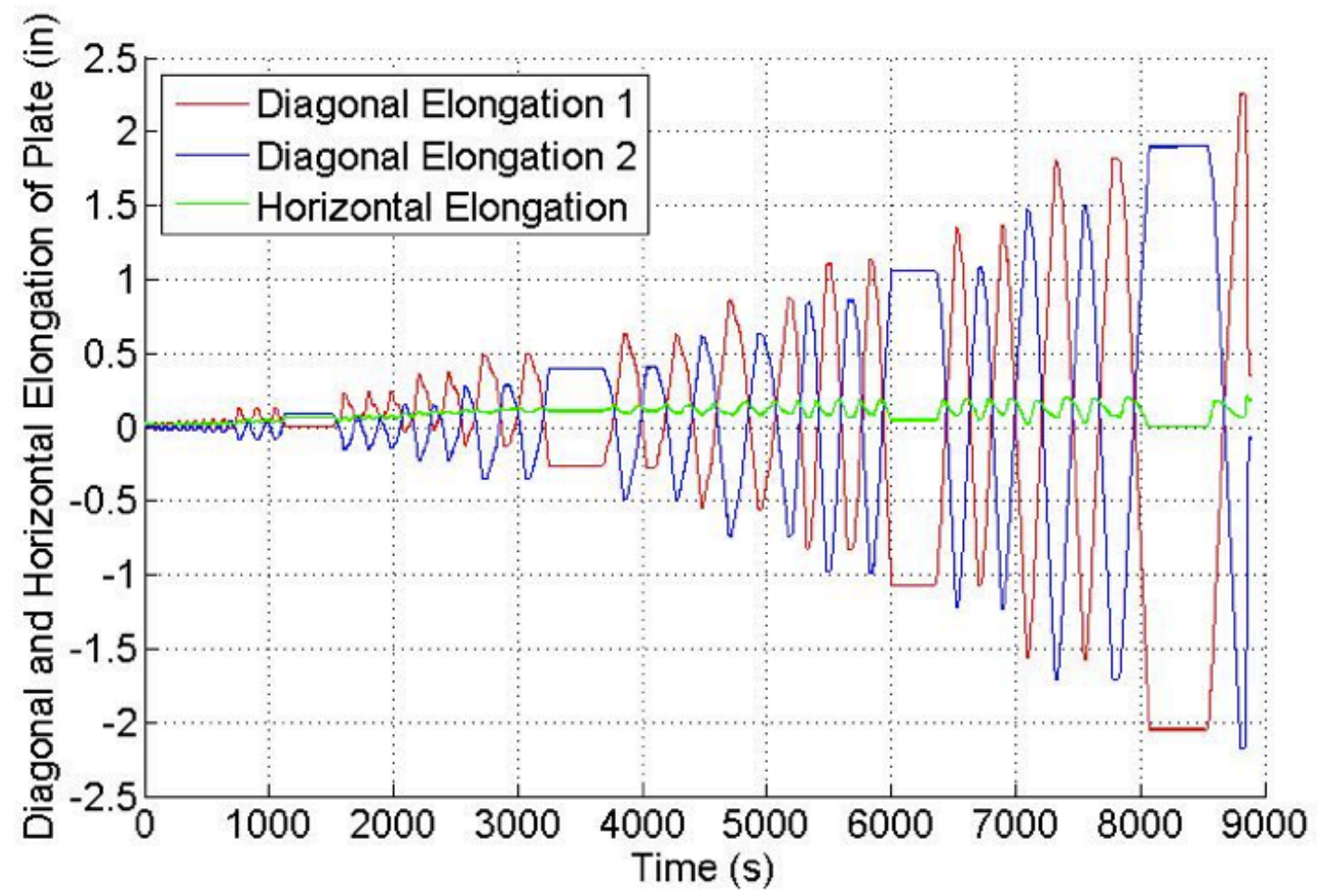

Figure 194. Diagonal and Horizontal Elongation of Plate versus Time - Test 6

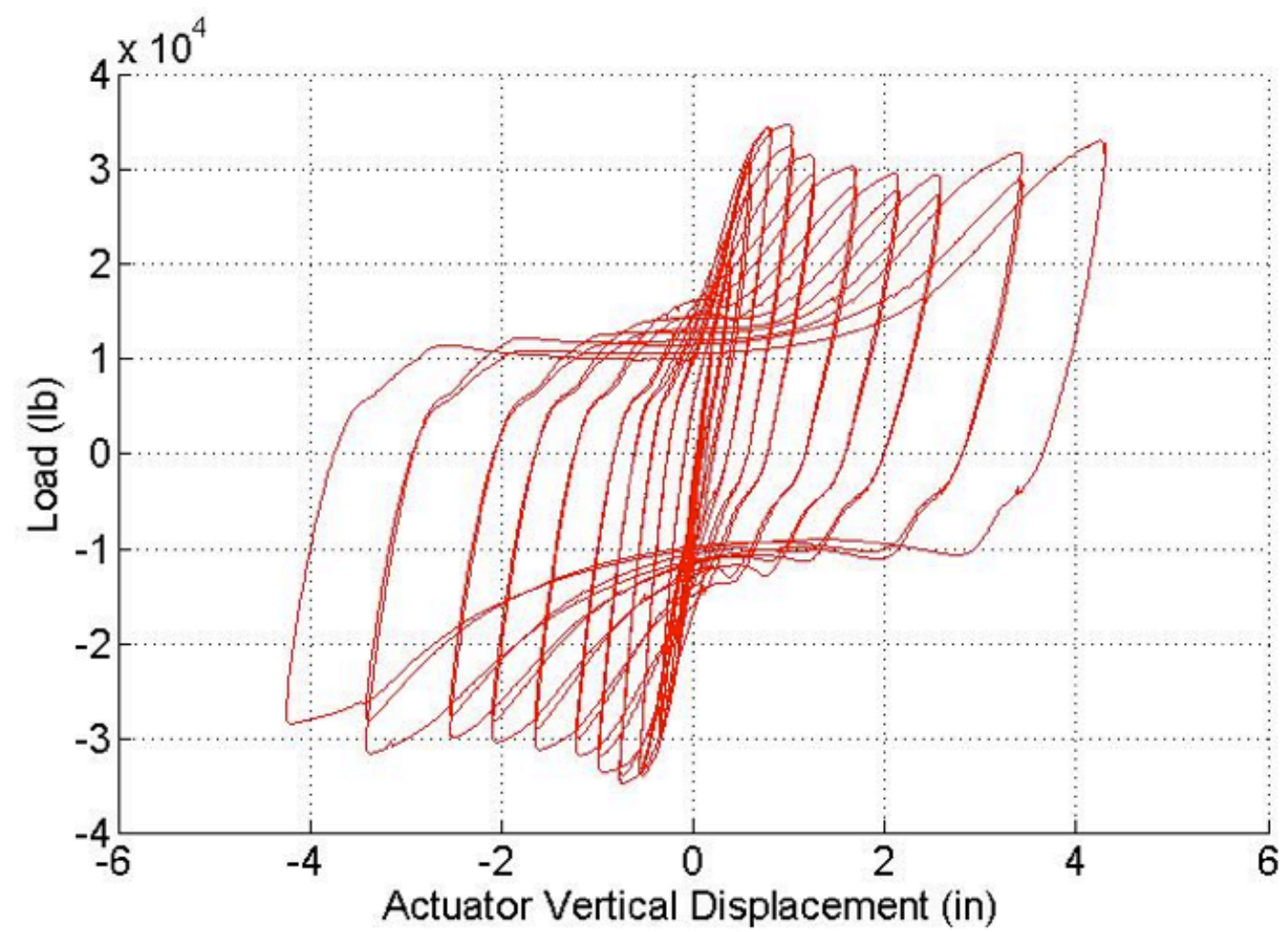

Figure 195. Load versus Actuator Vertical Displacement - Test 6 


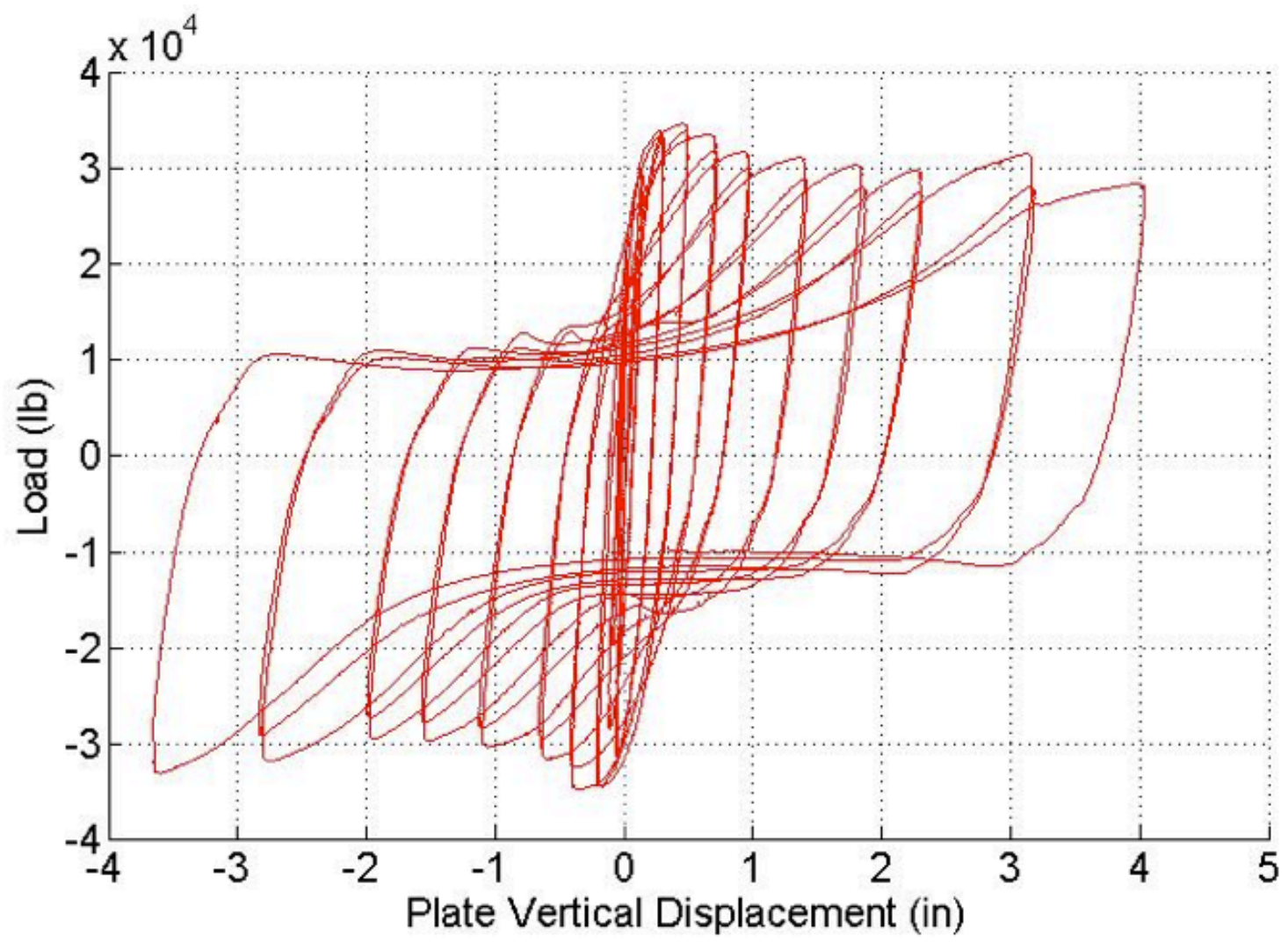

Figure 196. Load versus Plate Vertical Displacement - Test 6

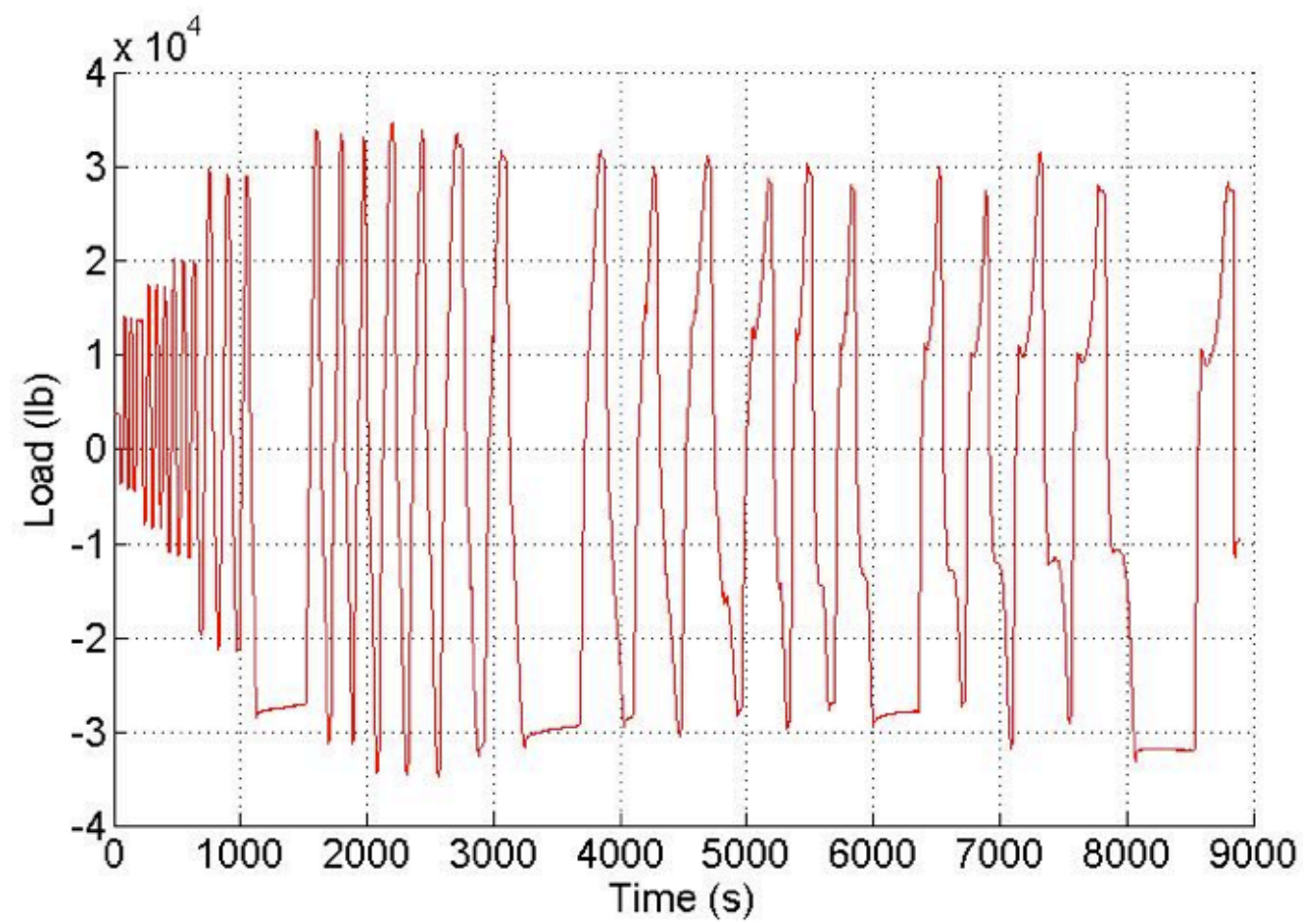

Figure 197. Load versus Time - Test 6 
TEST 7.

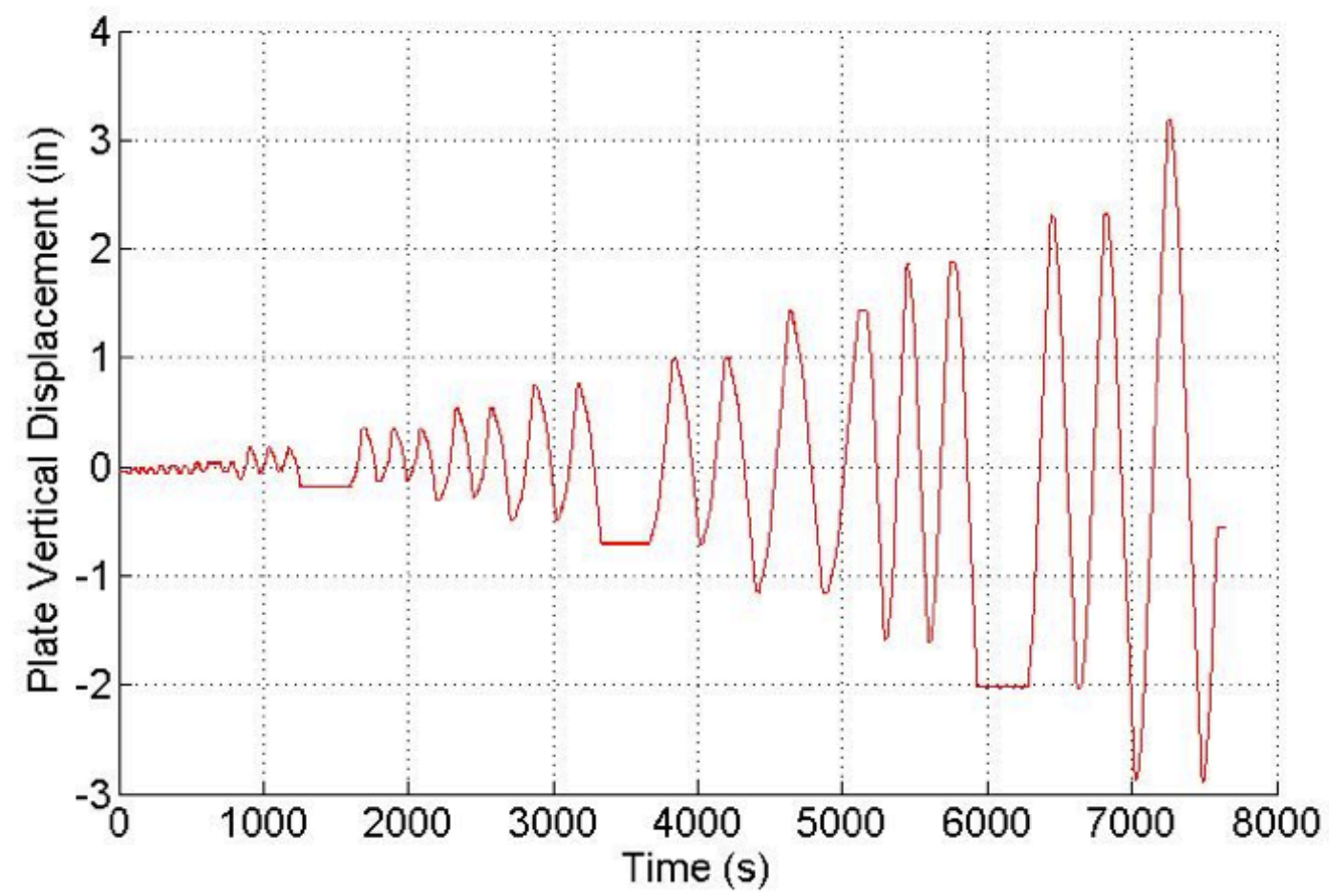

Figure 198. Plate Vertical Displacement versus Time - Test 7

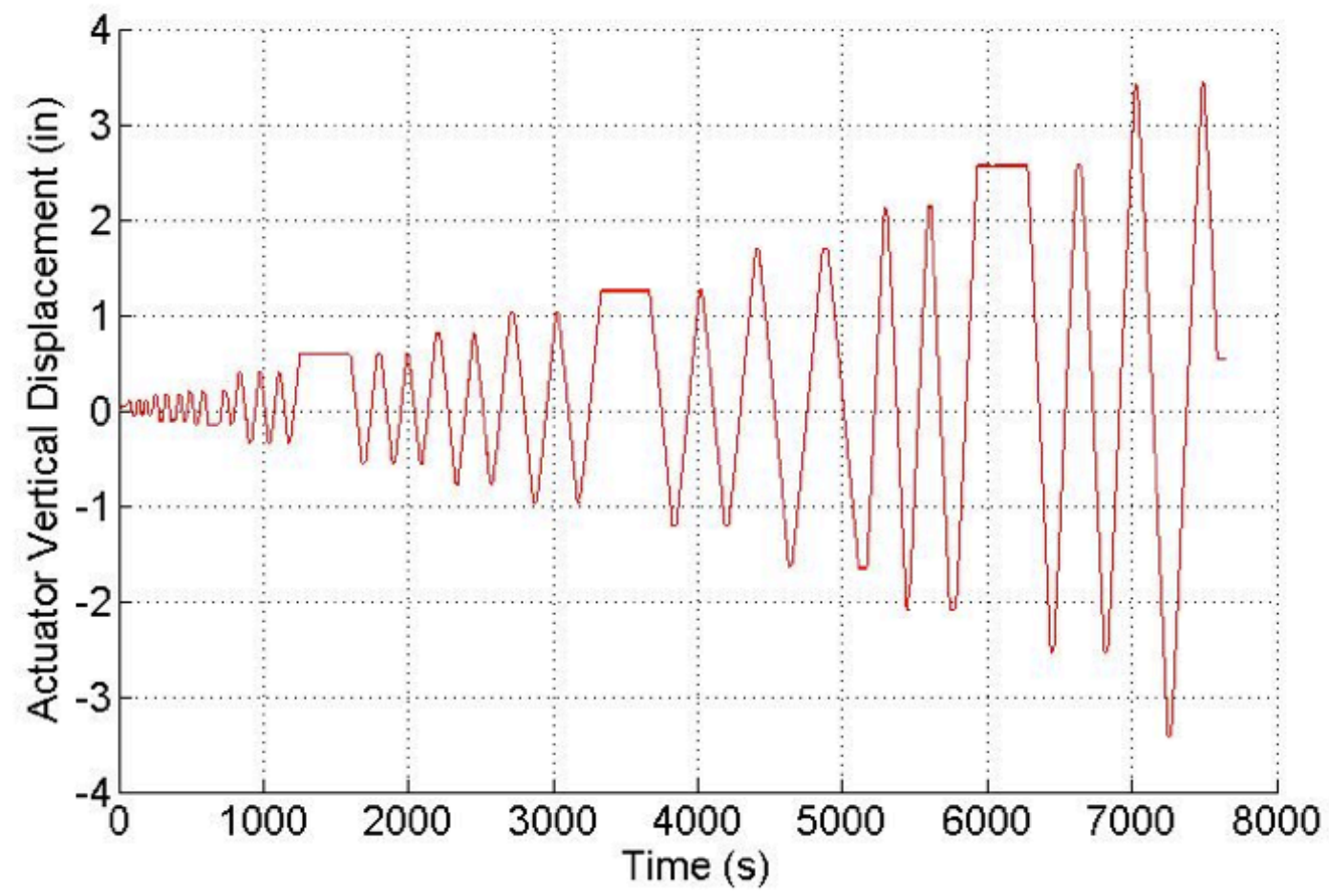

Figure 199. Actuator Vertical Displacement versus Time - Test 7 


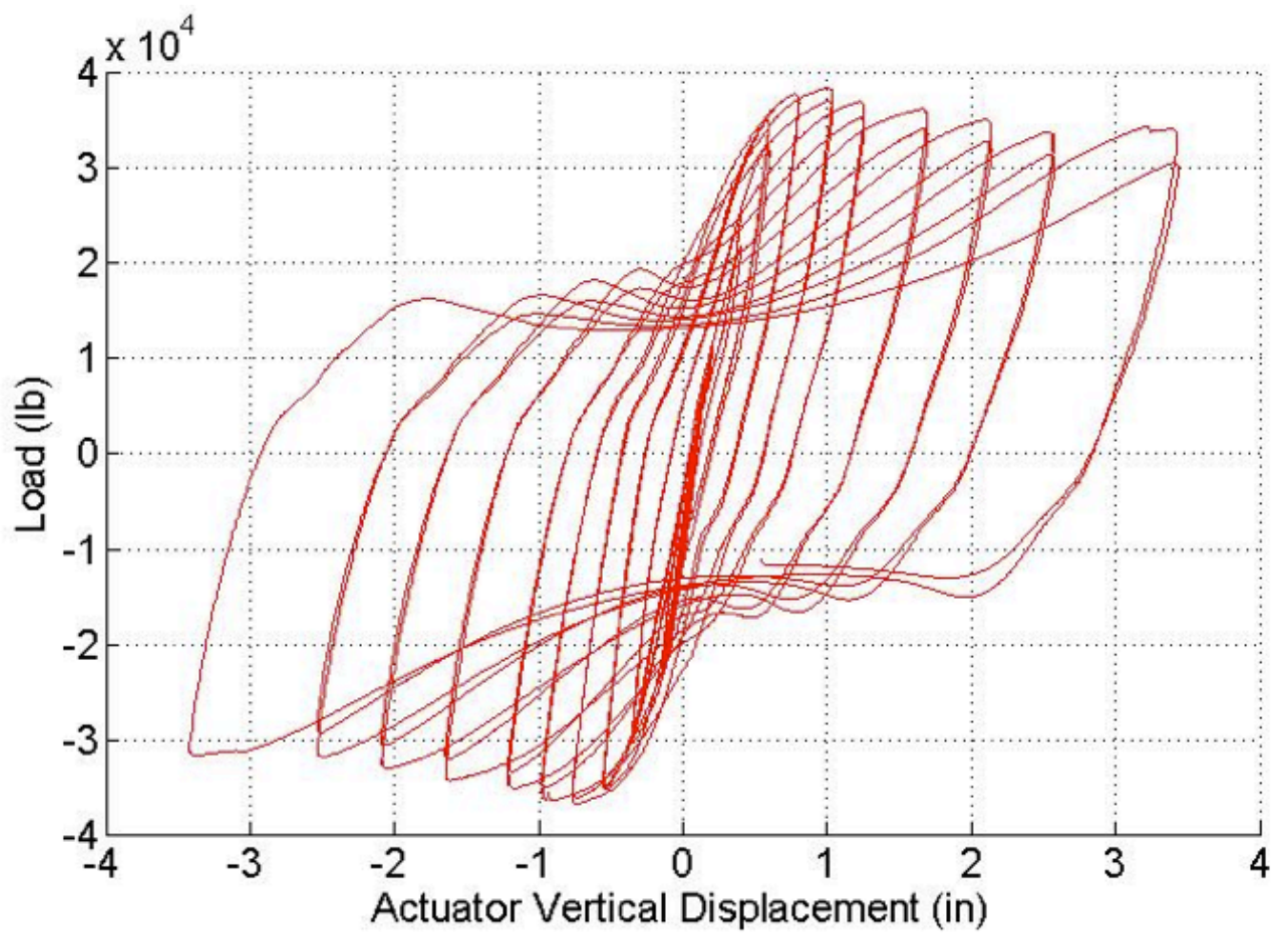

Figure 200. Load versus Actuator Vertical Displacement - Test 7

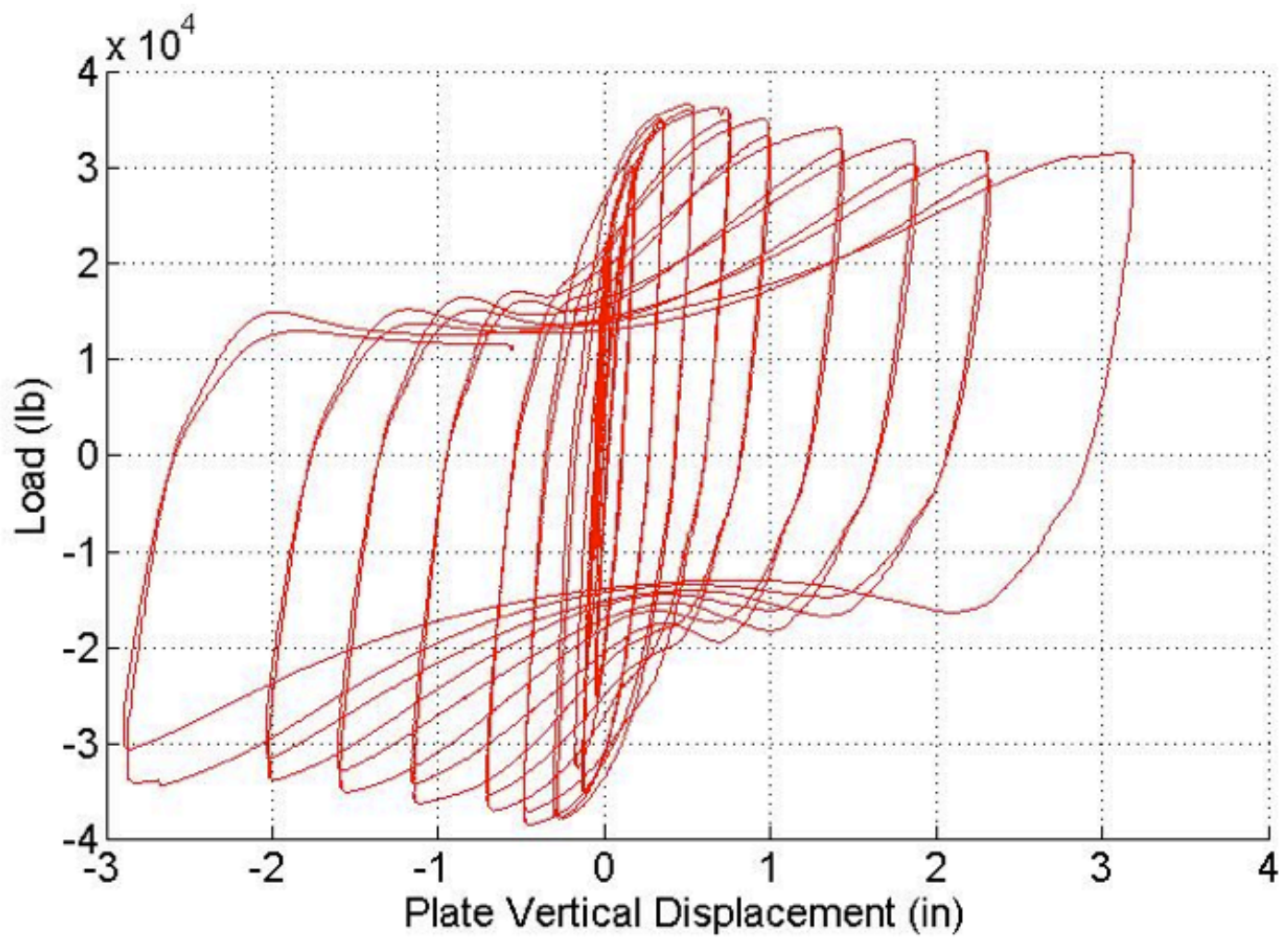

Figure 201. Load versus Plate Vertical Displacement - Test 7 


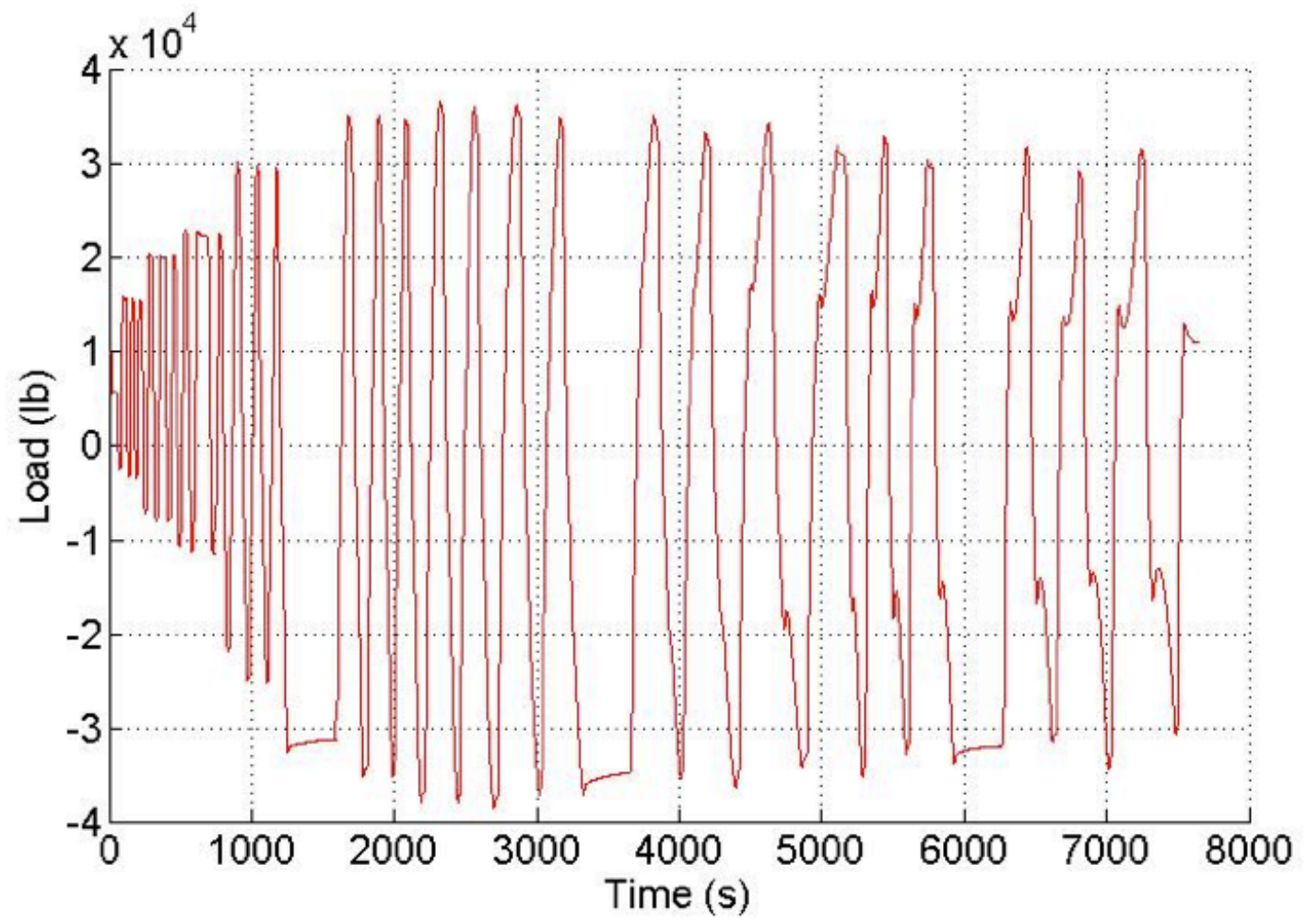

Figure 202. Load versus Time - Test 7

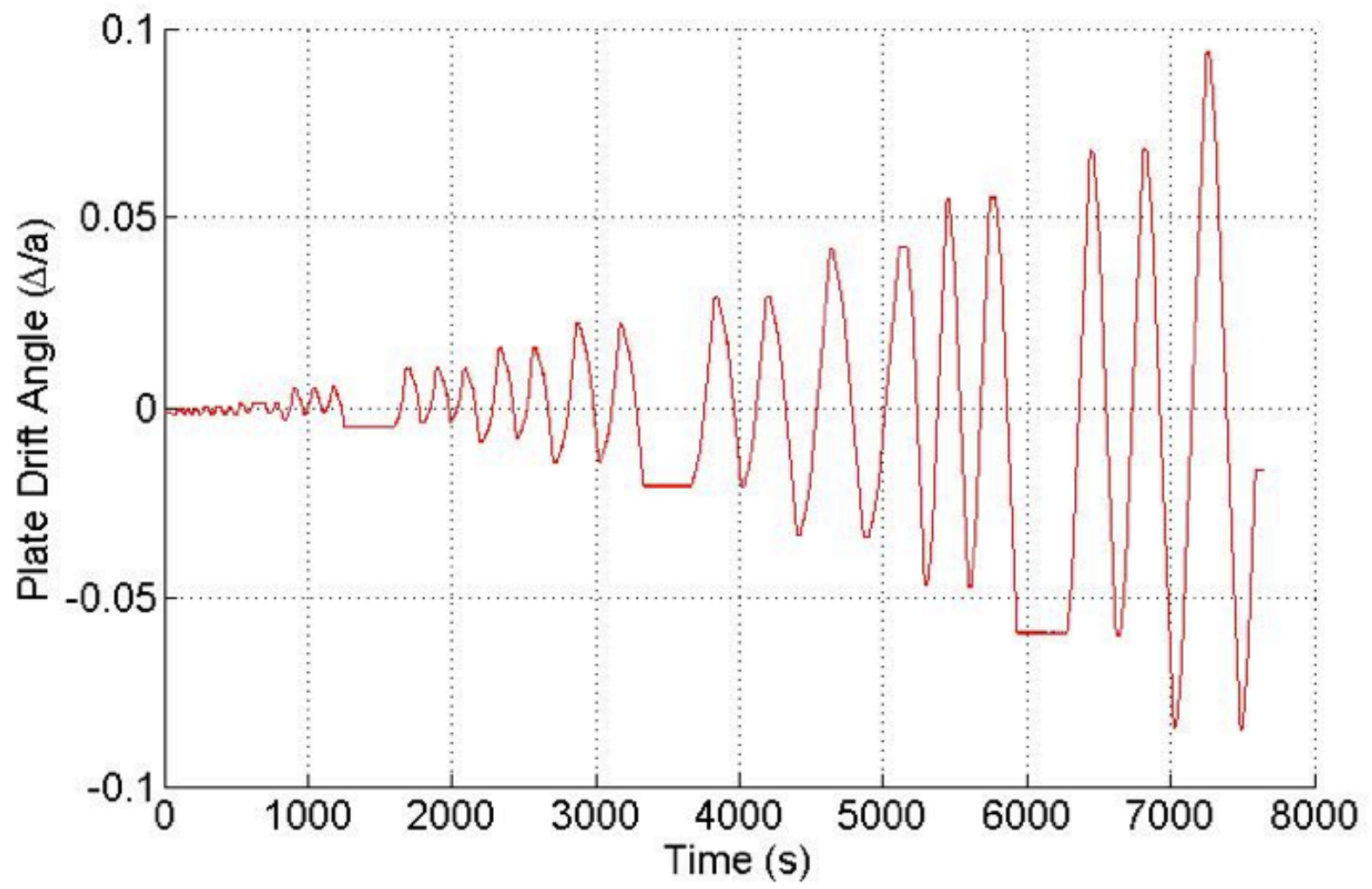

Figure 203. Plate Drift Angle versus Time - Test 7 


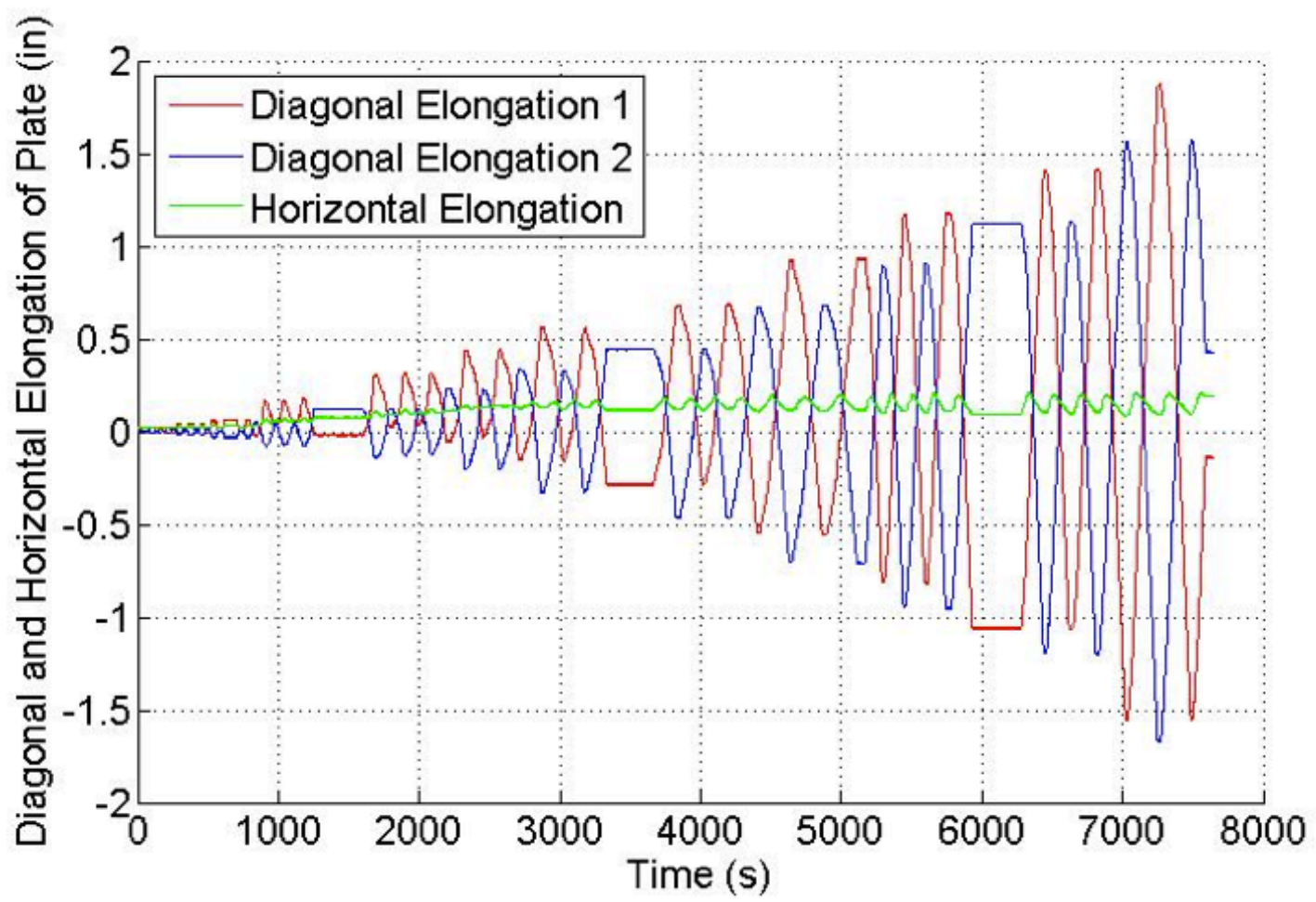

Figure 204. Diagonal and Horizontal Elongation of Plate versus Time - Test 7

TEST 8.

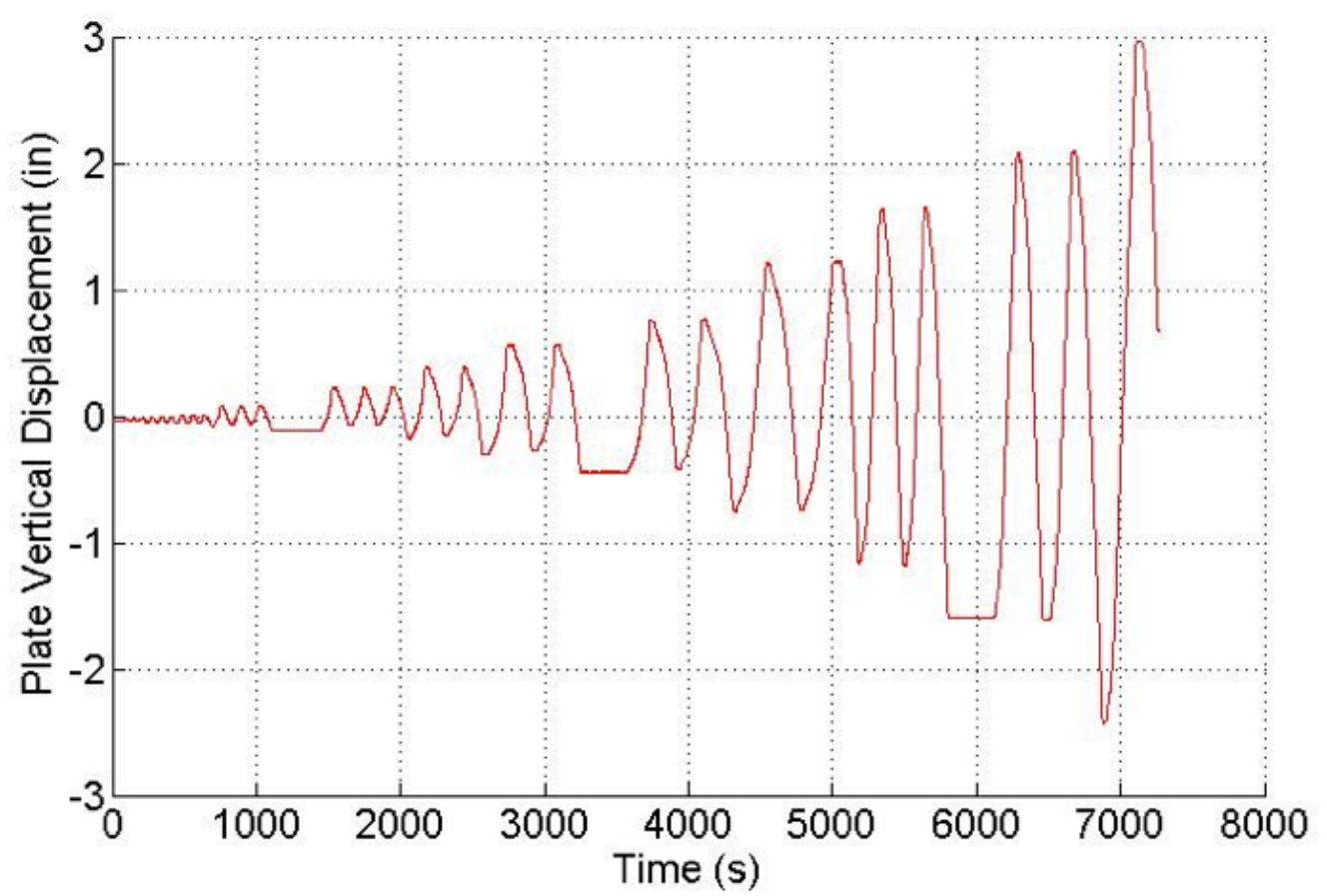

Figure 205. Plate Vertical Displacement versus Time - Test 8 


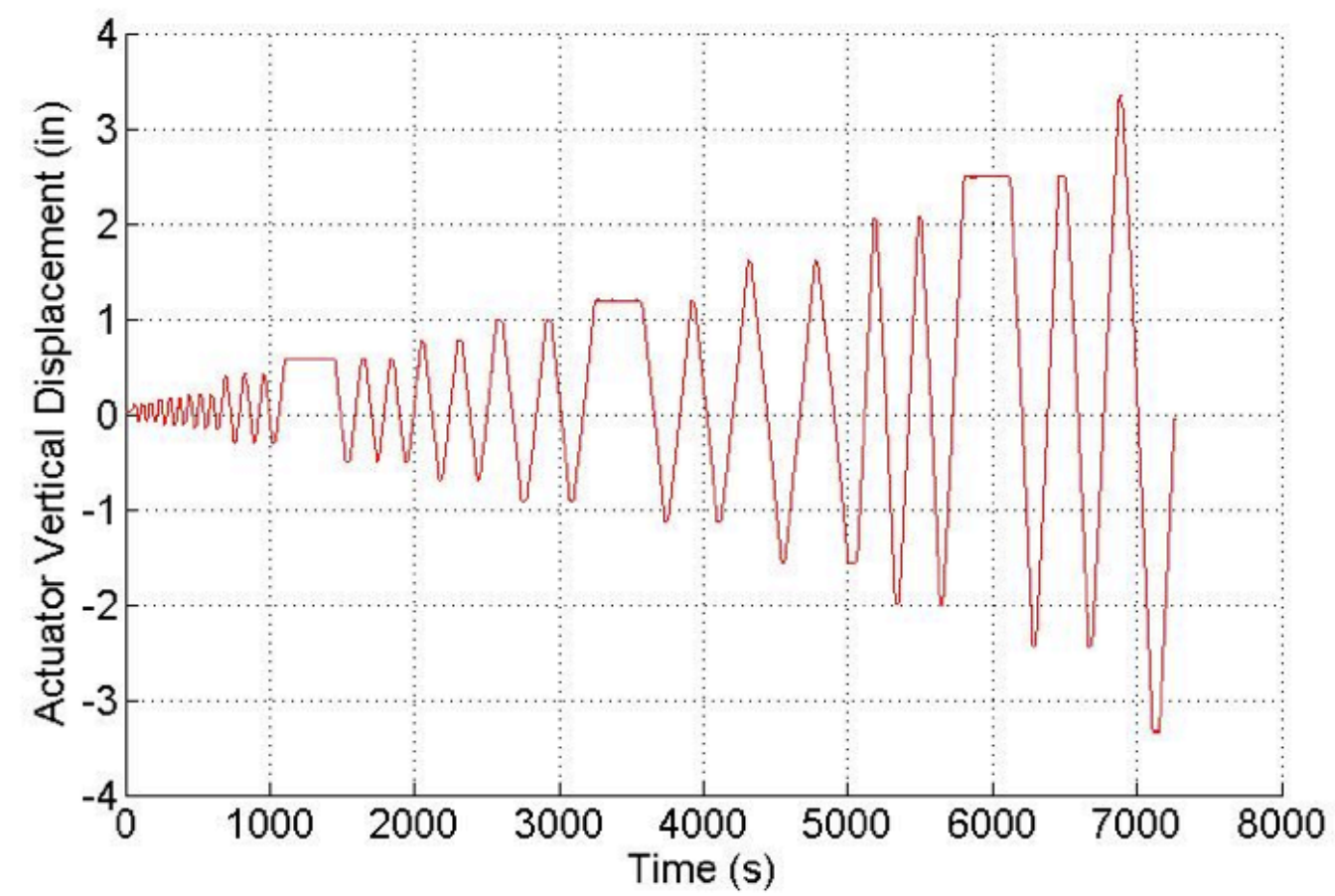

Figure 206. Actuator Vertical Displacement versus Time - Test 8

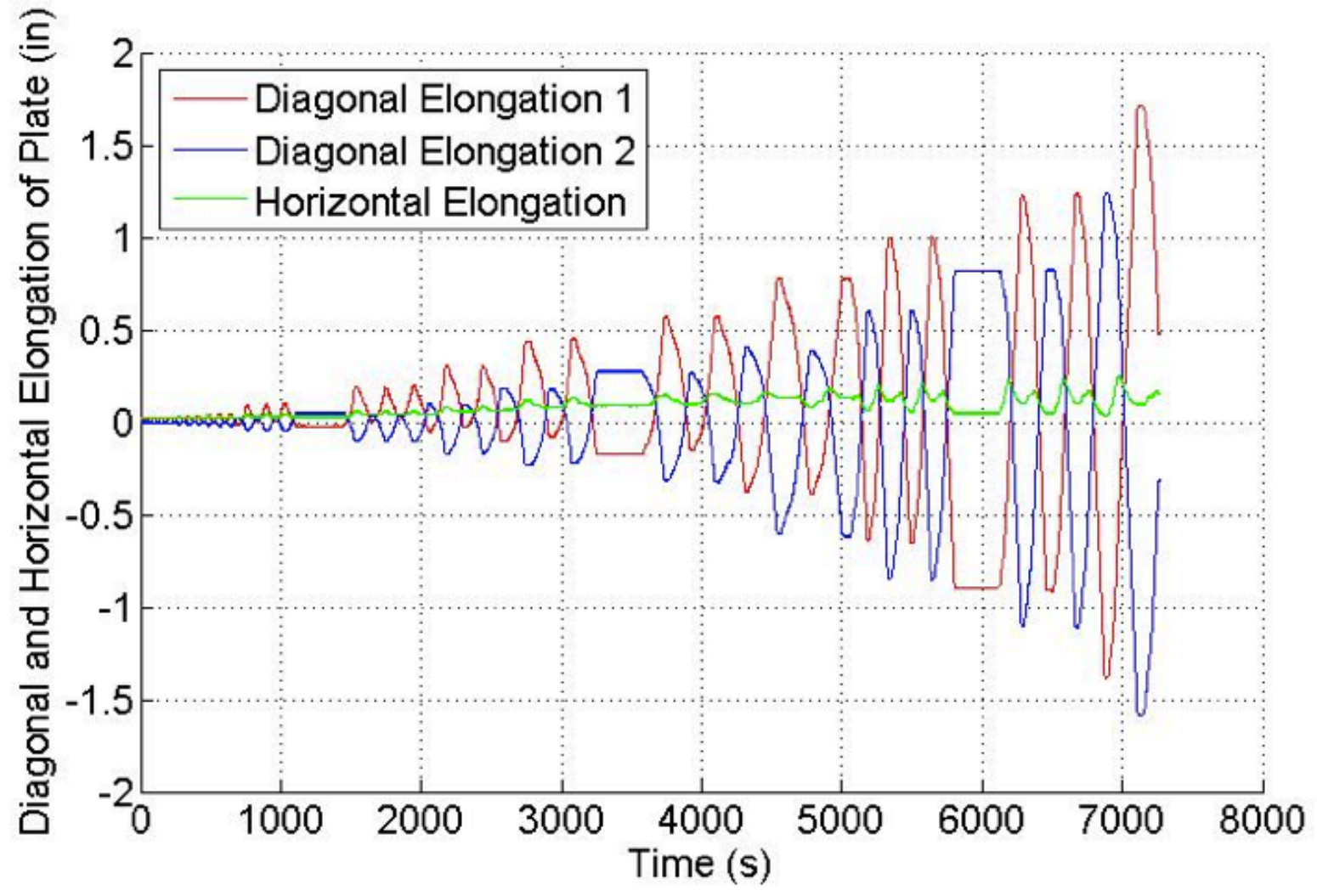

Figure 207. Diagonal and Horizontal Elongation of Plate versus Time - Test 8 


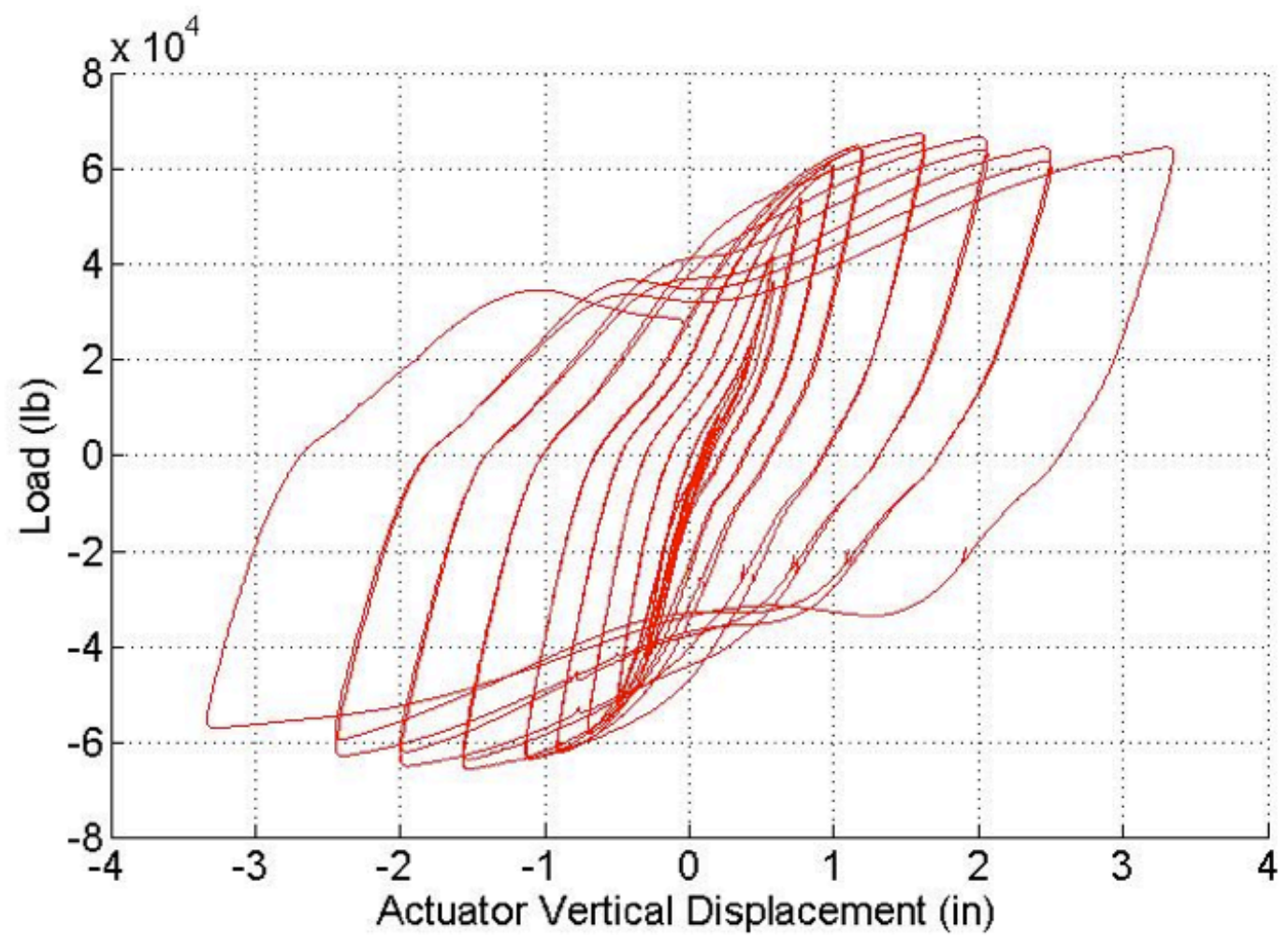

Figure 208. Load versus Actuator Vertical Displacement - Test 8

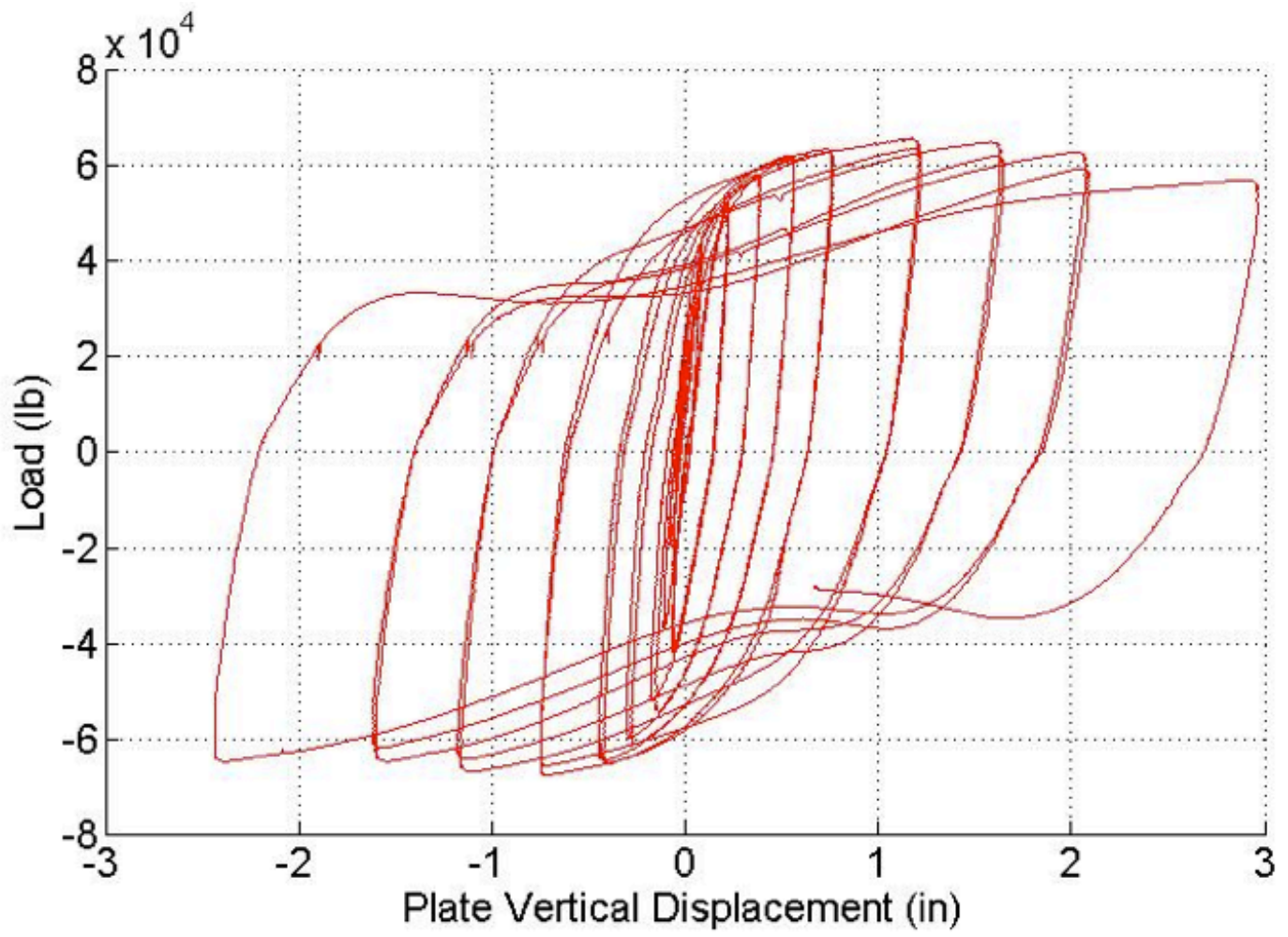

Figure 209. Load versus Plate Vertical Displacement - Test 8 


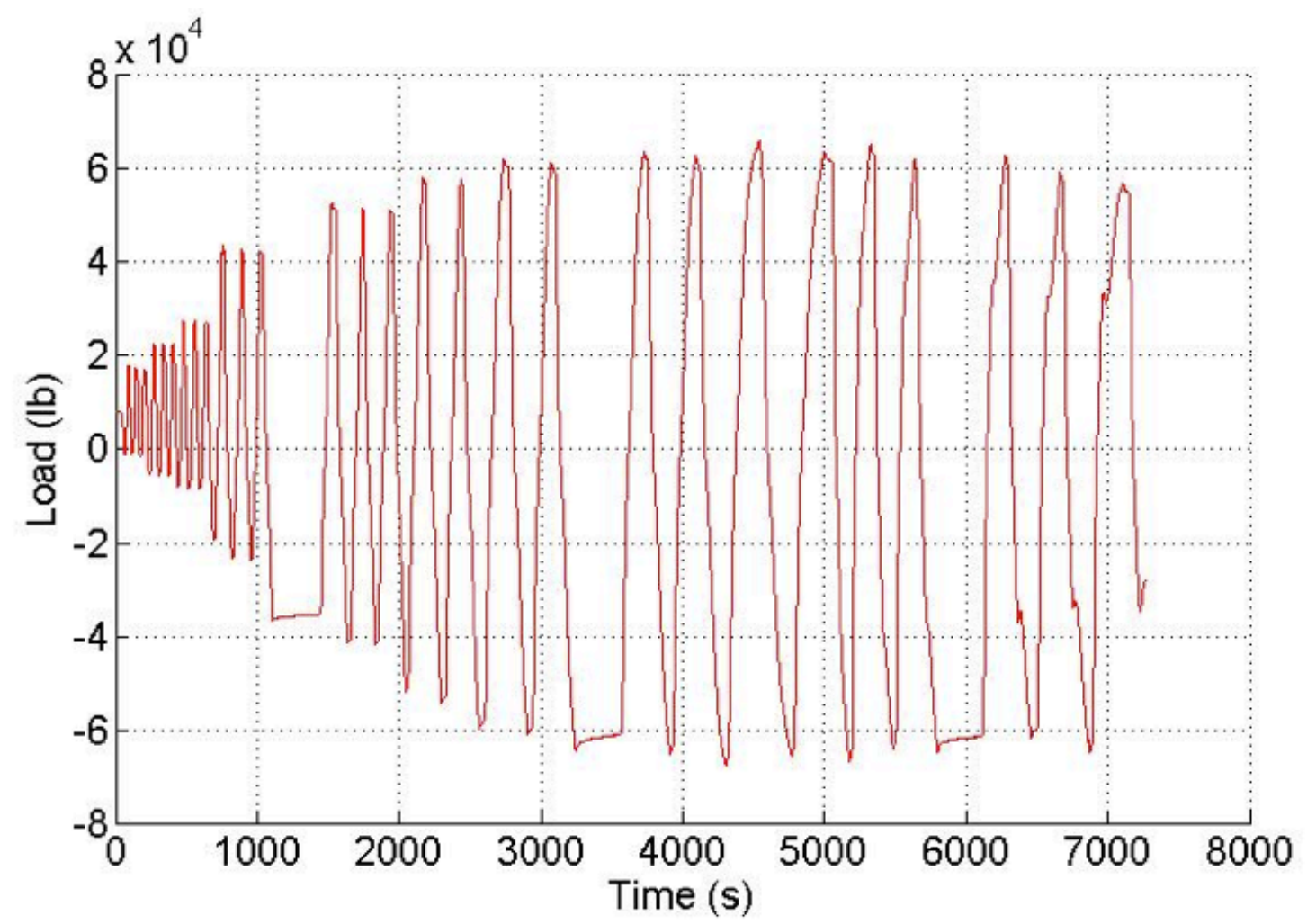

Figure 210. Load versus Time - Test 8

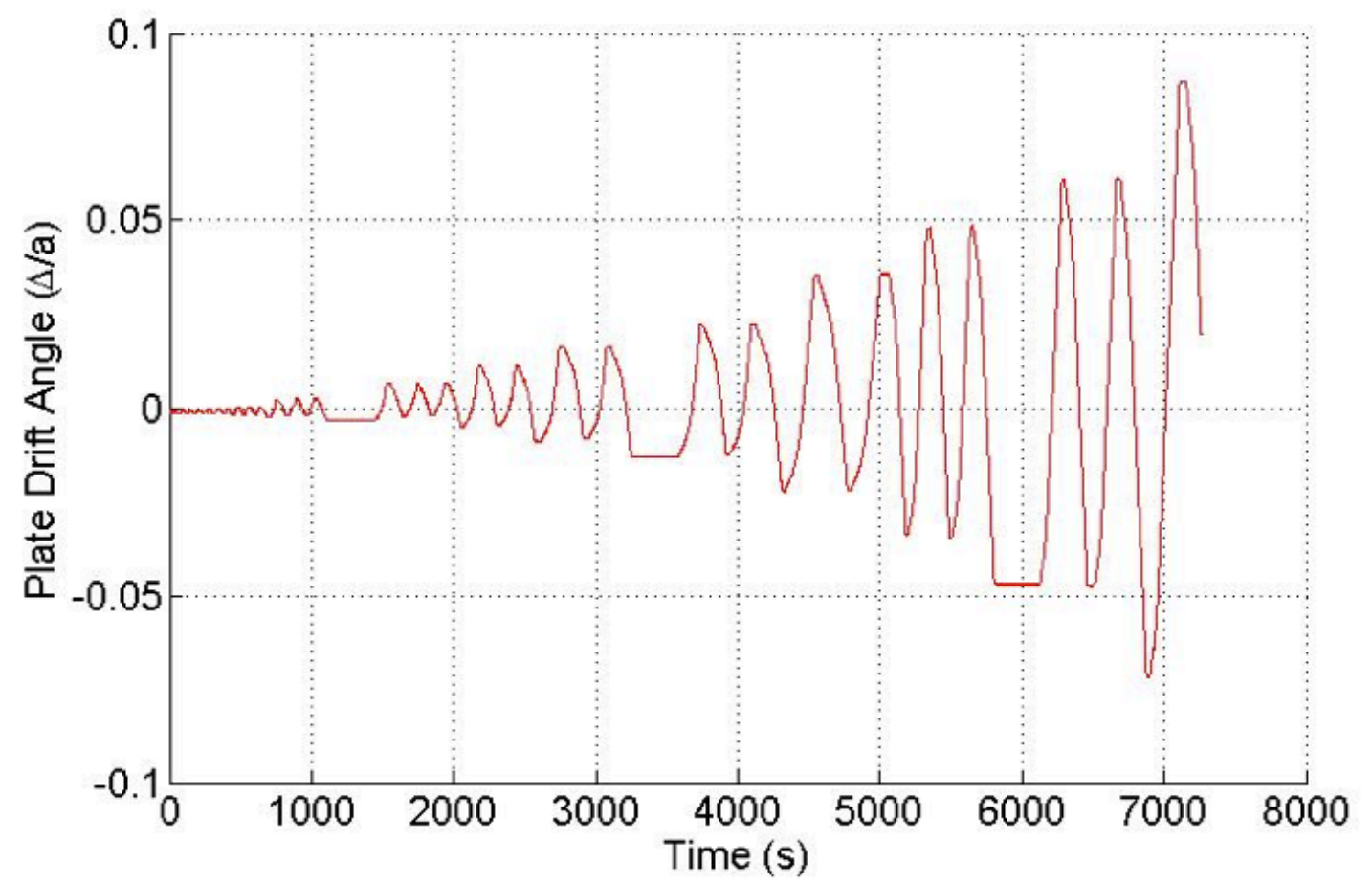

Figure 211. Plate Drift Angle versus Time - Test 8 


\section{APPENDIX E: Photogrammetry point clouds}

TEST 1 - Specimen 2-0.5-1.

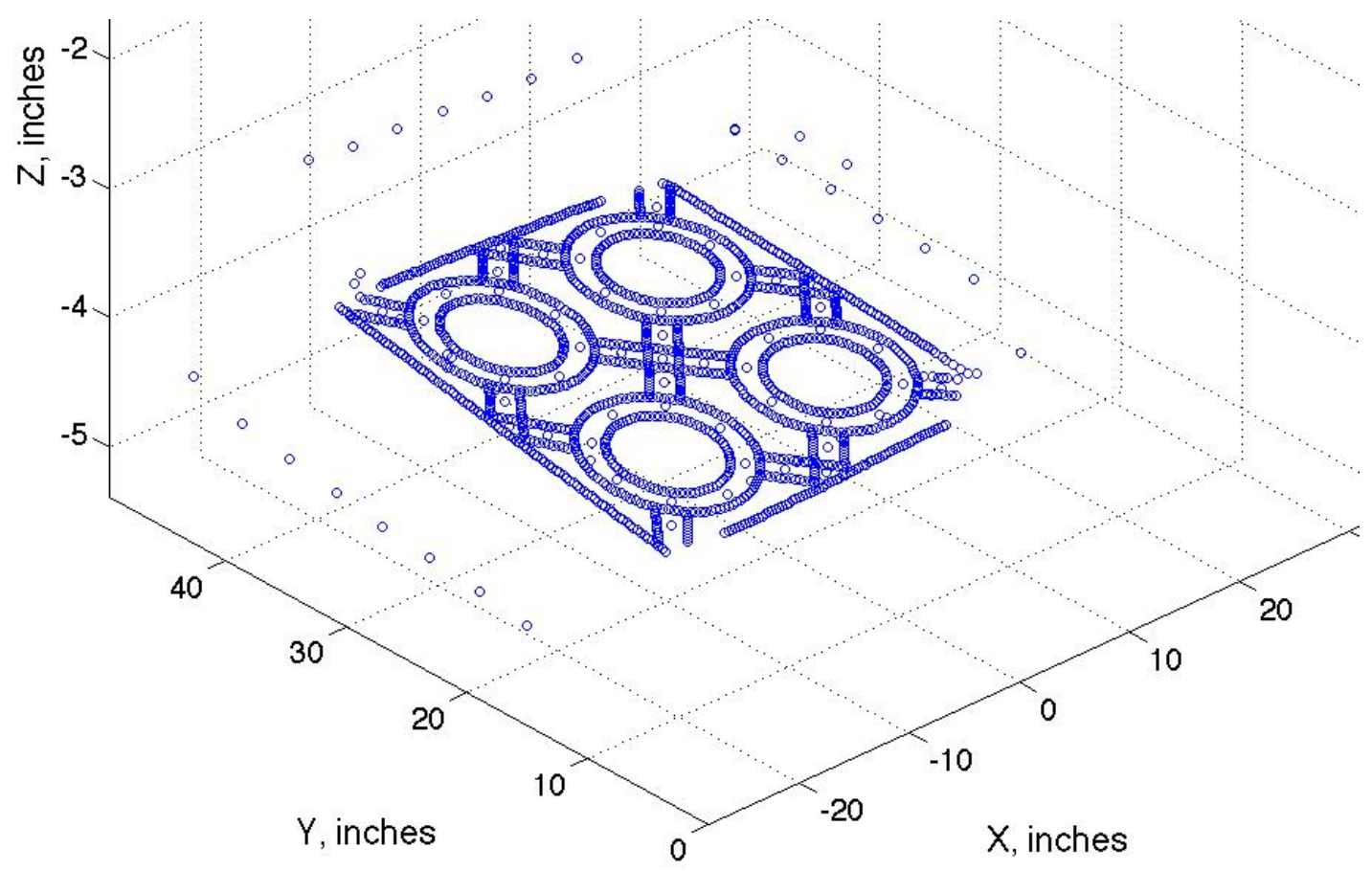

Figure 212. Point cloud - Specimen 2-05-0.1 - 0.3\% drift

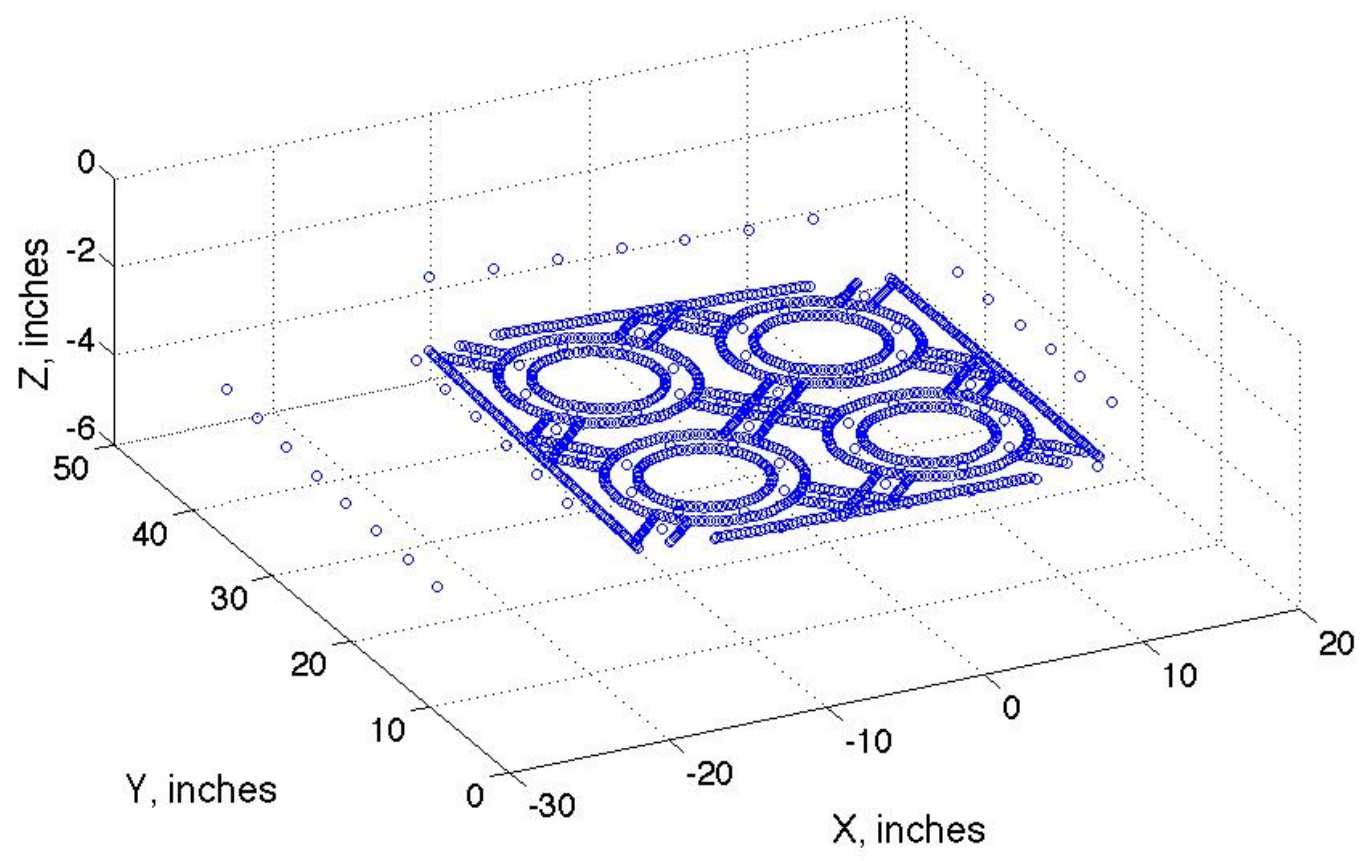

Figure 213. Point cloud - Specimen 2-05-0.1 - 0.8\% drift 


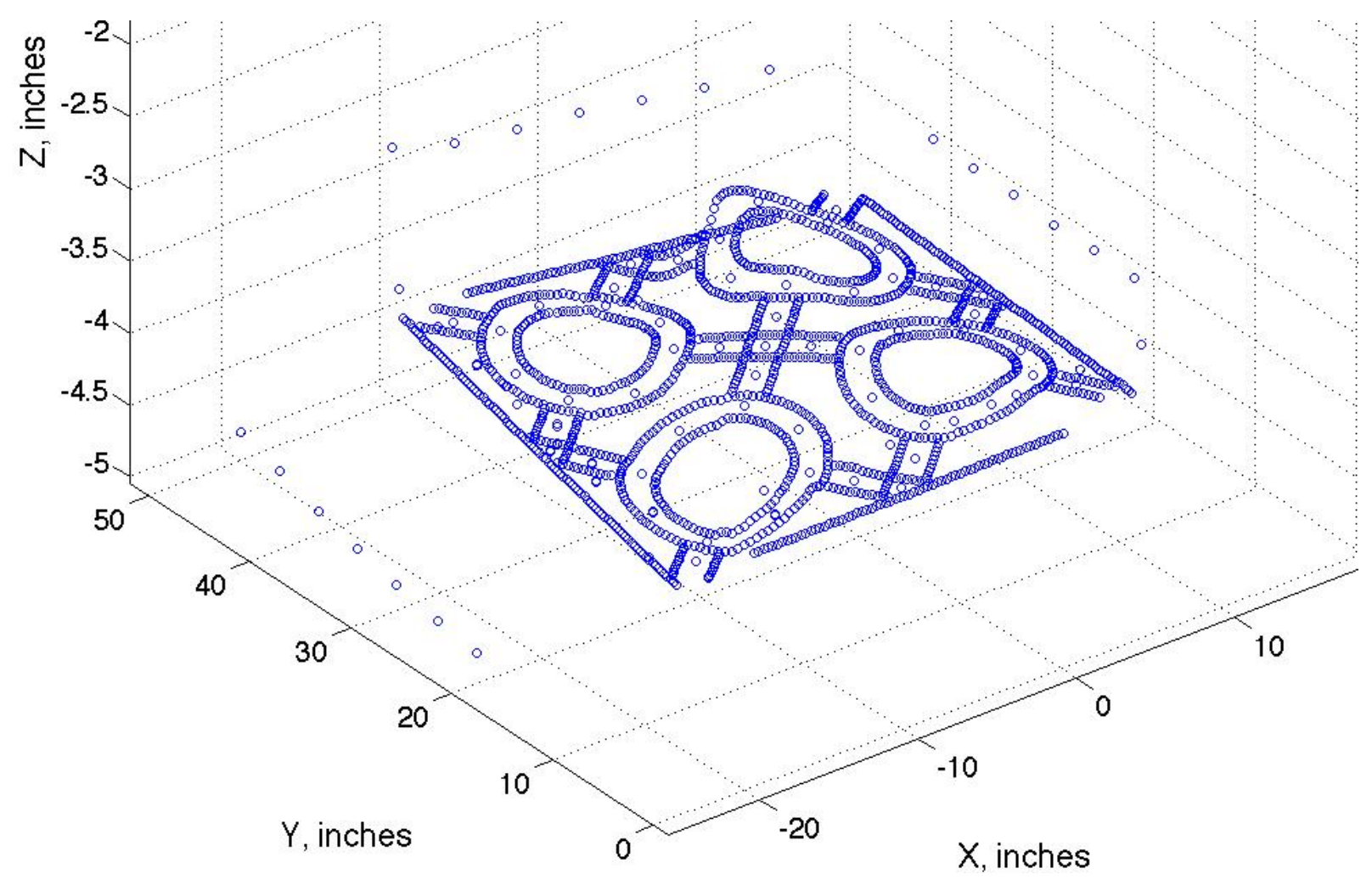

Figure 214. Point cloud - Specimen 2-05-0.1 - 2.8\% drift

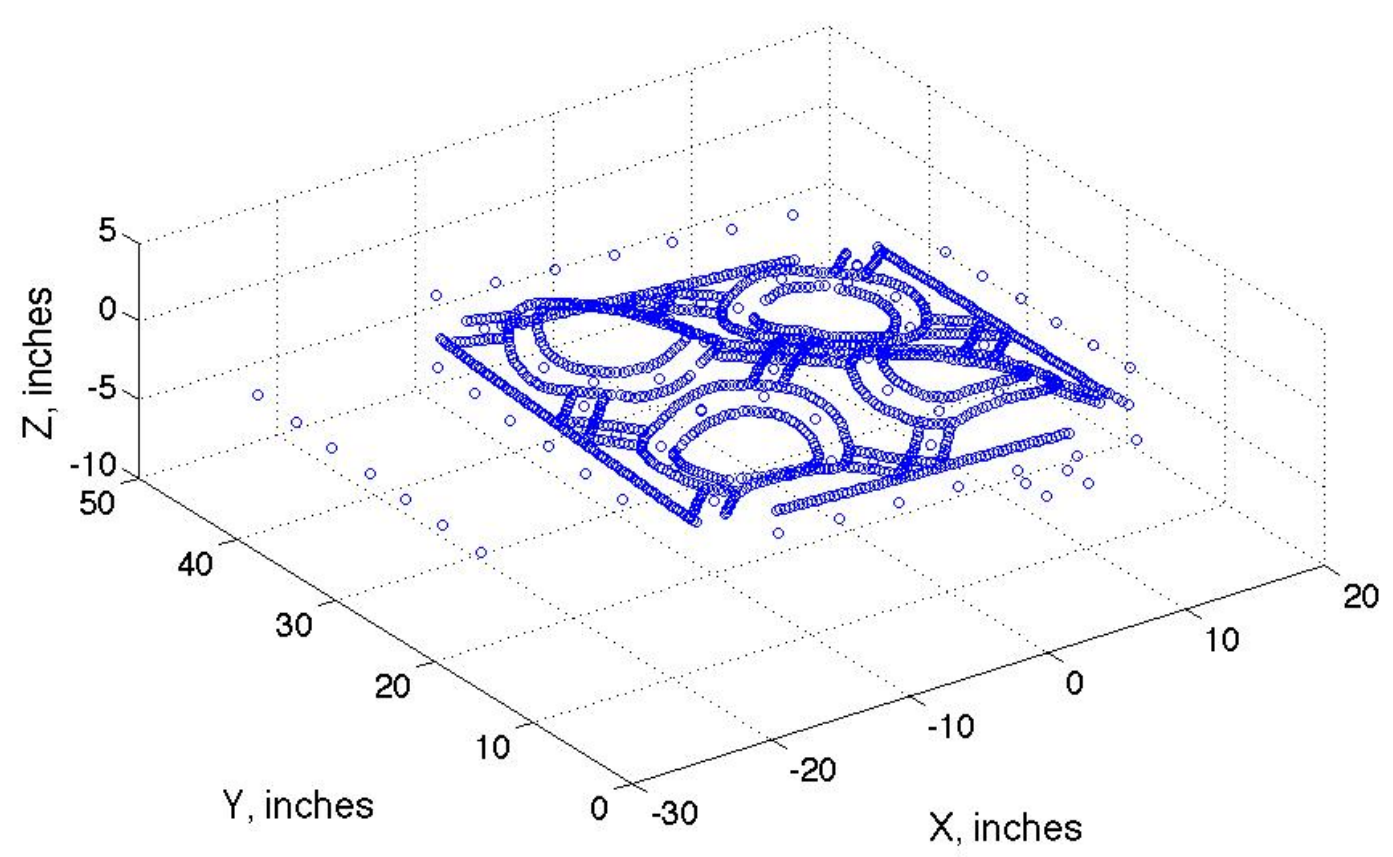

Figure 215. Point cloud - Specimen 2-05-0.1 - 6.7\% drift 


\section{TEST 2 - Specimen “Solid Plate”}

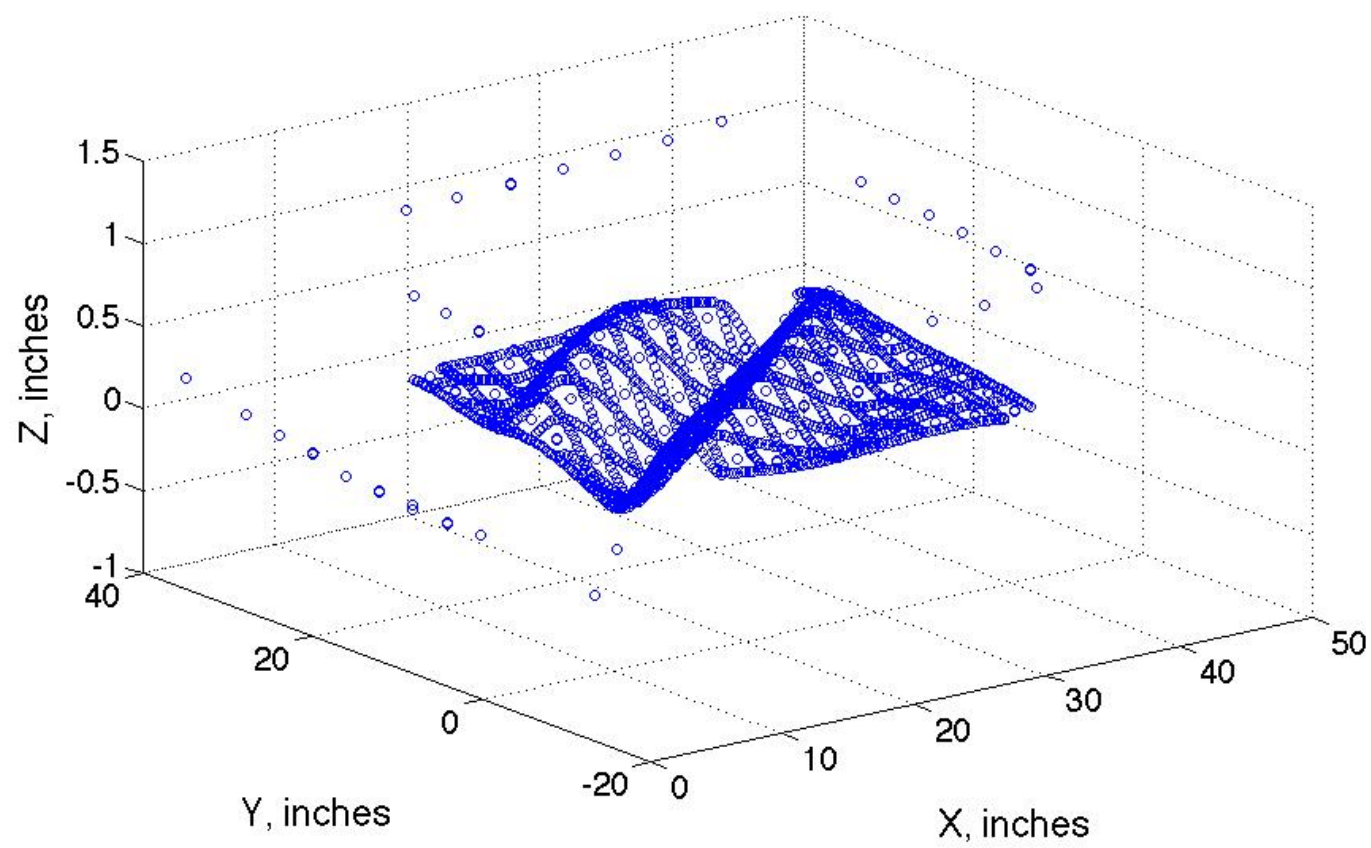

Figure 216. Point cloud - Specimen "Solid Plate" - 0.5\% drift

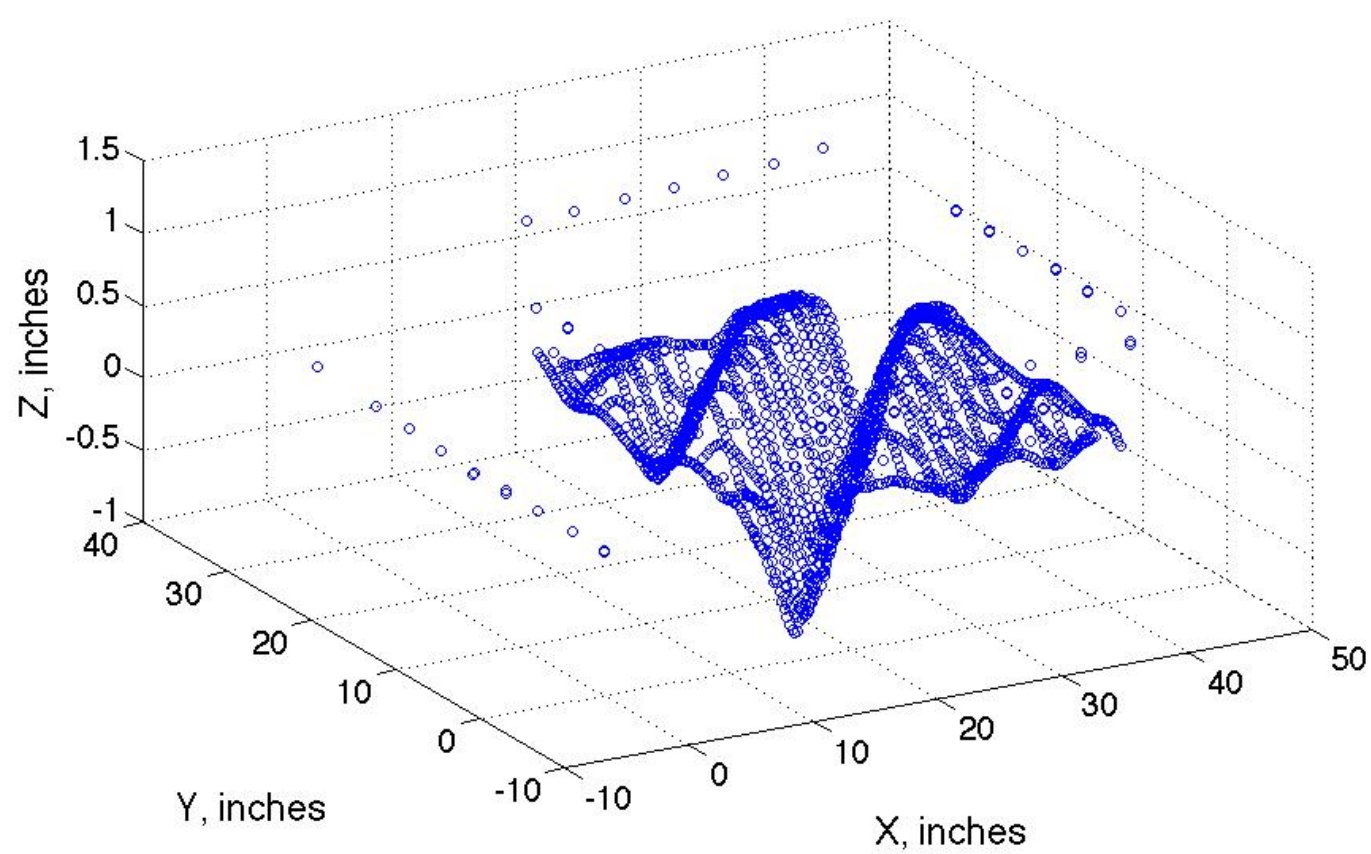

Figure 217. Point cloud - Specimen "Solid Plate" - 2.2\% drift 


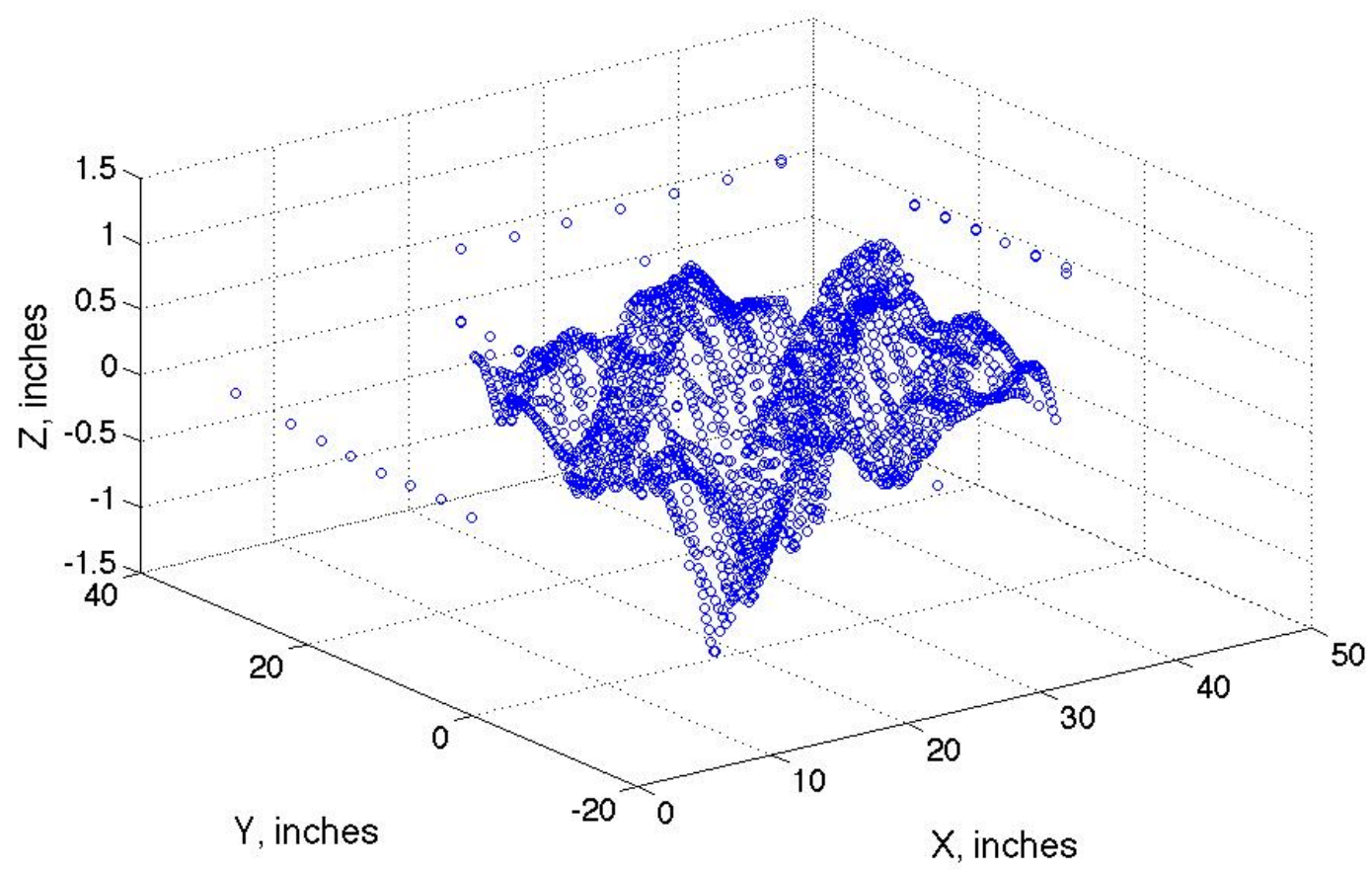

Figure 218. Point cloud - Specimen "Solid Plate" - 6\% drift

TEST 3 - Specimen 2-0.5-1.

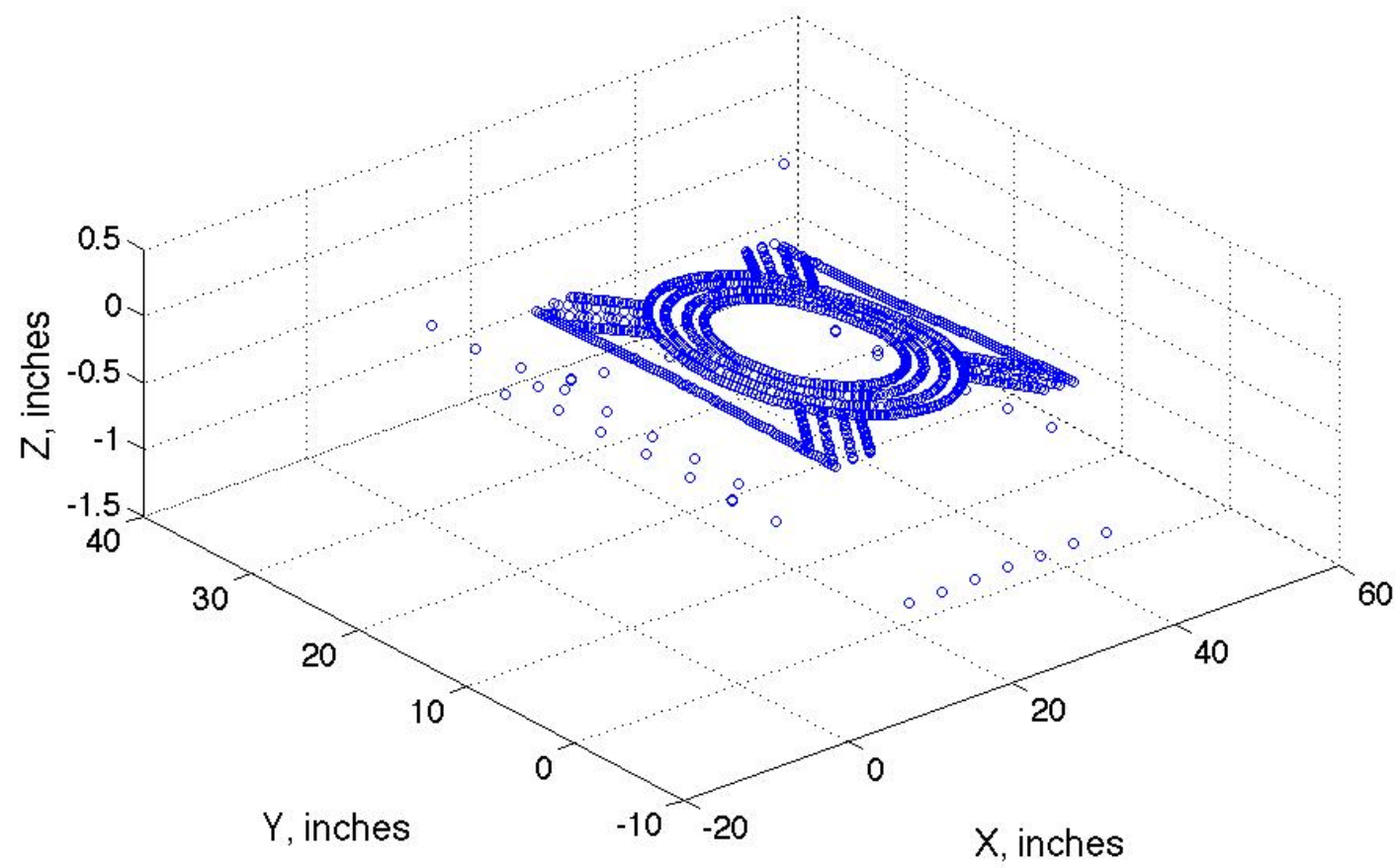

Figure 219. Point cloud - Specimen 2-0.5-1 - 0.9\% drift 


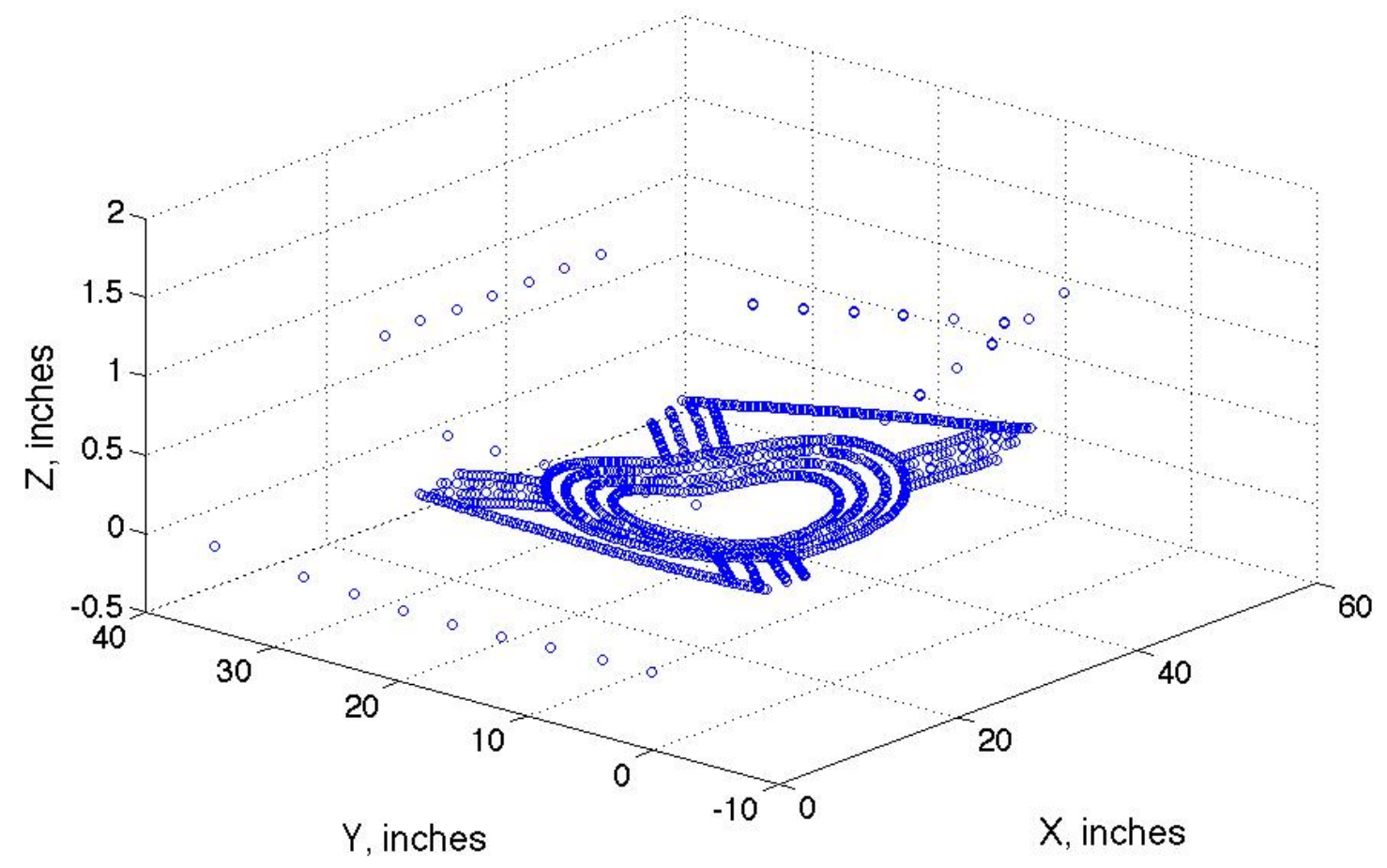

Figure 220. Point cloud - Specimen 2-0.5-1 - 2.8\% drift

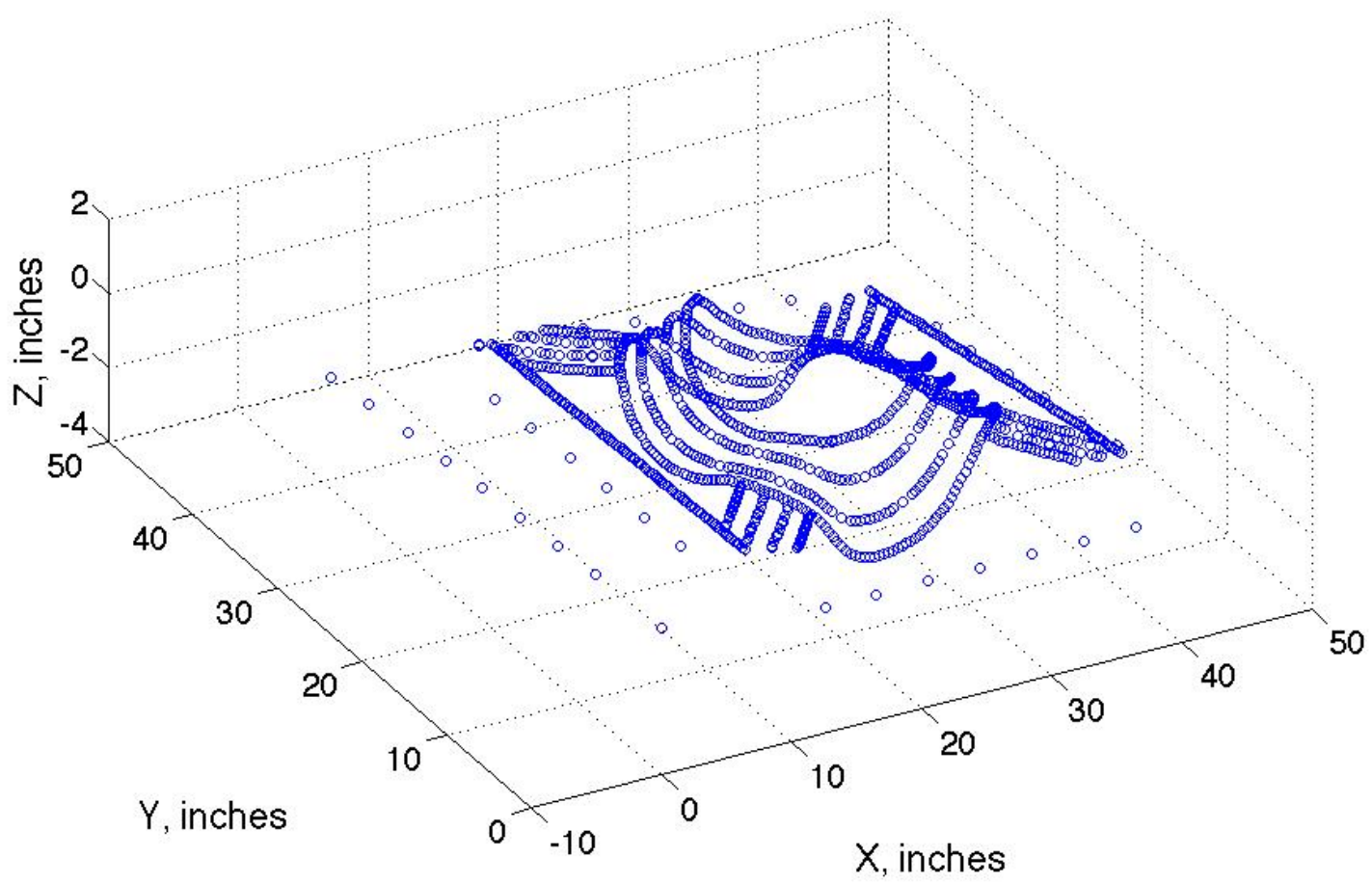

Figure 221. Point cloud - Specimen 2-0.5-1 - 6.8\% drift 


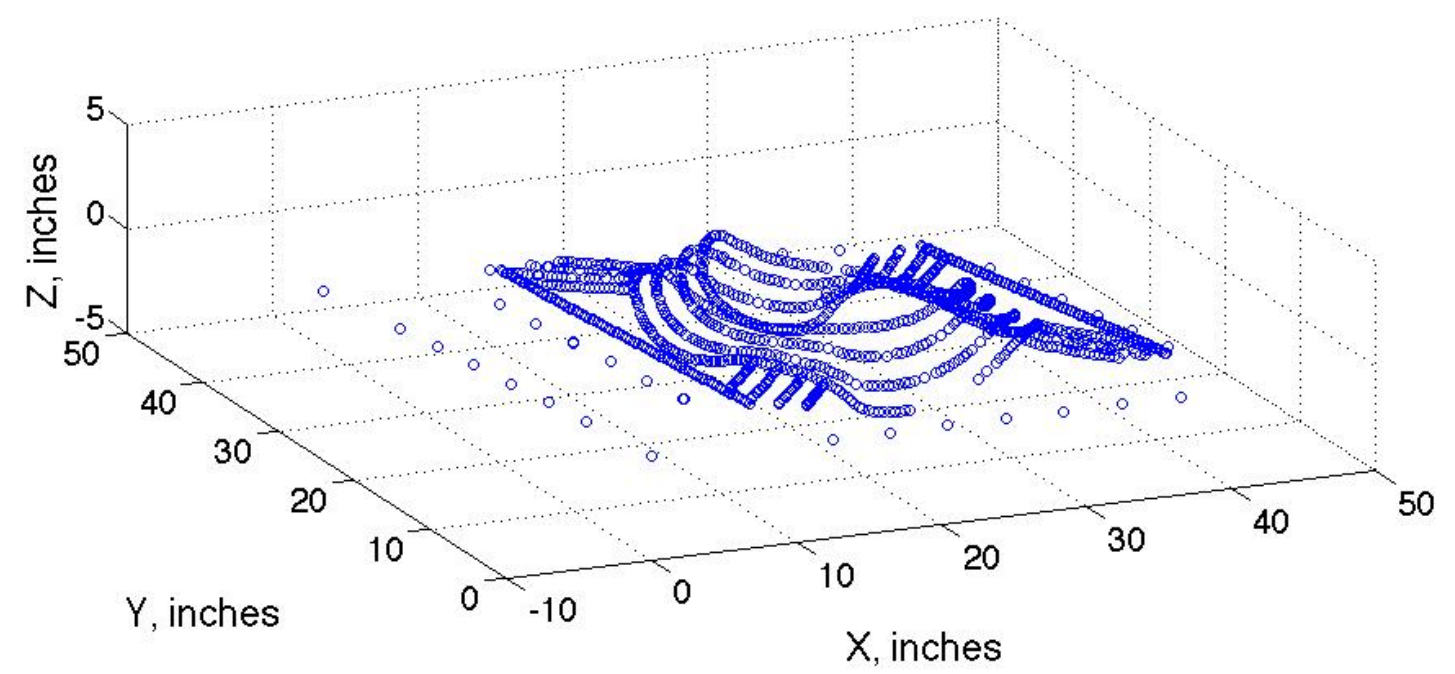

Figure 222. Point cloud - Specimen 2-0.5-1 - 11.9\% drift

TEST 4 - Specimen 3-0.5-1.

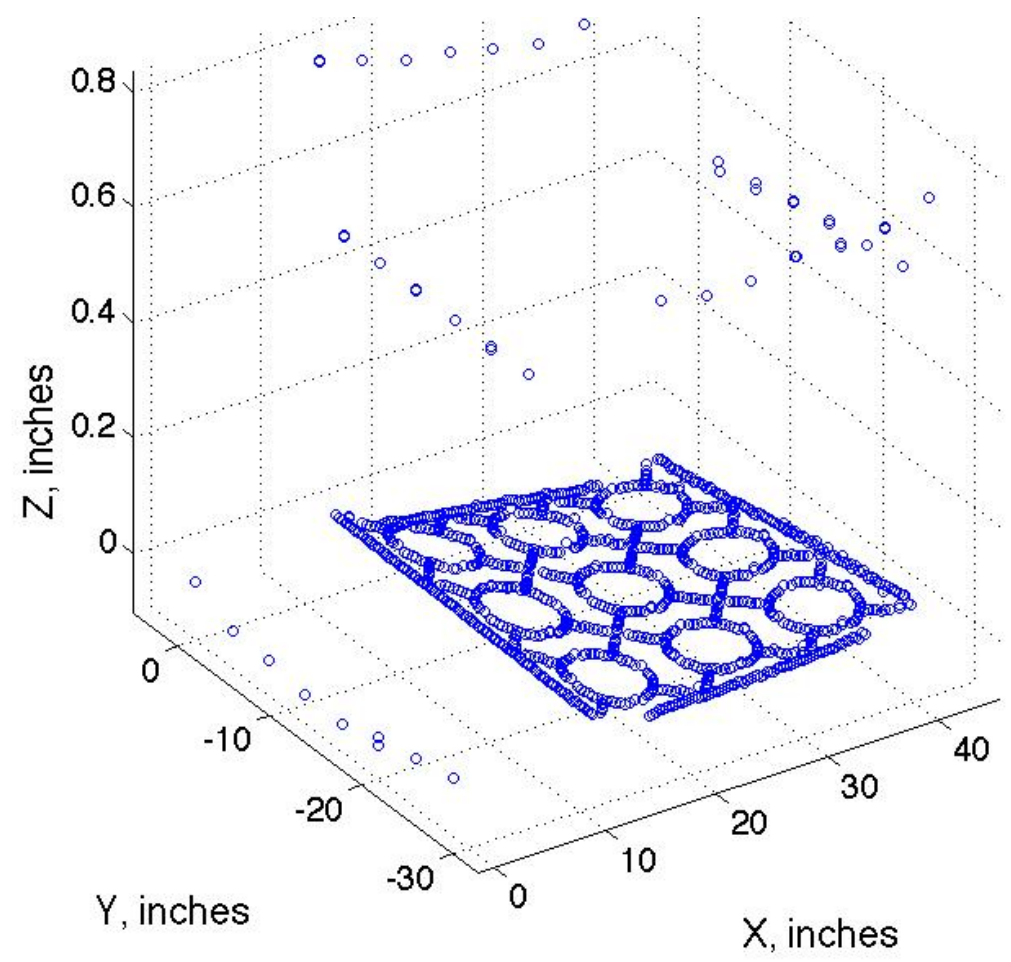

Figure 223. Point cloud - Specimen 3-0.5-1 - 0.4\% drift 


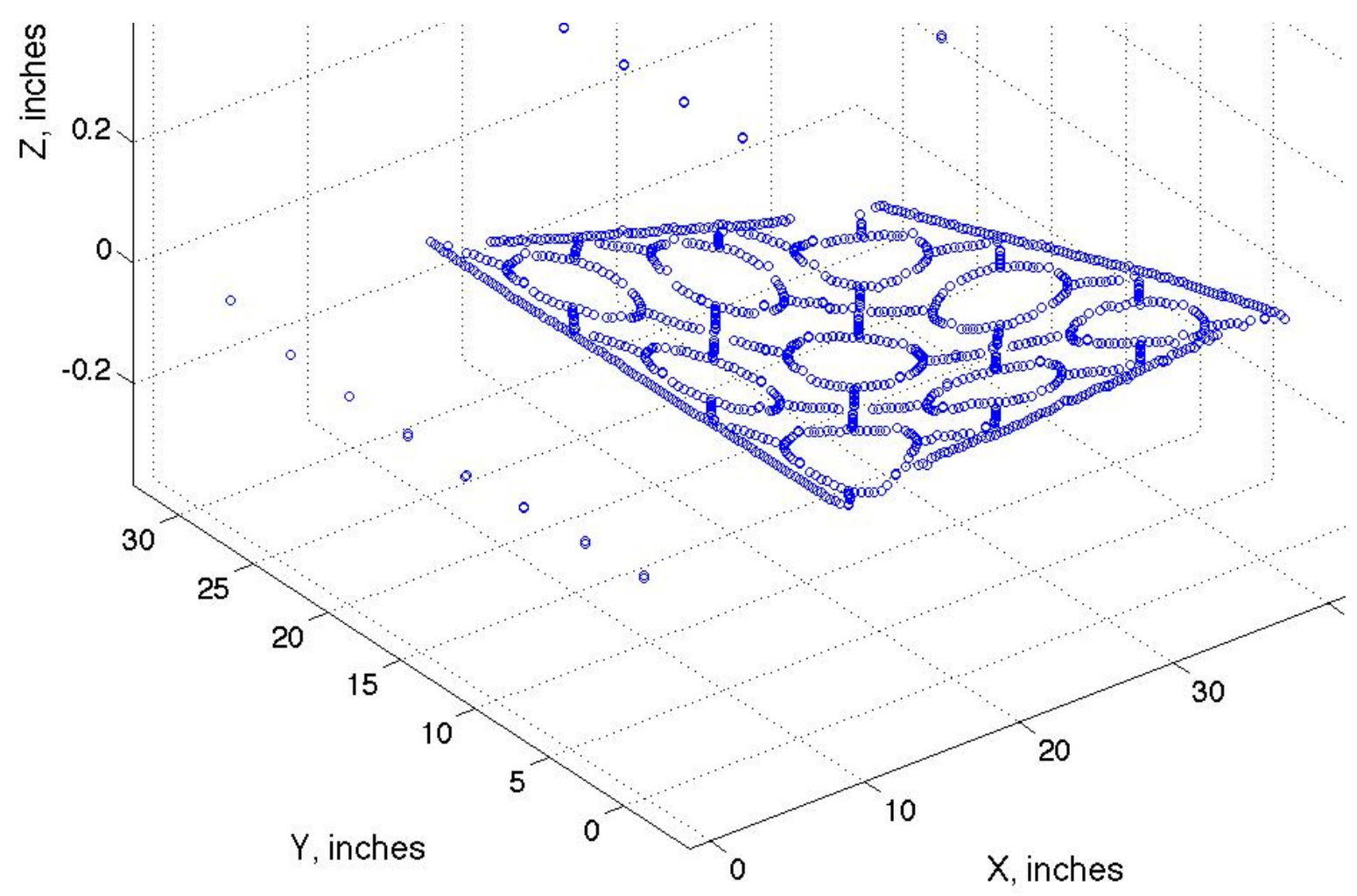

Figure 224. Point cloud - Specimen 3-0.5-1 - 1.6\% drift

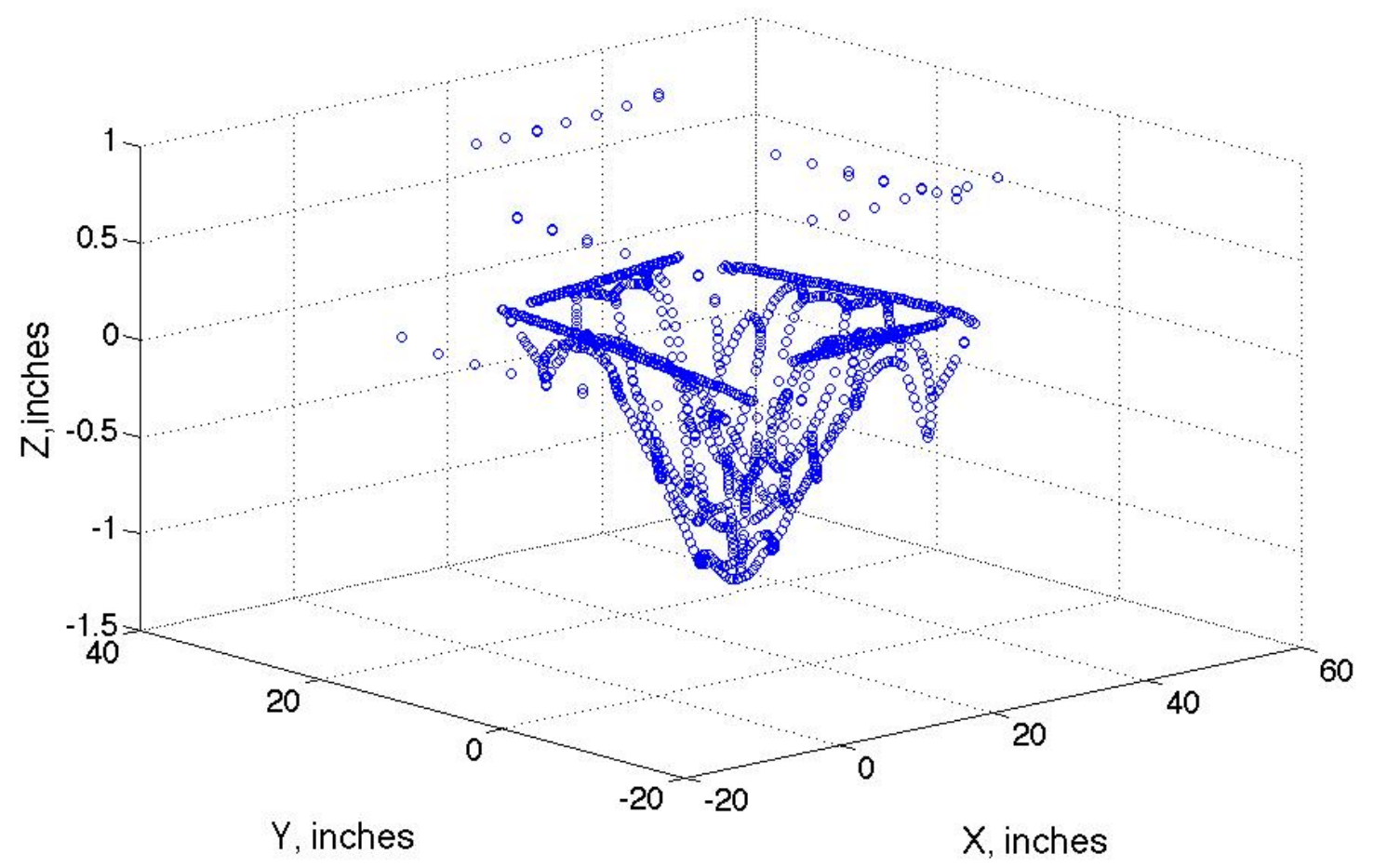

Figure 225. Point cloud - Specimen 3-0.5-1 - 3.2\% drift 
TEST 5 - Specimen 2-0.25-0.81.

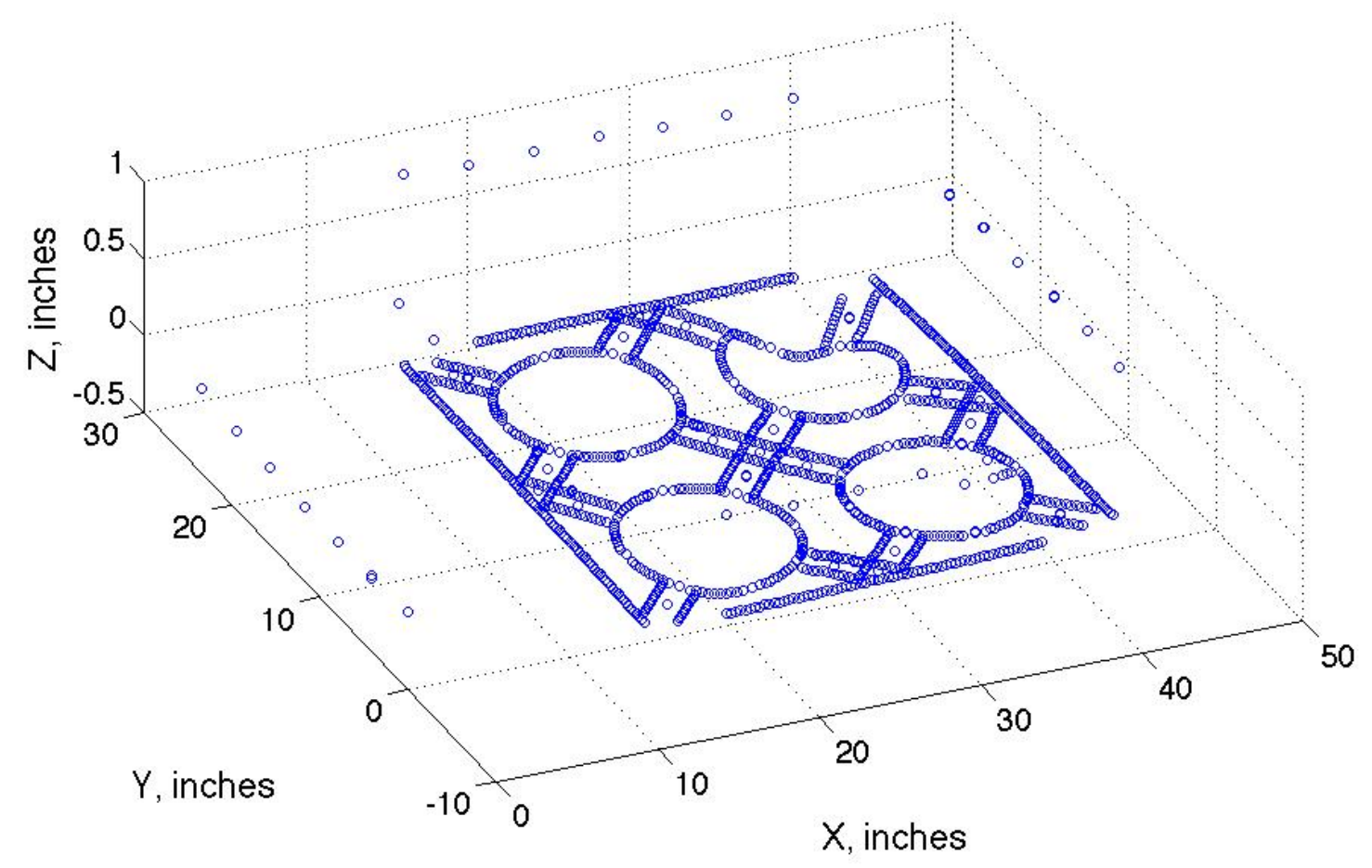

Figure 226. Point cloud - Specimen 2-0.25-0.81-1.2\% drift

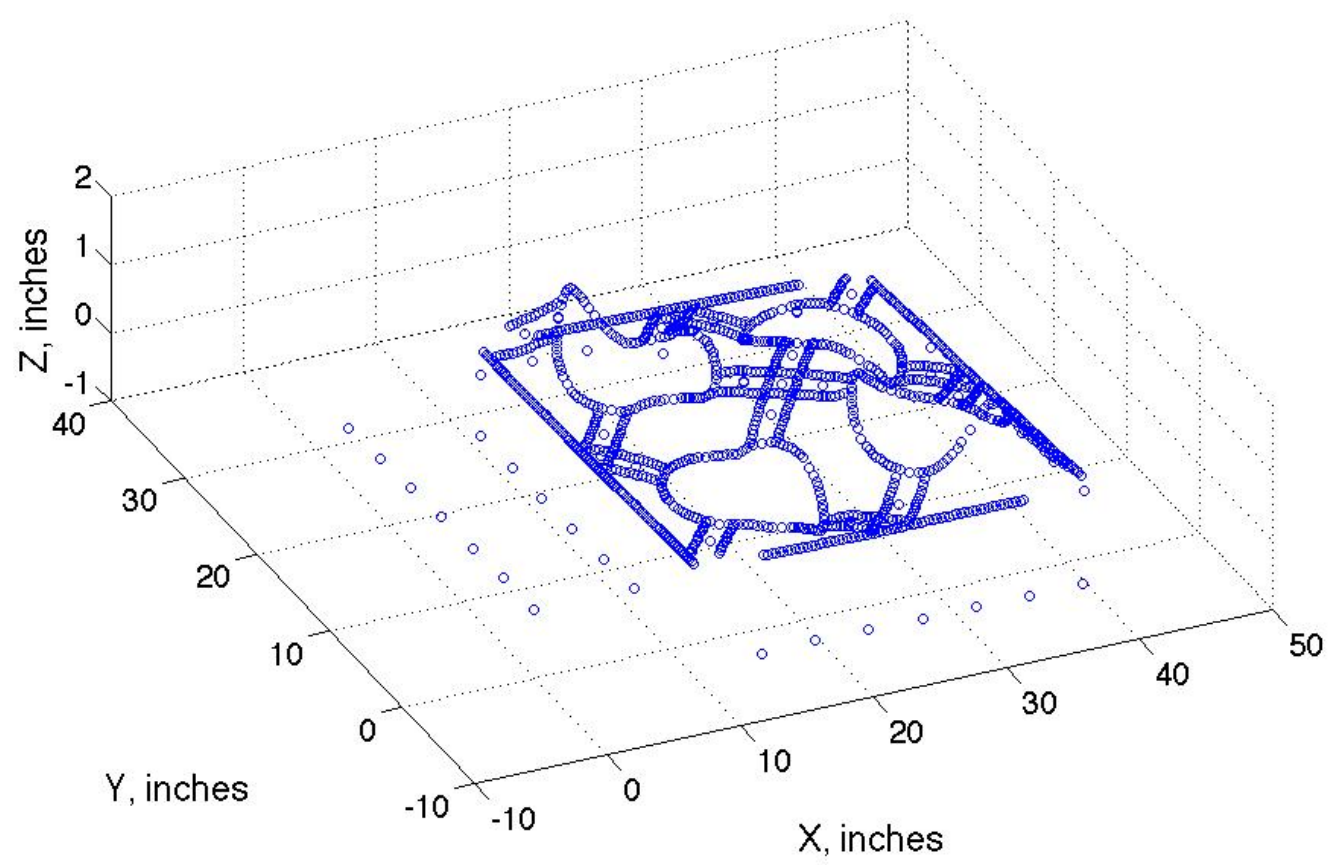

Figure 227. Point cloud - Specimen 2-0.25-0.81 - 3\% drift 


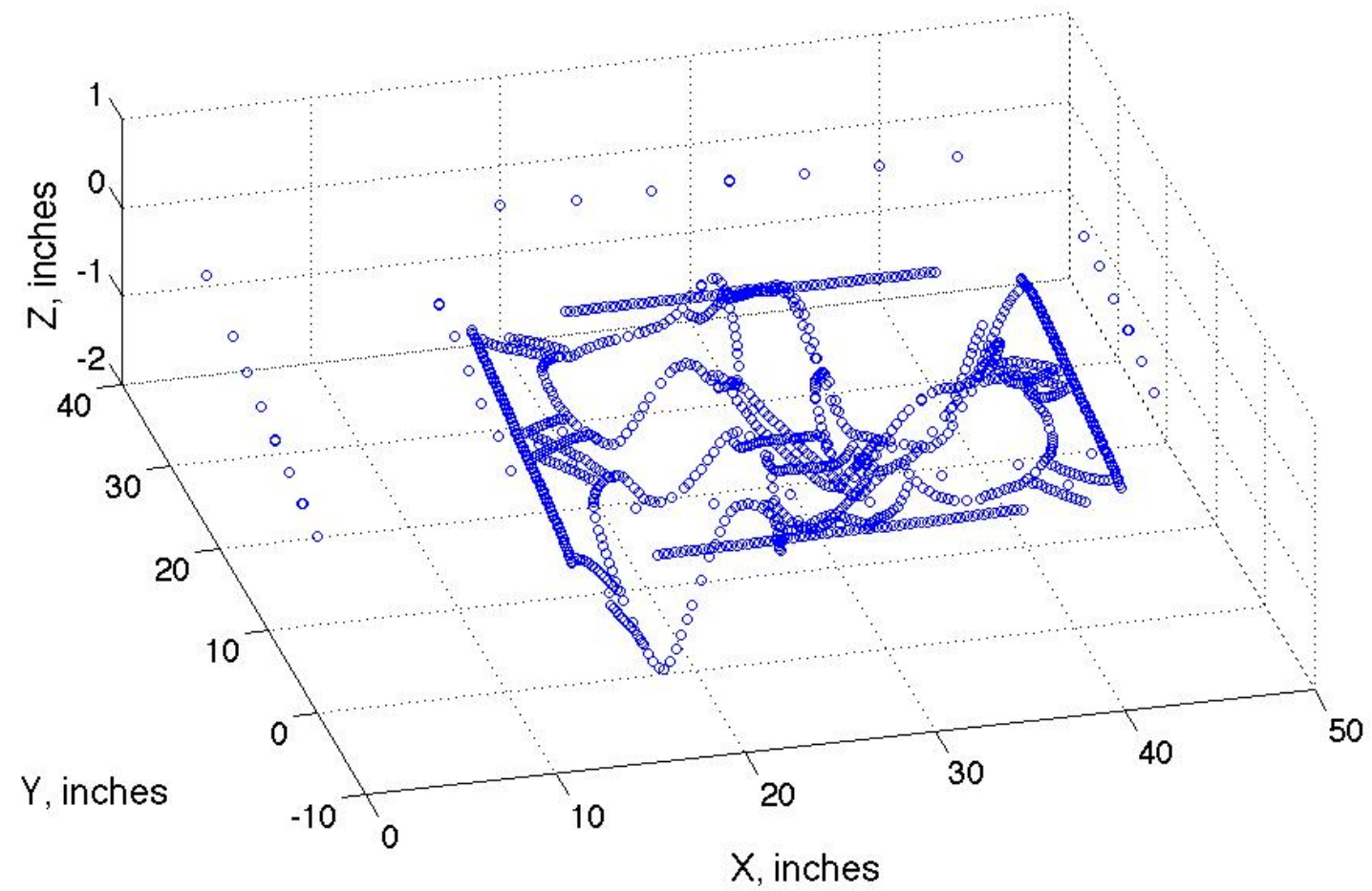

Figure 228. Point cloud - Specimen 2-0.25-0.81-6.8\% drift

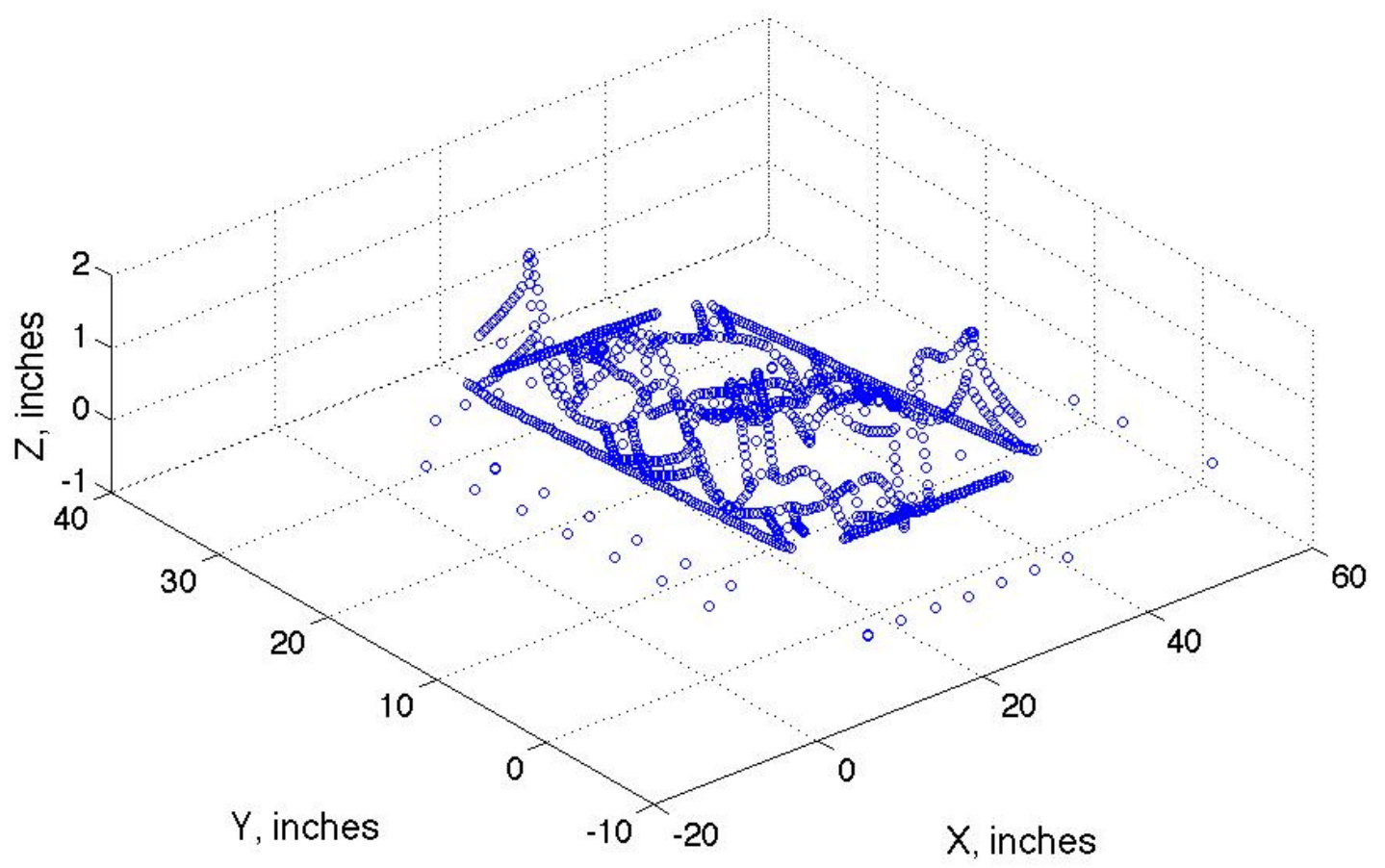

Figure 229. Point cloud - Specimen 2-0.25-0.81 - 11.9\% drift 
TEST 6 - Specimen 2-0.25-1.

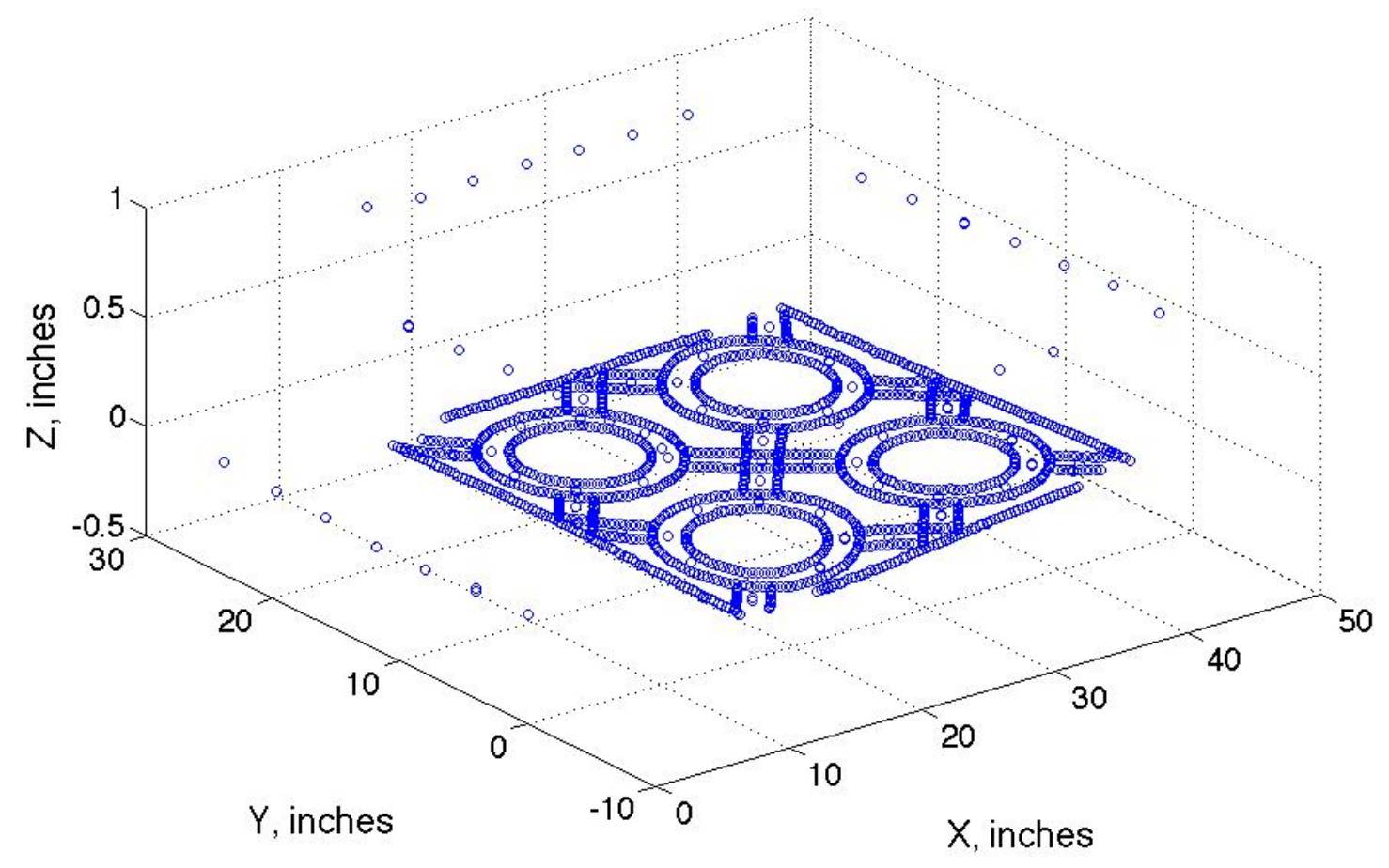

Figure 230. Point cloud - Specimen 2-0.25-1 - 0.9\% drift

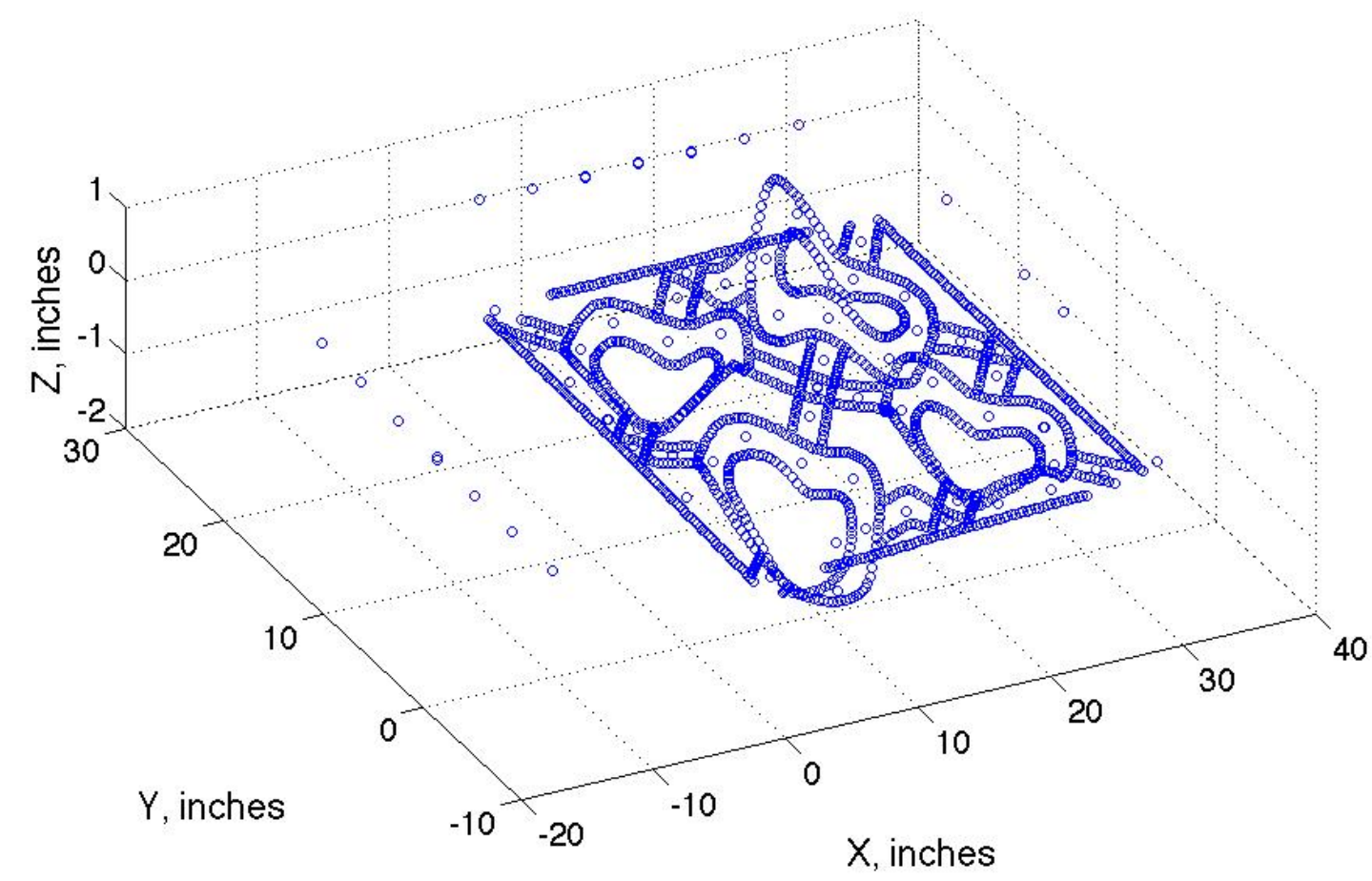

Figure 231. Point cloud - Specimen 2-0.25-1 - 2.8\% drift 


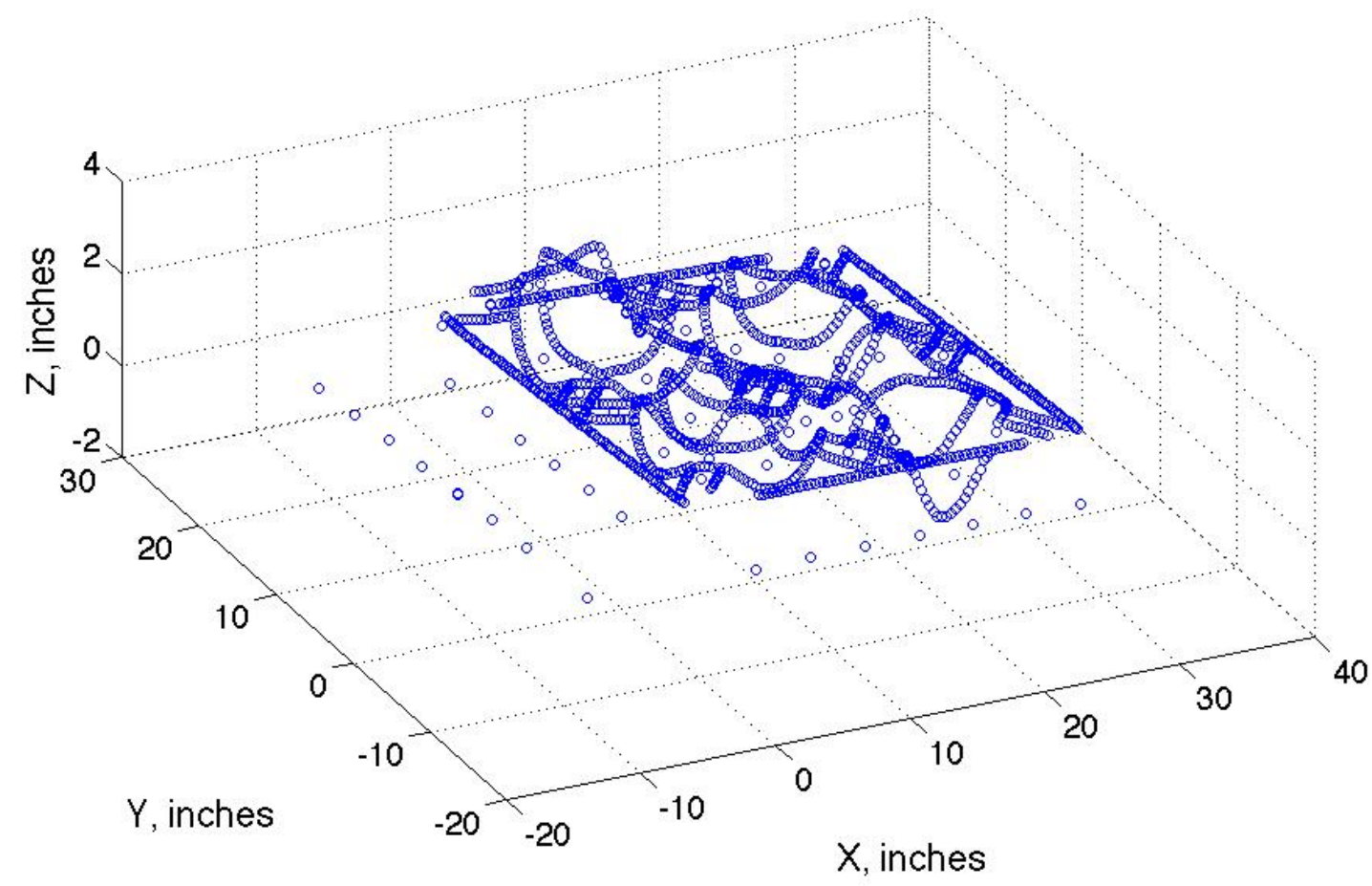

Figure 232. Point cloud - Specimen 2-0.25-1 - 6.8\% drift

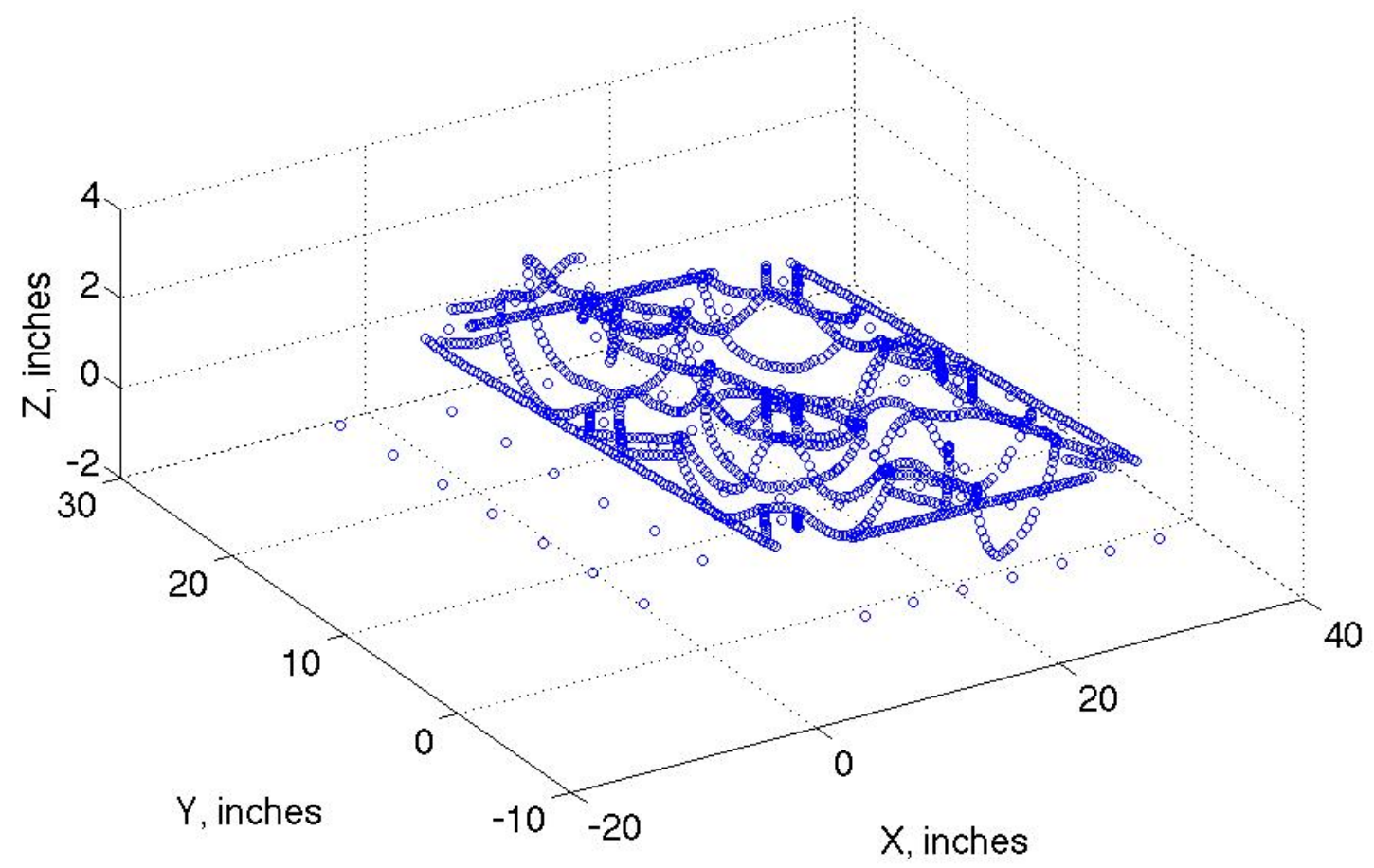

Figure 233. Point cloud - Specimen 2-0.25-1 - 11.9\% drift 
TEST 7 - Specimen 3-0.25-1.

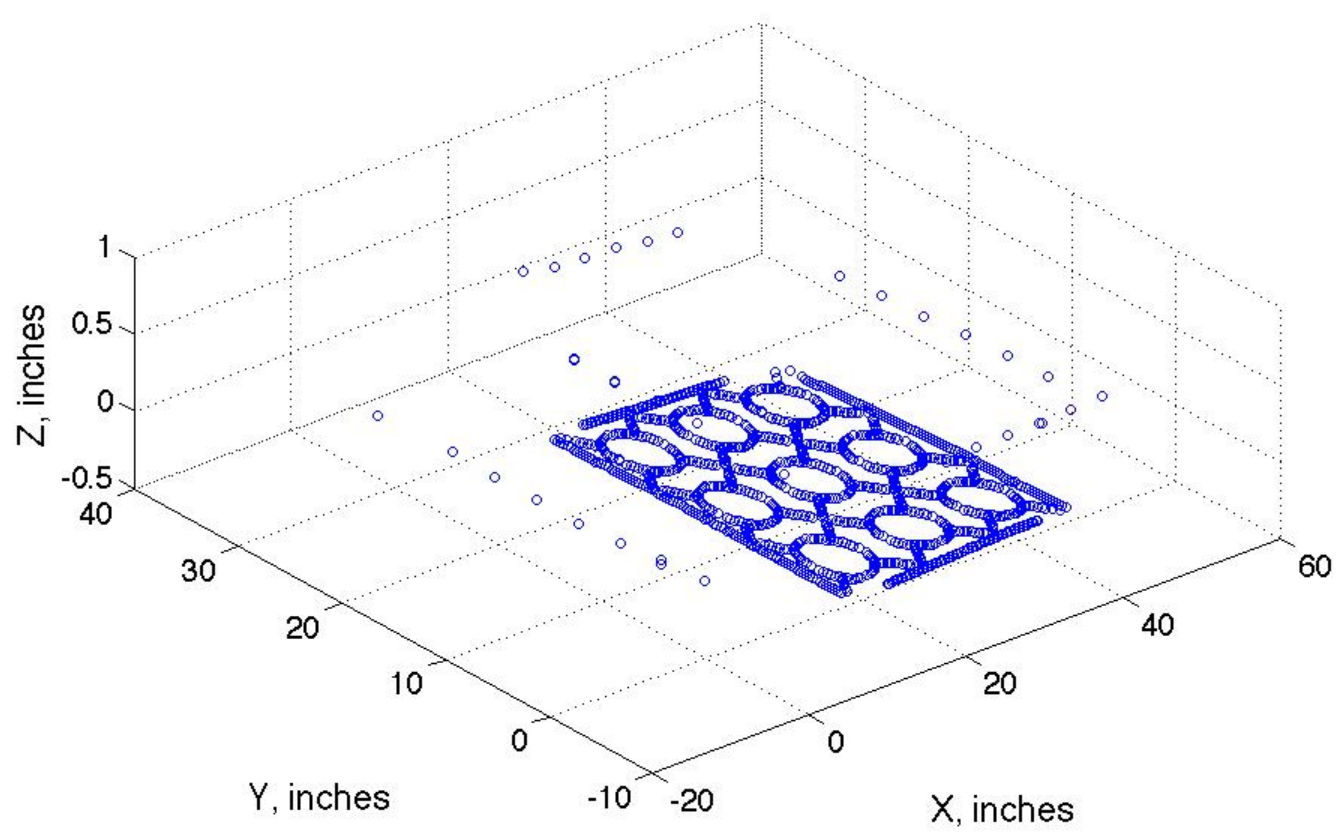

Figure 234. Point cloud - Specimen 3-0.25-1 - 1.1\% drift

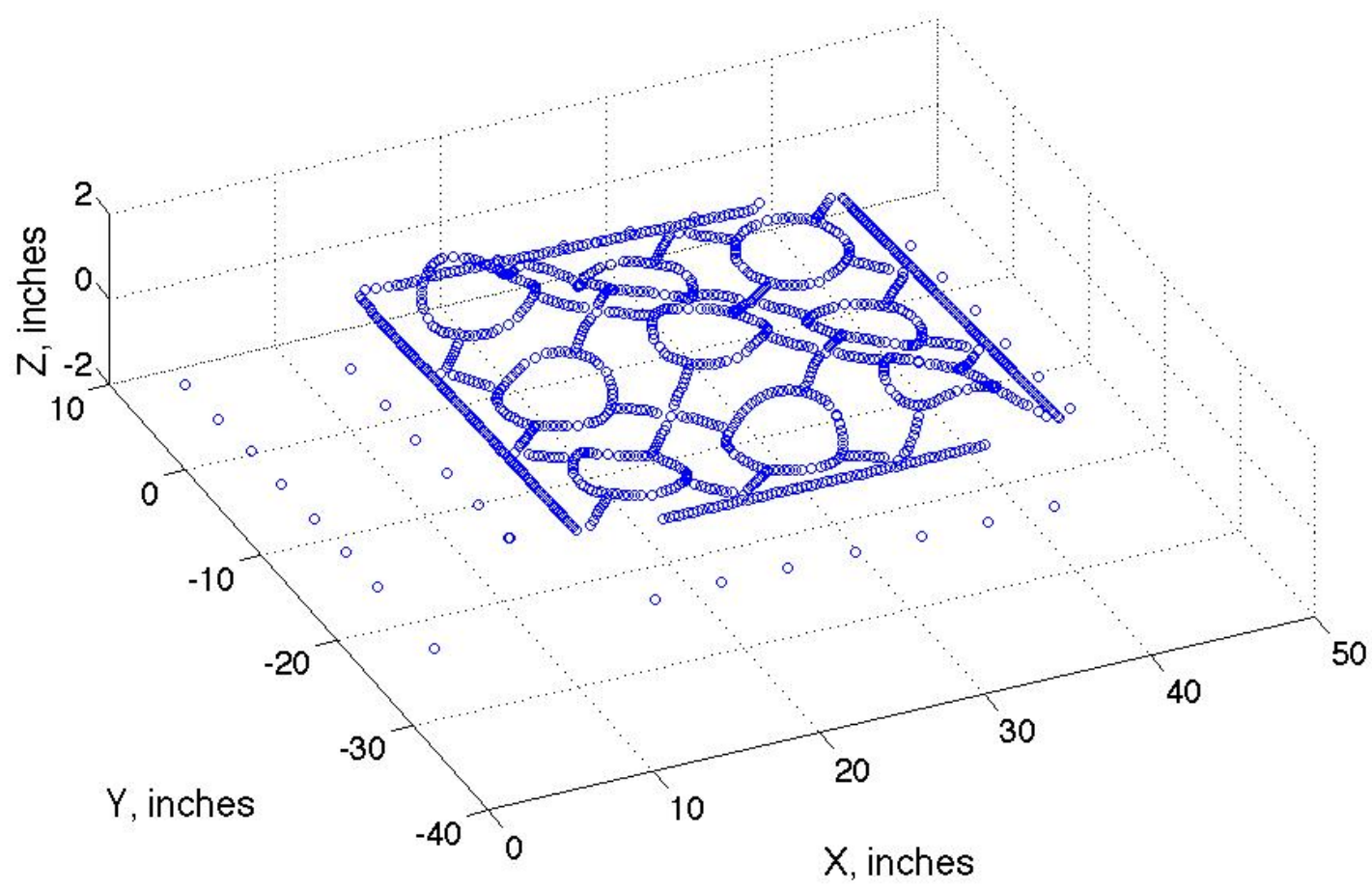

Figure 235. Point cloud - Specimen 3-0.25-1 - 2.9\% drift 


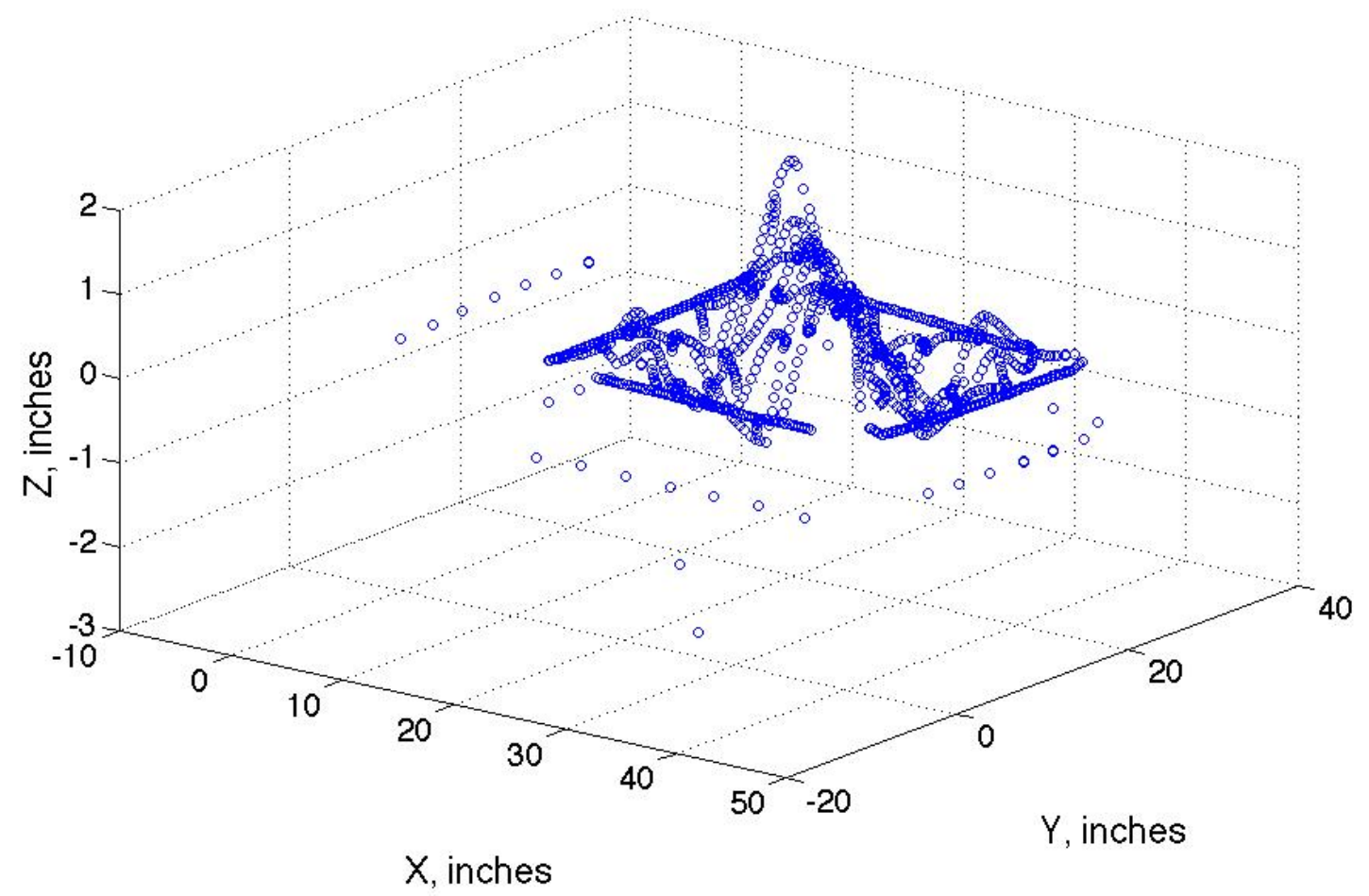

Figure 236. Point cloud - Specimen 3-0.25-1 - 6.8\% drift

TEST 8 - Specimen 3-0.375-1.

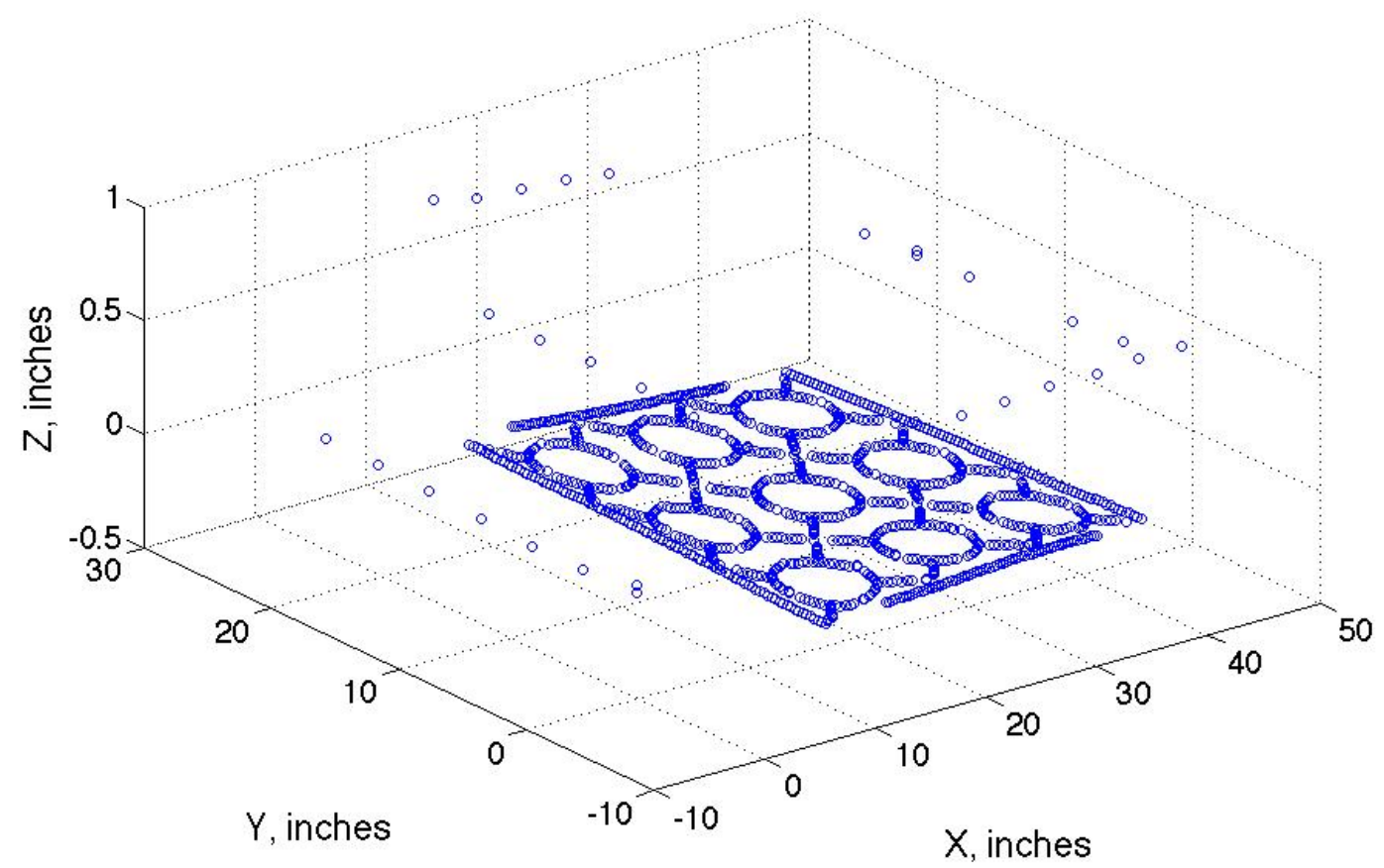

Figure 237. Point cloud - Specimen 3-0.375-1 - 0.7\% drift 


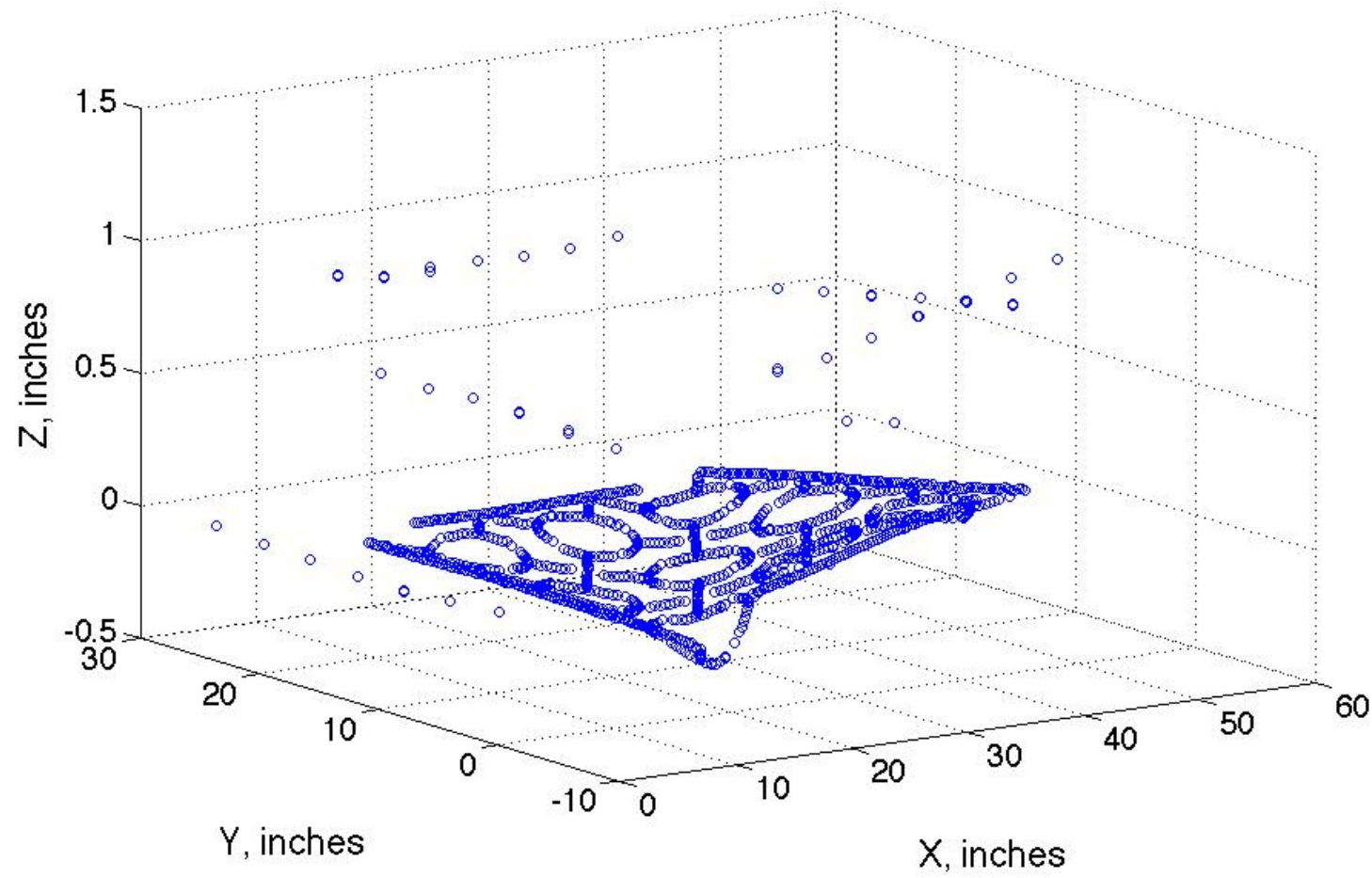

Figure 238. Point cloud - Specimen 3-0.375-1 - 2.2\% drift

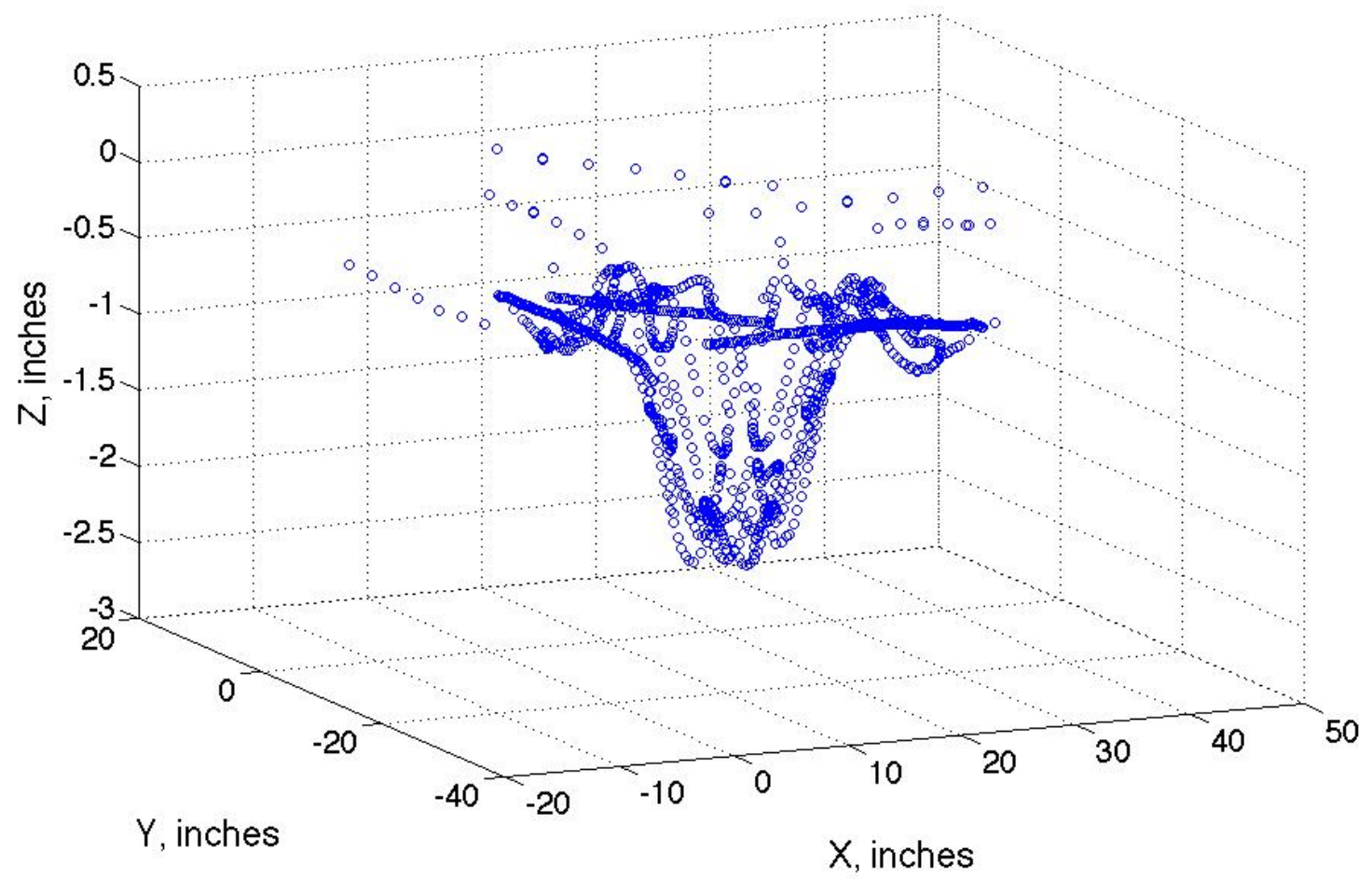

Figure 239. Point cloud - Specimen 3-0.375-1 - 6.1\% drift 


\section{APPENDIX F: Citation of copyrighted works}

Fig. 2 [public domain] Maurya A. (2012) Computational Simulation and Analytical Development of Buckling Resistant Steel Plate Shear Wall (BR-SPSW), Report, The Charles E. Via, Jr. Department of Civil and Environmental Engineering, Blacksburg, VA, USA. Digital Libraries and Archives, University Libraries, Virginia Polytechnic Institute and State University. http://scholar.lib.vt.edu/theses/available/etd-08102012-091024/ (accessed Oct. 15, 2012)

Fig. 3 [public domain]

Berman, J. W., and Bruneau, M. (2005) "Experimental Investigation of Light-Gauge Steel Plate Shear Walls", ASCE Journal of Structural Engineering, Vol. 131, No. 2. Digital Libraries and Archives, University Libraries, Virginia Polytechnic Institute and State University. http://ascelibrary.org/doi/abs/10.1061/\%28ASCE\%2907339445\%282005\%29131\%3A2\%28259\%29 (accessed Oct.15, 2012)

Fig. 4 [public domain]

Berman, J. W., and Bruneau, M. (2005) "Experimental Investigation of Light-Gauge Steel Plate Shear Walls", ASCE Journal of Structural Engineering, Vol. 131, No. 2. Digital Libraries and Archives, University Libraries, Virginia Polytechnic Institute and State University. http://ascelibrary.org/doi/abs/10.1061/\%28ASCE\%290733-

9445\%282005\%29131\%3A2\%28259\%29 (accessed Oct.15, 2012)

Fig. 5 [public domain]

Chen, S.-J., and Jhang, C. (2011) "Experimental Study of Los-Yield-Point Steel Plate Shear Wall Under In-Plane Load", Journal of Constructional Steel Research, Vol. 67, pp. 977-985. Digital Libraries and Archives, University Libraries, Virginia Polytechnic Institute and State University.

http://su8bj7jh4j.search.serialssolutions.com/?ctx_ver=Z39.882004\&ctx_enc=info\%3Aofi\%2Fenc\%3AUTF-

8\&rfr_id=info:sid/summon.serialssolutions.com\&rft_val_fmt=info:ofi/fmt:kev:mtx:journal \&rft.genre=article\&rft.atitle=Experimental+study+of+low-yieldpoint+steel+plate+shear+wall+under+inplane+load\&rft.jtitle=Journal+of+Constructional+Steel+Research\&rft.au=Chen\%2C+Sheng -Jin\&rft.au=Jhang\%2C+Chyuan\&rft.date=2011\&rft.pub=Elsevier+Ltd\&rft.issn=0143974X\&rft.eissn=18735983\&rft.volume $=67 \&$ rft.issue $=6 \&$ rft.spage $=977 \&$ rft.epage $=985 \&$ rft id $=$ info: doi $/ 10.1016$ \%2Fj.jcsr.2011.01.011\&rft.externalDocID=doi_10_1016_j_jcsr_2011_01_011 (accessed Oct.15, 2012) 
Fig. 6 [public domain]

Hitaka, T. and Matsui, C. (2003) "Experimental Study on Steel Shear Wall With Slits", Journal of Structural Engineering, ASCE, Vol. 129, No. 5. Digital Libraries and Archives, University Libraries, Virginia Polytechnic Institute and State University.

http://ascelibrary.org/doi/abs/10.1061/\%28ASCE\%290733-

9445\%282003\%29129\%3A5\%28586\%29 (accessed Oct.15, 2012)

Fig. 7 [public domain]

Vian, D., Bruneau, M., Tsai K.C., Lin, Y.C. (2009) "Special Perforated Steel Plate Shear Walls with Reduced Beam Section Anchor Beam. I: Experimental Investigation", Journal of Structural Engineering, ASCE, Vol. 135, No. 3, pp. 211-220. Digital Libraries and Archives, University Libraries, Virginia Polytechnic Institute and State University.

http://ascelibrary.org/doi/abs/10.1061/\%28ASCE\%290733-

9445\%282003\%29129\%3A5\%28586\%29 (accessed Oct.15, 2012)

Fig. 8 [public domain]

Ma, X., Borchers, E., Peña, A., Krawinkler, H., and Deierlein, G. (2010). Design and Behavior of Steel Shear Plates With Openings as Energy-Dissipating Fuses, Blume Earthquake Engineering Center, TR 173, Stanford University, Stanford, CA. Digital Libraries and Archives, University Libraries, Virginia Polytechnic Institute and State University.

http://su8bj7jh4j.search.serialssolutions.com/?ctx_ver=Z39.88-

2004\&ctx_enc=info\%3Aofi\%2Fenc\%3AUTF-

8\&rfr_id=info:sid/summon.serialssolutions.com\&rft_val_fmt=info:ofi/fmt:kev:mtx:journal \&rft.genre=article\&rft.atitle=Earthquake+resilient+steel+braced+frames+with+controlled +rocking+and+energy+dissipating+fuses\&rft.jtitle=Steel+Construction\&rft.au=Deierlein $\%$ $2 \mathrm{C}+$ Gregory\&rft.au $=$ Krawinkler\%2C+Helmut\&rft.au=Ma\%2C+Xiang\&rft.au=Eatherton\%2 C+Matthew\&rft.date=2011-08-01\&rft.pub=WILEY - VCH+Verlag\&rft.issn=1867-

0520\&rft.eissn $=1867-$

0539\&rft.volume $=4 \&$ rft.issue $=3 \&$ rft.spage $=171 \&$ rft.epage $=175 \&$ rft_id=info:doi $/ 10.1002$

\%2Fstco.201110023\&rft.externalDocID=STC0201110023 (accessed Oct. 15, 2012)

Fig. 10 [public domain]

Maurya A. (2012) Computational Simulation and Analytical Development of Buckling Resistant Steel Plate Shear Wall (BR-SPSW), Report, The Charles E. Via, Jr. Department of Civil and Environmental Engineering, Blacksburg, VA, USA. Digital Libraries and Archives, University Libraries, Virginia Polytechnic Institute and State University.

http://scholar.lib.vt.edu/theses/available/etd-08102012-091024/ (accessed Oct. 15, 2012)

Fig. 11 [public domain]

Maurya A. (2012) Computational Simulation and Analytical Development of Buckling Resistant Steel Plate Shear Wall (BR-SPSW), Report, The Charles E. Via, Jr. Department of Civil and Environmental Engineering, Blacksburg, VA, USA. Digital Libraries and Archives, University Libraries, Virginia Polytechnic Institute and State University. 
http://scholar.lib.vt.edu/theses/available/etd-08102012-091024/ (accessed Oct. 15, 2012)

Fig. 18 [public domain]

Caccese, V., Elgaaly, M., and Chen, R. (1993) "Experimental Study of Thin Steel-Plate Shear Walls Under Cyclic Load", Journal of Structural Engineering, ASCE, Vol. 119, No. 2. http://ascelibrary.org/doi/abs/10.1061/\%28ASCE\%2907339445\%281993\%29119\%3A2\%28573\%29 (accessed Oct. 15, 2012)

Fig. 19 [public domain] Li, C.-H., Tsai, K.-C, Lin, C.-H., and Chen, P.-C. (2010) "Cyclic Tests of Four Two-Story Narrow Steel Plate Shear Walls - Part 2: Experimental Results and Design Implications", Earthquake Engineering and Structural Dynamics, Vol. 39, pp. 801-826.

http://su8bj7jh4j.search.serialssolutions.com/?ctx_ver=Z39.882004\&ctx_enc=info\%3Aofi\%2Fenc\%3AUTF8\&rfr_id=info:sid/summon.serialssolutions.com\&rft_val_fmt=info:ofi/fmt:kev:mtx:journal \&rft.genre $=$ article \&rft.atitle $=$ Cyclic + tests + of + four + twostory+narrow+steel+plate+shear+walls.+Part $+2 \% 3 \mathrm{~A}+$ experimental+results+and+design + implications\&rft.jtitle=Earthquake+Engineering $\% 26+$ Structural + Dynamics\&rft.au $=\mathrm{Li} \% 2$ $\mathrm{C}+$ Chao-Hsien\&rft.au=Tsai\%2C+Keh-Chyuan\&rft.au $=$ Lin\%2C+ChihHan\&rft.au=Chen\%2C+Pei-Ching\&rft.date=2009\&rft.issn=0098-8847\&rft.eissn=10969845\&rft.epage $=$ n\%2Fa\&rft_id=info:doi/10.1002\%2Feqe.964\&rft.externalDBID $=$ n $\% 2 F a \&$ rft.externalDocID=10_1002_eqe_964 (accessed Oct. 15, 2012)

Table 1 [public domain]

Chen, S.-J., and Jhang, C. (2011) "Experimental Study of Los-Yield-Point Steel Plate Shear Wall Under In-Plane Load", Journal of Constructional Steel Research, Vol. 67, pp. 977-985. Digital Libraries and Archives, University Libraries, Virginia Polytechnic Institute and State University.

http://su8bj7jh4j.search.serialssolutions.com/?ctx_ver=Z39.88-

2004\&ctx_enc=info\%3Aofi\%2Fenc\%3AUTF-

8\&rfr_id=info:sid/summon.serialssolutions.com\&rft_val_fmt=info:ofi/fmt:kev:mtx:journal \&rft.genre=article\&rft.atitle=Experimental+study+of+low-yieldpoint+steel+plate+shear+wall+under+inplane+load\&rft.jtitle=Journal+of+Constructional+Steel+Research\&rft.au=Chen\%2C+Sheng -Jin\&rft.au $=J$ hang\%2C + Chyuan\&rft.date $=2011 \&$ rft.pub $=$ Elsevier + Ltd\&rft.issn $=0143-$ 974X\&rft.eissn $=1873-$ 5983\&rft.volume $=67 \&$ rft.issue $=6 \&$ rft.spage $=977 \&$ rft.epage $=985 \&$ rft_id=info:doi $/ 10.1016$ \%2Fj.jcsr.2011.01.011\&rft.externalDocID=doi_10_1016_j_jcsr_2011_01_011 (accessed Oct. 15,2012 ) 